\title{
Investigating the Role of G-protein Bias in the Anti- Nociceptive and Side Effect Profiles of Two Novel Mu Opioid Receptor Agonists Kurkinol and Kurkinorin
}

\section{Amy Frances Alder}

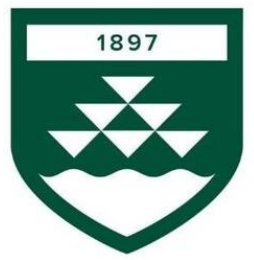

TE HERENGA WAKA

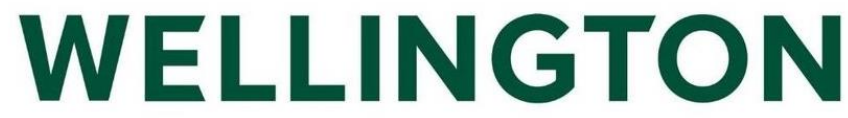

VICTORIA UNIVERSITY OF WELLINGTON

School of Biological Sciences

Te kura Matauranga Koiora 



\section{Abstract}

Chronic pain is a major problem worldwide, affecting 1 in 5 New Zealanders resulting in a decreased quality of life for the patient and a large socioeconomic problem costing an estimated \$13$\$ 14.5$ billion a year. Current therapeutics target the mu opioid receptor ( $\mu$ receptor) and include drugs such as morphine and fentanyl. While these drugs are highly effective in the treatment of strong acute pain, their long-term use is associated with tolerance to the analgesic effects and increasing rates of side effects such as respiratory depression and constipation. Due to their high abuse liability, they are also known to cause dependence and addiction when prescribed for extended periods. This is believed to have played a role in the opioid crisis in the United States and highlights the need for improved therapeutics for the treatment of chronic pain.

One mechanism that has been proposed to generate $\mu$ receptor agonists for the treatment of chronic pain with reduced analgesic tolerance and safer side effects is the development of G-protein biased agonists. Such agonists selectively activate the canonical G-protein signalling to a greater extent than the non-canonical $\beta$-arrestin2 pathway. This is based on previous work in $\beta$-arrestin2 knockout mice where the antinociceptive effects were increased, while side effects, including respiratory depression, tolerance, and constipation are reduced, increasing the therapeutic window. In this thesis, we aimed to assess the anti-nociceptive and side effect behavioural profiles of two novel $\mu$ receptor agonists, kurkinol (bias $=0.14$ ) and kurkinorin (bias $=0.57$ ), with a varying bias for the $\mathrm{G}$ protein pathway to assess the role of this paradigm.

Evaluation of the behavioural profile of kurkinol and kurkinorin revealed that G-protein bias was correlated to increased anti-nociceptive potency and reduced tolerance in wildtype C57BL/6J mice. Furthermore, the anti-nociceptive potency of morphine was increased, and tolerance decreased in in $\beta$-arrestin2 knockout mice. While the level of tolerance was reduced for kurkinorin. However, in the chemotherapy-induced model of neuropathic pain, tolerance to kurkinol and kurkinorin developed at the same rate as morphine. Overall this work showed a poor correlation between Gprotein bias and therapeutic window. With the G-protein selective kurkinol inducing worse respiratory depression, constipation, and motor coordination impairment compared to kurkinorin. Interestingly, respiratory depressive and constipation effects of kurkinol were not prevented in the $\beta$-arrestin2 knockout mice indicating that they are induced through the G-protein pathway.

These results highlight the change that has occurred in the biased agonism field over the last 4 years, with the lack of reproducibility of key experiments and poor translation of G-protein biased $\mu$ receptor agonists resulting in improved therapeutic windows both clinically and pre-clinically. Moreover, recent research has shown that pathway efficacy (i.e. partial agonism) and not G-protein bias is responsible for the behavioural profiles of compounds previously identified as G-protein biased. We therefore decided to further investigate the cell signalling profiles of our two novel agonists to 
assess them for partial agonism and to assess downstream signalling molecules activated by G-protein and $\beta$-arrestin2.

This revealed cell-specific inhibition of membrane hyperpolarisation in Hek293 and CHO cells stably expressing the human $\mu$ receptor, with kurkinol found to be the most potent in both cell lines, followed by kurkinorin, morphine, and [D-Ala ${ }^{2}, \mathrm{~N}-\mathrm{MePhe}^{4}$, Gly-ol]-enkephalin (DAMGO). However, no differences were identified between the $\mu$ receptor agonists in the activation of the inwardly rectifying channels in the $\mathrm{CHO}$ cell line. The assessment of PCREB (phosphorylated CAMP response-element binding protein) as a $\beta$-arrestin2 dependent pathway revealed poor activation by kurkinorin while kurkinol was a potent activator. Bias factors generated from this data showed poor correlations to therapeutic windows. While the differences in CREB phosphorylation was shown to have a stronger correlation to therapeutic windows generated from the behavioural data.

Overall this thesis has identified kurkinorin as a $\mu$ receptor agonist that induces potent antinociception with reduced side effects, without strong-G-protein bias. We also show that the highly selective $\mu$ receptor agonist kurkinol has improved anti-nociception with a worse side effect profile adding to the growing body of literature showing bias is not a good predictor in its current state. Furthermore, the discrepancies between cell lines, differential activation of subcellular pathways, and lack of reproducibility between bias equations indicate that the field has massively oversimplified a complex system. Which has, most likely, resulted in the poor translation of in vitro bias factors to clinically available $\mu$ receptor agonists for chronic pain. 


\section{Acknowledgements}

The last three and a half years have been a full of highs and lows, just as predicted by all the $\mathrm{PhD}$ memes on the internet. There are a lot of people who have helped me get through the lows I am very thankful for.

Firstly, to my supervisor Dr Bronwyn Kivell for placing me in your lab as an honours student and allowing me to grow as a scientist. Opioid research is never where I saw myself going, but I am very glad to have ended up here. I appreciate you taking the time to answer my questions and always having your door open, despite how busy you are, especially in these last few months. To my secondary supervisor Dr Janet Pitman, thank you for all the help with reading and editing of this thesis, your attention to detail and kind words were greatly appreciated. A special thanks to Emeritus Professor John Miller, your references for travel grants and help with the respiratory depression have been invaluable. Finally, to Professor Tom Prisinzano and his lab at the University of Kentucky for the supply of novel compounds that have allowed this thesis to happen.

Throughout the four years that I have been in the Kivell lab, there have been a lot of lab members that have come and gone that I am very grateful for. From the original members when I joined the lab; Susan, Stephen, Diana, Adam, Ricky, and Phyllida thank you for helping me when I was new to research in my honour's year. To the lab members and volunteers who joined the lab along the way; Afnan, Bria, Christa, Janine, Lisa, Matthew, Nikki, Rabia, and Sheein. To my newest desk mate, Britt, thank you for being a great summer and now fourth-year student I am very happy you joined the pain research group, doubling our member numbers to two. To our long-running token male, Andy, your ability to stay calm no matter what madness was going on was an absolute lifesaver at times. My former flatmate and travel buddy Kendra, I am so glad that you joined our lab. Thank you for the endless wine (or rum) during the good times and the bad times, for forcing me to leave and eat fries rather than throwing my computer down the stairs, and the constant stories and hilarity that, this would have been a much quieter experience without you here. Finally, to Kelly, the longestrunning member of the lab and the hardest working person I have ever met, you taught me how to be a proper scientist, to question every result and always run an experiment properly no matter how much more painful it made it, tried to teach me work-life balance and to take time off (even though you never did yourself). You have made this journey so much more fun, from covering my desk in pictures of cats to late nights in town and drinking too much, this experience would not have been the same without you.

I would also like to thank other key members of the SBS family for helping me along the way. To my original post-grad buddy Jen, even though you are doing your PhD in Melbourne now it has been great to continue the journey with you via messenger. To my friend and life coach, Varun, thank you for all the inappropriate workplace humour, constant judgement and bullying of my poor life 
decisions, and the great research and life advice along the way. Daniel, thank you for all the annoyance that you gave me over the years you were here, which I strangely miss now that you have graduated. Your dad jokes, ridiculous comments, and lunch schedule could always make a bad day a little bit better. To everyone else in SBS including, Matire, Mike, and Jess thank you for sharing this experience with me.

I would also like to thank all the technical staff; Craig, Derek, Lee, Nevil, Paul, Pisana, and Sushila. Thank you for your help fixing and training me how to use the equipment, especially the Friday afternoon emergency diaphragm changes by Nevil and Craig. Stacey, for the maintenance of the animal colony especially my GMO lines, I know they were a pain to keep straight. To the admin team at SBS; Adrian, Lesley, Mark, Mary, Paul, Sandra, Stephen, and Patricia from the faculty office. Thank you for helping with the paperwork, access, and extensions when needed, you all made the process so much easier.

I would like to thank my family; Mum, Dad, Breanne, Sarah, and all the family cats. Thank you for always encouraging and believing in me, more than I did at some points, and for putting up with me always being too busy to visit even though we all live in the same city. To my grandma, I know you always wanted me to become a vet so that I could look after all the family cats for free, even though that's not the path I chose I hope you are still proud of me. I wish you were here to see this. To my partner Scott, without you being there to hear my rants, put up with my late-night breakdowns and moments of self-doubt I most certainly wouldn't have made it to the end with the level of mental stability that I have now, you and our cat Pinky can calm me down like no one else. Thank you for being my rock throughout the last two years of $\mathrm{my} \mathrm{PhD}$ and always allowing me to put my lab work first, even when that meant accidentally waking you up before 7 am on the weekend. 


\section{Table of Contents}

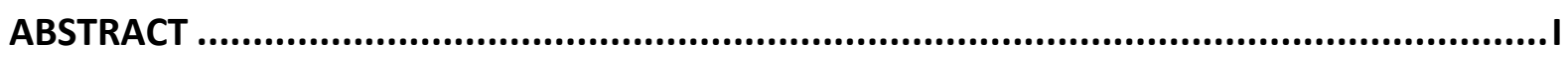

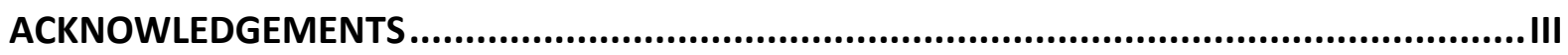

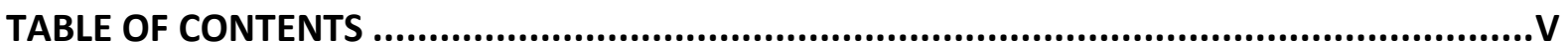

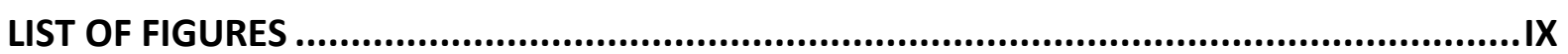

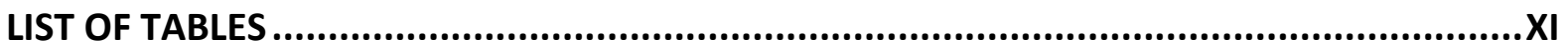

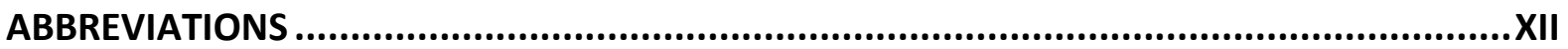

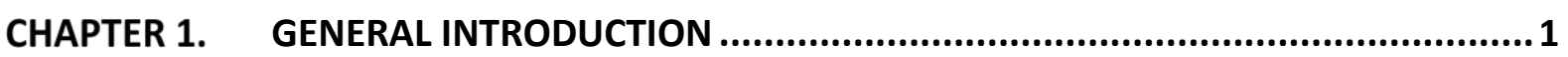

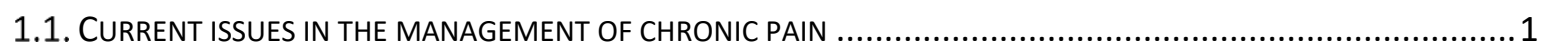

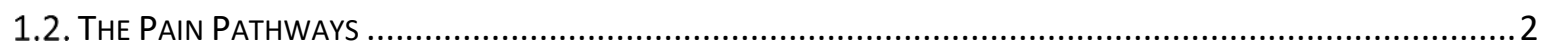

1.2.1. Nociceptors and Ion Channels Involved in Nociception .............................................. 2

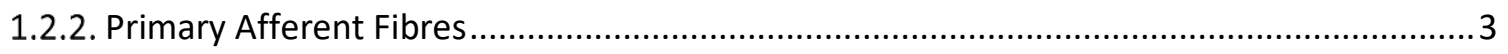

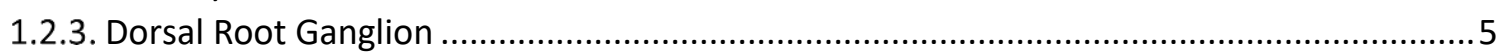

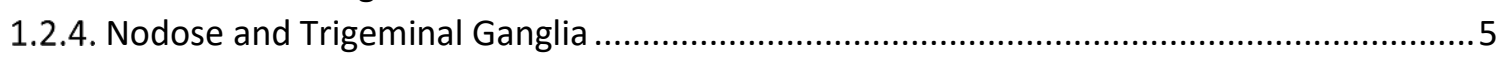

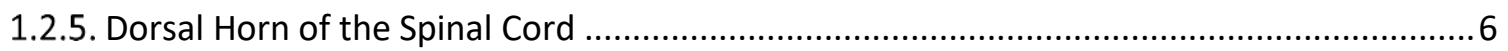

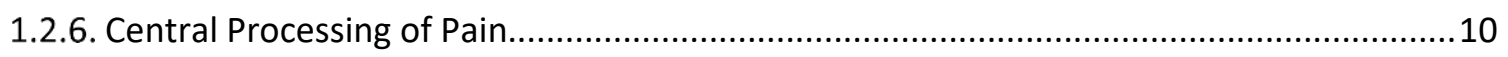

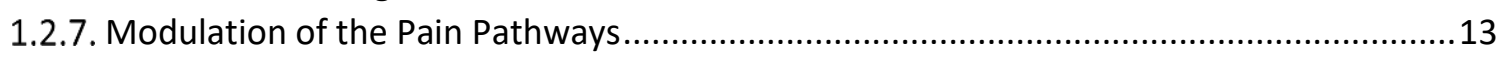

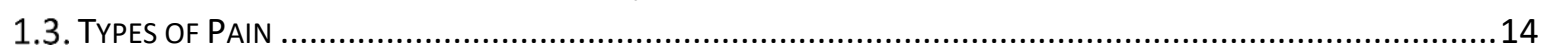

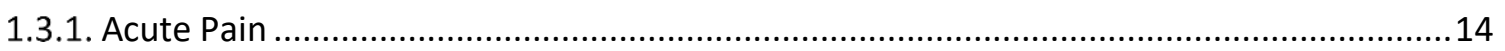

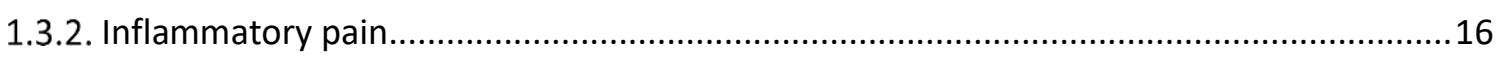

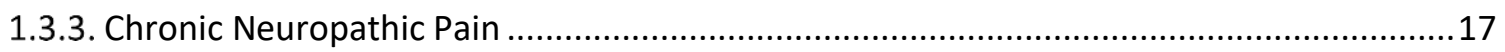

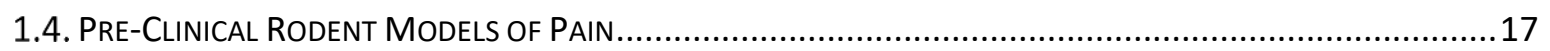

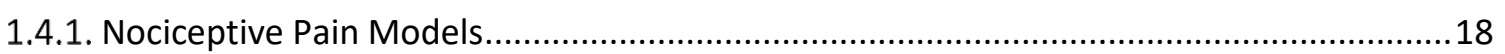

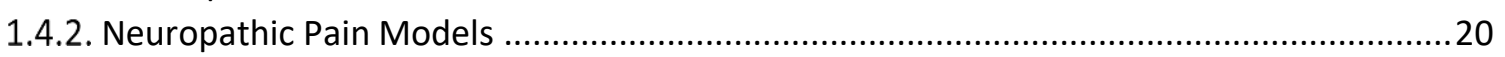

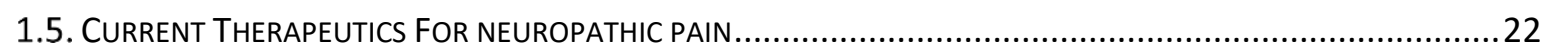

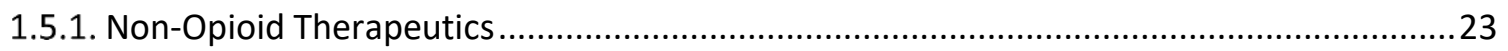

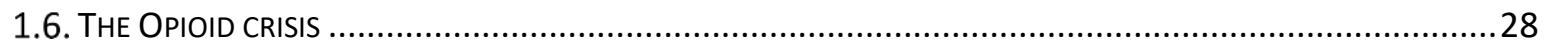

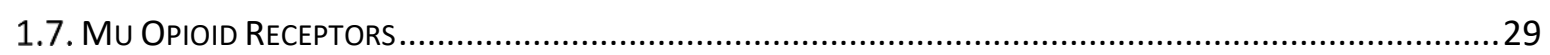

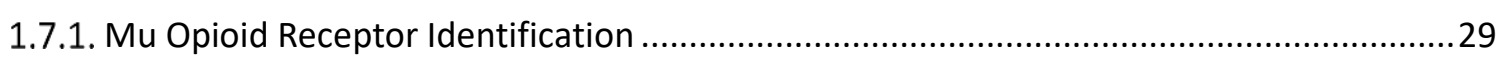

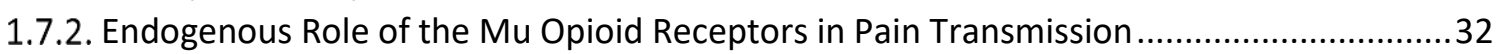

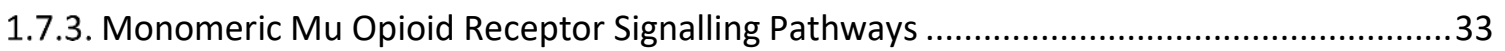

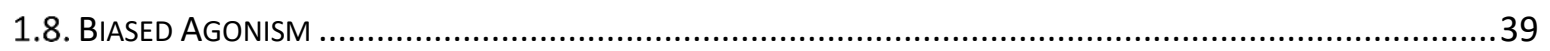

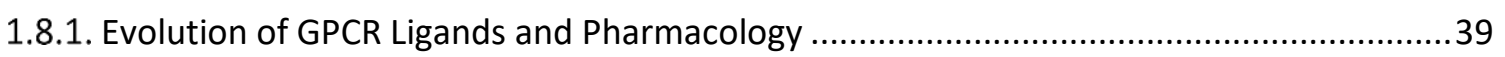

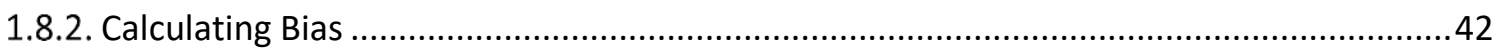

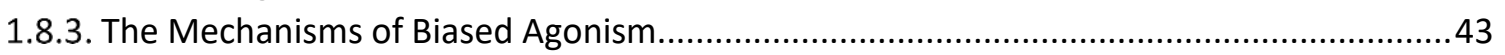

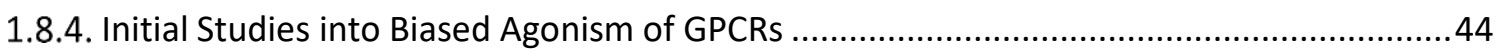

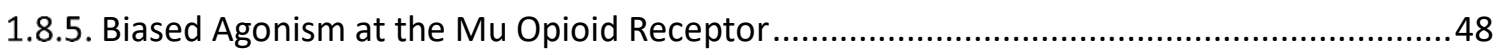

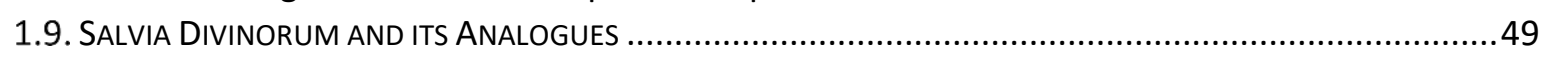

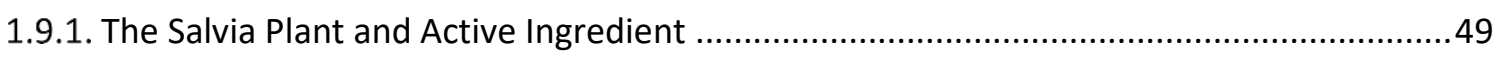

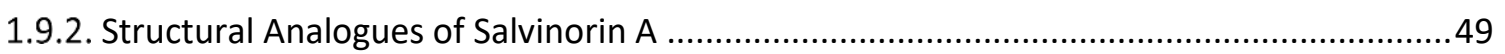

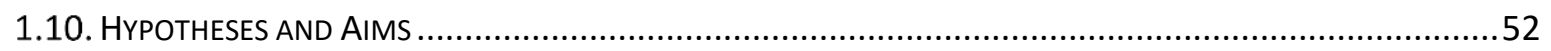

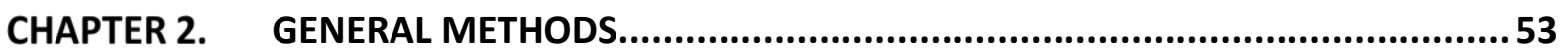

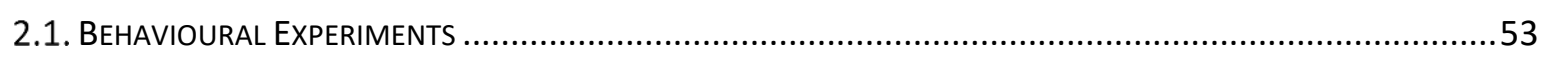

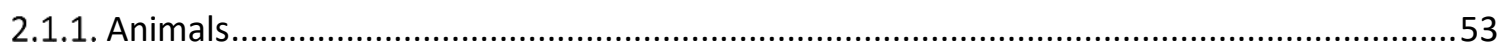




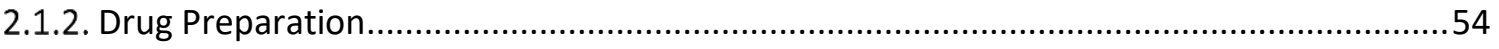

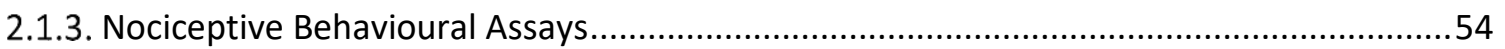

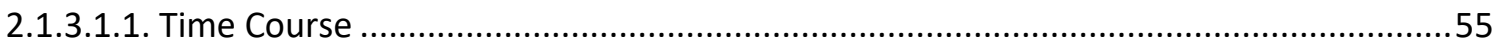

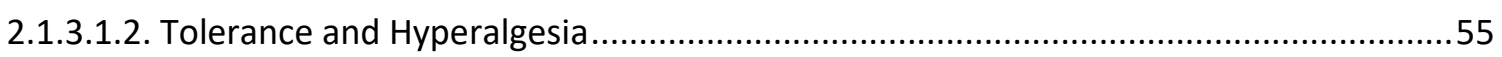

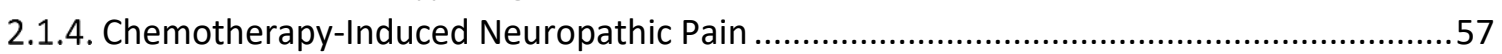

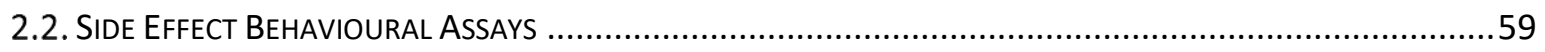

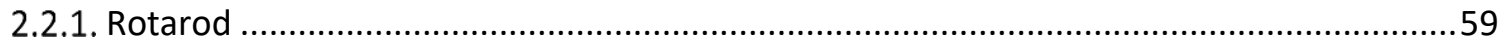

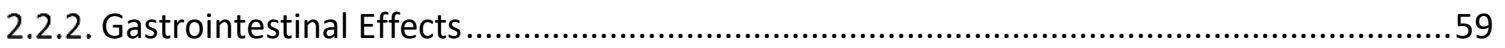

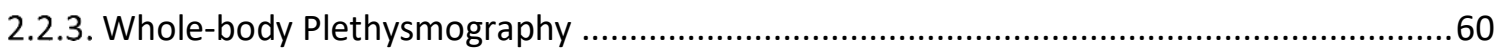

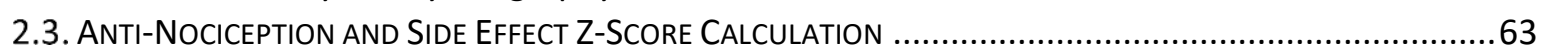

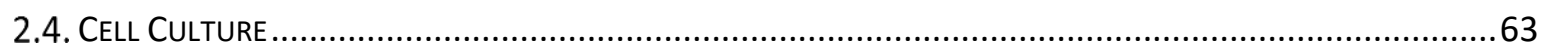

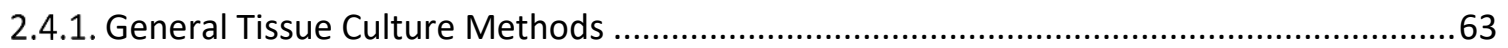

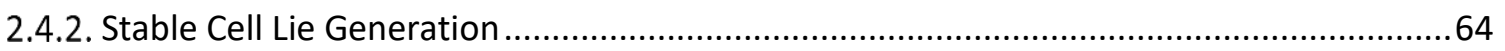

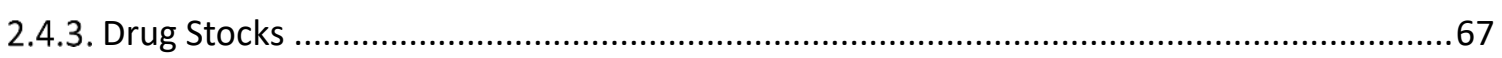

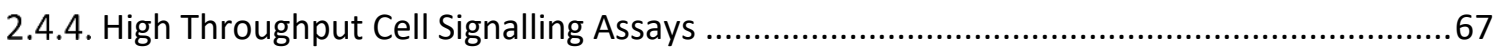

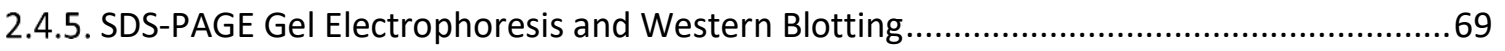

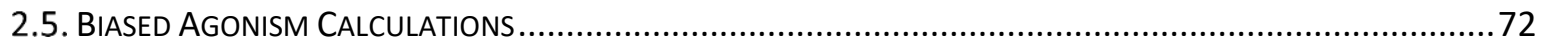

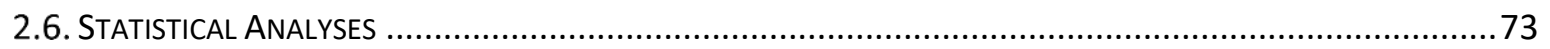

\section{CHAPTER 3. ANTI-NOCICEPTIVE EFFECTS OF KURKINOL AND KURKINORIN .................75}

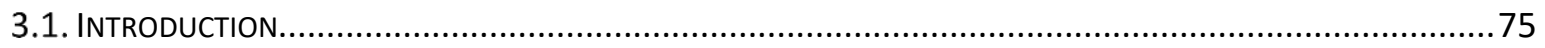

3.1.1. Regulation of Mu Opioid Receptors by $\beta$-arrestin2 and the Implications for Analgesia ....75

3.1.2. Pre-Clinical Effects of G-Protein Biased Agonists............................................................. 76

3.1.3. Anti-Nociceptive Effects of Novel Mu Opioid Receptor Agonists from the Structure of

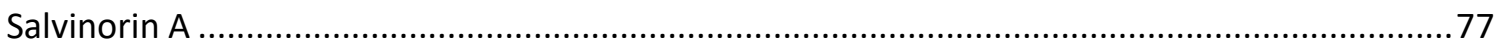

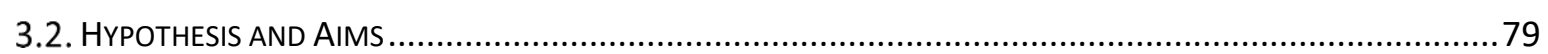

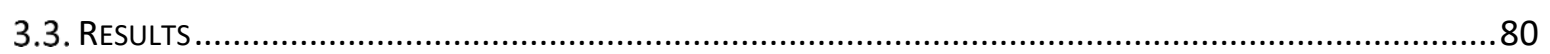

3.3.1. Dose response Effects of Kurkinol in the Hot water Tail-flick Assay.................................80

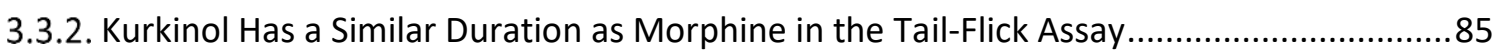

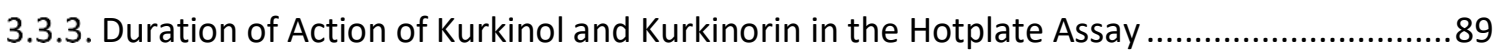

3.3.4. Effectiveness of Kurkinol and Kurkinorin in Chemotherapy-Induced Neuropathic Pain....93

3.3.5. Anti-Nociceptive Z-Scores Shows Improvements to Morphine with Kurkinol, But Not

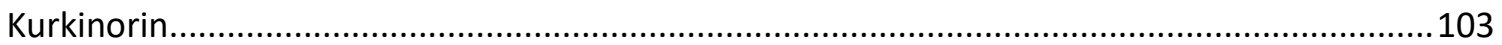

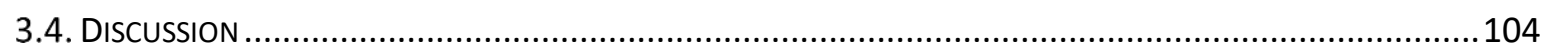

3.4.1. G-Protein Bias Correlated to Increased Potency and Decreased Tolerance in Spinally

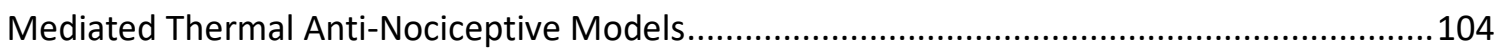

3.4.2. Kurkinol and Kurkinorin have Reduced Duration of Action in Supraspinal Thermally

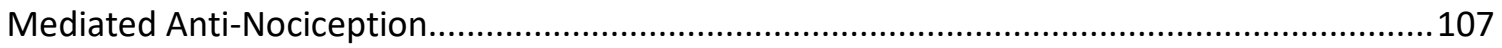

3.4.3. The Effect of G-protein Bias in Anti-Nociceptive Potency and Tolerance in Chemotherapy-

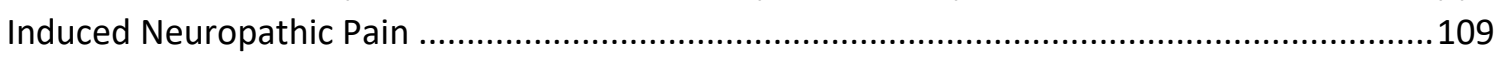

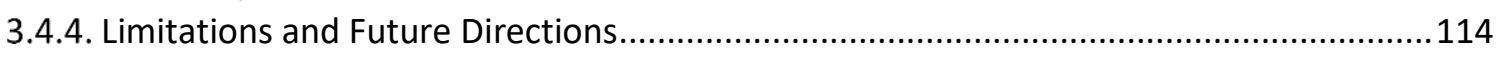

CHAPTER 4. SIDE EFFECT PROFILE OF KURKINORIN AND KURKINOL........................ 117

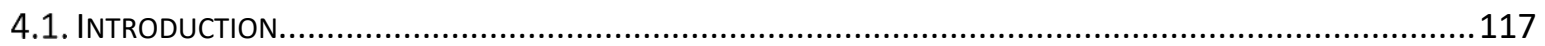

4.1.1. Mu Opioid Receptor Expression and the Induction of on-target Side Effects ..................117

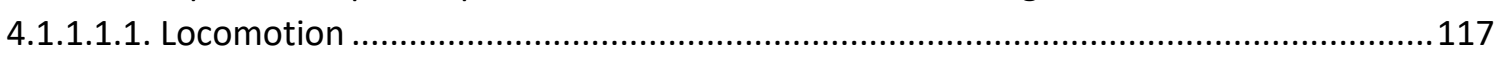

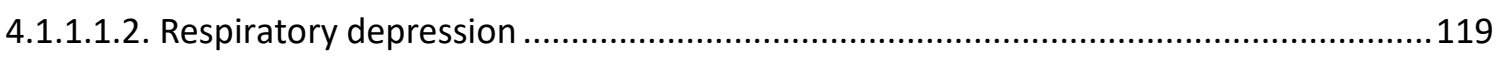

4.1.2. Side Effect profiles of Pre-clinical G-protein biased agonists ........................................121

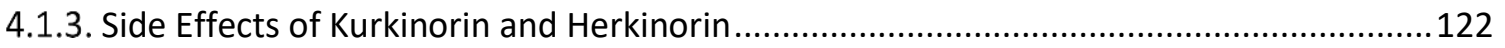

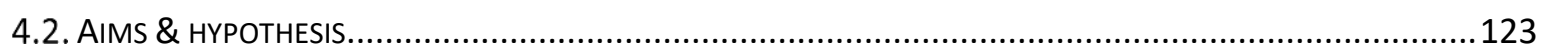




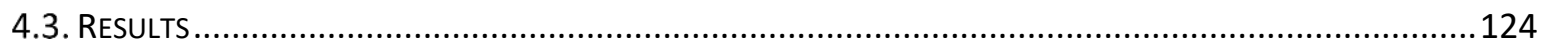

4.3.1. Kurkinol has a Reduced Duration of Motor Coordination Impairment ............................124

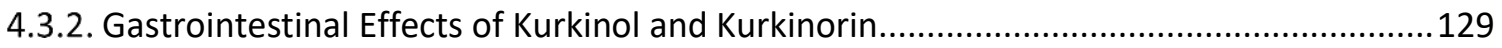

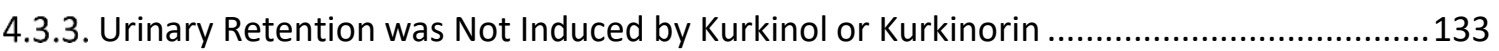

4.3.4. Respiratory Depression in C57BL/6J and $\beta$-arrestin2 Knockout Mice .............................135

4.3.5. Kurkinorin has an Improved Side Effect Z-score in C57BL/6J Mice ................................147

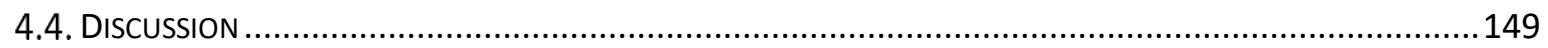

4.4.1. Improved Motor Coordination of Kurkinol Not Correlated to G-Protein Bias..................149

4.4.2. Kurkinol and Kurkinorin Induce Gastrointestinal Inhibition ...........................................152

4.4.3. Reduced $\beta$-arrestin2 Recruitment Does Not Improve the Respiratory Depressive Effects of

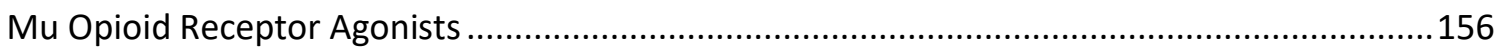

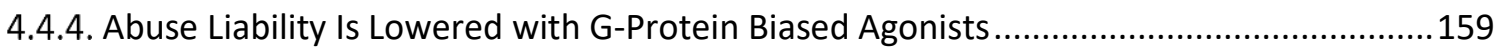

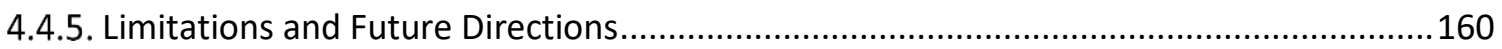

\section{CHAPTER 5. CELL SIGNALLING PROPERTIES OF KURKINOL AND KURKINORIN ............ 163}

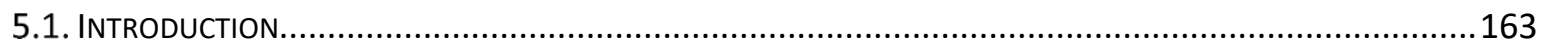

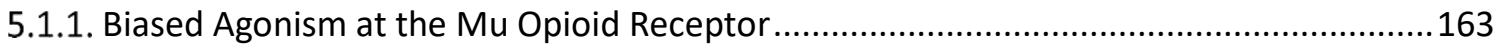

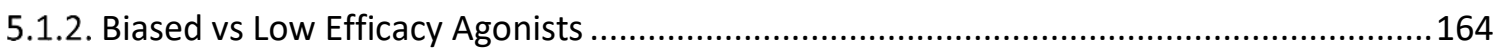

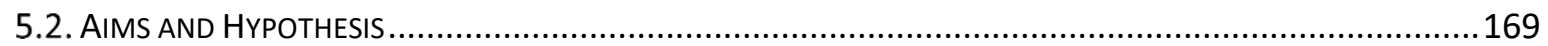

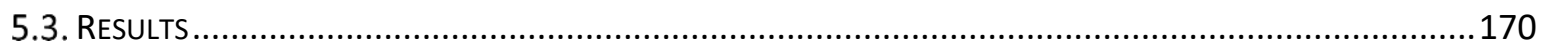

5.3.1. Activation of G-Protein Dependent Pathways by Kurkinol and Kurkinorin ......................170

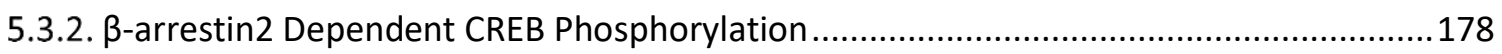

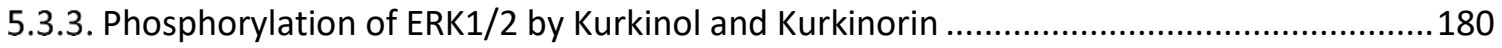

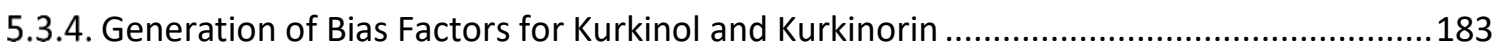

5.3.5. pCREB Efficacy Correlates to Therapeutic Window .......................................................... 187

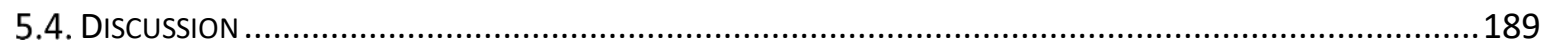

5.4.1. G-Protein Dependent Pathway Activation Matches Pre-Determined Bias ......................189

5.4.2. Kurkinorin is a Poor Activator of Mu Opioid Receptor-Induced CREB .............................192

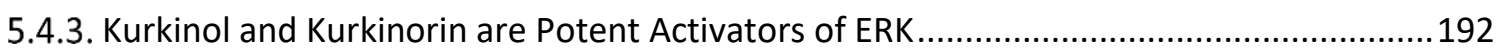

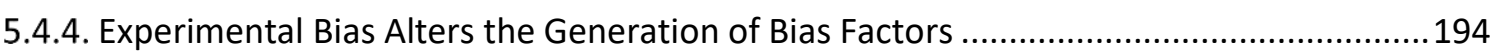

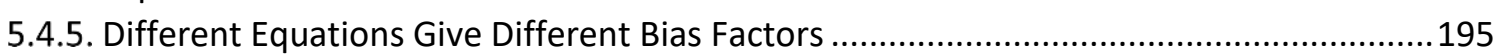

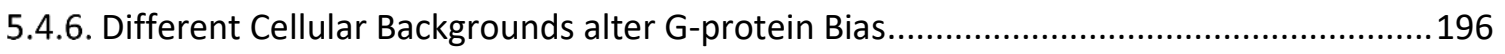

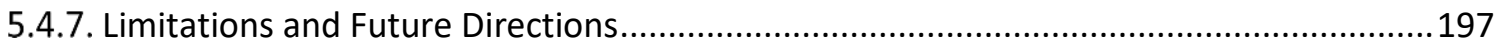

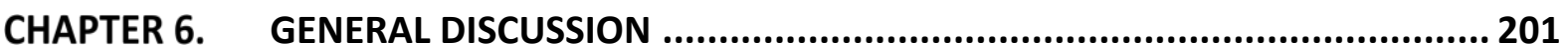

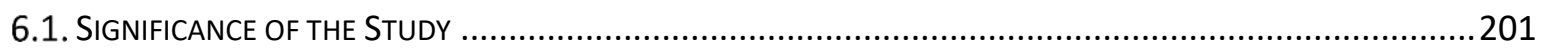

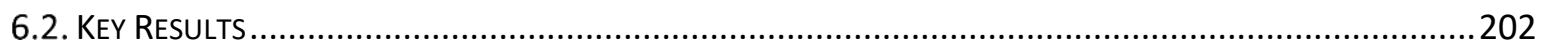

6.2.1. G-Protein Bias Correlates to the Anti-Nociceptive Profile but Not the Side Effect Profile

6.2.2. The Therapeutic Potential of Kurkinorin and Kurkinol ............................................2 203

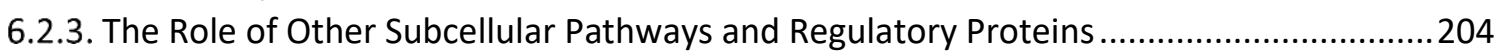

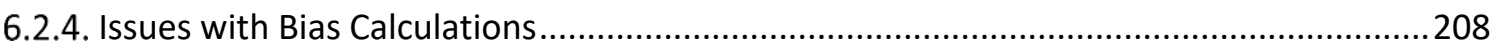

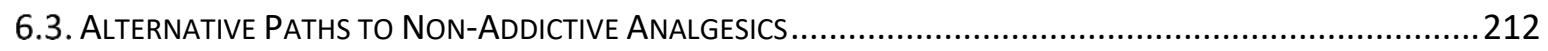

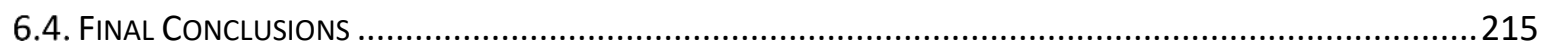

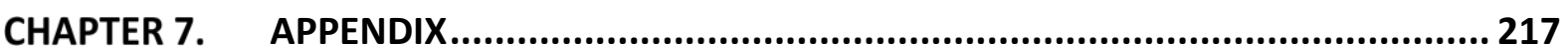

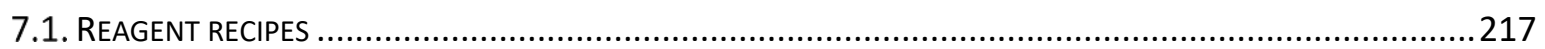

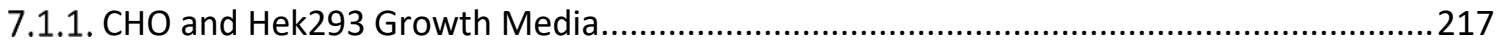

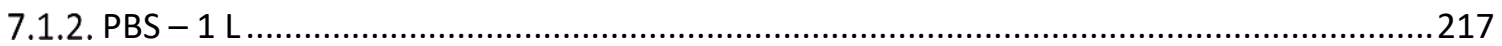

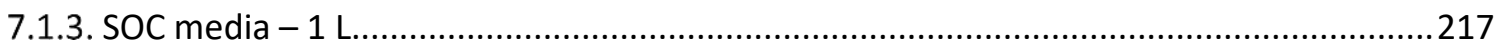




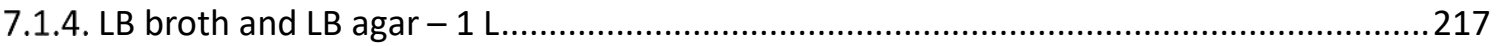

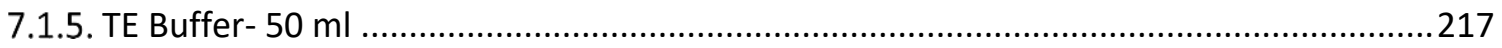

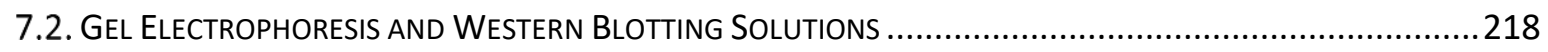

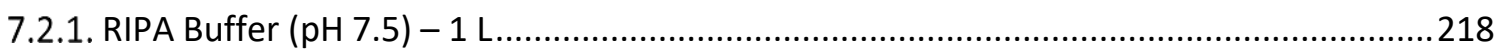

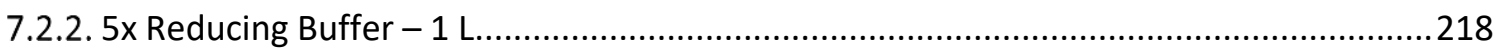

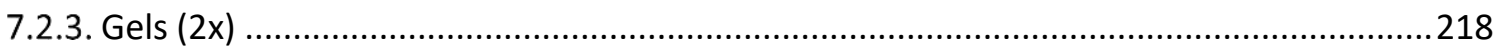

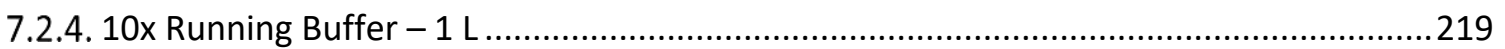

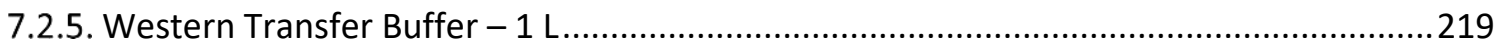

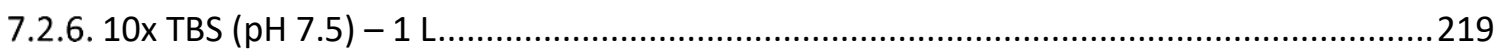

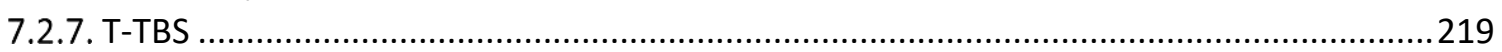

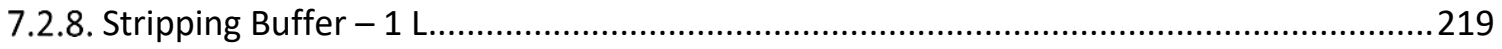

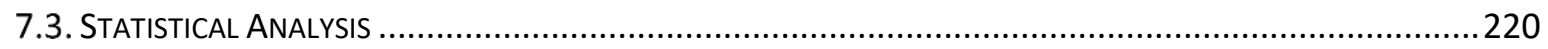

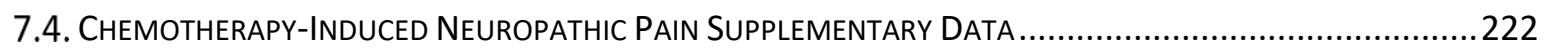

7.4.1. Paclitaxel Induced Comparable Disease Between Treatment Groups ...............................222

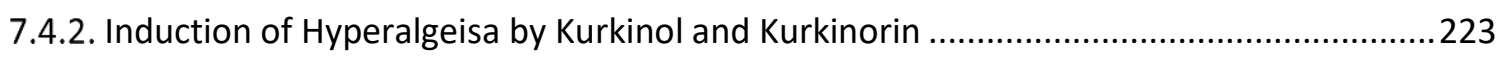

7.5. THE EFFECT OF KURKINOL AND KURKINORIN ON CORE BOdY TEMPERATURE .........................................22

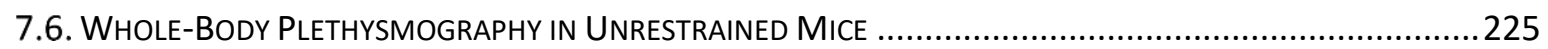

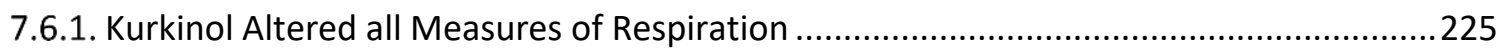

7.6.2. Effects of Kurkinorin on Further Respiratory Measures ....................................................226

7.6.3. $\beta$-arrestin2 Knockout Vehicle Controls from Whole-body Plethysmography in Unrestrained Mice

7.6.4. Further Respiratory Effects of Morphine, Kurkinol, and Kurkinorin in $\beta$-arrestin2 Knock

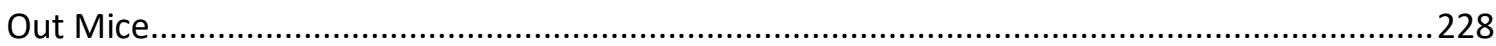

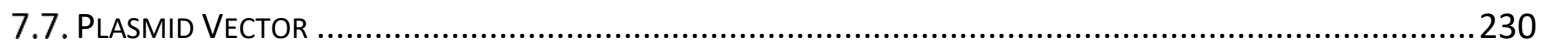

7.8. HeK293 \&AND CHO CELLS StABLy EXPRESSING THE HUMAN Mu OPIOID ReCEPTOR.................................230

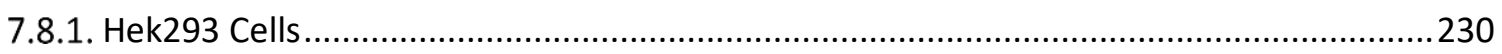

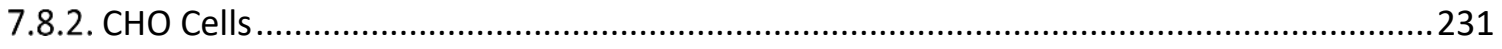

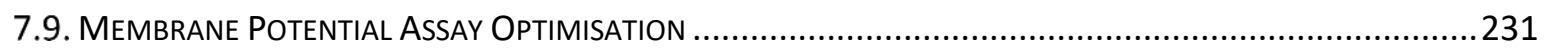

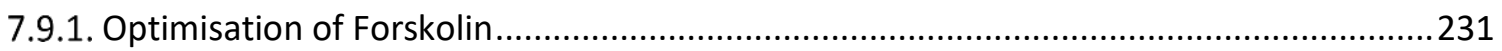

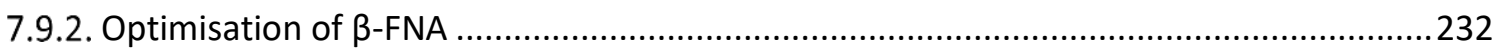

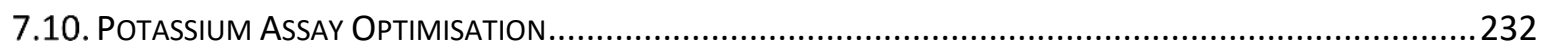

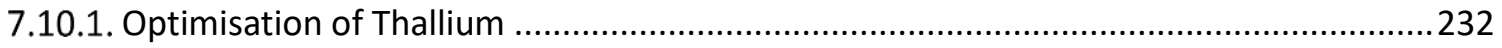

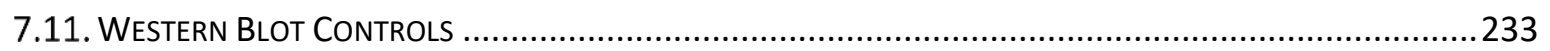

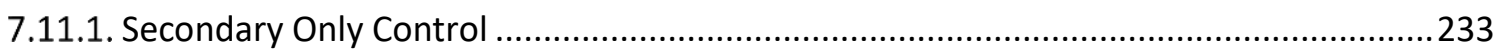

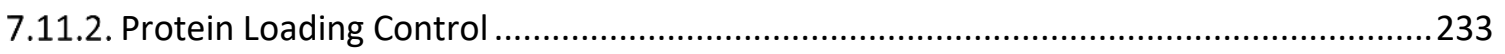

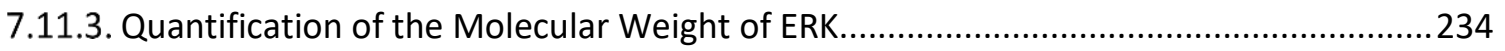

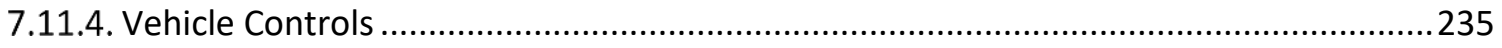

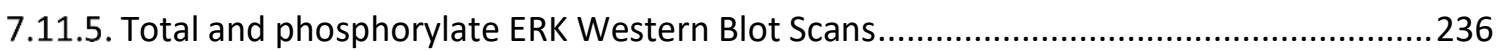

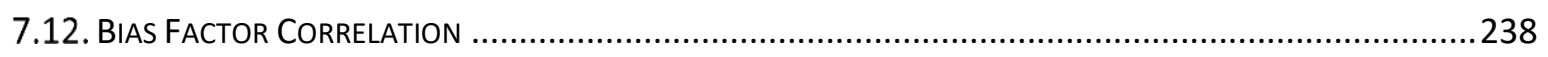

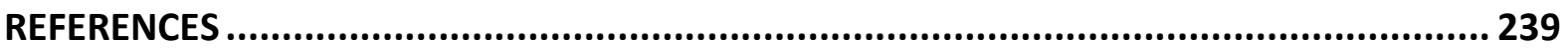




\section{List of Figures}

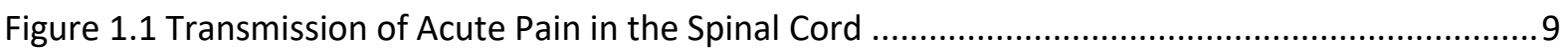

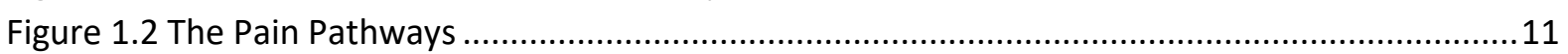

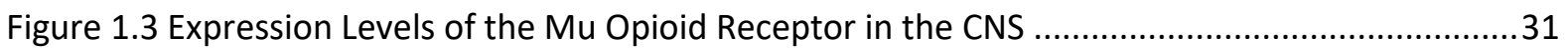

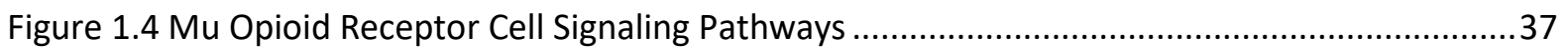

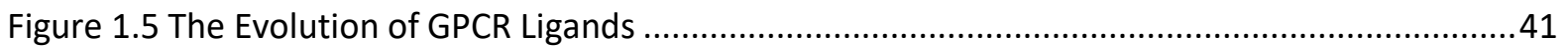

Figure 1.6 Chemical Structure of Known and Novel Mu Opioid Receptor Ligands ............................51

Figure 2.1 Dose response tolerance tail-flick procedure ...............................................................56

Figure 2.2 Schematic Representation of Chemotherapy-Induced Neuropathic Pain...........................58

Figure 3.1 Potency and Efficacy of Kurkinol Centrally Mediated Anti-Nociceptive Effects ..................81

Figure 3.2 $\beta$-Arrestin2 Knock Out Mice Increase the Potency of Morphine, but not Kurkinorin or

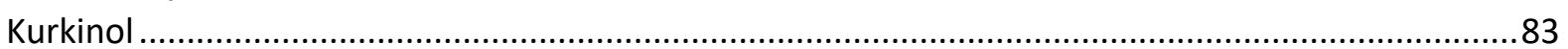

Figure 3.3 Duration of Action of Kurkinol in the Hot water Tail-flick Assay .........................................86

Figure 3.4 Anti-Nociceptive Effects of Mu Opioid Receptor Agonists in Mu Opioid Receptor Knock

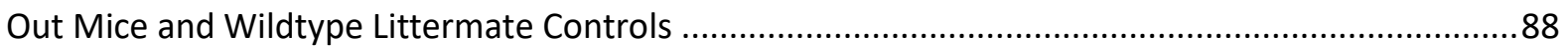

Figure 3.5 Kurkinol and kurkinorin has a Similar Duration of Action to Morphine in the Hotplate

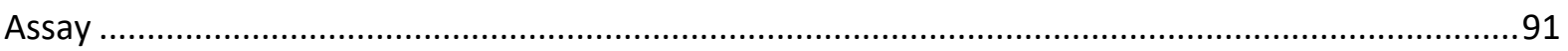

Figure 3.6 Overall Effect of Kurkinorin and Kurkinol on Supraspinally Mediated Anti-nociception ....92

Figure 3.7 Paclitaxel Produced Stable Disease by Day 15 in Male and Female C57BL/6J Mice ...........93

Figure 3.8 Kurkinol is more Potent than Morphine at Alleviating Chemotherapy-Induced Neuropathic

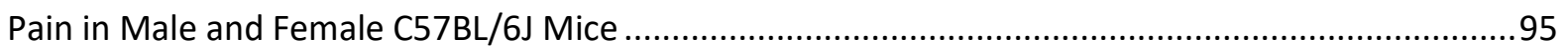

Figure 3.9 Anti-Nociception Induced by Kurkinol and Kurkinorin is Mediated by the Mu Opioid

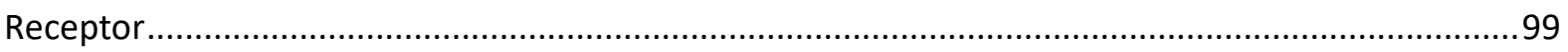

Figure 3.10 Effect of Sex on the Induction of Tolerance to Mechanical Allodynia ............................102

Figure 3.11 Kurkinol and Kurkinorin Show the Same Behavioural Pattern as Morphine ...................103

Figure 4.1 Kurkinol Has a Shorter Duration of Motor Coordination Impairment..............................127

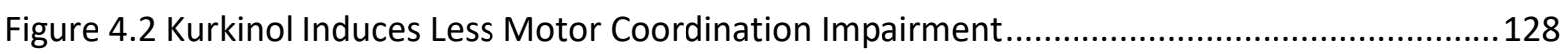

Figure 4.3 Sex Does Not Affect the Overall Accumulation of Feces ...............................................130

Figure 4.4 Sex Does Not Play a Role in the Inhibition of the Small Intestine...................................132

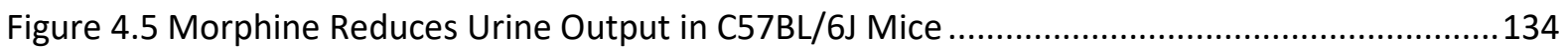

Figure 4.6 Kurkinol Induces Potent Respiratory Depression in Male and Female C57BL/6J Mice .....139

Figure 4.7 Kurkinorin Produces Greater Respiratory Depression in Female C57BL/6J Mice..............141

Figure $4.8 \beta$-arrestin2 Knock Out Mice Show a Small Statistical Improvement in Respiratory Measures

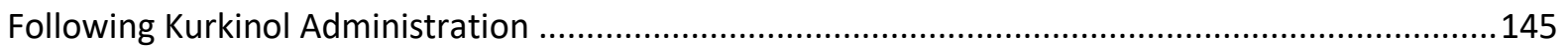

Figure 4.9 Kurkinol Induced Changes in Tidal Volume were Reduced in $\beta$-arrestin2 Knock Out Mice

146

Figure 4.10 Kurkinorin Has an Improved Z-score Profile Compared to Morphine .............................148

Figure 5.1 Kurkinol and Kurkinorin Potently Inhibit Membrane Depolarisation ................................171

Figure 5.2 Kurkinol and Kurkinorin are Potent Inhibitors of Membrane Depolarisation ...................172

Figure 5.3 All the Mu Opioid Receptor Agonists are Equipotent Activators of the GIRK Channels....176

Figure 5.4 Kurkinol and Kurkinorin Significantly Differ in the Activation of pCREB............................178

Figure 5.5 Kurkinol and Kurkinorin are Both Potent Bi-phasic Activators of pERK..............................181

Figure 5.6 Dynosore Reduced both Early and Late Phase Activation of pERK in Hek293 Cells Stably

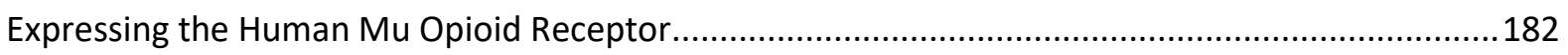

Figure 5.7 Kurkinol Shows Increased Selectivity for G-Protein Dependent Pathways ......................183

Figure 5.8 Decreased CREB phosphorylation Selectivity Correlates to Improved Therapeutic Windows

Figure 5.9 Increased Therapeutic Windows Correlate to Increased G-Protein Bias .........................188 
Figure 5.10 Summary of Pathway Selectivity at the Mu Opioid Receptor for Morphine, Kurkinol, and Kurkinorin.

Figure 6.1 Alternate Theories to Explain the Behavioural Profiles of "G-Protein" Biased Mu Opioid Receptor Agonists

Figure 7.1 Treatment Groups Did Not Significantly Differ in the Induction of Neuropathic Pain on Day 15

Figure 7.2 Chronic Treatment with Kurkinol or Kurkinorin did not Reverse Disease Progression or Induce Hyperalgesia to Mechanical or Cold Allodynia

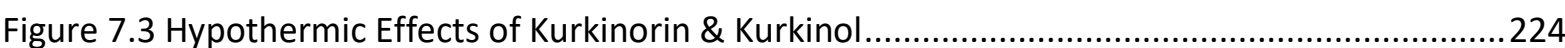

Figure 7.4 Kurkinol Negatively Impacts Multiple Measures of Respiration in C57 Bl/6 mice ...........225

Figure 7.5 Kurkinorin has a Reduced Effect on Respiratory Measures in C57 Bl/6 mice .................226

Figure 7.6 Respiratory Measures are not Affected by the Removal of $\beta$-arrestin2 .......................227

Figure 7.7 The Respiratory Effects of Morphine are not Affected by Deletion of $\beta$-arrestin2 ..........229

Figure 7.8 Human Mu Opioid Receptor Plasmid Vector Map......................................................230

Figure 7.9 Hek293 Cells Stably Expressing the Human Mu Opioid Receptor ................................230

Figure 7.10 CHO Cells Stably Expressing the Human Mu Opioid Receptor ..................................231

Figure 7.11 Dose Response effects of Forskolin on Membrane Potential....................................231

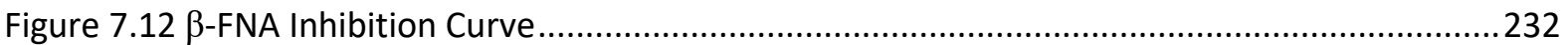

Figure 7.13 Optimisation of Thallium for the Potassium Assay in Human Mu Opioid Receptor CHO

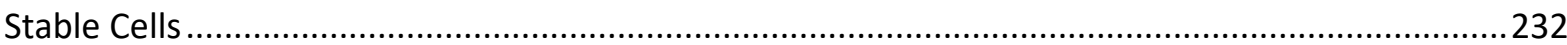

Figure 7.14. Secondary Only Control for Anti-mouse Cy5 and Anti-rabbit Cy5 .............................233

Figure 7.15 Protein Loading Analysis Shows a Strong Correlation between Density and Protein

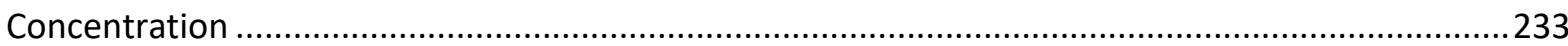

Figure 7.16 Quantification of the Molecular Weight the Protein Analysed ...................................234

Figure 7.17 Vehicle Treated Cells Show No Significant Change in Baseline pERK levels ...................235

Figure 7.18 Time Course Experiment; Full Scans of pERK....................................................236

Figure 7.19 Time Course Experiment; Full Scans of Total ERK.................................................236

Figure 7.20 Antagonist Experiment; Full Scans of pERK ......................................................237

Figure 7.21 Antagonist Experiment; Full Scans of Total ERK ....................................................237

Figure 7.22 There is Poor Correlation Between Therapeutic Window and the Bias Factors Generated

Using the Simplified Bias Equation 238 


\section{List of Tables}

Table 1.1 Primary Afferent Fibers are Classified by Axon Diameter and Level of Myelination .............. 4

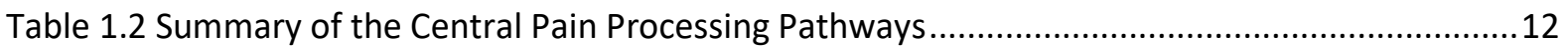

Table 1.3 lon channels Involved in Nociception ..........................................................................15

Table 1.4 Summary of Chemotherapy Agents that Induce Neuropathic Pain in Humans and Rodents

Table 1.5 Summary of Current and Newly Developed Treatments for Chronic Pain ...........................27

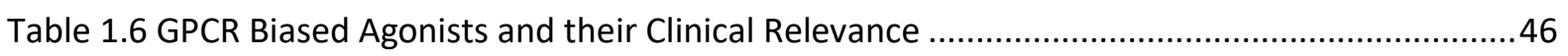

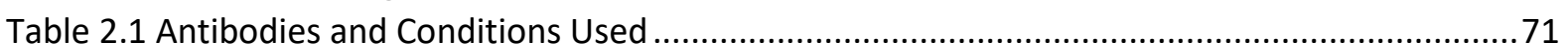

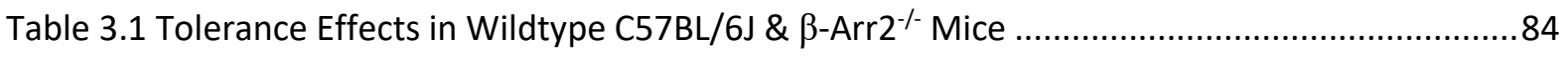

Table 3.2 Kurkinorin is Significantly more Potent at Inhibiting Cold Allodynia in Male Mice ..............96

Table 3.3 Combined Dose Response Effects on Mechanical and Cold Allodynia in the Paclitaxel Model

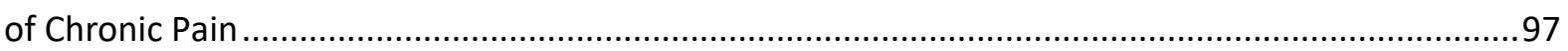

Table 3.4 Summary of Kurkinol and Kurkinorin Anti-Nociceptive Effects .......................................106

Table 3.5 Summary of Main Effects of Sex on the Anti-Nociceptive Effects of Kurkinol and Kurkinorin

108

Table 3.6 Summary of the Anti-Nociceptive Profiles of G-Protein Biased Mu Opioid Receptor Agonists

Table 4.1 Mu Opioid Receptor Location and Associated Side Effects ............................................118

Table 4.2 Summary of the Side Effect Profiles of Kurkinol and Kurkinorin ........................................151

Table 4.3 Summary of the Side Effect Profiles of G-Protein Biased Mu Opioid Receptor Agonists ...154

Table 4.4 Effect of Sex in the Induction of Side Effects by Morphine, Kurkinol, and Kurkinorin.........158

Table 5.1 Summary of Biased Mu Opioid Receptor Agonists .........................................................166

Table 5.2 $\mathrm{EC}_{50}$ and $\mathrm{E}_{\max }$ of Kurkinol and Kurkinorin in the Membrane Potential Assay in Hek293 and

$\mathrm{CHO}$ cells Stably Expressing the Human Mu Opioid Receptor...................................................174

Table 5.3 Effect of Cell Background on the $\mathrm{EC}_{50}$ and $\mathrm{E}_{\max }$ Values in the Membrane Potential Assay.175 Table 5.4 Kurkinol and Kurkinorin offer no Increase in Potency and Efficacy of GIRK channel activation

Table 5.5 Potency and Efficacy of Kurkinol and Kurkinorin at Activating pCREB in CHO Cells Stably

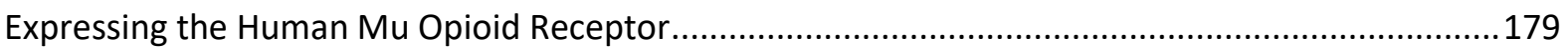

Table 5.6 Comparison of the Bias Factors Generated from Two Different Cell Backgrounds ............186

Table 6.1 Behavioural Effects of RGS Proteins that Regulate the Mu Opioid Receptor.....................207

Table 7.1 Chapter 3 Statistical Test Assumption Information ........................................................220

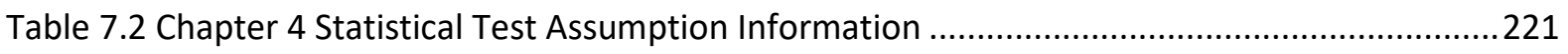




\section{Abbreviations}

AC

ACC

ANOVA

ASIC

ATP

AUC

BRET

$\mathrm{Ca}^{2+}$

CAMP

CB1

CB2

$\mathrm{CCl}$

$\mathrm{CHO}$

CINP

CNS

CREB

DAMGO

DMSO

$\delta$ receptor

DRG

$\mathrm{EC}_{50}$

$E D_{50}$

$E_{\max }$

ERK1/2

eVF

FCS

FDA

FSK

G.I

GABA

GIRK

GPCR

GRK

GRP

GTP

Hek293

i.c.v.

i.p.

i.t.

i.v.

IASP

$\mathrm{ID}_{50}$

IHC

JNK

$\mathrm{K}^{+}$

$\mathrm{K}$ receptor
Adenylyl cyclase

Anterior cingulate cortex

Analysis of variance

Acid-sensing ion channels

Adenosine triphosphate

Area under the curve

Bioluminescent resonance energy transfer

Calcium

Cyclic adenosine monophosphate

Cannabinoid type 1

Cannabinoid type 2

Chronic constriction injury

Chinese hamster ovary

Chemotherapy-induced neuropathic pain

Central nervous system

cAMP response-element binding protein

[D-Ala2,N-Me-Phe4,Gly5-ol]-enkephalin

Dimethyl sulfoxide

Delta opioid receptor

Dorsal root ganglion

Median effective concentration (potency)

Median effective dose (potency)

Maximum response (efficacy)

Extracellular signal-regulated kinase 1 and 2

Electronic von Frey

Foetal calf serum

Food and drug administration

Forskolin

Gastrointestinal tract

$\gamma$-aminobutyric acid

G-protein inwardly rectifying potassium channel

G-protein coupled receptor

G-protein coupled receptor kinase

GPCR specific phosphatases

Guanine triphosphate

Human embryonic kidney 293

Intracerebroventricular

Intraperitoneal

Intrathecal

Intravenous

International Association for the Study of Pain

Median inhibitory dose (potency)

Immunohistochemistry

c-Jun N-terminal kinase

Potassium

Kappa opioid receptor 


\begin{tabular}{|c|c|}
\hline LC & Locus coeruleus \\
\hline MAPK & Mitogen-activated protein kinase \\
\hline$\mu$ receptor & Mu opioid receptor \\
\hline$\mu$ receptor $\%$ & $\mu$ receptor knock out mice \\
\hline MPE & Maximum possible effect \\
\hline $\mathrm{mVF}$ & Manual von Frey \\
\hline $\mathrm{Na}^{+}$ & Sodium \\
\hline nor-BNI & nor-binaltorphimine \\
\hline NTS & Nucleus tract solitarius \\
\hline OIRD & Opioid-induced respiratory depression \\
\hline PBS & Phosphate buffered saline \\
\hline PFC & Prefrontal cortex \\
\hline PKA & Protein kinase $A$ \\
\hline PKC & Protein kinase $\mathrm{C}$ \\
\hline PTX & Paclitaxel \\
\hline Px & Pertussis Toxin \\
\hline RTN/pFRG & Retro-trapezoid and parafacial respiratory group \\
\hline RVM & Rostral ventrolateral medulla \\
\hline s.c. & Subcutaneous \\
\hline Sal B & Salvinorin B \\
\hline Sal A & Salvinorin A \\
\hline SEM & Standard error of the mean \\
\hline TRP & Transient receptor potential \\
\hline Veh & Vehicle \\
\hline VG $\mathrm{Ca}^{2+}$ & Voltage-gated calcium channel \\
\hline VG $K^{+}$ & Voltage-gated potassium channel \\
\hline VG $\mathrm{Na}^{+}$ & Voltage-gated sodium channel \\
\hline $\mathrm{V}_{\min }$ & Minute volume \\
\hline $\mathrm{V}_{\mathrm{T}}$ & Tidal volume \\
\hline VTA & Ventral tegmental area \\
\hline VUW & Victoria University of Wellington \\
\hline Wt & Wildtype littermate \\
\hline$\beta 2 A R$ & $\beta 2$-adrenergic receptor \\
\hline$\beta$-arr2 ${ }^{--}$ & $\beta$-arrestin2 knockout mice \\
\hline$\beta-F N A$ & $\beta$-funaltrexamine \\
\hline
\end{tabular}




\section{Chapter 1. General Introduction}

Acute pain is a biological warning system that allows for the detection of noxious stimuli and potentially life-threatening tissue damage or injury. According to the International Association for the Study of Pain (IASP), chronic pain can be further classified into nociceptive, neuropathic, and nociplastic pain. Whereby nociceptive pain arises from actual or threatened damage to non-neuronal tissue, neuropathic pain results from a lesion or disease in the somatosensory nervous system, and nociplastic pain has no clear link to tissue damage or disease/lesion (IASP, 2019). While acute pain may range from mild to severe, it does not last longer than 3-6 months. Chronic pain, which persists for $>6$ months or beyond the normal healing time, is no longer classified as beneficial and can lead to further discomfort and impact on the quality of life (reviewed in Treede et al., 2015). The current definition of pain is a distressing sensory and emotional experience associated with potential or actual tissue damage (Williams et al., 2016). However, experience of painful stimuli is subjective and can be influenced by emotion and attention (Bushnell et al., 2013), society (Craig, 2015, Hadjistavropoulos et al., 2011), environment (Bushnell et al., 2015), and the gender of the person experiencing the stimuli (Boerner et al., 2018). Despite years of research, pain is still difficult to classify and treat due to the subjective nature of its perception. The consensus has been to classify pain into three major categories acute nociceptive, inflammatory, and chronic/neuropathic nociceptive pain (Williams et al., 2016).

\subsection{Current issues in the management of chronic pain}

According to a 2018/2019 survey compiled by the New Zealand Ministry of Health, chronic pain affects 763,000 (19.4\%) New Zealand adults daily (Ministry of Health, 2019). This is an increase from 16.2\% in the 2011/12 health survey (Ministry of health, 2012). In the United States, an estimated 50 million adults (20.4\%) suffer from chronic pain and a further 19.6 million (8\%) had high-impact chronic pain ( $\mathrm{NIH}, 2016)$. Rates of chronic pain in Australia are also high with 3.24 million adults (15.4\%) affected, and the prevalence is predicted to rise to 5.23 million in 2050 (Deloitte, 2019).

Individuals who suffer from chronic pain are likely to experience comorbidities such as major depression (Gunn et al., 2012, Stein et al., 2006), anxiety (Carleton et al., 2018), and suicidal behaviour (El-Gabalawy et al., 2011). For those living with chronic pain, there is also a large impact on the quality of life with adverse effects on work (Breivik et al., 2006, Pike et al., 2016), sleep (McCracken et al., 2002), and the ability to maintain stable relationships (Smith et al., 2001). Therefore, the rising rates of chronic pain have a large socioeconomic burden with loss of productivity and healthcare costs. These costs are calculated based on the direct costs (hospital visits, pharmaceuticals, insurance payments, welfare support), indirect costs (loss of productivity and decrease in employment of sufferers and informal carers), and the intangible costs (decreases in quality of life of sufferers and families). Generally, these numbers are available from government private institution reports. 
However, in smaller countries or ones that do not directly track the cost of chronic pain, these numbers are inferred from other countries' data. In the United States, the direct cost and that associated with lower work productivity was estimated to be $\$ 635$ billion a year, which is more than heart disease (\$309 billion) and cancer (\$243 billion) combined (Gaskin et al., 2012). In Australia, the total socio-economic cost of chronic pain was reported to be $\$ 139.3$ billion in 2018 and is expected to rise to \$215.6 billion by 2050 (Deloitte, 2019). The cost of chronic pain in New Zealand in 2016 estimated to be between $\$ 13.2$ and $\$ 14.8$ billion, and the costs are predicted to rise to between $\$ 21.2$ - \$24.3 billion by 2048 (Moore et al., 2018).

Despite the high prevalence and financial cost of chronic pain, $40 \%$ of Pan-Europeans suffering from chronic pain reported insufficient treatment (Breivik et al., 2006). This is supported by physicians who also expressed low satisfaction with their currently available treatment options (Green et al., 2001). Due to the complex nature of pain when it has taken a chronic course, all aspects must be examined, including; physiological, psychological, and social. To provide the correct pharmaceutical treatment for chronic pain, the cause; i.e. neuropathic, cancer, or chronic inflammation associated pain, needs to be determined. This is normally paired with psychological interventions, such as mindfulness or cognitive-behavioural therapy. Overall, there is no single treatment that fits each case of chronic pain. Individual plans must be made for all sufferers of chronic pain which increases costs and decreases the ability to treat it. Due to the ineffectiveness of treatments, catastrophising of pain is common. This is defined as an exaggerated mental mindset during an actual or anticipated painful experience. It includes ruminating on pain, magnifying pain, and pain helplessness, which is where sufferers believe the pain will only get worse (Sullivan et al., 2001). The combination of poor treatment, social isolation, pain poverty, and comorbidities leads to a significant decrease in the quality of life of people suffering from chronic pain.

\subsection{The Pain Pathways}

Chronic pain is a maladaptive response that occurs as a result of stimuli that were previously non-noxious or with no identifiable cause. While pain pathways involved in acute pain transmission are well described, less is known about the cause of chronic pain. This is specifically true of the transition between acute and chronic pain states. It is important to understand the pain pathways that are activated in acute pain states in addition to understanding the changes that may allow that occur during the transition to chronic pain.

\subsubsection{Nociceptors and Ion Channels Involved in Nociception}

A range of receptors and ion channels are involved in the detection and translation of painful stimuli into electrical signals and are collectively known as nociceptors (Sherrington, 1952, Sherrington, 1903). Nociceptors are located on free nerve endings of primary afferent fibres that 
innervate the periphery, including in the skin, muscle, joints, and viscera. Unlike receptors that respond to non-noxious stimuli, nociceptors have a high threshold for activation and many types that are specialised to respond to thermal or mechanical stimuli. They can also be polymodal and respond to multiple noxious stimuli. The differential expression of these nociceptors on primary afferent fibres determines the sensitivity to noxious stimuli (Ramsey et al., 2006).

The family of Transient Receptor Potential (TRP) cation channels that control neuron excitability is one of the largest families of ion channels. A subset of which are involved in the transmission of noxious heat, cold, chemical and mechanical damage, nociceptive TRP channels. There are seven subfamilies of TRP channels, namely; anarkin, vanilloid, melastatin, canonical, no mechanoreceptor, mucilipin, and polycistin TRP channels. These receptors all have important physiological roles, including determining noxious thermal, chemical, and mechanical stimuli. Upon activation by noxious stimuli, the TRP ion channels open allowing the flow of calcium $\left(\mathrm{Ca}^{2+}\right)$ and sodium $\left(\mathrm{Na}^{+}\right)$ions into the nociceptor, which depolarises the membrane (Mulier et al., 2017, Pedersen et al., 2005). If the hypo-polarisation is of sufficient amplitude, it causes the opening of voltage-gated $\mathrm{Na}^{+}$ channels and the efflux of potassium $\left(\mathrm{K}^{+}\right)$allowing the propagation of the action potential along the axon. The frequency, temporal summation, and modulation received from descending pain pathways in response to noxious stimuli provide information on the intensity of the pain (Woolf et al., 2007).

Nociceptors also respond to compounds that are released in response to tissue damage, inflammation, and necrosis. These include; histamine, bradykinins, interleukin-1 $\beta$ (II-1 $\beta$ ) tumour necrosis factor $\alpha$ (TNF $\alpha)$, Substance $P$, serotonin, and glutamate. These molecules can interact with several TRP ion channels, acid-sensitive ion channels (ASIC) and receptors (G-protein coupled receptors (GPCR), receptor tyrosine kinases (RTKs)) on the membrane of nociceptors to induce depolarization and nociception (reviewed inBasbaum et al., 2009).

\subsubsection{Primary Afferent Fibres}

Noxious stimuli are detected in the periphery and transduced into electrical signals by the free nerve endings of the main nociceptor axon. The electrical signal is passed as an "all or nothing" action potential up the axon. The speed of transmission of an action potential is dependent on the diameter of the axon and if the sensory neuron is myelinated or not (Gasser et al., 1927). A large proportion of nociceptors are small unmyelinated axons called C-fibres which have high thresholds for activation and conduct at velocities of $0.4-1.4 \mathrm{~m} / \mathrm{s}$ (Gasser, 1941). Transmission through these fibres is supported by a non-myelinating version of Schwann cells, with bundles of multiple axons (Remak Bundles) encompassed in the sheath of one cell (Griffin et al., 2008). C-fibre nerve endings are diffuse within the periphery and activated by noxious mechanical, thermal, and chemical stimuli. Because of this disbursed localisation, they lead to diffuse pain sensations with poor spatial localisation (Bessou et al., 1969). Sharp or fast-onset pain is mediated by the myelinated A-fibre nociceptors with conduction velocities of $5-70 \mathrm{~m} / \mathrm{s}$ (Gasser, 1941). A-fibre nociceptors are further classified into the 
small diameter $A \delta$-fibres and the large diameter $A \beta$-fibres, with unmyelinated nerve endings that cluster in small distinct regions that respond precisely to stimuli (Kruger et al., 1981). Aס-fibres typically respond to noxious heat and mechanical stimuli with conduction speeds of $2-30 \mathrm{~m} / \mathrm{s}$ (Burgess et al., 1967, Gasser, 1941). Distinctly, the A $\beta$-fibres are thought to be non-nociceptive, responding to low threshold touch, stretch vibration, and hair movement with conduction speeds of $30-70 \mathrm{~m} / \mathrm{s}$ (Gasser, 1941). However there is some evidence of $A \beta$-fibres to transmit nociceptive signals in vivo animal models, including in monkey (Perl., 1968, Treede, Meyer, and Campbell., 1998) and cat (Brugess. and Perl., 1967) hairy skin. While in human's high frequency A $\beta$-fibre stimulation can result in pain and an associated reflex (Bai et al., 2015, Willer at al., 1978, Willer and Albe-Fessard, 1983) (Summarised in Table 1). In the peripheral nervous system, Schwann cells are responsible for the myelination of neuronal axons. A single Schwann cell wraps a sheath around a section of the axon to generate the myelin layer. The gaps that form between two myelinating glial cells are known as the Node of Ranvier. These areas contain ion channels that aid in the propagation of action potentials down the axon (Salzer, 2015).

Table 1.1 Primary Afferent Fibers are Classified by Axon Diameter and Level of Myelination

\begin{tabular}{c|c|c|c|c} 
& Size $(\mu \mathrm{m})$ & Myelin level & $\begin{array}{c}\text { Transmission } \\
\text { speed }(\mathrm{m} / \mathrm{s})\end{array}$ & Sensation \\
\hline C-fibre & $0.5-2$ & Unmyelinated & $0.4-1.4$ & $\begin{array}{c}\text { Slow, diffuse, dull } \\
\text { pain }\end{array}$ \\
\hline Ad-fibre & $1-5$ & Thin & $2-30$ & $\begin{array}{c}\text { Sharp, acute, } \\
\text { localised pain }\end{array}$ \\
\hline A -fibres & $6-12$ & High & $30-70$ & $\begin{array}{c}\text { Non-noxious light } \\
\text { touch, some } \\
\text { nociceptors }\end{array}$ \\
\hline
\end{tabular}




\subsubsection{Dorsal Root Ganglion}

The primary afferent neurons carry the afferent nociceptive signals towards the spinal column. They enter the spinal column, via the intervertebral neural foramina, and form the dorsal root ganglion (DRG). The DRG contains the soma of the body's sensory neurons which are 'pseudounipolar' with a single bifurcating axon that results in two distinct branches; the distal and proximal processes that enter the spinal column or peripheral nervous system respectively (Lieberman, 1976). Once the signal reaches the bifurcation point on the soma of the primary afferent neuron, the signal can either be impeded or propagated towards the entry zone of the DRG (Gemes et al., 2013). The layers of satellite glial cells that separate the soma of sensory neurons inhibits cross-talk between different sensory inputs, while also releasing chemicals involved in neuronal activation. These chemicals include; bradykinin, cytokines, chemokines, and adenosine-5'-triphosphate (ATP) and influence the transmission of afferent nociceptive signals within the DRG (Hanani, 2005). The soma within the DRG also provides metabolic support for the axon as well as generating receptors and ion channels that are transported down the axons to provide functional support to the transmission of electrical impulses (Devor, 1999) (Summarised in Fig. 1.1).

\subsubsection{Nodose and Trigeminal Ganglia}

The inferior ganglion of the vagus nerve (nodose ganglion) and the trigeminal ganglion are other areas that are important in the transmission of painful stimuli. The nodose ganglion is located within the jugular foramen, where the vagus nerve exits the skull. It contains sensory neurons that project from the gastrointestinal, cardiac, and respiratory organs to the medulla oblongata via the solitary tract nucleus and vagus nerve (Hisa, Lyon, and Malmgren., 1985, Kalia and Mesulam, 1980). The presence of both neurotransmitters (substance $P$, vasoactive intestinal peptide, cholecystokinin, calcitonin gene-related peptide, and Leu-enkephalin and nociceptors (vanilloid receptor type 1, purinoceptor 3, and ASIC nociceptors) that can respond to inflammatory mediators and neurotrophic factors makes this ganglion key in the transmission of visceral pain (Caterina et al., 1997, Chen et al., 1998, Hisa et al., 1994, Lundberg et al., 1985, Vulchanova et al., 1997).

The trigeminal ganglion is the sensory ganglia of the trigeminal nerve and is situated in Meckel's cave on the temporal bone (Yousry et al., 2005). It contains 3 main branches the ophthalmic (sensory), maxillary (sensory), and mandibular (sensory and motor). Within these areas the trigeminal ganglion is essential for releasing many neurotransmitters and peptides including calcitonin generelated peptide, ATP, nitric oxide and cytokines that play important roles in peripheral and central sensitization with primary headaches and cluster migraines (Belin, Ran, and EdVinsson, 2020, Messlinger and Russo, 2019). 


\subsubsection{Dorsal Horn of the Spinal Cord}

Once the afferent nociceptive signal has passed the soma of the primary afferent fibre, the signal enters the central nervous system (CNS) via the dorsal root entry zone. Bundles of C-fibres and A-type fibres carrying this nociceptive signal run longitudinally along the spinal column for a few segments, known as the track of Lissauer, before penetrating the dorsal horn of the spinal cord (LaMotte, 1977, Lissaure, 1885, Ramon Y Cajal, 1909, Ranson, 1913, 1914). Once in the dorsal horn, the sensory neurons synapse with second order neurons within distinct layers of the dorsal horn grey matter. These layers of neurons are known as Rexed laminae and can be divided into ten layers. The neurons within each layer perform specific functions, with Layers I to VI involved in the transmission of pain (Rexed, 1952). The myelinated high threshold Aס-fibres typically innervate Layer I and $V$ of the Rexed laminae and synapse with bipolar or unipolar neurons respectively. The A $\beta$-fibres are typically low-threshold mechanoreceptor fibres that innervate Layer IV and synapse with unipolar neurons. Unmyelinated C-fibres typically innervate at Layer II of the dorsal horn and synapse with multipolar secondary neurons (Mense, 1990). All three fibre types synapse with and are regulated by the inhibitory interneurons and islet cells in Layers II and III. If the hyperpolarisation overcomes the negative regulation by these cells the second order neurons, excitatory interneurons, regulate the motor response through the inhibitory and excitatory motor neurons in Layer $\mathrm{V}$ and carry the nociceptive signal across the central canal to the contralateral side of the spinal cord before projecting to higher brain regions (Fig. 1.1). 


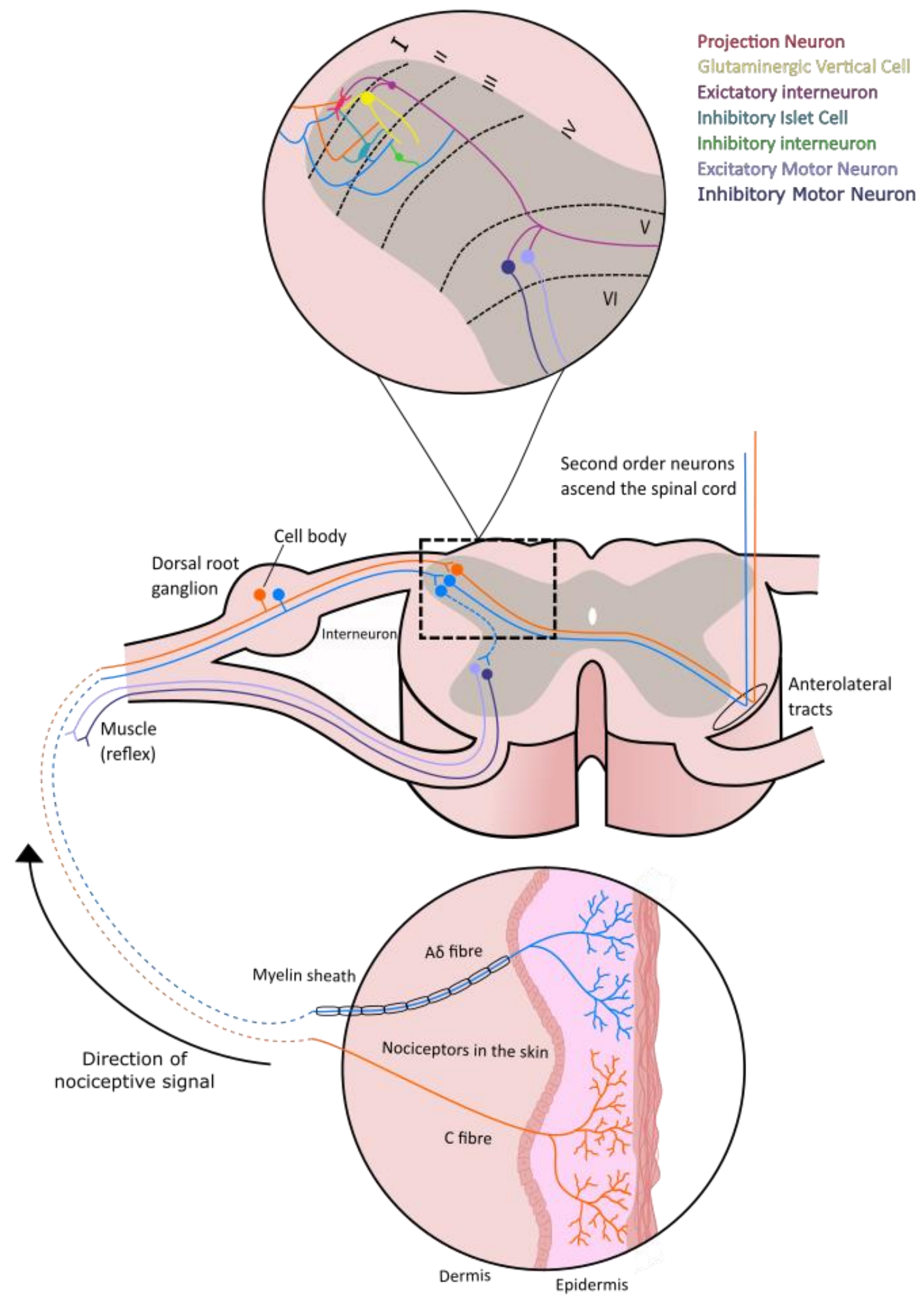


Figure 1.1 Transmission of Acute Pain in the Spinal Cord

Painful sensory information is transmitted from the periphery via both small unmyelinated C-fibers (blue) and the large myelinated A fibres (yellow). These sensory neurons, known as nociceptors, have free un-capsulated nerve endings in the periphery that detect noxious stimuli in the skin, muscles, and viscera. They then synapse with second order neurons in the dorsal horn of the spinal cord, with Cfibers terminating in Laminae II, and A $\delta$ fibres in Laminae I and V. Second order neurons then cross to the contralateral side before ascending to higher brain regions (adapted from Dubin et al., 2010, Peirs et al., 2016). 


\subsubsection{Central Processing of Pain}

Once the secondary neuron within the spinal column has crossed the central canal to the contralateral side, there are multiple pathways through which the sensory information can be transmitted to the brain, including, the spinothalamic (Fig. 1.2 A; blue), spinoreticular (Fig. 1.2 A; black), spinohypothalamic (Fig. 1.2 A; green), and spinomesencephalic tracks (Fig. 1.2 A; orange). Each pathway terminates in different brain regions and is involved in the transmission or inhibition of different components of pain. The spinothalamic tract is an ascending pathway that provides information on temperature, crude touch, and pain to the somatosensory cortex. This occurs via termination of the second order neurons in the thalamus where synapses are formed with third order neurons that project to the somatosensory cortex (Hodge et al., 1990). The spinoreticular tract is another ascending pathway that projects to the reticular formation of the medulla, and then to the pons before projection to the thalamus, hypothalamus, and limbic system. This allows for the autonomic, reflective, motivational, and emotional aspects of pain. The spinohypothalamic track is believed to be involved in the autonomic and endocrine response to pain. Neurons within this pathway terminate in the hypothalamus, thalamus, superior colliculus, and reticular formations in both the medulla and pons. The spinomesencephalic tact is often separated into three prominent pathways; spinotectal (superior colliculus, movement of head and eyes towards painful stimuli), spinoannular (periaqueductal grey, modulation of pain), and the spinoparabrachial tract (parabrachial regions, learned avoidance behaviour) (Bourne et al., 2014, Lamont et al., 2000, Willis, 1985) (Table 1.2). 


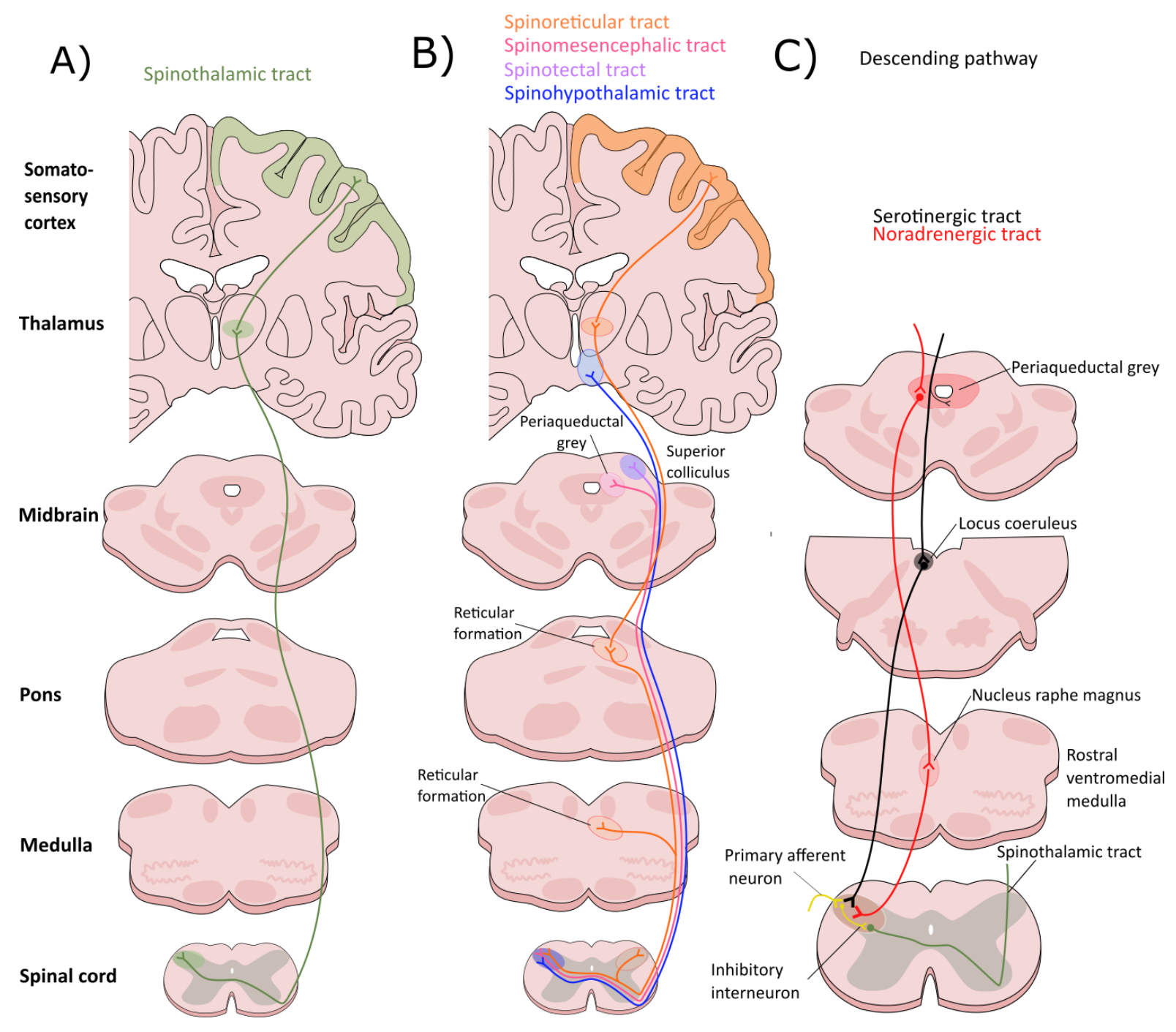

Figure 1.2 The Pain Pathways

There are four main pathways through which the transmission of noxious stimuli occurs. (A, B) This includes the spinothalamic, spinoreticular, spinohypothalamic, and spinomesencephalic pathways. (C) The main control of the painful stimuli comes from the descending pathway which elicits its control through the PAG and inhibitory serotonergic and noradrenergic neurons which synapse on to the second order neurons, primary afferent neurons, or inhibitory interneurons in the $\mathrm{DH}$ of the spinal cord (adapted from Kandel et al., 2000). 


\begin{tabular}{c|c|c|c}
\hline Ascending Vs & Termination & Component of Pain \\
\hline Spinothalamic & Ascending & $\begin{array}{c}\text { Thalamus \& Somatosensory } \\
\text { cortex, Insular, anterior } \\
\text { cingulate }\end{array}$ & $\begin{array}{c}\text { Location of Temperature, } \\
\text { crude touch, pain }\end{array}$ \\
\hline Spinoreticular & Ascending & $\begin{array}{c}\text { The reticular formation, } \\
\text { pons, hypothalamus, } \\
\text { thalamus, limbic system } \\
\text { Spinohypothalamic }\end{array}$ & $\begin{array}{c}\text { Autonomic, reflective, } \\
\text { motivational, and } \\
\text { emotional }\end{array}$ \\
\hline Spinomesencephalic & Ascending & $\begin{array}{c}\text { Hypothalamus, thalamus, } \\
\text { superior colliculus, and } \\
\text { reticular formations }\end{array}$ & $\begin{array}{c}\text { Autonomic and endocrine } \\
\text { response }\end{array}$ \\
\hline Superiotectal; \\
Pathway
\end{tabular}

There are multiple ascending pain pathways responsible for the transmission of different components of noxious stimuli. These include; the spinothalamic, spinoreticular, spinohypothalamic and spinomesencephalic pathways. 


\subsubsection{Modulation of the Pain Pathways}

Many theories have been proposed over the last few centuries to explain how the transmission of painful stimuli is modulated. These include; the Specificity Hypothesis, Gate Control Theory, Central Control System, and the Descending Pain Pathway.

The Specificity Hypothesis suggests that different sensory inputs are transmitted by specific and independent neural circuits. This theory was supported by the discovery of nociceptive-specific neurons that only transmit in response to one noxious stimulus (Christensen et al., 1970). The Gate Control Theory of pain modulation was proposed by Melzack \& Wall (1965) and was built on clinical and electrophysiological evidence. This theory states that both large $A \beta$ fibres (touch) and small sensory fibres (C-fibres) synapse with secondary neurons (T-cells) within the substantia gelatinosa of the dorsal horn of the spinal cord, before projecting to the brain. Prolonged high-intensity stimulations from the C-fibres were thought to "unbalance" the input from the large A $\beta$ fibres allowing presynaptic inhibition to be removed from the T-cell, thus "opening the gate". If the firing of the T-cell reached a critical pre-set level, the integrated signal would be interpreted as "pain". This theory explained the phenomena of licking, touching, or stimulating an injured nerve with Transcutaneous Nerve Electrical Stimulation to reduce pain perception. The Central Control System was also proposed by Melzak and Wall (1965) as a way to reset the gate based on external factors, which has since been shown to exist in the form of the descending pain pathway (Duan et al., 2018, Mendell, 2014).

The Descending Pain Pathway is an endogenous analgesic system that modulates nociceptive transmission in response to the endogenous opioids, including enkephalin and endomorphin. The role of the descending pain pathway was identified during an investigation into alternate pain treatments in the 1960s. They believed discrete, deliberate lesions in the brain would alleviate pain states. However, anti-nociceptive effects in rodents were inconsistent. The research then moved towards the placement of electrodes to identify key pain processing areas. When placed in the periaqueductal grey (PAG) of rats, activation of the electrodes abolished pain responses in a naloxone-sensitive manner (Hosobuchi et al., 1997, Mayer et al., 1971). This effect was found to partially occur through the blockade of the nociceptive signal at the level of the spinal cord (Mayer et al., 1974). Similarly, microinjection of morphine into the PAG of rats (Lewis et al., 1977) and rhesus monkeys (Pert et al., 1974) induced potent anti-nociceptive effects.

Since the discovery of the PAG's role in the descending pain pathway, the full neuronal circuit has been elucidated. The PAG neurons descend into the rostral ventromedial medulla (RVM). Here serotonergic neurons, classified as pain inhibitory “off' neurons, that project to Lamina's I, II, IV, and $V$ of the dorsal horn of the spinal cord via the dorsolateral funiculus and trigeminal dorsal horn (Adams, 1976, Fields et al., 1995, Heinricher et al., 1989). The PAG also projects to the locus coeruleus via noradrenergic neurons before passing into the dorsal horn of the spinal cord. When the serotonergic and noradrenergic neurons enter the dorsal horn, they synapse with spinothalamic, primary afferent, 
or interneurons to modulate the transmission of pain (reviewed in Basbaum et al., 1978, Bourne et al., 2014) (Fig. 1.2 B).

\subsection{Types of Pain}

\subsubsection{Acute Pain}

Acute nociceptive pain is induced by tissue injury and the subsequent activation of nociceptors at the site of the local tissue damage, known as nociceptive pain (IASP, 2019). This type of pain can be caused by trauma, surgical interventions, and some diseases, but does not impair the body's ability to heal. Acute pain normally resolves within the period of healing (Loeser et al., 1999). This sharp and fast transducing pain can be elicited by a variety of thermal, chemical, and mechanical stimuli within the skin, muscles, joints, tendons, or bones. In response to this painful stimulus, a withdrawal reflex can be initiated if experienced in areas such as the limbs, and the stimuli will be avoided in the future.

Noxious stimuli are detected in the primary afferent nociceptors by a range of high threshold nociceptors. The receptors and ion channels present on a nociceptor determines what that nociceptor responds to. For example, nociceptive TRP channels respond to noxious thermal (TRP ankyrin 1, melastatin 8, vanilloid 1, and vanilloid 4), chemical (TRP ankyrin 1, melastatin 8, vanilloid 1), and mechanical (TRP ankyrin 1, canonical, vanilloid 1, and vanilloid 4) stimuli. Other channels involved in sensing of pain include the two-pore $\mathrm{K}^{+}$ion channels, which play a critical role in determining neuronal excitability, as they counteract the action potentials at nerve terminals by 'leaking' $\mathrm{K}^{+}$to hyperpolarise the membrane (Hille, 2001, Tsantoulas et al., 2014). Evidence has shown these channels to be involved in heat (TASK1), cold (TASK3), and mechanical pain transmission (TREK1, TRAAK, TRESK) (reviewed in Li et al., 2015). As a result of these ion channels being activated by their respective ligands, $\mathrm{K}^{+}$leakage is decreased and the generation of an action potential in response to a noxious stimulus can occur more readily (Enyedi et al., 2010). The detection of chemical stimuli has also been shown to act through the polymodal functions of some of the previously mentioned receptors, such as the TRPV1 channel detecting capsaicin (Hayes et al., 2000), TRPM8, and menthol (Liu et al., 2013), whereas TRPA1 detects isothiocyanates and thiosulfinates that are present in mustard (e.g. wasabi) (Jordt et al., 2004) and allium (e.g. garlic) (Bautista et al., 2005). Other channels involved in nociception are the ASIC that detect changes in $\mathrm{pH}$ and the piezo proteins that detect noxious mechanical stimuli (Bagriantsev et al., 2014, Wemmie et al., 2013) (Table 1.3).

The overall result of activating these different ion channels is to cause a change in the membrane potential of the primary afferent neuron. This then allows the activation of the voltagegated sodium (VG Na+) and $\mathrm{Ca}^{2+}\left(\mathrm{VG} \mathrm{Ca}^{2+}\right)$ channels to further depolarise the membrane allowing the threshold for the generation of an AP to be met, therefore leading to the transmission of noxious stimuli. 


\begin{tabular}{|c|c|c|}
\hline & $\begin{array}{c}\text { Receptor/channel } \\
\text { subtype }\end{array}$ & Type of Pain \\
\hline \multirow{5}{*}{ TRP Channels } & Ankyrin 1 & Thermal, chemical, mechanical \\
\hline & Canonical 1 & mechanical \\
\hline & Melastatin 8 & Thermal, chemical \\
\hline & Vanilloid 1 & Thermal, chemical, mechanical \\
\hline & Vanilloid 4 & Thermal, mechanical \\
\hline \multirow{5}{*}{ Two-pore } & TASK1 & Thermal \\
\hline & TASK3 & Thermal \\
\hline & TREK1 & Mechanical \\
\hline & TRAAK & Mechanical \\
\hline & TRESK & Mechanical \\
\hline \multirow{3}{*}{$\begin{array}{l}\text { Acid Sensing Ion } \\
\text { Channels (ASIC) }\end{array}$} & ASIC1 & Low $\mathrm{pH}$ \\
\hline & ASIC2 & Low $\mathrm{pH}$ \\
\hline & ASIC3 & Low pH \\
\hline Piezo Proteins & Peizo2 & Mechanical \\
\hline
\end{tabular}

Many ion channels and receptors are involved in the transmission of pain. These can include TRP, two-pore $K^{+} A S I C$, and Piezo proteins. They are highly specific and normally respond to one type of noxious stimuli. 


\subsubsection{Inflammatory pain}

The induction of pain is not always directly associated with the injury and/or disease responsible for the transduction of noxious stimuli but rather is elicited from endogenously generated factors, known as inflammatory pain. Inflammation occurs in response to a localised area of external injury or systemically in response to trauma, disease, or severe infections. It is a result of a complex network of signals released by immune cells and is identified by five cardinal signs; redness, heat, swelling, pain, and loss of function (Scott et al., 2004). Sensitisation of the peripheral nociceptor then occurs by one of three mechanisms; (1) direct activation of cation channels, (2) GPCR activation, (3) or transcriptional alterations to the cell. These serve to lower the firing threshold of the nociceptor, so it activates at a lower stimulus intensity (Yaksh et al., 2015).

After tissue damage occurs, inflammatory mediators are released from the blood, damaged tissue, and local (e.g. keratinocytes and mast cells) and migrating inflammatory cells (e.g. macrophages and neutrophils) (Kim. et al., 2011). This includes the release of substance P and calcitonin gene-related peptide from nociceptive terminals, and cytokines, nerve growth factor (NGF), tumour necrosis factor $\alpha$, serotonin, and histamine from the innate immune cells. These function to increase blood vessel permeability allowing the infiltration of more circulating immune cells and fluid causing oedema (Woolf. et al., 1997). While prostaglandins, such as prostaglandin E2 increase Na1.8 currents by binding to prostaglandin E receptors, a $\mathrm{G}_{\mathrm{s}}$ coupled GPCR, to active PKA. This leads to the phosphorylation of TRPV1 receptors and VG $\mathrm{Na}^{+}$currents. This pro-inflammatory molecule is particularly important in the development of sensitisation as prostaglandin synthesis inhibitors reduce sensitization (reviewed in Scheibel et al., 2011).

The TRP vanilloid 1 and TRP ankyrin 1 channels can also be activated by lipids (Gregus et al., 2012, Okun et al., 2011) and arachidonic acid (Sisignano et al., 2012) released from the damaged tissue and cells. Circulating immune cells, including macrophages, also produce many cytokines and chemokines that can act on nociceptors to induce pain. These include IL-1 $\beta$ (Ferreira et al., 1988), IL6 (increases prostaglandins), tumour necrosis factor $\alpha$ (increases neuronal excitability) (Cunha et al., 1992), bradykinin (increases vascular permeability) (Manning et al., 1991), and brain-derived neurotrophic factor (modulates glutaminergic, $\curlyvee$-aminobutyric acid (GABA)-ergic and peptidergic transmission) (Lever et al., 2001). Transcriptional changes also occur as a result of inflammation. These include upregulation of $\mathrm{VG} \mathrm{Na}^{+}$channels and downregulation of $\mathrm{VG} \mathrm{K}^{+}$channels which function to hypopolarise peripheral nociceptors to a hyperexcitable state (Hoeijmakers et al., 2015, Tsantoulas, 2015). The inflammatory process discourages movement and contact with the affected area to limit further damage and increase recovery and healing. However, the increased sensitivity over time can lead to the development of primary hyperalgesia (increased sensitivity to pain) (Choi et al., 2016, Lewin et al., 1994). 


\subsubsection{Chronic Neuropathic Pain}

Unlike acute and inflammatory pain states, chronic pain has no protective function. It is a maladaptive response that lasts $>6$ months past the time of normal healing. As previously described, this can be due to a lesion or disease the body cannot heal (neuropathic pain). Neuropathic pain is a symptom of many diseases, including multiple sclerosis (MS) (O'Connor et al., 2008), HIV/AIDS (Miaskowski et al., 2011), diabetes (Callaghan et al., 2012), and chemotherapy-induced peripheral neuropathies (CIPN) (Staff et al., 2017). Alternately, there nociplastic pain occurs where there is no clear link to lesions or disease. In these cases, the dysregulation of sensory/integration pathways or the remapping of the pain pathways is believed to be the cause of the pain and includes conditions such as fibromyalgia and migraines (Cohen et al., 2014). It is often characterised by spontaneous pain (e.g. burning, tingling, numbness), as well as maladaptive responses such as hyperalgesia and allodynia.

The abnormal firing of nociceptors can occur in response to both peripheral or central sensitisation and the resulting changes in synaptic plasticity. Sensitisation is defined by the International Association for the Study of Pain (IASP) as an 'increased response to subthreshold stimuli in the peripheral or central nociceptors' (IASP., 2019). Peripheral sensitisation occurs in response to inflammatory mediators leading to sensitisation at the peripheral terminal of the nociceptor (see Section 1.3.2). In central sensitisation, the nociceptive pathways start responding to stimuli that do not normally elicit a pain response, known as allodynia (Ramer et al., 1998, Thibault et al., 2014). It can also produce secondary hyperalgesia, where non-inflamed tissue responds as if inflamed by changing the response generated by normal inputs. Overall, the centrally mediated changes in nociceptive firing mean the sensation of pain is no longer linked to the presence, intensity, or duration of noxious stimuli. Perception of painful stimuli instead represents increased responsiveness or gain in the nociceptive system that occurs by increasing membrane potential, removing/reducing inhibition, or changing synaptic efficacy (Ji et al., 2003, Latremoliere et al., 2009).

\subsection{Pre-Clinical Rodent Models of Pain}

A myriad of different anti-nociceptive behavioural tests have been developed to test multiple factions of the pain. These models take advantage of thermal, mechanical, chemical, and electrical noxious stimuli that elicit measurable pain behaviours. To fully assess the anti-nociceptive effects in rodents, multiple models must be used to cover the complexity of pain signalling and different presentations (Deuis et al., 2017). Many factors can influence the outcomes of pain assays. These include; stress-related anti-nociception in response to behavioural apparatus (reviewed in Butler et al., 2009) or the presence of an unknown male (Sorge et al., 2014). Another confounding factor in the analysis of pain in rodents is the effect of gender, with male animals traditionally used exclusively due to the variation on the pain-like behaviour introduced by the female reproductive cycle (Bartley et al., 
2013, Craft, 2007). With further complications of lower body fat in female mice potentially affecting lipophilic compound distribution, potency, duration of action, and efficacy. Other areas of pharmacokinetics are also altered such as membrane transport and liver metabolism (reviewed in Greenspan et al., 2007). While altered inflammatory responses driven by differential gonadal hormone regulation may also differentiate male and female sensitisation (Rosen et al., 2016). However, the National Institutes of Health $(\mathrm{NIH})$ in the United States of America, announced a policy in 2015 aiming to integrate sex as a biological variable into pre-clinical and clinical studies to fully understand the effect of sex on biological functioning, disease progression, and the response to treatment (Clayton et al., 2014).

\subsubsection{Nociceptive Pain Models}

Nociceptive pain models are highly utilised to study the anti-nociceptive effects of novel compounds. The ability to induce pain-like behaviour with a short duration of a nociceptive stimuli means anti-nociception can be quickly identified and repeated measurements are taken to assess the duration of action, tolerance, or hyperalgesia effects of a compound over time with no tissue damage.

\subsubsection{Thermal Nociceptive Pain Models}

Thermal nociceptive pain can be elicited in response to either hot or cold stimuli at the level of the spinal cord (spinally mediated) or higher brain regions (supraspinally mediated). Common thermal pain models utilising hot nociceptive stimuli include the tail-flick assay, the hot-plate assay, and the Hargreaves test. While nociceptive cold stimuli-based assays include the cold plate, dynamic cold plate, and acetone evaporation test.

The tail-flick test was first described in D'amour et al. (1941) and involves the application of heat to the tail until a withdrawal response, or 'flick', is recorded. The heat stimulus can be induced by inserting the distal end of the tail into a hot water bath, generally set to $50^{\circ} \mathrm{C}$. To automate the experiment and remove human error a radiant heat, induced by a focused light beam, can also be used. The tail-flick withdrawal response is a spinal reflex, however, supraspinal processing is believed to be involved in the delay of the spinally mediated flick response via the descending pain pathway and associated higher brain regions (Fields et al., 1983, Irwin et al., 1951, Jensen et al., 1986).

The hot-plate assay was first described by Woolfe et al. (1944). The authors placed a rodent on a plate heated to $50^{\circ} \mathrm{C}-55^{\circ} \mathrm{C}$ and timed the latency to pain-like behaviour to determine the thermal threshold. Multiple pain-like behaviours may be elicited, including; stamping, jumping and shaking or licking of the hind paws (Espejo et al., 1993). The dynamic hot-plate was developed based on the standard hot-plate. In this modified assay the animals are placed on the hot-plate apparatus at a nonnoxious temperature, which is gradually increased until a pain-like behaviour is seen. The temperature at which the response occurs is taken as the thermal pain threshold (Ogren et al., 1984, Tjolsen et al., 
1991). This model is generally considered a measure of supraspinal mediated anti-nociception, as the transection of the rat spinal cord abolishes the withdrawal response (Giglio et al., 2006).

The cold-plate assay is the inverse experiment to the hot-plate assay previously discussed. In this assay, the animals are placed on a metal plate that is cooled to a nociceptive temperature, between $-5^{\circ} \mathrm{C}$ and $15^{\circ} \mathrm{C}$, and the time to pain behaviour is recorded. These behaviours are similar to the hot-plate assay with the addition of weight shifting/repositioning to reduce contact with the surface (Allchorne et al., 2005). A cooling ramp of the temperature can also be used to determine the cold withdrawal threshold (Yalcin et al., 2009).

The acetone evaporation test was first described in 1994 and is used as a measure of cold allodynia. During this experiment, animals are placed in a chamber with a mesh floor to allow acetone to be applied to the plantar surface of the hind paw, the cooling effect of the acetone evaporating on the skin evokes a pain-like behaviour that can present as flicking, shaking, or licking of the paw (Carlton et al., 1994, Choi et al., 1994). The time spent responding to the thermal stimuli can be used to track the induction of cold allodynia over time in response to neuropathic pain states, including chemotherapy-induced neuropathic pain (CINP) and sciatic nerve ligation (Paton et al., 2017, Shahid et al., 2017).

\subsubsection{Mechanical Nociceptive Pain}

The induction of aversive behaviour in response to mechanical stimuli is used to assess allodynia or the induction of hyperalgesia in multiple models of pain, including chemotherapy-induced neuropathic pain and the development of hyperalgesia with chronic opioid administration (Deuis et al., 2014, Ferrini et al., 2017). Mechanical sensory thresholds are measured using one of two tests: the von Frey filaments (VF), or the Randall-Selitto test.

The manual (m) VF test was developed by Maximillian Von Frey (1896). Each monofilament is made from nylon and delivers a pre-determined force based on its width. When applied to the plantar surface of the hind paw, the filament buckles at the pre-determined force. If a positive pain behaviour is elicited, i.e. lifting, shaking or licking of the paw, the next lowest filament is used. If no response is elicited, the next highest filament is used. This is known as the "up-down" method (Bonin et al., 2014, Chaplan et al., 1994). The electronic (e) VF test operates under the same principle as the mVF test, except that a single filament is applied to the plantar surface of the hind paw until the withdrawal reflex is observed, recording the force at which this occurs. The scale of increase in force is therefore continuous, rather than in steps, increasing the test's accuracy (Deuis et al., 2015, Lu et al., 2013).

The Randal-Selitto or paw-pressure test assesses the response to mechanical pressures stimulation via a clamp-like device on the paw or tail (Randall et al., 1957). The clamp device has one flat side and one with a single spike. As the clamp is shut on the paw or tail, a slow increase in pressure is applied until a tail-withdrawal or vocalisation reaction is given by the animal. Vocalisation as an endpoint is ethically limiting as rodents only audibly vocalise when in severe pain. Work into using 
ultrasonic vocalisation has been investigated, but has proven to be variable (Han et al., 2005, Wallace et al., 2005).

\subsubsection{Neuropathic Pain Models}

Neuropathic pain refers to pain that is caused by injury to the nervous system and has been defined as "pain arising as a direct consequence of lesion or disease affecting the somatosensory system" (Treede et al., 2008, IASP., 2019). It is not a single disease with one underlying mechanism, but a syndrome that is caused by many diseases. These include; diabetic neuropathy, viral infection (e.g. HIV), nerve damage, inflammatory/autoimmune disorders (e.g. multiple sclerosis), or chemotherapy treatment (Colleoni et al., 2010). Due to the wide range of causes and underlying mechanisms, there is no perfect pre-clinical neuropathic pain model that can identify compounds that effectively treat all pain conditions. Instead, there is a wide variety of models that allow the investigation of a singular cause.

\subsubsection{Chemotherapy-induced Neuropathic Pain}

The progression of cancer by constricting nerves or invading secondary sites can cause pain syndromes. However, chemotherapy drugs used to treat cancer can also induce peripheral neuropathy in $30-40 \%$ of patients (Banach et al., 2016). Categories of neurotoxic chemotherapy agents that induce neuropathic pain include; platinum, taxanes, vinca alkaloids, and thalidomide (Table 3.1). Animal models of these disease phenotypes have been developed using the chemotherapy agent's cisplatin, oxaliplatin, paclitaxel, and vincristine. These chemotherapy agents are systemically administered leading to the development of thermal and mechanical allodynia.

Paclitaxel (PTX) is commonly used to induce CINP in rodents via damage to the peripheral nerves and DRG. While a single injection can induce neuropathic pain, chronic administration with four injections over 6 days produces more sustained mechanical and thermal hyperalgesia. The mechanism through which this hyperalgesia is induced by PTX is not known. However, many mechanistic changes have been identified and are believed to play a role. This includes the microtubule dysfunction resulting in decreased cell stability and impaired transport of lipids, proteins, and ion channels (Shemesh et al., 2010). As a result, nerves become demyelinated and the endings degenerate. With no way to regenerate, the sensory innervations of the periphery are permanently reduced, particularly in the skin where regeneration is required with the turnover of the epidermis (Boehmerle et al., 2014, Gornstein et al., 2017, Siau et al., 2006). PTX can also damage mitochondria in peripheral nerves and the DRG causing decreased ATP, an increase in reactive oxygen species (Xiao et al., 2011, Zheng et al., 2011), and a release of $\mathrm{Ca}^{2+}$ from mitochondria (Boehmerle et al., 2006, Kidd et al., 2002) causing hyperexcitability and dysfunctional signalling. Genetic changes can also be induced, with the upregulation of ion channels required for the maintenance of membrane potential and hyperpolarisation, such as VG $\mathrm{Ca}_{3.2}$ and $\mathrm{Na}_{1.7}$ channels (Li et al., 2017). While the immune system is pushed to activate macrophages in the DRG and peripheral nerves causing peripheral sensitisation, 
while microglia and astrocytes are activated in the spinal cord to induce neuroinflammation (ZaksZilberman et al., 2001, Zhang et al., 2016).

Table 1.4 Summary of Chemotherapy Agents that Induce Neuropathic Pain in Humans and Rodents

\begin{tabular}{|c|c|c|c|c|}
\hline & Drug Name & $\begin{array}{c}\text { Types of Cancer } \\
\text { treated }\end{array}$ & $\begin{array}{l}\text { Neuropathy } \\
\text { incidence }\end{array}$ & $\begin{array}{l}\text { General Mechanism of CINP } \\
\text { Induction }\end{array}$ \\
\hline Platinum & $\begin{array}{l}\text { Cisplatin } \\
\text { Oxaliplatin }\end{array}$ & $\begin{array}{l}\text { Lung, ovarian, } \\
\text { bladder, } \\
\text { testicular, } \\
\text { colorectal }\end{array}$ & $70-100 \%$ & $\begin{array}{c}\text { Altered excitability of peripheral } \\
\text { neurons } \\
\text { Neuroinflammation }\end{array}$ \\
\hline Taxanes & Paclitaxel & $\begin{array}{l}\text { Brest, ovarian, } \\
\text { lung, prostate }\end{array}$ & $11-87 \%$ & $\begin{array}{c}\text { Altered excitability of peripheral } \\
\text { neurons } \\
\text { Neuroinflammation }\end{array}$ \\
\hline $\begin{array}{l}\text { Vinca } \\
\text { Alkaloids }\end{array}$ & Vincristine & $\begin{array}{l}\text { Lung, brain, } \\
\text { bladder, } \\
\text { testicular }\end{array}$ & $>20 \%$ & $\begin{array}{c}\text { Altered excitability of peripheral } \\
\text { neurons } \\
\text { Neuroinflammation }\end{array}$ \\
\hline $\begin{array}{l}\text { Proteasome } \\
\text { Inhibitors }\end{array}$ & Bortezomib & $\begin{array}{l}\text { Multiple } \\
\text { myeloma }\end{array}$ & $20-30 \%$ & $\begin{array}{c}\text { Altered excitability of peripheral } \\
\text { neurons } \\
\text { Neuroinflammation }\end{array}$ \\
\hline Others & Thalidomide & $\begin{array}{l}\text { Multiple } \\
\text { myeloma }\end{array}$ & $20-60 \%$ & $\begin{array}{c}\text { Altered excitability of peripheral } \\
\text { neurons }\end{array}$ \\
\hline
\end{tabular}

Data summarised from Banach et al. (2016), Zajaczkowska et al. (2019) 


\subsubsection{Other Neuropathic Pain models}

Other behavioural neuropathic pain models have been developed to investigate the cause and to find effective treatments for other chronic pain conditions. The distal sympathetic sensory neuropathy caused by diabetes in humans can be studied using multiple murine models, including genetic models (e.g. non-obese diabetic (NOD), insulin-resistant ob/ob mice) that spontaneously develop diabetes and diabetic neuropathy (Hinder et al., 2013, Schmidt et al., 2003). However, the most common model is the streptozotocin-induced diabetic neuropathy. This chemical kills the insulin-secreting Langerhans islet cells to rapidly cause hyperglycaemia and diabetic neuropathy (Jakobsen et al., 1976). While the most reliable animal model of Human Immunodeficient Virusassociated Sensory Neuropathy is induced by the interaction of the viral protein gp120 with the myelin sheath of the sciatic nerve resulting in mechanical allodynia (Wallace et al., 2007).

Nerve injury models can be used to assess both peripherally and centrally mediated neuropathic pain states based on the nerve that is injured. Spinal injuries are commonly induced by contusion, constriction, crushing, or hemisecting of the spinal cord (Marques et al., 2009, Tanabe et al., 2009). However, the most common nerve injuries are assessed using the peripheral sciatic nerve models. Chronic constriction injuries $(\mathrm{CCl})$ can be induced by tying ligatures on the sciatic nerve, which causes intra-neural oedema and severs the nerve axons (Bennett et al., 1988). The introduction of a cuff on the nerve, in place of ligatures, has reduced the variability in pain scores induced by manual tightening of ligatures (Benbouzid et al., 2008). Another variation on this model is the partial sciatic nerve injury model developed by Seltzer et al. (1990). In this model, one third to half of the nerve is tightly ligated to induce injury. All three variations of the model result in allodynia to mechanical and thermal stimuli a short period after the surgery (Costa et al., 2019, Samur et al., 2018).

\subsection{Current Therapeutics For neuropathic pain}

Effective treatments for chronic pain are hard to identify due to its complex presentation. Current treatment options are prescribed based on the cause of chronic pain. For non-cancer chronic pain, first line options are non-opioid based pharmaceuticals, including gabapentinoids and antidepressants. However, in $15-20 \%$ of patients, the non-opioid treatment options are ineffective or the side effects too severe. In these cases, the second line treatments, opioid based pharmaceuticals, are prescribed. For patients in palliative care or those with cancer/chemotherapy-induced chronic pain, the first line treatment options are opioids due to the intensity of the pain. Opioid based therapeutics are also heavily limited in their use due to their addictive properties, analgesic tolerance with chronic use, and side effects, such as respiratory depression and constipation. The ineffectiveness of both non-opioid and opioid based therapeutics highlights the need for effective analgesics with a large therapeutic window and reduced side effects. 


\subsubsection{Non-Opioid Therapeutics}

\subsubsection{Gabapentinoids}

Anti-convulsant drugs, such as the gabapentinoids, gabapentin, and pregabalin, are often first line treatments for neuropathic pain (Attal et al., 2010) (Table 1.4). These compounds are derivates of the inhibitory neurotransmitter GABA and induce their analgesic effects by binding to the $\alpha 2 \delta$ subunit of voltage-dependent calcium channels. This produces neuron-specific actions in the dorsal horn of the spinal cord and higher brain areas resulting in activation of the descending pain pathway and inhibition of the serotonergic system and inflammatory mediators (Alles et al., 2018, Chincholkar, 2018). In animal models of neuropathic pain, gabapentinoids consistently take 30 mins to induce antinociceptive effects. However, in human's, anti-nociception takes a minimum of one day to develop, with the maximum benefits occurring two weeks after treatment is started. Overall the average number of patients needed to treat 1 patient with $50 \%$ pain relief in peripheral neuropathic pain and painful diabetic neuropathy of $4.3(2.8-8.6)$ while postherpetic neuralgia has a number needed to treat of $4.3(3.3-6.1)$ (Sindrup., et al., 2005). The dosing in humans usually ranges between $300-600$ $\mathrm{mg} /$ day for pregabalin and $1200-3600 \mathrm{mg} /$ day for gabapentin. Dizziness, oedema, headaches, weight gain, and concentration deficits are common side effects induced by gabapentin and pregabalin. This leads to a discontinuation rate of around $20 \%$ for patients on $600 \mathrm{mg} /$ day of pregabalin (Alles et al., 2018, Attal et al., 2010, Kremer et al., 2016).

\subsubsection{Anti-depressants}

Many different anti-depressant compounds have been found effective in treating neuropathic pain at doses lower than that for treating major depression. This includes tricyclic anti-depressants, which modulates monoaminergic responses and inhibit serotonin/noradrenaline reuptake. An example of this is amitriptyline ( $25-150 \mathrm{mg} /$ day), which is used to treat diabetic neuropathy and postherpetic neuralgia (Saarto et al., 2007). Randomised control trials have shown that tricyclic antidepressants have an average number needed to treat of 2.3 (2.1-2.7) (Sindrup., et al., 2005). However, tricyclic anti-depressants can also cause drowsiness, dizziness, constipation, nausea, blurred vision, ataxia, and palpitations (Attal et al., 2010). Their use is also limited in patients with cardiac issues, glaucoma, and dysuria due to their anticholinergic effects, with the largest risk factor being sudden death from cardiac arrythmia (Roose, 2000, Sindrup., et al., 2005). The adverse effects associated with tricyclic anti-depressants gives a number needed to harm (reciprocal of number needed to treat) of $13.6(9.8-22.5)$ and results in treatment withdrawal in up to $20 \%$ of patients (Saarto et al., 2007, Sindrup., et al., 2005).

Selective serotonin-noradrenalin reuptake inhibitors, such as duloxetine $(60-120 \mathrm{mg} /$ day) and venlafaxine (150 - $225 \mathrm{mg} /$ day), are another class of anti-depressants that have clinical utility in the treatment of neuropathic pain. Selective serotonin-noradrenalin reuptake inhibitors bind the serotonin and noradrenaline reuptake receptors causing an increase of the associated 
neurotransmitter in the synaptic cleft activating the descending pain pathway to induce antinociception. Like the gabapentinoids, the onset of the anti-nociceptive effect is not immediate, instead, taking a few days to a week. Their side effects of include, decreased libido, nausea, insomnia, reduced appetite, and hypertension. Despite the safer side effect profile of the selective serotoninnoradrenalin reuptake inhibitors compared to tricyclic anti-depressants, there are still discontinuation rates of up to $15 \%$ when used to treat neuropathic pain with a number needed to harm of 21.5 (11.2 - 270) (Hayashida et al., 2019, Kremer et al., 2016, Sindrup., et al., 2005).

Selective serotonin reuptake inhibitors, such as fluoxetine and citalopram, are better tolerated than tricyclic anti-depressants and selective serotonin-noradrenalin reuptake due to the reduced anticholinergic and adrenergic effects. However, they show limited efficacy in the treatment of neuropathic pain conditions such as painful diabetic neuropathy compared to other antidepressants and are not recommended for treatment (Attal et al., 2010, Kremer et al., 2016) (Table 1.4).

\subsubsection{Cannabinoids}

Cannabinoids, including $\Delta 9$-tetrahydrocannabinol, cannabidiol, endocannabinoids, and synthetic cannabinoids, recently become a drug of interest for the treatment of chronic pain. These compounds act through the cannabinoid receptor type 1 and/or type 2 (CB1 \& CB2), which are members of the GPCR superfamily of receptors that are enriched throughout the CNS (Mackie, 2005). Cannabinoids have previously been shown to be effective at treating spasticity in multiple sclerosis patients, fibromyalgia, and neuropathic pain (Pascual et al., 2018). Currently available cannabinoids for chronic pain include Cesamet ${ }^{\mathrm{TM}}$ (active component is $\Delta 9$-tetrahydrocannabinol; Valeant Pharmaceuticals), Marinol ${ }^{\text {TM }}$ (active component is $\Delta 9$-tetrahydrocannabinol; Solvay Pharmaceuticals), and Sativex ${ }^{\mathrm{TM}}$ (active components are $\Delta$-tetrahydrocannabinol and cannabidiol; GW Pharmaceuticals) (Table 1.4). Currently, the only cannabinoid based therapeutic in New Zealand is Sativex ${ }^{\mathrm{TM}}$, which can be prescribed for the treatment of spasticity in multiple sclerosis. Other cannabinoid-based products that meet the pharmaceutical grade as determined by the Ministry of Health can be applied for via Pharmac.

Despite the positve evidence of the analgesic effects of cannabinoids, a recetent metaanalysis of randomised control trials by Fisher et al. (2020) found litte evidence that cannabis, or cannabinoid based therapeutics, reduced pain intesnsity in any chronic pain condition. The authors also stated under reporting of adverse events in treatment arms of randomised control trials, with some evidence of higher adverse events in treatmnet groups. These compunds are further limited by the induction of cardiovascular disorders, psychosis, schizophrenia, mood disorders, and the psychoactive effects of $\Delta 9$-tetrahydrocannabinol (Cohen et al., 2019). With more evidence arising of negative long-term effects of cannabinoids with high levels of THC (>10\%) (Di Fort et al., 2019). 


\subsubsection{New Therapeutic Targets}

A range of specific toxins is currently yielding some of the most exciting leads for the development of non-opioid therapeutics. These compounds are being investigated for their ability to induce analgesia, whilst not inducing tolerance or addiction to more effectively treat chronic pain states.

The marine cone snail represents a group of more than 700 species of snails, which have become a focus for drug discovery through the active components of their venom, known as conotoxins. The most well-known conotoxin for the treatment of pain is $\omega$-MVIIA or Ziconotide. This is an inhibitor of $\mathrm{VG} \mathrm{Ca}^{2+}$ channels and has been approved by the Food and Drug Administration (FDA) for severe chronic pain and due to an inability to cross the blood brain barrier, is administered intrathecally (Miljanich, 2004). Unfortunately, commonly encountered side effects of its use include confusion, hallucination, meningitis, and depression that severely limits its utility and adherence (Nair et al., 2018). X-MrlA (norepinephrine transporter) (Nielsen et al., 2005), w-CVID (VG Ca ${ }^{2+}$ inhibitor)(Adams et al., 2003), and Contulakin-G (Neurotensin receptor) (Craig et al., 1999) are compounds isolated from cone snail venom that are currently in human clinical trials for the treatment of various chronic pain disorders.

Spider venoms are another source of toxins that have been exploited for their potential antinociceptive effects. It has been shown that peptides isolated from the venom of spiders can modulate the activity of multiple receptors and channels involved in the development and maintenance of neuropathic pain, including TRP channels, ASICs, GPCRs and VG Na${ }^{+}, \mathrm{K}^{+}$, and $\mathrm{Ca}^{2+}$ channels (King et al., 2013). One such toxin, Pha1 $\beta$, has been isolated from the venom of the Brazilian Spider, Phoneutria nigriventer. This toxin has been reported to selectively inhibit VG $\mathrm{Ca}^{2+}$ channels and antagonise the TRPA1 channels to inhibit neuronal excitability and induce anti-nociception (Tonello et al., 2017, Vieira et al., 2005). In animal models of pain, Pha1ß induces anti-nociceptive effects in inflammatory, nociceptive, chemotherapy-induced neuropathic pain, mechanical hypersensitivity, and acute thermal pain with a larger therapeutic window than the $\omega$-MVIIA (Castro-Junior et al., 2013, de Souza et al., 2013, Rigo et al., 2013, Souza et al., 2008) (Table 1.4).

\subsubsection{Opioids for Chronic Pain}

Opioid receptors were one of the first targets for the treatment of severe acute and chronic pain with the milk of the unripe pods from the poppy, Papaver somniferum, in use for its analgesic properties as early as the $15^{\text {th }}$ century BC (Norn et al., 2005). The active component of the poppy was first purified by Friedrich Wilhelm Adam Sertürner in 1805 (Jurna, 2003). Since then, opioids derived from the opium poppy such as morphine and codeine, as well as semi-synthetic (oxycodone), and fully synthetic (fentanyl, sufentanil) opioids have been used to treat Chronic pain (Table 1.4). These compounds, and most opioids, are mu-opioid receptor ( $\mu$ receptor) agonists and are widely considered the most effective analgesic for severe pain with a number needed to treat for oxycodone 
and tramadol in peripheral neuropathic pain of $2.6(1.7-6)$ and $3.5(2.4-6.4)$ respectively (Lowinson, 2005, Sindrup., et al., 2005, Toblin et al., 2011).

Despite the analgesic effects of these compounds, they have limited utility in the respite of chronic pain due to the induction of analgesic tolerance (Chu et al., 2006). A six-month double-blind randomised control trial showed the induction of tolerance opioid induced hyperalgesia in non-cancer chronic pain (Rowbotham and Wallace., 2020). The high rate of opioid induced adverse events across long-term randomised clinical trials has recently been shown by Krebs et al., (2018). In this study patients with chronic back, hip, or knee osteoarthritis pain were shown to have higher rates of adverse events when treated with opioid (morphine, oxycodone, or hydrocodone/acetaminophen) vs nonopioid therapeutics (paracetamol) or a nonsteroidal anti-inflammatory drug). A meta-analysis of randomised control trails for non-cancer chronic pain similarly showed increased adverse events, mainly vomiting, opioid vs non-opioid therapeutic arms (Busse et al., 2018). Tolerance to the analgesic, but not side effects reduces the therapeutic window over time increasing the risk of respiratory depression, accidental overdose, and opioid misuse and abuse (Martins et al., 2015). Constipation is another common side effect associated with chronic opioid use, affecting up to $50 \%$ of patients (Pappagallo, 2001). Other common side effects include urinary retention, nausea, vomiting, and pruritus (Ruan, 2007). Morphine is also known to induce effects on physical activity levels, with spontaneous locomotion/hyperactivity common in rodents following doses up to $20 \mathrm{mg} / \mathrm{kg} / \mathrm{i}$.p. (Hirabayashi and Alam, 1981, Izuka and Hirabayashi., 1983). While morphine-induced sedation has been shown post-operatively during titration in humans (Aubrun et al., 2004, Paqueron et al., 2002, Tveita et al., 2008). 


\begin{tabular}{|c|c|c|c|c|c|}
\hline & Line of Treatment & Example Drugs & Target & Use & $\begin{array}{c}\text { FDA } \\
\text { Approved }\end{array}$ \\
\hline Gabapentinoids & $\begin{array}{l}1^{\text {st }} \text { line for non-cancer } \\
\text { chronic pain }\end{array}$ & Gabapentin, Pregabalin & VG Ca ${ }^{2+}$ Channels & $\begin{array}{c}\text { Non-cancer chronic } \\
\text { pain }\end{array}$ & Yes \\
\hline Ani-depressants & $\begin{array}{l}1^{\text {st }} \text { line for non-cancer } \\
\text { chronic pain }\end{array}$ & $\begin{array}{c}\text { TCAs; amitriptyline } \\
\text { SSNRIs; duloxetine, venlafaxine } \\
\text { SSRIs; fluoxetine citalopram }\end{array}$ & $\begin{array}{c}\text { monoaminergic responses } \\
\text { Serotonin \& noradrenaline } \\
\text { reuptake } \\
\text { Serotonin reuptake } \\
\end{array}$ & $\begin{array}{l}\text { Non-cancer chronic } \\
\text { pain }\end{array}$ & Yes \\
\hline Cannabinoids & Recommended $2^{\text {nd }}$ line & $\begin{array}{l}\text { Cesamet } \\
\text { Marinol } \\
\text { Sativex }\end{array}$ & Cannabinoid Receptors & $\begin{array}{l}\text { Neuropathic pain } \\
\text { CIPN } \\
\text { Spasticity in MS }\end{array}$ & Yes \\
\hline Conotoxins & - & $\begin{array}{c}\text { Ziconotide } \\
\text { X-MrlA } \\
\text { Contulakin-G } \\
\end{array}$ & $\begin{array}{c}\mathrm{VG} \mathrm{Ca}^{2+} \\
\text { norepinephrine transporter } \\
\text { Neurotensin receptor } \\
\end{array}$ & $\begin{array}{c}\text { Severe chronic pain } \\
\text { N/A } \\
\text { N/A } \\
\end{array}$ & $\begin{array}{c}\text { Yes } \\
\text { Phase II } \\
\text { Phase Ib } \\
\end{array}$ \\
\hline Spider Venom & N/A & Pha1ß & $\mathrm{VG} \mathrm{Ca}^{2+}$ Channels & N/A & Pre-clinical \\
\hline Opioids & $\begin{array}{c}2^{\text {nd }} \text { line: non-cancer } \\
\text { chronic pain } \\
1^{\text {st }} \text { line: cancer chronic } \\
\text { pain }\end{array}$ & Morphine, Fentanyl, Sufentanil & $\mu$ receptor & $\begin{array}{l}\text { Non-cancer chronic } \\
\text { pain, } \\
\text { Cancer chronic pain }\end{array}$ & Yes \\
\hline
\end{tabular}

Due to the complexity of chronic pain states, there is no single treatment that can be prescribed and there are a range of possible first-and second-line therapeutics that can be trialed. Novel targets and drug sources are also currently being investigated to improve on current options.

\section{Abbreviations:}

N/A; Not applicable

TCAs; Tricyclic anti-depressants

SSRIs; Selective serotonin reuptake inhibitors

SSNRIs; Selective serotonin-noradrenalin reuptake inhibitors

VG $\mathrm{Ca}^{2+}$ Voltage gated calcium channels 


\subsection{The Opioid crisis}

Opioids, such as morphine and fentanyl are highly addictive compounds due to their expression in and activation of the reward pathway. This is due to high levels of $\mu$ receptor expression on GABA-ergic neurons in the mesolimbic system, including in the ventral tegmental area (VTA) and nucleus accumbens. The mesolimbic system can also be stimulated via the pre-frontal cortex (PFC), hippocampus and amygdala which also express high levels of $\mu$ receptor. Activation in these areas inhibits the release of GABA causing disinhibition of dopaminergic neurons and increased levels of dopamine in the synaptic cleft (Listos et al., 2019). Increased dopamine within the reward pathway causes the euphoric sensation associated with drug use providing positive reinforcement and increasing the risk of abuse, misuse and addiction (Compton et al., 2006, Di Chiara et al., 1988b).

The highly addictive nature of $\mu$ receptor analgesics coupled with their over-prescription has been a contributing factor to the dramatic increase in the rates of opioid abuse and overdose with both prescription and illicit opioids in the United States. This is due to prescription opioids being a gateway to further illicit drug use and addiction. A comprehensive systematic review of papers published between 2000 and 2013 on opioid use, misuse and abuse in the United States, Denmark, and Norway showed that $21-29 \%$ of patients were abusing and a further $8-12 \%$ were addicted to their prescription opioids (Vowles et al., 2015). Furthermore, 39\% of heroin users in Kings County, Washington (USA) admitted being 'hooked on prescription opioids' before abusing heroin (Peavy et al., 2012). While it was reported that in Cumberland County, Maine (USA), misuse of OxyContin increased the risk of further illicit drug use (Grau et al., 2007). Increased rates of opioid misuse led to over 47,000 opioid related overdoses between 2017 and 2018 alone, 32\% of these deaths involved prescription opioids (Wilson et al., 2020).

There is a large misconception that the opioid epidemic is a problem that is exclusive to the United States. However, in Europe 455,000 patients, out of a total of 22.8 million, prescribed opioids for chronic pain are believed to be dependent upon their medication (Kraus et al., 2016). There is an estimated opioid death rate of 1.8 per 100,000 people averaged over 30 European countries (Marotta et al., 2018). In Australia, the rate of prescription opioid overdose deaths has increased by 2.5 fold from 2001-2012, with more deaths attributed to prescription opioids than heroin (Roxburgh et al., 2017). Rates of opioid use, misuse and abuse are not readily available for the New Zealand population. However, Adamson et al. (2012) estimated the nationwide abuse of opioids to be 9142 individuals or $0.325 \%$ of the adult population. This information was obtained via snowball sampling of people receiving opioid substitution treatment. New Zealand has also seen a spike in the number of opioid overdose deaths. Between 2001 and 2012 there was a 33\% increase in opioid overdoses, with more than half (178 of 325 deaths) of these deaths classified as an accidental overdose of prescription opioids prescribed by a healthcare official including methadone, morphine, or codeine (Shipton et al., 2017). The number of patients misusing their prescription opioids and increasing numbers of opioid 
related deaths worldwide highlights the necessity for developing safer, non-addictive analgesics for the treatment of chronic pain.

\subsection{Mu Opioid Receptors}

\subsubsection{Mu Opioid Receptor Identification}

The $\mu$ receptor, identified in 1976 and was later identified as a (Martin et al., 1976). Opioid receptors are seven-transmembrane domain receptor that belongs to the class $A$, rhodopsin-like, subfamily of the GPCRs. The $\mu$ receptor selectively couples to the $\mathrm{G}_{\mathrm{i} / 0}$ heterotrimeric $\mathrm{G}$-proteins to translate extracellular signals to intracellular responses (Fredriksson et al., 2003, Milligan et al., 2006). It is encoded by the gene Opioid Peptide Receptor Mu (OPRM) in humans (Bare et al., 1994, Mestek et al., 1995, Wang et al., 1994), mice (Kaufman et al., 1995, Min et al., 1994), and rats (Chen. et al., 1993). Comparison of the genetic sequence of the four main opioid receptors, namely kappa ( $\mathrm{k}$ receptor), $\mu$ receptor, delta ( $\delta$ receptor), and nociceptin opioid peptide (NOP) receptors reveals a $75 \%$ homology in the transmembrane domains and a 65\% homology in the intracellular loops. The largest sequence divergence occurs in the $\mathrm{N}$ - and C-terminal domains and the extracellular loops, with only $37 \%$ sequence homology, allowing for selectivity of different endogenous peptides.

Many physiological systems are regulated by opioids that activate the $\mu$ receptor including the hypothalamic-pituitary-adrenal axis involved in stress response (Bali et al., 2015), immune function (Bhargava et al., 1994, Simpkins et al., 1984), the reward pathway (Akil et al., 1997, Belluzzi et al., 1977), and responses to pain (Basbaum et al., 1984, Büscher et al., 1976). The multifaceted physiological roles of $\mu$ receptor are due to their expression throughout the CNS and peripheral nervous systems. In the peripheral nervous system, $\mu$ receptors are highly expressed on the primary afferent fibres and in the DRG (Fields et al., 1980, Stein et al., 1989). High levels of $\mu$ receptor can also be found in the adrenal glands of humans (Peng et al., 2012), and in the submucosal and myenteric plexus of the enteric nervous system in dogs (Allescher et al., 1989), rats (Bagnol et al., 1997) and humans (Lupp et al., 2011, Peng et al., 2012, Sternini et al., 2004). $\mu$ receptors are highly expressed in the CNS of humans and rodents. In situ hybridisation studies in rats showed abundance in the anterior cingulate, frontal parietal cortex, piriform cortex, striatum, hippocampus, nucleus accumbens, amygdala, locus coeruleus, PAG, and Laminae I-II of the dorsal horn of the spinal cord (Delfs et al., 1994, Maekawa et al., 1994, Mansour et al., 1987). In humans, expression of the gene that encodes the $\mu$ receptor (opioid peptide receptor mu 1: OPRM1) has been identified in the cerebellum, nucleus accumbens, caudate nucleus, and spinal cord (Peng et al., 2012). In Situ, hybridisation methodologies have enabled the accurate location of OPRM1 in the human brain to include the cortex, hippocampus, nucleus accumbens, striatum, thalamus, hypothalamus, substantia nigra, PAG, and cerebellum (Peckys et al., 1999). 
$\mu$ receptor are located on a range of neuronal types in a tissue dependent manner. In the DRG and dorsal horn of the spinal cord, $\mu$ receptor are present on peptidergic C-fibre neurons and excitatory interneurons (Kemp et al., 1996). While in the brain they are present on GABAergic (Ben Hamida et al., 2019, Vaughan et al., 1997), noradrenergic, dopaminergic (Li et al., 2016, Margolis et al., 2014), and serotonergic (Berger et al., 2006) neurons. 


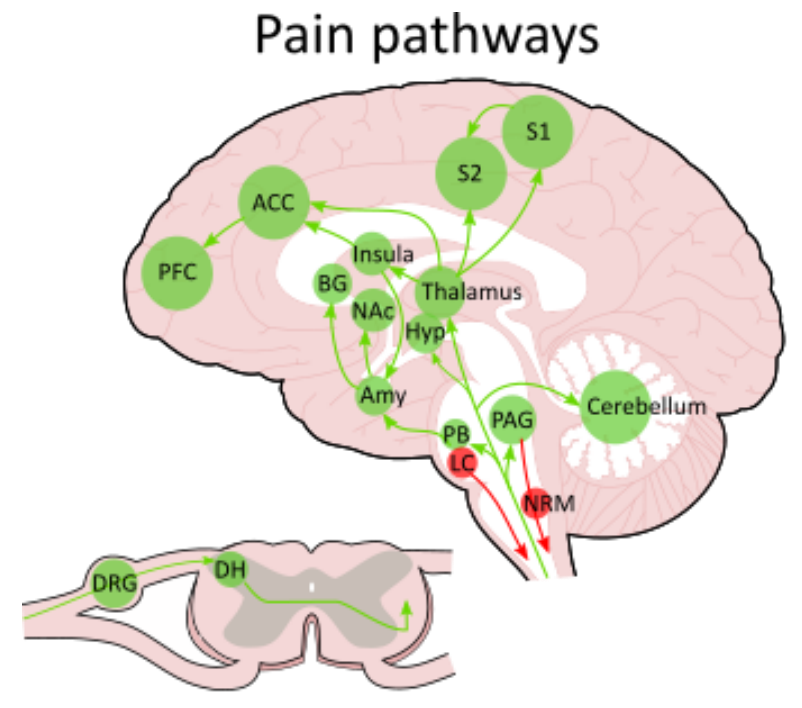

Ascending pathways

Descending pathway

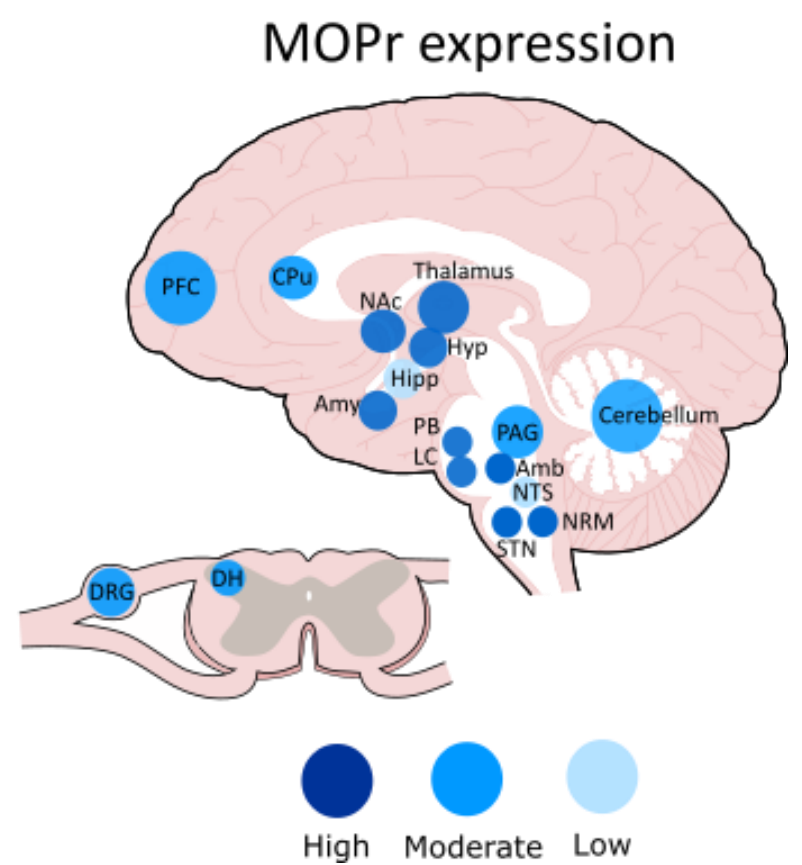

High Moderate Low

Figure 1.3 Expression Levels of the Mu Opioid Receptor in the CNS

There are many areas within the brain and spinal cord associated with the ascending and descending pathways. These include the DRG, PAG, amygdala, hypothalamus, thalamus, and PFC. The expression of $\mu$ receptor heavily overlaps with these regions with moderate to high levels of expression in the DRG, $D H, L C, P A G$, hippocampus and hypothalamus. Figure adapted from Cahill et al. (2014) with $\mu$ receptor expression based on RT-PCR, radioligand binding, and in situ hybridization in humans and rodents Delfs et al. (1994), Mansour et al. (1987), Peckys et al. (1999), Peng et al. (2012).

Abbreviations: ACC; anterior cingulate cortex, $A M B$; nucleus ambiguous, Amy, amygdala, $C P u$; caudate putamen, DH; dorsal horn, DRG; dorsal root ganglion, Hippo; hippocampus, Hypo; hypothalamus, LC; locus coerulus, NAc; nucleus accumbens, NRM; nucleus raphes medianus, NTS; nucleus tract solitarius, PAG; periaqueductal grey, PB; parabrachial nucleus, S1; primary somatosensory cortex, S2; secondary somatosensory cortex, STN spinal trigeminal nucleus 


\subsubsection{Endogenous Role of the Mu Opioid Receptors in Pain Transmission}

The endogenous peptide ligands for the opioid receptors are cleaved from three precursors, proenkephalin, prodynorphin, and proopiomelanocortin, which go on to form multiple endogenous opioids. Proopiomelanocortin is produced in the arcuate nucleus and nucleus of the solitary tract (Bloom et al., 1978). Cleavage into proopiocorticotropin results in the formation of the endorphin peptide family, including $\beta$-endorphin which show selectivity for the $\mu$ receptor (Akil et al., 1997). The cleavage of proenkephalin into Met- and Leu-enkephalin also results in endogenous opioids with high affinity for $\mu$ receptor and $\delta$ receptor (Akil et al., 1997, Field et al., 1997, Hughes et al., 1975, Noda et al., 1982). These peptides are primarily synthesised in the anterior lobe of the pituitary gland and are released in response to corticotropin-releasing factor in stressful or painful events (Nikolarakis et al., 1986). When administered intracerebrally (i.c), $\beta$-endorphin induced potent anti-nociception in the hot-plate, tail-flick, and acetic acid writhing tests in mice with a potency $18-33$ times greater than morphine (Loh et al., 1976). Intraventricular (i.v.c) injection in rats and hamsters also showed dose dependent increases in anti-nociception in the tail-flick assay (Tseng et al., 1979). The enkephalins have also been shown to induce anti-nociceptive effects in rats and mice after i.c.v administration (Belluzzi et al., 1976, Büscher et al., 1976). When the gene for the precursors of $\beta$-endorphins (Pomc/- Jackson Laboratory: B6.129S2-Pomc ${ }^{\text {tm1Low }} / \mathrm{J}$ ) and enkephalin (Penk- ${ }^{- \text {; }}$ Jackson Laboratory: B6.129Penk-rs ${ }^{\text {tm1Pig/J }}$ ) were deleted in vivo, the animals responded normally to morphine with antinociception induced in the hot-plate and acetic writhing assays (König et al., 1996, Rubinstein et al., 1996). However, in Pomc ${ }^{-1}$ mice, mild swim-stress fails to induce anti-nociceptive effects unlike wildtype (Wt) mice (Rubinstein et al., 1996). The Penk ${ }^{-1-}$ also exhibited differences to controls in supraspinal (hot-plate), but not spinal (tail-flick, intraplantar formalin) mediated anti-nociception assays (König et al., 1996). Together, these results indicate the importance of both the endorphins and enkephalins in the endogenous pain pathways.

Two other endogenous ligands have also been identified in the bovine and human brain, endomorphin 1 and 2 (Hackler et al., 1997, Zadina et al., 1997). When given intrathecally (i.t) or i.v.c they produce anti-nociception in both the hot-plate and tail-flick assays in mice (reviewed in Tseng, 2002). However, the precursor gene for these two $\mu$ receptor selective opioid peptides has never been identified and their legitimacy has been questioned (Terskiy et al., 2007).

The generation of a $\mu$ receptor knock out ( $\mu$ receptor ${ }^{-1}$ ) mouse (available at the Jackson Laboratory; OPRM $1^{\text {tm1kff }}$ ) also helped to establish the role of the $\mu$ receptor system in anti-nociception. By knocking out the OPRM1 gene and assessing the behavioural effects of morphine, Matthes et al. (1996) were able to show that the $\mu$ receptor was the target for the anti-nociceptive properties of morphine. In response to the endogenous ligands, it was also shown that there was an increased sensitivity to hot-plate, tail pressure and intraplantar formalin tests in $\mu$ receptor/- compared to $\mathrm{Wt}$ 
littermate controls (Martin et al., 2003). This increase in sensitivity with the loss of the $\mu$ receptor is most-likely due to the large expression of the receptors in descending pain pathway, including the PAG, rostral ventromedial medulla, the dorsal horn of the spinal cord. The $\mu$ receptor is also located within the ascending pain pathways, including in the primary afferent fibres, DRG, the hypothalamus, thalamus, PFC, and the amygdala where they act to stop the transmission of the nociceptive signal from the periphery to the CNS (Peckys et al., 1999, Peng et al., 2012) (expression levels summarised in Fig. 1.3). The expression within the pain pathways and the effects of removing the endogenous opioids and/or $\mu$ receptor indicate that the activation of the descending pain pathway is depended on the $\mu$ receptor and its downstream effects.

\subsubsection{Monomeric Mu Opioid Receptor Signalling Pathways}

\subsubsection{G-Protein Signalling Pathway}

Binding of agonists to the $\mu$ receptor results in the activation of the canonical cell signalling pathway via the phosphorylation multiple serine/threonine on the cytoplasmic terminal. This can include the GRK2/3 mediated phosphorylation of $\mathrm{Ser}^{375}$ resulting in a flow on cascade resulting in the phosphorylation of $\mathrm{Thr}^{370}$, $\mathrm{Thr}^{376}$, and $\mathrm{Thr}^{379}$. However, phosphorylation can also occur at $\mathrm{Thr}^{370}, \mathrm{Ser}^{363}$ , $\mathrm{Ser}^{354}, \mathrm{Thr}^{375}, \mathrm{Thr}^{370}$, and $\mathrm{Thr}^{394}$ in response to a wide range of kinases that includes CAMKII, PKA, and PKC (Deng et al., 2000, Feng and Wang., 2011, Illing et al., 2014, ; Koch et al., 1997; Kovoor et al., 1997, Pak et al., 1997; Wolf et al., 1999, 1998 , Zhang et al., 1996). Once these residues have been phosphorylated the $\mathrm{G}_{1 / 0}$ protein subunit can be activated by the exchange of guanosine diphosphate for guanosine triphosphate (GTP), leading to dissociation of the heterotrimeric $G$ Protein into the $G_{\alpha}$ and $G_{\beta \gamma}$ subunits. Each subunit functions independently have differing effects. The $G_{\beta \gamma}$ subunit stays associated with the cell membrane to interact directly with G-protein gated inwardly rectifying potassium (GIRK) channels ( $\mathrm{Kir}_{3}$ ), upregulating their activity causing to induce $\mathrm{K}^{+}$efflux. (Huang et al., 1995, Kahanovitch et al., 2014, Sadja et al., 2003, Torrecilla et al., 2002). The $G_{\beta \gamma}$ also decrease the pore opening on the VG $\mathrm{Ca}^{2+}$ channels to reduce $\mathrm{Ca}^{2+}$ conductance (Bourinet et al., 1996, Ortiz-Miranda et al., 2005, Zamponi et al., 1998). This changes the Membrane Potential resulting in hyperpolarisation and decreased neuronal excitability.

The $G_{\alpha i}$ subunit remains associated with the cell membrane via palmytoilation upon $\mu$ receptor activation where it inactivates adenylyl cyclase (AC) to inhibit the formation of cyclic adenosine monophosphate (CAMP). CAMP is a second messenger molecule that regulates $\mathrm{Ca}^{2+}$ influx and activates protein kinase A (PKA) to regulate cAMP Response Element Binding Protein (CREB). Therefore, when CAMP is inhibited, the cAMP dependent $\mathrm{Ca}^{2+}$ influx, together with PKA activation and CREB phosphorylation is also inhibited (Hsia et al., 1984, Minneman et al., 1976, Taussig et al., 1993).

Furthermore, $\mathrm{G}$ protein activation can induce the activation of mitogen-activated protein kinase (MAPK) pathways, including extracellular regulated protein kinase $1 / 2$ (ERK1/2) via the $G_{\beta \gamma}$ 
subunit (Belcheva et al., 1998, Li et al., 1996, Romero et al., 2012). Once activated, a subset of phosphorylated ERK (pERK) is translocated to the nucleus where it regulates the activity of transcription factors involved in cell survival, division, and motility. Cytoplasmic substrates of pERK1/2 include phosphoprotein phosphatases, cAMP phosphodiesterases, cytoskeletal proteins, apoptotic proteins, and regulatory cell signalling proteins to further influence cell motility, growth, proliferation and survival (Roskoski, 2012). Overall the effect of this signalling cascade is neuronal hyperpolarisation and anti-nociception. 


\subsubsection{1. $\beta$-arrestin2 Signalling Pathway}

$\beta$-arrestin is a family of four semi-bisymmetric scaffold proteins that aid in the transmission of signalling cascades from the plasma membrane to intracellular responses. The first two members of the family are arrestin1 and arrestin4, also known as the visual arrestins as they are almost exclusively expressed in the retina (Kuhn, 1978). $\beta$-arrestin1 (arrestin2) and $\beta$-arrestin2 (arrestin3) are ubiquitously expressed throughout the body and can interact with heteromeric GPCRs to inhibit Gprotein signal transduction and physically scaffold other signal transduction proteins. With the $\mu$ receptor able to recruit both $\beta$-arrestin1 and $\beta$-arrestin2 in an agonist dependent manner as a part of the non-canonical pathway (Bohn et al., 2004, Groer et al., 2011).

Once recruited to the $\mu$ receptor, $\beta$-arrestin aids in its desensitisation and inhibits continuous G-protein signalling. This occurs in a two-step process whereby the active receptor is phosphorylated by a second messenger associated kinase, protein kinase $C$ (PKC), protein kinase $A(P K A)$, or G-protein receptor kinases 2 and 3 (GRK) (Shukla et al., 2011, Terman et al., 2004, Wang, 2000). The cytoplasmic C-terminal is differentially phosphorylated by these kinases in an agonist dependent manner, with over 20 identified sites of phosphorylation reviewed in (Koch et al., 2008). More recently, phosphospecific anti-bodies and mutagenesis of the Ser/Thr residues of the C-terminal have both identified the $\mathrm{Thr}^{370}$ and $\mathrm{Ser}^{375}$ residues as potential phosphorylation sites involved in the recruitment of $\beta$ arrestin2 to $\mu$ receptor (Doll et al., 2011, El Kouhen et al., 2001). Once $\beta$-arrestin2 has been recruited to the $\mu$ receptor, the $C$-terminus binds directly to the heavy chain and $\beta$-adaptin subunit of the adapter-protein 2 (AP2) targeting the $\mu$ receptor to clathrin-coated pits (Laporte et al., 2000, Laporte et al., 1999, Zhang et al., 1996). This ultimately leads to internalisation of $\mu$ receptor in clathrin-coated vesicles. Once $\mu$ receptor are internalised, the two possible fates are recycling to the membrane or degradation. Receptor recycling occurs via the de-phosphorylation of receptors by GPCR specific phosphatases (GRP) that pass the endosome from Rab5 to Rab4 positive compartments (Sonnichsen, 2000). The Rab4 positive endosome then returns the receptors to the cell membrane (van der Sluijs et al., 1992, Zerial et al., 2001). In the absence of GRP de-phosphorylation, the $\mu$ receptor remains bound to $\beta$-arrestin2 and the endosome Rab5 positive, this leads to the formation of the late endosome, targeting to lysosomes and receptor degradation (Mousa et al., 2013). The coupling of $\beta$ arrestin2 to MOR's is also believed to cause functional uncoupling of G-proteins from the GPCR to inhibit the G-protein signalling cascade (Lefkowitz, 1998, Ren et al., 2005).

More recent advances in $\mu$ receptor signalling have revealed that $\beta$-arrestin can also link GPCRs to other effector molecules such as the Scr family kinases and MAPK pathways. In particular, they activate ERK1/2, JNK3 (c-Jun N-terminal kinase 3) and p38 to send G-protein independent signals that lead to receptor desensitization and trafficking via translational and cytoplasmic changes (Hanson et al., 2008, Luttrell et al., 1999, Seo et al., 2011, Yamdeu et al., 2011). 
A)

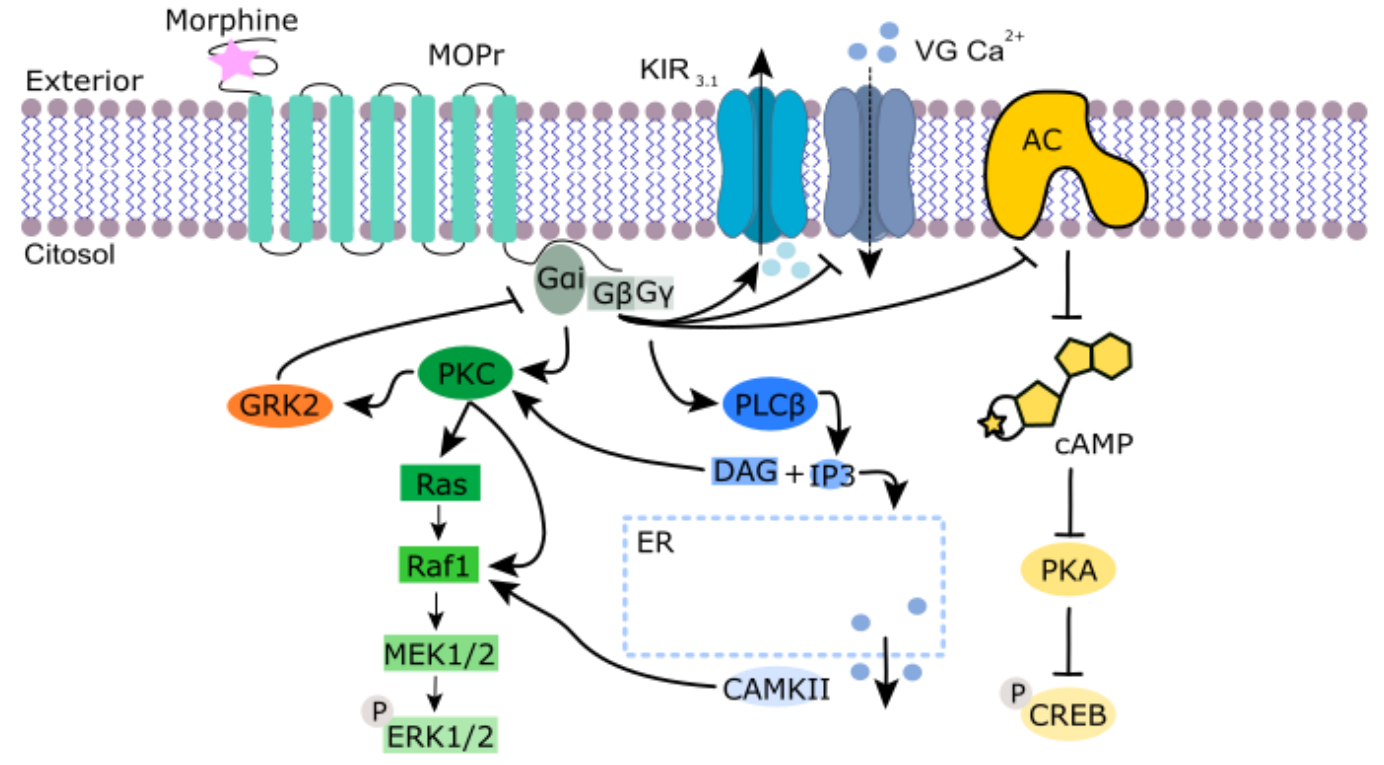

๗ั

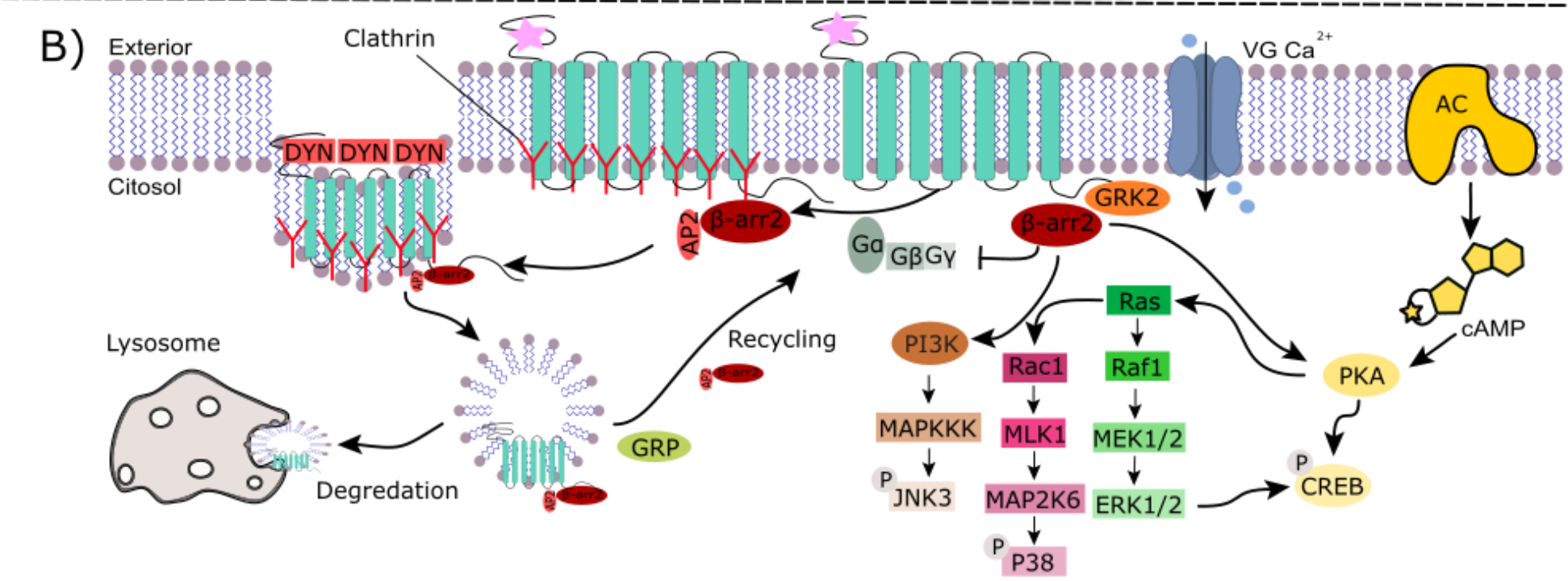


Figure 1.4 Mu Opioid Receptor Cell Signaling Pathways

Binding of $\mu$ receptor ligands to the receptor results in a conformational change that allows the activation of both the (A) G-protein and (B) $\beta$-arrestin2 pathways. The G-protein pathway causes the hyperpolarisation of the membrane and analgesia. Autoregulation of the pathway occurs via recruitment of the scaffold protein $\beta$-arrestin. This pathway causes the direction of receptors to clathrin-coated pits for endocytosis inducing either acute or long-term tolerance.

(Al-Hasani et al., 2013, Williams et al., 2013).

\section{Abbreviations:}

AP2 = adaptor protein 2

$C A M=$ calmodulin

CAMKII = calmodulin-dependent protein

kinase II

$D A G=$ diacylglycerol

$D Y N=$ dynamin

w $\quad G R K 2=G$-protein regulator kinase 2

$G R P=G$-protein regulator phosphatase

IP3 = Inositol triphosphate

$P I 3 K=$ phosphoinositide 3 kinase

$P K A=$ protein kinase $A$

$P L C=$ protein kinase $C$

$P L C \beta=$ phospholipase $C \beta$ 


\subsubsection{Opioid Dimerization and Differential Cell Signalling}

GPCR's have been shown to exist as dynamic protein complexes that form as a result of dimerization or oligomerization. This is known to occur in two forms, homodimers (same receptor) and heterodimers (different receptors). The dimerization of opioid receptors has generated interest as a potential target for the development of novel opioid therapeutics. The research into opioid receptor dimerization has been pioneered by Devi and colleagues. They initially discovered the homodimerization of the $\delta$ receptor and its heterodimerisation with the $\mathrm{k}$ receptor leading to altered signalling properties (Cvejic et al., 1997, Jordan et al., 1999). Since then, the $\mu$ receptor has shown to dimerise with $\mu$ receptor, $\delta$ receptor, $\mathrm{k}$ receptor, cannabinoid receptor 1 (CB1), and Neurokinin 1 receptors (Pan et al., 2002, Pfeiffer et al., 2002, Rios et al., 2006, Wang et al., 2005a).

While the $\mu$ receptor has been shown to form heterodimers with opioid-like receptor 1 and $\mathrm{k}$ receptor, the most studied heterodimer is $\delta$ receptor $-\mu$ receptor. Several pharmacological studies have shown administration of $\delta$ receptor agonists could diminish the induction of morphine tolerance and increase anti-nociceptive effects of morphine when co-administered supraspinally. Interestingly effects are decreased when administered spinally suggesting the presence of heterodimers (Abdelhamid et al., 1991, Porreca et al., 1987). This was then shown in vitro when the receptors were co-immunoprecipitated (Gomes et al., 2000) and an increase in bioluminescence energy resonance transfer signal was seen in co-expressing cells (Gomes et al., 2004). The $\mu$ receptor- $\delta$ receptor heterodimers have also been isolated from the spinal cords of Wt mice, but not $\delta$ receptor $\%$ mice (Gomes et al., 2004). An increase in expression levels was also reported in multiple pain associated CNS regions (e.g. RVM, hypothalamus, and DRG) in mice chronically treated with morphine (Gupta et al., 2010). Despite the evidence pointing to their existence and their potential to improve analgesia, the mechanism through which this occurs is not known. Current evidence has shown that in the presence of $\delta$ receptor specific agonists and antagonist the binding of morphine and other $\mu$ receptor agonists is enhanced, as is ERK1/2 phosphorylation (Gomes et al., 2000), G-protein recruitment, the inhibition of cAMP (Gomes et al., 2004), and changes in $\mathrm{Ca}^{2+}$ signalling (Charles et al., 2003). These results indicate that both the activation and inhibition of the $\delta$ receptor in the heterodimer enhances $\mu$ receptor coupling to the $\mathrm{G}_{\mathrm{i} / \mathrm{o}}$ protein and therefore the anti-nociceptive effects of $\mu$ receptor agonists.

The $\mathrm{k}$ receptor- $\mu$ receptor is another heterodimer that may hold the potential to induce potent anti-nociception with reduced side effects, with the angiogenic and depressive effects of $k$ receptor agonism negating the addictive effects of $\mu$ receptor agonists. Many dual agonists have been generated to target the $\mathrm{k}$ receptor $-\mu$ receptor heterodimer with a mix of full agonism, partial agonism, and antagonism of one or both receptors have shown potent anti-nociceptive effects with reduced side effects including hyperlocomotion and lowering morphine-induced conditioned place preference and tolerance, while also reducing the rewarding effects of cocaine and heroin (Brice-Tutt et al., 2020, 
Ferracane et al., 2020, Stevenson et al., 2004, Wang et al., 2009). This includes the partial k receptor agonist - partial $\mu$ receptor agonist/antagonist nalmefene. Human clinical trials in which morphine and nalmefene were co-administered revealed an improved analgesia post-surgery for males, while also reducing the tolerance and abuse liability of morphine (Crain et al., 2000). While there is little evidence of $\mu$ receptor and $\mathrm{k}$ receptor co-activation and the effect on downstream signalling pathways, the two receptors are known to co-localise within the spinal cord and DRG of male and female rats (Chakrabarti et al., 2010, Ji et al., 1995) Moreover, their co-expression increased morphine's anti-nociception in the females (Liu et al., 2011). Indicating that the improved behavioural profile of dual agonists is likely due to their co-activation and potential alteration of subcellular signalling within the CNS.

Heterodimerisation of $\mu$ receptor has also been shown to occur with non-opioid GPCR. This includes the CB1 receptor, where the increase in potency of morphine when co-administered with $\Delta 9$ tetrahydrocannabinol is believed to be due to heterodimer formation (Cichewicz, 2004). Increased bioluminescence resonance energy transfer and fluorescence resonance energy transfer signals were seen in vitro when $\mu$ receptor and CB1 receptors were co-expressed suggesting the formation of heterodimers (Hojo et al., 2008, Rios et al., 2006). Functionally, this significantly reduced the levels of ERK1/2 phosphorylation in response to activation of both receptors (Rios et al., 2006). While NK1 and $\mu$ receptor have been shown to heterodimerise in vitro, no effect on ligand binding or signalling was observed. However, activation of the NK1 receptor with substance P or the NK1 selective ligand, L779,976, resulted in the phosphorylation and internalisation of both receptors (Pfeiffer et al., 2003, Pfeiffer et al., 2002). The ability of NK1 antagonists to reduce the development of anti-nociceptive tolerance in rats led to the suggestion that a combined NK1 antagonist- $\mu$ receptor agonist could improve the treatment of neuropathic pain (Kalso, 2005, Powell et al., 2003).

\subsection{Biased Agonism}

\subsubsection{Evolution of GPCR Ligands and Pharmacology}

As our knowledge on receptor activation and signal transduction has advanced, so have the theories and models to explain these advancements. This includes the traditional pharmacology theory of the two-state model, orthosteric and allosteric modulators, and the discovery of inverse agonists. More recent advancements have led to the formation of the theory of biased agonism to explain the ability of receptors to selectively couple to more than one transduction pathway (Fig.1.5).

\subsubsection{Traditional Pharmacology}

Initially, receptors were thought to be a simple "on"/"off" switch that allowed for the activation of a single signal transduction pathway via a conformational state shifting, known as the two-state model. This accounted for the known ligands that bound the orthosteric, or active, binding sites. Full agonists at the orthosteric sites bound to target receptors to elicit the maximal response of a signalling pathway, whereas partial agonists only elicit a submaximal response. Antagonists at the 
orthosteric site, that inhibit the binding of other ligands at the orthosteric site, were also explained by this model. Up until the early 2000s, these were the only known receptor ligands types due to the limitations of the technology at the time (Fig 1.5). With the development of functional receptor screens, the basal activity of receptors was able to be measured. As such ligands that bound to sites other than the orthosteric site, known as the allosteric binding site, were identified. Allosteric ligands can be both full or partial positive or negative modulators that regulate the response to orthosteric ligands and endogenous activity of the receptor. Furthermore, the improvement in ligand screens and cell signalling systems has led to the discovery of inverse agonists. These bind to constitutively active receptors to inhibit or antagonise their activity without affecting ligand-binding interactions (reviewed in Kenakin, 2001) (Fig 1.5). Over the last few decades, advancements in our knowledge of cell signalling have allowed the understanding of receptor pharmacology to develop further. 


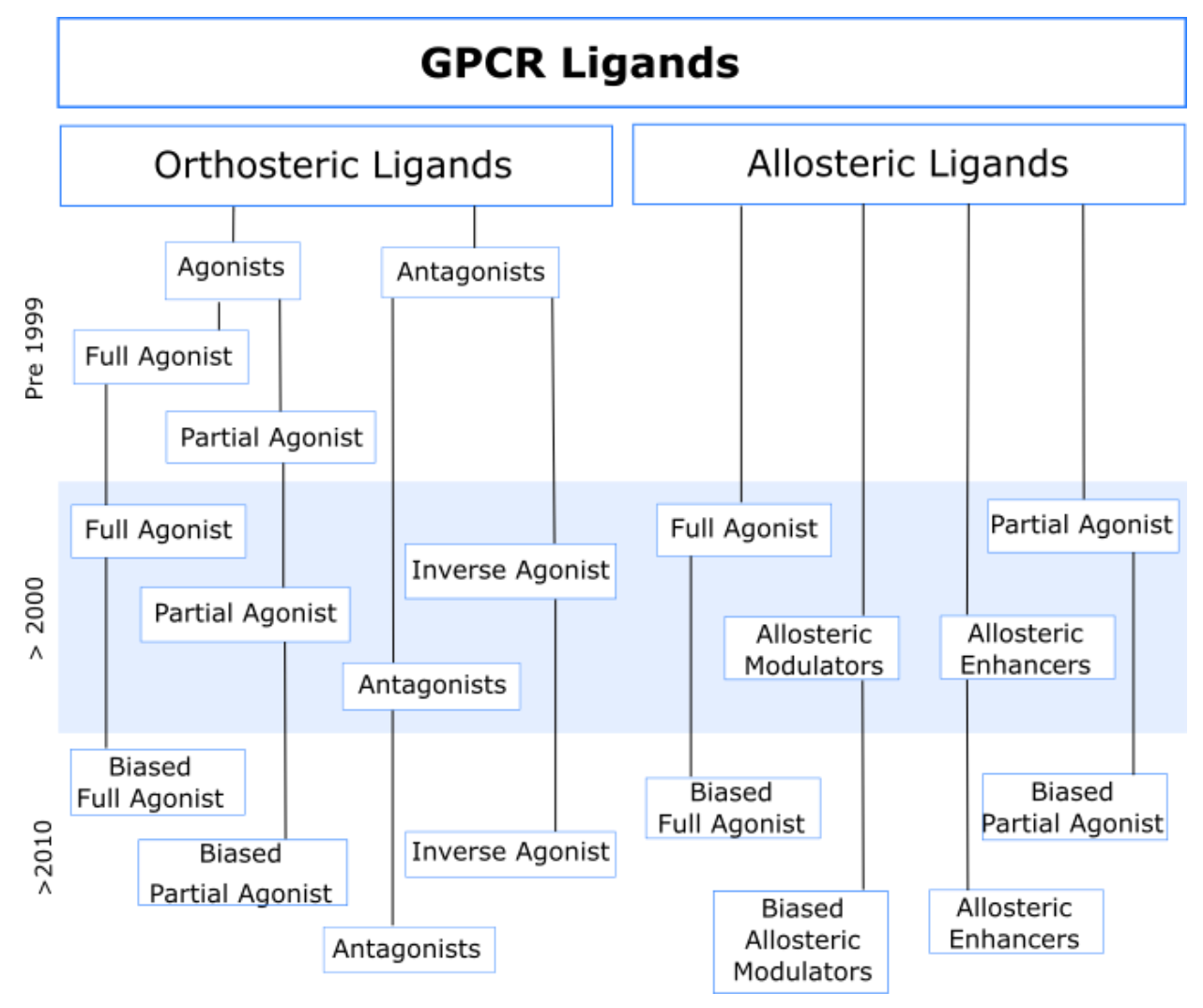

Figure 1.5 The Evolution of GPCR Ligands

Before 1995, the only ligands that were known to bind to GPCR were the agonists and antagonists at the orthosteric binding site. With the development of improved ligand screening in the 2000s, ligands were identified that inhibited the base activity of a receptor, known as inverse agonists. This also allowed for the identification of the allosteric binding site and ligands, including; modulators, enhancers, and partial or full agonists. Since this time screening of biased ligands at both the orthosteric and allosteric binding sites have further added to the GPCR ligand repertoire and increase the ways to take advantage of GPCR signalling complexity. (adapted from Kenakin, 2001). 


\subsubsection{Biased Agonism}

$\beta$-arrestin was first identified by Kühn et al. (1984) as a $48 \mathrm{kDa}$ protein with the ability to bind the rhodopsin receptor in response to light and receptor phosphorylation after recruitment of the Gprotein. This protein was later shown to also 'arrest' the activation of the G-protein pathway (Wilden et al., 1986). These proteins were later identified in other mammalian systems, including $\beta$-arrestin involved in the regulation of the $\beta 2$-adrenergic receptor (Lohse et al., 1990). It wasn't until 1999 that Luttrell et al. (1999) showed that $\beta$-arrestin could act as a scaffold protein to recruit c-Src tyrosine kinase, ERK1/2, JNK3, ubiquitin kinases, CAMP phosphodiesterases and other cytosolic proteins (Beaulieu et al., 2005, Luttrell et al., 2001, McDonald et al., 2000, Perry et al., 2002, Shenoy et al., 2001).

The discovery that receptors were not just a simple "on"/"off" switch for a single pathway, but rather activate multiple signalling pathways in both a ligand and tissue dependent manner increased the complexity of receptor transduction systems (Jin et al., 2001, Laugwitz et al., 1996). It was hypothesised that the differential recruitment of signalling molecules, or "biased agonism", is due to the ability of ligands to stabilize unique receptor conformation states to couple different intracellular signalling pathways (Kenakin, 1995a, b). It was further hypothesized that different pathways induced different physiological responses, providing a cell signalling mechanism that may allow the development of compounds with improved desired effects while reducing on-target side effects. Both orthosteric and allosteric ligands can induce biased signalling at GPCRs. With biased allosteric ligands able to act independently to the activity of the natural ligand and alter the response to the natural ligand when both occupy a GPCR simultaneously (Kenakin et al., 2013, Leach et al., 2015).

\subsubsection{Calculating Bias}

To determine the bias factor of a compound in each receptor-signalling system the ability to activate both pathways in question is assessed via both the maximal effect $\left(E_{\max }\right)$ and the potency at which they are activated ( $\mathrm{EC}_{50}=$ the concentration that produces $50 \%$ maximal response). This data is then entered into one of the many variations of bias equations to determine the relative ability to activate one pathway over another.

The first model used to quantify bias was based on an operational model of pharmacological agonism (Black et al., 1983). This model aimed to describe the agonist-concentration effect curves and behaviour for both pathways in question. The strength of the Black and Leff model is the inclusion of parameters that are receptor ( $\mathrm{K}_{\mathrm{A}}$; agonist-receptor dissociation constant) and cell-receptor $\left(\mathrm{K}_{\mathrm{E}}\right.$ : transduction of agonist-receptor complexes into pharmacological responses, $n$ : transducer slope, $\tau$ : transducer ratio) specific. Therefore, to quantify agonism in this system all ligands must be acting on the same receptor in the same cell system, reducing the introduction of bias as a factor of cell or receptor specific pathway activation (Kenakin et al., 2012). 
Due to the heavy constraints applied to the Black and Leff model, many alternate equations have been designed to reduce constraints and simplify the calculation. This includes the relative intrinsic efficacy (RA) model, which uses the concentration to induce the same response in different pathways. As with the Black and Leff model, the equation includes the $\mathrm{E}_{\max }$ and $\mathrm{EC}_{50}$ values of each pathway relative to a reference ligand (Ehlert et al., 1999, Griffin et al., 2007). This method of calculating bias can be further strengthened by expressing the agonists $E_{\max }$ and $\mathrm{EC}_{50}$ values as a proportion of the maximal response ( $\max$ ) in the system, not the response of the most effective ligand. When this value is compared to the reference ligand, bias can be measured as the anti-log of the values between each pathway (Kenakin, 2017). As the RA models have no specific parameters for cellreceptor variations it allows data from the different receptor and cell types to be entered in the same equation (De Deurwaerdère et al., 2004, Schattauer et al., 2012). This increases the ability to draw data from the literature and removes the need for one group to perform all necessary cell signalling assays to quantify bias.

\subsubsection{The Mechanisms of Biased Agonism}

The best researched, and subsequently predominant, theory into the mechanism through which a biased agonist induces its effects is through the stabilization of differential receptor conformational states. Crystallographic analysis of multiple GPCRs in their active states, including $\mu$ receptor, $\beta 2$ adrenergic receptors $\left(\beta_{2} A R\right)$, and the $M 2$ muscarinic acetylcholine receptor in their active and inactive states reveals a conformational change in the cytoplasmic TM6 domain. Specifically, the TM6 domain moved away from the central helical bundle to create an opening allow G-protein binding (Kruse et al., 2012, Kruse et al., 2013, Manglik et al., 2012). The recruitment of $\beta$-arrestin to the rhodopsin receptor induced a similar movement in the TM6 domain, however, subtle differences were reported in the TM1, 4, 5, and 7 domains. These differences in conformational states may provide a mechanism for the differential recruitment and signalling at GPCR (Kang et al., 2015, Szczepek et al., 2014). Other studies have reported similar trends in the conformational changes of $\mu$ receptor and $\beta_{2} A R$, which are stabilized in response to biased ligands (Okude et al., 2015, Ye et al., 2016). Together, this provides evidence to support the theory that biased agonism is induced by stabilizing different receptor conformational states.

A more recent theory into the mechanism of biased agonism is the theory of "Kinetic Bias". While the importance of kinetic and temporal measures on in vitro receptor behaviour and analysis had been assessed before (Charlton et al., 2010, Unett et al., 2013), the first paper to explicitly discuss the role of "kinetic bias" was published by Herenbrink et al. (2016). The authors believed that the mathematical quantification of bias via equations, such as the Black and Leff operational model and the simplified method for bias quantification, did not sufficiently control for the system and operational bias. This is supported by the inconsistency between published bias factors of the same 
compound. Using the dopamine $D_{2}$ receptor as an example system they showed that biased agonists had reached maximal occupancy significantly slower and exhibited slower dissociation kinetics (Herenbrink et al., 2016). The authors concluded that although conformational stabilization is the underlying fundamental mechanism of bias, ligand binding kinetics and assay endpoints must be taken into consideration when assessing the potential biased agonism of a compound.

\subsubsection{Initial Studies into Biased Agonism of GPCRs}

The phenomenon of biased agonism was initially studied in the $\beta$-adrenergic system in vitro, with phosphorylation and internalization of the $\beta_{2} A R$, a $G_{S}$ coupled GPCR, being dependent on the recruitment of $\beta$-arrestin1 (Freedman et al., 1996, Goodman et al., 1996, Lohse et al., 1990). Despite the in vitro evidence for the role of $\beta$-arrestin1 internalization, a physiological consequence in vivo was identified. Conner et al. (1997) further exploited this through a $\beta$-arrestin1 knockout ( $\beta$-arr $1^{-1}$ ) mouse model and assessed the cardiac responses to $\beta_{2} A R$ agonists. Heart rate and blood pressure remained similar to control mice, but an increase in the ejection fraction was recorded in the 6 -arr $1^{-}$ . Since then, Carvedilol, a clinically available $\beta$-blocker prescribed for heart failure, was found to induced it's well known cardioprotective effects via $\beta$-arrestin1 bias at the $\beta_{2}$ AR (Kim et al., 2008, Wisler et al., 2007).

Many other clinically relevant GPCRs can be targeted with biased agonism (Table 1.5). These include the chemokine receptor CXCR3, which is a $G_{q} G P C R$, involved in the migration of T-cells in response to inflammation and is implicated in allergic contact dermatitis. Small molecule agonists that are biased for the G-protein pathway have been shown to reduce T-cell migration in a mouse model of allergic contact dermatitis. In contrast, $\beta$-arrestin biased small molecules increased T-cell migration. (Smith et al., 2018). G-protein bias at the $\mathrm{G}_{\mathrm{i} / \mathrm{o}}$ cannabinoid and opioid receptors (kappa, mu, and delta) has also shown promise in producing analgesics with reduced side effects (Table 1.5) 


\begin{tabular}{|c|c|c|c|c|c|}
\hline & Biased ligand & Bias & Used to treat & Therapeutic Implications & Stage \\
\hline Adenosine $A_{1}$ & $\operatorname{VCP} 746[1,2]$ & $\begin{array}{c}\mathrm{Ca}^{2+} \\
\text { Signalling }\end{array}$ & $\begin{array}{c}\text { Cardio-protection in ischemic } \\
\text { injury }\end{array}$ & Reduced bradycardia & Pre-clinical \\
\hline $\begin{array}{c}\text { Adenosine } \\
\text { A3 }\end{array}$ & $\begin{array}{c}(\mathrm{N}) \text { methanocarba } 5 \\
\text { uronamide }[1,2]\end{array}$ & $\beta$-arr2/Gi/o & $\begin{array}{c}\text { Cardio-protection in ischemic } \\
\text { injury }\end{array}$ & Improved desensitisation & Pre-clinical \\
\hline $\begin{array}{l}\text { Angiotensin } \\
\quad \text { II } 1\end{array}$ & $\begin{array}{l}\text { TRV120023 [3, 4] } \\
\text { TRV027 [5] }\end{array}$ & $\beta$-arr2 & Cardiovascular diseases & $\begin{array}{l}\text { Reduced cellular injury and preserve renal } \\
\text { function }\end{array}$ & $\begin{array}{c}\text { Pre-clinical } \\
\text { Failed } \\
\text { Phase II }\end{array}$ \\
\hline Apelin & CMF-01 [6] & G-protein & Heart failure & Increased cardiac contractility & Pre-clinical \\
\hline $\begin{array}{c}\boldsymbol{\beta 2 -} \\
\text { adrenergic }\end{array}$ & $\begin{array}{l}\text { Carvedilol }[7,8] \\
\text { Nadolol }[9]\end{array}$ & $\begin{array}{c}\beta \text {-arr2 } \\
\text { G-protein }\end{array}$ & $\begin{array}{l}\text { Heart failure } \\
\text { Asthma }\end{array}$ & $\begin{array}{c}\text { Cardioprotective } \\
\text { Reduced hyper-responsiveness }\end{array}$ & $\begin{array}{l}\text { Approved } \\
\text { Phase II }\end{array}$ \\
\hline $\begin{array}{c}\text { Cannabinoid } \\
1\end{array}$ & $\operatorname{LY} 2828360[10,11]$ & G-protein & Neuropathic pain & $\begin{array}{l}\text { Reduced tolerance and attenuates opioid } \\
\text { withdrawal }\end{array}$ & Pre-clinical \\
\hline Chemokine & CXCR3; VUF11418 [12] & G-protein & Chronic inflammation & A decrease in T-cell recruitment & Pre-clinical \\
\hline $\begin{array}{c}\text { Dopamine } \\
\text { D1 }\end{array}$ & $\begin{array}{l}\text { Fenoldopam [13] } \\
\text { Noncatechol [14] }\end{array}$ & G-protein & $\begin{array}{c}\text { Hypertension } \\
\text { Parkinson's Disease }\end{array}$ & $\begin{array}{l}\text { Reduced tachycardia and vasoconstriction } \\
\text { No dyskinesia }\end{array}$ & $\begin{array}{l}\text { Approved } \\
\text { Pre-clinical }\end{array}$ \\
\hline D2 & $\begin{array}{c}\text { 94A [15] } \\
\text { MLS1547 [16] }\end{array}$ & $\begin{array}{c}\beta \text {-arr2 } \\
\text { G-Protein }\end{array}$ & $\begin{array}{c}\text { Schizophrenia } \\
\text { Parkinson's disease }\end{array}$ & Reduced extrapyramidal symptoms & $\begin{array}{l}\text { Pre-clinical } \\
\text { Pre-clinical }\end{array}$ \\
\hline Endothelin & Macitentan $[17,18]$ & $\beta$-arr2 & Cancer adjunct & Reduce chemoresistance & Approved \\
\hline $\begin{array}{l}\text { Opioid } \\
\text { Delta }\end{array}$ & BMS 986187 [19] & G-protein & Chronic pain/depression & Reduced side effects & Pre-clinical \\
\hline
\end{tabular}




\begin{tabular}{|c|c|c|c|c|c|}
\hline Kappa & $\begin{array}{l}\text { Nalfurafine }[20,21] \\
\text { Mesyl Sal B [22, 23] } \\
\text { Triazole } 1.1[24,25]\end{array}$ & G-protein & $\begin{array}{l}\text { Anti-pruritic } \\
\text { Anti-addiction } \\
\text { Pain }\end{array}$ & Reduced side effects & $\begin{array}{l}\text { Available } \\
\text { Pre-clinical } \\
\text { Pre-clinical }\end{array}$ \\
\hline Mu & $\begin{array}{c}\text { TRV130 [26-28] } \\
\text { PZM21 [29, 30] } \\
\text { Mitragynine }[27,30]\end{array}$ & G-protein & Pain & Analgesia with reduced side effects & $\begin{array}{l}\text { Approved } \\
\text { Pre-clinical } \\
\text { Pre-clinical }\end{array}$ \\
\hline $\begin{array}{l}\text { Serotonin } \\
5-\mathrm{HT}_{1 \mathrm{~A}}\end{array}$ & $\begin{array}{c}\text { F15599 }[29,30] \\
\text { Befiradol }[29,30]\end{array}$ & $\begin{array}{c}\text { ERK1/2 } \\
\text { Go G- } \\
\text { protein }\end{array}$ & $\begin{array}{l}\text { Rhett's Syndrome } \\
\text { Parkinson's Disease }\end{array}$ & $\begin{array}{c}\text { Reverses breathing deficits, Alleviates } \\
\text { dyskinesia }\end{array}$ & $\begin{array}{l}\text { Phase I } \\
\text { Phase II }\end{array}$ \\
\hline $5-\mathrm{HT} 2_{\mathrm{A}}$ & $\begin{array}{l}\text { Thioridazine [30] } \\
\text { Cyclobenzaprine [30] } \\
\text { Trimipramine [30] }\end{array}$ & PLC via $G_{q}$ & $\begin{array}{l}\text { Anti-psychotics } \\
\text { Anti-depressant }\end{array}$ & Not known & Approved \\
\hline
\end{tabular}

Summary of therapeutic targets of biased agonism and examples of biased agonists at each receptor.

\section{References:}

1. Valant, et al. 2014.

2. Baltos, et al. 2016

3. Boerrigter, et al. 2011.

4. Boerrigter, et al. 2012

5. Tarigopula, et al. 2015

6. Read, et al. 2016.

7. Kim, et al. 2008.

8. Wisler, et al. 2007.

9. Hanania, et al. 2008.

10. Lin, et al. 2018.

11. lyer et al. 2020

12. Smith, et al. 2018.

13. Conroy, et al. 2015

14. Martini, et al. 2019.
15. Urs, et al. 2016.

16. Free, et al. 2014

17. Rosano, et al. 2014.

18. Maguire. 2016.

19. Stanczyk, et al. 2019.

20. Schattauer, et al. 2017.

21. Yagi, et al. 2018.

22. Zhou, et al. 2013.

23. Lovell, et al. 2015

24. Soergel, et al. 2014.

25. Singla, et al. 2019.

26. Kruegel, et al. 2016

27. Manglik., et al. 2016.
28. Kudla, et al. 2019.

29. Váradi, et al. 2016

30. Iglesias, et al. 2016 


\subsubsection{Biased Agonism at the Mu Opioid Receptor}

$\beta$-arrestin2 was first shown to be involved in $\mu$ receptor induced anti-nociception when it was shown that $\beta$-arrestin2 knockout $\left(\beta\right.$-arr $\left.{ }^{-/}\right)$mice had a larger and longer anti-nociceptive effect in the hot-plate test compared to their Wt littermates, while also having a reduced effect on core temperature (Bohn et al., 1999). Further work on the $\beta$-arr $2^{\%}$ line showed a reduction in other side effects, including spontaneous locomotion, constipation, tolerance, and respiratory depression (Bohn et al., 2000, Bohn et al., 2003, Bohn et al., 2002, Raehal et al., 2005). Further, G-protein bias was shown to increase the therapeutic window between the potency in thermal anti-nociception, as measured by the hot-plate and tail-flick assays and respiratory depression as measured by $\mathrm{O}_{2}$ saturation and respiratory frequency. The compound with the highest G-protein bias, namely SR-17018 (bias $=40$ ), exhibited a therapeutic window of 105 , compared to 13 with morphine. Inversely, an increase in $\beta$ arrestin2 bias was reported to correlate to a decreased therapeutic window for the compound SR11501 (bias $=0.81$ ) providing a therapeutic window of 4 (Schmid et al., 2017). Together, this work suggested that generating compounds preferentially activated the G-protein pathways could provide an avenue to generate $\mu$ receptor agonists for chronic pain with reduced side effects and has resulted in the development and screening of hundreds of novel $\mu$ receptor ligands.

To identify compounds that exhibit biased agonism, a variety of methodologies have been employed, including high throughput screening of known compounds, or the generation of novel compounds either synthetically or via chemical modification of scaffold structures. PZM21 is a novel synthetic molecule based on the computational docking of novel structures to the $\mu$ receptor. Quantification of bias showed potent G-protein activation with minimal $\beta$-arrestin2 recruitment (Manglik. et al., 2016). Behavioural investigations have not been conclusive with some publications showing increased analgesic effects with decreases in constipation, respiratory depression, hyperactivity, and abuse potential (Kudla et al., 2019, Manglik. et al., 2016). Conversely, other studies have reported significant respiratory depression and tolerance (Hill et al., 2018, Kudla et al., 2019). Further chemical modifications to the PZM21 structure have been conducted to understand the structure activity relationship between ligands and biological responses to $\mu$ receptor agonists (Ma et al., 2020, Ma et al., 2019).

One of the only $\mu$ receptor $\mathrm{G}$-protein biased agonists to make it to clinical trials is a drug made by Trevena Ltd., TRV130, under the brand name Olinvo $^{\circledR}$ (oliceridine). Pre-clinical cell signalling and behavioural analysis reported that TRV130 was G-protein biased with an improved therapeutic window between analgesic and respiratory depressive effects in mice (Chen et al., 2013). TRV130 proceeded through human clinical trials, before being submitted to the FDA for intravenous use for moderate to severe pain (Singla et al., 2017, Singla et al., 2019, Soergel et al., 2014, Viscusi et al., 2016). The application to the FDA was declined in October 2018, with doubts surrounding safety and the improvement on current therapeutics. This decision was backed by contradictory independent 
pre-clinical research, with prominent induction of respiratory depression and high abuse potential identified (Altarifi et al., 2017, Austin Zamarripa et al., 2018). Recently, Trevena resubmitted its application to the FDA, addressing some of the original concerns (Trevena, 2020). This included the reanalysis of data from the original phase II trials allowing the identification of an improved hypercapnic ventilatory response compared to morphine (Dahan et al., 2020). Further phase III clinical trials were also run, again identifying a clinical improvement in respiratory depression with lower rates of treatment interruption due to the induction of respiratory depression (Ayad et al., 2020). This application has since been approved, under the name Olynvink ${ }^{\circledR}$, for treatment of postoperative pain when no other options are available (FDA, 2020).

\subsection{Salvia Divinorum and its Analogues}

\subsubsection{The Salvia Plant and Active Ingredient}

Salvia divinorum is a member of the Lamiaceae family of sage plants endemic to Oaxaca, Mexico (Wasson, 1962). Traditionally the leaves of the plant were used as a hallucinogen in traditional rituals. They were also commonly used in the treatment of headaches, diarrhoea, and a disease known as panzón de barrego (swollen belly) (Valdes et al., 1983). The active ingredient of S. divinorum was identified as a non-nitrogenous neoclerodane diterpene called salvinorin A (Sal A) (Ortega et al., 1982). Since its discovery, Sal A has been identified as a potent and selective $\mathrm{k}$ receptor agonist, the first nonnitrogenous opioid selective ligand (Roth et al., 2004). Preclinical rodent studies have shown Sal A reduces the rewarding properties of cocaine and has anti-nociceptive properties (Kivell et al., 2014). However, a short duration of action, aversive, pro-depressive, anxiogenic, and hallucinogenic effects mean it can never be used clinically (Butelman et al., 2009, Carlezon et al., 2006, Valdes et al., 1983, Zhang et al., 2005). Despite this, it does provide a novel scaffold for the chemical synthesis of analogues with reduced on-target side effects.

\subsubsection{Structural Analogues of Salvinorin A}

Structural modification of Sal A was performed to produce analogues for the treatment of cocaine addiction and/or non-addictive opioids for chronic pain with reduced side effects. The C2 position was a focus for modification due to its role in ligand binding and ability to improve the pharmacokinetics of the analogues (Beguin et al., 2006, Kane et al., 2006).

One such C2 modification was the addition of a benzoyl group at the C2 position which resulted in a 47-fold loss in $\mathrm{k}$ receptor affinity while increasing the affinity for $\mu$ receptor and $\delta$ receptor by 25 - and 5 -fold respectively (Harding et al., 2005). This compound, herkinorin, was the first nonnitrogenous $\mu$ receptor-selective agonist developed. In vitro assessment via cellular trafficking, biotinylated receptor internalisation, immunofluorescent quantification of cell surface receptors, and pERK1/2 immunoblotting were evaluated as indirect measures of $\beta$-arrestin2 recruitment. This 
revealed herkinorin as a poor recruiter of $\beta$-arrestin2 (Groer et al., 2007, Lamb et al., 2012). In vivo assessment of the anti-nociceptive properties showed a reduction in acute pain in the intradermal formalin assay. Furthermore, herkinorin $(10 \mathrm{mg} / \mathrm{kg})$ induced less tolerance after 5 days of administration than morphine $(10 \mathrm{mg} / \mathrm{kg})$ in the same assay (Lamb et al., 2012). Contralateral injections of herkinorin $(10 \mathrm{mg} / \mathrm{kg})$ did not reduce pain behaviours in the formalin test (Lamb et al., 2012). However, the use of this compound is limited due to its reduced bioavailability and half-life due to its strong similarity to the Sal A structure.

Further structural modification of herkinorin at the C2 position was undertaken to improve the bioavailability, half-life, and central availability and selectivity of herkinorin. This led to the identification of a second non-nitrogenous, MOR-selective agonist, kurkinorin (Crowley et al., 2016). A single degree of saturation, via the addition of a double bond at the $\mathrm{C} 2$ position, distinguishes kurkinorin and caused a $>8,000$ fold increase in the selectivity for the $\mu$ receptor compared to the $\mathrm{k}$ receptor and an increase in potency compared to herkinorin $\left(\mathrm{EC}_{50}=1.2 \pm 0.2 \mathrm{nM}\right.$ and $39.0 \pm 4.0 \mathrm{nM}$ respectively) (Crowley et al., 2016). In vitro analysis of the inhibition of forskolin-induced cAMP accumulation (HitHunter) and $\beta$-arrestin2 recruitment (PathHunter) followed by the simplified bias calculation, showed that kurkinorin was a G-protein biased agonist (bias factor $=0.57$ ).

To further probe the structural activity relationship between the novel $\mu$ receptor scaffold and a reduction in side effects with an increase in G-protein bias, more chemical modifications were made to kurkinorin. The addition of an alcohol group to the C4 position on the benzene group further increased the selectivity by $>10,000$ for the $\mu$ receptor, compared to the $\mathrm{k}$ receptor, potency $\left(E D_{50}=0.03 \pm 0.01 \mathrm{nM}\right)$ and G-protein bias (bias factor $\left.=0.14\right)$, relative to its predecessors (Crowley et al., 2020). This novel $\mu$ receptor agonist, kurkinol, and kurkinorin will be the focus of study in this thesis. 


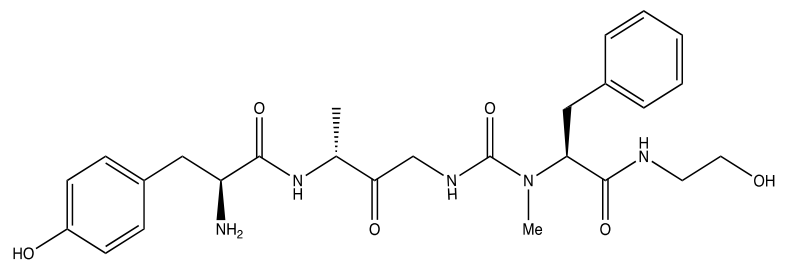

DAMGO*

$\mathrm{EC}_{50}=0.6 \mathrm{nM}$

Selectivity $>16,000$

Bias $=1$

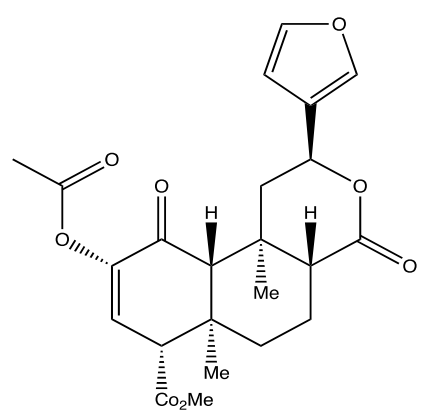

Sal A**

$\mathrm{EC}_{50}=0.21 \mathrm{nM}$

Selectivity $>10,000$

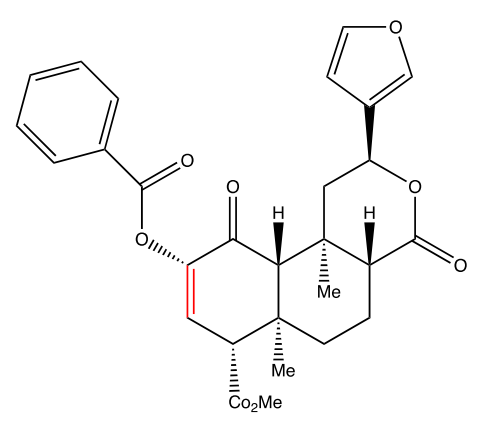

Kurkinorin**

$\mathrm{EC}_{50}=1.2 \mathrm{nM}$

Selectivity $>8,000$

Bias $=0.57$

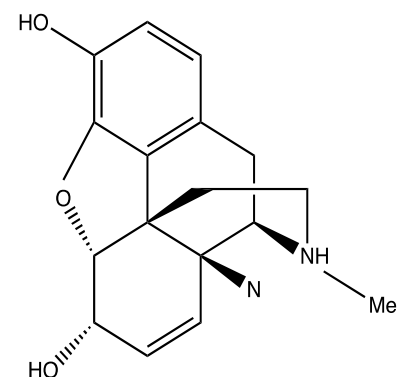

Morphine*

$\mathrm{EC}_{50}=5 \mathrm{nM}$

Selectivity $=65$

Bias $=0.36$

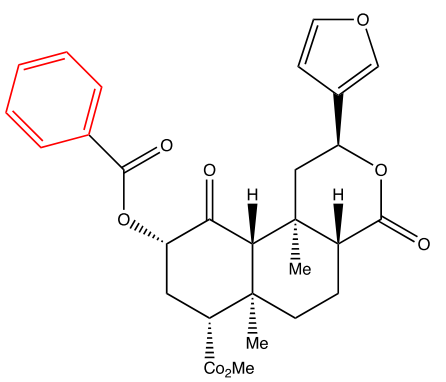

Herkinorin*

$\mathrm{EC}_{50}=40 \mathrm{nM}$

Selectivity $=4.25$

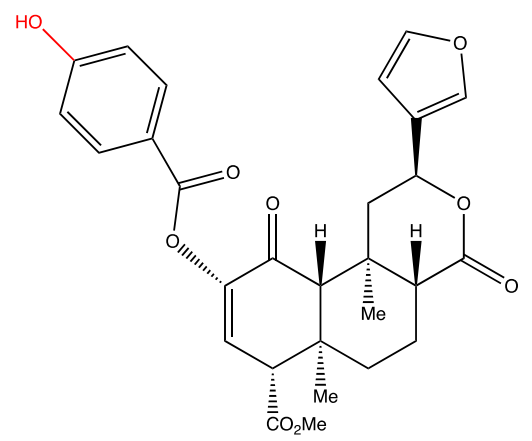

Kurkinol***

$\mathrm{EC}_{50}=0.03 \mathrm{nM}$

Selectivity $>10,000$

Bias $=0.14$

Figure 1.6 Chemical Structure of Known and Novel Mu Opioid Receptor Ligands

The structure of the traditional $\mu$ receptor agonist Morphine and full agonist, DAMGO, both differ significantly to the $\mathrm{k}$ receptor agonist salvinorin A, and its synthetic analogues herkinorin, kurkinorin, and Kurkinol that are selective $\mu$ receptor agonists. $E C_{50}$ values determined from the DiscoverX HitHunter $c A M P$ inhibition Assay in $\mathrm{CHO}$ cells expressing the human $\mu$ receptor. Selectivity for $\mu / \kappa$ determined from the DiscoverX HitHunter CAMP inhibition Assay in CHO cells expressing the human $\mu$ receptor vs. $k$ receptor- $h$.

*(Crowley et al., 2016) **(Sherwood et al., 2017) ***(Crowley et al., 2020). 


\subsection{Hypotheses and Aims}

We, and others, hypothesise that developing $\mu$ receptor agonists that are biased towards the G-protein pathway can lead to the discovery of potent, efficacious analgesics with improved safety profiles. The experiments included within this thesis aimed to investigate the differing pre-clinical antinociceptive profiles of two structurally unique $\mu$ receptor agonists, kurkinol and kurkinorin, that exhibit differing bias factors. We further probed the role of $\beta$-arrestin2 signalling in the behavioural profiles of $\mu$ receptor agonists by utilising the $\beta$-arr $2^{-/}$mice. From previous literature, we hypothesised that increased G-protein bias would correlate to more potent anti-nociceptive effects and reduced side effect induction. The mechanism(s) through which biased agonism results in improved antinociception and side effects were further investigated using in vitro studies to link the role of other subcellular molecules to behavioural changes.

\section{Specific aims:}

1. To evaluate the Sal A analogues, kurkinol and kurkinorin, for their ability to modulate acute thermal anti-nociception and nociceptive-like behaviours in the chemotherapy-induced model of neuropathic pain.

2. To evaluate the side effect profile of kurkinol and kurkinorin in pre-clinical models of tolerance, hyperalgesia, motor coordination impairment, constipation and respiratory depression.

3. To explore the role of $\beta$-arrestin2 in pre-clinical models of anti-nociception and side effects using $\beta$-arrestin2 knockout mice and the novel agonists kurkinol and kurkinorin in comparison to morphine.

4. To evaluate the cell signalling properties of kurkinol and kurkinorin in human $\mu$ receptor $\mathrm{CHO}$ and Hek293 cells to identify other pathways associated with the anti-nociceptive and side effects of kurkinorin and kurkinol. 


\section{Chapter 2. General Methods}

\subsection{Behavioural Experiments}

\subsubsection{Animals}

Adult (8-12 weeks unless otherwise stated) male and female C57 Bl/6 mice (20 - 30 g) were sourced from the centralised animal breeding facility at Victoria University of Wellington (VUW), with the initial breeding pairs originating from the animal facility at Mallaghan Research Institute. Mu-

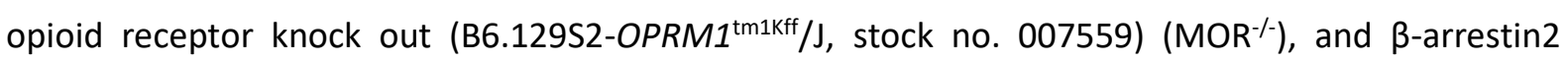
knockout (B6.129X1(Cg)-Arrb2 ${ }^{\text {tmRjl}} / J$, stock no. 023852) $\left(\beta\right.$-arr2 $\left.2^{--}\right)$were purchased from Jackson Laboratory (Maine, USA). The homozygous stock of all genetically modified animals was used to maintain the line, with the homozygous offspring from heterozygous breeding pairs and their $\mathrm{Wt}$ littermates used for experiments.

All mice were housed within the VUW small animal facility in groups of up to 6 mice in closed top cages with enrichment in the form of huts or tubes. Food (Irradiated Rat and Mouse, Specialty Feeds, Western Australia) and water were provided ad libitum except during experimental procedures. The animal housing facility was maintained at a constant temperature of $20-22^{\circ} \mathrm{C}$ and $50 \%$ humidity. Lights were set to a 12 -hr light/dark cycle, with lights on at $7 \mathrm{am}$. All experiments were undertaken at least $1 \mathrm{hr}$ into the light cycle. All procedures were carried out in the presence of white noise to mask the effects of background noise.

Before all behavioural experiments, mice were handled for a minimum of three days to reduce the effect of handling stress. During this time, the mice were habituated to the experimental room for $30 \mathrm{~min}$ in the presence of white noise, followed by $5-15$ mins of habituation to the behavioural being utilised to reduce stress. All experiments were carried out per the New Zealand Animal Welfare Act, 1999, and approved by the VUW Animal Ethics Committee (opioids for chronic pain; approval numbers 21480 (May 2017 - December 2017), 25751 (February 2018 - 2021). Following the three R's efforts were made to reduce animal numbers (Russell et al., 1959). All vehicle-treated animals from rotarod, faecal accumulation, and whole-body plethysmography were reused in subsequent experiments following a 7-day stand-down period. Due to the rapid onset of tolerance following $\mu$ receptor agonist treatment, any animal that received morphine, kurkinorin, or kurkinol was culled after the experiment. To further reduce numbers multiple behavioural measures were assessed within a single experiment where possible. For example, the induction of tolerance and hyperalgesia were assessed within the dose response tail-flick experiment. 


\subsubsection{Drug Preparation}

The novel $\mu$ receptor agonists kurkinorin and kurkinol were kindly supplied by Prof. Thomas Prisinzano (University of Kansas, Lawrence, Kansas, USA). Both compounds were semi-synthesised from Sal A as previously described (Crowley et al., 2020, Crowley et al., 2016). Before in vivo use, all compounds were identified as $>95 \%$ pure via high-performance liquid chromatography. Due to low solubility of the compounds, kurkinorin and kurkinol were dissolved in the solvent (dimethyl sulfoxide) DMSO, before the addition of tween- 80 and $0.9 \%$ saline (ratio of $2: 1: 7$ ). Morphine sulphate solution came pre-dissolved in $0.9 \%$ saline (Hospira, NZ). It was subsequently diluted in DMSO, tween-80, and $0.9 \%$ saline (2:1:7). Gabapentin, a fist line clinical treatment for chronic pain, was purchased from Sigma Aldrich (Auckland, NZ) and dissolved in a 2:1:7 ratio of DMSO, tween-80, and $0.9 \%$ saline respectively. The $\mu$ receptor antagonist $\beta$-funaltraxamine ( $\beta$-FNA) was purchased from Sigma as a solid and was first dissolved in DMSO before the addition of tween-80 and $0.9 \%$ saline. All compounds were injected at a volume of $1 \mu \mathrm{L} / \mathrm{g}$ for i.p. injections and $0.5 \mu \mathrm{L} / \mathrm{g}$ for s.c. injections used in the dose response assays.

\subsubsection{Nociceptive Behavioural Assays}

\subsubsection{Tail-flick}

The hot water tail-flick assay was performed in male and female $\mathrm{C} 57 \mathrm{BI} / 6$ mice, $\mathrm{MOR}^{-1 /}$, and $\beta$ arr2/- mice (8 -16 weeks old, 20 - $30 \mathrm{~g}$ ). Before experimentation, all mice were habituated to the Plexiglas restrainer (internal diameter of $24 \mathrm{~mm}$ ) for a total of $15 \mathrm{~min}$ over three consecutive days. On experimental day 3 , baseline tail-flick latencies were taken by placing the mouse in the Plexiglas restrainer and submerging the last $2 \mathrm{~cm}$ of the tail into a $50^{\circ} \mathrm{C}$ water bath and the latency to elicit a tail withdrawal. This was determined using a manual stopwatch that allowed the measurement of seconds and split seconds. A 10 s cut off was used to avoid tissue damage. Mice with an average baseline tail withdrawal latency of greater than $4 \mathrm{sec}$ were excluded from the experiment. Once baseline recordings had been acquired, mice were administered the test compounds and the latency to elicit a tail-flick measurement determined at regular intervals. The percent maximum possible effect (\% MPE) was calculated as follows;

$$
\% M P E=100 \times\left(\frac{\text { test latency }- \text { baseline latency }}{10-\text { baseline latency }}\right)
$$




\subsection{Time Course}

Following the determination of baselines latencies, mice received a single i.p. injection of morphine (10 or $5 \mathrm{mg} / \mathrm{kg}$ ), kurkinol ( 5 or $2.5 \mathrm{mg} / \mathrm{kg}$ ), or vehicle (DMSO: tween-80: saline, 2:1:7). In experiments where antagonists were used mice received $5 \mathrm{mg} / \mathrm{kg} / \mathrm{s}$.c. $\beta$-FNA 24-hrs before the experiment. The latency to flick was then measured 5, 10, 15, 30, 45, 60, 90, 120, and 150 min postinjection and the \%MPE determined at each time point.

\subsection{Tolerance and Hyperalgesia}

To determine the potency and to evaluate tolerance and hyperalgesia, cumulative within animal dose response evaluations were performed as previously described (Crowley et al., 2016). In brief, escalating doses of $\mu$ receptor agonists was delivered s.c. (vol. of $5 \mu \mathrm{L} / \mathrm{g}$ ) every $30 \mathrm{~min}$ (max effect) and the tail withdrawal latencies determined. As soon as the tail-flick latency was measured, the next dose of the compound or vehicle was administered. To reduce the number of injections at a single site, injections were delivered in the left and right flank, and scruff of the neck. The antinociceptive effects of morphine were assessed at $0.5,1,5,10,15,20,25,30 \mathrm{mg} / \mathrm{kg} / \mathrm{s}$.c., and the more potent kurkinol at $0.1,0.3,0.6,1,3,5,7,9,11 \mathrm{mg} / \mathrm{kg} / \mathrm{s.c}$. The \%MPE was calculated for each dose and a linear regression generated to determine the potency $\left(\mathrm{ED}_{50}\right)$ and $\mathrm{ED}_{80}$ determined using GraphPad Prism V.7 (GraphPad Software Inc., La Jolla, CA, USA). The $\mathrm{ED}_{80}$ doses (morphine $=10 \mathrm{mg} / \mathrm{kg} / \mathrm{s}$.c., kurkinol $=5 \mathrm{mg} / \mathrm{kg} / \mathrm{s}$.c.) were administered daily for 8 days with the induction of tolerance and hyperalgesia evaluated on alternate days (Fig 3.1).

The onset of hyperalgesia was assessed on days $0,2,4,6$, and 8 using the $m V F$ filaments (fibres 2 through 9) (Touch Test Sensory Evaluator Kit for Animal Research, \#58011, Stoelting, IL, USA). The simplified up-down method was used to determine the paw withdrawal threshold (PWT) using the previously described protocol (Bonin et al., 2014). In brief, each filament was pressed at a right angle against the plantar surface of footpad starting with filament 5 . If a positive paw withdrawal was seen, the next lower filament was used. Conversely, the next higher filament is used if the paw is not withdrawn within $3 \mathrm{~s}$. This procedure was continued until 5 measurements had been taken for both left and right hind paws in duplicate. The paw withdrawal threshold was then generated by averaging the withdrawal thresholds of each hind paw.

On day 9 the mice were challenged with a second cumulative dose response assay with the following doses of morphine; $0.5,1,5,10,15,20,25,30 \mathrm{mg} / \mathrm{kg} / \mathrm{s} . c$. and kurkinol; 0.1, 0.3, 0.6, 1, 3, 5, $7,9,11 \mathrm{mg} / \mathrm{kg} / \mathrm{s}$.c.. Linear regression was then performed so that the potency $\left(E D_{50}\right)$ at day 9 could be determined. This was compared to the values seen on day 1 , allowing for anti-nociceptive tolerance to be evaluated. 


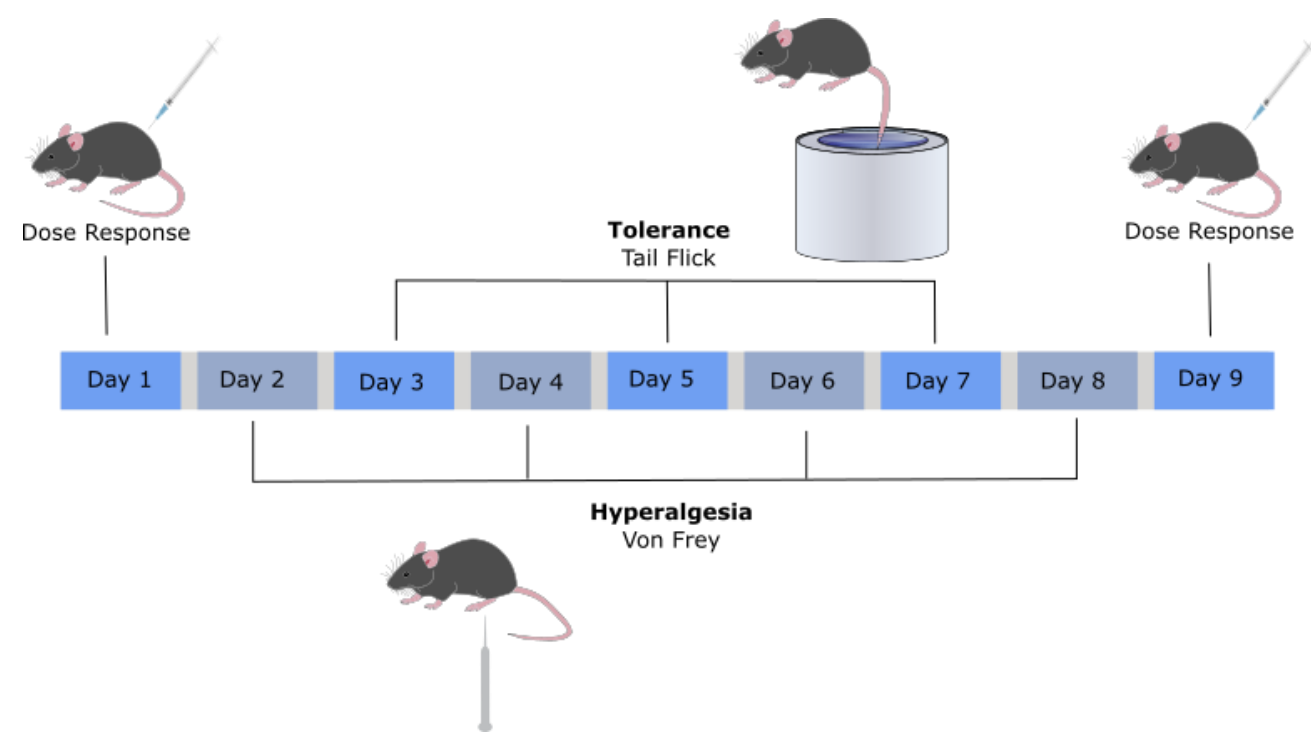

Figure 2.1 Dose response tolerance tail-flick procedure

Changes in tail-flick latency and mechanical allodynia following morphine and kurkinol on days 1 and

9. With the induction of tolerance tracked on days 3, 5, \& 7 and hyperalgesia assessed on day 0, 2, 4, 6, and 8 . 


\subsubsection{Hotplate}

The incremental HotPlate Analgesia Meter (iHAMP) (IITC, Woodland Hills, CA, USA) was set to $50^{\circ} \mathrm{C}$ and the thermal nociceptive threshold determined. Mice were habituated to the Plexiglas enclosure and iHAMP at a non-nociceptive temperature $\left(25^{\circ} \mathrm{C}\right)$ for $10 \mathrm{~min}$ a day for 3 days. On the experimental day, the hotplate was set to $50^{\circ} \mathrm{C}$ and 3 baseline measurements taken. The timer was started when all 4 paws were flat on the hotplate, and stopped when jumping, licking or flicking of either of the hind paws occurred, with a 30 second cut off to avoid tissue damage. Mice with an average baseline of $<5 \mathrm{~s}$ continued to the experiment. Injections of vehicle, morphine (10 or 5 $\mathrm{mg} / \mathrm{kg} /$ i.p.), kurkinorin (10 or $5 \mathrm{mg} / \mathrm{kg} /$ i.p.), or kurkinol ( 5 or $2.5 \mathrm{mg} / \mathrm{kg} / \mathrm{i}$. .p.) were then administered i.p. and measurements taken at $15,30,45,60,90$, and $120 \mathrm{~min}$. The $\%$ change was then calculated as follows;

$$
\% \text { change }=\left(\frac{\text { test latency }- \text { baseline latency }}{\text { baseline latency }}\right) \times 100
$$

\subsubsection{Chemotherapy-Induced Neuropathic Pain}

Mice ( $8-16$ weeks old) were habituated to the transparent plastic chambers on a metal mesh stand for 10 min over 3 consecutive days. On day zero, baseline eVF (mechanical allodynia) and acetone (cold allodynia) measurements were taken in duplicate for both left and right hind paws. The eVF measurements were taken first, with the force $(\mathrm{g})$ required to induce paw withdrawal assessed. Directly followed by the placement of acetone on the plantar surface of the hind paw, where the time responding to evaporation of acetone was recorded. The average of the 4 mechanical and cold allodynia measurements were used as baseline thresholds to assign animals into matched treatment groups, PTX or vehicle. All animals then received an i.p. injection of PTX (4 mg/kg) or vehicle at 10 $\mu \mathrm{L} / \mathrm{g}$. PTX and vehicle were also administered on days 2,4 , and 6 to give a cumulative dose of 16 $\mathrm{mg} / \mathrm{kg}$. The induction of neuropathic pain was tracked with mechanical and cold allodynia on days 0 , $2,4,7,11,13$, and 15, with all behavioural measurements taken before PTX injections (Fig. 3.2).

On day 15 all PTX treated animals were divided into groups matched by mechanical and cold allodynia to ensure even disease induction and ensure no group looked better due to reduced disease induction. Each group was assigned either; vehicle, morphine, gabapentin, kurkinorin, or kurkinol. All treatments were blinded to the experimenter to avoid bias. A within animal cumulative dose response was then performed. Escalating doses were delivered s.c. with a single mechanical and cold measurement taken from the left and right hind paws $30 \mathrm{~min}$ after injections. Doses for each treatment group were as follows; morphine; 1, 5, 10, 15, 20, 25, $30 \mathrm{mg} / \mathrm{kg} / \mathrm{s}$.c. gabapentin; 3, 5, 10, 20, 30, 40 mg/kg/s.c. kurkinorin; 1, 4, 5, 10, 15, 20, 30 mg/kg/s.c. kurkinol; .5, 1, 3, 5, 7, 9 mg/kg/s.c. Vehicle - vehicle mice did not participate in the dose response. 
Following the dose response, the average PWT and time responding to acetone were calculated for each dose and a linear regression generated using GraphPad Prism to generate the $E D_{50}$ and $E D_{80}$ for both measurements. The $\mathrm{ED}_{50}$ dose was then chronically administered daily from day 17 to 37 ( 20 days) with all mice receiving the same compound as in the dose response. Animals were then sacrificed on day 37. The brain, spinal cord and sciatic nerve were collected and banked in OCT in the $-80^{\circ}$ for future analysis of $\mu$ receptor localisation.

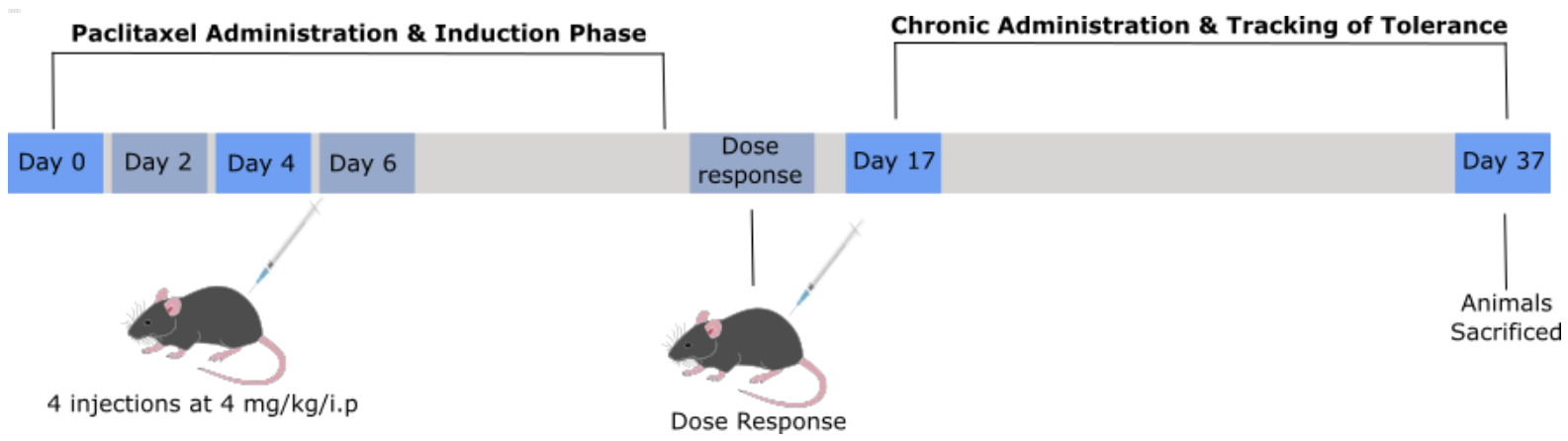

Figure 2.2 Schematic Representation of Chemotherapy-Induced Neuropathic Pain

PTX (4 mg/kg) or vehicle was administered on days $0,2,4$, and 6 with the induction of allodynia to mechanical and cold allodynia tracked on days $0,2,4,6,7,11,13$, and 15. A within animal dose response was conducted on day 15 and the ED 50 dose administered chronically from day $17-37$ to track the induction of tolerance to cold and mechanical allodynia. On day 37 all animals were sacrificed for tissue collection. 


\subsection{Side Effect Behavioural Assays}

\subsubsection{Rotarod}

Motor-coordination impairment was assessed using a 5 lane accelerating rotarod (barrel diameter $30 \mathrm{~mm}$, Harvard Apparatus) set to accelerate from 3-400 rpm over $300 \mathrm{~s}$. Mice were trained in groups of 4, with training sessions carried out in quadruplicate over 5 days (20 training sessions) leading up to the experiment. Training consisted of placing mice on the barrel, set to $3 \mathrm{rpm}$, with the acceleration started after a few seconds. Latency to fall was automatically recorded for each lane, with a maximal time of $5 \mathrm{~min}$. On the experimental day, baselines were recorded in triplicate with the first baseline excluded as a trial, the other baseline measurements were averaged. Any mouse with an average latency to fall under $240 \mathrm{~s}(4 \mathrm{~min}$ ) were excluded from the study. Animals with an average of $>240 \mathrm{~s}$ then received an i.p. injection of vehicle (DMSO: tween-80: 0.9\% saline, 2:1:7) or $\mu$ receptor agonist and measurements taken at 15, 30, 45, 60, 90, 120, 180, 240 min post-injection. The latency to fall following drug administration was calculated for each mouse as follows;

$$
\% \text { baseline }=\left(\frac{\text { test latency }- \text { baseline latency }}{\text { baseline latency }}\right) \times 100
$$

\subsubsection{Gastrointestinal Effects}

\subsubsection{Metabolic Chamber}

Mice were habituated to the metabolic chamber (Techniplast, NSW, Australia) for $1 \mathrm{hr}$ to reduce stress before the experiment, with free access to food and water overnight. On the experimental day, mice were administered either morphine, kurkinol, kurkinorin, or vehicle and were placed in the metabolic chamber with access to water, but not food, for $6 \mathrm{hr}$. The metabolic chamber separates the faeces and urine into two individual cylinders and the content was weighed on an analytical balance every hour for 6 hours. The accumulation of excrement was then plotted as a factor of time.

\subsubsection{Charcoal Meal Assay}

The charcoal meal assay was used to assess the transit through the small intestine as previously described (Raehal et al., 2005). In brief, mice were fasted for $24 \mathrm{hr}$ to ensure the gastrointestinal (G.I) tract was empty by removing access to food and insertion a wire floor (to prevent eating of faeces or bedding). Mice were then administered morphine ( 5 or $10 \mathrm{mg} / \mathrm{kg} / \mathrm{i}$. p.), kurkinol ( 2.5 or $5 \mathrm{mg} / \mathrm{kg} /$ i.p.), kurkinorin ( 5 or $10 \mathrm{mg} / \mathrm{kg} /$ i.p.), or vehicle $20 \mathrm{~min}$ before a bolus of charcoal meal ( $5 \%$ aqueous suspension of charcoal in a $10 \%$ Gum Arabic solution, vol. of $10 \mu \mathrm{l} / \mathrm{g}$ ) was administered by oral gavage. Animals were then euthanised via $\mathrm{CO}_{2}$ asphyxiation, followed by cervical dislocation. The total length of the small intestine was measured (duodenum to ileum) and the distance the charcoal meal travelled determined. Percent transit distance was calculated as follows; 


$$
\% \text { transit }=\left(\frac{\text { charcoal distance }}{\text { small intestine length }}\right) \times 100
$$

\subsubsection{Whole-body Plethysmography}

The effect of $\mu$ receptor agonists on respiration was assessed using whole-body plethysmography in unrestrained mice as previously described (Lewanowitsch et al., 2006, Lim et al., 2014, Zysman-Colman et al., 2016). Before the experimental day, individual mice were habituated to the Plexiglas chamber (Diameter; $6 \mathrm{~cm}$ ) for $10 \mathrm{~min}$, every day for 14 days to reduce the effect of stress and production of faeces and urine which can affect temperature and humidity. During this procedure, mice were placed in a Plexiglas restrainer that was heated to $30^{\circ} \mathrm{C}\left( \pm 2^{\circ} \mathrm{C}\right)$ and kept at a constant humidity by passing carbogen gas $\left(5 \% \mathrm{O}_{2}\right.$ in $\left.\mathrm{CO}_{2}, \mathrm{BOC}, \mathrm{NZ}\right)$ through a scintillated glass bead humidifier in a water bath set to $75^{\circ} \mathrm{C}$. After every measurement, the chamber temperature, relative humidity, and barometric pressure were recorded and later used in calculations to determine respiratory depression. All recordings were generated using a PowerLab 26T and the data analysed using the LabChart8 software (ADInstruments, Dunedin, NZ).

To reduce the loss of heat through the Plexiglas restrainer an insulating layer of bubble wrap and tinfoil was wrapped around the chamber exterior. Plastic tubing connects the carbogen tank to a pressure regulator to control the flow into the chamber (black ball $=30$; silver ball $=70$ ) before passing into the scintillated glass humidifier in a water bath set to $75^{\circ} \mathrm{C}$. The heated and humidified carbogen then exits via a second tube and enters the plethysmography chamber, a 75 Watt lamp is placed on the bench and the distance to the tubing used to control the temperature of the carbogen between the water bath and chamber. The flow rate of carbogen is further regulated with three-way Leuer lock valves. The third piece of plastic tubing was used to connect the chamber to the pressure transducer, which was in turn connected to a reference chamber. Other ports in the chamber allowed for a calibration syringe to be attached, and a temperature and humidity probe to be inserted (Compact Digital Multimeter, Digitech). Once the chamber has been set up the PowerLab was connected to a laptop for data acquisition and the pressure transducer connected from "output 1 " to "input 1" of the PowerLab via a BNC cable.

The PowerLab was then turned on and the LabChart software opened to calibrate the pressure transducer using the following steps. The Bridge Amp range set to $20 \mathrm{mV}$, the low pass set at $10 \mathrm{~Hz}$ and 'inversion' function turned on. The baseline trace was then zeroed manually. Once the software settings were enabled a $1 \mathrm{~mL}$ syringe, containing $200 \mu \mathrm{L}$ of air (average volume of mouse lungs) was injected into the chamber, resulting in a sudden change in pressure. This was used to calibrate the software to a known volume. The following LabChart settings were set: unit conversion was turned on and the "background trace" or "zero region" selected (-0.05-0.05 mV) and set to $0 \mathrm{mV}$, the "increased pressure region" was then selected $(2-3 \mathrm{mV})$ and set to $1 \mathrm{mV}$. The difference in pressure $(\mathrm{mV})$ in the 
between the "zero region" and "increased pressure region" was recorded for calculating the respiratory function. The amplifier was re-zeroed to complete the calibration step, allowing the recordings to begin.

\subsubsection{Experimental Day}

Before performing respiratory recordings, mice were weighed, and their core body temperature recorded using a rectal thermometer (Acorn series, Singapore) lubricated with petroleum jelly, followed by a 10 min rest period to reduce the effect of any stress on respiration. Mice were then placed in the recording chamber to habituate for $10 \mathrm{~min}$, with baseline measurements taken at 3, 7, and $9 \mathrm{~min}$. The mouse was then removed from the chamber, injected with morphine (5 $\mathrm{mg} / \mathrm{kg} /$ i.p., kurkinol (2.5 mg/kg/i.p.), kurkinorin ( $5 \mathrm{mg} / \mathrm{kg} /$ i.p.), or vehicle (DMSO, tween-80, 0.9\% saline, 2:1:7) and placed back into the chamber. Recordings were taken at 5, 10, 15, 20, 30, 45, 50, and $60 \mathrm{~min}$ post-injection. During the recordings, the mice were monitored, and notes dropped on the trace to track when the mouse was moving and still to exclude the effect of movement on later calculations. At the end of the time course, the mice were removed, and the core temperature was taken with the rectal thermometer to track the possible induction of opioid-induced temperature changes.

\subsubsection{Trace Analysis \& Respiratory Calculations}

All respiratory traces were blinded by a second party before analysis to remove bias. Five secs of the clean trace was used from every time point to analyse the respiratory frequency, pressure deflection, and cycle time as previously described (Lim et al., 2014). The following columns were set up in the DataPad on the LabChart software to assess these factors; pressure deflection due to each tidal volume $\left(\mathrm{P}_{\mathrm{T}}\right)$ was measured with the 'average cyclic height' function (minimum peak detection set to $1 \mathrm{~m} / \mathrm{sec}$ ), the frequency (F) was measured using 'event count', and the total cycle time ( $\left.T_{\text {tot }}\right)$ was assessed with the period function. A macro was then generated within the programme to assess the average inspiration $\left(T_{i}\right)$ and expiration $\left(T_{e}\right)$ time from 9 consecutive peaks.

All data gained from analysing the trace, plus the animal weight $(\mathrm{kg})$, relative humidity, chamber temperature, and the barometric pressure was then entered in an excel spreadsheet to generate the tidal volume $\left(\mathrm{V}_{\mathrm{T}}\right)(\mathrm{mL})$ (Eq. 1). From this the following parameters were also determined; $V_{T}$ as a function of weight $(\mathrm{mL} / \mathrm{kg})$ (Eq. 2), minute volume $(\mathrm{mL} / \mathrm{min} / \mathrm{kg}$ ) (Eq. 3), inspiratory duty cycle (\%) (Eq. 4), inspiratory flow rate (mL/s) (Eq. 5), and ratio of inspiration to expiration time (Eq. 6). All respiratory factors were then expressed as a percent of baseline and plotted against time using GraphPad Prism V7. 


\section{Equation 1.}

$$
V_{T}(m L)=\left(\frac{P_{T}}{P_{K}}\right) \times\left(V_{K}\right) \times\left(\left(T_{C O R E}\left(P_{B}-P_{C}\right)\right) \div\left(T_{C O R E}\left(P_{B}-P_{C}\right)\right)-T_{C}\left(P_{B}-P_{C O R E}\right)\right)
$$

Where;

$V_{T}=$ tidal volume

$P_{T}=$ pressure deflection due to tidal volume

$P_{K}=$ pressure deflection due to each $\mu$ L injection

$V_{K}=$ volume of each calibration injection

$T_{C O R E}=$ core temperature of each mouse

$P_{B}=$ barometric pressure

$P_{C}=$ water vapor pressure (chamber temperature $X$ relative humidity in chamber)

$P_{C O R E}=$

pressure at body temperature (water vapor pressure at body temperature $\times 1$ )

Equation 2.

$V_{T}\left(\frac{m L}{k g}\right)=\frac{V_{T}(m l)}{\text { Body weight }(k g)}$

\section{Equation 3.}

Minute Volume $\left(\frac{\mathrm{mL}}{\min } / \mathrm{kg}\right)=\frac{\left(V_{T} \times F\right)}{\text { Body weight }(\mathrm{kg})}$

\section{Equation 4.}

Inspiratory Duty Cycle $(\%)=\frac{T_{i}}{T_{\text {tot }}}$

\section{Equation 5.}

Inspiratory Flow Rate $(\mathrm{mL} / \mathrm{s})=\frac{V_{T}}{T_{i}}$

\section{Equation 6.}

Ratio of inspiration time to expiration time $=\frac{T_{i}}{T_{e}}$ 


\subsection{Anti-Nociception and Side Effect Z-Score Calculation}

Z-scores are a mathematical tool used to normalise the means from multiple related data sets allowing direct comparisons between experiments. The Z-scores for the anti-nociceptive and side effects of morphine, kurkinol, and kurkinorin were generated for male and female mice individually and combined as previously described (Guilloux et al., 2011, Labots et al., 2016). In brief, Z-scores were generated for individual mice using the test value from each mouse $(\chi)$ compared to the mean $(\mu)$ and standard deviation $(\sigma)$ of the control group as follows;

$$
Z=\frac{\chi-\mu}{\sigma}
$$

Individual Z-scores were then averaged within each experiment. Anti-nociceptive and sideeffect scores were then generated for each compound based on 4 and 9 different measures respectively. For example, the anti-nociceptive Z-score for males was calculated as follows;

$$
\text { Anti-nociceptive score }=\frac{Z_{T F} Z_{H P} Z_{P T X-V F} Z_{P T X-\text { acetone }}}{4}
$$

Where $Z_{T F}$ is the tail-flick Z-score, $Z_{H P}$ is the hotplate Z-score, $Z_{\text {PTX-VF }}$ is the Paclitaxel von Frey Z-score, $Z_{\text {PTX-acetone }}$ is the Paclitaxel acetone Z-score. Excel (Microsoft Office 365 V.1908, Wash., U.SA) was then used to plot radar charts of the anti-nociceptive and side effect scores for each compound to directly compare their behavioural profiles.

\subsection{Cell Culture}

\subsubsection{General Tissue Culture Methods}

All cells were grown in a humidified and temperature-controlled $\left(37^{\circ} \mathrm{C}\right)$ incubator (Bioline, $\mathrm{NSW}$, Australia) with $5 \% \mathrm{CO}_{2}$. Frozen cell stocks were maintained by regularly freezing down aliquots of cells in the log phase of growth $\left(1 \times 10^{6}\right.$ cells $\left./ \mathrm{mL}\right)$ in freeze media containing cell-specific base media, 10\% dimethyl sulfoxide (DMSO), 10\% Foetal Calf Serum (FCS) (ICP biological, Auckland, NZ), and 1\% PenStrep (Penicillin G Sodium $5000 \mathrm{U} / \mathrm{mL}$ and streptomycin sulphate $5000 \mathrm{U} / \mathrm{mL}$ in $0.8 \%$ saline (ThermoFischer Scientific). Cryovials were then placed inside a specialised chamber, 'Mr. Frosty' for (ThermoFischer Scientific) to ensure gradual freezing of approximately $-1^{\circ} \mathrm{C} / \mathrm{min}$ when placed at $-80^{\circ} \mathrm{C}$ overnight. Cryovials were then transferred to a liquid $\mathrm{N}_{2}$ dewar for long-term storage. 


\subsubsection{Chinese Hamster Ovary (CHO) Cells Growth \& Passaging}

Chinese Hamster Ovary- $\mathrm{K} 1(\mathrm{CHO})$ cells are derived as a subclone of the original $\mathrm{CHO}$ cell line generated from a biopsy of an ovary from a Chinese hamster in 1958 (Puck et al., 1958), and were purchased from American Type Culture Collection (ATCC, Manassas, VA, USA) (passage 1). Frozen cell stocks were removed from the liquid $\mathrm{N}_{2}$ dewar and placed in a $37^{\circ} \mathrm{C}$ water bath, once thawed cells were immediately placed in $10 \mathrm{~mL}$ of Ham's F-12K (Kaighn's) Medium (F-12K) (Thermo Fischer Scientific) supplemented with 10\% FCS and centrifuged (Appendix 7.1.1) at $300 \mathrm{~g}$ for $5 \mathrm{~min}$. The supernatant was removed, and the pellet resuspended in $7 \mathrm{~mL}$ of growth media and placed in a T25 flask (Falcon, Thermo Fischer Scientific). The following day cells were checked and either fed by removing the old media replacing it with fresh growth media if $<70 \%$ confluent or passaged into a 100 $\mathrm{mm}$ tissue culture petri dish (Corning ${ }^{\circledR}$, Thermo Fischer Scientific) if $>70 \%$ confluent.

Cells in the log phase of growth at $80-90 \%$ confluent were passaged and reseeded at an experiment and/or seeding density. This occurred by removing the media from the cells and washing briefly with $1 \mathrm{~mL}$ of phosphate-buffered saline (PBS, pH 7.4; $137 \mathrm{mM} \mathrm{NaCl}, 2.7 \mathrm{mM} \mathrm{KCl}, 10 \mathrm{mM}$ $\mathrm{Na}_{2} \mathrm{HPO}_{4}, 1.8 \mathrm{mM} \mathrm{KH} \mathrm{PO}_{4}$, Appendix 7.1.2), 1-2 mL of TrypLE ${ }^{\mathrm{TM}}$ Express Enzyme (Life Technologies, Auckland, NZ) was added to dislodge the cells. After $5 \mathrm{~min}$, cells were checked and if detached $9 \mathrm{~mL}$ of growth media was added and the flask surface rinsed a few times to ensure cell detachment. The cell suspension was then transferred to a $15 \mathrm{~mL}$ falcon tube for centrifugation at $300 \mathrm{~g}$ for $5 \mathrm{~min}$. The pellet was then resuspended in $2 \mathrm{~mL}$ of growth media and $300 \mu \mathrm{L}$ seeded into a new $100 \mathrm{~mm}$ tissue culture petri dish containing $10 \mathrm{~mL}$ of growth media.

\subsubsection{Human Embryonic Kidney 293 cells Growth \& Passaging}

Human embryonic kidney 293 (Hek293) cells, an adherent immortalised cell line, were purchased from AATC (Manassas, VA, USA). The Hek-293 cell line was maintained in the same manner as the $\mathrm{CHO}$ cell line (Section 2.1.2.2) with a modification to the growth media, Hek-293 cells were grown in Dulbecco's Modified Eagle's Medium (DMEM) (Thermo Fischer Scientific) supplemented with 10\% FCS and 1\% PenStrep (Appendix 7.1.1). The human $\mu$ receptor-Hek293 stable cell line had previously been generated in the lab, and the media supplemented with geneticin (G418, 1\%) to maintain the human $\mu$ receptor expression and were used up to passage 35.

\subsubsection{Stable Cell Lie Generation}

\subsubsection{Plasmid Preparation}

Previously generated bacterial stocks of chemically competent $E$. coli MAX Efficiency ${ }^{\circledast} \mathrm{DH} 5 \alpha^{\mathrm{TM}}$ (Invitrogen) expressing OPRM1 FLAG-tagged (N-terminal) plasmid (cDNA resource centre, Pennsylvania) was removed from the $-80^{\circ} \mathrm{C}$. A flame sterilised metal loop was then used to take a scraping of the bacteria, which was subsequently streaked onto LB ampicillin agar plates (ampicillin $50 \mu \mathrm{g}$. Appendix 7.1.4) or an LB agar plate (Appendix 7.1.4) (negative control). The plates were then incubated inverted for $14-18 \mathrm{hrs}$, in a stationary incubator set to $37^{\circ} \mathrm{C}$. After the incubation period, 
and if no growth occurred on the negative control, a single colony was selected and incubated in $2 \mathrm{ml}$ of LB broth (Appendix 7.1.4) for $8 \mathrm{hrs}\left(200 \mathrm{rpm}\right.$ at $\left.37^{\circ} \mathrm{C}\right)$. Once the incubation was complete $200 \mu \mathrm{l}$ of cell suspension was inoculated in $200 \mathrm{ml}$ of LB broth with Ampicillin $(100 \mu \mathrm{g} / \mathrm{ml})$ and returned to the shaking incubator $\left(300 \mathrm{rpm}\right.$ at $37^{\circ} \mathrm{C}$ ) for $12-16$ hours to allow further growth.

Plasmid preparation was then performed on the resulting bacterial culture using the HiSpeed ${ }^{\circledR}$ Plasmid Maxi Kit (Qiagen, BioStrategy Auckland, NZ) following the manufacturer's protocol. Briefly, the pellet of transformed bacteria $(200 \mathrm{ml})$ was centrifuged at $6,000 \mathrm{~g}$ for $15 \mathrm{~min}$ at $4^{\circ} \mathrm{C}$. The supernatant was then discarded the pellet was re-suspended in $10 \mathrm{ml}$ of P1 buffer containing RNase A and lysis blue. The cell suspension was then transferred to a $50 \mathrm{ml}$ falcon tube and $10 \mathrm{ml}$ of P2 buffer added, mixed thoroughly and then left to incubate at room temperature for $5 \mathrm{~min}$. Due to the addition of Lysis blue to P1 buffer, the solution became blue. At the end of the incubation $10 \mathrm{ml}$ of pre-chilled of P3 buffer was added and the solution mixed by inversion until colourless, it was then added to the QIAfilter cartridge and incubated for a further $10 \mathrm{~min}$. Throughout this incubation period, the HiSpeed Maxi Tip was placed over a waste container and equilibrated with $10 \mathrm{ml}$ of QBT buffer. The lysate was then filtered through the HiSpeed Maxi Tip via the application of constant pressure on the plunger to trap the DNA. QC buffer $(60 \mathrm{ml})$ was then passed through the filter to wash the DNA, which was then eluted into a $50 \mathrm{ml}$ tube with $15 \mathrm{ml}$ of QF buffer. The DNA was then precipitated with $10.5 \mathrm{~mL}$ of isopropanol (>99.5\%, Sigma Aldrich) over $5 \mathrm{~min}$. The precipitated DNA was then transferred to a 30 $\mathrm{ml}$ syringe with the QIAprecipitator Module attached to the outlet nozzle, the solution was filtered through to a waste container trapping the DNA within the filter. The trapped DNA was then washed with $2 \mathrm{ml}$ of 70\% ethanol before being quickly air-dried twice. The tip of the QIAprecipitator was then dried with a paper towel to prevent the transfer of ethanol. A new $5 \mathrm{ml}$ syringe was then attached to the QIAprecipitator and $1 \mathrm{ml}$ of TE buffer was added to elute the DNA into a sterile $1.5 \mathrm{ml}$ microcentrifuge tube. Subsequently, the eluted DNA was filtered through the QIAprecipitator for a second time into the same $1.5 \mathrm{ml}$ microcentrifuge tube to maximise the DNA yield.

The yield of DNA obtained was determined using a Nanodrop ND-1000 spectrophotometer (BioLab, Auckland, NZ) that was initialized with distilled water and then blanked with TE buffer (0.5 M EDTA, $1 M$ Tris. Appendix 7.1.5). The eluted DNA ( $1 \mu \mathrm{l})$ was then pipetted onto the Nanodrop and the yield of DNA determined. If the yield was above $500 \mathrm{ng} / \mu \mathrm{l}$ and the absorbance was between 1.8-1.9 the DNA was then used for the transfection of $\mathrm{CHO}$ cells. 


\subsubsection{Transfection of $\mathrm{CHO}$ Cells with the Human Mu Opioid Receptor}

CHO cells were transfected $24 \mathrm{hrs}$ after plating onto a $100 \mathrm{~mm}$ dish if $90 \%-100 \%$ confluent. Cells in $10 \mathrm{~mL}$ Petri dishes were transfected using $30 \mu \mathrm{L}$ of Lipofectamine 2000 (Invitrogen) made up to $500 \mu \mathrm{L}$ with Opti-MEM (Invitrogen). In a second tube, $1 \mu \mathrm{g} / \mathrm{mL}$ of $\mathrm{N}$-terminal FLAG-tagged OPRM1 plasmid DNA was made up to $500 \mu \mathrm{L}$ with Opti-MEM. After a 5 min incubation, the two tubes were mixed and incubated for $30 \mathrm{~min}$. During this period the cell culture growth media was replaced with antibiotic-free medium (F-12K, 10\% FCS) and $1 \mathrm{ml}$ of the transfection reagent added to each petri dish. After $24 \mathrm{hr}$ the transfection reagent was removed and fresh antibiotic-free growth media added to the cultures. Twenty-four hr later the cells were detached using trypsin (0.25\%) (Sigma Aldrich), spun down, and counted using trypan blue (0.4\%) (Sigma Aldrich) and a haemocytometer (Neubauer, ThermoFisher). They were then resuspended in base $\mathrm{CHO}$ media containing the selection antibiotic, G418 (1\%), and seeded at 1 cell/well in a 96 well plate. Single cells that proliferated and reached 80$90 \%$ confluency were then transferred to a 24 well plate and allowed to reach confluency before passaging to a 12 well, then 6 well plate, and eventually, cells were transferred into a T25 flask.

To determine the expression levels of the "stable" lines cells were passaged using enzymefree cell dissociation buffer (ThermoFisher, 13151014, Wellington, New Zealand) and plated into 12well plates containing poly-D lysine coated $18 \mathrm{~mm}$ coverslips at $3 \times 10^{5}$ cells $/ \mathrm{mL}$. Twelve to $24 \mathrm{hrs}$ later cells were fixed with ice-cold 1:1 acetone-methanol for $7 \mathrm{~min}$. The acetone-methanol was immediately removed and washed $3 \times 5$ min in PBS. Cells were then blocked in $1 \%$ bovine serum albumin (BSA) in PBS containing $0.01 \%$ tween for $1 \mathrm{hr}$ before the addition of anti-FLAG antibody (Sigma, F1804) (1:500) in the same blocking reagent. The plates were then incubated overnight at $4^{\circ} \mathrm{C}$. The primary antibody was then removed and the cells washed $3 \mathrm{X}$ for $5 \mathrm{~min}$ in PBS before the addition of goat anti-mouse Cy5 $(1: 1,00)$ (Amersham) for $1 \mathrm{hr}$. The secondary was then removed and the cells washed for a further $3 \times 5$ min with PBS. The slides were tapped to remove any excess liquid before the mounting in prolong $^{\mathrm{TM}}$ gold antifade with DAPI (ThermoFisher; P36941). The cells were then imaged using an Olympus FV3000 (Olympus, Auckland, NZ). The cells were then imaged using the $647 \mathrm{~nm}$ laser and Cy5 filter (650 nm excitation, $667 \mathrm{~nm}$ emission, bandwidth; 630 - 690nm) and 405 lasers and DAPI filter (358 nm excitation, $461 \mathrm{~nm}$ emission, bandwidth; $340-480 \mathrm{~nm}$ ). Secondary only controls and nontransfected cells were used to assess background fluorescence. Monoclonal cell cultures with moderate to high expression levels were then passaged into $100 \mathrm{~mm}$ tissue culture Petri dishes to generate frozen stocks. 


\subsubsection{Drug Stocks}

Tissue culture drug stocks were generated in a biohazard hood to ensure sterility and at a concentration range of $10 \mathrm{mM}-30 \mathrm{mM}$. Morphine (Hospira, NZ) was purchased as a $10 \mathrm{mg} / \mathrm{mL}$ solution in $0.9 \%$ saline; all stocks were made by diluting the saline solution in tissue culture grade dimethyl sulfoxide (DMSO) to give a vehicle of $1 \%$ saline in DMSO. DAMGO, Morphine, kurkinol, and kurkinorin stocks were all generated by weighing out $1 \mathrm{mg}$ on an analytical balance and dissolving in DMSO and $1 \%$ saline to produce the desired stock concentration.

\subsubsection{High Throughput Cell Signalling Assays}

\subsubsection{FLIPR Membrane Potential Assay}

The FLIPR Membrane Potential assay kit was purchased from Molecular Devices (San Jose, CA, USA). Either Hek293 or $\mathrm{CHO}$ cells stably expressing the human $\mu$ receptor were passaged and seeded at 100,000 cells $/ 90 \mu \mathrm{L}$ in antibiotic-free low serum media and placed in a $37^{\circ} \mathrm{C}$ incubator overnight. The next day the cells were checked and if $90-100 \%$ confluent the assay was set up. The fluorescent assay dye was made following the manufactures instructions and added to the wells at a volume of $90 \mu \mathrm{L}$ per/well. The plate was then returned to the incubator for $1 \mathrm{hr}$. During this incubation period, a dilution series (1:3) was generated for each compound to $5 \mathrm{X}$ the final concentration in low serum media with forskolin (final conc. $=10 \mathrm{mM}$ ). The final concentrations for DAMGO were 100, 33, 11, 3.7, $1.2,0.41,0.13 \mu \mathrm{M}$, morphine was $200,100,33,11,3.7,1.2,0.41 \mu \mathrm{M}$, kurkinol was $33,11,3.7,1.2$, $0.41,0.13,0.045 \mu \mathrm{M}$, and kurkinorin; 100, 33, 11, 3.7, 1.2, 0.41, $0.13 \mu \mathrm{M}$. At the end of the $1 \mathrm{hr}$ incubation the plate was placed into the Enspire 2300 multilabel plate reader (PerkinElmer, Japan) $\left(37^{\circ} \mathrm{C}\right)$ and 5 baseline fluorescence measurements recorded $(E x=530 \mathrm{~nm}, \mathrm{Em}=565 \mathrm{~nm})$. The plate was then removed and $20 \mu \mathrm{L}$ of compound, forskolin (FSK) GTP alone, or vehicle (0.1\% DMSO) was added to each well (in triplicate) and 10 measurements taken.

All data were normalised to baseline measurements to reduce variation and the percent inhibition of FSK-induced depolarisation calculated for each time point. The percent FSK-inhibition from 5 min after compound addition was plotted against Log [dose M] and non-linear regression (4parameter) was used to fit a curve in GraphPad Prism V.7 to generate $\mathrm{EC}_{50}$ and $\mathrm{E}_{\max }$ values.

\subsubsection{Potassium Assay}

The $\mathrm{K}^{+}$assay kit was purchased from Molecular Devices (San Jose, CA, USA). CHO cells stably expressing the human $\mu$ receptor were passaged and seeded into a black, plastic bottom 96 well plate at a concentration of 90,000 cells/well in $90 \mu \mathrm{L}$ and incubated overnight at $37^{\circ} \mathrm{C}$. The next day the loading buffer was prepared as described in the manufacturer's instructions. As suggested in the manufacturer's protocol, probenecid (Sigma, Auckland, NZ) was added to the loading buffer at a concentration of $5 \mathrm{mM}$ (final con. $=2.5 \mathrm{mM} /$ well) to block organic anion transporters. The loading buffer was then added at $90 \mu \mathrm{L} /$ well and the plate placed back into the incubator for $1 \mathrm{hr}$. All compound dilution series (1:3) were made at $5 X$ the final concentration in chloride free buffer 
containing $3 \mathrm{mM}$ thallium sulphate $\left(\mathrm{Ti}_{2} \mathrm{SO}_{4}\right)$. The final concentrations were; DAMGO; 5, 1.6, 0.53, 0.17, 0.06, 0.02 , and $0.0068 \mu \mathrm{M}$, morphine; $10,3.3,1.1,0.36,0.12,0.04$, and $0.013 \mu \mathrm{M}$, Kurkinol; 2, 0.66, $0.22,0.07,0.02,0.008$, and $0.0027 \mu \mathrm{M}$, and kurkinorin; $5,1.6,0.53,0.17,0.06,0.02$, and $0.0068 \mu \mathrm{M}$. At the end of the $1 \mathrm{hr}$ incubation period, the plate was moved to the ClarioStar Plus plate reader (BMG LabTech, Ortenburg, Germany), heated to $37^{\circ} \mathrm{C}$, and 5 baseline fluorescent measurements recorded $(E x=470 \mathrm{~nm}, \mathrm{Em}=575 \mathrm{~nm})$. The plate was then removed and $20 \mu \mathrm{L}$ of compound or vehicle $(0.1 \%$ DMSO) was added to each well and 10 more measurements were taken. Each dose response was run in triplicate over 7 experimental replicates.

The baseline for each well was subtracted to reduce variance and the percent fluorescence increase from vehicle calculated for each time point. Percent increase of vehicle from $5 \mathrm{~min}$ after drug addition, where the curve stabilised, was plotted against Log [dose M] and non-linear regression (4parameter) used to fit a curve in GraphPad Prism to generate the concentration $\mathrm{EC}_{50}$ and $\mathrm{E}_{\max }$ values.

\subsubsection{PCREB Assay}

The CisBio pCREB (Ser133) homogenous time-resolved fluorescence (HTRF) assay (PerkinElmer, Japan) was used to assess the activation of pCREB by morphine, DAMGO, kurkinol, and kurkinorin according to the manufacturer's instructions. In brief, human $\mu$ receptor $\mathrm{CHO}$ cells were plated in a 384 well HTRF plate at 80,000 cells/well and placed in the incubator overnight $\left(37^{\circ} \mathrm{C}\right)$. The next day the dilution series of DAMGO $(5,2.5,1.25,0.625,0.312 \mu \mathrm{M})$, morphine $(5,2.5,1.25,0.625$, $0.312 \mu \mathrm{M})$, kurkinol $(2.5,1.25,0.625,0.312,0.152 \mu \mathrm{M})$, and kurkinorin $(10,5,2.5,1.25,0.625 \mu \mathrm{M})$ were made to a final volume of $50 \mu \mathrm{L}$ ( $2 \mathrm{X}$ stock) in serum-free media. $5 \mu \mathrm{L}$ of media was then removed from each well and $5 \mu \mathrm{L}$ of drug added to each well. The plate was returned to the incubator $\left(37^{\circ} \mathrm{C}\right)$ for $30 \mathrm{~min}$. The cell supernatant was removed at the end of the incubation and immediately replaced with $8 \mu \mathrm{L}$ of supplemented lysis buffer (1X). The plate was then placed on an orbital shaker and rocked for $1 \mathrm{hr}$ at room temperature. Once homogenised $2 \mu \mathrm{L}$ of antibody solution (d2 \& Eu Cryptate antibodies, 1:1) was added to each well. The plate was then covered with a plate seal and incubated overnight at room temperature. The next day the seal was removed at the plate read on the ClarioStar Plus plate reader (BMG LabTech), with the fluorescent emission determined at $665 \mathrm{~nm}$ and $620 \mathrm{~nm}$. The HTRF ratio of acceptor to donor emission signal was calculated for each well as follows;

$$
\text { Ratio }=\frac{\text { signal } 665 \mathrm{~nm}}{\text { signal } 620 \mathrm{~nm}} \times 10^{4}
$$

This was then plotted as a function of Log [dose M] in GraphPad Prism 7 and non-linear regression (4parameter) performed to fit a curve to the data. This was used to generate the $\mathrm{EC}_{50}$ and $\mathrm{E}_{\max }$ values. Negative controls and positive control lysate were run with each assay to ensure the accuracy of the results. 


\subsubsection{SDS-PAGE Gel Electrophoresis and Western Blotting}

\subsubsection{Protein Preparation}

SDS-PAGE gel electrophoresis and Western blotting were used to assess the time of pERK activation in response to $\mu$ receptor agonists. Hek293 cells stably expressing the human $\mu$ receptor were plated at 100,000 cells/plate, or to be confluent the next day. If $>70 \%$ confluent the cells were serum-starved for an hour before treatment with the kurkinol $(10 \mu \mathrm{M})$, kurkinorin $(10 \mu \mathrm{M})$, DAMGO $(1 \mu \mathrm{M})$, morphine $(10 \mu \mathrm{M})$, or vehicle for $5,10,30,40,50,60,90,120,150$, or $180 \mathrm{~min}$ for time course experiments. For antagonist pre-treated experiments the cells were pre-treated with pertussis toxin (Px) for $18 \mathrm{hr}(100 \mathrm{ng} / \mathrm{ml})$ or dynosore $(50 \mu \mathrm{M})$ for $1 \mathrm{hr}$. The drug treatments were then performed in the presence of the respective antagonists for 0,10 , or $180 \mathrm{~min}$ at the previously mentioned doses of DAMGO, morphine, kurkinol, or kurkinorin.

The cells were then lysed with radioimmunoprecipitation assay (RIPA) buffer (10 mM Tris$\mathrm{HCl}, 150 \mathrm{mM} \mathrm{NaCl}, 1$ mM EDTA, 1\% Triton-X-100, 0.1\% SDS, 1\% Sodium deoxycholate; Appendix 7.2.1) and $1 \%$ phosphatase (sodium orthovanadate, sodium molybdate, sodium tartrate, imidazole) and protease inhibitor cocktails (AEBSF, Aprotinin, Bestatin, E-64, Leupeptin, Pepstatin A) (Sigma-Aldrich, USA) for $60 \mathrm{~min}$ at $4{ }^{\circ} \mathrm{C}$. The cell lysate was then spun down for $30 \mathrm{~min}$ at $4{ }^{\circ} \mathrm{C}$ and the DNA pellet removed before protein quantification.

\subsubsection{Protein Quantification}

The level of protein was then quantified using the Biorad protein assay according to the manufacturer instructions. In brief, standards were prepared in RIPA buffer $(1000,750,500,250,125$, 25 , and $0 \mu \mathrm{g} / \mathrm{ml}$ ) and loaded onto a 96-well plate in triplicate alongside each sample. After this $200 \mu \mathrm{l}$ of the assay, the dye was added to each well. This was incubated at $37^{\circ} \mathrm{C}$ for $30 \mathrm{~min}$ before absorption $(595 \mathrm{~mm})$ reading in the Enspire 2300 multilabel plate reader (PerkinElmer, Japan). The triplicate readings were then averaged and then graphed to produce a standard curve, which was subsequently used to determine the protein concentration. The protein samples were then diluted to the required concentration $(50 \mathrm{\mu g} / \mathrm{ml})$ and reduced in reducing buffer containing $(62.5 \mathrm{mM}$ Tris- $\mathrm{HCl}, 2 \% \mathrm{SDS}, 20 \%$ Glycerol, $1 \%$ Bromophenol Blue; Appendix 7.2.2) containing $\beta$-mercaptoethanol (9\%) and loaded into a 15 well sodium dodecyl sulphate- polyacrylamide (SDS-PAGE) gel electrophoresis. 


\subsubsection{SDS-PAGE and Western Blotting}

SDS-PAGE gels were hand cast and consisted of a $10 \%$ separating gel and $4 \%$ stacking gel (Appendix 7.2.3). Protein samples were loaded into the wells at a volume of $20 \mu$ lalongside the molecular weight marker ( $3 \mu \mathrm{l}$ ) (Precision Plus Protein ${ }^{\mathrm{TM}}$ Dual Colour Standards, \#1610374, Bio-Rad) and the electrophoresis run at $120 \mathrm{~V}$ for $1.5 \mathrm{hrs}$ in running buffer ( $3.5 \mathrm{mM} \mathrm{SDS}, 25 \mathrm{mM}$ Tris- $\mathrm{HCl}, 190$ mM Glycine; Appendix 7.2.4) using the Mini-PROTEAN Tetra Cell system (Bio-Rad, Hercules, CA, USA). Western blot transfer was then carried out using low fluorescence polyvinylidene difluoride (PVDF) membrane (pore size: $0.45 \mu \mathrm{m}$ ) (Immobilon-FL, Millipore, Billerica, MA, USA), which had been presoaked in transfer buffer (190 mM Glycine, $25 \mathrm{mM}$ Tris-HCl, 20\% methanol; Appendix 7.2.5) for a minimum of $5 \mathrm{~min}$. The transfer cassette was then assembled by layering; a sponge, filter paper, protein gel, transfer membrane, filter paper, and sponge, in the aforementioned order. This was then placed into the transfer tank (Mini Trans-Blot Cell, Bio-Rad, Hercules, CA, USA) with an ice pack and transfer buffer before electrophoresis at $20 \mathrm{~V}$ overnight (17 hrs).

Once the transfer was complete the membranes were removed and washed $3 \mathrm{X}$ in Trisbuffered saline (TBS, pH 7.5; $20 \mathrm{mM}$ Tris, $150 \mathrm{mM} \mathrm{NaCl}$ ) and blocked with TBS containing 5\% BSA for $1 \mathrm{hr}$ at room temperature. Membranes were then incubated with the relevant primary antibodies for phosphorylated protein, mouse monoclonal anti-phospho-ERK42/44 (1:500) (Santa Cruz, supplied by ThermoFischer), in T-TBS (0.1\% Tween-20 in TBS) containing 5\% BSA at $4{ }^{\circ} \mathrm{C}$ overnight. The membranes were then labelled with secondary antibodies, goat anti-rabbit IgG Cy3 (1:5000) (Amersham) or antimouse Cy5 (1:5000) (Amersham), in blocking buffer at room temperature for an hour and subsequently washed in T-TBS 3 X. Stained membranes were then imaged using the Typhoon FLA 9500 laser scanner (Fujifilm, Tokyo, Japan) (635nm laser, LPR filter). The primary-secondary complexes were then removed with $3 \mathrm{~mL}$ stripping buffer (20 mM SDS, $0.05 \mathrm{M}$ Tris $\mathrm{HCl}, 0.75 \% \beta$-mercaptoethanol) for $40 \mathrm{~min}$. The membranes were washed $3 \mathrm{X}$ for $20 \mathrm{~min}$ in excess T-TBS. The membrane was re-blocked for $1 \mathrm{hr}$ at room temperature and the primary antibody for the total protein, rabbit monoclonal ERK42/44 primary antibody (Cell Signalling Technology, supplied by ThermoFischer) (1:1000), applied in T-TBS containing $5 \%$ BSA overnight at $4{ }^{\circ} \mathrm{C}$. The secondary antibody was then applied and scanned on the FLA-500 as described as above (see Table 2.1 for further antibody details).

\subsubsection{Data Analysis}

Western blots were analysed using the ImageJ software. A region of interest was selected around the bands and the intensity of the determined. All band densities were corrected against the background and loading irregularities by normalising all density values to total ERK1/2 of the same condition. The data obtained were also compared to the density of the vehicle condition and all results expressed as the mean fold increase compared to the vehicle to allow comparison between blots. The linear migration of visualised bands was used to identify the proteins of interest (Appendix Table 7.3). 
Table 2.1 Antibodies and Conditions Used

\begin{tabular}{|c|c|c|c|c|c|c|c|c|c|}
\hline \multirow[b]{2}{*}{ Protein } & \multirow{2}{*}{$\begin{array}{l}\text { Size } \\
(\mathrm{kDa})\end{array}$} & \multicolumn{2}{|c|}{ Blocking } & \multicolumn{3}{|c|}{ Primary antibody } & \multicolumn{3}{|c|}{ Secondary antibody } \\
\hline & & Solution & $\begin{array}{c}\text { Time } \\
\text { /Conditions }\end{array}$ & Solution & $\begin{array}{l}\text { Dilution } \\
\text { factor }\end{array}$ & $\begin{array}{c}\text { Time/ } \\
\text { Conditions }\end{array}$ & Solution & $\begin{array}{l}\text { Dilution } \\
\text { factor }\end{array}$ & $\begin{array}{c}\text { Time/ } \\
\text { Conditions }\end{array}$ \\
\hline $\begin{array}{c}\text { pERK } \\
1 / 2\end{array}$ & $\begin{array}{l}42, \\
44\end{array}$ & $\begin{array}{c}5 \% \text { BSA in } \\
\text { T-TBS }\end{array}$ & $1 \mathrm{hr} / \mathrm{RT}$ & $\begin{array}{l}\text { Mouse monoclonal pERK 1/2 } \\
\text { (Santa Cruz, supplied by } \\
\text { Thermofisher, Auckland, NZ) }\end{array}$ & 1:500 & Overnight $/ 4^{\circ} \mathrm{C}$ & $\begin{array}{l}\text { Goat anti-mouse } \\
\text { Cy5 (GE Healthcare, } \\
\text { Auckland, NZ) }\end{array}$ & $1: 5000$ & $\begin{array}{c}1 \mathrm{hr} / \mathrm{RT} \\
\text { dark }\end{array}$ \\
\hline ERK $1 / 2$ & $\begin{array}{l}42, \\
44\end{array}$ & $\begin{array}{c}5 \% \text { BSA in } \\
\text { T-TBS }\end{array}$ & $1 \mathrm{hr} / \mathrm{RT}$ & $\begin{array}{l}\text { Rabbit monoclonal ERK 1/2 } \\
\text { (Cell Signalling, supplied by } \\
\text { Thermofisher, Auckland, NZ) }\end{array}$ & 1:1000 & Overnight $/ 4^{\circ} \mathrm{C}$ & $\begin{array}{l}\text { Goat anti-rabbit Cy5 } \\
\text { (GE Healthcare, } \\
\text { Auckland, NZ) }\end{array}$ & $1: 5000$ & $\begin{array}{c}1 \mathrm{hr} / \mathrm{RT} \\
\text { dark }\end{array}$ \\
\hline $\begin{array}{c}\alpha- \\
\text { tubulin }\end{array}$ & 50 & $\begin{array}{c}5 \% \text { BSA in } \\
\text { T-TBS }\end{array}$ & $1 \mathrm{hr} / \mathrm{RT}$ & $\begin{array}{l}\text { Rabbit monoclonal } \alpha \text {-tubulin } \\
\text { (ab18251, Abcam, } \\
\text { Melbourne, Australia }\end{array}$ & $1: 5000$ & $1 \mathrm{hr} / \mathrm{RT}$ & $\begin{array}{c}\text { Goat anti-rabbit Cy5 } \\
\text { (GE Healthcare, } \\
\text { Auckland, NZ) }\end{array}$ & $1: 5000$ & $\begin{array}{c}1 \mathrm{hr} / \mathrm{RT} \\
\text { dark }\end{array}$ \\
\hline
\end{tabular}

$B S A=$ bovine serum albumin

$T B S=$ tris-buffered saline

$T$-TBS $=$ TBS with $0.1 \%$ Tween 20

$R T=$ room temperature 


\subsection{Biased Agonism Calculations}

To assess the bias of kurkinol and kurkinorin the two variations of the ratiometric $R A_{i}$ model were used. In the firsts instance, the bias factor was calculated using the $\mathrm{EC}_{50}$ and $\mathrm{E}_{\max }$ values obtained from the dose response experiments as described in (Rajagopal et al., 2010), with the $E_{\max }$ values expressed as a percent of the reference ligand DAMGO as previously described (Kenakin, 2017). The bias factor $(\beta)$ was then calculated as follows for the ratiometric analysis:

$$
\beta=\log \left(\left(\frac{R_{\text {max }- \text { path } 1} E C_{50-\text { path } 2}}{R_{\text {max }- \text { path } 2} E C_{50-p a t h 1}}\right)_{L i g} \times\left(\frac{R_{\max -\text { path } 1} E C_{50-p a t h 2}}{R_{\max -\text { path } 2} E C_{50-p a t h 1}}\right)_{\text {Ref }}\right)
$$

If the Hill slopes generated from the cellular data are not significantly different from one another this method is analogous to the operational models of bias. However, if the Hill slopes do significantly differ between different treatments the $\mathrm{EC}_{50}$ values that this calculation relies on are not accurate (Kenakin et al., 2013). To account for this, all Hill slopes were restrained to 1 before the use of the calculation. Using this calculation, a bias factor of 0 is balanced, less than 0 is $\beta$-arrestin2 biased, and greater than 0 is $\mathrm{G}$-protein biased.

The second method used to calculate bias, known as the simplified bias calculation, was performed as previously described (Crowley et al., 2020, Crowley et al., 2016) using the same normalised data described above. Using this calculation, a bias factor of 1 is balanced, less than 1 is $\beta$ arrestin2 biased and greater than 1 is G-protein biased.

$\log$ (bias factor)

$$
\begin{aligned}
& =\log \left(\frac{E_{\max -\text { test }} E C_{50 \text {-control }}}{E C_{50-\text { test }} E_{\text {max-control }}}\right)_{G \text {-protein }} \\
& -\log \left(\frac{E_{\max -\text { test }} E C_{50-\text { control }}}{E C_{50-\text { test }} E_{\text {max-control }}}\right)_{\beta \text {-arrestin }}
\end{aligned}
$$




\subsection{Statistical Analyses}

Two-way and One-way analysis of variance tests (ANOVA) were conducted using GraphPad Prism, ensuring all data passed the D'Agostino Pearson omnibus K2 normality test. For data with 2 independent variables (i.e. sex and drug), that may affect the behavioural output overtime, Three-way mixed ANOVAs were used. This analysis was performed using SPSS statistics (Statistical Package for the Social Sciences) (V.26, IBM, Armonk, NY, USA). The data was checked for normality (Shapiro-Wilk test with standardised residuals) and homogeneity (Levene's test of equality of error variances). Where the assumptions were not met, the data was transformed based on the skew, with slightly negative data transformed using square root, while moderately negative data was corrected with a logarithmic transformation. Slightly positive data was first corrected with a reflect, followed by a square root transformation. If the data could not be corrected using the following transformations the ratio of the variance were assessed. If the smallest to largest variance was found to be less than 3 , ANOVA analysis was still performed due to tolerance of ANOVAs to withstand unequal variance. Once all other assumptions were met, the sphericity of the data was assessed using Mauchly's test. If $p>$ 0.05 the data was assumed to be meet sphericity requirements and no corrections were applied. However, if $p<0.05$ the assumption was not met and the Greenhouse-Geisser correction was applied. If the data met all test assumptions and the ANOVA was significant post-hoc testing was performed using the Bonferroni multiple corrections test. To simplify the results sections the technical detail of these statistical analyses have been reported in the appendix (Appendix 7.3, Table 7.1 and Table 7.2). 


\section{Chapter 3. Anti-Nociceptive Effects of Kurkinol and Kurkinorin}

\subsection{Introduction}

The lack of current therapeutics to treat chronic pain and worsening opioid crisis around the world has led to a great need for non-addictive opioids that are still highly efficacious (Breivik et al., 2006, Shipton et al., 2017). By biasing the signalling pathway activated at the $\mu$ receptor towards the G-protein pathway, over the $\beta$-arrestin2 pathway, we may be able to reduce the tolerance of opioids while maintaining potent analgesic effects (Bohn et al., 1999). In the current chapter, we aimed to investigate the hypothesis that G-protein bias determines the anti-nociceptive potency and tolerance of $\mu$ receptor agonists using our structurally unique Sal $A$ analogues active at the $\mu$ receptor, kurkinol and kurkinorin.

\subsubsection{Regulation of Mu Opioid Receptors by $\beta$-arrestin2 and the Implications for Analgesia}

The multifaceted role of $\beta$-arrestin 2 in the analgesic effects of $\mu$ receptor agonists was initially discovered via the loss of morphine-induced side effects in the $\beta$-arr2 ${ }^{-/-}$mice (Bohn et al., 1999). Since this discovery, more work has gone into elucidating the role of $\beta$-arrestin2 in analgesia. Selective inhibition of $\beta$-arrestin2 in the PAG using siRNA knockdown increases the anti-nociceptive effects of morphine and reduces the onset of tolerance in mice (Li et al., 2009). A similar delay in anti-nociceptive tolerance to morphine in the tail-flick assay is also seen in rats when $\beta$-arrestin2 is knocked down in the spinal cord (Przewlocka et al., 2002). In the reverse experiment, the overexpression of $\beta$-arrestin2 in the PAG of rats abolishes the anti-nociceptive effects of morphine in the hotplate assay (Jiang et al., 2006, Yang et al., 2011). Further, RNA silencing of $\beta$-arrestin2 in the brain via i.c.v injection of lentivirus containing the anti-gene resulted in decreased $\beta$-arrestin2 expression and reduced anti-nociceptive tolerance (Bu et al., 2015). This identified 2 central areas of nociceptive transmission, the spinal cord and PAG, as key areas of $\mu$ receptor induced anti-nociception and tolerance that is regulated via $\beta$ arrestin2.

The $\beta$-arr $2^{--}$mouse line and siRNA knockdown has also been used to assess the effects of $\beta$ arrestin2 on the signalling of $\mu$ receptor agonists in different neuronal populations involved in nociception. In the PAG and brain stem of $\beta$-arr ${ }^{-/-}$mice, the selective $\mu$ receptor agonist, DAMGO, was shown to increase $\mathrm{G}$-protein coupling at the $\mu$ receptor compared to the $\mathrm{Wt}$ littermate controls (Bohn et al., 2000, Bohn et al., 1999). While primary neuronal DRG cultures from $\beta$-arr $2^{-/-}$mice showed increased coupling between $\mu$ receptor and VG $\mathrm{Ca}^{2+}$ channels, which reduced receptor recycling and increased cell surface expression. However, treatment with DAMGO and morphine still resulted in the inhibition of the voltage-gated $\mathrm{Ca}^{2+}$ channels (Walwyn et al., 2007). Furthermore, acute treatment of 
enkephalin results in rapid desensitisation of the GIRK channels in the LC of $\beta$-arr2 ${ }^{--}$and Wt littermate controls. However, when both GRK2 and ERK1/2 (Wt) or ERK1/2 ( $\beta$-arr2 $\left.{ }^{-/}\right)$were inhibited, GRIK desensitisation was almost fully abolished, suggesting that in the LC, desensitisation can occur in a $\beta$ arrestin2 independent manner (Dang et al., 2006, Dang et al., 2009). In the same LC neuronal model, the dependence of $\mu$ receptor trafficking on $\beta$-arrestin2-dynamin interactions were assessed. The authors showed that resensitisation in response to acute morphine is independent of $\beta$-arrestin2 dynamin. However, when neurons are chronically treated with morphine, the development of cellular tolerance is abolished in LC neurons from $\beta$-arr $2^{-/}$mice. Moreover, disruption of either $\beta$-arrestin2 or dynamin in Wt neurons accelerated the resensitisation of $\mu$ receptor (Dang et al., 2011). This suggests that under chronic conditions, $\beta$-arrestin2 functions to slow the return of internalised receptors to the membrane, inducing anti-nociceptive tolerance. Connor et al. (2015) showed that $\beta$-arrestin2 deletion did not affect the coupling of $\mu$ receptors to voltage gated $\mathrm{Ca}^{2+}$ channels in the PAG of vehicle or chronic morphine treated mice. Furthermore, the authors showed that cellular tolerance to $\mathrm{Ca}^{2+}$ channels did not occur in the $\beta$-arr ${ }^{-\%}$ as it did in the Wt littermate controls, providing further evidence on the cellular mechanism through which $\beta$-arrestin2 induces anti-nociceptive tolerance. While the cellular adaptations that occur with opioid withdrawal, mainly increases in GABA transporter type 1 currents, were not affected by the deletion of $\beta$-arrestin2 in the PAG (Connor et al., 2015). However, not all neuronal populations and $\mu$ receptor agonists are regulated by $\beta$-arrestin 2 in the same manner. For example, methadone impairs resensitisation to the same extent in LC neurons from $\beta$-arr ${ }^{-/}$and Wt mice. While in peripheral sensory fibres, siRNA knockdown of $\beta$-arrestin2 results in the inhibition of cAMP accumulation in the absence of $\mu$ receptor activation, which implicates $\beta$-arrestin2 as the inhibitory, regulatory mechanism for inhibiting constitutive $\mu$ receptor activity (Sullivan et al., 2016).

The differential regulatory role of $\beta$-arrestin2 throughout the CNS increases the difficulty of developing analgesics with limited $\beta$-arrestin2 recruitment. While it appears that in pain associated CNS areas (i.e. PAG, DRG, and LC) reduced $\beta$-arrestin2 increases potency and decrease tolerance. The differential cellular regulation by methadone and morphine suggests that the translation of the theory into clinically available compounds may not be straight forward.

\subsubsection{Pre-Clinical Effects of G-Protein Biased Agonists}

Many novel G-protein biased $\mu$ receptor agonists have been generated to develop better therapeutics for chronic pain. These include TRV130 and TRV0109101, which both have reduced tolerance in thermal models of anti-nociception, with a similar duration of action to morphine (Table 3.6). While PZM21 has a large reduction in potency and a similar duration of action compared to morphine (Table 3.6). To fully assess the potential of G-protein bias at the $\mu$ receptor, researchers at The Scripts Research Institute generated a library of novel compounds with varying bias factors, called the SR compounds. In vivo assessment of thermal anti-nociception and respiratory depression resulted 
in a correlation between increased G-protein bias and larger therapeutic windows. Despite many reported G-protein biased compounds, very few novel $\mu$ receptor agonists have been screened through chronic models of pain (Table 3.6). This leaves a gap in our knowledge of the clinical utility of these compounds in the more complex chronic pain states.

More work has also gone into the screening of current $\mu$ receptor therapies for G-protein bias. This includes the identification of Loperamide (peripherally restricted anti-diarrheal) and Buprenorphine (opioid replacement therapy) as G-protein biased agonists with improved side effect profiles. These effects are now believed to be due to their recently discovered G-protein bias (Burgueño et al., 2017, Emerich et al., 1998, He et al., 2013, Ray et al., 2005, Ray et al., 2008, Tayrouz et al., 2001, Thompson et al., 2015) (Table 3.6).

\subsubsection{Anti-Nociceptive Effects of Novel Mu Opioid Receptor Agonists from the Structure of Salvinorin A}

Sal $A$ has been shown to induce potent anti-nociception in vivo via the $\mathrm{k}$ receptor, however, it has a short duration of action and can induce side effects such as anxiety and depression (Butelman et al., 2009, Zhang et al., 2005). Many structural analogues of Sal A have been made by altering the C2 and C16 positions. These include Mesyl Sal B, 16-Ethynyl Sal A, and 16-Bromo Sal A (Harding et al., 2005, Riley et al., 2014). These compounds are effective in reducing drug-seeking behaviour, reducing cocaine-induced reinstatement of self-administration in Sprague Dawley rats, and induce potent antinociceptive effects in the hot water dose response tail-flick assay (Riley et al., 2014, Simonson et al., 2015). The addition of a benzene ring to the $C 2$ position to make herkinorin (bias $=0.95$ ) and subsequent insertion of the double bond to make kurkinorin (bias $=0.57$ ) increased the selectivity and bias factor at the $\mu$ receptor. When screened in preclinical rodent models, herkinorin induced less anti-nociceptive tolerance than morphine in peripheral models of pain but did not produce centrally mediated effects and was therefore not further assessed (Lamb et al., 2012) (Table 3.6). Unlike its predecessor, kurkinorin (10 mg/kg/s.c.) induces potent centrally mediated anti-nociception in the hot water tail-flick assay in mice with a similar duration of action to morphine $(10 \mathrm{mg} / \mathrm{kg} / \mathrm{i} . \mathrm{p}$.$) , overcoming$ the reduced bioavailability and CNS penetration of Sal A and herkinorin. Due to the bias factors of kurkinorin (bias $=0.57$ ) and morphine (bias $=0.36$ ), it was believed that kurkinorin would have improved potency and reduced tolerance. In the dose response tolerance tail-flick assay. Kurkinorin $\left(E D_{80}=9.7 \mathrm{mg} / \mathrm{kg} / \mathrm{s}\right.$.c. $)$ was in fact equipotent with morphine $\left(E D_{80}=9.9 \mathrm{mg} / \mathrm{kg} / \mathrm{s}\right.$.c. $)$. Following 8 days of administration, kurkinorin $\left(E D_{80}=8.0 \mathrm{mg} / \mathrm{kg} / \mathrm{s}\right.$.c.) induced mild anti-nociceptive tolerance that was significantly reduced to morphine $\left(E D_{80}=16.6 \mathrm{mg} / \mathrm{kg} / \mathrm{s}\right.$.c.) (Crowley et al., 2016). Kurkinorin has also been shown to reduce pain scores during phase 1 (nociceptive) and 2 (inflammatory) pain in the intradermal formalin assay to a similar extent to morphine (Kivell lab unpublished data). From what is currently known about the in vivo effects, kurkinorin appears to follow the paradigm of biased 
agonism with increased G-protein recruitment correlating to improved anti-nociception and tolerance (Table 3.6). To fully assess this, the structure of kurkinorin was modified with an alcohol group added to the $\mathrm{C} 6$ position of the benzene ring, making kurkinol, a heavily G-protein biased agonist, kurkinol. 


\subsection{Hypothesis and Aims}

Biased agonism at the $\mu$ receptor has been proposed to generate potent, highly efficacious analgesics for the treatment of pain. In this study, we aimed to investigate the ability of two novel $\mu$ receptor agonists with varying G-protein biases to induce analgesia in acute and chronic pre-clinical models of anti-nociception. With a secondary aim of investigating the bioavailability and duration of action of these compounds due to the profile of the parent structures, Sal A and herkinorin. We hypothesised that the more G-protein biased agonist, kurkinol, would have improved anti-nociceptive effects and reduced tolerance compared to the intermediately biased kurkinorin. Furthermore, we believed that anti-nociceptive profiles of kurkinol and kurkinorin would be altered in $\beta$-arrestin2 knockout mice, with increases expected in potency alongside decreased tolerance.

Aims:

1. Determine the duration of action of kurkinol and kurkinorin in spinal and supraspinal models of acute thermal anti-nociception utilising the hot water tail-flick and hotplate assays.

2. Assess the role of G-protein bias in the anti-nociceptive potency and tolerance to acute thermal stimuli using the hot water tail-flick assay in C57BL/6J and $\beta$-arr2 ${ }^{-1-}$ mice.

3. Investigate the ability of $\mathrm{G}$-protein biased $\mu$ receptor agonists to induce anti-nociception and tolerance in chronic, neuropathic pain states using the chemotherapy-induced neuropathic pain model in C57BL/6J mice. 


\subsection{Results}

\subsubsection{Dose response Effects of Kurkinol in the Hot water Tail-flick Assay}

The hot water tail-flick assay was used to assess the spinal potency of kurkinorin and kurkinol in an acute thermal model of pain. Non-linear regression was used to fit a curve to the dose response data to generate the potency $\left(E C_{50}\right)$ and efficacy $\left(E_{\max }\right)$ of kurkinol, which were then compared to the standard $\mu$ receptor agonist, morphine. Before One and Two-way repeated measures ANOVA statistical analysis, the normalcy of all data was assessed using the D'Agostino \& Pearson omnibus K2 normality test.

\subsubsection{Kurkinol is More Potent and Shows Reduced Tolerance in the Tail-Flick Assay}

Kurkinol $\left(E D_{50}=2.37 \mathrm{mg} / \mathrm{kg} / \mathrm{s}\right.$.c. $)$ was found to be 2.7 fold more potent than morphine $\left(E D_{50}=\right.$ $6.40 \mathrm{mg} / \mathrm{kg} / \mathrm{s} . \mathrm{c}$.) on the first dose response day $\left(\mathrm{F}_{(3,28)}=135.5, p<0.0001, p<0.0001\right)$ (Fig. $3.1 \mathrm{~A}$, closed symbols). The animals then received daily injections of morphine $(10 \mathrm{mg} / \mathrm{kg} / \mathrm{s} . \mathrm{c}$.), kurkinol (5 $\mathrm{mg} / \mathrm{kg} / \mathrm{s}$.c.), or vehicle with the induction of tolerance tracked on days 3,6 , and 9 . Kurkinol showed no decrease in anti-nociceptive tolerance over the 9 day period compared to vehicle treated mice, while a significant reduction in tail withdrawal latency with morphine was seen from day $6\left(F_{(8,84)}=\right.$ 25.71, $p<0.0001, p<0.0001$ ) (Fig. 3.1 B). When animals were challenged with a second dose response on day 9 (Fig. $3.1 \mathrm{~A}$, open symbols), morphine had induced a 2.6 fold decrease in potency compared to day $1\left(E_{50}=16.67 \mathrm{mg} / \mathrm{kg} / \mathrm{s}\right.$.c., $\left.p<0.0001\right)$, whereas kurkinol showed no significant difference $\left(\mathrm{ED}_{50}\right.$ $=3.62 \mathrm{mg} / \mathrm{kg} / \mathrm{s}$.c., $p>0.9999$ ). Hyperalgesia was also measured with Von Frey filament on days 0,2 , 4,6 , and 8 (Fig. 3.1 C). Both morphine and kurkinol induced a significant decrease in paw withdrawal threshold, hyperalgesia, compared to vehicle from day $4(p<0.05)$ of chronic administration with no significant difference between the two $\mu$ receptor agonists by day $8\left(F_{(8,72)}=5.55, p<0.0001, p\right\rangle$ 0.9999) (Table 3.1).

The $\mathrm{ED}_{80}$ and $\mathrm{ED}_{50}$ doses of morphine $(10 \& 5 \mathrm{mg} / \mathrm{kg}$ ) and kurkinol $(5 \& 2.5 \mathrm{mg} / \mathrm{kg}$ ) from this experiment were used to inform additional preclinical tests to evaluate both anti-nociceptive effects and side effects. The $\mathrm{ED}_{80}$ and $\mathrm{ED}_{50}$ doses of kurkinorin $(10 \& 5 \mathrm{mg} / \mathrm{kg}$ ) were already determined utilising the same experimental protocol (previously conducted in the Kivell lab by Dr Kelly Paton and Richard Anderson III, Crowley et al., 2016). 
A)

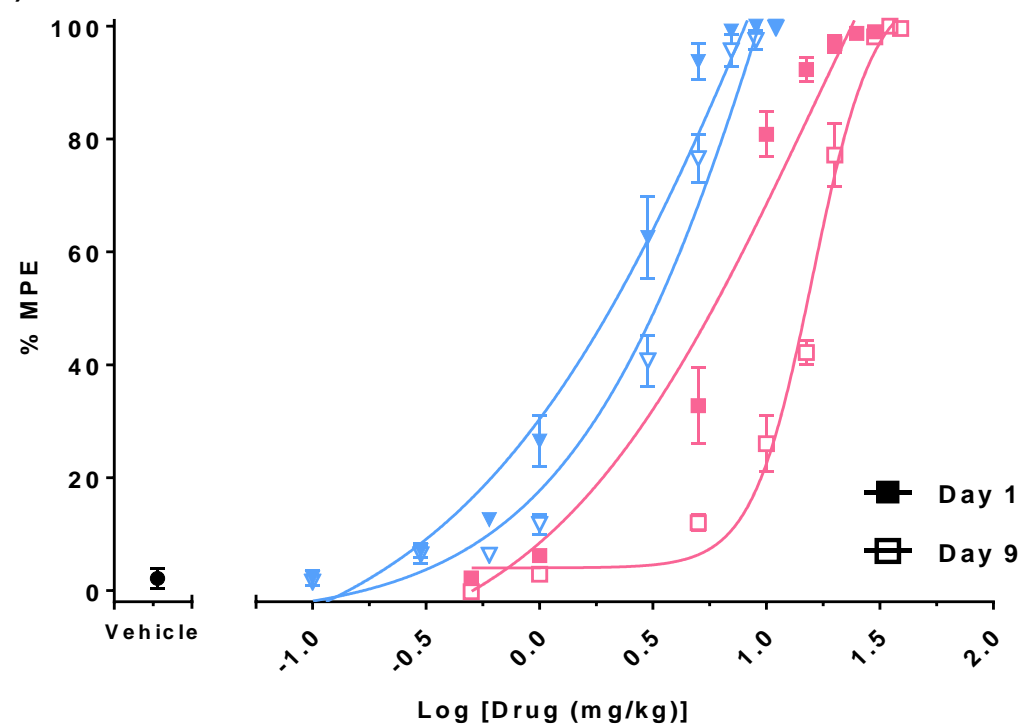

B )

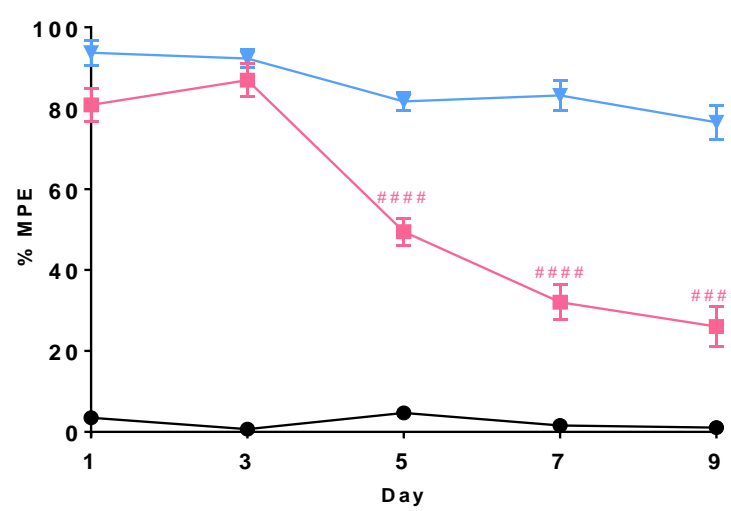

C)

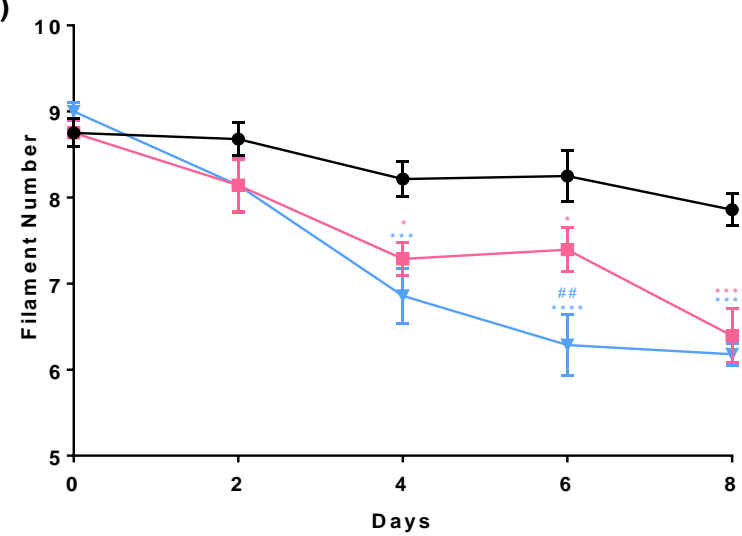

Vehicle

Morphine (10)

$\neg$ Kurkinol (5)

Figure 3.1 Potency and Efficacy of Kurkinol Centrally Mediated Anti-Nociceptive Effects

The anti-nociceptive potency of kurkinol was assessed in comparison to morphine using a cumulative dosing scheme in C57BL/6J mice on day 1 (closed symbols) and following 8 days sub-chronic dosing (open symbols). The induction of $(\mathbf{A}, \mathbf{B})$ tolerance and $(\boldsymbol{C})$ hyperalgesia was tracked over the 9 days. $(\boldsymbol{A}$, B) On day 1 kurkinol was shown to be significantly more potent than morphine and induced no significant tolerance by day 9. (C) Kurkinol induced the same level of hyperalgesia as morphine by day 8. Two-way repeated measures ANOVA with Bonferroni multiple corrections test, ${ }^{*} p<0.05$,

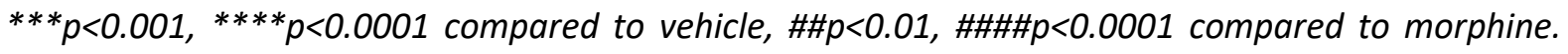
Data are presented as a percentage of the maximal possible effect (\%MPE). Data are shown as mean \pm SEM ( $N=7-8$ per group). Doses in brackets in $\mathrm{mg} / \mathrm{kg} / \mathrm{s} . \mathrm{c}$. 


\subsubsection{Anti-Nociceptive Tolerance to Acute Thermal Stimuli is Reduced in $\beta$ - arrestin2 Knock-Out Mice}

Previous work has shown that the deletion of $\beta$-arrestin2 increases the anti-nociceptive potency and reduced tolerance in an acute thermal model of pain. We, therefore, conducted the hot water dose response tolerance tail-flick experiment in $\beta$-arr2 ${ }^{--}$and $\mathrm{Wt}$ littermate controls to further investigate the roles of $\mathrm{G}$-proteins and $\beta$-arrestin2. (Fig. 3.2). On day 1, morphine was shown to be 1.8 fold more potent in $\beta$-arr2 ${ }^{--}$mice $\left(E D_{50}=5.02 \mathrm{mg} / \mathrm{kg} / \mathrm{s} . c\right.$. Vs $E D_{50}=7.88 \mathrm{mg} / \mathrm{kg} / \mathrm{s}$.c.) $(p=0.036)$. When challenged with a second dose response on day 9, morphine-induced a fold 2.6 fold decrease in potency in Wt mice, while the $\beta$-arr2 ${ }^{-/}$mice showed a 1.4 fold improvement in anti-nociceptive tolerance to the Wt littermate controls $\left(W_{t}: E_{50}=13.55 \mathrm{mg} / \mathrm{kg} / \mathrm{s}\right.$. c., $\beta-\mathrm{arr}^{-1-}: \mathrm{ED}_{50}=9.12 \mathrm{mg} / \mathrm{kg} / \mathrm{s}$.c., $p<0.05$ ) (Fig. 3.2 A, Table 3.1).

When the induction of tolerance to opioid-induced anti-nociception was tracked between the dose response days a significant decrease in tail withdrawal latencies was observed in morphine treated Wt mice from day 4 compared to vehicle. While no significant decrease in tail withdrawal latency compared to vehicle was observed in the $\beta$-arr ${ }^{--}$mice, with a significant increase in tail withdrawal latency compared to Wt mice also observed from day $4\left(\mathrm{~F}_{(12,72)}=6.49, p<0.0001, p<0.05\right)$ (Fig. 3.2 B). The more G-protein biased kurkinol showed no difference in potency between $\beta$-arr2 ${ }^{-/-}$ and Wt littermate controls on day 1 or day $9\left(F_{(3,16)}=2.35, p=0.1107\right)$ (Fig 3.2 C, Table 3.1). During tolerance tracking between dose response days neither $\beta$-arr ${ }^{--}$nor the $\mathrm{Wt}$ littermate controls induced significant tolerance, with no difference between genotypes $\left(F_{(12,64)}=6.004, p<0.0001, p\right\rangle$ 0.9999) (Fig 3.2 D). The deletion of $\beta$-arrestin2 did not affect the potency of kurkinorin on day 1 compared to their Wt controls (Wt: $\mathrm{ED}_{50}=5.81 \mathrm{mg} / \mathrm{kg} / \mathrm{s}$.c., $\beta-\mathrm{arr}^{-1-}: \mathrm{ED}_{50}=5.32 \mathrm{mg} / \mathrm{kg} / \mathrm{s}$.c.). However, there was a significant induction of tolerance in Wt mice on day 9, with a 1.8-fold decrease in potency compared to day 1 . This was reduced by 1.7 fold in the $\beta$-arr2 ${ }^{-/}$mice $\left(W t: E D_{50}=10.71 \mathrm{mg} / \mathrm{kg} / \mathrm{s}\right.$.c., $\beta$ $\operatorname{arr}^{-1-:} \mathrm{ED}_{50}=6.02 \mathrm{mg} / \mathrm{kg} / \mathrm{s} . \mathrm{c}$.) $\left(F_{(3,16)}=7.17, p=0.0029, p<0.05\right)$ (Fig. $3.2 \mathrm{E}$, Table 3.1). Tracking of tolerance with tail-flick measurements on alternate days showed no significant effect of kurkinorin on anti-nociceptive tolerance, no further analysis was performed $\left(F_{(12,64)}=1.28, p=0.2513\right)$ (Fig 3.2 F). 
A)

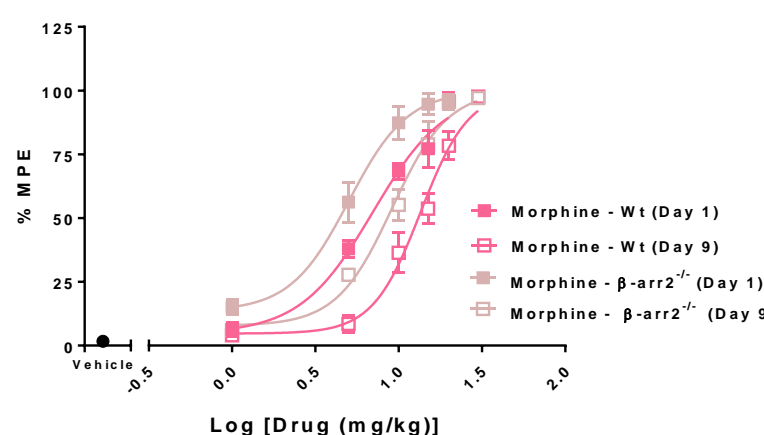

C)

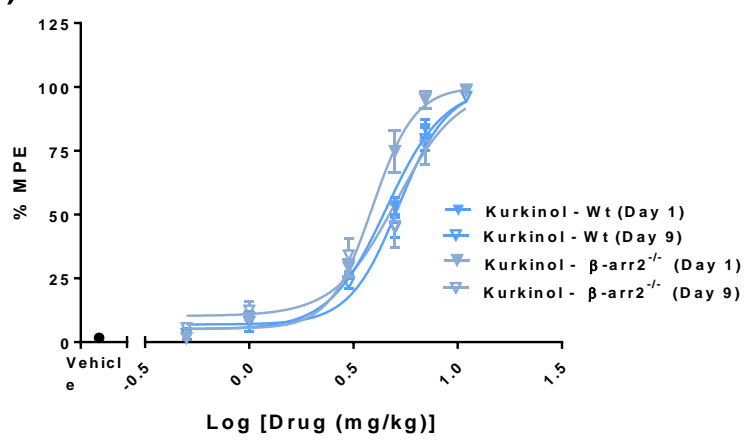

E)

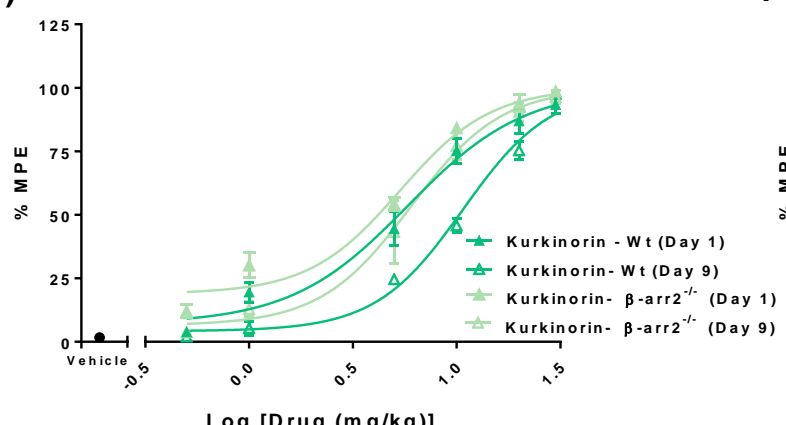

B )

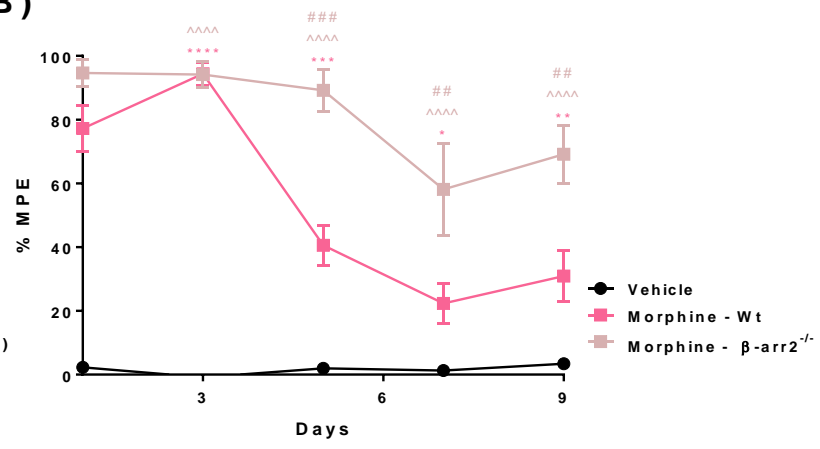

D)

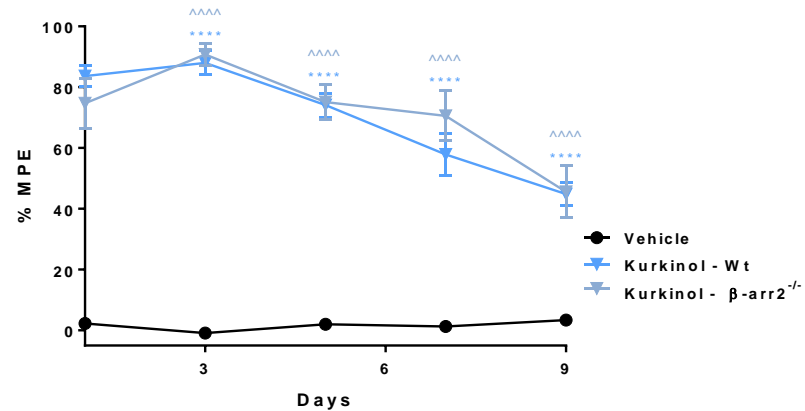

F)

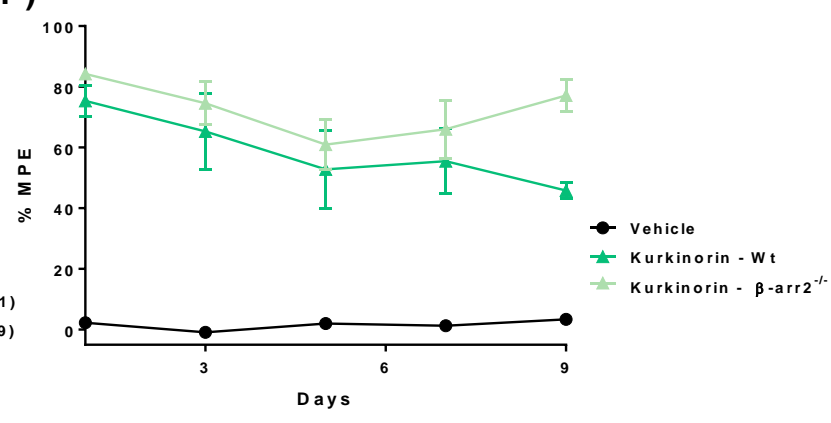

Figure 3.2 $\beta$-Arrestin2 Knock Out Mice Increase the Potency of Morphine, but not Kurkinorin or Kurkinol

The role of $\beta$-arrestin2 in the development of anti-nociceptive tolerance was assessed using the dose response tolerance hot water tail-flick assay in $\beta$-arr $2^{-}$. (A, B) $\beta$-arrestin2 was shown to be involved in the potency and tolerant effects of morphine, with an increase in potency in the $\beta$-arr $2^{-1}$ mice on day 1 with reduced tolerance on day $9 .(C, D)$ However, no increase in potency was seen for kurkinorin, but day 9 kurkinorin induced reduced tolerance in $\beta$-arr-1- mice. (E, F) The most G-protein biased $\mu$ receptor agonists kurkinol was not affected by the deletion of $\beta$-arrestin2.Two-way repeated measures ANOVA with Bonferroni multiple corrections test, ${ }^{*} p<0.05,{ }^{* *} p<0.01,{ }^{* * *} p<0.001,{ }^{* * * *} p<0.0001$ compared to

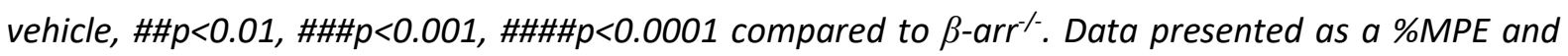
shown as mean \pm SEM ( $N=5-6$ per group). Doses in brackets in $\mathrm{mg} / \mathrm{kg} / \mathrm{s}$.c. 
Table 3.1 Tolerance Effects in Wildtype C57BL/6J \& $\beta$-Arr2 $\%$ Mice

\begin{tabular}{|c|c|c|c|c|c|c|c|c|c|c|c|c|c|}
\hline & Genotype & $\begin{array}{c}\mathrm{ED}_{50} \pm \mathrm{SEM} \\
\text { Day } 1 \\
(\mathrm{mg} / \mathrm{kg} / \mathrm{s.c.})\end{array}$ & \multicolumn{2}{|c|}{$\begin{array}{l}p \text { value to } \\
\text { morphine }\end{array}$} & \multicolumn{2}{|c|}{$\begin{array}{l}p \text { value to } \\
\text { Wt } \\
\text { littermate } \\
\text { control }\end{array}$} & $\begin{array}{c}\mathrm{ED}_{50} \pm \mathrm{SEM} \\
\text { Day } 9 \\
(\mathrm{mg} / \mathrm{kg} / \mathrm{s.c} .)\end{array}$ & \multicolumn{2}{|c|}{$\begin{array}{c}p \text { value to day } \\
1\end{array}$} & \multicolumn{2}{|c|}{$\begin{array}{c}p \text { value to } \mathrm{Wt} \\
\text { littermate control }\end{array}$} & \multicolumn{2}{|c|}{$\begin{array}{l}p \text { value to } \\
\text { morphine }\end{array}$} \\
\hline Morphine & $\mathrm{C} 57 \mathrm{BL} / 6 \mathrm{~J}$ & $6.40 \pm 0.58$ & - & - & - & - & $16.67 \pm 0.57$ & & $* * * *$ & - & - & - & - \\
\hline Kurkinorin ${ }^{+}$ & C57BL/6J & $5.00 \pm 0.51$ & & n.s & - & - & $8.00 \pm 0.11$ & & $*$ & - & - & & $* *$ \\
\hline Kurkinol & C57BL/6J & $2.37 \pm 0.36$ & $<0.0001$ & $* * * *$ & - & - & $3.62 \pm 0.38$ & $>0.9999$ & n.s & - & - & $<0.0001$ & $* * * *$ \\
\hline Morphine & $\begin{array}{c}\text { Wt } \\
\beta \text {-arr2 }\end{array}$ & $\begin{array}{l}7.88 \pm 1.53 \\
5.02 \pm 1.01\end{array}$ & - & - & $\begin{array}{c}- \\
=0.036\end{array}$ & $\begin{array}{ll}- \\
*\end{array}$ & $\begin{array}{c}13.55 \pm 3.08 \\
9.12 \pm 3.19\end{array}$ & $\begin{array}{l}=0.0005 \\
=0.0801\end{array}$ & $\begin{array}{l}* * * \\
\text { n.s }\end{array}$ & $\begin{array}{c}- \\
>0.9999\end{array}$ & $\begin{array}{c}- \\
=0.0273\end{array}$ & - & - \\
\hline Kurkinorin & $\begin{array}{c}\text { Wt } \\
\beta \text {-arr2 }\end{array}$ & $\begin{array}{l}5.81 \pm 1.23 \\
5.32 \pm 1.02\end{array}$ & $\begin{array}{l}=0.9344 \\
>0.9999\end{array}$ & $\begin{array}{l}\text { n.s } \\
\text { n.s }\end{array}$ & $\begin{array}{c}- \\
=0.6231\end{array}$ & $\begin{array}{l}- \\
\text { n.s }\end{array}$ & $\begin{array}{c}10.74 \pm 0.73 \\
6.02 \pm 1.39\end{array}$ & $\begin{array}{l}=0.0269 \\
>0.9999\end{array}$ & $\begin{array}{l}* \\
\text { n.s }\end{array}$ & $\begin{array}{c}- \\
=0.0274\end{array}$ & $\begin{array}{l}- \\
*\end{array}$ & $\begin{array}{l}<0.0001 \\
=0.1714\end{array}$ & $\begin{array}{c}* * * * \\
\text { n.s }\end{array}$ \\
\hline Kurkinol & $\begin{array}{c}\mathrm{Wt} \\
\beta-\mathrm{arr2}^{-/-}\end{array}$ & $\begin{array}{l}5.07 \pm 0.23 \\
3.89 \pm 0.27\end{array}$ & $\begin{array}{l}>0.9999 \\
>0.9999\end{array}$ & $\begin{array}{l}\text { n.s } \\
\text { n.s }\end{array}$ & $\begin{array}{c}- \\
>0.9999\end{array}$ & n. & $\begin{array}{l}5.21 \pm 0.67 \\
5.07 \pm 0.19\end{array}$ & $\begin{array}{l}>0.9999 \\
>0.9999\end{array}$ & $\begin{array}{l}\text { n.s } \\
\text { n.s }\end{array}$ & $\begin{array}{c}- \\
>0.9999\end{array}$ & $\begin{array}{l}- \\
\text { n.s }\end{array}$ & $\begin{array}{l}<0.0001 \\
=0.0287\end{array}$ & $\begin{array}{c}* * * * \\
*\end{array}$ \\
\hline
\end{tabular}

The $E D_{50}$ doses $(\mathrm{mg} / \mathrm{kg} / \mathrm{s.c.}$ ) on day 1 and day 9 of the hot water dose response tolerance tail-flick are compared between mouse strains and genotypes. Oneway ANOVA with Bonferroni multiple corrections test. $n . s=$ non-significant. Data shown as mean \pm SEM (N = 5-8 per group).

${ }^{+}$(Crowley et al., 2016)

Wt $=$ Wildtype littermate control. $\beta$-arr $2^{-}=\beta$-arrestin2 knockout. 


\subsubsection{Kurkinol Has a Similar Duration as Morphine in the Tail-Flick Assay}

The spinally mediated hot water tail-flick assay was further utilised to assess both the onset of anti-nociception and its duration compared to morphine. Morphine was found to have significant anti-nociceptive effects from $15-120 \mathrm{~min}$ at $10 \mathrm{mg} / \mathrm{kg} / \mathrm{i}$.p. and $15-90 \mathrm{~min}$ at $5 \mathrm{mg} / \mathrm{kg} / \mathrm{i} . \mathrm{p}$. $(\mathrm{F}(45,270)=$ 20.3, $p<0.0001, p<0.05$ ) (Fig. 3.3 A). While kurkinol had a faster onset of anti-nociceptive effects with a significant increase compared to vehicle seen from $5 \mathrm{~min}$. There was an overall reduction in duration of action with anti-nociception lasting until $90 \mathrm{~min}(5 \mathrm{mg} / \mathrm{kg} / \mathrm{i}$. .p.) and $60 \mathrm{~min}(2.5 \mathrm{mg} / \mathrm{kg} /$ i.p., $p<0.05)$. The anti-nociceptive effects of kurkinol, from 5-60 min, was partially inhibited following prior administration of the $\mu$ receptor selective antagonist, $\beta$-FNA ( $5 \mathrm{mg} / \mathrm{kg} / \mathrm{i}$.p., $24 \mathrm{hrs}$ pre-treatment) $(p<0.05)$. However, a significant anti-nociceptive effect was also found in $\beta$-FNA pre-treated kurkinol mice between $15-45 \mathrm{~min}(p<0.05)$ suggesting either the dose used was not high enough for complete inhibition of the $\mu$ receptor or another mechanism of action may be partially responsible for the antinociceptive effect of this compound.

Area under the curve (AUC) analysis was then used to assess the overall anti-nociceptive effects of kurkinol and morphine, with both showing significantly increased anti-nociception compared to vehicle-treated mice $\left(\mathrm{F}_{(5,29)}=144.4, p<0.0001, p<0.0001\right)$ (Fig $3.3 \mathrm{~B}$ ). Kurkinol (5 $\mathrm{mg} / \mathrm{kg} / \mathrm{i}$.p.) was also shown to have significantly lower anti-nociceptive effects than the equivalent dose of morphine $(10 \mathrm{mg} / \mathrm{kg} / \mathrm{i}$. ., $p<0.0001)$. Once again $\beta$-FNA pre-treated animals had a significant reduction to the equivalent dose $(p<0.0001)$, while no significant difference to vehicle treatment was seen $(p=0.20)$. 

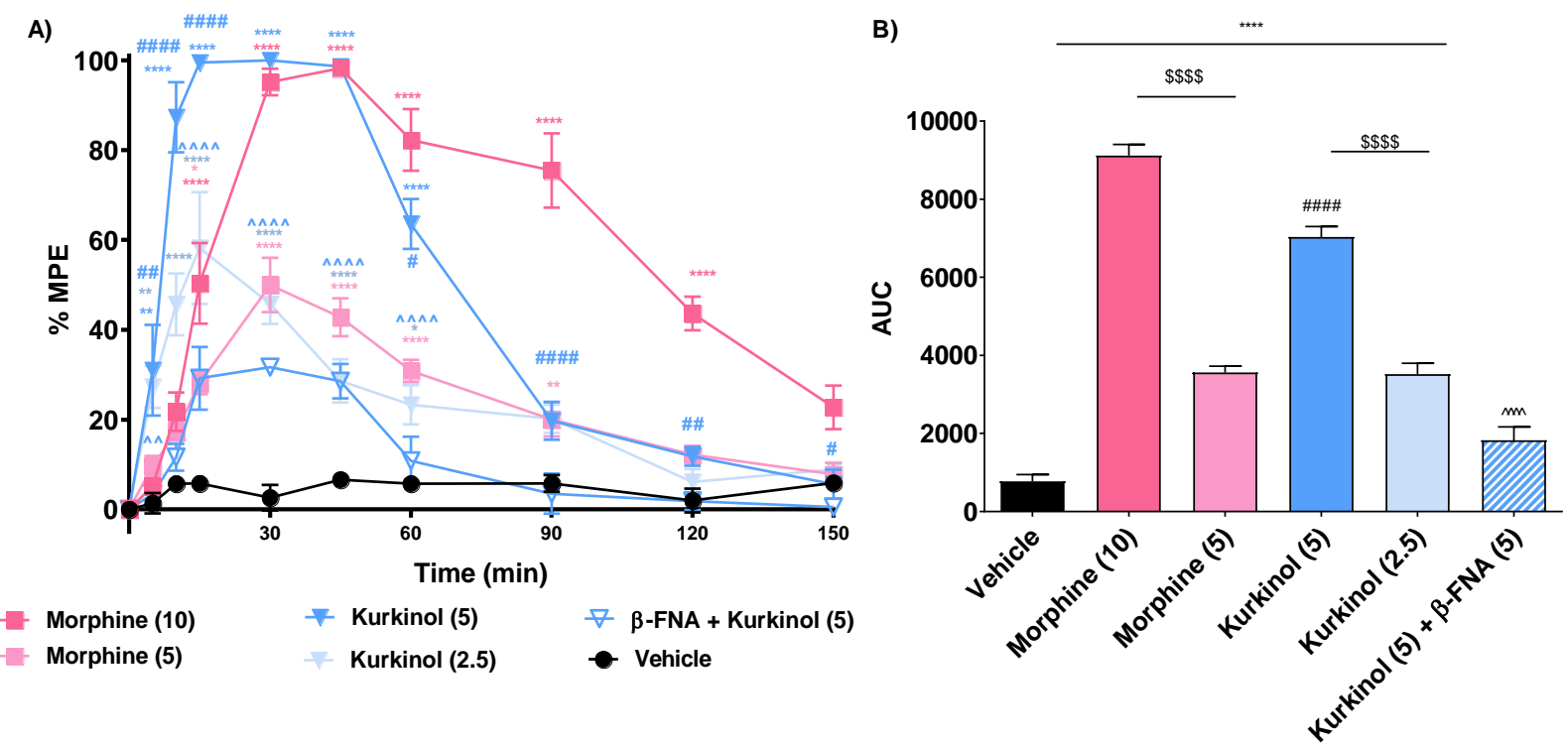

Figure 3.3 Duration of Action of Kurkinol in the Hot water Tail-flick Assay

Onset and duration of the spinally mediated anti-nociceptive effects of kurkinol and morphine were assessed in the hot water tail-flick assay. (A) Kurkinol had rapid onset with a slight reduction in duration of action. Prior administration with the $\mu$ receptor antagonist $\beta$-FNA $(5 \mathrm{~m} / \mathrm{kg})$ significantly reduced the anti-nociceptive effects of kurkinol. (B) AUC analysis showed a significant decrease in the antinociceptive effect of kurkinol (5 mg/kg/i.p.) compared to morphine (10 mg/kg/i.p.) and $\beta$-FNA pretreated mice. (A) Two-way repeated measures ANOVA with Bonferroni multiple corrections test. (B) One-way repeated measures ANOVA with Bonferroni multiple corrections test, ${ }^{* *} p<0.01,{ }^{* * *} p<0.001$, $* * * * p<0.0001$, drug compared to vehicle, $\# p<0.05, \# \# p<0.01, \# \# \# p<0.001, \# \# \# \#<0.0001$ compared to morphine, $\$ \$ \$ \$ p<0.0001 E D_{80}$ compared to $E D_{50}$ dose, $\wedge \wedge \wedge \wedge p<0.0001$ kurkinol compared to kurkinol+ $\beta$-FNA. Data presented as \%MPE at each time point and shown as mean \pm SEM $(N=5-7$ per group). Doses in brackets in $\mathrm{mg} / \mathrm{kg} / \mathrm{i}$.p. 


\subsubsection{The Anti-Nociceptive Effects of Kurkinol and Kurkinorin are Reversed in Mu Opioid Receptor Knock-Out Mice}

The selectivity of kurkinorin and kurkinol for the $\mu$ receptor, and therefore the mechanism through which the anti-nociceptive effects are induced, was investigated using $\mu$ receptor ${ }^{-/}$mice and their Wt littermate controls. A single injection of vehicle or the morphine $(10 \mathrm{mg} / \mathrm{kg} / \mathrm{i} . \mathrm{p}$.$) , kurkinol (5$ $\mathrm{mg} / \mathrm{kg} /$ i.p.), or kurkinorin ( $10 \mathrm{mg} / \mathrm{kg} /$ i.p.) were administered and the anti-nociceptive effects assessed at $30 \mathrm{~min}$ (Fig 3.4 A). No significant difference was identified between vehicle-treated $\mu$ receptor ${ }^{-1}$ and Wt mice, indicating that the removal of the $\mu$ receptor does not affect the ability of the mice to interpret nociceptive stimuli $\left(F_{(7,40)}=96.07, p<0.0001, p>0.9999\right)$. Significant anti-nociception was found with morphine, kurkinol, and kurkinorin in Wt animals $(p<0.0001)$, which was significantly reduced in the $\mu$ receptor $^{-1-}$ mice $(p<0.0001)$. These results suggest that partial inhibition of antinociception by $\beta$-FNA ( $5 \mathrm{~m} / \mathrm{kg}$ ) is likely due to the low dose and not off target effects. Together indicating that the anti-nociceptive properties of kurkinol and kurkinorin are induced through their action at the $\mu$ receptor.

To ensure the anti-nociceptive effects of kurkinorin were not partially mediated through the $\mathrm{\kappa}$ receptor, $\mu$ receptor ${ }^{-/-}$mice were pre-treated with the $\mathrm{k}$ receptor selective antagonist, Nor-BNI (10 $\mathrm{mg} / \mathrm{kg} / \mathrm{i}$. . ., $24 \mathrm{hrs}$ pre-treatment), and a single tail-flick measurement taken $30 \mathrm{~min}$ postadministration (Fig 3.4 B). Nor-BNI treatment did not affect the tail-flick latency of Wt or $\mu$ receptor ${ }^{-1}$ animals, so all vehicle-treated animals were pooled into a single group based on genotype ( $p>0.9999)$. In Wt mice kurkinorin induced potent anti-nociception, with no effect of Nor-BNI pre-treatment $\left(\mathrm{F}_{55}\right.$, $\left.{ }_{48)}=329.5, p<0.0001, p<0.9999\right)$. In $\mu$ receptor ${ }^{-1 /}$, mice there was a significant decrease in the antinociceptive effects compared to their Wt littermate controls, with no further reduction in antinociception in Nor-BNI treated animals ( $p=0.2858$ ). 

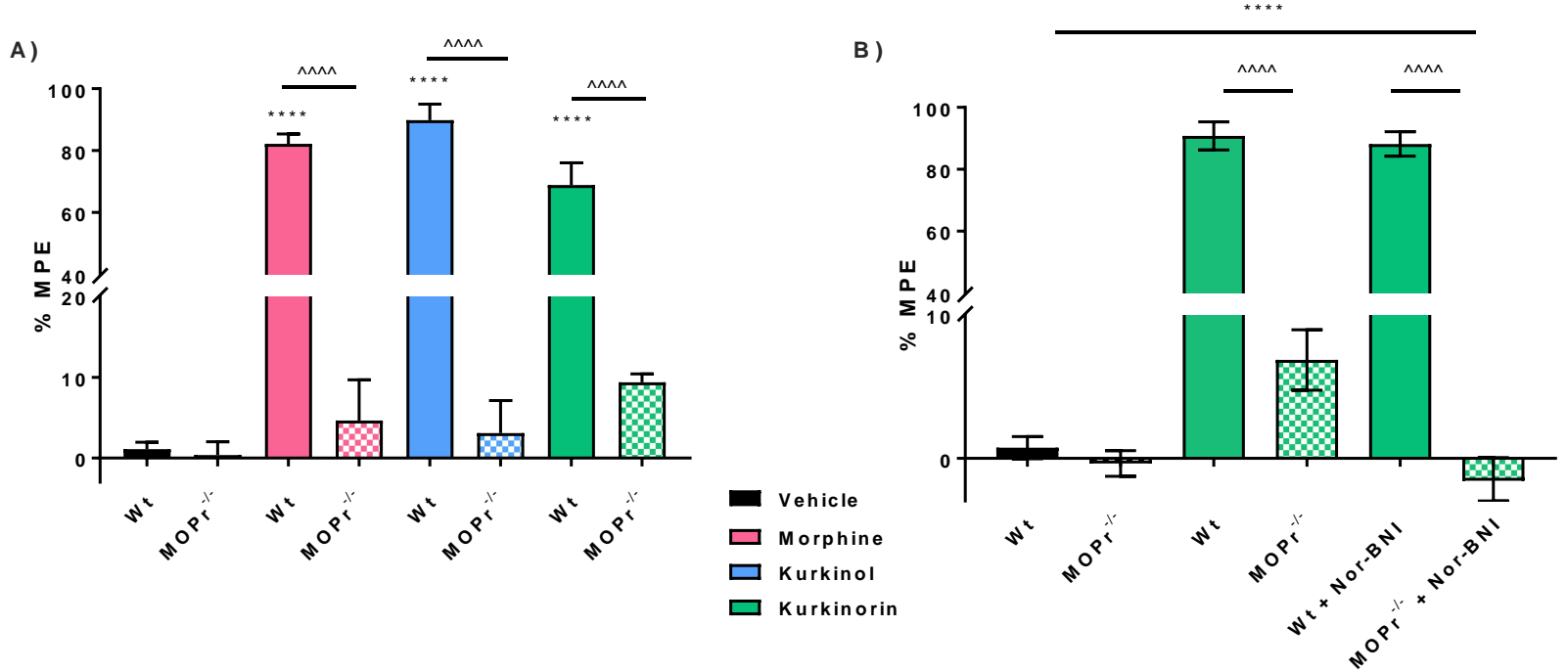

Figure 3.4 Anti-Nociceptive Effects of Mu Opioid Receptor Agonists in Mu Opioid Receptor Knock Out Mice and Wildtype Littermate Controls

A single dose of vehicle, morphine (10 mg/kg/i.p.), kurkinol (5 mg/kg/i.p.), or kurkinorin (10 mg/kg/i.p.) was administered to $\mu$ receptor ${ }^{-1}$ and the anti-nociceptive effects measured 30 mins later. All data is presented as a \%MPE. (A) All Wt animals treated with the $\mu$ receptor agonists had strong antinociceptive responses at $30 \mathrm{~min}$. These effects were significantly reduced in the $\mu$ receptor-1- mice, indicating all agonists examined induce their anti-nociceptive effects through the $\mu$ receptor. (B) Pretreatment of $\mu$ receptor-- and Wt mice with the $\mathrm{k}$ receptor antagonist Nor-BNI (10 mg/kg/i.p., $24 \mathrm{hrs}$ pre-treatment) did not affect tail-flick latency of kurkinorin. One-way repeated measures ANOVA with Bonferroni multiple corrections test, ${ }^{* * * *} p<0.0001$, drug compared to vehicle, $\wedge^{\wedge \wedge \wedge} p<0.0001 \mu$ receptor-1- compared to Wt littermate controls. Data are shown as mean \pm SEM ( $N=5-6$ per group). Doses in brackets in $\mathrm{mg} / \mathrm{kg} / \mathrm{i} . \mathrm{p}$. 


\subsubsection{Duration of Action of Kurkinol and Kurkinorin in the Hotplate Assay}

To understand the effect of sex, treatment, and time on thermal supraspinal pain evoked in the hotplate assay, Three-way mixed ANOVAs were conducted. Unfortunately, the morphine data did not meet the criteria of equal variance to perform this parametric test as the ratio of variance was greater than 3. Therefore, non-parametric tests were investigated. However, the data also did not meet the requirements for these tests. To overcome this issue, the Three-way mixed ANOVA was performed both with and without the data with unequal variance $(30 \mathrm{~min}$ and $45 \mathrm{~min}$ postadministration). No significant difference was found between the two analyses, so it was determined to be robust. From this point, the analysis was conducted without the data with uneven variance. All other data met the assumptions for ANOVA analysis and was performed as previously described (Chapter 2, Section 2.6).

The anti-nociceptive effects of morphine ( $10 \mathrm{mg} / \mathrm{kg} / \mathrm{i} . \mathrm{p}$.) were found to be affected by the sex of the animals, with a greater overall effect in females $\left(F_{(3,0.104)}=3.789, p=0.014, p=0.009\right)$ (Fig. 3.5 J). The sex of the animals also interacted with morphine to affect anti-nociception over time, with morphine $(5 \mathrm{mg} / \mathrm{kg} /$ i.p.) more effective in males at $15 \mathrm{~min}, 60 \mathrm{~min}$ and $90 \mathrm{~min}(p<0.05)$ (Fig. $3.5 \mathrm{~A})$. Interestingly, at $10 \mathrm{mg} / \mathrm{kg} / \mathrm{i}$.p., the opposite was seen with females more affected at $15 \mathrm{~min}$ and 120 $\min \left(F_{(6,0.413)}=7.517, p<0.0001, p<0.05\right)$ (Fig. $\left.3.5 \mathrm{D}\right)$. Overall morphine had a duration of action of 90 and $120 \mathrm{~min}$ at 5 and $10 \mathrm{mg} / \mathrm{kg} / \mathrm{i} . p$. respectively $\left(\mathrm{F}_{(6,0.78)}=14.307, p<0.0001, p<0.0001\right)$ (Fig. $\left.3.5 \mathrm{G}\right)$. Kurkinol had a shorter duration of action than morphine with the anti-nociceptive effects lasting for only $45 \mathrm{~min}$. This reduced anti-nociceptive effect was found to be dependent on the $\mu$ receptor as pretreatment with the antagonist $\beta$-FNA resulted in a full reversal of the anti-nociceptive effects $\left(F_{(15}\right.$, $127.117)=8.478, p<0.0001, p<0.05$ ). Unlike morphine, no effect of sex was found with kurkinol (main effect; $F_{(2,5.2)}=2.6, p=0.119$, Interaction over time; $\left.F_{(10,9.45)}=0.945, p=0.757\right)$. The anti-nociceptive duration of kurkinorin was found to be similar to that of morphine, with effects lasting until $90 \mathrm{~min}$ and $45 \mathrm{~min}$ at $10 \mathrm{mg} / \mathrm{kg} /$ i.p. and $5 \mathrm{mg} / \mathrm{kg} /$ i.p. respectively $\left(\mathrm{F}_{(15,5.545)}=4.44, p<0.0001, p<0.05\right)$. Once again, pre-treatment with $\beta$-FNA reduced the anti-nociceptive effects of kurkinorin $(10 \mathrm{mg} / \mathrm{kg} / \mathrm{i}$. .p.) indicating that the anti-nociceptive effect is due to the actions at this receptor (Fig. $3.5 \mathrm{I}$ ). While kurkinorin and the sex of the animals did not influence anti-nociception over time $\left(\mathrm{F}_{(15,1.462)}=1.172, p\right.$ $=0.303)$, a main effect of sex on anti-nociception was identified, with males $(5 \mathrm{mg} / \mathrm{kg} / \mathrm{i}$. .p. $)$ showing increased effects $\left(\mathrm{F}_{(3,7.611)}=3.451, p=0.034, p<0.0001\right)$ (Fig. $\left.3.5 \mathrm{~L}\right)$. When the main anti-nociceptive effects of each compound were compared, irrespective of sex, morphine, kurkinol, and kurkinorin all produced a strong anti-nociception compared to vehicle treated mice (Fig. 3.6 A). With dosing effects also present for all treatments. Furthermore, $\beta$-FNA significantly reduced the anti-nociceptive effects of Kurkinorin (10 mg/kg/i.p.) and kurkinol ( $5 \mathrm{mg} / \mathrm{kg} / \mathrm{i}$.p.) such that no change was identified to vehicle treated mice $\left(F_{(8,85)}=50.79, p<0.0001\right)$. 
To ensure that the anti-nociceptive effects of kurkinol and kurkinorin are due to their action at the $\mu$ receptor the hotplate assay was also conducted in $\mu$ receptor ${ }^{-}$mice and Wt littermate controls (Fig. 3.6 B). In the Wt animals' morphine, kurkinol, and kurkinorin all produced strong antinociception as in the C57BL/6J mice $(p<0.05)$. These effects were significantly reduced in the $\mu$ receptor $^{-/}$mice, with no anti-nociceptive effect compared to the vehicle controls and interaction $\left(F_{(42,}\right.$ 240) $=10.8, p<0.0001, p<0.9999)$. This confirms that all the analgesic effects seen in the hotplate assay are dependent on the action at the $\mu$ receptor. 
A)

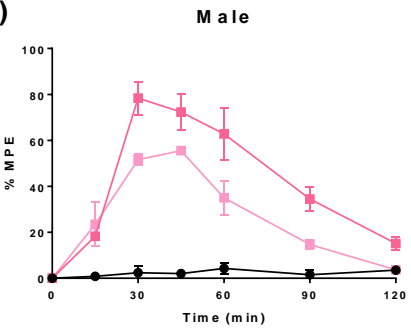

D)

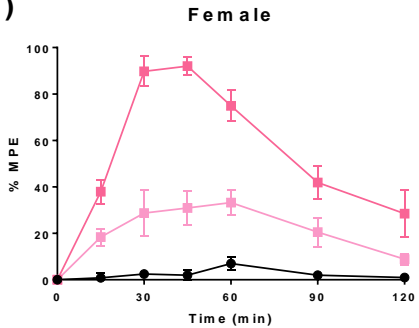

G)

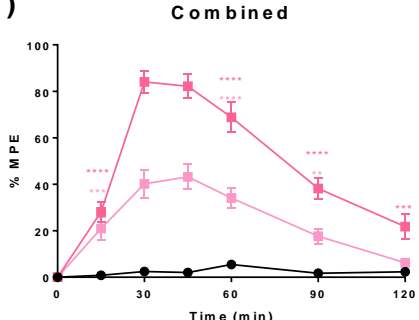

J)

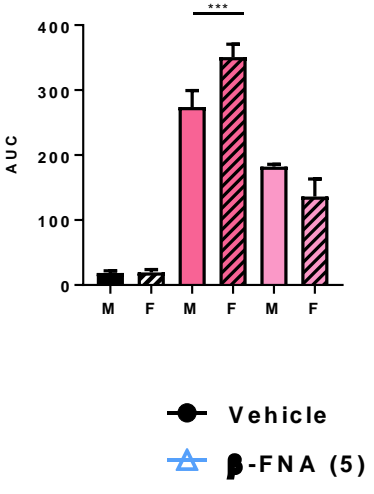

B)

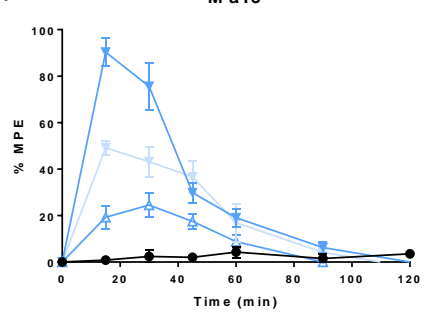

E)

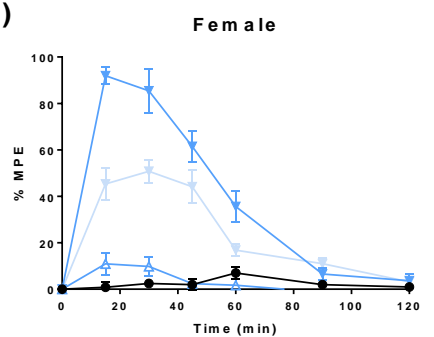

H)

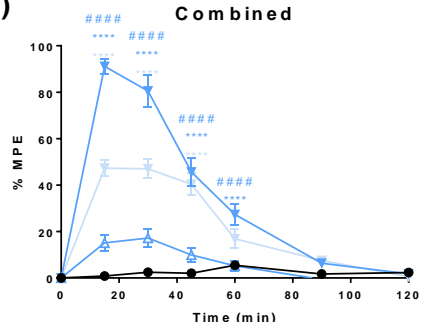

K)

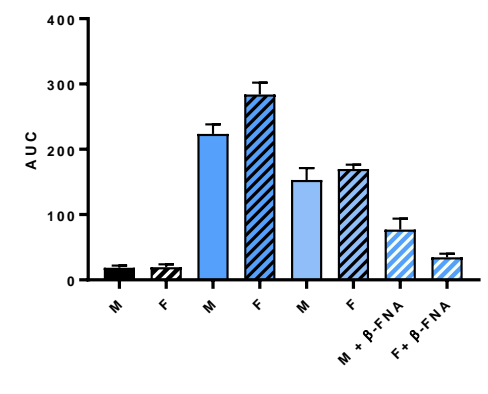

Morphine (10) $\rightarrow$ Kurkinol (5)

Morphine (5)
C)

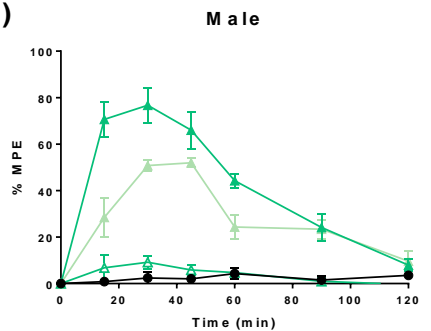

F)

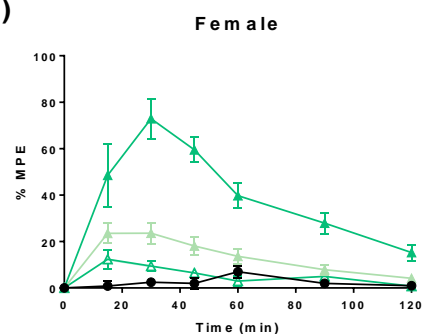

I)

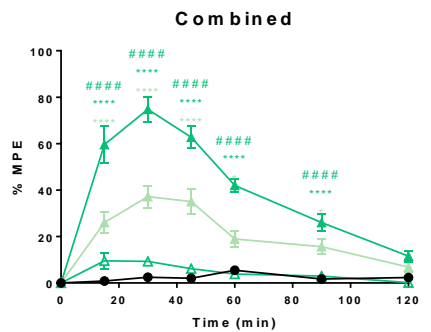

L)



\pm Kurkinorin $(10)$

$=$ Kurkinorin (5)

Figure 3.5 Kurkinol and kurkinorin has a Similar Duration of Action to Morphine in the Hotplate Assay

(A-C) Male time course effects. (D-F) Female time course effects. (G) Morphine produced potent antinociception until 90 and $120 \mathrm{~min}$ at 5 and $10 \mathrm{mg} / \mathrm{kg} / \mathrm{i} . \mathrm{p}$. respectively. $(\mathrm{H})$ While kurkinol had a reduced duration of action at $2.5 \mathrm{mg} / \mathrm{kg} / \mathrm{i}$.p. (45 $\mathrm{min}$ ) and $5 \mathrm{mg} / \mathrm{kg} / \mathrm{i} . \mathrm{p}$. (60 min). (I) Kurkinorin at both doses was effective until $90 \mathrm{~min}$. (J-L) Sex was found to affect morphine $(10 \mathrm{mg} / \mathrm{kg} / \mathrm{i} . \mathrm{p}$.) and kurkinorin (5 $m g / k g / i . p$.$) with improved anti-nociception in females and males respectively. Three-way mixed$ ANOVA with Bonferroni multiple corrections ${ }^{*} p<0.05,{ }^{* * *} p<0.001,{ }^{* * * *} p<0.0001$, (G-I) drug compared to vehicle (J-L) male compared to female, $\wedge \wedge \wedge \wedge p<0.0001$ drugs compared to $\beta$-FNA. Data are shown as mean \pm SEM (A-C) N = 5-6 per group, $(D) N=5-6$ males and 5-6 females per group. Doses in brackets in $\mathrm{mg} / \mathrm{kg} / \mathrm{i} . \mathrm{p}$. 
A)

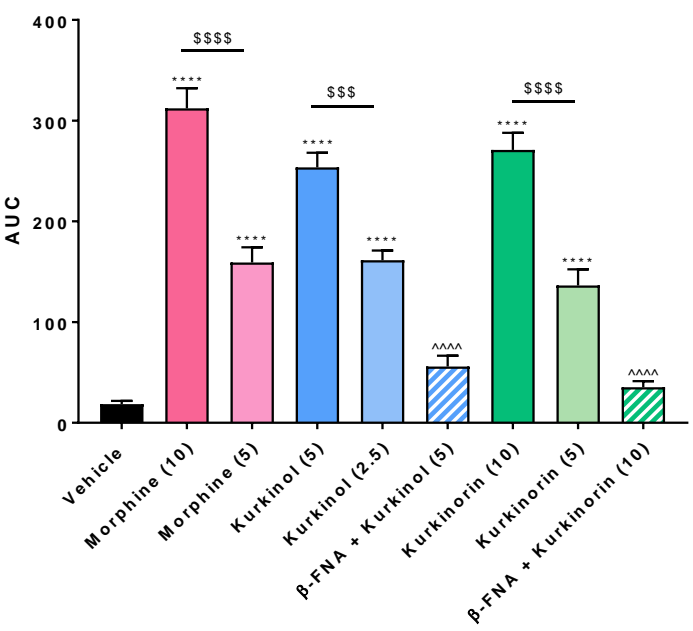

B )

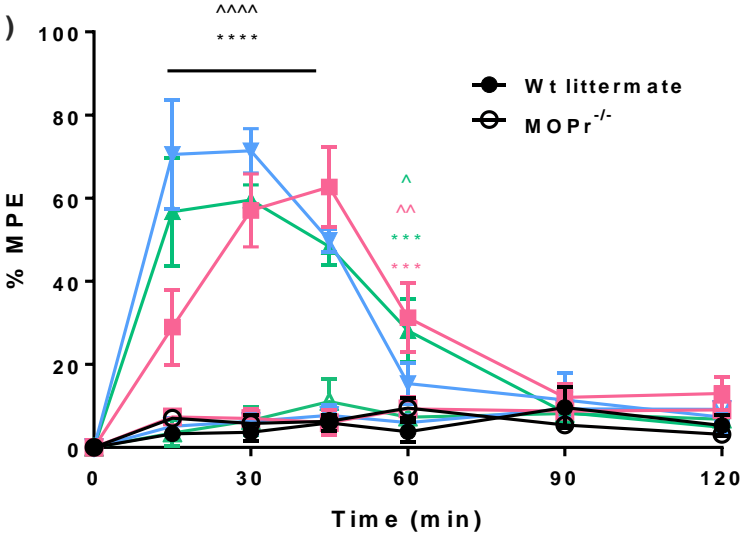

Figure 3.6 Overall Effect of Kurkinorin and Kurkinol on Supraspinally Mediated Anti-nociception

(A) Combined male and female data evaluating the effects of kurkinol and kurkinorin in the hotplate assay show both novel $\mu$ receptor agonists have similar anti-nociceptive effects to morphine, with a significant reduction by $\beta$-FNA pre-treatment. (B) $\mu$ receptor-1- mice showed no anti-nociceptive effect with morphine (10 mg/kg/i.p.), kurkinol (5 mg/kg/i.p.), or kurkinorin (10 mg/kg/i.p.) compared to Wt littermate controls. (A) One-way or (B) Two-way repeated measures ANOVA with Bonferroni multiple corrections test, ${ }^{* * *} p<0.001, * * * * p<0.0001$, drug compared to vehicle, $\$ \$ \$ p<0.001, \$ \$ \$ p<0.0001$ $E D_{80}$ compared to $E D_{50}, \wedge p<0.05, \wedge \wedge p<0.01, \wedge \wedge \wedge \wedge p<0.0001 \mu$ receptor ${ }^{/-}$or $\beta$-FNA compared to control. Data are shown as mean \pm SEM ( $N=5-7$ per group). Doses in brackets in $\mathrm{mg} / \mathrm{kg} /$ i.p. 


\subsubsection{Effectiveness of Kurkinol and Kurkinorin in Chemotherapy- Induced Neuropathic Pain}

\subsubsection{Paclitaxel-Induced Allodynia Does Not Differ Between Sexes}

To induce chronic, neuropathic pain in mice we utilised the preclinical model of paclitaxelinduced allodynia in both male and female C57BL/6J mice. Paclitaxel (PTX) (4 mg/kg/i.p.) or vehicle (1; 1; 18, ethanol; kolliphor; saline) was administered on days $0,2,4$, and 6 (cumulative dose; $16 \mathrm{mg} / \mathrm{kg}$ ). The development of mechanical and cold allodynia was tracked on days $0,2,4,7,11,13$, and 15, with behaviour conducted before PTX injections on days 0,2 , and 4 .

To determine whether the induction of mechanical and cold allodynia differed between male and female animals Three-way mixed ANOVA with Bonferroni corrections test were conducted. This identified a significant induction of mechanical allodynia from day 2, with a $40 \%$ decrease in mechanical threshold from day 0 and a stable $77 \%$ reduction from by day 15 when the sexes were combined $\left(\mathrm{F}_{(4.952,7.338)}=22.362, p<0.0001, p<0.05\right)$ (Fig. 3.7 A). However, no significant effect sex was identified to alter the induction of mechanical allodynia over time $\left(\mathrm{F}_{(4.952,0.066)}=0.201, p=0.961\right)$. Similarly, no statistically significant effect of sex was found for the induction of cold allodynia, despite female mice responding for an average of $25 \%$ on day 7 , with an average increased responding time of $11 \%$ from day $2-15\left(F_{(2.903,0.049)}=0.266, p=0.844\right)$. When the two sexes were combined an increase in cold allodynia was seen from day $2-15\left(F_{(2.903,4.517)}=24.648, p<0.0001, p<0.0001\right)$ (Fig. 3.7 B).

A)

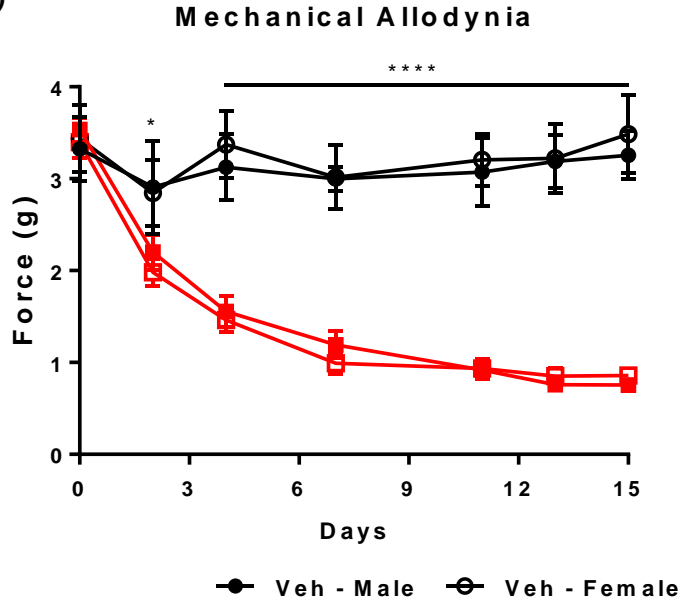

B )

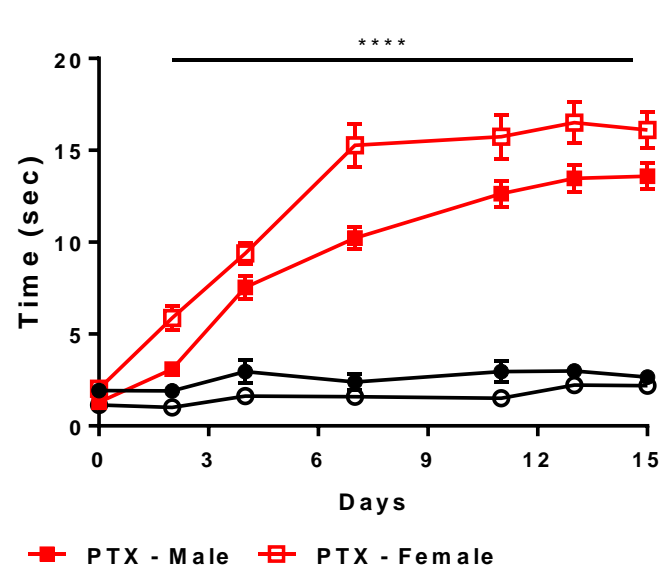

Figure 3.7 Paclitaxel Produced Stable Disease by Day 15 in Male and Female C57BL/6J Mice

(A) Measures of mechanical allodynia performed on days $0-15$ showed male and female mice developed allodynia from day 2, with no effect of sex. (B) Mice also developed a sensitivity to cold stimuli from day 2, with no statistical difference between genders. Three-way mixed ANOVA with Bonferroni multiple corrections. Data are shown as mean \pm SEM. ${ }^{*} p<0.05,{ }^{* * * *} P<0.0001$ (Vehicle $N=$ 5 males \& 6 females, PTX $N=30$ males \& 30 females). 


\subsubsection{Kurkinol is Significantly More Potent than Morphine in the Acute Phase of the Chemotherapy-Induced Neuropathic Pain Model}

Dose response effects of all compounds were assessed regarding both mechanical and cold allodynia on day 15. Before the dose response, the mice were grouped based on the induction of mechanical and cold allodynia to ensure the results were not confounded by higher or lower starting values (Appendix Fig 7.1). The dose response curves of kurkinol and kurkinorin were assessed in response to morphine and gabapentin, a non-opioid treatment prescribed for chronic pain.

Analysis of the ability of morphine, kurkinol, kurkinorin, and gabapentin to alleviate mechanical allodynia in male and female mice indicated a significant effect of gender on potency. However, no differences were identified between the sexes of a given treatment, suggesting that sex does not play a role in modulating their effects in the treatment of mechanical allodynia $\left(F_{(5,35)}=0.60\right.$, $p=0.0004, p>0.9999$ ) (Fig. 3.8 A, B, Table 3.2). Between the sexes, the pattern of potency remained the same, with kurkinorin and morphine being equipotent while kurkinol was more potent (Table 3.2). A significant effect of sex was also identified for cold allodynia, with kurkinorin found to be 1.4 fold more potent in males $\left(I_{50}=10.17 \mathrm{mg} / \mathrm{kg} / \mathrm{s}\right.$.c. $)$ compared to females $\left(I_{50}=14.62 \mathrm{mg} / \mathrm{kg} / \mathrm{s}\right.$.c. $)\left(F_{(5,35)}=\right.$ 1.43, $p=0.0022, p=0.0035$ ) (Fig. 3.9 C, D, Table 3.2). In both sexes and measures of mechanical and cold allodynia kurkinorin was found to be equipotent with morphine, while kurkinol was significantly more potent (Table 3.2).

The dose response curves generated for each sex were then combined to assess their overall potency in alleviating mechanical and cold allodynia (Fig. 3.8). In doing this, kurkinol $\left(E_{50}=1.56\right.$ $\mathrm{mg} / \mathrm{kg} / \mathrm{s}$.c.) was found to be more potent at alleviating mechanical allodynia than both morphine $\left(E D_{50}\right.$ $=9.26 \mathrm{mg} / \mathrm{kg} / \mathrm{s}$.c. $)$ and gabapentin $\left(E D_{50}=4.22 \mathrm{mg} / \mathrm{kg} / \mathrm{s}\right.$.c.), while kurkinorin $\left(E_{50}=9.11 \mathrm{mg} / \mathrm{kg} / \mathrm{s} . c\right.$. $)$ was equipotent with morphine $\left(F_{(3,44)}=11.12, p<0.0001\right)$ (Fig. 3.8 E, Table 3.3). Cold allodynia showed the same pattern of potency, with kurkinol $\left(E_{50}=1.91 \mathrm{mg} / \mathrm{kg} / \mathrm{s}\right.$.c.) having increased potency compared to morphine $\left(E D_{50}=10.5 \mathrm{mg} / \mathrm{kg} / \mathrm{s}\right.$.c.), while kurkinorin $\left(E D_{50}=11.69 \mathrm{mg} / \mathrm{kg} / \mathrm{s} . \mathrm{c}\right.$.) was equipotent $\left(F_{(3,44)}=8.39, p=0.0002\right)$ (Fig. 3.8 E, Table 3.3). 
Mechanical Allodynia

Male

A)

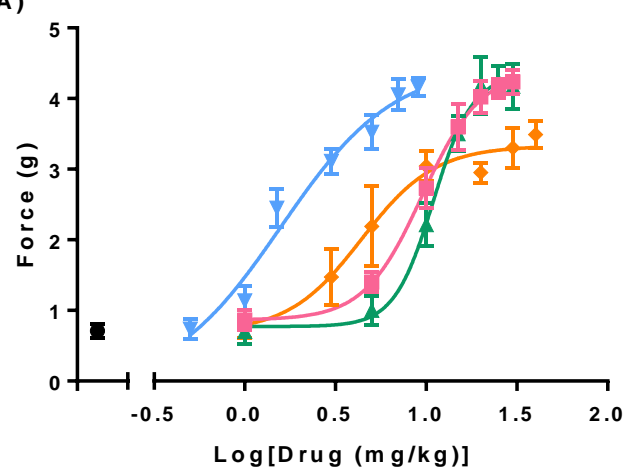

C)

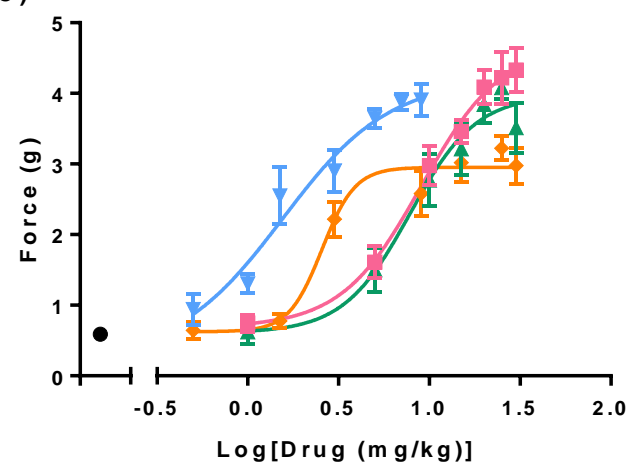

E)

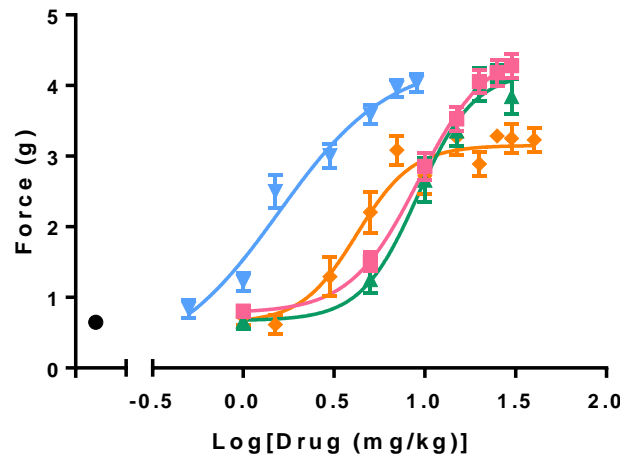

Cold Alodynia

M a le

B)

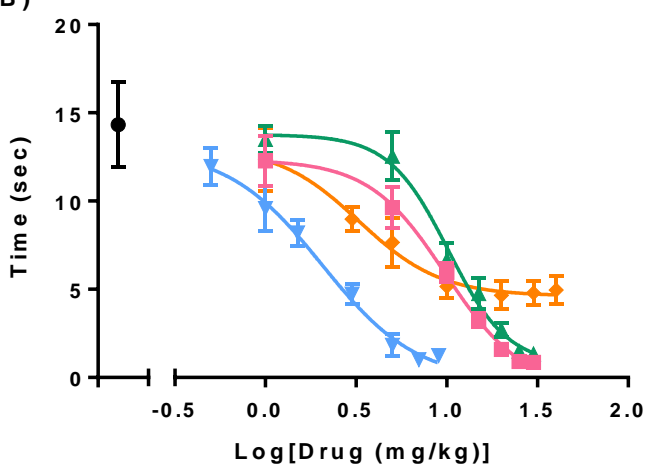

D)

Fem ale

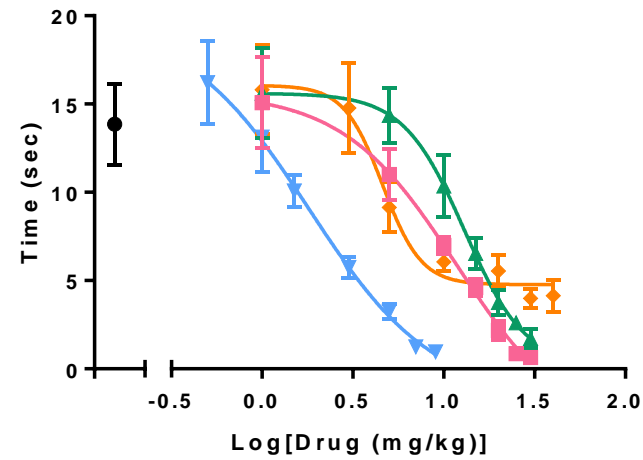

F)

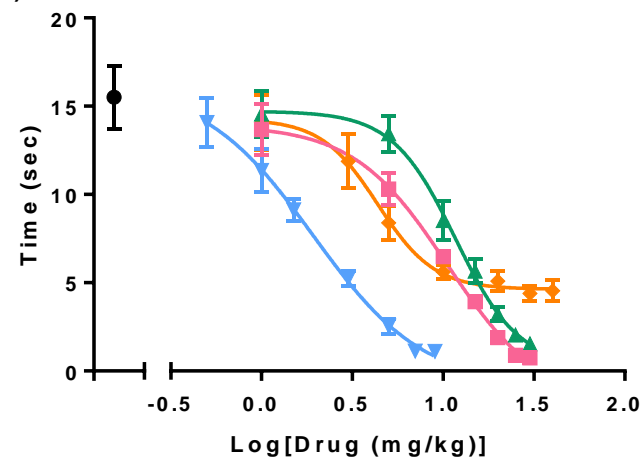

- Vehicle $\rightarrow$ Morphine $\rightarrow$ Gabapentin $\rightarrow$ Kurkinol $\quad$ Kurkinorin

Figure 3.8 Kurkinol is more Potent than Morphine at Alleviating Chemotherapy-Induced Neuropathic Pain in Male and Female C57BL/6J Mice

The potency and efficacy of kurkinol and Kurkinorin were assessed in male $(\boldsymbol{A}, \mathbf{B})$ and female $(\boldsymbol{C}, \boldsymbol{D})$ C57BL/6J mice in the paclitaxel-induced neuropathic pain model. (E, F) The data from male and female mice was then combined to assess the overall potency and efficacy. Data are presented as an average of duplicate results. Data are shown as mean \pm SEM ( $N=5-6$ males \& 6 females per group). 
Table 3.2 Kurkinorin is Significantly more Potent at Inhibiting Cold Allodynia in Male Mice

\begin{tabular}{|c|c|c|c|c|c|c|c|c|c|c|c|c|}
\hline \multirow[b]{3}{*}{ Morphine } & \multicolumn{2}{|c|}{$\mathrm{ED}_{50} \pm \mathrm{SEM}(\mathrm{mg} / \mathrm{kg} / \mathrm{s} . \mathrm{c})}$. & \multirow{2}{*}{\multicolumn{2}{|c|}{$P$ value }} & \multicolumn{2}{|c|}{$R^{2}$ value } & \multicolumn{2}{|c|}{$\mathrm{ID}_{50} \pm \mathrm{SEM}(\mathrm{mg} / \mathrm{kg} / \mathrm{s} . \mathrm{c})}$. & \multirow{2}{*}{\multicolumn{2}{|c|}{$P$ value }} & \multicolumn{2}{|c|}{$R^{2}$ value } \\
\hline & \multirow{2}{*}{$\begin{array}{c}\text { Males } \\
9.52 \pm 1.74\end{array}$} & \multirow{2}{*}{$\begin{array}{c}\text { Females } \\
9.04 \pm 1.13\end{array}$} & & & \multirow{2}{*}{$\begin{array}{l}\text { Males } \\
0.8771\end{array}$} & \multirow{2}{*}{$\begin{array}{c}\text { Females } \\
0.8292\end{array}$} & \multirow{2}{*}{$\begin{array}{c}\text { Males } \\
9.82 \pm 1.52\end{array}$} & \multirow{2}{*}{$\begin{array}{c}\text { Females } \\
8.08 \pm 1.64\end{array}$} & & & \multirow{2}{*}{$\begin{array}{l}\text { Males } \\
0.8608\end{array}$} & \multirow{2}{*}{$\begin{array}{c}\text { Females } \\
0.9943\end{array}$} \\
\hline & & & $>0.9999$ & n.s & & & & & $>0.9999$ & n.s & & \\
\hline Gabapentin & $4.40 \pm 3.10$ & $2.55 \pm 1.58$ & $>0.9999$ & n.s & 0.6218 & 0.7695 & $3.28 \pm 1.15$ & $4.66 \pm 1.33$ & $>0.9999$ & n.s & 0.5589 & 0.9939 \\
\hline Kurkinol & $4.59 \pm 0.76$ & $1.55 \pm 0.66$ & $>0.9999$ & n.s & 0.8603 & 0.7890 & $2.05 \pm 0.28$ & $1.75 \pm 1.45$ & $>0.9999$ & n.s & 0.8423 & 0.9625 \\
\hline Kurkinorin & $10.98 \pm 0.40$ & $7.80 \pm 2.38$ & $>0.9999$ & n.s & 0.8377 & 0.7412 & $10.17 \pm 2.16$ & $14.62 \pm 1.17$ & $=0.0035$ & $* *$ & 0.8670 & 0.9855 \\
\hline
\end{tabular}

Nonlinear regression of the dose response effects was used to calculate the $E D_{50}$ and $I D_{50}$ values for mechanical and cold allodynia. No significant effect of sex was found for mechanical allodynia. Kurkinorin is more potent at inhibiting cold allodynia in males compared to females. Two-way repeated measures ANOVA with Bonferroni multiple corrections test. ${ }^{* *} P<0.001$, n.s $=$ non-significant. Doses in $\mathrm{mg} / \mathrm{kg} / \mathrm{s.c}$. ( $\mathrm{N}=5-6$ males and 6 females per group). 
Table 3.3 Combined Dose Response Effects on Mechanical and Cold Allodynia in the Paclitaxel Model of Chronic Pain

\begin{tabular}{|c|c|c|c|c|c|c|c|c|c|c|c|c|}
\hline \multirow[b]{2}{*}{ Morphine } & \multirow{2}{*}{$\begin{array}{l}\mathrm{ED}_{50} \pm \mathrm{SEM} \\
(\mathrm{mg} / \mathrm{kg} / \mathrm{s} \text {.c. })\end{array}$} & \multicolumn{2}{|c|}{$\begin{array}{l}P \text { value to } \\
\text { morphine }\end{array}$} & \multicolumn{2}{|c|}{$\begin{array}{l}P \text { value to } \\
\text { gabapentin }\end{array}$} & \multirow{2}{*}{$\begin{array}{c}\mathbf{R}^{2} \\
\text { value } \\
0.8502\end{array}$} & \multirow{2}{*}{$\begin{array}{c}\begin{array}{c}\mathrm{ID}_{50} \pm \mathrm{SEM} \\
(\mathrm{mg} / \mathrm{kg} / \mathrm{s} . c .)\end{array} \\
10.5 \pm 1.12\end{array}$} & \multicolumn{2}{|c|}{$\begin{array}{l}P \text { value to } \\
\text { morphine }\end{array}$} & \multicolumn{2}{|c|}{$\begin{array}{l}P \text { value to } \\
\text { gabapentin }\end{array}$} & \multirow{2}{*}{$\begin{array}{c}\begin{array}{c}\mathbf{R}^{\mathbf{2}} \\
\text { value }\end{array} \\
0.7997\end{array}$} \\
\hline & & - & - & - & - & & & - & - & - & - & \\
\hline Gabapentin & $4.22 \pm 1.75$ & $=0.2906$ & n.s & - & - & 0.6824 & $4.35 \pm 0.87$ & $=0.1111$ & n.s & - & - & 0.5461 \\
\hline Kurkinol & $1.56 \pm 0.38$ & $<0.0001$ & $* * * *$ & $=0.0310$ & $*$ & 0.8229 & $1.91 \pm 0.89$ & $=0.0445$ & * & $=0.9767$ & n.s & 0.7739 \\
\hline Kurkinorin & $9.11 \pm 1.19$ & $>0.9999$ & n.s & $=0.1331$ & n.s & 0.7560 & $11.69 \pm 2.93$ & $=0.3623$ & n.s & $=0.0015$ & $* *$ & 0.7649 \\
\hline
\end{tabular}

Nonlinear regression of the dose response effects was used to calculate the $E D_{50}$ values for all treatment groups. Kurkinol was shown to be more potent than both morphine and gabapentin in alleviating mechanical and cold allodynia. One-way repeated measures ANOVA with Bonferroni multiple corrections test. * $p<0.05, * * P<0.01, * * * * 0.0001$, n.s $=$ non-significant. Doses in $\mathrm{mg} / \mathrm{kg} / \mathrm{s} . \mathrm{c}$. $(\mathrm{N}=5-6$ males and 6 females per group 


\subsubsection{Blockade of the Mu Opioid Receptor inhibits the Anti-Nociceptive properties of Kurkinol and Kurkinorin}

To ensure the anti-nociceptive effects of kurkinol and kurkinorin in paclitaxel-induced neuropathic pain are dependent on the $\mu$ receptor male $\mathrm{C} 57 \mathrm{BL} / 6 \mathrm{~J}$ mice were treated with the selective $\mu$ receptor antagonist $\beta$-FNA (5 mg/kg/s.c.) on day 14 (24 hrs pre-treatment, Fig 3.9). This led to a rightward shift of the dose response curves for morphine, kurkinol, and kurkinorin in both mechanical (Fig. 3.9 A) and cold (Fig. 3.9 C) allodynia. Furthermore, a decrease in mechanical threshold is seen for morphine (9.5 mg/kg/s.c.), kurkinol (1.5 mg/kg/s.c.), and kurkinorin $\left(9 \mathrm{mg} / \mathrm{kg} / \mathrm{s} . c\right.$.) $\left(F_{(5,30)}=9.12, p<\right.$ $0.0001, p<0.01$ ) (Fig. 3.9 B). The effects of morphine on cold allodynia were also significantly reduced with $\beta$-FNA pre-treatment $\left(F_{(5,30)}=5.26, p=0.0014, p=0.0404\right)$. While kurkinol and kurkinorin showed no difference ( $p>0.9999$ ). This suggests that antagonism with $5 \mathrm{mg} / \mathrm{kg} / \mathrm{i} . \mathrm{p}$. $\beta$-FNA is not enough to fully reverse the anti-nociceptive effects in this model. 

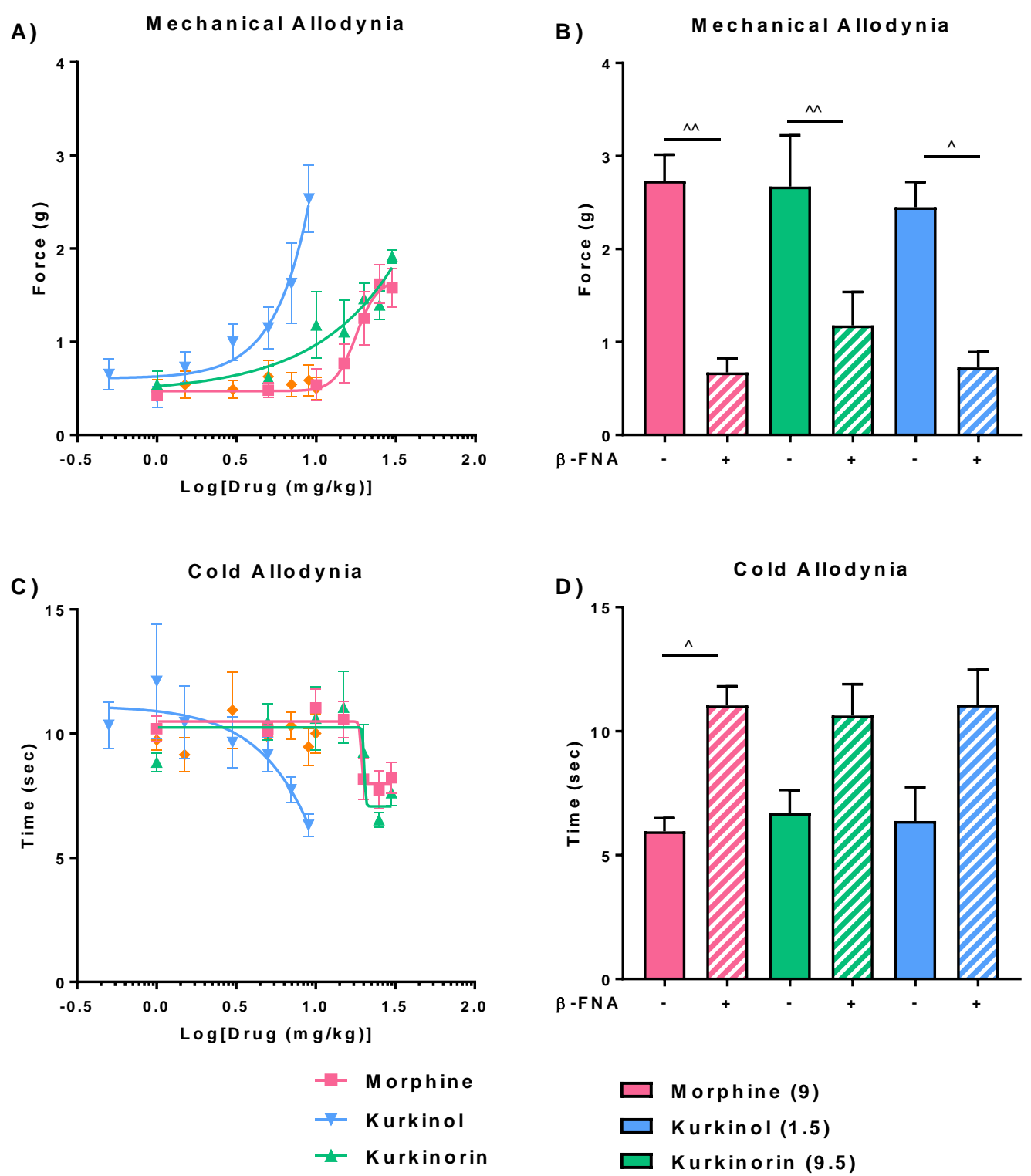

Figure 3.9 Anti-Nociception Induced by Kurkinol and Kurkinorin is Mediated by the Mu Opioid Receptor

Male C57BL/6J mice were administered the $\mu$ receptor antagonist $\beta$-FNA (5 mg/kg/s.c.) 24 hrs before the cumulative dose response to assess the role of $\mu$ receptor in anti-nociception. (A) The dose effects of morphine, kurkinol and kurkinorin on mechanical allodynia were significantly reduced with none forming a full dose response curve. (B) Responses at the $E D_{50}$ dose are is significantly reduced for all $\beta$ FNA pre-treated animals. (C) Dose dependent effects on cold allodynia were also reduced with $\beta$-FNA pre-treatment. (D) At the $E D_{50}$ dose, the time spent responding to acetone was significantly increased compared to untreated. One-way repeated measures ANOVA with Bonferroni multiple corrections test, $\wedge^{\wedge} p<0.05, \wedge^{\wedge} p<0.01$ drug compared to $\beta$-FNA pre-treatment. Data are shown as mean \pm SEM $(N=6$ per group). Doses in brackets in $\mathrm{mg} / \mathrm{kg} / \mathrm{s}$.c. 


\subsubsection{Kurkinol and Kurkinorin Do Not Show Reduced Tolerance to Neuropathic Pain}

For the chronic phase of the experiment, MICE remained in their original treatment groups and were administered the $\mathrm{ED}_{50}$ dose determined by the cumulative dose response mechanical allodynia in males (Table 3.2). Behavioural measurements were taken $30 \mathrm{~min}$ after the injection of each compound to assess the induction of tolerance from day 17 - 37. Mechanical and cold allodynia measurements were also taken before the compound administration to assess the induction of hyperalgesia. The interaction of sex and treatment on mechanical and cold allodynia measurements over time were assessed via Three-way mixed ANOVA with Bonferroni correction tests.

Tolerance to mechanical allodynia was found to occur independently of sex, either a main effect $\left(F_{(5,0.068)}=0.135, p=0.984\right)$ or a factor of time $\left(F_{(36.404,1.842)}=1.216, p=0.147\right)$ (Fig. 3.10 A, B). When the data from both sexes were combined morphine, gabapentin, kurkinol, and kurkinorin produced significant anti-nociception from day $17-33$, with no compound showing improvement in the development of tolerance $\left(\mathrm{F}_{(36.404,11.473)}=7.857, p<0.0001, p<0.05\right)$ (Fig. $\left.3.10 \mathrm{C}\right)$. As with mechanical allodynia, the development of tolerance to cold allodynia with chronic dosing of morphine, gabapentin, kurkinol, and kurkinorin occurred independently of sex, as a main effect of $\operatorname{sex}\left(F_{(5,6.068)}=\right.$ $0.326, p=0.895)$ and a factor of time $\left(\mathrm{F}_{(20.907,38.849)}=6.457, p<0.0001\right)$ (Fig. $\left.3.10 \mathrm{E}, \mathrm{F}\right)$. When grouped independently of sex, morphine and gabapentin produced anti-nociception until day 27 . While a delay 4 and 6-day delay in the return to baseline measurement of PTX-vehicle treated mice was observed for kurkinol and kurkinorin respectively (Fig. 3.10 E).

The development of hyperalgesia to mechanical and cold stimuli and the interaction between sex and the drug on the development of hyperalgesia over time was also assessed. No induction of mechanical hyperalgesia was identified as a main effect of $\operatorname{sex}\left(F_{(5,0.385)}=1.478, p=0.211\right)$, and the drugs did not interact with sex to affect mechanical withdrawal thresholds over time $\left(F_{(25,0.792)}=0.901\right.$, $p=0.601)$. Furthermore, no compounds were shown to induce mechanical hyperalgesia, irrespective of $\operatorname{sex}\left(F_{(25,0.97)}=1.078, p=0.368\right)$ (Appendix Fig. 7.2). However, an overall effect of the drugs on cold hyperalgesia was identified, upon further investigation, no significant change in cold thresholds in response to drug treatments was identified over time $\left(\mathrm{F}_{(25,0.007)}=1.597, p=0.038, p>0.9999\right)$. No main effects of $\operatorname{sex}\left(F_{(5,0.421)}=0.084, p=0.646\right)$, nor the compounds and sex interacting to affect allodynia over time were identified $\left(\mathrm{F}_{(25,0.138)}=1.202, p=0.20\right)$ (Appendix Fig. 7.2). 
A)

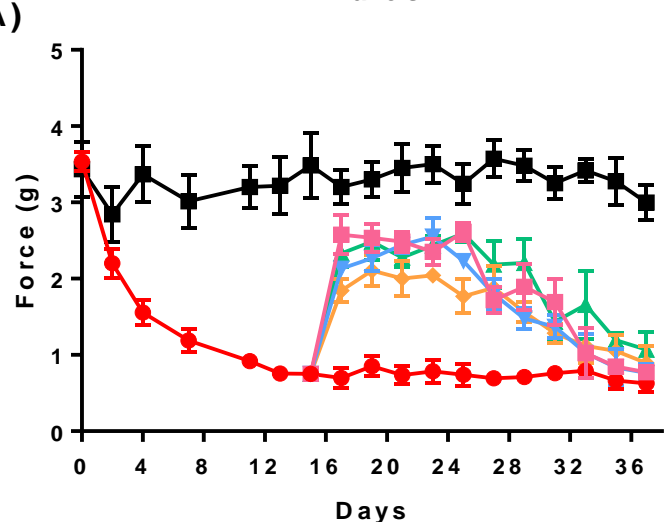

官

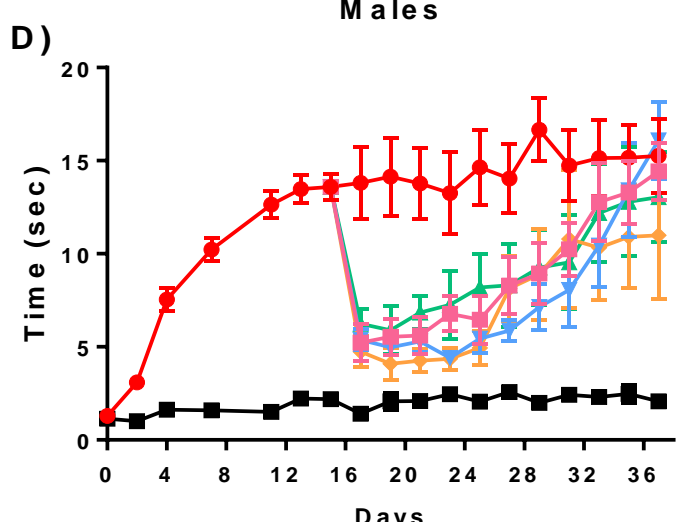

B
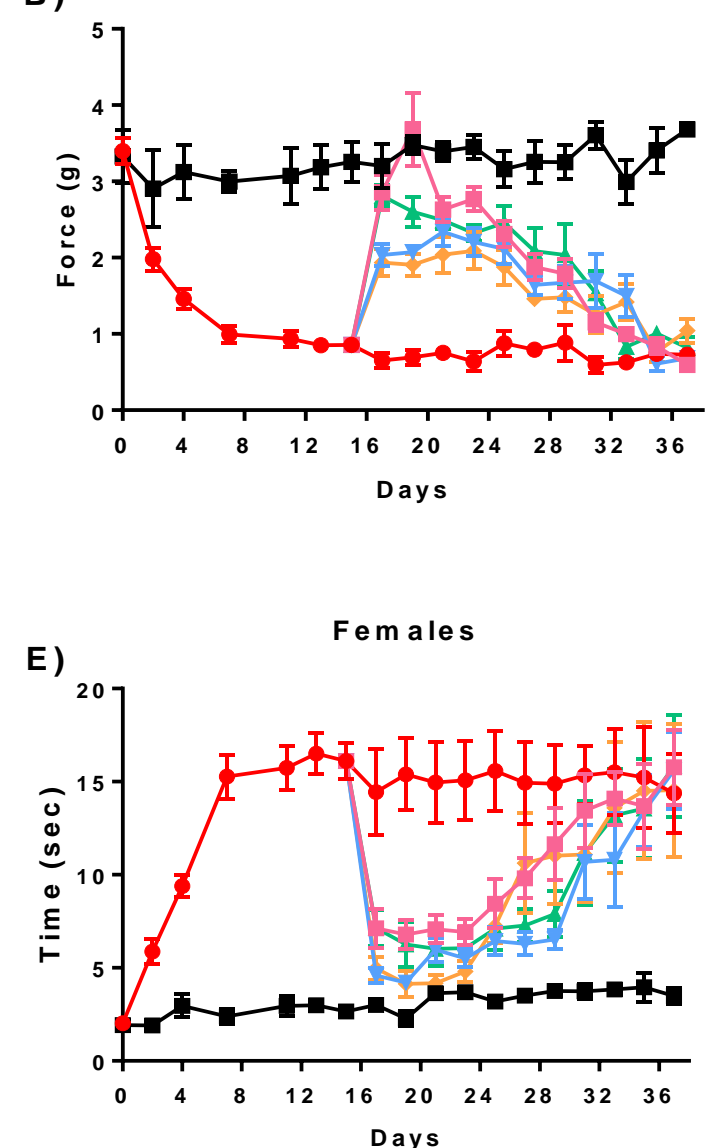

C)
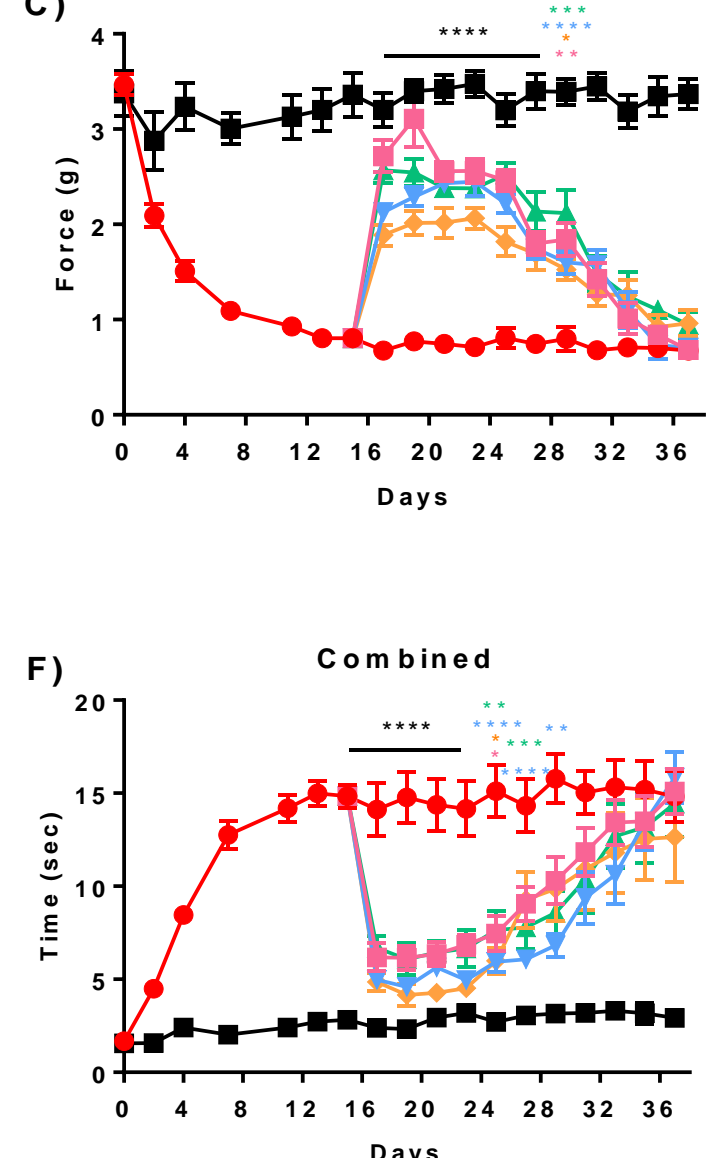

$\rightarrow$ Veh/Veh $\rightarrow$ PTX/Veh $\neg$ Morphine $\neg$ Gabapentin $\rightarrow$ Kurkinol $\neg$ Kurkinorin 
Figure 3.10 Effect of Sex on the Induction of Tolerance to Mechanical Allodynia

On day 17 of PTX-induced neuropathic pain, daily administration of vehicle, morphine, gabapentin, kurkinol, or kurkinorin was initiated and the reversal of mechanical and cold allodynia assessed. (A) Time course of anti-nociception in male mice. (B) Time course analysis of anti-nociception in female mice. (C) When the sexes were combined a significant effect was seen over time, with neither kurkinol nor kurkinorin showing any major improvements in the development of anti-nociceptive tolerance (day 17 - 33). (D) Time course of anti-nociceptive effects in male mice. (E) Time course of anti-nociceptive effects in female mice. (F) When the sexes were combined, kurkinorin (day 17-31) had a 4-day delay in the development of tolerance in measures of cold allodynia, while kurkinol (day 17 - 33) showed a 6-day delay compared to morphine and gabapentin (day 17-27). Three-way mixed ANOVA with Bonferroni multiple corrections test. Data are shown as mean \pm SEM. ${ }^{*} p<0.05,{ }^{* *} p<0.01,{ }^{* * *} p<0.001,{ }^{* * * *} p<0.0001$ drug compared to PTX/Veh (N=5 -6 males \& 6 females per group). Doses in brackets in $\mathrm{mg} / \mathrm{kg} / \mathrm{i} . \mathrm{p}$. 


\subsubsection{Anti-Nociceptive Z-Scores Shows Improvements to Morphine with Kurkinol, But Not Kurkinorin}

Generation of Z-scores was used to evaluate the induction anti-nociception following administration of $5 \mathrm{mg} / \mathrm{kg}$ morphine, kurkinol, or kurkinorin. The directionality of the Z-score indicates the level to which anti-nociception is induced, with values $>0$ indicating a decrease in pain-like behaviours, while $\leq 0$ occurs if no anti-nociception is induced. As the tail-flick was only performed in males, it has been excluded from the combined Z-scores and overall male Z-score to allow the comparison to the female data. Slight differences in the anti-nociceptive profiles can be seen between male and female mice, with kurkinol and morphine being more effective in the hotplate in females compared to males (Fig. 3.11 A, B). The overall anti-nociceptive Z-scores for morphine (male $=4.41 \mathrm{Vs}$ female $=3.75)$ and kurkinol (male $=9.45$ Vs female $=11.96$ ) are also larger in females. While kurkinorin shows the opposite, with a higher anti-nociceptive Z-score in males (male $=3.6 \mathrm{Vs}$ female $=2.90$ ) (Fig. 3.13 A \& B). When the Z-scores from male and female mice were combined, morphine (Z-score $=5.47$ ) and kurkinorin (Z-score $=4.73$ ) induced a similar level of anti-nociception at $5 \mathrm{mg} / \mathrm{kg}$, while kurkinol had a larger Z-score indicating a greater anti-nociceptive effect (Z-score = 10.08) (Fig. 3.11 C).
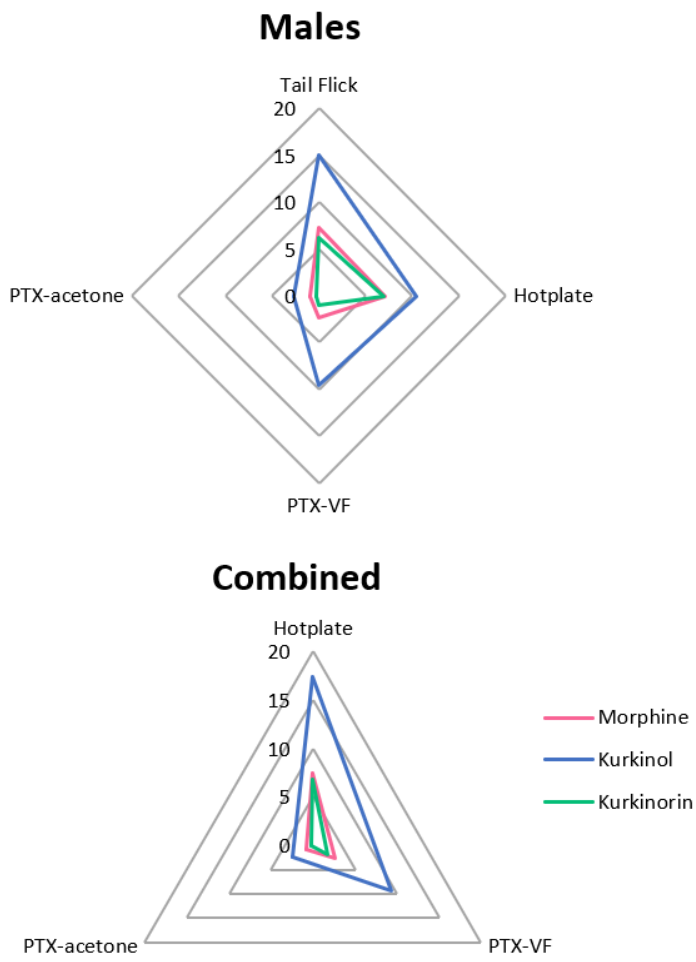

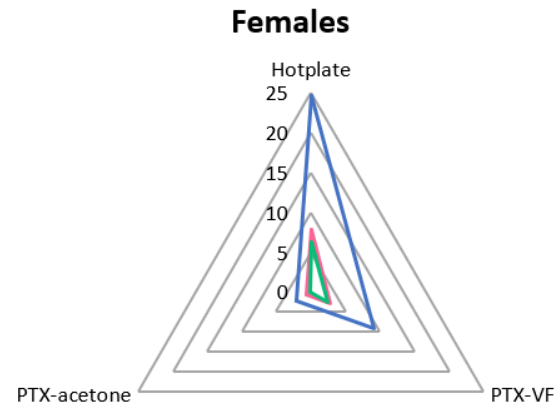

Figure 3.11 Kurkinol and Kurkinorin Show the Same Behavioural Pattern as Morphine

Z-score analysis of the anti-nociceptive effects of morphine, kurkinol, and kurkinorin showed a similar pattern of effect between behavioural assays in (A) males, (B) females, (C) and when the sexes were combined. However, kurkinorin was found to have a slight reduction in anti-nociceptive ability compared to morphine and kurkinol. Abbreviations:

$$
\text { PTX }=\text { paclitaxel } \quad V F=\text { Von Frey Filaments }
$$




\subsection{Discussion}

It has been widely postulated that $\mathrm{G}$-protein biased $\mu$ receptor agonists may hold the key to developing analgesics for the treatment of severe acute and chronic pain with reduced tolerance at the $\mu$ receptor (Bohn et al., 1999, Chen et al., 2013, DeWire et al., 2013). This is of great importance due to the current lack of effective treatments for chronic pain and the worsening opioid crisis, in part caused by the over prescription of opioids such as oxycodone and morphine (Breivik et al., 2006, Peavy et al., 2012, Shipton et al., 2017, Sullivan et al., 2001, Vowles et al., 2015). Kurkinol and kurkinorin are two novel compounds with varying G-protein bias (Crowley et al., 2016). In this chapter, we aimed to investigate the effects that the bias of the compounds had on their anti-nociceptive effects in vivo in preclinical rodent behavioural models.

\subsubsection{G-Protein Bias Correlated to Increased Potency and Decreased Tolerance in Spinally Mediated Thermal Anti-Nociceptive Models}

The dose response effects of the $\mu$ receptor agonists, kurkinol and morphine were assessed using the hot water tail-flick assay as a measure of acute thermal anti-nociception. In this assay, kurkinol was found to be significantly more potent than morphine with no difference in efficacy and no significant tolerance. Comparatively, kurkinorin was previously shown to be equipotent with morphine with a moderate induction of tolerance compared to morphine (Crowley et al., 2016). These results show that in acute thermal nociceptive models of anti-nociceptive tolerance G-protein bias correlates to an increase in potency and decreased tolerance (Table 3.4 and 3.6).

To further assess the anti-nociceptive role of $\beta$-arrestin 2 the hot water dose response tail-flick assay was conducted in $\beta$-arr2 ${ }^{-/}$mice to evaluate tolerance. In the absence of $\beta$-arrestin2, the potency of morphine was significantly increased compared to Wt littermate controls, while neither kurkinol nor kurkinorin showed a difference in potency between genotypes. As in C57BL/6J mice, Wt littermate controls showed tolerance to morphine and kurkinorin over the 9 days while kurkinol did not. These tolerance effects of morphine were significantly reduced in the $\beta$-arr $2^{-}$mice, while kurkinorin produced no tolerance (Table 3.4). Previous literature had identified the leftward shift and reduced tolerance to morphine in $\beta$-arr2 ${ }^{-/-}$mice (Bohn et al., 1999, Raehal et al., 2011), GRK3 ${ }^{-1-}$ mice (Glück et al., 2014, Grecksch et al., 2011, Terman et al., 2004), and in knock-in mice with limited $\beta$-arrestin2 recruitment (Kliewer et al., 2019). While the lack of potency changes following kurkinol administration in both $\beta$-arr2 ${ }^{-/}$mice and their Wt littermate controls can be attributed to the G-protein bias, kurkinorin was predicted to have a slight increase in potency in these mice. However, only morphine showed a leftward shift in dose response curve, with a $2 \mathrm{mg} / \mathrm{kg}$ difference between the $\mathrm{ED}_{50}$ dose in the $\beta$-arr $2^{--}$mice and their Wt littermate controls on day 1 . This indicating the differences in antinociceptive potency may be too subtle to see when $\beta$-arrestin2 recruitment is already limited, as shown in the cell signalling data from kurkinol and kurkinorin. Assessing $\beta$-arrestin2 biased agonists in 
this model may allow better identification of the role of $\beta$-arrestin in the development of antinociceptive tolerance.

The hot water tail-flick assay was also used in a repeated measures fashion to assess the duration of action of a single dose. In this assay, kurkinol had a significantly faster onset of action compared to morphine ( $5 \mathrm{~min}$ vs $10 \mathrm{~min}$ ) while, overall, having a shorter duration of action at both the high ( $45 \mathrm{~min}$ vs $120 \mathrm{~min}$ ) and low doses (45 $\mathrm{min}$ vs $90 \mathrm{~min}$ ). This may be due to the high resemblance to the parent compound Sal A, which is known to have low bioavailability and a short duration of action in mice as it is quickly metabolised to the inactive product Salvinorin B (John et al., 2006, Kutrzeba et al., 2009, McCurdy et al., 2006, Schmidt et al., 2005).

The addition of the alcohol group also may have increased the recognition by degradation enzymes, resulting in a shorter half-life and increased clearance compared to its structural analogue kurkinorin, which has a duration of $120 \mathrm{~min}$ at $10 \mathrm{mg} / \mathrm{kg} / \mathrm{i} . \mathrm{p}$. (Crowley et al., 2016). When animals were pre-treated with the $\mu$ receptor specific antagonist $\beta$-FNA $(5 \mathrm{mg} / \mathrm{kg} / \mathrm{i}$. .p., $24 \mathrm{hrs})$ the antinociceptive effects of kurkinol (5 mg/ $\mathrm{kg} /$ i.p.) were significantly reduced, however, a significant antinociceptive effect was still induced (Table 3.4). To assess whether this was due to latent $\mathrm{k}$ receptor affinity or insufficient dosing of the antagonist, the anti-nociceptive effects were also assessed in $\mu$ receptor $^{-/}$mice at $30 \mathrm{~min}$, the time of peak anti-nociception. Kurkinorin was also assessed in $\mu$ receptor $^{-/}$mice, as the prior hot water tail-flick experiments carried out on kurkinorin by Nirajomhoan Shivaperumal used the mixed opioid antagonist naloxone rather than a $\mu$ receptor selective antagonist, the $\mathrm{k}$ receptor induced anti-nociception was not known (Crowley et al., 2016). Morphine, kurkinol, and kurkinorin all induced strong anti-nociception in Wt mice which was reduced in $\mu$ receptor $^{-/}$mice. Although no statistical difference was observed between vehicle and kurkinorin in $\mu$ receptor $^{-/}$mice, a trend towards anti-nociception was observed. Therefore, the experiment was performed in $\mu$ receptor ${ }^{-/}$mice with nor-BNI $(10 \mathrm{mg} / \mathrm{kg} / \mathrm{i}$.p.) pre-treatment to assess the role of $\mathrm{k}$ receptor. This showed no difference to animals who did not receive nor-BNI, indicating that the antinociceptive effects in the tail-flick assay are induced solely via the $\mu$ receptor. 
Table 3.4 Summary of Kurkinol and Kurkinorin Anti-Nociceptive Effects

\begin{tabular}{|c|c|c|c|c|c|c|c|c|c|c|c|c|}
\hline & \multicolumn{2}{|c|}{$\begin{array}{l}\text { Dose response } \\
\text { (Day 1) }\end{array}$} & \multicolumn{2}{|c|}{$\begin{array}{c}\text { Tolerance } \\
\text { (Day 2) }\end{array}$} & \multicolumn{2}{|c|}{ Tail-flick time course } & \multicolumn{2}{|c|}{ Hotplate time course } & \multicolumn{2}{|c|}{ CINP Acute } & \multicolumn{2}{|c|}{ CINP Tolerance } \\
\hline & Wt & $\beta$-arr $/-$ & $\mathbf{W t}$ & $\beta$-arr ${ }^{-/}$ & Wt & $\begin{array}{c}\mu \\
\text { receptor }^{-/-}\end{array}$ & Wt & $\begin{array}{c}\mu \\
\text { receptor } \\
/-\end{array}$ & Mechanical & Cold & Mechanical & Cold \\
\hline Morphine & $\begin{array}{c}7.0 \\
\mathrm{mg} / \mathrm{kg}\end{array}$ & $\begin{array}{c}5.02 \\
\mathrm{mg} / \mathrm{kg}\end{array}$ & $\begin{array}{l}16.67 \\
\mathrm{mg} / \mathrm{kg}\end{array}$ & $\begin{array}{c}9.12 \\
\mathrm{mg} / \mathrm{kg}\end{array}$ & $\begin{array}{c}5=90 \mathrm{~min} \\
10=120 \\
\min \end{array}$ & $\begin{array}{c}\text { Fully } \\
\text { reversed }\end{array}$ & $\begin{array}{c}5=90 \mathrm{~min} \\
10=120 \\
\min \end{array}$ & $\begin{array}{l}\text { Fully } \\
\text { reversed }\end{array}$ & $9.26 \mathrm{mg} / \mathrm{kg}$ & $\begin{array}{c}10.5 \\
\mathrm{mg} / \mathrm{kg}\end{array}$ & Day 33 & Day 27 \\
\hline Kurkinol & $\begin{array}{c}2.3 \\
\mathrm{mg} / \mathrm{kg}\end{array}$ & $\begin{array}{c}3.89 \\
\mathrm{mg} / \mathrm{kg}\end{array}$ & $\begin{array}{c}3.67 \\
\mathrm{mg} / \mathrm{kg}\end{array}$ & $\begin{array}{c}5.07 \\
\mathrm{mg} / \mathrm{kg}\end{array}$ & $\begin{array}{c}2.5=60 \\
\min \\
5=90 \mathrm{~min}\end{array}$ & $\begin{array}{c}\text { Fully } \\
\text { reversed }\end{array}$ & $\begin{array}{c}2.5=45 \\
\min \\
5=45 \mathrm{~min}\end{array}$ & $\begin{array}{l}\text { Fully } \\
\text { reversed }\end{array}$ & $1.56 \mathrm{mg} / \mathrm{kg}$ & $\begin{array}{c}1.91 \\
\mathrm{mg} / \mathrm{kg}\end{array}$ & Day 33 & Day 33 \\
\hline Kurkinorin & $\begin{array}{c}5.81 \\
\mathrm{mg} / \mathrm{kg}\end{array}$ & $\begin{array}{c}5.32 \\
\mathrm{mg} / \mathrm{kg}\end{array}$ & $\begin{array}{c}8.0 \\
\mathrm{mg} / \mathrm{kg}\end{array}$ & $\begin{array}{c}6.02 \\
\mathrm{mg} / \mathrm{kg}\end{array}$ & $\begin{array}{c}5=60 \mathrm{~min} \\
10=120 \\
\text { min* }\end{array}$ & 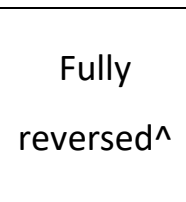 & $\begin{array}{c}5=60 \mathrm{~min} \\
10=90 \\
\min \end{array}$ & $\begin{array}{l}\text { Fully } \\
\text { reversed }\end{array}$ & $9.11 \mathrm{mg} / \mathrm{kg}$ & $\begin{array}{l}11.69 \\
\mathrm{mg} / \mathrm{kg}\end{array}$ & Day 33 & Day 31 \\
\hline Gabapentin & n.d & n.d & n.d & n.d & n.d & n.d & n.d & n.d & $4.22 \mathrm{mg} / \mathrm{kg}$ & $\begin{array}{c}4.35 \\
\mathrm{mg} / \mathrm{kg}\end{array}$ & Day 33 & Day 27 \\
\hline
\end{tabular}

Summary of when sexes are combined looking at the differences between compounds and genotypes

Dose response tolerance show doses in in $\mathrm{mg} / \mathrm{kg} / \mathrm{s}$.c., all other values are in $\mathrm{mg} / \mathrm{kg} / \mathrm{i}$. $\mathrm{p}$,

CINP tolerance; Days = loss of anti-nociception compared to vehicle

*(Crowley et al., 2016)

$\wedge$ Naloxone in place of $\beta$-FNA

n.d $=$ not determined 


\subsubsection{Kurkinol and Kurkinorin have Reduced Duration of Action in Supraspinal Thermally Mediated Anti-Nociception}

To assess the duration of action of kurkinol and kurkinorin in supraspinal anti-nociception the hotplate $\left(50^{\circ} \mathrm{C}\right)$ assay was utilised. The effects were also compared between male and female animals to assess interactions between sex and treatment on anti-nociception. This identified differential effects of sex between the treatment groups, with morphine affecting females to a greater extent at the $10 \mathrm{mg} / \mathrm{kg} /$ i.p. dose while the males were more affected at $5 \mathrm{mg} / \mathrm{kg} / \mathrm{i} . \mathrm{p}$. No effect of sex on antinociception over time was found for either kurkinol or kurkinorin, however, there was a simple effect of treatment and sex such that kurkinorin produced stronger anti-nociception in males. The finding that the $5 \mathrm{mg} / \mathrm{kg} / \mathrm{i}$.p. morphine and kurkinorin had a greater effect on males fits with our current understanding of sex anti-nociception interactions at the $\mu$ receptor (Table 3.5). In rodents, the existence of sex differences is more established. Multiple studies show that morphine is more efficacious in males in a variety of pre-clinical anti-nociception models. These include; inflammatory models (Loyd et al., 2008, Wang et al., 2006), visceral models (Ji et al., 2006, Neelakantan et al., 2015), tail-flick (Kepler et al., 1989, Kest et al., 1999), and hotplate assays (Cicero et al., 1997, Posillico et al., 2015). In humans, the existence of sex differences in $\mu$ receptor analgesia is controversial, with some reports stating no sex difference (Bijur et al., 2008, Niesters et al., 2010) and others show morphine is less efficacious in females than males (Cepeda et al., 2003).

With sexes combined morphine produced potent anti-nociception from 15 - 90 min and 120 min post-administration for the 5 and $10 \mathrm{mg} / \mathrm{kg} /$ i.p. doses. Kurkinol had an early onset at $15 \mathrm{~min}$, while the anti-nociceptive duration was only $45 \mathrm{~min}$ for both 2.5 and $5 \mathrm{mg} / \mathrm{kg} / \mathrm{i}$.p. doses. The $5 \mathrm{mg} / \mathrm{kg} / \mathrm{i} . \mathrm{p}$. kurkinorin induced strong anti-nociception from $15-60 \mathrm{~min}$, this was extended to $90 \mathrm{~min}$ at the 10 $\mathrm{mg} / \mathrm{kg} / \mathrm{i} . \mathrm{p}$. dose. As in the tail-flick assay, the duration of action is reduced in the kurkinol and kurkinorin treated mice compared to morphine (Table 3.4 and 3.6). However, the duration of action is still significantly increased compared to Sal A which has shown to induce no increase in hotplate latency in rats at $10 \mathrm{mg} / \mathrm{kg} / \mathrm{i} . \mathrm{p}$. (Wang et al., 2008) and a $30 \mathrm{~min}$ duration of action in mice at a 2 $\mathrm{mg} / \mathrm{kg} / \mathrm{i}$. . . dose (Kivell lab unpublished data). The anti-nociceptive effects were shown to occur through the $\mu$ receptor as $24 \mathrm{hr}$ pre-treatment with $\beta$-FNA (to avoid transient $\mathrm{k}$ receptor agonism following acute administration) reduced the anti-nociception. However, the effects were not fully reduced by this dose of $\beta$-FNA. While some papers have shown that $2-10 \mathrm{mg} / \mathrm{kg} / \mathrm{i} . \mathrm{p}$ is able to reverse the effects of morphine in the tail flick assay (Zagon et al., 1985, 19869), the doses used can be as high as $40 \mathrm{mg} / \mathrm{kg}$. Unfortunately, we did not have access to this amount of $\beta$-FNA and could not realistically afford to purchase the volume required for this dose. However, we did have access to the $\mu$ receptorI- mice. To ensure the effects were mediated by this receptor we also conducted the experiment in this mouse line resulting in the complete ablation of anti-nociception with all $\mu$ receptor agonists in the $\mu$ receptor ${ }^{-/}$mice. 
Table 3.5 Summary of Main Effects of Sex on the Anti-Nociceptive Effects of Kurkinol and Kurkinorin

\begin{tabular}{|c|c|c|c|c|c|c|c|c|c|c|}
\hline & \multicolumn{2}{|c|}{ Hotplate time course } & \multicolumn{4}{|c|}{ CINP Acute } & \multicolumn{4}{|c|}{ CINP Chronic } \\
\hline & \multirow{2}{*}{$\begin{array}{c}\text { Male Vs } \\
\text { Female }\end{array}$} & \multirow[b]{2}{*}{$p$ value } & \multicolumn{2}{|c|}{ Mechanical } & \multicolumn{2}{|c|}{ Cold } & \multicolumn{2}{|c|}{ Mechanical } & \multicolumn{2}{|c|}{ Cold } \\
\hline & & & Male Vs Female & $p$ value & $\begin{array}{c}\text { Male Vs } \\
\text { Female }\end{array}$ & $p$ value & $\begin{array}{l}\text { Male Vs } \\
\text { Female }\end{array}$ & $p$ value & $\begin{array}{l}\text { Male Vs } \\
\text { Female }\end{array}$ & $p$ value \\
\hline Morphine & $\phi>0^{\wedge}$ & $=0.009$ & $q=0^{\lambda}$ & $>0.9999$ & $q=0^{\lambda}$ & $>0.9999$ & $q=0^{\lambda}$ & $>0.9999$ & $q=0^{\lambda}$ & $>0.999$ \\
\hline Kurkinol & $\phi=0^{\lambda}$ & $>0.9999$ & $q=0^{\lambda}$ & $>0.9999$ & $q=\hat{0}$ & $>0.9999$ & $q=0^{\lambda}$ & $>0.9999$ & $q=0^{\lambda}$ & $>0.999$ \\
\hline Kurkinorin & $q=\sigma^{\lambda}$ & $>0.9999$ & $q=\sigma^{\lambda}$ & $>0.9999$ & $q<\lambda$ & $=0.0035$ & $\phi=0^{\lambda}$ & $>0.9999$ & $q=\sigma^{\lambda}$ & $>0.999 \mathrm{c}$ \\
\hline Gabapentin & n.d & n.d & $\phi=\hat{O}$ & $>0.9999$ & $\phi=\hat{O}$ & $>0.9999$ & $q=\hat{O}$ & $>0.9999$ & $\phi=0^{\lambda}$ & $>0.999$ \\
\hline
\end{tabular}

灾

$$
\begin{aligned}
& q=\text { Female } \\
& \delta \text { = Male }
\end{aligned}
$$

n.d $=$ not determined 


\subsubsection{The Effect of G-protein Bias in Anti-Nociceptive Potency and Tolerance in Chemotherapy-Induced Neuropathic Pain}

\subsubsection{Induction Phase}

The CINP model is a well-established model of neuropathic pain both in the literature and Kivell lab. It is a non-surgical method of inducing neuropathic pain with reproducible induction of mechanical and cold allodynia. The disease phenotype in rodents closely mimics the peripheral sensitivity seen in humans where a 'glove and stocking' distribution in the hands and feet is predominant (Starobova et al., 2017), allowing a good translation from rodents to humans. During the induction phase of CINP male and female mice show no difference in the induction of mechanical allodynia, as was found here. Unlike mechanical allodynia, a sex difference is commonly observed with the induction of cold allodynia (Naji-Esfahani et al., 2016, Wang et al., 2018, Kivell Lab Unpublished data). While a visual trend of increased sensitivity can be seen in the female mice, a statistically significant difference was not observed for cold allodynia (Table 3.5).

\subsubsection{Acute Phase}

In the acute phase the dose response effects of kurkinol and kurkinorin were compared to morphine and gabapentin, a first line non-opioid analgesic for chronic pain (Attal et al., 2010). The dose response measurements for mechanical allodynia showed that the sex did not influence their potency. Overall the combined potency revealed kurkinol was significantly more potent than morphine and gabapentin, while kurkinorin was equipotent with morphine (Table 3.4 and 3.6). Interestingly kurkinorin was 1.4 fold more potent at alleviating cold allodynia in males $\left(E D_{50}=10.17\right.$ $\mathrm{mg} / \mathrm{kg} / \mathrm{s}$.c.) than females $\left(E D_{50}=14.62 \mathrm{mg} / \mathrm{kg} / \mathrm{s} . c\right.$.) , this is consistent with previous literature stating higher doses of $\mu$ receptor agonists are required to produce equivalent anti-nociception in female rodents (Barrett et al., 2002, Collins et al., 2016). However, no such difference was found for morphine or kurkinol (Table 3.5). The overall pattern of anti-nociception to cold allodynia was the same as seen with mechanical allodynia; kurkinol was more potent, while kurkinorin was equipotent with morphine and less potent than gabapentin. Again, the potency of kurkinol and kurkinorin fit with the theory of biased agonism, with reduced $\beta$-arrestin2 recruitment associated with increased potency (Bohn et al., 2000, Bohn et al., 1999). In the hot water tail-flick assay the $\mathrm{ED}_{50}$ dose of morphine and kurkinorin is significantly lower than what was required to induce the same response in the CINP model. This is a phenomenon that has been previously documented in the $\mathrm{CCl}$ model of neuropathic pain with both 10 and $30 \mathrm{mg} / \mathrm{kg} \mathrm{mg} / \mathrm{kg} / \mathrm{s}$.c. morphine having reduced effects compared to sham animals. Indicating that in an environment of abnormal nociception and neuronal hyperexcitability larger doses are required to overcome this damage (Ochiai et al., 2016). Despite this fact, the dose of gabapentin required to alleviate mechanical and cold allodynia is significantly lower than what is required in humans (1200 - $3600 \mathrm{mg} /$ day) and what is published in CCI models of neuropathic pain, with effective doses ranging from $17.9-100$ mg/kg (Atwal et al., 2019, Gao et al., 2019, Li et al., 2019, Zulazmi et al., 
2015). To date the only published data on gabapentin in the paclitaxel-induced neuropathic pain model has used an i.c.v dose of $100 \mu \mathrm{g} /$ mouse, making the extrapolation to i.p. injection difficult (Juarez-Salinas et al., 2018). Overall indicating a lower dose of i.p. gabapentin is enough to induced reliable anti-nociception in this model.

\subsubsection{Chronic Phase}

For the chronic phase of the CINP model animals continued in the same treatment group from the acute phase, with the $E D_{50}$ dose determined from the male mechanical dose response administered daily from day 17. Ideally, the dose of the drugs would have been administered at sex specific $E D_{80}$ doses, unfortunately, due to limitations in the supply of kurkinorin, the $E D_{50}$ from the male mechanical dose response had to be used for all animals.

Over the 21 days of chronic administration, all compounds assed began to develop tolerance, independent of sex, with no significant improvements for either kurkinol or kurkinorin. Effective antinociception to mechanical allodynia was lost on day 33 for all compounds while tolerance to cold allodynia developed on day 27 for morphine and gabapentin, day 33 for kurkinol and day 31 for kurkinorin. The induction of tolerance to both mechanical and cold allodynia at the same rate as morphine is an unexpected result as gabapentin showed no tolerance in CINP in rats (Mangaiarkkarasi et al., 2015), other pre-clinical rodent models, and clinically in humans (Chu et al., 2011, Field et al., 1997, Putzke et al., 2002). Previous work has shown that the target of gabapentin, the $\alpha 2 \delta$-1 subunit of the voltage-gated $\mathrm{Ca}^{2+}$ channel, is increased in the dorsal horn of the spinal cord following PTX treatment, resulting in increased intracellular $\mathrm{Ca}^{2+}$ and nociceptor hyperresponsiveness. The mechanism of anti-nociception is therefore believed to be the regulation of atypical $\mathrm{Ca}^{2+}$ signalling (Xiao et al., 2007). Subsequent use of gabapentin in the Kivell Lab has shown no tolerance in the same model of chronic pain, with the experimenter the only different variable. Together this suggests an experimenter or environmental cause of the tolerance.

While the acute effects of kurkinol and kurkinorin line up with the theory of biased agonism the chronic phase does not. Neither compound had any significant delay in the development of tolerance to mechanical allodynia (day 33), while a slight increase in anti-nociceptive effects was seen for cold allodynia (kurkinol; day 33, kurkinorin; day 31). This is in direct opposition to the hot water tail-flick dose response tolerance assay where the degree of G-protein bias correlated to decreased tolerance (Crowley et al., 2016). The difference between the models is the state of the pain pathways, with the hot water dose response acting on an undamaged spinothalamic pain pathway. While PTX treatment results in the loss of peripheral nerve fibres, demyelination, and dysregulated $\mathrm{Ca}^{2+}$ signalling in the peripheral nerves, DRG and dorsal horn of the spinal cord, as well as inflammation in the DRG and dorsal horn of the spinal cord. This induces hyperexcitability of excitatory neurons within the ventral lateral PAG and inhibition of inhibitory neurons to send faciliatory signals through the spinothalamic pathway in response to thermal non-noxious and noxious stimuli (Li et al., 2017, 
Samineni et al., 2017, Xiao et al., 2011). Further dysregulation can be seen in the reorganisation of 166 brain regions, such that connections between the striatum and hindbrain, including key areas of pain circuitry (e.g. PAG, parabrachial nucleus, raphe nucleus, and the trigeminal nerve) are increased. The PAG also loses connections to limbic areas and increases the connections with the raphe (Ferris et al., 2019). The full dysregulation of the pain pathways from the terminal ends of nociceptors to the dysregulation of the descending pathway may override any benefits G-protein bias has in reducing tolerance. Especially with regards to increases in $\mathrm{Ca}^{2+}$ channels and signalling throughout the descending pain pathway, as the inhibition of $\mathrm{Ca}^{2+}$ channels is a key mechanism of $\mu$ receptor agonists. When $\beta$-arrestin2 is deleted from the system $\mu$ receptor increase their coupling to VG $\mathrm{Ca}^{2+}$ channels to inhibit internalisation, but not the induction of cellular tolerance (Walwyn et al., 2007). It may also be possible that the cellular mechanism of tolerance in the CINP model is dependent on GRK2-ERK1/2 mediated desensitisation of ion channels not receptor internalisation (Dang et al., 2009). Overall, this indicates that decreased $\beta$-arrestin2 recruitment may not result in decreased anti-nociceptive tolerance when used long-term for the treatment of chronic pain. 
Table 3.6 Summary of the Anti-Nociceptive Profiles of G-Protein Biased Mu Opioid Receptor Agonists

\begin{tabular}{|c|c|c|c|c|c|c|c|}
\hline & $\begin{array}{l}\text { Bias } \\
\text { Factor }\end{array}$ & $\begin{array}{c}\text { Thermal Dose } \\
\text { Response }\end{array}$ & $\begin{array}{c}\text { Thermal Duration } \\
\text { of Action }\end{array}$ & $\begin{array}{c}\text { Anti-Nociceptive } \\
\text { Tolerance }\end{array}$ & $\begin{array}{c}\text { Inflammatory } \\
\text { (Formalin Assay) }\end{array}$ & $\begin{array}{l}\text { Surgical Anti- } \\
\text { Nociception }\end{array}$ & Chronic Pain \\
\hline Buprenorphine & $\begin{array}{c}1.8- \\
2.8[1]\end{array}$ & $\begin{array}{c}\text { More potent than } \\
\text { morphine }[2-6]\end{array}$ & $\begin{array}{l}\text { Longer duration of } \\
\text { action }[3,4,6]\end{array}$ & No tolerance [3-6] & $\begin{array}{c}\text { Reduces phase I \& } \\
\text { II }[2,6]\end{array}$ & $\begin{array}{c}\text { Effective reversal } \\
\qquad[5]\end{array}$ & $\begin{array}{l}\text { Effective in SNL, } \\
\text { CCl, CINP }[3,4,6]\end{array}$ \\
\hline Loperamide & $\begin{array}{c}0.5-1 \\
{[1,7]}\end{array}$ & $\begin{array}{c}\text { More potent than } \\
\text { morphine [8-10] }\end{array}$ & $\begin{array}{l}\text { Longer duration of } \\
\text { action [8-10] }\end{array}$ & $\begin{array}{c}\text { Tolerance in SNL } \\
{[11]}\end{array}$ & $\begin{array}{c}\text { Reduces phase II } \\
\text { [12] }\end{array}$ & n.d & Effective in SNL [11] \\
\hline Mitragynine 3 & n.d & $\begin{array}{c}\text { More potent than } \\
\text { morphine [13] }\end{array}$ & $\begin{array}{l}\text { Same duration of } \\
\text { action [13] }\end{array}$ & n.d & n.d & n.d & n.d \\
\hline TRV130 & $\begin{array}{c}3-4 \\
{[14,15]}\end{array}$ & $\begin{array}{l}\text { More potent than } \\
\text { morphine }[15,16]\end{array}$ & $\begin{array}{c}\text { Same duration of } \\
\text { action }[15,16]\end{array}$ & $\begin{array}{c}\text { No tolerance }[15, \\
16]\end{array}$ & n.d & n.d & n.d \\
\hline TRV0109101 & n.d & $\begin{array}{c}\text { More potent than } \\
\text { morphine [17] }\end{array}$ & n.d & $\begin{array}{c}\text { same tolerance as } \\
\text { morphine [17] }\end{array}$ & n.d & $\begin{array}{l}\text { Same effect as } \\
\text { morphine [17] }\end{array}$ & n.d \\
\hline PZM21 & $\begin{array}{c}3.23 \\
{[15]}\end{array}$ & $\begin{array}{l}\text { Less potent than } \\
\text { morphine }[15,18 \text {, } \\
19]\end{array}$ & $\begin{array}{l}\text { Similar duration of } \\
\text { action }[15,18,19]\end{array}$ & $\begin{array}{l}\text { same tolerance as } \\
\text { morphine }[15,18, \\
19]\end{array}$ & $\begin{array}{c}\text { Reduces phase I \& } \\
\text { II }[15,18]\end{array}$ & n.d & n.d \\
\hline $\begin{array}{c}\text { SR-11501, - } \\
\text { 14969, -15908, - } \\
51099, \&-14968\end{array}$ & $\begin{array}{c}5-55 \\
{[20]}\end{array}$ & $\begin{array}{l}\text { Similar potency to } \\
\text { morphine }[20,21]\end{array}$ & $\begin{array}{l}\text { Longer duration of } \\
\text { action }[20,21]\end{array}$ & n.d & n.d & n.d & n.d \\
\hline SR-17018 & $\begin{array}{l}40- \\
102 \\
{[20]}\end{array}$ & $\begin{array}{l}\text { Similar potency to } \\
\text { morphine }[20,22 \text {, } \\
23]\end{array}$ & $\begin{array}{l}\text { Longer duration of } \\
\text { action }[20,22]\end{array}$ & $\begin{array}{l}\text { No tolerance [20, } \\
\text { 22] }\end{array}$ & n.d & n.d & n.d \\
\hline
\end{tabular}




\begin{tabular}{|c|c|c|c|c|c|c|c|}
\hline Herkinorin & $\begin{array}{l}0.95 \\
{[24]}\end{array}$ & n.a & No effect [24] & $\begin{array}{l}\text { Reduced tolerance } \\
\qquad[25]\end{array}$ & $\begin{array}{c}\text { Contralateral; both } \\
\text { phases } \\
\text { Ipsilateral; no } \\
\text { effect [25] }\end{array}$ & n.d & n.d \\
\hline Kurkinorin & $\begin{array}{l}0.32 \\
{[24]}\end{array}$ & $\begin{array}{c}\text { More potent than } \\
\text { morphine [24] }\end{array}$ & $\begin{array}{c}\text { Similar duration of } \\
\text { action to morphine } \\
\text { [24] }\end{array}$ & $\begin{array}{l}\text { Reduced tolerance } \\
\qquad[24]\end{array}$ & $\begin{array}{c}\text { Reduces phase I \& } \\
\text { II [n.p] }\end{array}$ & n.d & Effective in CINP \\
\hline Kurkinol & $\begin{array}{l}0.14 \\
{[26]}\end{array}$ & $\begin{array}{c}\text { More potent than } \\
\text { morphine [26] }\end{array}$ & $\begin{array}{l}\text { shorter duration of } \\
\text { action to morphine } \\
{[26]}\end{array}$ & No tolerance [26] & n.d & n.d & Effective in CINP \\
\hline
\end{tabular}

Bias Factors reported as values $>1$ are G-protein biased agonists, except for herkinorin, kurkinorin, and kurkinol where $<0$ is G-protein biased

\section{Abbreviations:}

$\mathrm{CCl}=$ chronic constriction injury

CINP = chemotherapy induced neuropathic pain

n. $a=$ not applicable

n.d = not determined

n.p. = not published

$S N L=$ sciatic nerve ligation

\section{References:}

1. Burgueño, et al. 2017.

2. Yamamoto, et al. 2006.

3. Gades, et al. 2000

4. Cowan, et al. 1977.

5. Roughan and Flecknell. 2002

6. Christoph, et al. 2005.

7. Thompson, et al. 2015.

8. Ray, et al. 2005.

9. Tayrouz, et al. 2001.

10. Emerich, et al. 1998.

11. He, et al. 2013.

12. Ray and Yaksh. 2008.

13. Váradi, et al. 2016.

14. DeWire, et al. 2013.

15. Manglik., et al. 2016.

16. Zamarripa, et al. 2018

17. Koblish, et al. 2017.

18. Hill, et al. 2018.

19. Kudla, et al. 2019.

20. Schmid, et al. 2017.

21. Schwienteck, et al. 2019

22. Grim, et al. 2020.
23. Gillis, et al. 2020

24. Crowley, et al. 2016.

25. Lamb, et al. 2012.

26. Crowley, et al. 2020. 


\subsubsection{Limitations and Future Directions}

A large source of variation in behavioural tests is the experimenter and environment, with stress-induced anti-nociception the largest concern. This can be caused by a multitude of factors including the environment, apparatus noise, scuffing/injection, and restraint required to perform the assay. To mitigate these factors animals were habituated to the room, apparatus noise, scuffing, and any restraint in the presence of white noise for a minimum of 3 days. One large, uncontrollable, environmental change during this thesis was the movement to a new animal facility causing slight differences in dose response tolerance $\mathrm{ED}_{50}$ values in $\mathrm{Wt}$ littermate controls and decreased duration of action in Wt from $\mu$ receptor ${ }^{-1-}$ breeding in the hotplate. The same trend occurred at each facility, indicating the results are a true representation of the drug's effects.

In the initial stages of this thesis, the behavioural effects of morphine and kurkinol were only assessed in male $\mathrm{C} 57 \mathrm{BL} / 6 \mathrm{~J}$ mice. This includes the dose response tolerance hot water tail-flick assay, which was used to inform the doses in subsequent pain and side effect assays. Due to the known sex differences of $\mu$ receptor agonists in preclinical rodent models of anti-nociception, it would have been beneficial to also assess the dose response effects in female C57BL/6J mice (Cicero et al., 1997, Kepler et al., 1989, Kest et al., 1999, Posillico et al., 2015). In the paclitaxel experiment, both male and female mice were assed, but the same dose of drug was administered to both male and female mice, despite the shown sex differences. This may have skewed the tolerance data, in future running these behavioural assays at a sex specific dose will show if decreases in duration of action in the hotplate assay are overcome at an equivalent, sex specific, dose and if tolerance differs between sexes.

The measurements for hot water tail-flick, hotplate, and acetone in the CINP model are all reliant on the reaction time of the experimenter to determine when pain behaviour occurs or is terminated. To reduce these effects, baseline measurements are taken to normalise to both experimenter reaction time and nociceptive threshold of each animal. The implementation of an apparatus with an automatic stop when pain behaviours occur, such as the IITC tail-flick analgesia meter that stops when a beam break occurs, would further improve accuracy. Thermal nociceptive thresholds are also influenced by the temperature of the skin temperature at the area assessed (e.g. tail, foot) and core temperature (Hole et al., 1993, Tjølsen et al., 1989) and were not controlled for in these experiments. As morphine and other $\mu$ receptor agonists are known to dose-dependently induce hypo- and hyperthermia this could affect tail-flick latency, paw withdrawal, or shaking (Muraki et al., 1987). In future, the monitoring tail or paw temperature would help control temperature thermal measurements.

CINP is a simple, reproducible, and non-surgical method of inducing neuropathic pain with a disease a progression and phenotype comparable to what occurs in humans. However, the rates are not with $11-87 \%$ of patients treated with PTX developing neuropathic pain compared to $100 \%$ of rodents (Banach et al., 2016, Zajączkowska et al., 2019). High rodent induction rates are necessary to 
avoid unethical use of animals but comes at the expense of clinical translation due to slight variation in the pathological process. Furthermore, the outcome measures are evoked by the VF filaments or acetone. Where human disease typically presents as spontaneous, continuous, or paroxysmal pain with low rates of mechanical (57\%) and thermal (33\%) hypersensitivity (Maier et al., 2010). Adding a spontaneous measure of nociception to rodent models, such as mouse grimace scale (Langford et al., 2010) or spontaneous burrowing (Andrews et al., 2012, Deacon, 2009), would improve the translation of the model. Due to the complexity of the pathological processes leading to neuropathic pain multiple models of pre-clinical neuropathic pain should be utilised. This could be a surgical model such as $\mathrm{CCl}$ to the sciatic nerve or non-surgical model such as HIV-induced neuropathy where there are currently few effective treatments (Phillips et al., 2010).

While the CINP pain model was conducted in both sexes, the decision was made to use one dose, the male mechanical $E D_{50}$, to simplify the daily experimental procedure. Given no significant interaction of sex and potency implies a relative dose for both sexes were used. Further, the absence of sex differences in the chronic phase indicates no overall impact on the integrity of the experiment. As already mentioned, this experiment was run at the $\mathrm{ED}_{50}$ dose rather than the $\mathrm{ED}_{80}$ as the volume of kurkinorin required for the $\mathrm{ED}_{80}$ was not available. In future, this experiment should be repeated using the $\mathrm{ED}_{80}$ doses. Repeating CINP in $\beta$-arr2 ${ }^{--}$mice would also allow the role of $\beta$-arrestin2 in antinociceptive tolerance to be investigated further, as both kurkinorin and kurkinol had no improvements compared to morphine.

Throughout this thesis, a large volume of tissue was collected from the tail-flick dose response and CINP experiments. This includes: the L4-L6 spinal cord of $\beta$-arr2 ${ }^{-1}$ mice from the dose response tolerance tail-flick assay and the L4-L6 spinal cord, DRG, left sciatic nerve, and the PAG from CINP. In future immunohistochemistry $(\mathrm{IHC})$ can be used to assess the level $\mu$ receptor localisation and expression within the L4-L6 spinal cords of $\beta$-arr2 ${ }^{--}$and Wt littermate controls and C57BL/6L mice from the tail-flick and CINP experiments will allow the correlation to tolerance to be assessed in both models. Further, Co-labelling of the VG-Ca ${ }^{2+}$ channels and $\mu$ receptor would allow the evaluation of both mechanisms of cellular tolerance to be assessed and potentially identify the mechanism through which the different levels of tolerance are induced.

Furthermore, the spinal cord, DRG, and sciatic nerve could be used to investigate the PTX disease progression. This could include markers of neuroinflammation such as Glial Fibrillary Acidic Protein to assess astrocyte activation, ionized calcium binding adaptor molecule 1 for the activation of microglia, and the infiltration of macrophages into the DRG with CD68. Furthermore, the cytokines they release, including TNF $\alpha, I L-1 \beta$, and CCL2, are believed to play an important role in the peripheral sensitisation that occurs with PTX treatment and can also be assessed via IHC. Other changes that could be assessed is the altered localisation of ion channels within the periphery and spinal cord. With the upregulation of the $\alpha 2 \delta-1$ subunit of the VG Ca ${ }^{2+}$ channel, $\mathrm{Na}^{+}$channels $\mathrm{NaV}_{1.7}$ and $\mathrm{NaV}_{1.6}$ and 
decreases in the TRAAK and TREK-1 potassium channels associated with the neuronal hyperexcitability. While the level of axonal degradation in the sciatic nerve and DRG could be assessed via transcription factor 3 and sterile alpha and TIR motif-containing protein 1 markers of neuronal damage and black gold staining for the level of myelination. Any difference seen between PTX and treatments providing information on pathogenesis and potential targets for phenotype reversal. 


\section{Chapter 4. Side Effect Profile of Kurkinorin and Kurkinol}

\subsection{Introduction}

The small therapeutic window between the analgesic and on-target side effects of current $\mu$ receptor analgesics is a major limiting factor in the treatment of strong acute and chronic pain. Especially considering this window is consistently reduced with the onset of analgesic tolerance. It is well established that tolerance to side effects does not develop at the same rate as analgesic tolerance, or at all (Martins et al., 2015). On-target side effects (i.e. side effects elicited through the binding of the $\mu$ receptor) can include; addiction (Compton et al., 2006), respiratory depression (Martins et al., 2015), coordination impairment, sedation (Aubrun et al., 2001), constipation, urinary retention, nausea, and vomiting (Ruan, 2007). The high abuse liability and addiction to prescription opioids is a major contributor to the opioid crisis worldwide with respiratory depression being the cause of death in opioid overdose cases (Marotta et al., 2018, Shipton et al., 2017, Vowles et al., 2015). In this chapter, we aimed to investigate the effect that G-protein bias has on the side effect profile of kurkinol and kurkinorin to determine whether G-protein bias was associated with improved side effects. To evaluate this, we used the rotarod assay to assess motor coordination impairment, faecal accumulation and the charcoal meal assay to assess the induction of constipation, urinary retention with urine accumulation, and whole-body plethysmography to assess the induction of respiratory depression.

\subsubsection{Mu Opioid Receptor Expression and the Induction of on-target Side Effects}

The $\mu$ receptor is widely located throughout the peripheral and central nervous system, in areas associated with pain and other biological functions. It is this wide expression that results in the induction of on-target side effects upon the systemic administration of $\mu$ receptor agonists such as morphine and fentanyl.

\subsubsection{Central Nervous System}

\subsection{Locomotion}

Morphine and other $\mu$ receptor agonists have dose dependent effects on locomotion in rodents, with low doses causing hyperactivity and motor-coordination impairment (Cartmell et al., 1991, Stone et al., 2014), while higher doses result in sedation (Stone et al., 2014). These effects are postulated to occur via increases in dopamine and serotonin, alongside the activation of muscarinic cholinergic receptors in the VTA (Di Chiara et al., 1988a, Tao et al., 1994, Wasserman et al., 2013). The dopamine increase is caused by the inhibition of GABAergic interneurons, disinhibiting the dopaminergic neurons, and increasing dopamine release in the ventral and dorsal striatum (Di Chiara 
et al., 1988a, b) (Table 4.1). The selective activation of post-synaptic dopamine D1, D2, and D3 receptors is believed to induce the dose dependent locomotor effects, with the D1 and D2 receptors increasing locomotion while D3 receptor activation inhibits locomotion (Missale et al., 1998). Interestingly, morphine induces the same level of dopamine release in the striatum of $\beta$-arr2 ${ }^{-/-}$mice and Wt littermate controls, while spontaneous locomotion is reduced in the open field test (Bohn et al., 2003). This indicates that it may not be the $\mu$ receptor recruitment of $\beta$-arrestin 2 that is responsible for the induction of motor coordination deficits following morphine administration.

Table 4.1 Mu Opioid Receptor Location and Associated Side Effects

\begin{tabular}{c|c} 
& $\mu$ receptor Location \\
\hline Locomotion & $\begin{array}{c}\text { VTA [1-3] } \\
\text { ventral and dorsal striatum [3] }\end{array}$ \\
\hline $\begin{array}{c}\text { Respiratory } \\
\text { Depression }\end{array}$ & $\begin{array}{c}\text { Pons, medulla oblongata, LC, NTS, and ACC [4-7] } \\
\text { Carotid bodies [9] } \\
\text { Vagus nerve [10] }\end{array}$ \\
\hline Constipation & Submucosal and myenteric plexuses of the gastric antrum, ileum, and \\
distal colon [7, 10-13]
\end{tabular}

\section{References:}

1. Wasserman, et al. 2013.

2.Tao and Auerbach. 1994.

3.Di Chiara and Imperato. 1988.

4.Delfs, et al. 1994.

5.Mansour, et al. 1987.

6.Peckys and Landwehrmeyer. 1999.

7.Peng, et al. 2012.

8.Wharton, et al. 1980.

9.Lundberg, et al. 1979.

10. Bagnol, et al. 1997.

11.Gray, et al. 2006.

12.Lupp, et al. 2011.

13.Sternini, et al. 2004.

14. Machelska and Celik. 2020. 


\subsection{Respiratory depression}

Under normal conditions, the respiratory pattern is set by the pre-Bötzinger (inhalation) and retro-trapezoid and parafacial respiratory group (RTN/pFRG) (exhalation) in the ventrolateral medulla (Janczewski et al., 2006, Lumsden, 1923, Onimaru et al., 2003, Smith et al., 1991). However, they do not act in complete isolation, with the pons, particularly the Kölliker-Fuse nucleus, LC, and PB, heavily influencing respiratory rhythm (Rybak et al., 2004). $\mu$ receptor are densely located throughout many of the respiratory centres, including the pons, medulla oblongata, LC, NTS, and ACC (Delfs et al., 1994, Mansour et al., 1987, Peckys et al., 1999, Peng et al., 2012) (Table 4.1). As well as in the carotid bodies (Lundberg et al., 1979a, Wharton et al., 1980) and vagus nerve (Lundberg et al., 1979b) that aid in sensing blood oxygen levels (Table 4.1).

The mechanism through which $\mu$ receptor agonists induce respiratory depression is not fully elucidated, with current evidence indicating the inhibition of the RTN/pFRG, Kölliker-Fuse nucleus, NTS, and parabrachial nucleus as key areas. When DAMGO is applied to the pre-Bötzinger complex in isolation, neuronal excitability is decreased and the respiratory rate slows, indicating opioid sensitivity. When both pre-Bötzinger complex and RTN/pFRG are included the respiratory rate is slowed and a change in pattern occurs, with inhalation periodically skipped, known as a quantal pattern. This occurs as the opioid insensitive RTN/pFRG continues to fire inducing exhalation and phasic excitatory signals to the pre-Bötzinger complex. However, opioid-induced hyperpolarisation of the pre-Bötzinger complex results in inconsistent firing and skipped inhalation (Mellen et al., 2003). $\mu$ receptor agonists further disrupt the respiratory pattern via the inhibition of the GABAergic neurons in the Kölliker-Fuse nucleus causing a delay in the exchange between inspiration and expiration, increasing the cycle time of a single breath (Dutschmann et al., 2006, Dutschmann et al., 2004). Furthermore, the microinjection of DAMGO into this area decreases tidal volume in a GIRK dependent manner (Levitt et al., 2015). The activation of $\mu$ receptor in the NTS (Cerritelli et al., 2016, Zhang et al., 2011) and, the parabrachial nucleus (Prkic et al., 2012) have also been implicated in opioid-induced respiratory depression (OIRD).

The role of $\beta$-arrestin 2 in the OIRD is currently unclear. Initially, morphine was found to have little effect on respiratory frequency in $\beta$-arr2 ${ }^{--}$mice (Raehal et al., 2005), however, these effects have never been replicated. Multiple studies have since shown that novel $\mu$ receptor $G$-protein biased agonists have reduced OIRD pre-clinically in male C57BL/6 mice and Sprague Dawley rats (DeWire et al., 2013, Manglik. et al., 2016, Schmid et al., 2017). Further independent studies of these same compounds have more recently shown opposing effects in male C57BL/6 and CD1 mice and male Sprague Dawley rats (Altarifi et al., 2017, Austin Zamarripa et al., 2018, Hill et al., 2018, Kudla et al., 2019). With no clear role of $\beta$-arrestin2 in OIRD, the clinical utility of G-protein biased agonists is being questioned. 


\subsubsection{Enteric nervous system}

The induction of constipation is common with both acute and chronic administration of $\mu$ receptor analgesics. The $\mu$ receptor is present throughout the enteric nervous system with high levels found within the submucosal and myenteric plexuses of the gastric antrum, ileum, and distal colon of dogs (Allescher et al., 1989), rats (Bagnol et al., 1997, Gray et al., 2006), Guinea pigs (Galligan et al., 1991, Lay et al., 2016), and humans (Lupp et al., 2011, Peng et al., 2012, Sternini et al., 2004) (Table 4.1). The neurons of the myenteric plexus release neurotransmitters that induce the contractions (Substance $p$ and acetylcholine) and relaxation (ATP, nitrous oxide, vasoactive intestinal peptide) of the longitudinal and circular muscles of the Gl tract (Roy et al., 1998, Thompson et al., 2014). While the submucosal plexus contains secretomotor neurons that release acetylcholine and vasoactive intestinal peptide to activate chloride channels on enterocytes, inducing osmosis of water into the gut lumen (Brookes, 2001). When $\mu$ receptor agonists are applied to this system $\mathrm{Ca}^{2+}$ and $\mathrm{Na}^{+}$channels are inhibited and GIRK channels activated, resulting in a decrease in the release of ATP, nitrous oxide, and vasoactive intestinal peptide. Together, this functions to increase muscle tone and decrease propulsion within the small intestine (Bauer et al., 1991, Morita et al., 1982, North et al., 1977, Smith et al., 2012, Surprenant et al., 1990). While in submucosal neurons, $\mu$ receptor activation inhibits acetylcholine and vasoactive intestinal peptide release, decreasing chloride and water movement into the ileum to further induce constipation (Gaginella et al., 1983). $\mu$ receptor-induced G.I effects are abolished in $\mu$ receptor ${ }^{-/}$mice and can be reduced with the administration of peripherally restricted $\mu$ receptor antagonists such as methylnaltrexone (Roy et al., 1998, Thompson et al., 2014).

In $\beta$-arr ${ }^{-/}$mice acute administration of morphine inhibiting smooth muscles contraction the same extent in the small intestine and colon of $\beta$-arr $2^{--}$mice as it does in Wt littermate controls (Akbarali et al., 2014, Kang et al., 2012). Interestingly, faecal output was still increased in $\beta$-arr $2^{-/}$mice compared to the Wt littermate controls (Raehal et al., 2005). The induction of tolerance to constipation is well documented in the S.I of rodents (Goldstein et al., 1973, Paton, 1957, Rezvani et al., 1983) and is also present in the ileum of $\beta$-arr $2^{-/-}$mice (Kang et al., 2012, Maguma et al., 2012). Indicating different regulatory mechanisms in the development of tolerance between the small intestine and colon with no dependence on $\beta$-arrestin2 recruitment.

\subsubsection{Mu Opioid Receptor Expression on Immune Cells}

$\mu$ receptor mRNA and/or protein has been identified in multiple immune cell lineages, including lymphocytes, monocytes, macrophages, granulocytes, splenocytes, astrocytes and microglia (reviewed in Machelska et al., 2020) (Table 4.1). In chronic pain states, such as the CCI model in mice, $\mu$ receptor positive immune cells are increased and secrete endogenous opioids to aid in antinociception (Celik et al., 2016). While chronic morphine treatment has been shown to induce the secretion of CRF and II-1 $\beta$ to induce the release of endogenous opioids causing anti-nociception (Cabot et al., 1997, Schafer et al., 1996). 
In the periphery, there is evidence that immune cells expressing the $\mu$ receptor play an antinociceptive role in response to endogenous opioids. Systemic, chronic administration of $\mu$ receptor agonists results in the suppression of multiple immune cell populations and their functions. This includes natural killer cells (Sacerdote et al., 1997, Yeager et al., 1995), decreased antibody production (Taub et al., 1991), inhibited T cell response (Pellis et al., 1986), enhanced apoptosis (Singhal et al., 1998, Yin et al., 1999), decreased phagocytosis (Rojavin et al., 1993, Tubaro et al., 1985), decreased IL$1 \beta$, IL-2, TNF- $\alpha$, IFN- $\gamma$, and increased TGF- $\beta 1$ and IL-10 production (Pacifici et al., 2000). The mass inhibition of the innate and adaptive immune systems results in higher rates of infection in opioid addicts, particularly HIV, hepatitis C, and pneumonia (Alter et al., 1999, Strathdee et al., 2010, Wiese et al., 2019, 2018). The expression of $\mu$ receptor on CNS immune cells, such as microglia and astrocytes, has also been suggested to play a role in the development of hyperalgesia. For example, chronic opioid administration has been correlated to an increase in the number of $M 2$ reactive microglia and pro-inflammatory markers (IL-1 $\beta$, IL-6, and TNF- $\alpha$ ) in the cerebral spinal fluid and spinal cord tissue of male Sprague Dawley rats, resulting in peripheral sensitisation (Hutchinson et al., 2008, Johnston et al., 2004).

\subsubsection{Side Effect profiles of Pre-clinical G-protein biased agonists}

There are many novel G-protein biased $\mu$ receptor agonists being developed for the treatment for chronic pain because of the reduced respiratory depression, constipation, and motor coordination impairment seen in the $\beta$-arr ${ }^{-/-}$mice, leading to the hypothesis that G-protein biased agonists could reduce the side effect profile of $\mu$ receptor agonists (Bohn et al., 2003, Raehal et al., 2005). Clinically available $\mu$ receptor agonists have also been screened to further investigate the role of G-protein biased signalling has on the prevalence of side effects. This includes the identification of Loperamide and Buprenorphine as G-protein biased agonists (refer to Chapter 5, Table 5.1) with overall improvements in side effect profiles compared to morphine (Cowan et al., 1977, Dahan, 2006, Kuo et al., 2015, Liles et al., 1992, Sadeque et al., 2000, Tan-No et al., 2003, Tompkins et al., 2014) (Table 4.3).

Two novel G-protein biased $\mu$ receptor agonists with reduced side effect profiles are TRV130 and PZM21. Initially, these compounds showed improved locomotor, G.I, respiratory, and reduced rewarding properties (DeWire et al., 2013, Manglik. et al., 2016). However, independent analyses have indicated increased abuse liability, respiratory depression, and constipation (Altarifi et al., 2017, Zamarripa et al., 2018, Hill et al., 2018, Kudla et al., 2019) (Table 4.3). This calls into question whether signalling bias seen in cellular assays translates to clinical use. Despite this, many novel compounds continue to be developed to probe the role of G-protein bias in the initiation of side effects, including the SR- G-protein biased agonists that have potent anti-nociceptive effects with reduced respiratory depression and abuse liability (Gillis et al., 2020a, Grim et al., 2020, Schmid et al., 2017, Schwienteck et al., 2019) (Table 5.3). 


\subsubsection{Side Effects of Kurkinorin and Herkinorin}

The side effect profiles for herkinorin and kurkinorin have been partially investigated. This has revealed no reduction in core temperature, significantly decreased rewarding effects in the conditioned place preference assay and limited motor coordination impairment previously conducted in the Kivell lab by Dr Kelly Paton and Nirajomhoan Shivaperumal (Crowley et al., 2016) (Table 4.3). While the lack of on-target effects of herkinorin is most likely due to the poor brain penetrance, the improved side effect profile of kurkinorin is believed to be due to reduced $\beta$-arrestin2 recruitment, as seen by a relative decrease in potency in the PathHunter assay compared to the level of cAMP inhibition it the HitHunter assay. Further assessment of other on-target side effects is required to fully understand the role of $\beta$-arrestin2 recruitment on the side effect profile of kurkinorin and kurkinol, and the potential effect that co-activation of $\mathrm{k}$ receptor may play. 


\subsection{Aims \& hypothesis}

The theory of biased agonism at the $\mu$ receptor promises to create pharmacotherapies with improved analgesic effects and reduced side effects, thus increasing the therapeutic window. In this chapter, we investigate the role of $\beta$-arrestin2 recruitment in side effect induction using the novel agonists, kurkinorin and kurkinol. We therefore assessed the induction of motor coordination impairment, gastrointestinal inhibition, and respiratory depression in pre-clinical behavioural models in $\mathrm{C} 57 \mathrm{BL} / 6 \mathrm{~J}$ and $\beta$-arr2 ${ }^{-1}$ mice. In line with the theory of biased agonism, kurkinol was hypothesised to show an improvement in therapeutic window, with a moderate improvement for kurkinorin. We further hypothesised the global deletion of $\beta$-arrestin2 would further decrease any side effects present.

Aims;

1. Evaluate the effects of kurkinol on motor coordination in male and female $\mathrm{C} 57 \mathrm{BL} / 6 \mathrm{~J}$ mice using the accelerating rotarod assay.

2. Determine the impact of G-protein bias on the G.I and urinary systems using the faecal accumulation, charcoal meal, and urinary retention assays in male and female C57BL/6J mice and $\beta$-arr2 ${ }^{-/-}$mice.

3. Examine the role of $\beta$-arrestin2 in the induction of respiratory depression using the novel $\mu$ receptor agonists kurkinol and kurkinorin in whole-body plethysmography using unrestrained C57BL/6J and $\beta$-arr2 ${ }^{-/}$mice. 


\subsection{Results}

\subsubsection{Kurkinol has a Reduced Duration of Motor Coordination Impairment}

The induction of motor coordination impairment was assessed over 180 min utilising the accelerating rotarod assay in both male and female mice after the administration of vehicle, morphine or kurkinol. The higher $10 \mathrm{mg} / \mathrm{kg} / \mathrm{i} . \mathrm{p}$. dose of morphine showed no significant differences in motor coordination with the sex of the mice over time $\left(F_{(4.223,4.838)}=1.617, p=0.199\right)$ (Fig $\left.4.1 \mathrm{~A}, \mathrm{C}\right)$. However, the $5 \mathrm{mg} / \mathrm{kg} / \mathrm{i}$.p. dose of morphine was found to have a greater overall impact on male mice $\left(F_{(1,11.111)}\right.$ $=4.834, p=0.035, p=0.011$ ) (Fig $4.1 \mathrm{E}$ ). When the data from the male and female mice were combined, morphine was found to impair motor coordination for the duration of the time course, with dosing effects observed between $15-30 \min \left(F_{(8.447,93.946)}=14.695, p<0.0001, p<0.05\right)$ (Fig. $\left.4.1 \mathrm{G}\right)$. In comparison, no effect of sex was seen following kurkinol administration in relation to time $\left(F_{(8.621}\right.$, 5.242) $=0.608, p=0.443)$ or a simple main effect $\left(\mathrm{F}_{(2,3.342)}=1.137, p=0.333\right)$ (Fig $\left.4.1 \mathrm{~B}, \mathrm{D}, \mathrm{H}\right)$. A shorter duration of impaired motor coordination was seen following kurkinol administration than what was observed with morphine when data obtained from both sexes were combined, with decreases observed between 15 and $60 \mathrm{~min}$ following $2.5 \mathrm{mg} / \mathrm{kg} / \mathrm{i}$.p. and up to $90 \mathrm{~min}$ at the $5 \mathrm{mg} / \mathrm{kg} /$ i.p. dose $\left(\mathrm{F}_{(8.612,120.997)}=23.030, p<0.0001, p<0.05\right)$ (Fig. $\left.4.1 \mathrm{~F}\right)$.

The data obtained from male and female mice AUC analysis was then combined to assess the overall effect and allow the direct comparison of the two compounds. In doing this, a $21 \%$ improvement in the overall anti-nociception was seen following kurkinol ( $5 \mathrm{mg} / \mathrm{kg} / \mathrm{i}$. . .) administration to the equivalent dose of morphine (10 mg/kg/i.p., $p=0.0043)$, with no difference between with the lower dose $(\mathrm{F}(4,58)=24.46, p<0.0001,, p=0.145)$ (Fig 4.2). 
A)

M a le

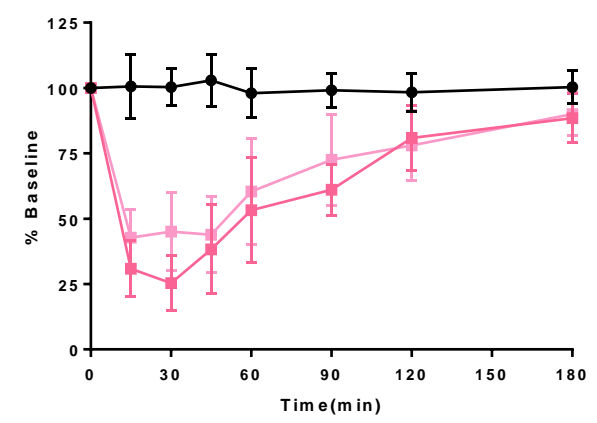

C)

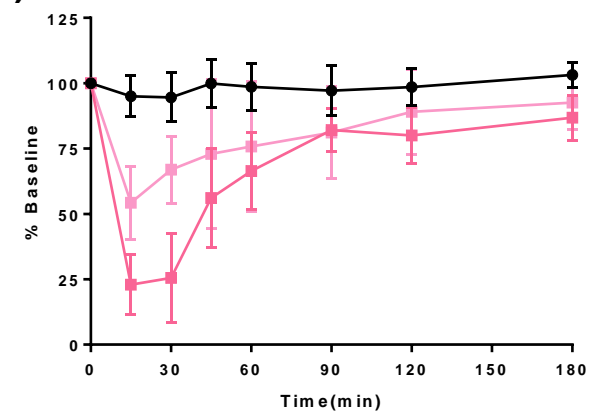

E)

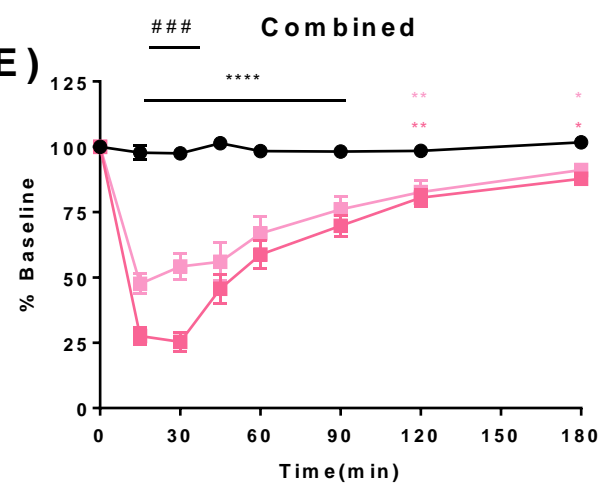

G )

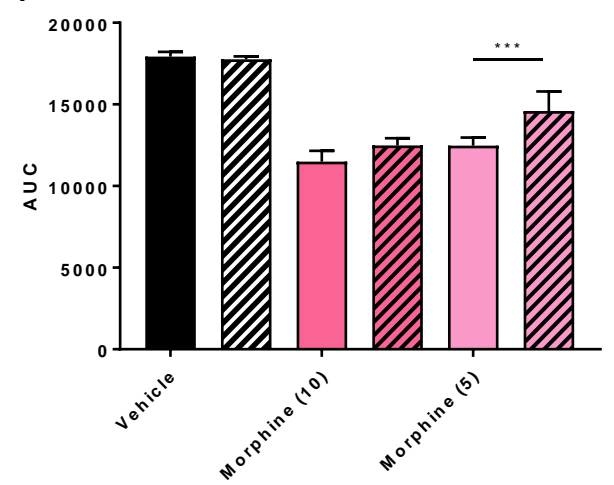

B )

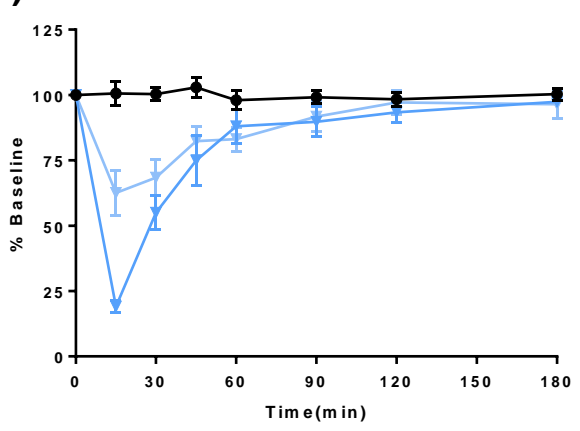

D)

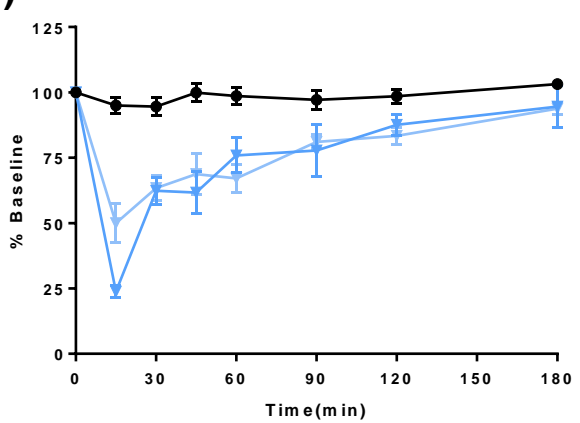

F )

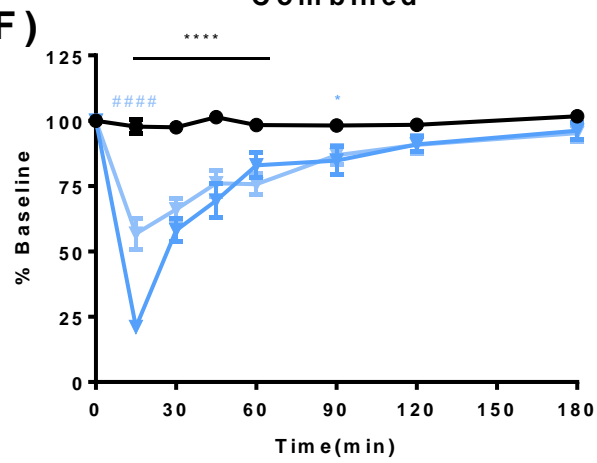

H)

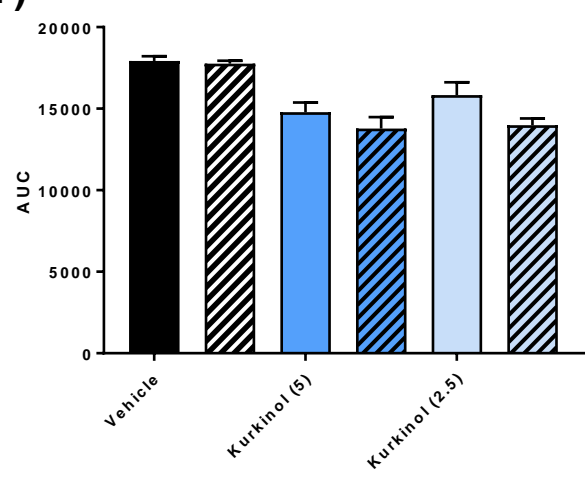

\footnotetext{
$\rightarrow$ Vehicle $\rightarrow$ Morphine (10) $\rightarrow$ Kurkinol (5) $\square$ Male

$\rightarrow$ Morphine (5) $\quad \rightarrow$ Kurkinol (2.5) Female
} 


\section{Figure 4.1 Kurkinol Has a Shorter Duration of Motor Coordination Impairment}

The induction of motor coordination impairment was assessed in male and female mice following morphine (5 or $10 \mathrm{mg} / \mathrm{kg} /$ i.p.) or kurkinol (2.5 or $5 \mathrm{mg} / \mathrm{kg} /$ i.p.) administration. (A, B) Time course of motor coordination impairment in males. $(C, D)$ Time course of motor coordination impairment in females. (E) Combining the sexes revealed impaired coordination over the 180 min time course with morphine (5 and $10 \mathrm{mg} / \mathrm{kg} / \mathrm{l} . \mathrm{p}$.). (F) While kurkinol only had reduced effects compared to 60 and 90 min at the $2.5 \mathrm{mg} / \mathrm{kg} /$ i.p. and $5 \mathrm{mg} / \mathrm{kg} / \mathrm{i}$.p. doses respectively. (G) Overall males were more impaired at $5 \mathrm{mg} / \mathrm{kg} / \mathrm{i}$.p. (H) While sex did not affect kurkinol induction of motor coordination impairment. Three-way mixed ANOVA with Bonferroni multiple corrections. ${ }^{*} p<0.05,{ }^{* *} p<0.01,{ }^{* * * *} p<0.0001$ compared to vehicle, $\# \# \# p<0.001, \# \# \# \# p<0.0001 E D_{50}$ compared to $E D_{80}$ dose. $N$; males $=7$, females = 5-6. Data presented as a percent of the baseline. Doses in brackets in $\mathrm{mg} / \mathrm{kg} / \mathrm{i}$.p. 


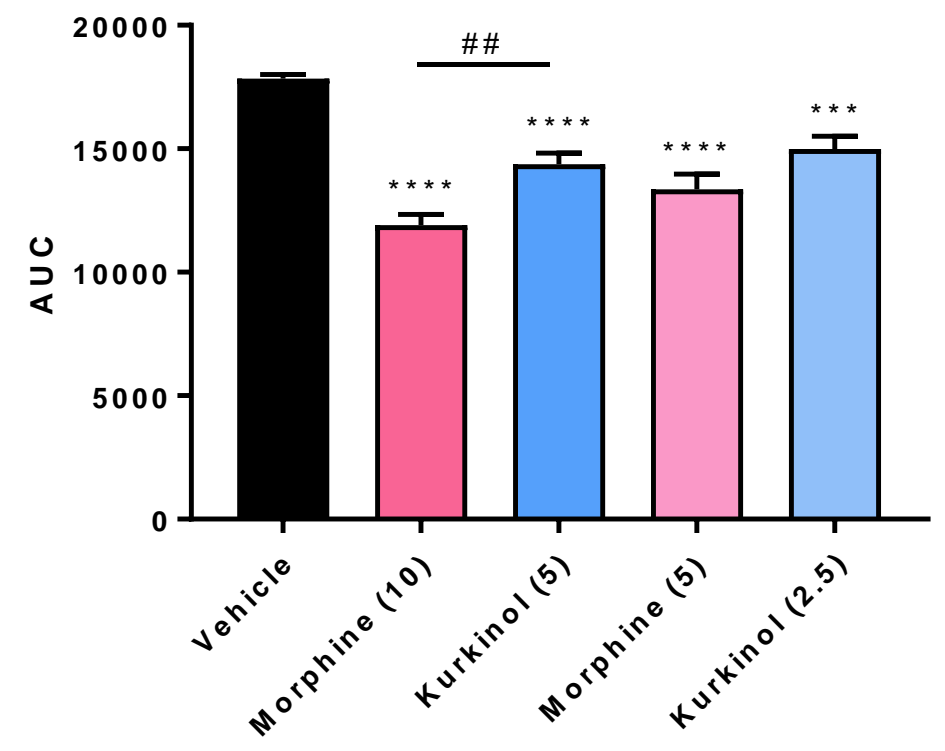

Figure 4.2 Kurkinol Induces Less Motor Coordination Impairment

When data from male and female mice were combined a significant improvement in AUC was seen following kurkinol ( $5 \mathrm{mg} / \mathrm{kg} / \mathrm{i}$.p.) administration and the equivalent dose of morphine (10 $\mathrm{mg} / \mathrm{kg} / \mathrm{i} . \mathrm{p}$.). One-way ANOVA with Bonferroni multiple corrections ${ }^{* * *} p<0.001,{ }^{* * * *} p<0.0001$ compared to vehicle, \#\#p<0.01, compared to morphine. $\mathrm{N}$; males =7, females $=5-6$. Doses in brackets in $\mathrm{mg} / \mathrm{kg} / \mathrm{i} . \mathrm{p}$. 


\subsubsection{Gastrointestinal Effects of Kurkinol and Kurkinorin}

Constipation is the most common side effect of chronic opioid use, occurring in $>50 \%$ of patients. Here we assess the ability of kurkinol and kurkinorin to inhibit G.I utilising both the faecal accumulation assay (overall G.I transit), and the charcoal meal assay (small intestine transit).

\subsubsection{Increased G-protein Recruitment Does Not Correlate to Decreased Faecal Accumulation}

To assess the effect of our drugs on G.I transit in both male and female mice the total weight of faeces produced over 6 hrs was measured.

The accumulation of faeces upon morphine administration was not affected by the sex of the animals overall, or over time (main effect; $\mathrm{F}_{(2,0.043)}=0.509, p=0.605$, Over time; $\mathrm{F}_{(4.971,0.42)}=2.022, p$ $=0.083)$ (Fig. 4.3 A, D, J, K). However, a decrease in faecal production was identified between $1-6 \mathrm{hr}$ when data obtained from male and female mice were combined $\left(F_{(4.917,0.187)}=8.971, p<0.0001, p<\right.$ 0.05). Significant differences between the $\mathrm{ED}_{50}$ and $\mathrm{ED}_{80}$ doses of morphine were also identified with a $37 \%$ increase at $5 \mathrm{hr}$ post-administration and 32\% increase $6 \mathrm{hrs}$ post-administration of the 5 $\mathrm{mg} / \mathrm{kg} /$ i.p. dose compared to $10 \mathrm{mg} / \mathrm{kg} /$ i.p. $(p<0.01$ ) (Fig. $4.3 \mathrm{G}$ ). Interestingly the more G-protein biased drug, kurkinol, also reduced faecal production throughout the 1 - $6 \mathrm{hr}$ at timepoints the $E \mathrm{D}_{80}$ anti-nociceptive dose $(5 \mathrm{mg} / \mathrm{kg} / \mathrm{i} . \mathrm{p})$. However, the $\mathrm{ED}_{50}$ anti-nociceptive dose of $2.5 \mathrm{mg} / \mathrm{kg} / \mathrm{i}$.p. only reduced faecal production in the mice at the $4 \mathrm{hr}$ time point $\left(\mathrm{F}_{(5.360,0.115)}=4.372, p=0.001, p<0.05\right)$ (Fig. $4.3 \mathrm{H})$. This effect was found to be independent of sex, both over time $\left(F_{(5.360,0.016)}=0.601, p=\right.$ 0.811 ) and as a simple main effect $\left(\mathrm{F}_{(2,0.135)}=1.452, p=0.248\right)$ (Fig $\left.4.3 \mathrm{~B}, \mathrm{E}, \mathrm{J}, \mathrm{K}\right)$. As with morphine and kurkinol, the reduced G.I transit in response to kurkinorin was found to be independent of sex over time $\left(\mathrm{F}_{(6.152,0.038)}=1.316, p=0.24\right)$ and as a main effect $\left(\mathrm{F}_{(2,0.097)}=1.049, p=0.361\right)(\mathrm{Fig} 4.3 \mathrm{C}, \mathrm{F}, \mathrm{J}, \mathrm{K})$. When the data from male and female mice were combined a similar pattern was identified than with the $\mathrm{ED}_{80}$ anti-nociceptive dose $(10 \mathrm{mg} / \mathrm{kg} / \mathrm{i}$. .p.) reducing the faecal accumulation between $1-6 \mathrm{hr}$, while the $\mathrm{ED}_{50}$ anti-nociceptive dose $(5 \mathrm{mg} / \mathrm{kg} / \mathrm{i}$.p.) only showing a significant reduction at the $3 \mathrm{hr}$ time point $\left(\mathrm{F}_{(6.152,0.118)}=4.073, p=0.001, p<0.05\right)$ (Fig. $\left.4.3 \mathrm{I}\right)$.

The cumulative weight of faeces over the $6 \mathrm{hr}$ period was then used to assess the overall reduction in G.I transit and to directly compare treatments. This identified significant reductions in total faeces production with morphine, kurkinorin $(10 \mathrm{mg} / \mathrm{kg} / \mathrm{i}$. .p.), and kurkinol $(5 \mathrm{mg} / \mathrm{kg} / \mathrm{i.p.}$.) compared to the vehicle-treated mice $\left(F_{(6,90)}=8.83, p<0.0001, p<0.05\right)$. While at the $E D_{50}$ dose, only morphine showed a significant transit impairment $(p=0.0178)$, while the faecal output of kurkinol and kurkinorin treated animals was not significantly different to the vehicle ( $p>0.05)$ (Fig. $4.3 \mathrm{~L}$ ). 
A)

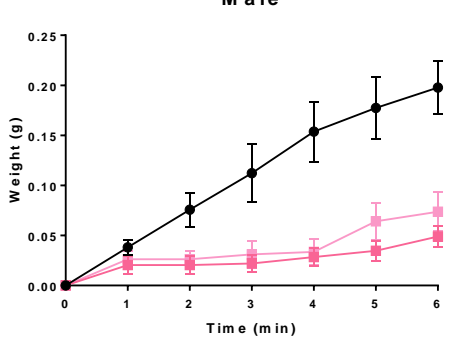

D )

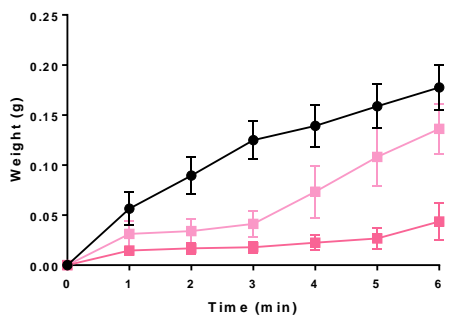

G )

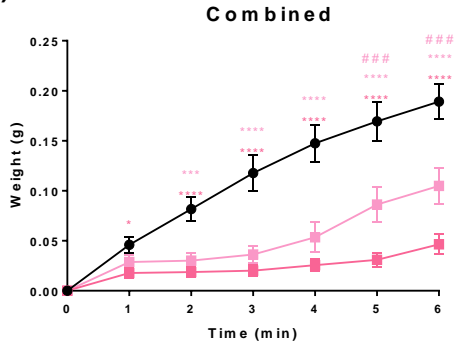

J)

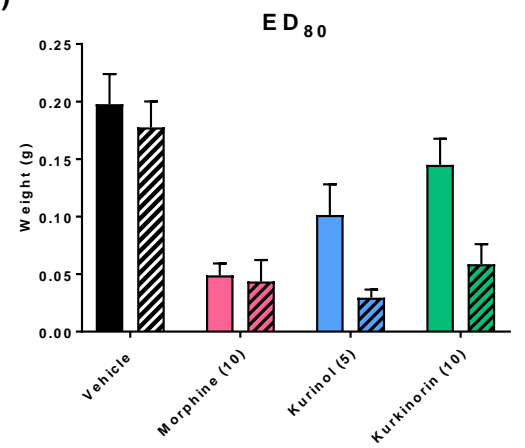

B )

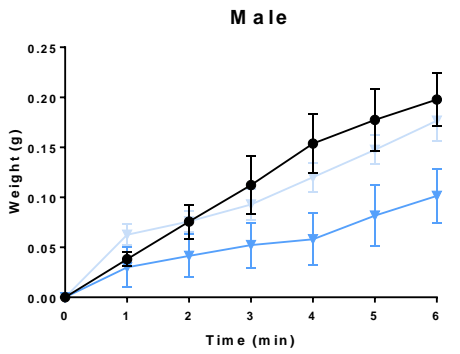

E)

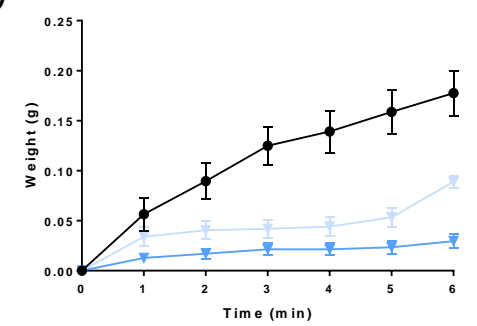

H)

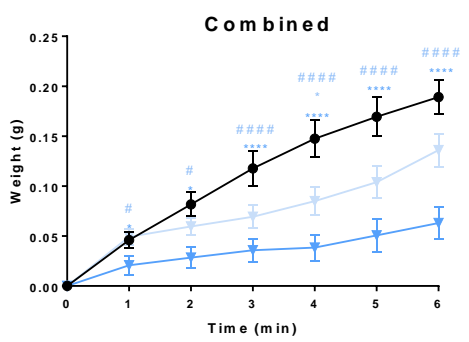

K)

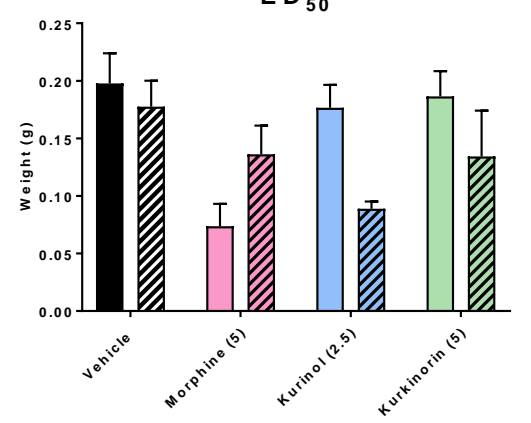

C)

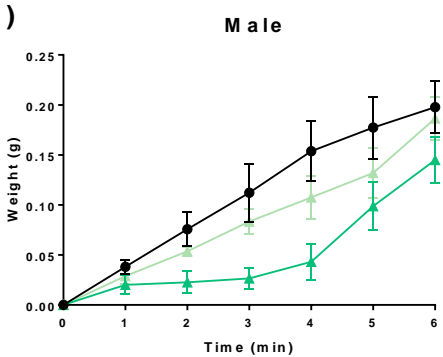

F)

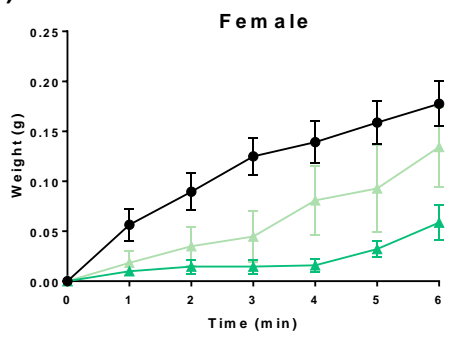

I)

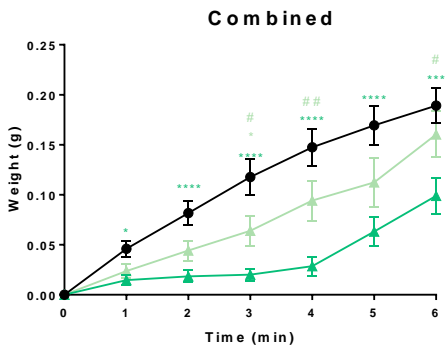

L)

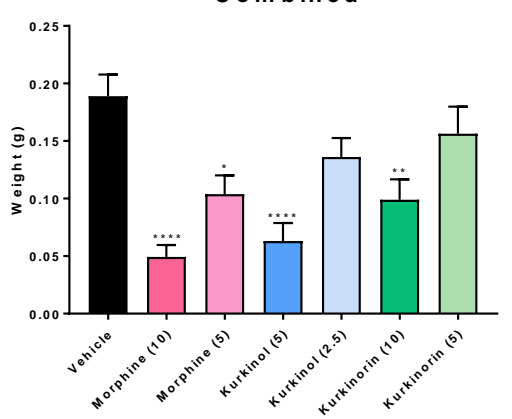

$\rightarrow$ Vehicle

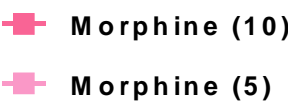

- Kurkinorin (10)
- Kurkinorin (5)

$\neg$ Kurkinol (5)

Male

Temale

\section{Figure 4.3 Sex Does Not Affect the Overall Accumulation of Feces}

(A-C) Faecal accumulation in males treated with morphine, kurkinol, or kurkinorin. (D-F) Faecal accumulation in females treated with morphine, kurkinol, or kurkinorin. (G) When the sexes were combined morphine (10\& $5 \mathrm{mg} / \mathrm{kg} / \mathrm{i}$. p.) induced significant constipation at both doses administered. (H) While kurkinol only induced an effect $5 \mathrm{mg} / \mathrm{kg} / \mathrm{i} . \mathrm{p}$. , (I) as did kurkinorin. (J, K). (L) Total weight of accumulated faeces at the $E D_{50}$ and $E D_{80}$ doses shows no interaction of sex and treatment. (A-J) Threeway mixed ANOVA or (L) One-way repeated measures ANOVA with Bonferroni multiple corrections test, ${ }^{*} p<0.05,{ }^{* *} p<0.01,{ }^{* * *} p<0.001,{ }^{* * * *} p<0.0001$ compared to vehicle, $\# p<0.05, \# \# p<0.01$, \#\#\#\#p<0.0001 compared to $E D_{80}$ dose. Doses in brackets in $\mathrm{mg} / \mathrm{kg} / \mathrm{i}$. . 


\subsubsection{Kurkinorin Does Not Significantly Alter Transit Through the Small Intestine}

Due to the differential control of the large and small intestine we also chose to directly assess the effect of morphine ( 5 or $10 \mathrm{mg} / \mathrm{kg} / \mathrm{i}$. p.), kurkinol ( 2.5 or $5 \mathrm{mg} / \mathrm{kg} / \mathrm{i}$. . .), and kurkinorin ( 5 or 10 $\mathrm{mg} / \mathrm{kg} / \mathrm{i}$.p.) on the transit through the S.I using the charcoal meal assay (Fig. 4.4).

In doing this we found that morphine, kurkinol, and kurkinorin were all unaffected by the sex of the mice $\left(\mathrm{ED}_{50}: F_{(3,54)}=2.85, p=0.045, \mathrm{ED}_{80}: F_{(3,53)}=0.73, p=0.533, p>0.9999\right)$ (Fig 4.4 A). When the data from male and female mice were combined, morphine and kurkinol were found to significantly reduce the $\mathrm{S} . \mathrm{I}$ transit at their respective $\mathrm{ED}_{80}$ doses. With a $62 \%$ reduction following morphine (10 mg/kg/i.p.) administration while kurkinol $(5 \mathrm{mg} / \mathrm{kg} / \mathrm{i}$. . $)$ reduced transit by $46 \%$ compared to vehicle-treated mice $(p<0.0001)$. Interestingly, the $\mathrm{ED}_{80}$ dose of kurkinorin (10 $\mathrm{mg} / \mathrm{kg} / \mathrm{i}$.p.) only produced a $23 \%$ reduction in transit with no significant difference identified to the vehicle-treated mice $\left(F_{(6,98)}=12.19, p<0.0001, p=0.1354\right)$. The induction of $S$.I inhibition was rescued at the $\mathrm{ED}_{50}$ dose of both kurkinol (2.5 mg/kg/i.p.), with the decrease in transit reduced to only $16 \%$ less than that of vehicle-treated animals. Once again, kurkinorin produced a $23 \%$ reduction in $\mathrm{S} . \mathrm{I}$ transit with no difference to vehicle-treated animals at the $E_{50}$ anti-nociceptive dose $(5 \mathrm{mg} / \mathrm{kg} / \mathrm{i}$.p.). Morphine (5 mg/kg/i.p.) was the only drug that inhibited S.I transit at the $E D_{50}$ anti-nociceptive dose, with a $37 \%$ reduction compared to vehicle-treated control mice ( $p=0.0003)$ (Fig. $4.4 \mathrm{~B})$.

This experiment was then repeated in female $\beta$-arr2 ${ }^{-/-}$mice and $\mathrm{Wt}$ littermate controls to assess the role of $\beta$-arrestin2 in S.I transit (Fig $4.4 \mathrm{C}$ ). The treatments were again found to affect intestinal transit in Wt mice $\left(\mathrm{F}_{(7,38)}=11.25, p<0.0001\right)$. The absence of $\beta$-arrestin2 had no significant effect on the transit of the S.I, with no differences identified between the genotypes with any drug ( $p$ $>0.9999)$. However, kurkinorin showed no difference to vehicle controls in Wt mice but did induce a significant impairment in the $\beta$-arr $2^{-/-}$mice $(p=0.027)$, indicating greater constipation in this strain. 
A)

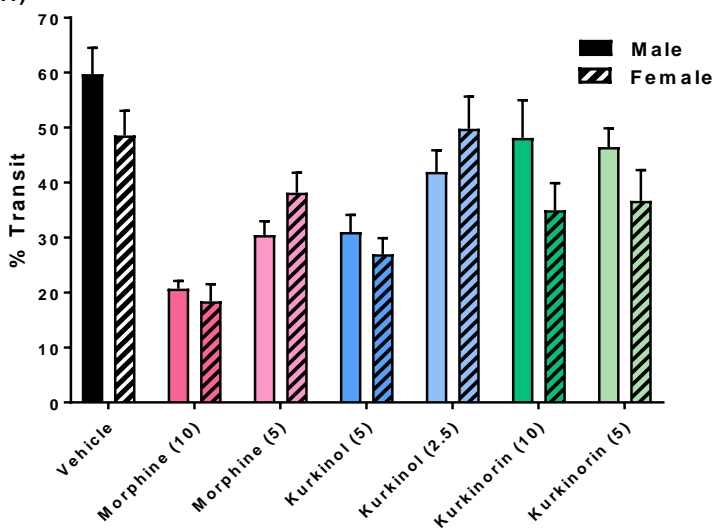

C)

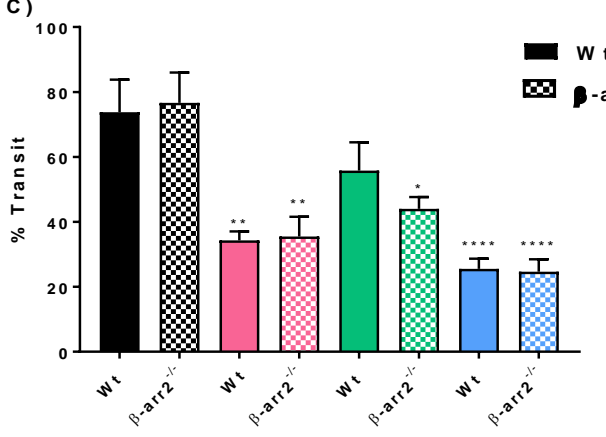

B )

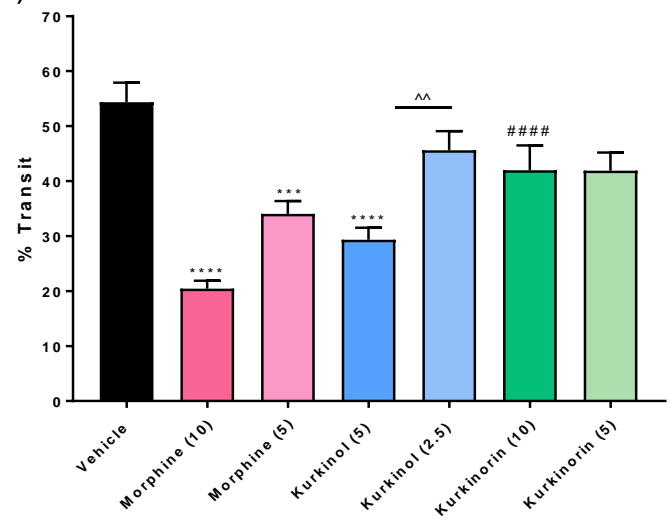

Figure 4.4 Sex Does Not Play a Role in the Inhibition of the Small Intestine

The percent transit (\% transit) of the charcoal meal through the small intestine was used to assess the effects of sex and $\mu$ receptor agonists on S.I inhibition. (A) The inhibition of small intestine transit after administration of morphine (5 or $10 \mathrm{mg} / \mathrm{kg} / \mathrm{i}$. p.), kurkinol (5 or $2.5 \mathrm{mg} / \mathrm{kg} / \mathrm{i}$. .p.), or kurkinorin (5 or 10 $\mathrm{mg} / \mathrm{kg} /$ i.p.) was not impacted by sex. (B) When data male and female mice were combined morphine at both (10 and $5 \mathrm{mg} / \mathrm{kg} / \mathrm{i} . \mathrm{p}$.) significantly reduced transit, while kurkinol only had an effect at 5 $\mathrm{mg} / \mathrm{kg} / \mathrm{i}$.p. Kurkinorin showed no reduction transit distance while showing improvement to morphine. (C) The absence of $\beta$-arrestin2 did not alter transit through the small intestine with the administration of the $\mu$ receptor agonists. $(A)$ Two-way or $(B, C)$ One-way ANOVA with Bonferroni multiple corrections

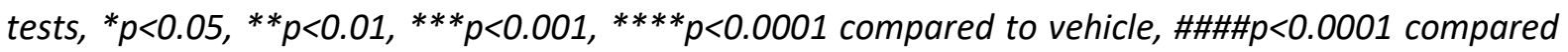
to Morphine, $\wedge^{\wedge} p<0.01$ compared to $E D_{80}$ dose. Data are shown as mean $\pm S E M(N=6-10$ males and 6-8 females per group). Doses in brackets in $\mathrm{mg} / \mathrm{kg} / \mathrm{i}$. p. 


\subsubsection{Urinary Retention was Not Induced by Kurkinol or Kurkinorin}

Urinary retention is induced in $10-84 \%$ of patients receiving mu-opioids post-operatively and often requires catheterization and longer hospital stays (Tomaszewski et al., 2014, Wroblewski et al., 1980). This side effect was assessed in tandem with the faecal accumulation study. Urine samples were collected every hour for 6 hrs post drug administration to assess the effects on urine production.

Over the $6 \mathrm{hr}$ period assessed, the vehicle-treated control male mice were shown to produce a significantly larger total volume of urine compared to their female counterparts. However, no sex differences were identified following administration of the $\mu$ receptor agonists $(F(2,1.295)=6.191, p$ $=0.005, p=0.002$ ) (Fig. $4.5 \mathrm{~J}, \mathrm{~K}$ ). When the data from male and female mice were combined morphine was found to impair urine production for the first hour at the lower $E_{50}$ dose, $5 \mathrm{mg} / \mathrm{kg} / \mathrm{i}$.p. and up to 4 hrs with the higher $E_{80}$ dose of $10 \mathrm{mg} / \mathrm{kg} / \mathrm{i} . \mathrm{p} .(F(6.859,0.577)=2.937, \mathrm{p}=0.008, \mathrm{p}<0.05)$ (Fig. 4.5 $\mathrm{G}$ ). Neither of the G-protein biased drugs, kurkinol or kurkinorin, produced urinary retention in the C57BL/6J mice over time (kurkinol; $F(6.658,0.211)=1.457, p=0.186$, kurkinorin; $F(7.051,0.0050)=$ 0.596, $p=0.758)(F i g ~ 4.5 \mathrm{H}, \mathrm{J})$. When the data from male and female mice were combined, an AUC analysis was performed to allow direct comparisons to be made between the ability of morphine, kurkinol, and kurkinorin to induce urinary retention. This data confirmed that no drug had a significant effect on urinary retention (Fig. $4.5 \mathrm{~L}$ ). 
A)

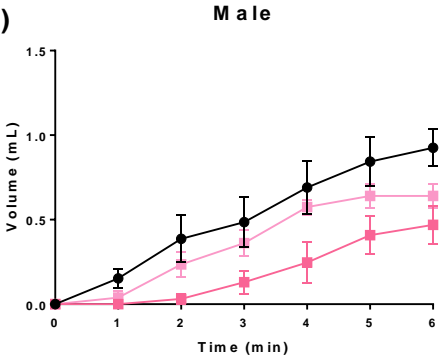

D)

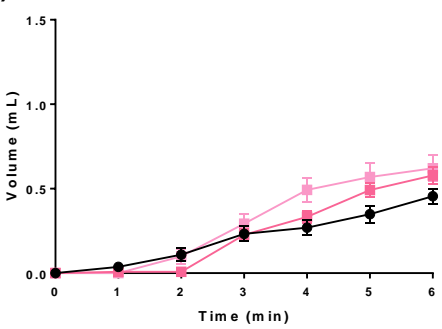

G)

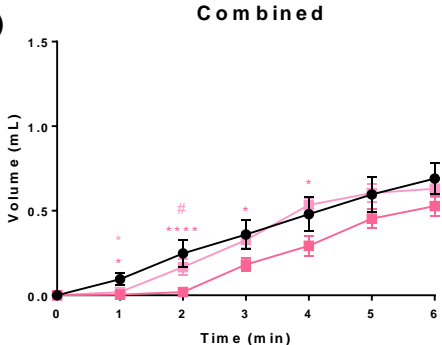

J)

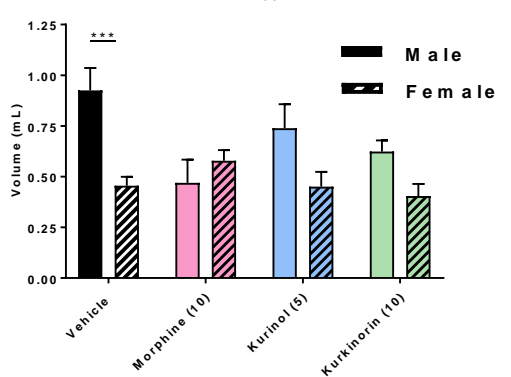

B )

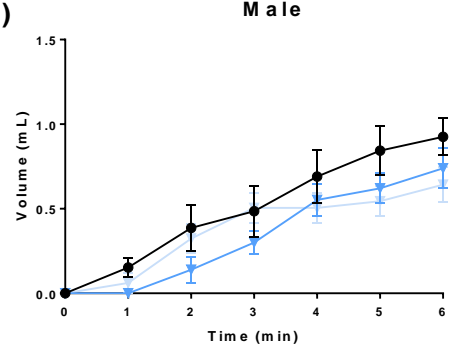

E)

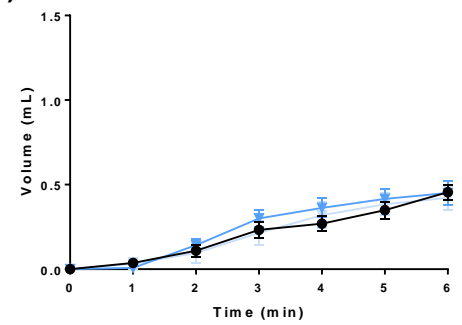

H)

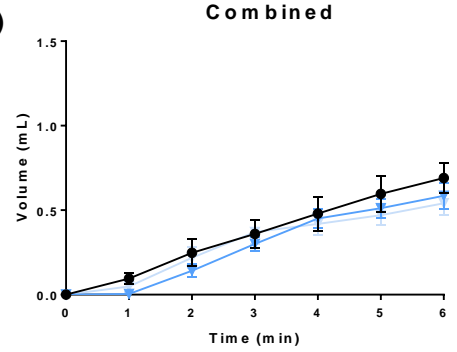

K)

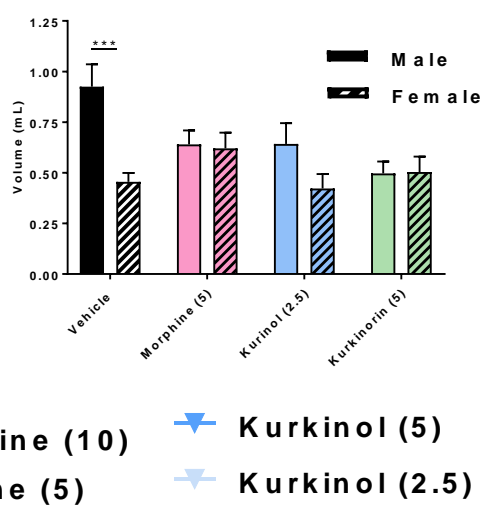

C)

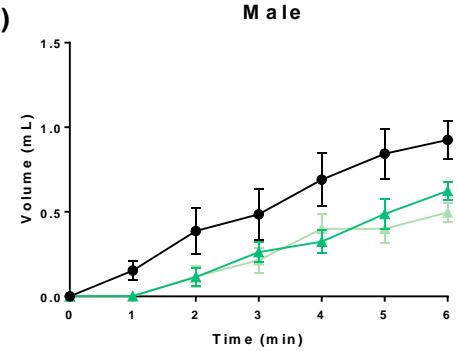

F)

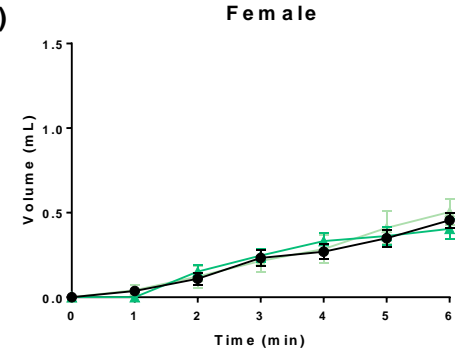

I)

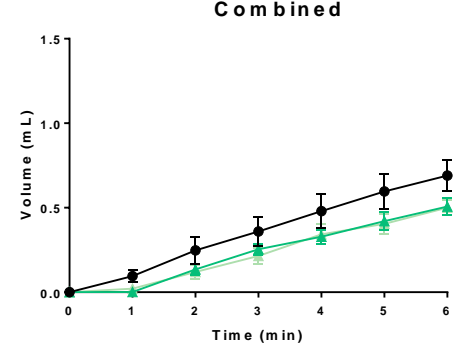

L)

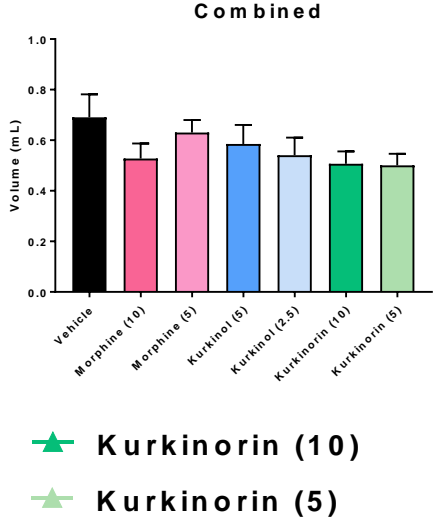

Figure 4.5 Morphine Reduces Urine Output in C57BL/6J Mice

(A-C) Time course effects of morphine, kurkinol, and kurkinorin in males (D-F) Time course effects of morphine, kurkinol, and kurkinorin in females. (G) Morphine $(10 \mathrm{mg} / \mathrm{kg} / \mathrm{i} . \mathrm{p})$ decreased urine output at 2 and $4 \mathrm{hr}$, with a significant dose effect at 4 hrs. $(\mathrm{H}, \mathrm{I})$ Kurkinorin and kurkinol did not affect urine output. (J, K) Only the vehicle showed a main effect of sex on urinary output. (L) Analysis of the total urine produced for each treatment over 6 hrs showed no significant difference compared to vehicle. (A-K) Three-way mixed ANOVA with Bonferroni multiple corrections test, ${ }^{*} p<0.05,{ }^{* * * *} p<0.0001$ compared to vehicle, $\# p<0.05$ compared to $E D_{80}$ dose. (L) One-way repeated measures ANOVA with Bonferroni multiple corrections test, ${ }^{* * *} p<0.001$ male compared to female. Data are shown as mean \pm SEM ( $N=6-8$ males and 6-8 females per group). Doses in brackets in $\mathrm{mg} / \mathrm{kg} / \mathrm{i} . \mathrm{p}$. 


\subsubsection{Respiratory Depression in $\mathrm{C} 57 \mathrm{BL} / 6 \mathrm{~J}$ and $\beta$-arrestin2 Knockout Mice}

To assess the respiratory effects of kurkinol and kurkinorin on respiratory depression wholebody plethysmography in unrestrained mice was performed in male and female $\mathrm{C} 57 \mathrm{BI} / 6 \mathrm{~J} \mathrm{mice}$. The respiratory frequency, tidal volume $\left(\mathrm{V}_{\mathrm{T}}\right)$ (volume of air taken in a single respiratory cycle), and minute volume $\left(V_{\text {min }}\right)$ (total volume of air over a minute) were then calculated from the raw trace and Threeway mixed ANOVAs with Bonferroni multiple corrections used to assess treatment and sex over time.

\subsubsection{Kurkinorin Only Induces Respiratory Depression in Female C57BL/6J Mice}

Kurkinol induced potent reductions in respiratory frequency between $5-60 \mathrm{~min}$, while morphine had a delayed onset with reductions seen between $15-60 \min \left(\mathrm{F}_{(21,0.120)}=3.922, p<0.0001\right.$, $p<0.05$ ) (Fig 4.6 C). This effect was independent of sex, with no differences identified at any time point $\left(\mathrm{F}_{(21,0.052)}=1.716, p=0.027, p>0.05\right)$ (Fig. 4.6 A, B). The sex of the mice also did not interact with kurkinol or morphine to affect $V_{T}$ over time $\left(F_{(11.392,0.377)}=1.754, p=0.063\right)$ (Fig. 4.6 C, D). However, the treatments did influence $V_{T}$ irrespective of sex, with morphine producing a stable decrease in tidal volume from 10 - 60 min while kurkinol had a slightly faster onset of action with effects observed from $5 \min \left(\mathrm{F}_{(11.329,0.401)}=1.866, p=0.045, p<0.05\right)(\mathrm{Fig} .4 .6 \mathrm{~F})$. Decreases in the $\mathrm{V}_{\min }$ were also observed with morphine at $10 \mathrm{~min}$ and between $40-60 \mathrm{~min}$. Again, kurkinol had a faster onset with significant decreases observed between $5-60 \min \left(F_{(12.667,0.768)}=1.806, p=0.038, p<0.05\right)$ (Fig. 4.6 I). With morphine and kurkinol having no significant differences in male and female mice in the $\mathrm{V}_{\text {min }}$ over time $\left(F_{(12.667,0.687)}=1.663, p=0.073\right)$ (Fig. $\left.4.6 \mathrm{G}, \mathrm{H}\right)$. The induction of respiratory depression by kurkinol was shown to occur via the $\mu$ receptor as pre-treatment with $\beta$-FNA ( $5 \mathrm{mg} / \mathrm{kg} / \mathrm{i} . \mathrm{p} ., 24 \mathrm{hrs}$ ) reversed all respiratory depression effects observed $(p<0.05)$ (Fig. $4.6 \mathrm{~A}-\mathrm{I})$.

Initially, kurkinorin also appeared to produce respiratory depression, with a significant decrease in frequency observed between $15-60 \min \left(F_{(21,0.135)}=4.439, p<0.000, p<0.05\right)$ (Fig. 4.7 C). With the sex of the animals not playing a role $(F(21,0.026)=0.840, p=0.670)$ (Fig. 4.7 A, B). An effect sex on kurkinorin induced changes in $\mathrm{V}_{T}$ was seen, with a significant decrease in $\mathrm{V}_{\mathrm{T}}$ observed between 20 - 50 min in female, but not male mice $\left(F_{(13.576,0.243)}=1.842, p=0.036, p<0.05\right)$ (Fig. 4.6 D, E). Interestingly, when the data from male and female mice was combined, no significant decreases in $V_{T}$ were identified. Instead kurkinorin showed improvements compared to morphine from $5-20$ min and 60 min following administration $\left(F_{(13.576,0.294)}=2.229, p=0.009, p<0.05\right)$ (Fig. $\left.4.7 \mathrm{~F}\right)$. Improvements in $V_{\min }$ was also observed following kurkinorin administration compared to morphine from $5-10 \min \left(F_{(12.595,0.527)}=1.780, p=0.03, p<0.05\right)$ (Fig. $\left.4.7 \mathrm{I}\right)$, with no influence of $\operatorname{sex}\left(\mathrm{F}_{(12.595,0.290)}\right.$ $=0.902, p=0.549)($ Fig. $4.7 \mathrm{G}, \mathrm{H})$. Once again, the respiratory effects of kurkinorin were shown to be depended on the $\mu$ receptor with pre-treatment of $\beta$-FNA ( $5 \mathrm{mg} / \mathrm{kg} / \mathrm{i} . \mathrm{p}$.) reducing all respiratory depressive effects $(p<0.05)$ (Fig. 4.7 A-I). 
Other respiratory measures were also assessed after the administration of $\mu$ receptor agonists, including inspiration time, expiration time, total cycle time, and inspiratory volume (Appendix 7.6). Analysis of this data revealed that morphine and kurkinol significantly altered all other respiratory measures (Appendix Fig 7.4). While the expiration time and total cycle time were the only other measures significantly altered following kurkinorin administration (Appendix Fig 7.5). No sex differences were identified for any of the above measures. 
A)

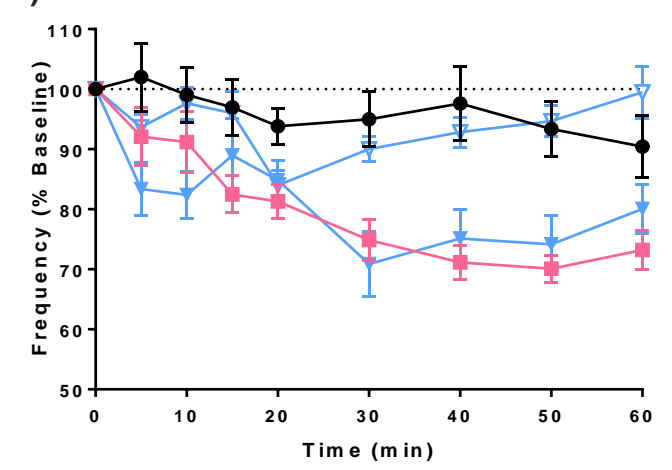

D)

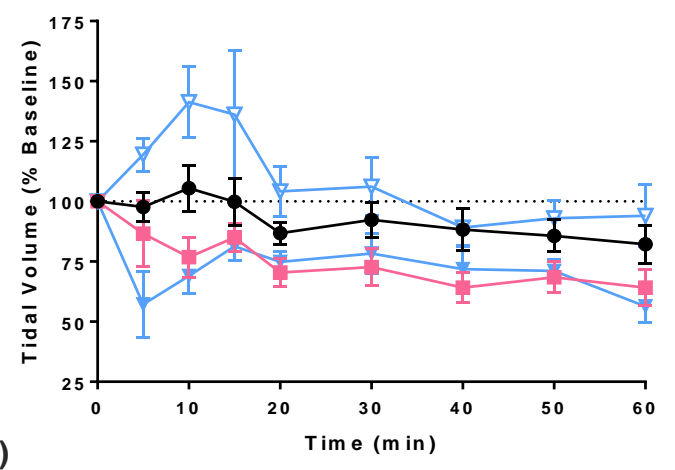

G)

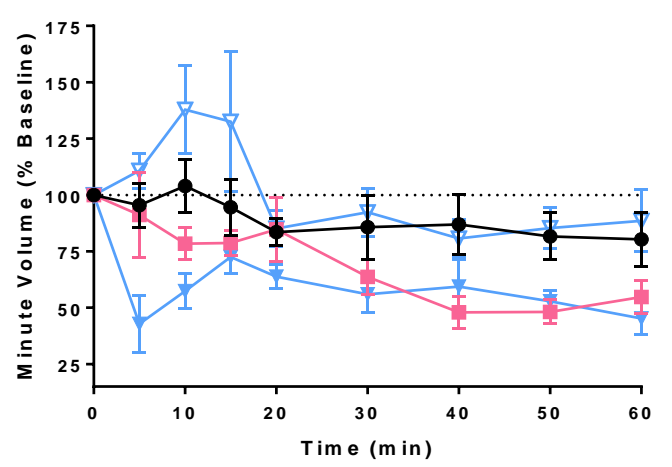

$\rightarrow$ Vehicle
B )

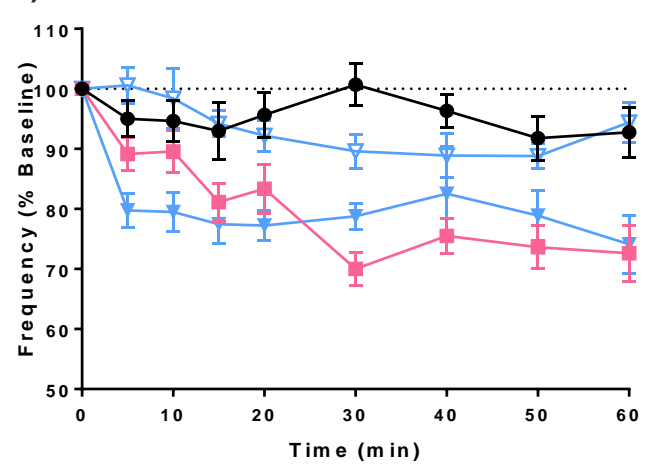

E)

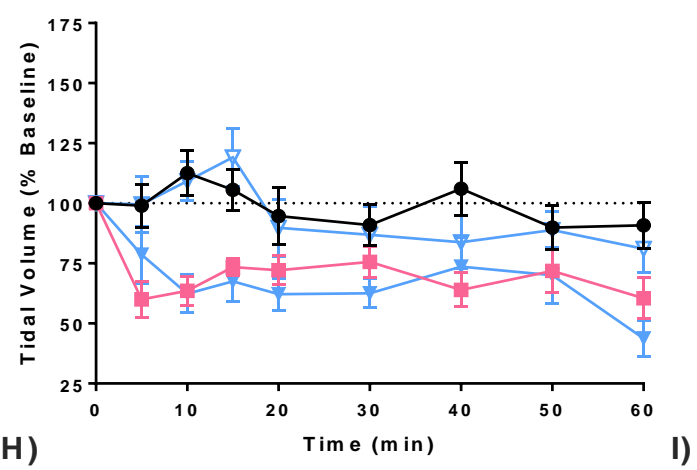

C)

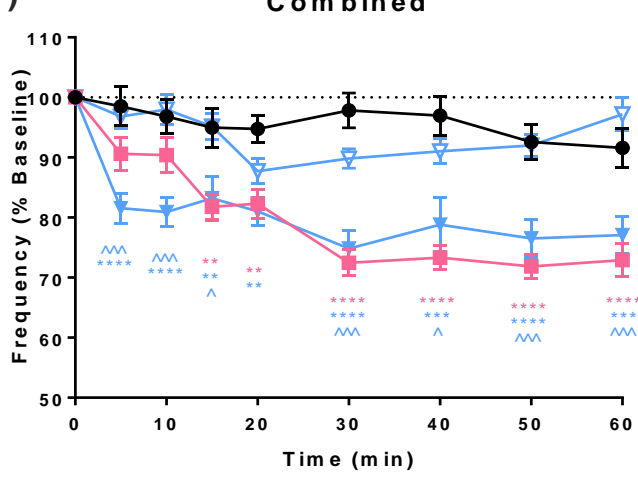

F)

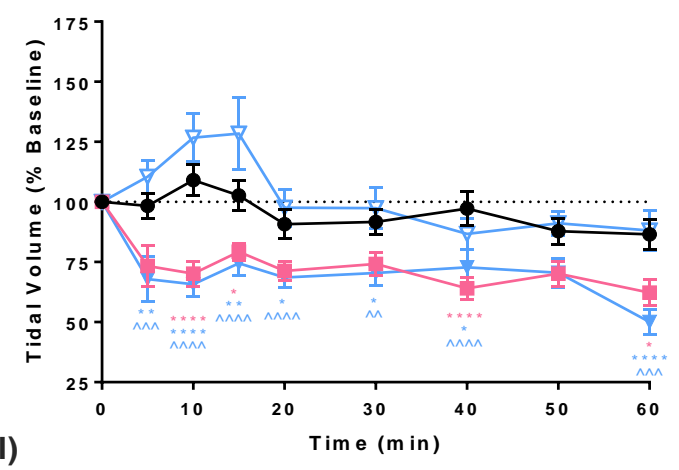

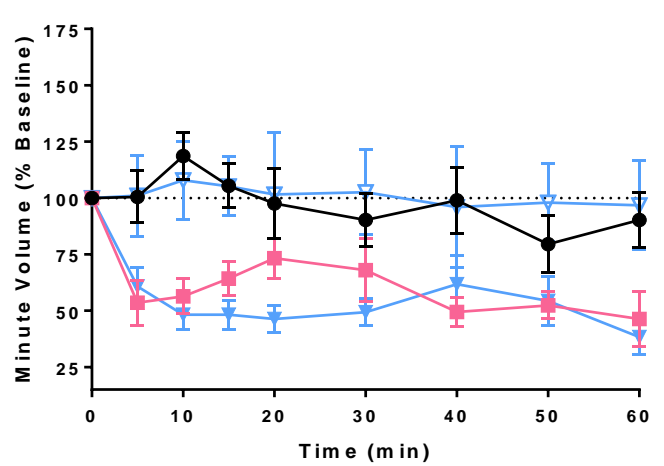

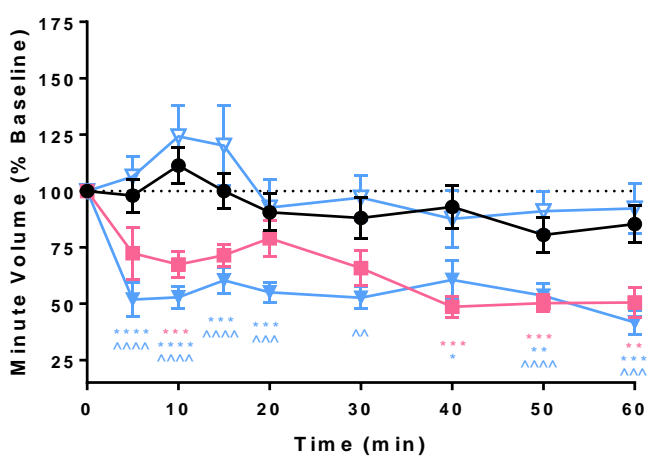

$\rightarrow$ Kurkinol (2.5)

$\nabla$ Kurkinol $(2.5 \mathrm{mg} / \mathrm{kg})+\beta-F N A$ 
Figure 4.6 Kurkinol Induces Potent Respiratory Depression in Male and Female C57BL/6J Mice

(A-B) Sex and treatment interacted to affect respiratory frequency over time, however, no differences between sex $s$ at a given time point were identified. (C) Overall a significant impact on frequency was seen earlier with kurkinol. (D) Effect of kurkinol and morphine on $V_{T}$ in males. (E) Effect of kurkinol and morphine on $V_{T}$ in females. (F) Kurkinol reduced $V_{T}$ measurements between $5-60$ min where morphine had a slower onset and showed effects from 10 -60 min. (G) Effect of kurkinol and morphine on $V_{\min }$ in males. (H) Effect of kurkinol and morphine on $V_{\min }$ in females. (I) Kurkinol significantly decreased $V_{\text {min }}$ over the 60 min time course, where morphine had a continuous reduction from 40-60 min. Three-way mixed ANOVA with Bonferroni multiple corrections test, * $p<0.05,{ }^{* *} p<0.01$, ${ }^{* * *} p<0.001,{ }^{* * * *} p<0.0001$ compared to vehicle, $\wedge^{\wedge} p<0.05, \wedge \wedge p<0.01, \wedge \wedge \wedge p<0.001, \wedge \wedge \wedge \wedge p<0.0001$ compared to $\beta$-FNA pre-treatment. Data are shown as mean \pm SEM ( $N=$ 5-7 males and 5-6 females per group). Doses in brackets in $\mathrm{mg} / \mathrm{kg} / \mathrm{i}$. . 
A)

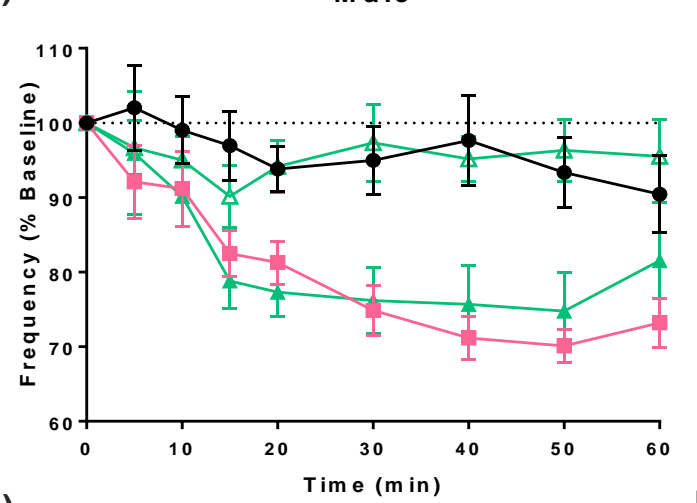

D)

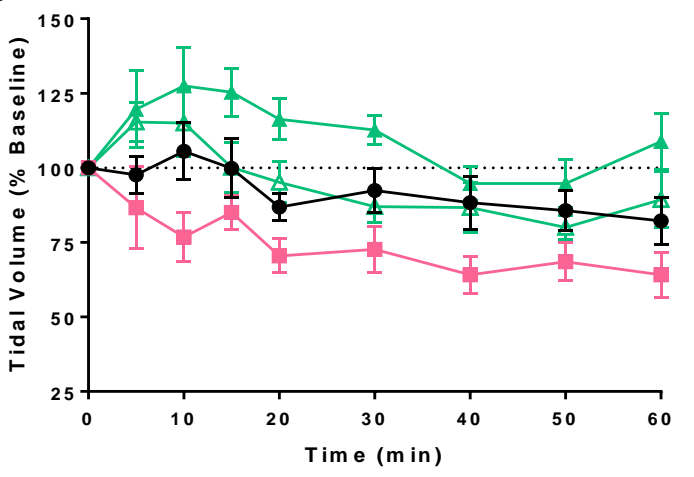

G )

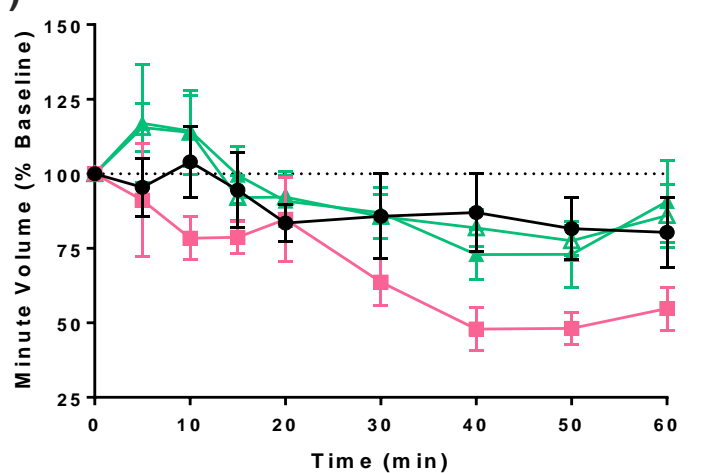

- Vehicle
B)

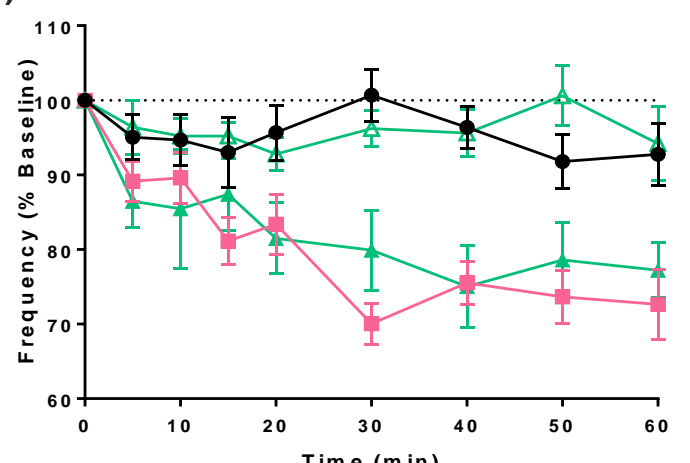

E)

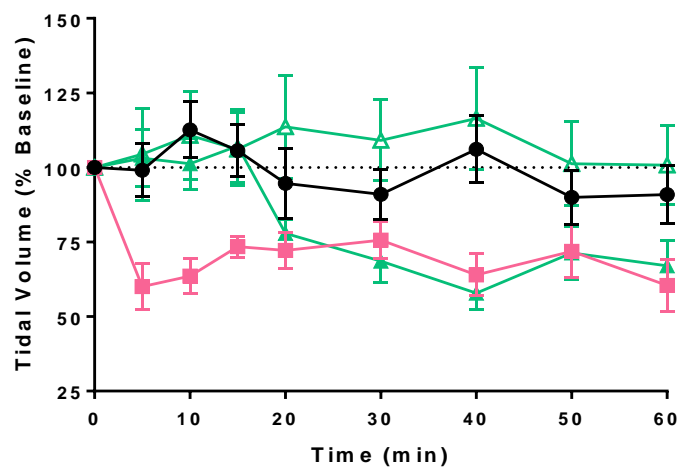

H)

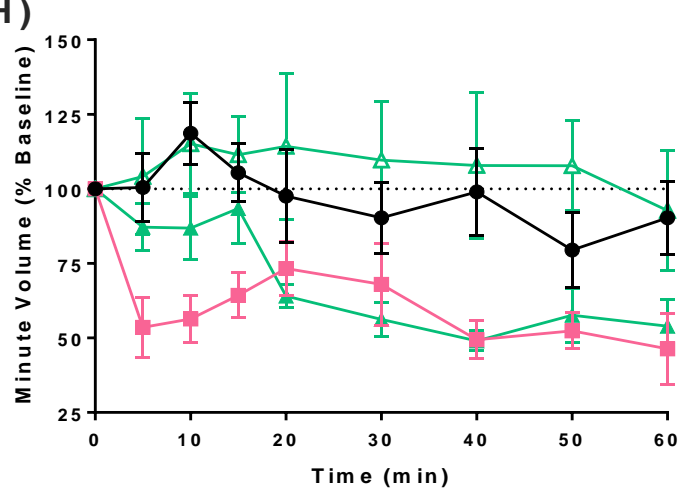

C)

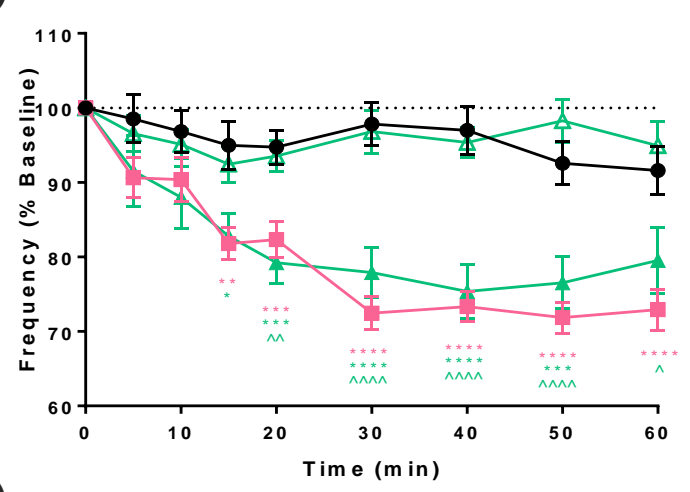

F)

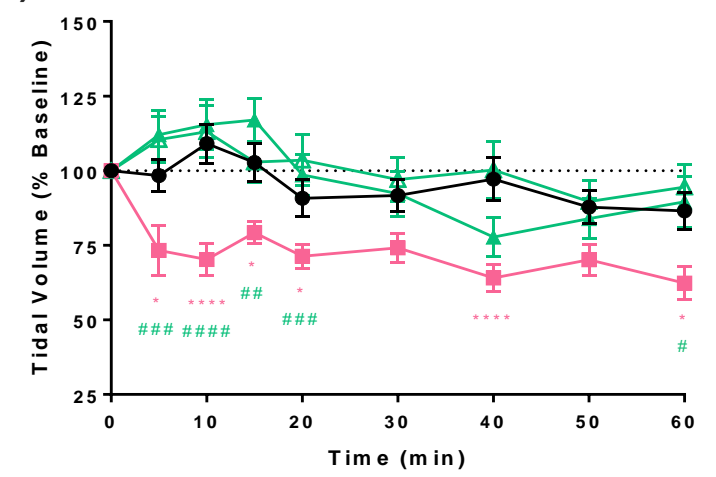

I)

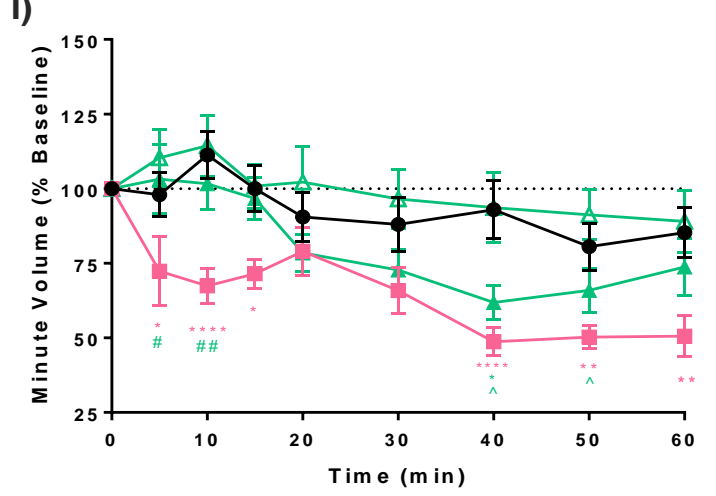

$\triangle$ Kurkinorin $(5)+\beta-F N A$ 
Figure 4.7 Kurkinorin Produces Greater Respiratory Depression in Female C57BL/6J Mice

(A) Effect of kurkinorin and morphine on frequency in male mice. (B) Effect of kurkinorin and morphine on respiratory frequency in female mice. (C) A significant impact on frequency was seen from $15-60 \mathrm{~min}$ with kurkinorin and morphine. (D-E) Kurkinorin induced a greater reduction in $V_{T}$ between $20-50$ min in female mice. (F) Overall kurkinorin did not affect $V_{T}$ where morphine showed reductions between $10-60 \mathrm{~min}$. (G) Effect of kurkinorin and morphine on $V_{\text {min }}$ in males. (H) Effect of kurkinorin and morphine on $V_{\min }$ in females. (I) Kurkinorin significantly decreased $V_{\min }$ at 40 min, where morphine continuous reductions from $40-60$ min. Three-way mixed ANOVA with Bonferroni multiple corrections test, ${ }^{*} p<0.05,{ }^{* *} p<0.01,{ }^{* * *} p<0.001,{ }^{* * * *} p<0.0001$ compared to vehicle, $\# p<0.05, \# \# p<0.01, \# \# \# p<0.001$ compared to morphine, $\wedge^{\wedge} p<0.05, \wedge \wedge p<0.01, \wedge \wedge \wedge \wedge p<0.0001$ compared to $\beta$-FNA pre-treatment. Data are shown as mean \pm SEM ( $N=$ 5-7 males and 5-6 females per group). Doses in brackets in $\mathrm{mg} / \mathrm{kg} / \mathrm{i} . \mathrm{p}$. 


\subsubsection{Respiratory Depression Occurs in $\beta$-arrestin2 Knockout Mice}

The role of $\beta$-arrestin 2 in OIRD was further investigated using whole-body plethysmography. With respiratory frequency, $\mathrm{V}_{\mathrm{T}}$, and $\mathrm{V}_{\text {min }}$ evaluated following administration of morphine, kurkinol, or kurkinorin in male $\beta$-arr $2^{-/-}$mice and $\mathrm{Wt}$ littermate controls. Before statistical analysis, vehicle-treated mice from both genotypes were compared. With no significant differences observed between $\beta$-arr2 ${ }^{1-}$ and $\mathrm{Wt}$ mice for respiratory frequency, $\mathrm{V}_{\mathrm{T}}$, or $\mathrm{V}_{\text {min. }}$. Based on this data, all vehicle-treated mice were combined (Fig 4.8, Fig 4.9) (Appendix 7.7).

Upon the administration of morphine and kurkinol, no significant change in respiratory frequency was identified over time (morphine; $\mathrm{F}_{(16,176)}=3.258, p=0.0613$, kurkinol; $\mathrm{F}_{(16,192)}=1.376, p$ $=0.1570$ ) (Fig. $4.8 \mathrm{~A}, \mathrm{D})$. While kurkinorin was shown to significantly reduce the respiratory frequency at $5 \mathrm{~min}$ and between $30-60 \mathrm{~min}$ for both genotypes, with an average decrease of $13 \%$ in the $\mathrm{Wt}$ littermate controls and $15 \%$ decrease in the $\beta$-arr $2^{-/}$mice over the 60 min period assessed $\left(F_{(16,176)}=\right.$ 1.819, $p=0.0318, p<0.05$ ) (Fig $4.8 \mathrm{G}$ ). The $\mathrm{V}_{\mathrm{T}}$ following the administration of morphine or kurkinol was found to have no significant effect over time despite an average decrease of $23 \%$ and $13 \%$ from vehicle-treated mice over the $60 \mathrm{~min}$ time course for morphine and kurkinol respectively (morphine; $\mathrm{F}_{(16,168)}=1.252, p=0.2342$, kurkinol; $\mathrm{F}_{(8,200)}=1.03, p=0.4271$ ) (Fig $4.8 \mathrm{~B}, \mathrm{E}$ ). Kurkinorin showed the same effect in male $\beta$-arr ${ }^{-/}$mice as it did in C57BL/6J mice, with no change in $V_{T}$ over 60 min $\left(F_{(16,168)}\right.$ $=0.7791, p=0.7076)\left(\mathrm{Fig} 4.7 \mathrm{H}\right.$ ). Despite an average decrease of $26 \%$ in $\mathrm{V}_{\min }$ over the $60 \mathrm{~min}$ period assessed no statistically significant effect was identified following morphine administration $\left(F_{(16,184)}=\right.$ $0.9967, p=0.4623$ ) (Fig $4.8 \mathrm{C}$ ). Kurkinorin also showed no change over time as expected $\left(\mathrm{F}_{(16,176)}=\right.$ $0.799, p=0.6855$ ) (Fig 4.8 I). On the other hand, kurkinol induced reduction in $V_{\min }$ between $5-60$ min, with an average decrease of $35 \%$ compared to the vehicle-treated mice. Interestingly, in the $\beta$ arr ${ }^{-1-}$ mice, a $29 \%$ decrease was observed at $40 \mathrm{~min}$, while showing an average $42 \%$ improvement in the $V_{\text {min }}$ reduction between $10-15$ min compared to the $\mathrm{Wt}$ littermate controls (Fig 4.8 F). Other respiratory measured assessed (inspiration time, expiration time, total cycle time, and inspiratory volume) also showed no significant effect of genotype following morphine, kurkinol, or kurkinorin administration (Appendix Fig 7.7).

Thus far, these results are inconclusive regarding the role of $\beta$-arrestin2 in the induction of respiratory depression. This is largely due to the lack of statistical significance identified, due to low group sizes. However, the data appears to show a non-significant trend towards reduced respiratory depression following kurkinol administration in the absence of $\beta$-arrestin2, while morphine and kurkinorin appear unaffected (Fig. 4.7). In order to further assess the mechanism of OIRD an AUC analysis was performed to assess the main effect on the 3 respiratory measures. In doing so, we were able to identify no effect of genotype on any morphine-induced changes respiratory measures (frequency; $\mathrm{F}_{(2,22)}=5.714, p=0.0099, p>0.05, \mathrm{~V}_{\mathrm{T}} ; \mathrm{F}_{(2,21)}=7.033, p=0.0046, p>0.05, \mathrm{~V}_{\text {min }} ; \mathrm{F}_{(2,23)}=$ 5.589, $p=0.0105, p<0.05$ ) (Fig 4.9 A, B, C). Similarly, the absence of $\beta$-arrestin2 did not alter the 
respiratory changes induced after the administration of kurkinol (respiratory frequency; $F_{(2,22)}=7.83$, $\left.p=0.0027, p>0.05, \mathrm{~V}_{\mathrm{T}} ; \mathrm{F}_{(2,21)}=1.803, p=0.1895, \mathrm{~V}_{\min } ; \mathrm{F}_{(2,22)}=1.302, p=0.2922\right)$ (Fig. $\left.4.9 \mathrm{G}, \mathrm{H}, \mathrm{I}\right)$. While decreases in respiratory frequency and $V_{\min }$ were not altered by genotype following kurkinol administration (frequency; $\mathrm{F}_{(2,24)}=6.714, p=0.0048, p>0.05, \mathrm{~V}_{\min } ;(2,26)=15.85, p<0.0001$ ) (Fig $4.9 \mathrm{D}$, $F)$, an effect was seen for $V_{T}$ with an $11 \%$ improvement observed in the $\beta$-arr2 ${ }^{-1-}$ mice $\left(F_{(2,25)}=7.137, p\right.$ $=0.0035, p<0.05)($ Fig $4.9 \mathrm{H})$. This result indicates that $\beta$-arrestin2 may play a role in OIRD of highly selective and potent $\mu$ receptor agonists such as kurkinol. 
A)

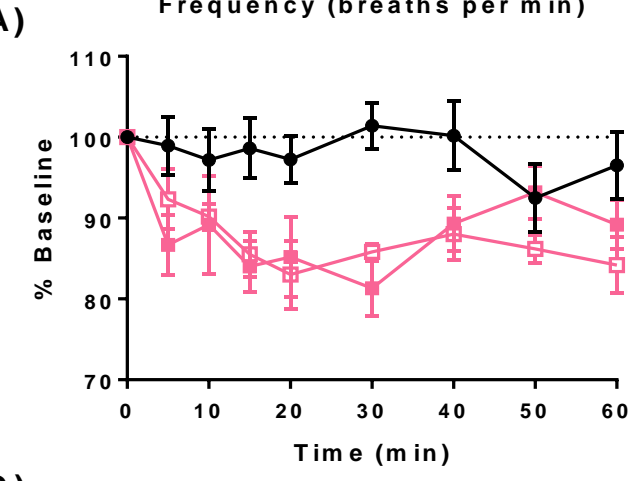

D)

$\stackrel{\leftrightarrow}{\$}$

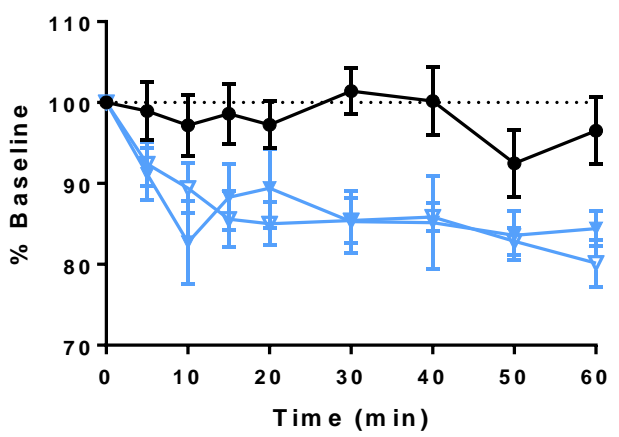

G )

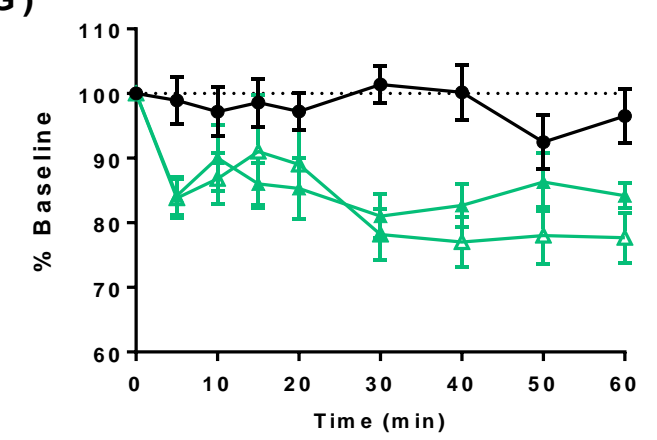

$\rightarrow$ Vehicle

- Morphine W t

$\square$ Morphine -arr $2^{-/-}$
B )

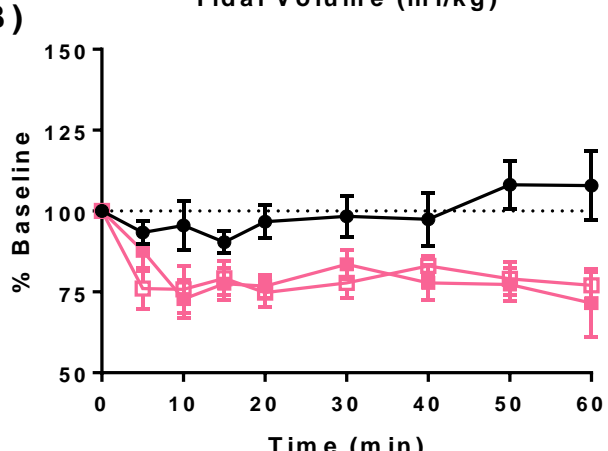

E)

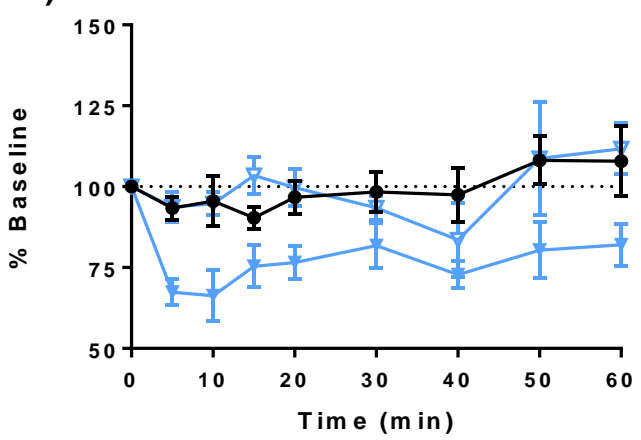

H)

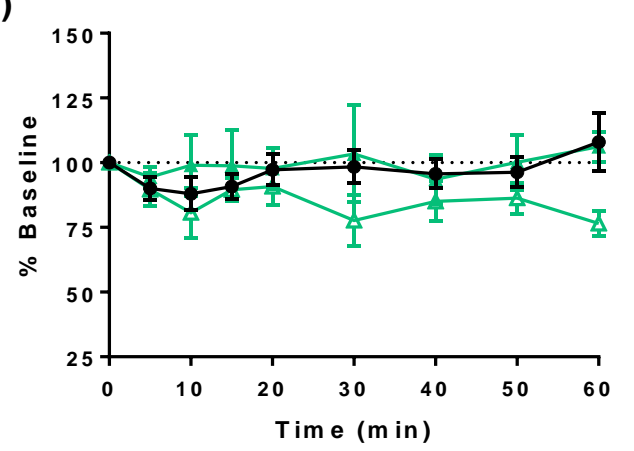

$\rightarrow$ Kurkinol W t

$\rightarrow$ Kurkinol -arr2 $2^{-/-}$
C) Minute volume $(\mathrm{m} \mathrm{I} / \mathrm{m} \mathrm{in} / \mathrm{kg})$

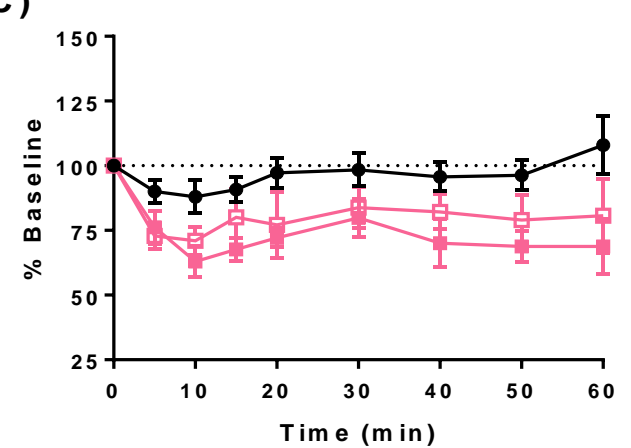

F )

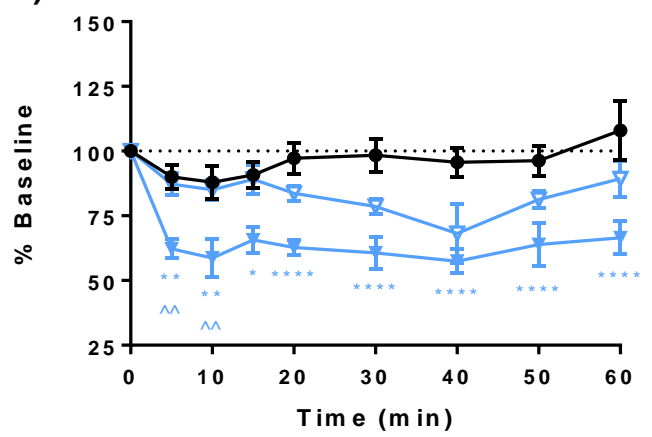

I)

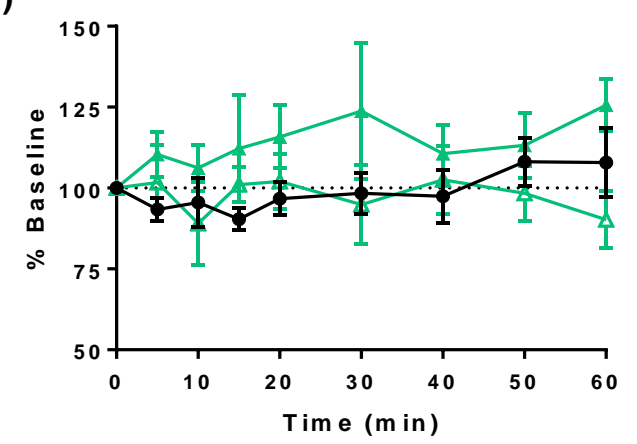

- Kurkinorin $\mathbf{W t}$

$\triangle$ Kurkinorin -arr $2^{-/-}$ 
Figure 4.8 B-arrestin2 Knock Out Mice Show a Small Statistical Improvement in Respiratory Measures Following Kurkinol Administration

Whole-body Plethysmography was repeated in $\beta$-arr $2^{-}$mice and Wt littermate controls to further assess the role of $\beta$-arrestin2 in the induction of OIRD.

Morphine-induced a change in (A) respiratory frequency (B) $V_{T}$ and $(C) V_{\min }$ over time with no effect of genotype on any measure. (D) Kurkinol decreased the respiratory frequency and (E) $V_{T}$ to the same level in $\beta$-arr ${ }^{-1-}$ mice and $W t$ littermate controls. (F) However, $V_{\min }$ was decreased to a greater extent in the Wt mice which was partially rescued in the $\beta$-arr ${ }^{\%}$ mice. (G) Kurkinorin induced reduced respiratory frequency over time, independent of the genotype. Neither (H) $V_{T}$ or (I) $V_{\min }$ were affected in Wt or $\beta$-arr2 ${ }^{-/}$mice who received kurkinorin. Two-way repeated measures ANOVA with Bonferroni multiple corrections test, ${ }^{*} p<0.05,{ }^{* *} p<0.01,{ }^{* * *} p<0.001,{ }^{* * * *} p<0.0001$ compared to vehicle, ${ }^{\wedge} p<0.01$ compared to $\beta$-arr2 ${ }^{-1}$ mice. Data are shown as mean $\pm S E M$ ( $N=6$-7 per group). Doses in brackets in $\mathrm{mg} / \mathrm{kg} / \mathrm{i} . \mathrm{p}$. 

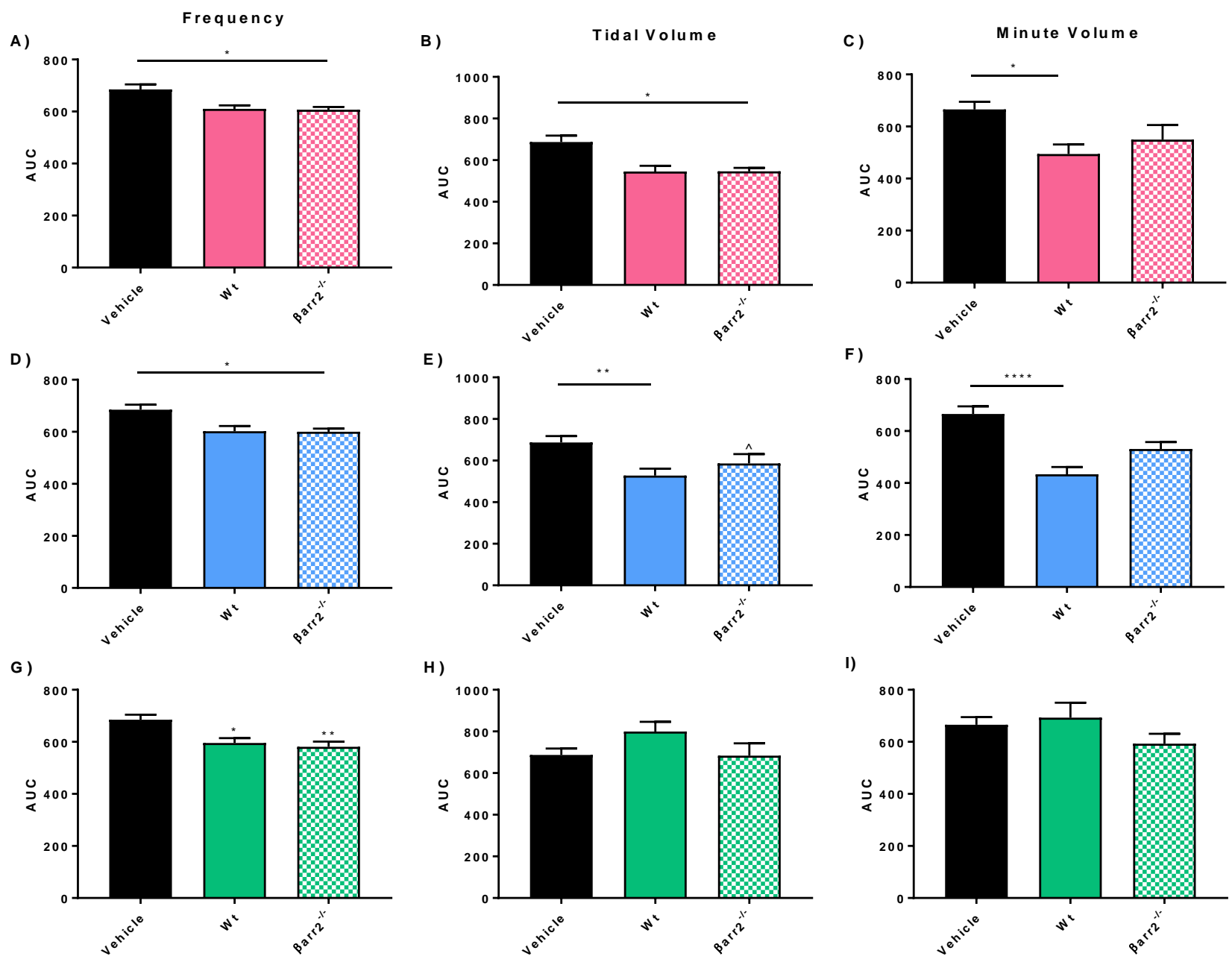

$\square$ Morphine (5) $\square$ Kurkinol (2.5) $\square$ Kurkinorin (5)

Figure 4.9 Kurkinol Induced Changes in Tidal Volume were Reduced in $\beta$-arrestin2 Knock Out Mice

AUC analysis was conducted on respiratory frequency, $V_{T}$, and $V_{\min }$ measurements to assess the overall respiratory effects of morphine, kurkinol, and kurkinorin, and the role of $\beta$-arrestin2 in these effects. (A) Respiratory frequency and $(B) V_{T}$ were both decreased over the 60 min recording, with differences between $W t$ and $\beta$-arr $2^{\%}$ mice. (C) $V_{\text {min }}$ was significantly reduced in the Wt but not $\beta$-arr $2^{\%}$ mice. (D) kurkinol reduced the respiratory frequency independent of genotype, however, both (E) $V_{T}$ and $(\boldsymbol{F}) V_{\min }$ were rescued in the $\beta$-arr $2^{-/}$mice. (G) Respiratory frequency was significantly decreased in both genotypes upon administration of kurkinorin, with no significant changes in $(\boldsymbol{H}) V_{T}$ and $(\boldsymbol{I}) V_{\min }$ across the 60 min assessed. One-way Mixed ANOVA with Bonferroni multiple corrections test, ${ }^{*} p<0.05$, ${ }^{* *} p<0.01, * * * * p<0.0001$ compared to vehicle, ${ }^{\wedge} p<0.05$ compared to $\beta$-arr ${ }^{\%}$ mice. Data are shown as mean \pm SEM ( $N=6-7$ males per group). Doses in brackets in $\mathrm{mg} / \mathrm{kg} / \mathrm{i}$. p. 


\subsubsection{Kurkinorin has an Improved Side Effect Z-score in C57BL/6J Mice}

Generation of the Z-scores was used to evaluate side effects following administration of morphine, kurkinol, and kurkinorin. The directionality of the Z-scores indicates the level to which the side effect is induced, with a value of $>0$ indicating no difference to the vehicle and a value $<0$ indicating a reduction compared to vehicle. In male mice, kurkinorin had improved rotarod, urinary retention, and S.I transit scores, with an overall increase in side effect score (Z-score $=-3.14)$ compared to morphine $(Z$-score $=-4.87)$ and kurkinol $(Z$-score $=-4.24)$ confirming the improved safety profile of this compound (Fig. 4.10 A). However, in female mice kurkinorin has a Z-score of -4.41, indicating the same level of side effects are induced as following morphine (Z-score $=-4.38)$ and kurkinol (Z-score $=$ -4.36) administration, reinforcing the sex differences of kurkinorin (Fig. 4.10 B). The Z-scores generated from male and female data was then combined to assess the overall Z-scores. This revealed that despite the induction of side effects in females, there was still an overall improvement in conditioned place preference, S.I transit, rotarod, and tolerance to acetone in CINP following kurkinorin administration (Fig. $4.10 \mathrm{C}$ ), with an improved side effect score (Z-score $=-2.96$ ) compared to morphine $(Z$-score $=-3.99)$ and kurkinol $(Z$-score $=-3.59)$. 


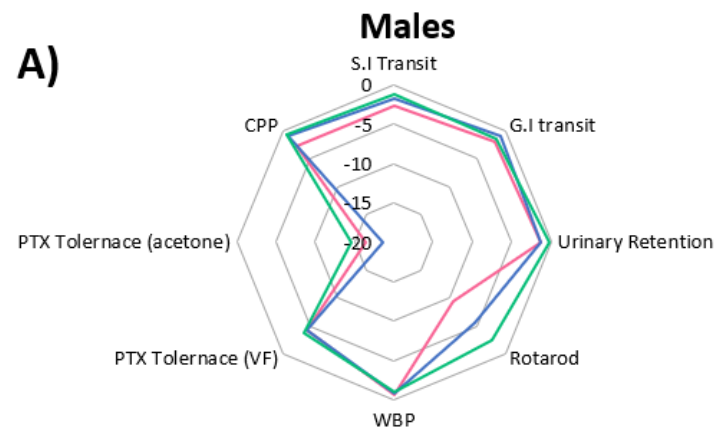

B)

\section{Females}

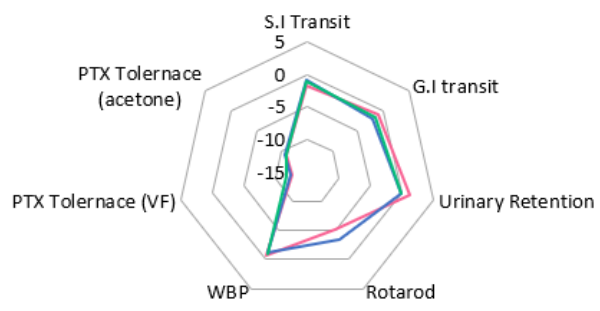

C) Combined

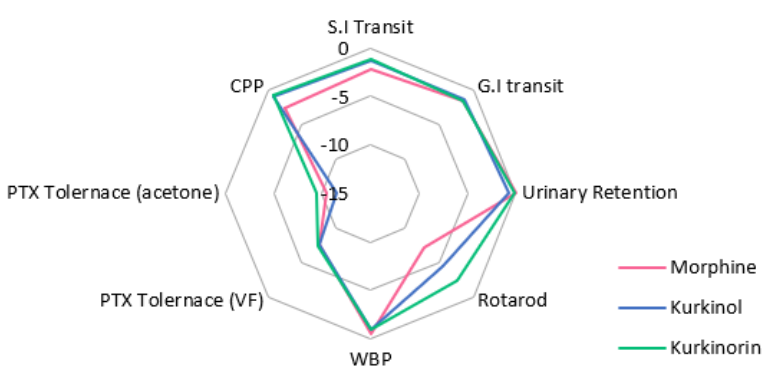

Figure 4.10 Kurkinorin Has an Improved Z-score Profile Compared to Morphine

Kurkinol has a reduced side effect Z-score in $(\boldsymbol{A})$ male mice and $(\boldsymbol{C})$ when the sexes are combined. (B) However, these side effects are produced to the same extent in female mice. Negative Z-scores indicate strong side effect induction. Therefore, the closer to the centre of the radial plot the greater the induction.

\section{Abbreviations:}

$C P P=$ conditioned place preference $W B P=$ whole-body plethysmography

$P T X=$ paclitaxel

G.I = gastrointestinal

S.I = small intestine 


\subsection{Discussion}

Currently, available $\mu$ receptor agonists develop tolerance, an effect that limits the effective treatment of chronic pain (Martins et al., 2015). The induction of dependence, addiction, and respiratory depression are of concern due to high rates of opioid-induced overdose and increasing illicit use of prescription opioids (Compton et al., 2006, Martins et al., 2015). Initial studies showed these effects were reduced with the removal of $\beta$-arrestin2 (Bohn et al., 2000, Raehal et al., 2005). However, this has not been replicated pharmacologically (Table 4.3) or in the $\beta$-arr $2^{-/-}$line. With Gprotein biased agonists, such as TRV130 and PZM21 still inducing respiratory depression and abuse liability in preclinical behavioural assays (Altarifi et al., 2017, Austin Zamarripa et al., 2018, Hill et al., 2018, Kudla et al., 2019). Moreover, the only other published work on the $\beta$-arr ${ }^{-/}$line found the same level of constipation and respiratory depression as in Wt littermate controls (Kliewer et al., 2019, 2020). Therefore, we aimed to investigate the side effect profile of kurkinol and kurkinorin, novel compounds with varying bias factors, in $\mathrm{Wt} C 57 \mathrm{BL} / 6 \mathrm{~J}$ mice and $\beta$-arr $2^{-1-}$ mice.

\subsubsection{Improved Motor Coordination of Kurkinol Not Correlated to G- Protein Bias}

The effect of G-protein bias on the induction of motor coordination impairment was assessed using the accelerating rotarod assay. No effect of sex on motor coordination was observed for kurkinol, however, morphine ( $5 \mathrm{mg} / \mathrm{kg} / \mathrm{i} . \mathrm{p}$.) was shown to affect males more than females (Table 4.4). When the sexes were combined, kurkinol induced the same level of motor coordination impairment as morphine with a shorter duration of action (45 $\mathrm{min}$ vs $180 \mathrm{~min}$ ). The reduced motor deficits observed reflect the reduced duration of effects in the tail-flick assay. This indicates that the shorter half-life is responsible for the reduced motor coordination impairment, not the G-protein bias. Interestingly, previous work has shown that the less G-protein bias, kurkinorin, only induced significant motor coordination impairments $15 \mathrm{~min}$ post-administration and had an overall improvement compared to morphine in the same assay (Crowley et al., 2016) (Table 4.2). The previously developed G-protein biased $\mu$ receptor agonists buprenorphine and TRV130 have also both been shown to induce motor coordination deficits to the same extent as morphine, with PZM21 the only compound to no observable locomotor effects (open field in mice) (Table 4.3).

The data we have generated in this study and that from the literature suggests that G-protein bias does not reduce the motor coordination impairment induced by $\mu$ receptor activation and is in direct opposition to what is stated under the theory of biased agonism. Moreover, it does not reflect what has been shown in $\beta$-arr2 ${ }^{--}$mice (Bohn et al., 2003). In the original study by Bohn et al, (2003) the measure of locomotion used was the open field assay, this assesses the induction of spontaneous locomotion/hyperactivity. The number of beam breaks over $180 \mathrm{~min}$ following morphine (10 $\mathrm{mg} / \mathrm{kg} / \mathrm{s}$.c.) administration was observed to be lower in the $\beta$-arr2 ${ }^{-/-}$mice compared to Wt littermate 
controls, with dose dependent decreases also observed at doses as high as $40 \mathrm{mg} / \mathrm{kg} / \mathrm{s} . \mathrm{c}$. Comparatively, the rotarod experiment is a measure of fine motor control and is, therefore, a more sensitive measure. This may explain why our highly G-protein biased $\mu$ receptor agonist, kurkinol, shows a strong reduction in fine motor control in the rotarod assay. Indicating that while an overall reduction in spontaneous locomotion occurs, a severe deficit in fine motor control may be present. Furthermore, the use of a global $\beta$-arr2 ${ }^{\%-}$ mice mouse alters multiple GPCR signalling pathways, particularly signalling through the dopamine receptors. Urs et al. (2011) investigated this potential explanation for the decrease in spontaneous locomotion in $\beta$-arr $2^{-/-}$mice. In doing so they were able to show that the formation of $\beta$-arr2-ERK1/2 signalling complexes at the D1 receptor, but not the $\mu$ receptor, was impaired in the $\beta$-arr $2^{-/-}$mice (Urs et al., 2011). While more recently, the D2 receptor has been shown to drive locomotor activity in the absence of G-protein signalling as measured in the open field assay in mice, further indicating that the reduction in locomotion in the $\beta$-arr $2^{-/}$mice is due to the action at the post-synaptic dopamine receptors (Donthamsetti et al., 2020). If true, $\mu$ receptor G-protein biased agonists would never elevate the locomotor and coordination impairment induced by $\mu$ receptor mediated dopamine release.

To fully elucidate the role of $\beta$-arrestin 2 recruitment at the $\mu$ receptor vs the D1, D2, and D3 receptors the level of fine motor coordination and spontaneous locomotion needs to be measured with the selective knockdown of $\beta$-arrestin2 in the neuronal populations that express each receptor. To achieve this a Cre mouse for each receptor would need to be generated and crossed with the floxed $\beta$-arr2 ${ }^{f / f}$ mouse line (Urs et al., 2016, Zhu et al., 2017) to generate neuronal-specific knockdown of $\beta$ arrestin2. 


\begin{tabular}{|c|c|c|c|c|c|c|c|c|}
\hline & \multirow{2}{*}{ Rotarod } & \multirow{2}{*}{$\begin{array}{c}\text { Faecal } \\
\text { accumulation }\end{array}$} & \multicolumn{2}{|c|}{ Charcoal Meal Assay } & \multirow{2}{*}{$\begin{array}{c}\text { Urinary } \\
\text { Retention }\end{array}$} & \multicolumn{2}{|c|}{ Whole-body Plethysmography } & \multirow{2}{*}{$\begin{array}{c}{ }^{* *} \text { Conditioned } \\
\text { place } \\
\text { preference }\end{array}$} \\
\hline & & & $\mathbf{W t}$ & $\beta$-arr $2^{-/-}$ & & Wt & ${ }^{* *} \beta$-arr2 ${ }^{--}$ & \\
\hline Morphine & $\begin{array}{l}\text { 5; } 180 \text { min } \\
\text { 10; } 180 \mathrm{~min}\end{array}$ & $\begin{array}{l}\text { 5; } 6 \text { hrs } \\
\text { 10; } 6 \text { hrs }\end{array}$ & $\begin{array}{l}\text { 5; decreased } \\
\text { 10; decreased }\end{array}$ & $\begin{array}{c}10 ; \\
\text { Decreased }\end{array}$ & $\begin{array}{c}\text { 5; } 1 \mathrm{hr} \\
\text { 10; } 1-4 \mathrm{hrs}\end{array}$ & $\begin{array}{c}\text { Reduced measures } \\
\text { of respiration }\end{array}$ & $\begin{array}{l}\text { Reduced all } \\
\text { measures }\end{array}$ & $\begin{array}{l}\text { High abuse } \\
\text { liability }\end{array}$ \\
\hline Kurkinol & $\begin{array}{l}2.5 ; 60 \mathrm{~min} \\
5 ; 90 \mathrm{~min}\end{array}$ & $\begin{array}{l}\text { 2.5; } 4 \mathrm{hr} \\
5 ; 6 \mathrm{hrs}\end{array}$ & $\begin{array}{l}\text { 10; no effect } \\
5 ; \text { decreased }\end{array}$ & $\begin{array}{c}\mathbf{5} \\
\text { Decreased }\end{array}$ & No change & $\begin{array}{c}\text { Reduced measures } \\
\text { of respiration }\end{array}$ & Improved $V_{T}$ and $V_{\text {min }}$ & $\begin{array}{c}\text { Low abuse } \\
\text { liability }\end{array}$ \\
\hline Kurkinorin & 10; 15 min* & $\begin{array}{c}5 ; 3 \mathrm{hr} \\
10 ; 6 \mathrm{hrs}\end{array}$ & $\begin{array}{c}\text { 5; no effect } \\
\text { 10; no effect }\end{array}$ & $\begin{array}{c}10 ; \\
\text { Decreased }\end{array}$ & No change & $\begin{array}{c}\text { Reduced respiratory } \\
\text { frequency }\end{array}$ & $\begin{array}{c}\text { Reduced respiratory } \\
\text { frequency }\end{array}$ & $\begin{array}{c}\text { Low abuse } \\
\text { liability* }\end{array}$ \\
\hline
\end{tabular}

All measures only presented for male mice to allow comparison to the *kurkinorin rotarod, ** $\beta$-arr $2^{--}$whole-body plethysmography, and conditioned place preference that have only been assessed in males.

*(Crowley et al., 2016) 


\subsubsection{Kurkinol and Kurkinorin Induce Gastrointestinal Inhibition}

Constipation is the most common side effect in patients prescribed mu-opioids for chronic pain and is one of the main reasons for discontinuation of pain medication (Webster, 2015). It is therefore an important side effect to investigate when developing novel opioids pain medications. To assess the effects of kurkinol and kurkinorin on G.I transit two assays were performed, the faecal accumulation assay that assesses overall transit, and the charcoal meal assay that specifically investigates transit through the S.I. Due to the nature of the separation of the faeces and urine in the faecal accumulation assay, the induction of urinary retention was also assessed by simply collecting both excrements every hour for 6 hrs.

\subsubsection{Improvements in Small Intestine Transit are not Present in Overall Transit}

Sex was found to not affect faecal output over time, however, when sexes were combined morphine, kurkinol, and kurkinorin all induced significant effects on faecal accumulation. Morphine (5 and $10 \mathrm{mg} / \mathrm{kg} /$ i.p.) induced a large reduction in faecal output. While kurkinol only reduced accumulation at $5 \mathrm{mg} / \mathrm{kg} / \mathrm{i} . p$. Interestingly, it was again kurkinorin $(5$ and $10 \mathrm{mg} / \mathrm{kg} / \mathrm{i}$. . .) that showed the most improved G.I side effect profile, with higher faecal output compared to the equivalent $E D_{50}$ and $\mathrm{ED}_{80}$ doses of morphine and kurkinol and no significant difference from the vehicle (Table 4.2). Interestingly, the reduction in faecal accumulation lasted significantly longer than the anti-nociceptive duration of action of all 3 compounds. This is due to the inhibition of gastric emptying and slowing of reabsorption resulting in constipation for hours after the $\mu$ receptor agonists anti-nociceptive effects have dissipated.

This opposes our hypothesis that the more G-protein biased $\mu$ receptor agonist, kurkinol, would show the best side effect profile. When $\beta$-arr $2^{-1-}$ mice were initially assessed in this assay by Raehal et al. (2005) morphine was shown to have reduced impairment of faecal accumulation in $\beta$ arr $^{-/}$mice compared to $\mathrm{Wt}$ littermate controls. However, the only G-protein biased $\mu$ receptor agonist with reduced impact on G.I transit is TRV0109101 (Koblish et al., 2017), with PZM21, buprenorphine, mitragynine-3, all showing severe inhibition of G.I transit. While TRV130 has been to both induce and not induce in various mouse and rat models (Table 4.3). Furthermore, a recent attempt to replicate the results of faecal accumulation in $\beta$-arr $2^{-/}$found that morphine inhibited faecal accumulation to an equal extent in both $\mathrm{Wt}$ and $\beta$-arr ${ }^{-/}$mice, adding to the controversy around the role of $\beta$-arrestin2 in G.I inhibition (Kliewer et al., 2020). This data is direct opposition to the original findings, highlighting the requirement for repeating experiments in multiple laboratories. Our data in the faecal accumulation assay appears to agree with the work of Kliewer et al. (2020), as our most Gprotein biased agonist, kurkinol induced the same level of G.I inhibition as morphine. These recent findings have added to the controversy surrounding the role of $\beta$-arrestin2 in G.I inhibition. 
To further investigate $\mu$ receptor induced constipation the charcoal meal assay was performed to look at the S.I when the compounds have the maximum possible effect (30 min). Again, no effect of sex on S.I transit was found, when the sexes were combined morphine ( 5 and $10 \mathrm{mg} / \mathrm{kg} / \mathrm{i}$. .p.) induced significant impairment, while kurkinol only induced slowed S.I transit at the $5 \mathrm{mg} / \mathrm{kg} / \mathrm{i} . \mathrm{p}$ dose $\left(\mathrm{ED}_{80}\right.$ dose in anti-nociceptive assays). Once again, the biggest improvement was seen with kurkinorin (10 and $5 \mathrm{mg} / \mathrm{kg} /$ i.p.), with no reduction in $\mathrm{G} . I$ transit observed at both $E D_{50}$ and $E D_{80}$ anti-nociceptive doses (Table 4.2). $\beta$-arrestin2 has previously been shown to have no impact on the S.I transit upon morphine administration, as no difference in transit was seen between $\beta$-arr $2^{-/-}$and $\mathrm{Wt}$ littermate controls (Raehal et al., 2005). When this experiment was replicated in female $\beta$-arr2 ${ }^{--}$and $\mathrm{Wt}$ littermate controls this same response was seen with no change in transit induced by morphine and kurkinol in the absence of $\beta$-arrestin. However, kurkinorin induced a stronger G.I inhibition in the $\beta$ arr ${ }^{-/-}$mice indicating that $\beta$-arrestin2 may have a protective role in the induction of constipation in the S.I. Another potential explanation for the absence of S.I transit inhibition with kurkinorin may be latent $\mathrm{k}$ receptor activity, as traditional $\mathrm{k}$ receptor agonists such as $\mathrm{U} 69,553$ and $\mathrm{U} 50,488 \mathrm{H}$ show little to no effect on S.I transit (La Regina et al., 1988, Porreca et al., 1984). The disparity between the effects on kurkinorin in the faecal accumulation and charcoal meal assays indicates that colonic transit may be impaired by kurkinorin, as morphine and other opioids are known to impair colonic transit to a greater extent (Ono et al., 2014, Raehal et al., 2005). This question can be easily answered in future by running the glass bead expulsion assay.

\subsubsection{The Mu Opioid Receptor Agonist Induced Urinary Retention is Limited in C57BL/6J Mice}

Opioids can also induce urinary retention resulting in the need for catheterisation causing further discomfort and increased infection risk (Tomaszewski et al., 2014, Wroblewski et al., 1980). The classical $\mu$ receptor agonists morphine and DAMGO administered i.t into the lumbosacral spine and i.c.v into the ventrolateral PAG of Sprague Dawley rats inhibits the spinobulbospinal pathway resulting in decreased bladder contractions and volume evoked bladder emptying (Dray et al., 1984, Matsumoto et al., 2004) in a naloxone sensitive manner (Igawa et al., 1993, Kontani et al., 1988). To our knowledge, this is the first investigation into the effect of G-protein bias on urinary retention. Our results have shown that in C57BL/6J mice $\mu$ receptor agonists have limited, sex independent, effects on urinary output with only morphine $(10 \mathrm{mg} / \mathrm{kg} / \mathrm{i}$.p.) having a significant effect. One of the few urinary retention studies conducted in mice has shown urinary retention to $\mu$ receptor agonists occurs with chronic administration and is more prevalent in males (Shuey et al., 2007). Therefore, a single dose may not be enough to induce urinary retention in mice, and a chronic dosing scheme and regular measurement may be required to assess its induction. 


\begin{tabular}{|c|c|c|c|c|c|c|c|}
\hline & $\begin{array}{l}\text { Bias } \\
\text { Factor }\end{array}$ & Locomotion & Abuse Liability & Withdrawal & Hyperalgesia & $\begin{array}{l}\text { Respiratory } \\
\text { depression }\end{array}$ & $\begin{array}{c}\text { Gastrointestinal } \\
\text { transit }\end{array}$ \\
\hline Buprenorphine & $\begin{array}{l}1.8- \\
2.8[1]\end{array}$ & $\begin{array}{l}\text { Equal to morphine } \\
\text { [2] }\end{array}$ & $\begin{array}{c}\text { Less than } \\
\text { morphine }[3,4]\end{array}$ & $\begin{array}{c}\text { Less than } \\
\text { morphine }[3,4]\end{array}$ & n.d & $\begin{array}{c}\text { Less than } \\
\text { morphine }[3,5,6]\end{array}$ & $\begin{array}{l}\text { Less than } \\
\text { morphine [6] }\end{array}$ \\
\hline Loperamide & $\begin{array}{c}0.5-1 \\
{[1,7]}\end{array}$ & n.d & $\begin{array}{c}\text { Equal to morphine } \\
{[8]}\end{array}$ & $\begin{array}{l}\text { More than } \\
\text { morphine [8] }\end{array}$ & n.d & $\begin{array}{c}\text { Less than } \\
\text { morphine [9] }\end{array}$ & $\begin{array}{l}\text { Equal to morphine } \\
{[10]}\end{array}$ \\
\hline Mitragynine-3 & N.R & n.d & $\begin{array}{c}\text { Less than } \\
\text { morphine [11] }\end{array}$ & $\begin{array}{c}\text { Less than } \\
\text { morphine [11] }\end{array}$ & n.d & $\begin{array}{l}\text { Equal to morphine } \\
\text { [11] }\end{array}$ & $\begin{array}{c}\text { Less than } \\
\text { morphine [11] }\end{array}$ \\
\hline TRV130 & $\begin{array}{c}3-4 \\
{[12,13]}\end{array}$ & $\begin{array}{l}\text { Equal to morphine } \\
\qquad[12,13]\end{array}$ & $\begin{array}{c}\text { Less than } \\
\text { morphine }[12,13] \\
\text { Equal to morphine } \\
{[14,15]}\end{array}$ & n.d & n.d & $\begin{array}{l}\text { Less than } \\
\text { morphine }[12,13] \\
\text { Greater than } \\
\text { morphine }[14,15]\end{array}$ & $\begin{array}{c}\text { Less than } \\
\text { morphine }[12,13] \\
\text { Equal to morphine } \\
\text { [14] }\end{array}$ \\
\hline TRV0109101 & N.R & n.d & n.d & n.d & $\begin{array}{l}\text { Less than } \\
\text { morphine [16] }\end{array}$ & n.d & $\begin{array}{c}\text { Less than } \\
\text { morphine [16] }\end{array}$ \\
\hline PZM21 & $\begin{array}{r}3.23 \\
{[15]}\end{array}$ & $\begin{array}{c}\text { Less than } \\
\text { morphine }[13,17]\end{array}$ & $\begin{array}{l}\text { Less than } \\
\text { morphine }[13,17]\end{array}$ & $\begin{array}{l}\text { Equal to morphine } \\
\qquad[17]\end{array}$ & $\begin{array}{l}\text { Equal to morphine } \\
\qquad[13,17,18]\end{array}$ & $\begin{array}{c}\text { Less than } \\
\text { morphine }[13,17] \\
\text { More than } \\
\text { morphine }[18]\end{array}$ & $\begin{array}{c}\text { Less than } \\
\text { morphine [13] }\end{array}$ \\
\hline $\begin{array}{c}\text { SR-11501, - } \\
14969\end{array}$ & $\begin{array}{c}0.81- \\
2.5[19]\end{array}$ & n.d & n.d & n.d & n.d & $\begin{array}{l}\text { Equal to morphine } \\
{[19]}\end{array}$ & n.d \\
\hline
\end{tabular}




\begin{tabular}{|c|c|c|c|c|c|c|c|}
\hline SR-14968 & $5.1[19]$ & n.d & $\begin{array}{c}\text { Less than } \\
\text { morphine }[19,20]\end{array}$ & n.d & n.d & $\begin{array}{l}\text { Equal to morphine } \\
\qquad[19]\end{array}$ & n.d \\
\hline $\begin{array}{c}\text { SR-15908, } \\
51099\end{array}$ & $\begin{array}{c}19,27 \\
{[19]}\end{array}$ & n.d & n.d & n.d & n.d & $\begin{array}{c}\text { Less than } \\
\text { morphine [19] }\end{array}$ & n.d \\
\hline SR-17018 & $\begin{array}{c}40- \\
102[19]\end{array}$ & n.d & n.d & $\begin{array}{c}\text { Less than } \\
\text { morphine [21] }\end{array}$ & n.d & $\begin{array}{c}\text { Less than } \\
\text { morphine }[19,22]\end{array}$ & n.d \\
\hline Herkinorin & $\begin{array}{c}0.95 \\
{[23,24]}\end{array}$ & n.d & $\begin{array}{l}\text { Less than } \\
\text { morphine }\end{array}$ & n.d & n.d & n.d & n.d \\
\hline Kurkinorin & $\begin{array}{r}0.32 \\
{[23]}\end{array}$ & $\begin{array}{c}\text { Less than } \\
\text { morphine [23] }\end{array}$ & $\begin{array}{c}\text { Less than } \\
\text { morphine [23] }\end{array}$ & n.d & n.d & $\begin{array}{l}\text { Less than } \\
\text { morphine }\end{array}$ & $\begin{array}{l}\text { Less than } \\
\text { morphine }\end{array}$ \\
\hline Kurkinol & $\begin{array}{c}0.14 \\
{[25]}\end{array}$ & $\begin{array}{l}\text { Less than } \\
\text { morphine }\end{array}$ & $\begin{array}{l}\text { Less than } \\
\text { morphine [n.p] }\end{array}$ & n.d & $\begin{array}{l}\text { Less than } \\
\text { morphine }\end{array}$ & Equal to morphine & Equal to morphine \\
\hline \multicolumn{8}{|c|}{$\begin{array}{l}n . d=\text { not determine } \\
n \cdot p=\text { not published } \\
N \cdot R=\text { not reported }\end{array}$} \\
\hline \multicolumn{3}{|c|}{$\begin{array}{l}\text { 1. Burgueño, et al. } 2017 . \\
\text { 2. Liles and Flecknell. } 1992 . \\
\text { 3. Tompkins, et al. } 2014 . \\
\text { 4. Cowan, et al. } 1977 . \\
\text { 5. Dahan. } 2006 . \\
\text { 6. Kuo, et al. } 2015 . \\
\text { 7. Thompson, et al. } 2015 . \\
\text { 8. Yanagita, et al. } 1979 .\end{array}$} & \multicolumn{2}{|c|}{$\begin{array}{l}\text { 9. Sadeque, et al. } 2000 . \\
\text { 10. Tan-No, et al. } 2003 . \\
\text { 11. Váradi, et al. } 2016 . \\
\text { 12. DeWire, et al. } 2013 . \\
\text { 13. Manglik., et al. } 2016 . \\
\text { 14. Altarifi, et al. } 2017 . \\
\text { 15. Zamarripa, et al. } 2018 . \\
\text { 16. Koblish, et al. } 2017 .\end{array}$} & & \multicolumn{2}{|c|}{$\begin{array}{l}\text { 17. Kudla, et al. } 2019 . \\
\text { 18. Hill, et al. } 2018 . \\
\text { 19. Schmid, et al. } 2017 . \\
\text { 20. Schwienteck, et al. } 2019 . \\
\text { 21. Grim, et al. } 2020 . \\
\text { 22. Gillis, et al. } 2020 . \\
\text { 23. Crowley, et al. } 2016 . \\
\text { 24. Lamb, et al. } 2012 . \\
\text { 25. Crowley, et al. } 2020\end{array}$} \\
\hline
\end{tabular}




\subsubsection{Reduced $\beta$-arrestin2 Recruitment Does Not Improve the Respiratory Depressive Effects of Mu Opioid Receptor Agonists}

The ability of kurkinol and kurkinorin to induce respiratory depression in male and female C57BL/6J mice was assessed using whole-body plethysmography in unrestrained mice and the respiratory frequency, $\mathrm{V}_{T}$, and $\mathrm{V}_{\min }$ assessed over $60 \mathrm{~min}$. This allowed us to assess the induction of acute respiratory depression and therefore the risk of death with overdosing. Due to this experimental design, we were not able to figure out the duration of the respiratory depressive effects of our novel compounds. This is also an important clinical measure to assess and a longer time course should be performed in the future. To avoid the induction of artefacts induced by $\mu$ receptor dependent hyperactivity only the $E D_{50}$ dose of morphine ( $5 \mathrm{mg} / \mathrm{kg} /$ i.p.), kurkinol $(2.5 \mathrm{mg} / \mathrm{kg} / \mathrm{i}$. . .), and kurkinorin $(5 \mathrm{mg} / \mathrm{kg} / \mathrm{i}$. p.) were assessed. To fully understand the respiratory depressive effects of G-protein biased agonists repeating the experiment at higher, sedative dose would need to be performed. Morphine and kurkinol both differently effected frequency in male and female mice over time. However, no difference was found between sexes at a given time point. By increasing the number of animals per group these effects may be teased apart.

Overall, morphine reduced respiratory frequency from $15 \mathrm{~min}$, with a faster onset from $5 \mathrm{~min}$ with kurkinol. Morphine and kurkinol did not show any further effect of sex on $V_{T}$ and $V_{\text {min. }}$. Combining the sexes revealed a faster onset of respiratory depression for both $V_{T}$ and $V_{\min }$ measures with kurkinol (5 $\mathrm{min}$ ) compared to morphine (10 $\mathrm{min}$ ). Kurkinorin also significantly decreased respiratory frequency in combined treatment groups from 15, with no effect of sex. A sex effect was observed with $V_{T}$, with only female mice more affected between $20-50$ min (Table 4.4). However, when the sexes were combined no overall decrease in $V_{T}$ was observed, with significant improvements to morphine between 5-20 $\mathrm{min}$ and at $60 \mathrm{~min}$. The increased respiratory depressive effects of $\mu$ receptor agonists in females has been well established in healthy human volunteers (Pleuvry et al., 1980, Sarton et al., 2000). Its presence in only the kurkinorin treated animals is interesting, however, previous reports of strain differences in the respiratory depressive effects of $\mu$ receptor agonists in rodents may play a part in this effect (Mohammed et al., 2013). Despite this, $V_{\text {min }}$ was not differently affected by sex, with a significant decrease seen at $40 \mathrm{~min}$ when data from both sexes were combined. The generation of respiratory rhythm is the first respiratory change to occur in response to $\mu$ receptor agonists. This occurs via the inhibition of the pre-Bötzinger complex and has been detected at sub-analgesic doses (Lalley, 2003, Lumsden, 1923). Thus, indicating that the anti-nociceptive dose of kurkinorin induces respiratory effects to a sub-analgesic level. To further assess the relationship between $\beta$-arrestin2 recruitment and OIRD the experiment was repeated in male $\beta$-arr ${ }^{-}$mice and their littermate controls. Key research in the field of biased agonism had established a diminished depressive effect on the respiratory frequency with morphine in $\beta$-arr2 ${ }^{-/-}$mice (Raehal et al., 2005). In opposition to this, we identified no difference in the depression of respiratory frequency in either genotype. 
Furthermore, the $V_{T}$ was not altered by removal of $\beta$-arrestin. Interestingly, minute volume was only decreased in Wt mice, however, there was also no significant improvement in $\beta$-arr $2^{-}$mice. While kurkinorin did not change in $\mathrm{V}_{\mathrm{T}}$ and $\mathrm{V}_{\min }$ in $\mathrm{C} 57 \mathrm{BL} / 6 \mathrm{~J}$ mice, the reduction of respiratory frequency was predicted to be reduced in the $\beta$-arr $2^{-/}$mice. As with morphine, no difference was identified indicating $\beta$-arrestin2 does not play a role in OIRD. However, the highly potent and G-protein biased kurkinol had an overall improvement in the $\mathrm{V}_{T} \beta$-arr2 ${ }^{-/}$mice, while $\mathrm{V}_{\min }$ was only decreased in the $\mathrm{Wt}$ littermate controls. The effects of morphine and kurkinol on the respiratory frequency and $V_{\min }$ are again in opposition to the current interpretation of the in vivo role of $\beta$-arrestin.

This is not the first case of a reported G-protein biased agonists inducing significant OIRD, as TRV130, PZM21, SR-11501, SR-14969, and SR-14968 all induce respiratory depression to the same level as morphine and fentanyl (see Table 4.3). This prompted a more detailed investigation into the role of $\beta$-arrestin2 in OIRD. When the c-terminal of the mouse $\mu$ receptor was mutated to impair $\beta$ arrestin2 recruitment to varying degrees, decreased $\beta$-arrestin2 recruitment was correlated to increased OIRD (Kliewer et al., 2019). Furthermore, the experiments assessing the respiratory depressive effects of morphine in $\beta$-arr ${ }^{-1-}$ has been repeated by 3 independent labs, in 3 countries, with all finding morphine-induced respiratory depression to the same level in $\beta$-arr $2^{-/-}$and $W t$ mice (Kliewer et al., 2020). The possibility that $\beta$-arrestin2 recruitment limits respiratory depressive effects of $\mu$ receptor agonists would explain the induction of respiratory depression by kurkinol and other $\mathrm{G}$ protein biased agonists (Table 4.3). 


\begin{tabular}{|c|c|c|c|c|c|c|c|c|c|c|c|c|c|c|}
\hline & \multirow{2}{*}{\multicolumn{2}{|c|}{ Rotarod }} & \multirow{2}{*}{\multicolumn{2}{|c|}{$\begin{array}{c}\text { Faecal } \\
\text { Accumulation }\end{array}$}} & \multirow{2}{*}{\multicolumn{2}{|c|}{ Charcoal Meal }} & \multirow{2}{*}{\multicolumn{2}{|c|}{$\begin{array}{c}\text { Urinary } \\
\text { Retention }\end{array}$}} & \multicolumn{6}{|c|}{ Whole-body Plethysmography } \\
\hline & & & & & & & & & \multicolumn{2}{|c|}{ Frequency } & \multicolumn{2}{|c|}{ Tidal Volume } & \multicolumn{2}{|c|}{ Minute Volume } \\
\hline & $\begin{array}{c}\text { Male } \\
\text { Vs } \\
\text { Female }\end{array}$ & P value & $\begin{array}{c}\text { Male } \\
\text { Vs } \\
\text { Female }\end{array}$ & $P$ value & $\begin{array}{c}\text { Male } \\
\text { Vs } \\
\text { Female }\end{array}$ & P value & $\begin{array}{c}\text { Male } \\
\text { Vs } \\
\text { Female }\end{array}$ & $P$ value & $\begin{array}{c}\text { Male } \\
\text { Vs } \\
\text { Female }\end{array}$ & P value & \begin{tabular}{|c|} 
Male \\
Vs \\
Female
\end{tabular} & P value & $\begin{array}{c}\text { Male } \\
\text { Vs } \\
\text { Female }\end{array}$ & $P$ value \\
\hline Vehicle & $q=\hat{O}$ & $>0.9999$ & $q=0^{\lambda}$ & $>0.9999$ & $q=0^{\lambda}$ & $>0.9999$ & $q<\hat{0}$ & $=0.0002$ & $q={ }^{\lambda}$ & $>0.9999$ & $q=\hat{0}$ & $>0.9999$ & $q=\hat{0}$ & $>0.9999$ \\
\hline Morphine & $\phi>\hat{0}$ & $=0.011$ & $\phi=\hat{O}$ & $>0.9999$ & $\phi=\hat{O}$ & $>0.9999$ & $q=\hat{O}$ & $>0.9999$ & $\phi=\hat{O}$ & $>0.9999$ & $\phi>0^{\lambda}$ & $=0.002$ & $q=\sigma^{\lambda}$ & $>0.9999$ \\
\hline Kurkinol & $q=0^{\lambda}$ & $>0.9999$ & $q=0^{\lambda}$ & $>0.9999$ & $q={ }^{\lambda}$ & $>0.9999$ & $q=\hat{0}$ & $>0.9999$ & $q=\widehat{0}$ & $>0.9999$ & $q=\hat{0}$ & $>0.9999$ & $q=\hat{0}$ & $>0.9999$ \\
\hline Kurkinorin & $q=\widehat{0}$ & $>0.9999$ & $q=\widehat{O}$ & $>0.9999$ & $q=\hat{O}$ & $>0.9999$ & $q=\hat{0}$ & $>0.9999$ & $q=\widehat{O}$ & $>0.9999$ & $q=\hat{O}$ & $>0.9999$ & $q=\hat{O}$ & $>0.9999$ \\
\hline
\end{tabular}

Morphine at the $5 \mathrm{mg} / \mathrm{kg} / \mathrm{i} . \mathrm{p}$. produced more motor coordination impairment in males than in females.

Female mice produce significantly less urine over 6 hrs than male counterparts, but no interaction of treatment and sex was seen.

Kurkinorin induced a greater reduction in tidal volume in female mice compared to their male counterparts.

$$
\begin{aligned}
& q=\text { Female } \\
& \hat{\sigma}=\text { Male }
\end{aligned}
$$




\subsubsection{Abuse Liability Is Lowered with G-Protein Biased Agonists}

The abuse liability of $\mu$ receptor agonists is one of the most important side effects to assess as no novel agonists will be approved for human use without lower abuse potential than the currently available therapeutic options. When the abuse liability of kurkinol and kurkinorin were assessed in conditioned place preference assay neither kurkinol ( $5 \mathrm{mg} / \mathrm{kg} / \mathrm{i}$. .p.) nor kurkinorin ( 5 and $10 \mathrm{mg} / \mathrm{kg} / \mathrm{i}$. . .) induced a place preference for the drug paired chamber, indicating significantly lowered abuse potential (Crowley et al., 2016, Kivell Lab unpublished data). Despite the often-repeated dogma that biased agonism can provide novel analgesics with reduced abuse liability, the original work by Bohn et al. (2003) showed morphine increases dopamine in the striatum of $\beta$-arr $2^{-/-}$mice, correlating to increased abuse liability compared to the Wt littermate controls. Similarly, the G-protein biased agonist TRV130 did not receive FDA approval for human use after the initial application was submitted in 2018 over concerns that the benefits did not outweigh the risks, including respiratory depression and high abuse potential (Altarifi et al., 2017, Austin Zamarripa et al., 2018). While other compounds such as PZM21, SR-14968, and buprenorphine all have reduced liability compared to current $\mu$ receptor analgesics they still show increases abuse liability compared to vehicle-treated mice in the conditioned place preference assay (see Table 4.3).

Together this indicates $\beta$-arrestin2 is needed to limit dopamine release in the striatum and therefore abuse liability. The lack of abuse liability of kurkinol and kurkinorin is therefore unexpected but may be explained by potential binding and activation of $\mathrm{k}$ receptor. The $\mathrm{k}$ receptor is present throughout the mesolimbic system causing aversion or reduced rewarding effects of other drugs that increase dopamine within NAc (Bals-Kubik et al., 1993, Spanagel et al., 1992). Further, activation of the $\mathrm{k}$ receptor system has been shown to have anti-addictive properties, with the selective agonism of the $\mathrm{k}$ receptor able to block cocaine-induced conditioned place preference in rats (Crawford et al., 1995, Mori et al., 2002) while also decreasing cocaine self-administration in rats (Glick et al., 1995, Schenk et al., 1999) and monkeys (Mello et al., 1998). Moreover, the scaffold structure that kurkinol and kurkinorin were derived from, Sal A, has been shown to reduce cocaine-primed reinstatement in rats (Morani et al., 2009), while other $\mathrm{k}$ receptor Sal $\mathrm{A}$ analogues have also been shown to reduce cocaine-primed reinstatement (Morani et al., 2013, Morani et al., 2012, Simonson et al., 2015). Therefore, any latent $\mathrm{k}$ receptor activation within the mesolimbic system by $\mu$ receptor selective Sal $\mathrm{A}$ analogues, kurkinol and kurkinorin could negate the $\mu$ receptor induced disinhibition of dopaminergic neurons, limiting the abuse liability. To assess this the conditioned place preference experiment would need to be run with nor-BNI pre-treatment, or assessment in $\mathrm{k}$ receptor ${ }^{\%}$ mice to assess whether blocking $\mathrm{k}$ receptor results in increased abuse potential. 


\subsubsection{Limitations and Future Directions}

As with behavioural measures of anti-nociception, stress can also impact the presentation of side effects. Stress can increase respiration (Isom et al., 1982, McGregor et al., 1994, Suess et al., 1980), slow S.I transit, and increase colonic propulsion (Enck et al., 1989, Williams et al., 1988). To mitigate these effects, mice in our studies were habituated to the experimental environment and scuffing and restraint were performed for 3 days before experimentation. Whole-body plethysmography habituations were extended to 2 weeks to ensure full relaxation under experimental conditions.

An interesting finding from this study was the increase in respiratory measures following the antagonism of the $\mu$ receptor with $\beta$-FNA. Moreover, kurkinorin is strongly respiratory depressive in females. This indicates that there may be an interaction between gender and biased agonism or kurkinorin and the $\mathrm{k}$ receptor. The lack of interaction between the gender and degree of bias has not been identified in any other behavioural profiles. Leading us to believe that there may be latent selectivity for the $\mathrm{k}$ receptor as sex differences are well documented at this receptor and traditional $\mathrm{k}$ receptor agonists have little to no effect on respiration while reversing the effects of $\mu$ receptor agonists (Castillo et al., 1986, Dosaka-Akita et al., 1993, Vankova et al., 1996). These sex differences have been attributed to the interaction between the oestrogen receptor/oestrous cycle and the $\mathrm{k}$ receptor. It has been shown that oestrogen-dependent sequestration of GRK2 leads to a decrease in pERK levels, thus inhibiting anti-nociception but not side effects, reducing the therapeutic window (Abraham et al., 2018). To investigate the role this receptor plays in the respiratory effects of kurkinorin the whole-body plethysmography experiment should be performed following either pretreatment with the $\mathrm{k}$ receptor antagonist, nor-BNI, or using the $\mathrm{k}$ receptor knock out mouse line. If an in vivo effect is seen with the absence of the $\mathrm{k}$ receptor the overall improvement in seen following kurkinorin administration in the rotarod assay (motor coordination impairment) and charcoal meal assay (S.I transit) should also be assessed.

Due to time and animal number limitations the role of $\beta$-arrestin2 in the induction of OIRD was only assessed using male $\beta$-arr $2^{--}$mice. However, as kurkinorin only showed significant respiratory effects in female mice and not male future experiments in the $\beta$-arr $2^{-}$mice will therefore be necessary to fully assess its role in the respiratory effects of kurkinorin. This should also be conducted for morphine and kurkinol to fully investigate the role that $\beta$-arrestin 2 has on the induction of OIRD.

So far, the abuse liability of kurkinol and kurkinorin has only been assessed in the conditioned place preference assay. This provides an idea of the abuse liability, but not whether the kurkinol or kurkinorin would be self-administered and display abuse potential. To assess this further addiction models, such as self-administration, drug discrimination, or the two-bottle choice would need to be performed. While self-administration and the drug discrimination assay are approved by the Victoria 
University Animal Ethics Committee in rats, the volume of drug required was not available. In future, either with the provision of larger drug volumes, these two experiments should be performed to fully understand the abuse potential of kurkinol and kurkinorin. Furthermore, since the original abuse liability and dependence of $\mu$ receptor agonists were assessed in the $\beta$-arr ${ }^{-/}$mouse line these experiments have not been replicated (Bohn et al., 2000, Bohn et al., 2003). Due to the recent work from Gillis et al. (2020a) and our inability to replicate the reduction in OIRD the in these mice it is important to reassess these assays.

Another behavioural measure that is reduced in both the $\beta$-arr $2^{-}$mice following the administration of morphine and the G-protein biased agonists TRV130 and PZM21 is the open field assay (DeWire et al., 2016, Kudla et al., 2019, Manglik et al., 2016). While we have shown that kurkinol reduces fine motor movement in the rotarod assay, there may be an overall decrease in the level of spontaneous locomotion, while kurkinorin may show an even greater reduction. Furthermore, the role of $\beta$-arrestin2 at the $\mu$ receptor is being questioned, with the global deletion of $\beta$-arrestin2 in the knock out mice thought to change the signalling at the dopamine receptors resulting in the reduced spontaneous locomotion in this assay (Donthamsetti et al., 2020, Urs et al., 2011). By assessing the spontaneous locomotion in the $\beta$-arr $2^{-/-}$mice and following the selective knock down of $\beta$-arrestin 2 at the $\mu$ receptor and the Dopamine D1 receptors to determine what receptor is responsible for these effects. Moreover, this highlights the lack of selectivity and the poor translation that a global knock out induces, calling into question the other data from the $\beta$-arr $2^{-/}$mice. To directly assess the role of $\beta$-arrestin2 signalling at the $\mu$ receptor specifically a cre-flox system to selectively knock down $\beta$ arrestin2 mice before the assessment of all behavioural measures assessed in this thesis. With the anti-nociceptive potency, tolerance, and respiratory depressive measures the most important. 


\section{Chapter 5. Cell Signalling Properties of Kurkinol and Kurkinorin}

\subsection{Introduction}

\subsubsection{Biased Agonism at the Mu Opioid Receptor}

The theory of biased agonism has dominated the field of opioid receptor research since its role in the regulation of $\mu$ receptor mediated anti-nociception and on-target side effects were originally identified. With the theory of biased agonism applied to the $\mu$ receptor in 1999, when it was shown that the genetic knockdown of $\beta$-arrestin2 in mice caused an increase in anti-nociceptive potency and a decrease in anti-nociceptive tolerance (Bohn et al., 1999). Since then other on-target side effects such as respiratory depression, motor coordination, and constipation have been associated with the recruitment of $\beta$-arrestin2 (Bohn et al., 2006, Raehal et al., 2011, Raehal et al., 2005), and hundreds of novel $\mu$ receptor agonists have been developed and screened for G-protein bias. From these in vitro screens, many have progressed to in vivo testing. However, these compounds have rarely resulted in marked improvements in side effects or translation into the clinical use (see chapter 3, section 1.1 \& chapter 4 , section 1.1 for review). In the last 5 years, this has led many researchers to question the methods utilised to question signalling bias and the control of confounding factors that can influence bias. This includes the cell background, species of $\mu$ receptor, reference ligand, pathways assessed, the cellular and receptor modifications that some assays require to measure pathway activation, and the different ways of calculating bias (Schattauer et al., 2017, Stahl et al., 2019, Stahl et al., 2015, Thompson et al., 2016).

While there has been some consistency in the methods for calculating bias at the $\mu$ receptor the assays require either the addition of large tags to the receptor and signalling molecules which can influence downstream signalling (DiscoverX PathHunter $\beta$-arrestin2 recruitment assay). While others require large signal amplification (DiscoverX HitHunter cAMP inhibition assay) or only directly assess receptor activation and not the level of pathway activation (GTP $\gamma \mathrm{S}$ assay) biasing the outcome of the calculations. The many ways to calculate bias are shown in Table 5.1, with the $\Delta \Delta \log (\tau K A)$ equation for the determination of bias the most commonly utilised. It is also clear from this data that the bias factors can still vary greatly for the same compound when different methods are used. This is most clearly seen with morphine where bias switches from G-protein to $\beta$-arrestin2 when the method of evaluating G-protein activation is altered (Ehrlich et al., 2019, Schmid et al., 2017, Thompson et al., 2015). While a change in $\mu$ receptor species switches fentanyl's bias (Ehrlich et al., 2019, Schmid et al., 2017) (Table 5.1). This suggests that data generation and application of bias calculation does not mitigate all confounding factors influencing the outcome (Kenakin, 2019). Experimentally, the use of a G-protein assay that requires high levels of artificial signal amplification compared to the $\beta$-arrestin2 
output, where little to no amplification occurs, known as system bias (e.g. cAMP vs $\beta$-arrestin2 recruitment). Failure to differentiate the system bias from ligand bias can result in the identification of false positive G-protein biased agonists (Lane et al., 2017, Michel et al., 2018, Onaran et al., 2017). Ideally, bias should only be performed on natively expressed protein, and in the specific cell types that are activated in vivo to ensure the correct identification of signalling bias.

\subsubsection{Biased vs Low Efficacy Agonists}

The lack of translatability at the $\mu$ receptor has also led to the development of alternate theories to explain the behavioural profiles of compounds previously determined as G-protein biased. Over the last 3 years, the theory of relative intrinsic efficacy as the cellular mechanism for the improved safety profiles of some $\mu$ receptor agonists that were previously identified biased agonists. Under this theory the traditional pharmacological and pharmacokinetic parameters of partial agonism, or low efficacy agonists, at both G-protein and $\beta$-arrestin2 dependent signalling pathways proposed as the mechanism.

Partial agonism and low efficacy agonists are well-established in the pharmacological literature, having been identified and studied since the 1970s. However, both system bias of highly amplifying G-protein assays and large receptor reserves in in vitro assays, where only a subset of receptor activation is required to produce a maximal response, may have masked the partial agonism at the $\mu$ receptor (Herenbrink et al., 2016, Kelly, 2013, Onaran et al., 2017). This alternate hypothesis on the mechanism of improved therapeutic effects must be considered when designing in vitro screens for bias determination. Not only will this allow for the immediate removal of the previously identified confounding variables, but also more reliably delineate the role of biased vs partial agonism at $\mu$ receptor. The recent publications by Yudin et al. (2019) and Gillis et al. (2020a) have further shown this with by correlating partial agonism at multiple $\mu$ receptor dependent pathways to the improved therapeutic windows of PZM21, TRV130, and SR-17018. 
Table 5.1 Summary of Biased Mu Opioid Receptor Agonists

\begin{tabular}{|c|c|c|c|c|c|c|}
\hline Ligand & Bias & $\begin{array}{l}\text { Bias } \\
\text { Factor }\end{array}$ & Bias Quantification & Assays & Cell Type & $\begin{array}{l}\text { Reference } \\
\text { Ligand }\end{array}$ \\
\hline Buprenorphine & G-protein & $\begin{array}{l}2.85[1] \\
1.84[2]\end{array}$ & $\begin{array}{c}\Delta \Delta \log R \\
\Delta \Delta \log \left(\tau K_{A}\right)\end{array}$ & $\begin{array}{l}\text { CAMP (h } \mu \text { receptor) vs } \beta \text {-arrestin2 recruitment (h } \mu \text { receptor) } \\
\text { cAMP (h } \mu \text { receptor) vs. } \beta \text {-arrestin2 recruitment (N.R.) }\end{array}$ & $\mathrm{CHO}$ & $\begin{array}{l}\text { Met- } \\
\text { Enkephalin } \\
\text { Morphine }\end{array}$ \\
\hline Endomorphin-1 & $\begin{array}{l}\text { Arrestin } \\
\text { G-protein } \\
\text { N.R }\end{array}$ & $\begin{array}{l}0.06[3] \\
-1.2[3] \\
\text { N.R }[4]\end{array}$ & $\begin{array}{c}\Delta \Delta \log \left(\tau K_{A}\right) \\
\Delta \Delta \log \left(\tau K_{A}\right) \\
\text { Operational Efficacy } \\
(\tau)\end{array}$ & $\begin{array}{l}\text { cAMP (h } \mu \text { receptor) vs. } \beta \text {-arrestin2 recruitment (N.R.) } \\
\text { GTP } \gamma \text { S (h } \mu \text { receptor) vs. } \beta \text {-arrestin2 recruitment (N.R.) } \\
\text { GTP } \gamma \text { ( } r \mu \text { receptor) vs. } \beta \text {-arrestin2 recruitment (N.R.) }\end{array}$ & $\begin{array}{l}\text { CHO } \\
\text { CHO } \\
\text { Hek293 }\end{array}$ & DAMGO \\
\hline Endomorphin-2 & $\begin{array}{l}\text { G-protein } \\
\text { Arrestin } \\
\text { Arrestin } \\
\text { G-protein }\end{array}$ & $\begin{array}{l}0.2[2] \\
-0.5[5] \\
\text { N.R [4] } \\
0.69[6]\end{array}$ & 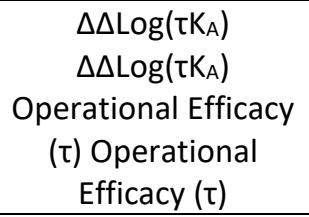 & $\begin{array}{l}\text { cAMP ( } h \mu \text { receptor) vs. } \beta \text {-arrestin2 recruitment (N.R.) } \\
\text { cAMP ( } h \mu \text { receptor) vs. } \beta \text {-arrestin2 recruitment (N.R.) } \\
\text { GTP } \gamma \text { S }(r \mu \text { receptor) vs. } \beta \text {-arrestin2 recruitment (N.R.) } \\
\text { GTP } \gamma \text { S }(r \mu \text { receptor) vs. } \beta \text {-arrestin2 recruitment (N.R.) }\end{array}$ & $\begin{array}{l}\text { CHO } \\
\text { Hek293 } \\
\text { Hek293 }\end{array}$ & $\begin{array}{l}\text { Morphine } \\
\text { Pfizer } \\
\text { standard-1 } \\
\text { Leu-enkephalin }\end{array}$ \\
\hline Fentanyl & $\begin{array}{c}\text { G-protein } \\
\text { G-protein } \\
\text { Arrestin } \\
\text { G-protein } \\
\text { Arrestin } \\
\text { Arrestin }\end{array}$ & $\begin{array}{c}1.65[1] \\
0.96[2] \\
0.18[7] \\
2.8[7] \\
0.5[7] \\
0.15[7]\end{array}$ & $\begin{array}{c}\Delta \Delta \log R \\
\Delta \Delta \log \left(\tau \mathrm{K}_{\mathrm{A}}\right) \\
\Delta \Delta \log \left(\tau \mathrm{K}_{\mathrm{A}}\right) \\
\Delta \Delta \log \left(\tau \mathrm{K}_{\mathrm{A}}\right) \\
\Delta \Delta \log \left(\tau \mathrm{K}_{\mathrm{A}}\right) \\
\Delta \Delta \log \left(\mathrm{\tau K}_{\mathrm{A}}\right)\end{array}$ & 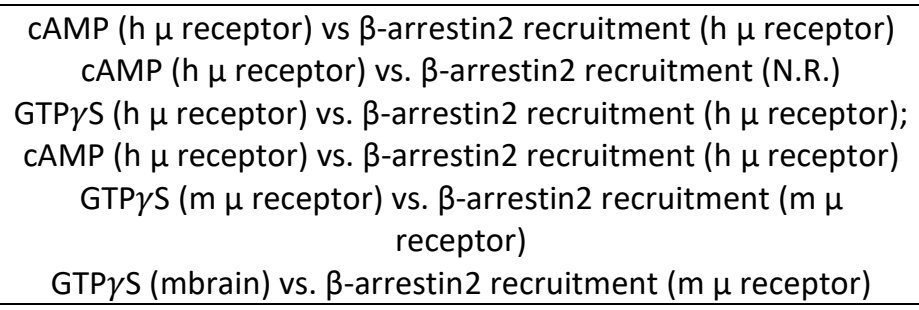 & $\begin{array}{c}\mathrm{CHO} \\
\mathrm{CHO} \\
\mathrm{CHO} / \mathrm{U} 2 \mathrm{OS} \\
\mathrm{CHO} / \mathrm{U} 2 \mathrm{OS} \\
\mathrm{CHO} / \mathrm{U} 2 \mathrm{OS} \\
\text { Brain slices }\end{array}$ & $\begin{array}{l}\text { Met- } \\
\text { Enkephalin } \\
\text { Morphine } \\
\text { DAMGO } \\
\text { DAMGO } \\
\text { DAMGO } \\
\text { DAMGO } \\
\end{array}$ \\
\hline Herkinorin & G-protein & $0.95[8]$ & $\Delta \Delta \log \left(\tau K_{A}\right)$ & cAMP (h $\mu$ receptor) vs. $\beta$-arrestin2 recruitment (h $\mu$ receptor) & $\mathrm{CHO}$ & DAMGO \\
\hline Kurkinol & G-protein & $0.14[9]$ & $\Delta \Delta \log \left(\tau K_{A}\right)$ & cAMP (h $\mu$ receptor) vs. $\beta$-arrestin 2 recruitment (h $\mu$ receptor) & $\mathrm{CHO}$ & DAMGO \\
\hline Kurkinorin & G-protein & $\begin{array}{l}0.32[8, \\
9]\end{array}$ & $\Delta \Delta \log \left(\tau K_{A}\right)$ & cAMP ( $h \mu$ receptor) vs. $\beta$-arrestin2 recruitment ( $h \mu$ receptor) & $\mathrm{CHO}$ & DAMGO \\
\hline Levorphanol & G-protein & $\begin{array}{l}-2.6 \\
{[10]}\end{array}$ & $\Delta \Delta \log \left(\tau K_{A}\right)$ & GTP $\gamma \mathrm{S}$ (h $\mu$ receptor) vs. $\beta$-arrestin2 recruitment (h $\mu$ receptor) & $\mathrm{CHO}$ & DAMGO \\
\hline Loperamide & G-protein & $\begin{array}{l}1.00[3] \\
0.97[3] \\
0.65[1]\end{array}$ & $\Delta \Delta \log \left(\tau K_{A}\right)$ & $\begin{array}{l}\text { GTP } \gamma S \text { (h } \mu \text { receptor vs. } \beta \text {-arrestin2 recruitment (N.R.) } \\
\text { cAMP ( } h \mu \text { receptor) vs. } \beta \text {-arrestin2 recruitment (N.R.) } \\
\text { cAMP (h } \mu \text { receptor) vs. } \beta \text {-arrestin2 recruitment (N.R.) }\end{array}$ & $\mathrm{CHO}$ & $\begin{array}{l}\text { Met- } \\
\text { Enkephalin } \\
\text { DAMGO } \\
\text { DAMGO }\end{array}$ \\
\hline $\begin{array}{l}\text { Mitragynine } \\
\text { Pseudoindoxyl }\end{array}$ & G-protein & N.R [11] & N.R & GTP $\gamma \mathrm{S}$ (m $\mu$ receptor) vs. $\beta$-arrestin 2 recruitment (N.R) & $\mathrm{CHO}$ & DAMGO \\
\hline
\end{tabular}




\begin{tabular}{|c|c|c|c|c|c|c|}
\hline MP103 & G-protein & $\begin{array}{l}0.02 \\
{[12]}\end{array}$ & $\Delta \Delta \log \left(\tau K_{A}\right)$ & cAMP ( $m \mu$ receptor) vs. $\beta$-arrestin2 recruitment ( $h \mu$ receptor) & Hek293/CHO & DAMGO \\
\hline MP105 & G-protein & $0.2[12]$ & $\Delta \Delta \log \left(\tau K_{\mathrm{A}}\right)$ & cAMP ( $h \mu$ receptor) vs. $\beta$-arrestin2 recruitment (N.R.) & Hek293/CHO & DAMGO \\
\hline Morphine & $\begin{array}{l}\text { Arrestin } \\
\text { Arrestin } \\
\text { G-protein } \\
\text { Arrestin } \\
\text { G-protein } \\
\text { Arrestin } \\
\text { G-protein }\end{array}$ & $\begin{array}{c}0.86[3] \\
-0.9[3] \\
1.3[7] \\
0.62[7] \\
0.8[7] \\
1.9[7] \\
1.99[1]\end{array}$ & $\begin{array}{c}\Delta \Delta \log \left(\tau K_{A}\right) \\
\Delta \Delta \log \left(\tau K_{A}\right) \\
\Delta \Delta \log \left(\tau K_{A}\right) \\
\Delta \Delta \log \left(\tau K_{A}\right) \\
\Delta \Delta \log \left(\tau K_{A}\right) \\
\Delta \Delta \log \left(\tau K_{A}\right) \\
\Delta \Delta \log \left(\tau K_{A}\right) \\
\Delta \Delta \operatorname{LogR}\end{array}$ & 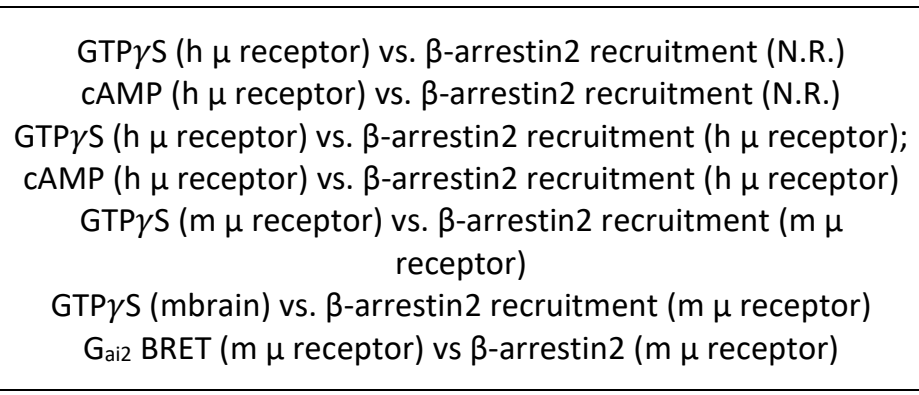 & $\begin{array}{l}\text { CHO } \\
\text { CHO } \\
\text { CHO/U2OS } \\
\text { CHO/U2OS } \\
\text { CHO/U2OS } \\
\text { CHO/U2OS } \\
\text { Brain slices/U2OS } \\
\text { CHO }\end{array}$ & $\begin{array}{c}\text { DAMGO } \\
\text { DAMGO } \\
\text { DAMGO } \\
\text { DAMGO } \\
\text { DAMGO } \\
\text { DAMGO } \\
\text { DAMGO } \\
\text { Met- } \\
\text { Enkephalin }\end{array}$ \\
\hline Oxycodone & G-protein & $1.89[1]$ & $\Delta \Delta \log R$ & 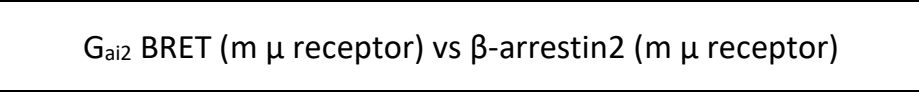 & DRG neurons & $\begin{array}{c}\text { Met- } \\
\text { Enkephalin }\end{array}$ \\
\hline Sufentanil & $\begin{array}{l}\text { Arrestin } \\
\text { Arrestin } \\
\text { Arrestin } \\
\text { G-protein }\end{array}$ & $\begin{array}{c}0.16[7] \\
0.06[7] \\
0.14[7] \\
1.9[7]\end{array}$ & $\Delta \Delta \log (\tau K A)$ & $\begin{array}{l}\text { GTP } \gamma \text { S (h } \mu \text { receptor) vs. } \beta \text {-arrestin2 recruitment (h } \mu \text { receptor); } \\
\text { cAMP (h }(\mathrm{h} \text { receptor) vs. } \beta \text {-arrestin2 recruitment (h } \mu \text { receptor) } \\
\text { GTP } \gamma \text { ( }(\mathrm{m} \mu \text { receptor) vs. } \beta \text {-arrestin2 recruitment (m } \mu \\
\text { receptor) } \\
\text { GTP } \gamma \text { (mbrain) vs. } \beta \text {-arrestin2 recruitment ( } \mathrm{m} \mu \text { receptor) }\end{array}$ & $\begin{array}{l}\mathrm{CHO} / \mathrm{U} 2 \mathrm{OS} \\
\mathrm{CHO} / \mathrm{U} 2 \mathrm{OS} \\
\mathrm{CHO} / \mathrm{U} 2 \mathrm{OS} \\
\text { Brain slices/U2OS }\end{array}$ & DAMGO \\
\hline PZM21 & G protein & $\begin{array}{l}3.23[1] \\
\quad N . R \\
{[13]}\end{array}$ & $\begin{array}{l}\Delta \Delta \log (\tau K A) \\
\Delta \Delta \log R\end{array}$ & $\begin{array}{l}\text { cAMP vs. } \beta \text {-arrestin2 recruitment } \\
\text { Gai2 BRET ( } m \mu \text { receptor) vs } \beta \text {-arrestin2 ( } m \mu \text { receptor) }\end{array}$ & $\begin{array}{l}\text { Hek293/U2OS } \\
\text { DRG neurons }\end{array}$ & $\begin{array}{c}\text { N.R } \\
\text { Met- } \\
\text { Enkephalin }\end{array}$ \\
\hline TRV0109101 & G-protein & $\begin{array}{l}3.23 \\
{[14]}\end{array}$ & N.R & cAMP (h $\mu$ receptor) vs. $\beta$-arrestin2 recruitment ( $h \mu$ receptor) & Hek293 & N.R \\
\hline TRV130 & $\begin{array}{c}\text { G-protein } \\
{[1,2,15]}\end{array}$ & $\begin{array}{l}3[2] \\
4.2[15] \\
4.02[1]\end{array}$ & $\begin{array}{c}\Delta \Delta \log (\tau K A) \\
R A_{i} \\
\Delta \Delta \log R\end{array}$ & $\begin{array}{l}\text { cAMP (h } \mu \text { receptor) vs. } \beta \text {-arrestin } 2 \text { recruitment (h } \mu \text { receptor) } \\
\text { cAMP (h }(\mathrm{h} \text { receptor) vs. } \beta \text {-arrestin2 recruitment (h } \mu \text { receptor) } \\
\text { Gai2 BRET (m } \mu \text { receptor) vs } \beta \text {-arrestin2 (m } \mu \text { receptor) }\end{array}$ & $\begin{array}{l}\text { CHO } \\
\text { CHO } \\
\text { DRG neurons }\end{array}$ & $\begin{array}{l}\text { Morphine } \\
\text { DAMGO } \\
\text { Met- } \\
\text { Enkephalin }\end{array}$ \\
\hline SR-11501 & $\begin{array}{l}\text { Arrestin } \\
{[7]}\end{array}$ & $\begin{array}{l}0.41[7] \\
0.12[7] \\
0.23[7]\end{array}$ & $\Delta \Delta \log \left(\tau K_{A}\right)$ & $\begin{array}{l}\text { GTP } \gamma \mathrm{S} \text { (h } \mu \text { receptor) vs. } \beta \text {-arrestin2 recruitment ( } \mathrm{h} \mu \text { receptor) } \\
\text { GTP } \gamma \mathrm{S} \text { (m } \mu \text { receptor) vs. } \beta \text {-arrestin2 recruitment ( } \mathrm{m} \mu \\
\text { receptor) } \\
\text { GTP } \gamma \mathrm{S} \text { (mbrain) vs. } \beta \text {-arrestin2 recruitment ( } \mathrm{m} \mu \text { receptor) }\end{array}$ & $\begin{array}{c}\mathrm{CHO} / \mathrm{U} 2 \mathrm{OS} \\
\mathrm{CHO} / \mathrm{U} 2 \mathrm{OS} \\
\text { Brain slices/U2OS }\end{array}$ & DAMGO \\
\hline SR-14969 & G-protein & $\begin{array}{l}11[7] \\
2.5[7] \\
2.9[7] \\
8.6[7]\end{array}$ & $\Delta \Delta \log \left(\tau_{\mathrm{A}}\right)$ & 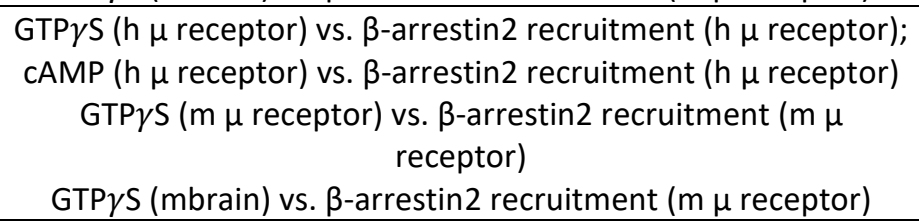 & $\begin{array}{l}\mathrm{CHO} / \mathrm{U} 2 \mathrm{OS} \\
\mathrm{CHO} / \mathrm{U} 2 \mathrm{OS} \\
\mathrm{CHO} / \mathrm{U} 2 \mathrm{OS} \\
\text { Brain slices/U2OS }\end{array}$ & DAMGO \\
\hline
\end{tabular}




\begin{tabular}{|c|c|c|c|c|c|c|}
\hline SR-14968 & G-protein & $\begin{array}{c}3[7] \\
5.1[7] \\
6.7[7] \\
34[7]\end{array}$ & $\Delta \Delta \log \left(\tau K_{A}\right)$ & $\begin{array}{l}\text { GTP } \gamma \mathrm{S} \text { (h } \mu \text { receptor) vs. } \beta \text {-arrestin2 recruitment (h } \mu \text { receptor); } \\
\text { cAMP (h } \mu \text { receptor) vs. } \beta \text {-arrestin2 recruitment (h } \mu \text { receptor) } \\
\text { GTP } \gamma \mathrm{S} \text { (m } \mu \text { receptor) vs. } \beta \text {-arrestin } 2 \text { recruitment ( } \mathrm{m} \mu \\
\text { receptor) } \\
\text { GTP } \gamma \mathrm{S} \text { (mbrain) vs. } \beta \text {-arrestin } 2 \text { recruitment ( } \mathrm{m} \mu \text { receptor) }\end{array}$ & $\begin{array}{c}\mathrm{CHO} / \mathrm{U} 2 \mathrm{OS} \\
\mathrm{CHO} / \mathrm{U} 2 \mathrm{OS} \\
\mathrm{CHO} / \mathrm{U} 2 \mathrm{OS} \\
\text { Brain slices/U2OS }\end{array}$ & DAMGO \\
\hline SR-15908 & G-protein & $\begin{array}{l}29[7] \\
19[7] \\
11[7] \\
55[7]\end{array}$ & $\Delta \Delta \log \left(\tau K_{\mathrm{A}}\right)$ & $\begin{array}{l}\text { GTP } \gamma \mathrm{S} \text { (h } \mu \text { receptor) vs. } \beta \text {-arrestin } 2 \text { recruitment (h } \mu \text { receptor); } \\
\text { cAMP (h } \mu \text { receptor) vs. } \beta \text {-arrestin2 recruitment (h } \mu \text { receptor) } \\
\text { GTP } \gamma \mathrm{S} \text { (m } \mu \text { receptor) vs. } \beta \text {-arrestin } 2 \text { recruitment ( } \mathrm{m} \mu \\
\text { receptor) } \\
\text { GTP } \gamma \mathrm{S} \text { (mbrain) vs. } \beta \text {-arrestin } 2 \text { recruitment ( } \mathrm{m} \mu \text { receptor) }\end{array}$ & $\begin{array}{c}\mathrm{CHO} / \mathrm{U} 2 \mathrm{OS} \\
\mathrm{CHO} / \mathrm{U} 2 \mathrm{OS} \\
\mathrm{CHO} / \mathrm{U} 2 \mathrm{OS} \\
\text { Brain slices/U2OS }\end{array}$ & DAMGO \\
\hline SR-51099 & G-protein & $\begin{array}{l}47[7] \\
27[7] \\
12[7] \\
55[7]\end{array}$ & $\Delta \Delta \log \left(\tau \mathrm{K}_{\mathrm{A}}\right)$ & $\begin{array}{l}\text { GTP } \gamma S \text { (h } \mu \text { receptor) vs. } \beta \text {-arrestin2 recruitment (h } \mu \text { receptor); } \\
\text { cAMP (h } \mu \text { receptor) vs. } \beta \text {-arrestin } 2 \text { recruitment ( } h \mu \text { receptor) } \\
\text { GTP } \gamma \mathrm{S} \text { (m } \mu \text { receptor) vs. } \beta \text {-arrestin } 2 \text { recruitment ( } \mathrm{m} \mu \\
\text { receptor) } \\
\text { GTP } \gamma \mathrm{S} \text { (mbrain) vs. } \beta \text {-arrestin } 2 \text { recruitment ( } \mathrm{m} \mu \text { receptor) }\end{array}$ & $\begin{array}{c}\mathrm{CHO} / \mathrm{U} 2 \mathrm{OS} \\
\mathrm{CHO} / \mathrm{U} 2 \mathrm{OS} \\
\mathrm{CHO} / \mathrm{U} 2 \mathrm{OS} \\
\text { Brain slices/U2OS }\end{array}$ & DAMGO \\
\hline SR-17018 & G-protein & $\begin{array}{c}85[7] \\
40[7] \\
30[7] \\
102[7]\end{array}$ & $\Delta \Delta \log \left(\tau K_{\mathrm{A}}\right)$ & 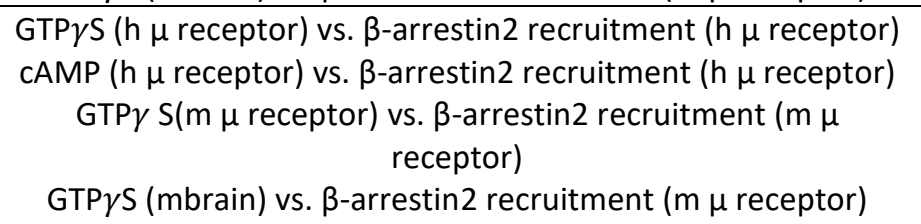 & $\begin{array}{c}\mathrm{CHO} / \mathrm{U} 2 \mathrm{OS} \\
\mathrm{CHO} / \mathrm{U} 2 \mathrm{OS} \\
\mathrm{CHO} / \mathrm{U} 2 \mathrm{OS} \\
\text { Brain slices/U2OS }\end{array}$ & DAMGO \\
\hline
\end{tabular}

Summary of $\mu$ receptor biased agonists that have been assessed in vivo or are clinically available. Hundreds of other compounds have been generated and not assessed in vivo, these are not the focus of this thesis and have therefore been excluded.

$N \cdot R=$ not reported

$\mathrm{h} \mu$ receptor = human $\mu$ receptor

$\mathrm{m} \mu$ receptor $=$ mouse $\mu$ receptor

$r \mu$ receptor $=$ rat $\mu$ receptor

BRET = bioluminescent resonance energy transfer

GTP $\gamma \mathrm{S}=\Upsilon$ irradiated guanine triphosphate

\section{References:}

1. Ehrlich, et al. 2019.

2. Burgueño, et al. 2017.

3. Thompson, et al. 2015.

4. McPherson, et al. 2010

5. Hothersall, et al. 2017.
6. Rivero, et al. 2012.

7. Schmid, et al. 2017.

8. Crowley, et al. 2016.

9. Crowley, et al. 2020.

10. Le Rouzic, et al. 2019

11. Váradi, et al. 2016.
12. Gutridge, et al. 2020

13. Manglik., et al. 2016

14. Koblish, et al. 2017.

15. DeWire, et al. 2013. 


\subsection{Aims and Hypothesis}

In this study, we aimed to investigate a range of different subcellular signalling pathways to determine whether this improves the correlation of signalling bias to improved anti-nociceptive effects and reduced side effects of kurkinol and kurkinorin. We further aimed to compare the effect different cell lines and bias equations on the generation of bias factors. We hypothesised that a more detailed cell signalling profile may generate a bias factor that is more representative of the cell signalling pathways activated upon receptor binding, therefore allowing for the correlation of specific cell signalling pathways to behavioural effects.

Aims;

1. Investigate the activation of $\mathrm{G}$-protein dependent pathways in human $\mu$ receptor $\mathrm{CHO}$ and Hek293 cells using the Molecular Devices Membrane Potential and Potassium Assays.

2. Assess the recruitment of $\beta$-arrestin2 using the downstream subcellular signalling molecule, CREB.

3. Assess the timing and role of G-protein and $\beta$-arrestin2 signalling pathways on the activation of ERK1/2 by kurkinol and kurkinorin in human $\mu$ receptor expressing $\mathrm{CHO}$ cells.

4. Utilise both the simplified method of bias calculation and the relative intrinsic efficacy model to determine the role of cell line and signalling pathway on the calculation of bias factors. 


\subsection{Results}

\subsubsection{Activation of G-Protein Dependent Pathways by Kurkinol and Kurkinorin}

\subsubsection{Membrane Potential Assay}

We assessed the inhibition of the VG $\mathrm{Ca}^{2+}$ channel using a Membrane Potential-sensitive dye (Molecular Devices) in both Hek293 (Fig. 5.1) and CHO (Fig 5.2) cells expressing the human $\mu$ receptor. The $\mathrm{EC}_{50}$ and $\mathrm{E}_{\max }$ values were determined from the dose response curves which were then compared to the prototypical $\mu$ receptor agonists DAMGO (reference ligand) and morphine.

In Hek293 cells stably expressing the human $\mu$ receptor, DAMGO, kurkinol, and kurkinorin were significantly more potent than morphine $(p<0.0001)$ with no increase or decrease in potency compared to DAMGO $\left(F_{(3,39)}=29.29, p<0.0001, p>0.05\right.$, Table 5.2). Differential effects were also found between $E_{\max }$ values, with morphine, kurkinol and kurkinorin having higher maximal effects than DAMGO $\left(F_{(3,42)}=7.860, P=0.0003, p<0.05\right)$ (Fig. 5.1 A, Table 5.2). Antagonism of the $\mu$ receptor with $\beta$-FNA $(10 \mu \mathrm{M})$ reversed the effects of the highest dose of all compounds assessed (Fig $5.1 \mathrm{~B}$ ). When this experiment was repeated in $\mathrm{CHO}$ cells stably expressing the human $\mu$ receptor, DAMGO, kurkinol, and kurkinorin were all more potent than morphine $\left(\mathrm{F}_{(3,33)}=19.78, p<0.0001, p<0.05\right)$. DAMGO was also found to have a significantly lower maximal efficacy compared to morphine $\left(F_{(3,37)}=3.483, p=\right.$ $0.0253, p<0.05$ ) (Fig. 5.2 A, Table 5.2). As with the Hek293 cells, the $\mu$ receptor specific antagonist $\beta$ FNA $(10 \mu \mathrm{M})$ reversed the effects, confirming signalling was mediated via the $\mu$ receptor (Fig $5.2 \mathrm{~B}$ ).

The $\mathrm{EC}_{50}$ and $\mathrm{E}_{\max }$ values were then compared between the two cell lines to assess the potential impact of cell type on second messenger signalling. This identified significant differences between the $\mathrm{EC}_{50}$ values, with DAMGO and morphine being more potent in the human $\mu$ receptor $\mathrm{CHO}$ cell line $\left(F_{(7,73)}=35.31, p<0.0001, p<0.01\right)$ (Table 5.3). However, no significant differences were identified between the $E_{\max }$ values between the cell lines $\left(F_{(7,79)}=5.151, p<0.0001, p>0.9999\right)$ (Table 5.3). 

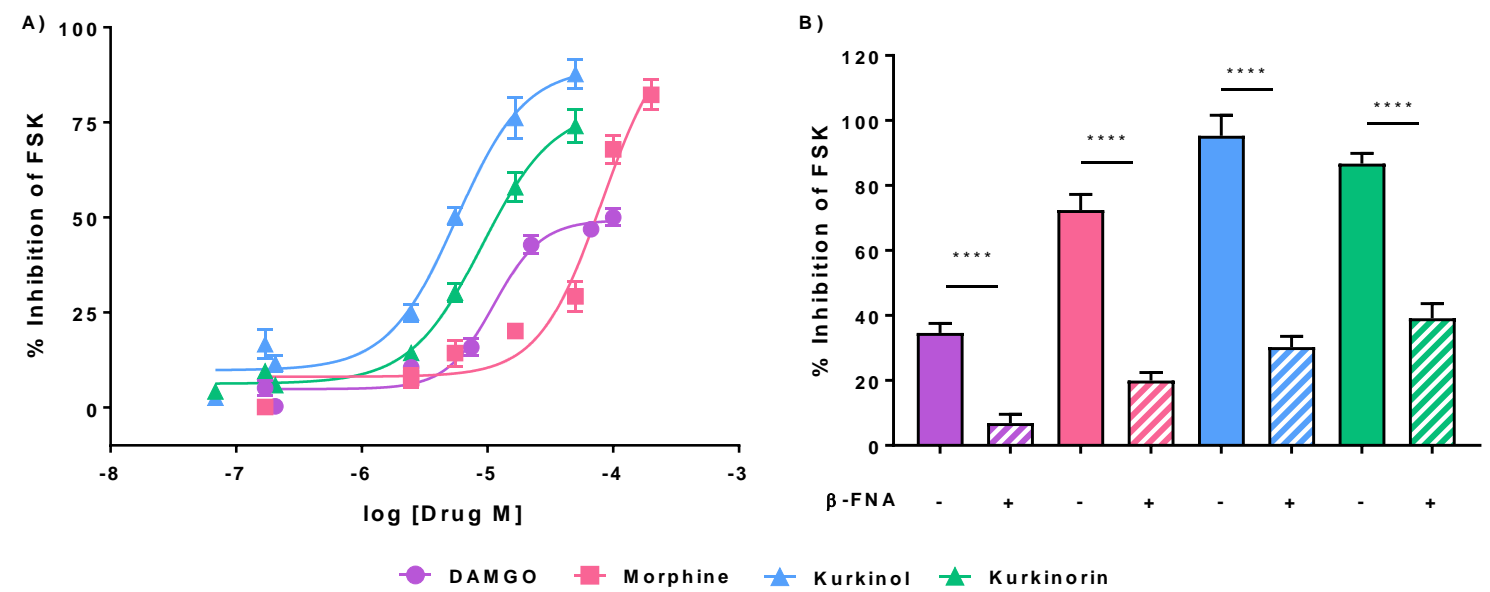

Figure 5.1 Kurkinol and Kurkinorin Potently Inhibit Membrane Depolarisation

(A) Kurkinol and kurkinorin potently inhibited forskolin (10 $\mu \mathrm{M})$ induced membrane depolarization in a dose-dependent manner in Hek293 cells stable stably expressing the human $\mu$ receptor using the Membrane Potential Assay (Molecular Devices). (B) When cells were incubated with the $\mu$ receptor specific antagonist $\beta$-FNA and the highest dose of the $\mu$ receptor agonists there was significant inhibition of FSK-induced depolarisation. One-way repeated measures ANOVA with Bonferroni multiple corrections test, ${ }^{* * * *} p<0.0001$ compared to $\beta$-FNA pre-treated. Each point presented as a percent inhibition of FSK induced change in Membrane Potential. All data are presented as mean \pm SEM from a mean of 4 experimental replicates, each conducted in triplicate. 
A)

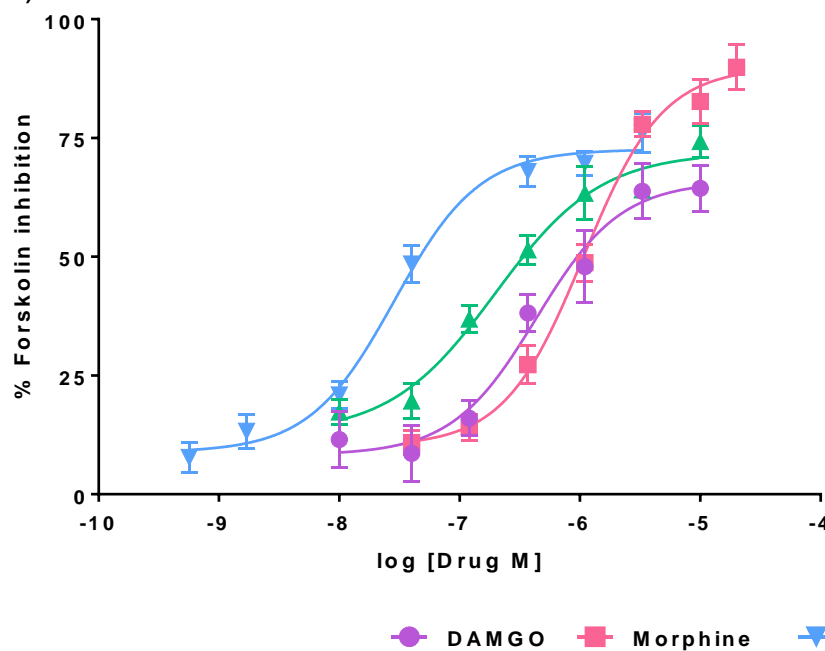

B )

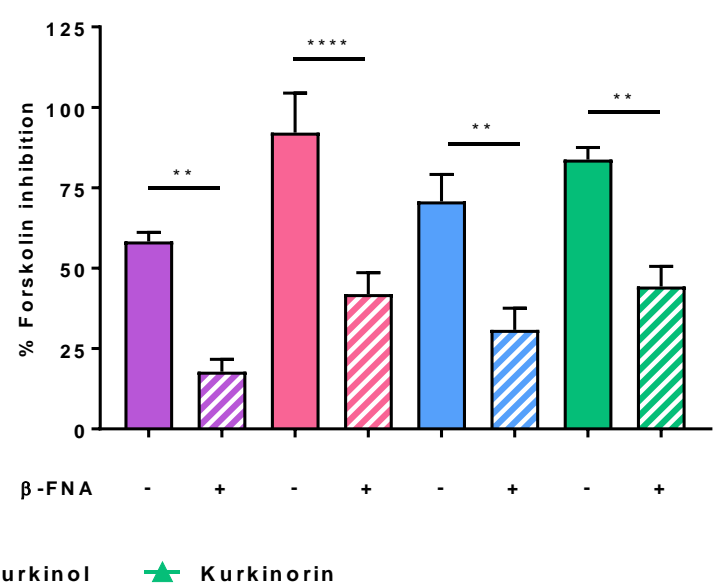

Figure 5.2 Kurkinol and Kurkinorin are Potent Inhibitors of Membrane Depolarisation

(A) Dose-dependent effects on membrane depolarisation were seen for all compounds in the Membrane Potential Assay when in CHO cells stably expressing the human $\mu$ receptor. (B) Coadministration of DAMGO $(10 \mu \mathrm{M})$, morphine $(20 \mu \mathrm{M})$, kurkinol $(10 \mu \mathrm{M})$, or kurkinorin $(3.3 \mu \mathrm{M})$ with $\beta$-FNA significantly reduced inhibition of forskolin-induced depolarisation. One-way repeated measures ANOVA with Bonferroni multiple corrections test ${ }^{*} p>0.05,{ }^{* *} p<0.01,{ }^{* * *} p<0.001$, $* * * * p<0.0001$ compared to B-FNA pre-treated. Each point presented as a percent inhibition of FSK induced change in Membrane Potential. All data are presented as mean \pm SEM from a mean of 4 experimental replicates, each conducted in triplicate. 
Table 5.2 EC $C_{50}$ and $E_{\max }$ of Kurkinol and Kurkinorin in the Membrane Potential Assay in Hek293 and CHO cells Stably Expressing the Human Mu Opioid Receptor

\begin{tabular}{|c|c|c|c|c|c|c|c|c|c|c|c|c|}
\hline \multirow[b]{2}{*}{ DAMGO } & \multirow{2}{*}{$\begin{array}{c}\text { Cell } \\
\text { Line } \\
\text { Hek293 } \\
\text { CHO }\end{array}$} & \multirow{2}{*}{$\begin{array}{c}\mathrm{EC}_{50} \pm \text { SEM } \\
(\mu \mathrm{M})\end{array}$} & \multicolumn{2}{|c|}{$\begin{array}{l}\text { P Value to } \\
\text { DAMGO }\end{array}$} & \multicolumn{2}{|c|}{$\begin{array}{l}\text { P Value to } \\
\text { Morphine }\end{array}$} & \multirow{2}{*}{$\begin{array}{c}\begin{array}{c}E_{\max } \pm \text { SEM } \\
(\% \mathrm{FSK})\end{array} \\
85.59 \pm 7.06 \\
68.73 \pm 4.41\end{array}$} & \multicolumn{2}{|c|}{$\begin{array}{l}\text { P Value to } \\
\text { DAMGO }\end{array}$} & \multicolumn{2}{|c|}{$\begin{array}{l}\text { P Value to } \\
\text { Morphine }\end{array}$} & \multirow{2}{*}{$\begin{array}{c}\mathbf{R}_{\mathbf{2}} \\
0.8617 \\
0.6115\end{array}$} \\
\hline & & & - & - & $\begin{array}{l}<0.0001 \\
=0.0229\end{array}$ & $\begin{array}{c}* * * * \\
*\end{array}$ & & - & $\begin{array}{l}- \\
-\end{array}$ & $\begin{array}{l}0.0041 \\
0.0169\end{array}$ & $\begin{array}{l}* * \\
*\end{array}$ & \\
\hline Morphine & $\begin{array}{c}\text { Hek293 } \\
\text { CHO }\end{array}$ & $\begin{array}{c}81.03 \pm 6.17 \\
1.07 \pm 0.12\end{array}$ & $\begin{array}{l}<0.0001 \\
0.0229\end{array}$ & $\begin{array}{c}* * * * \\
*\end{array}$ & - & - & $\begin{array}{l}58.34 \pm 5.10 \\
88.97 \pm 3.88\end{array}$ & $\begin{array}{l}0.0041 \\
0.0169\end{array}$ & $\begin{array}{l}* * \\
*\end{array}$ & - & - & $\begin{array}{l}0.9005 \\
0.8726\end{array}$ \\
\hline Kurkinol & $\begin{array}{c}\text { Hek293 } \\
\text { CHO }\end{array}$ & $\begin{array}{c}5.65 \pm 0.73 \\
0.028 \pm 0.0072\end{array}$ & $\begin{array}{l}0.2672 \\
0.0019\end{array}$ & $\begin{array}{l}\text { n.s } \\
* *\end{array}$ & $\begin{array}{l}<0.0001 \\
<0.0001\end{array}$ & $\begin{array}{l}* * * * \\
* * * *\end{array}$ & $\begin{array}{l}91.84 \pm 3.40 \\
79.37 \pm 5.38\end{array}$ & $\begin{array}{c}>0.9999 \\
0.5589\end{array}$ & $\begin{array}{l}\text { n.s } \\
\text { n.s }\end{array}$ & $\begin{array}{l}0.0002 \\
0.8255\end{array}$ & $\begin{array}{l}* * * \\
\text { n.s }\end{array}$ & $\begin{array}{l}0.8757 \\
0.8566\end{array}$ \\
\hline Kurkinorin & $\begin{array}{c}\text { Hek293 } \\
\mathrm{CHO}\end{array}$ & $\begin{array}{c}9.04 \pm 0.89 \\
0.19 \pm 0.017\end{array}$ & $\begin{array}{l}0.6888 \\
0.3104\end{array}$ & $\begin{array}{l}\text { n.s } \\
\text { n.s }\end{array}$ & $\begin{array}{l}<0.0001 \\
=0.0001\end{array}$ & $\begin{array}{l}* * * * \\
* * *\end{array}$ & $\begin{array}{l}78.37 \pm 4.64 \\
76.42 \pm 3.84\end{array}$ & $\begin{array}{l}>0.9999 \\
>0.9999\end{array}$ & $\begin{array}{l}\text { n.s } \\
\text { n.s }\end{array}$ & $\begin{array}{l}0.0456 \\
0.4021\end{array}$ & $\begin{array}{c}* \\
\text { n.s }\end{array}$ & $\begin{array}{l}0.9009 \\
0.7718\end{array}$ \\
\hline
\end{tabular}

Nonlinear regression of the dose response effects was used to calculate the $E C_{50}$ and $E_{\max }$ values for all $\mu$ receptor agonists in the human $\mu$ receptor expressing $\mathrm{CHO}$ cells. Kurkinol and kurkinorin were found to be more potent than morphine, while morphine had a higher $E_{\max }$ compared to DAMGO. One-way repeated measures ANOVA with Bonferroni multiple corrections test ${ }^{*} p>0.05,{ }^{* *} p<0.01,{ }^{* * *} p<0.001,{ }^{* * *} p<0.0001$. All data is a mean of 4 experimental replicates conducted in triplicate. Data presented as mean \pm SEM. 


\begin{tabular}{c|c|c|c|c|c|c|c|c|} 
& Hek293 EC $50 \pm$ SEM $(\mu \mathrm{M})$ & CHO EC $_{50} \pm$ SEM $(\mu \mathrm{M})$ & \multicolumn{2}{|c|}{ P Value } & $\begin{array}{c}\text { Hek293 } E_{\max } \pm \text { SEM } \\
\text { (\% FSK) }\end{array}$ & \multicolumn{2}{|c|}{$\begin{array}{c}\text { CHO E } E_{\max } \pm \text { SEM } \\
\text { (\% FSK) }\end{array}$} \\
\hline DAMGO & $81.03 \pm 8.41$ & $0.43 \pm 0.13$ & 0.0021 & $* *$ & $85.59 \pm 7.06$ & $68.73 \pm 4.41$ & $>0.9999$ & n.s \\
\hline Morphine & $11.24 \pm 6.17$ & $1.07 \pm 0.12$ & $<0.0001$ & $* * * *$ & $58.34 \pm 5.10$ & $88.97 \pm 3.88$ & $>0.9999$ & n.s \\
\hline Kurkinol & $9.04 \pm 0.89$ & $0.19 \pm 0.017$ & 0.8938 & n.s & $91.84 \pm 3.40$ & $79.537 \pm 5.38$ & $>0.9999$ & n.s \\
\hline Kurkinorin & $5.65 \pm 0.73$ & $0.028 \pm 0.0072$ & 0.3638 & n.s & $78.37 \pm 4.64$ & $76.42 \pm 3.84$ & $>0.9999$ & n.s
\end{tabular}

The effect of the cell background was then assessed by comparing the $E_{50}$ and $E_{\max }$ values from all $\mu$ receptor agonists in the human $\mu$ receptor expressing Hek293 and CHO cells. DAMGO and morphine were more potent in Hek293 cells, whit no differences between the kurkinol and kurkinorin. No effect on the Emax values was identified between the two cell lines. One-way repeated measures ANOVA with Bonferroni multiple corrections test $*^{* *} p<0.01,{ }^{* * *} p<0.001$, ज***p<0.0001. All data is a mean of 4 experimental replicates conducted in triplicate. Data presented as mean \pm SEM. 


\subsubsection{Potassium Assay}

GIRK channel ( $\left.\mathrm{K}_{\mathrm{ir} 3}\right)$ activation by the $\mathrm{G} \beta \gamma$ subunit was assessed using a potassium assay, which again utilises a voltage-sensitive fluorescent dye that exits the cell upon activation of the $\mathrm{K}_{\mathrm{ir} 3}$ channel and dose response effects were evaluated (Fig 5.3 A). However, no differences in potency or maximal efficacy were identified for DAMGO, morphine, kurkinol, or kurkinorin $\left(\mathrm{EC}_{50} ; \mathrm{F}_{(3,20)}=1.044, p=0.3945\right.$, $E_{\max } ; F_{(3,20)}=0.9782, p=0.4227$ ) (Fig $5.3 \mathrm{~A}$, Table 5.4). These effects were shown to be $\mu$ receptor dependent as administration of $\beta$-FNA significantly decreased $K_{\text {ir } 3}$ activation $\left(F_{(7,64)}=13.77, p<0.0001\right.$, p< 0.05) (Fig. 5.3 B).

A)

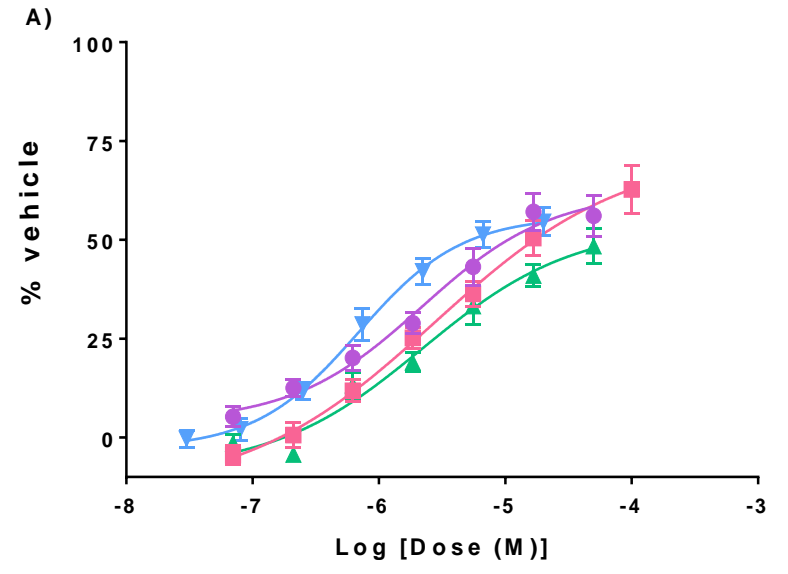

B )

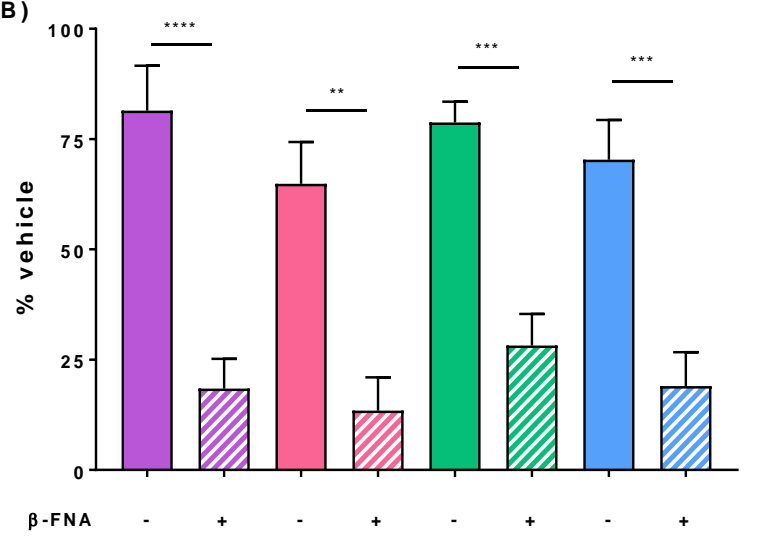

Figure 5.3 All the Mu Opioid Receptor Agonists are Equipotent Activators of the GIRK Channels

(A) Kurkinol and kurkinorin induce potent activation of the inwardly rectifying potassium channels in CHO cells stably expressing the human $\mu$ receptor as measured by the Molecular Devices Potassium Assay. (B) Treatment with the highest concentration of $\mu$ receptor agonist in the presence of the $\mu$ receptor antagonist $\beta$-FNA resulted in a significant reduction in potassium channel activation. Oneway repeated measures ANOVA with Bonferroni multiple corrections test ${ }^{* *} p<0.01,{ }^{* * *} p<0.001$, ****p<0.0001 compared to $\beta$-FNA pre-treated. Each point presented as percent inhibition of vehicle. All data are presented as mean \pm SEM from a mean of 7 experimental replicates, each conducted in triplicate. 
Table 5.4 Kurkinol and Kurkinorin offer no Increase in Potency and Efficacy of GIRK channel activation

\begin{tabular}{|c|c|c|c|c|c|c|c|c|c|c|c|}
\hline \multirow[b]{2}{*}{ DAMGO } & \multirow{2}{*}{$\begin{array}{c}\mathrm{EC}_{50} \pm \mathrm{SEM}(\mu \mathrm{M}) \\
2.17 \pm 1.29\end{array}$} & \multicolumn{2}{|c|}{ P Value to DAMGO } & \multicolumn{2}{|c|}{ P Value to Morphine } & \multirow{2}{*}{$\begin{array}{c}\begin{array}{c}E_{\max } \pm \text { SEM } \\
\text { (\% Veh) }\end{array} \\
56.8 \pm 8.08\end{array}$} & \multicolumn{2}{|c|}{ P Value to DAMGO } & \multicolumn{2}{|c|}{ P Value to Morphine } & \multirow{2}{*}{$\begin{array}{c}\mathbf{R}_{\mathbf{2}} \\
0.5495\end{array}$} \\
\hline & & n.s & n.s & n.s & n.s & & n.s & n.s & n.s & n.s & \\
\hline Morphine & $2.76 \pm 0.66$ & n.s & n.s & n.s & n.s & $81.9 \pm 16.9$ & n.s & n.s & n.s & n.s & 0.6520 \\
\hline Kurkinol & $0.68 \pm 0.25$ & n.s & n.s & n.s & n.s & $66.93 \pm 9.9$ & n.s & n.s & n.s & n.s & 0.6901 \\
\hline Kurkinorin & $2.22 \pm 1.62$ & n.s & n.s & n.s & n.s & $59.74 \pm 7.97$ & n.s & n.s & n.s & n.s & 0.6193 \\
\hline
\end{tabular}

Nonlinear regression of the dose response effects on the $K_{i r 3}$ channel was used to calculate the $E C_{50}$ and $E_{\max }$ values for all $\mu$ receptor agonists in the human $\mu$ receptor $\mathrm{CHO}$ cells. No significant differences were found between the $E C_{50}$ and $E_{\max }$ values of the compounds assessed. One-way repeated measures $A N O V A$. 


\subsection{2. $\beta$-arrestin2 Dependent CREB Phosphorylation}

The activation of CREB via phosphorylation is a downstream pathway of $\beta$-arrestin2 recruitment and signalling (Fig 5.4). We, therefore, utilised this non-amplifying method of assessing the ability of DAMGO, morphine, kurkinol and kurkinorin to signal via the $\beta$-arrestin2 pathway using the Cisbio (Melbourne, Australia) pCREB (Ser133) cellular kit in CHO cells stably expressing the human $\mu$ receptor. DAMGO and morphine were found to be equipotent, with kurkinol less potent and kurkinorin more potent than the two typical $\mu$ receptor agonists $\left(F_{(3,32)}=37.57, p<0.0001, p<0.001\right)$ (Table 5.5). However, there was no significant difference in the $E_{\max }$ produced by the different treatments $\left(\mathrm{F}_{(3,32)}=0.6969, \mathrm{p}=0.5608\right)$ (Table 5.5).

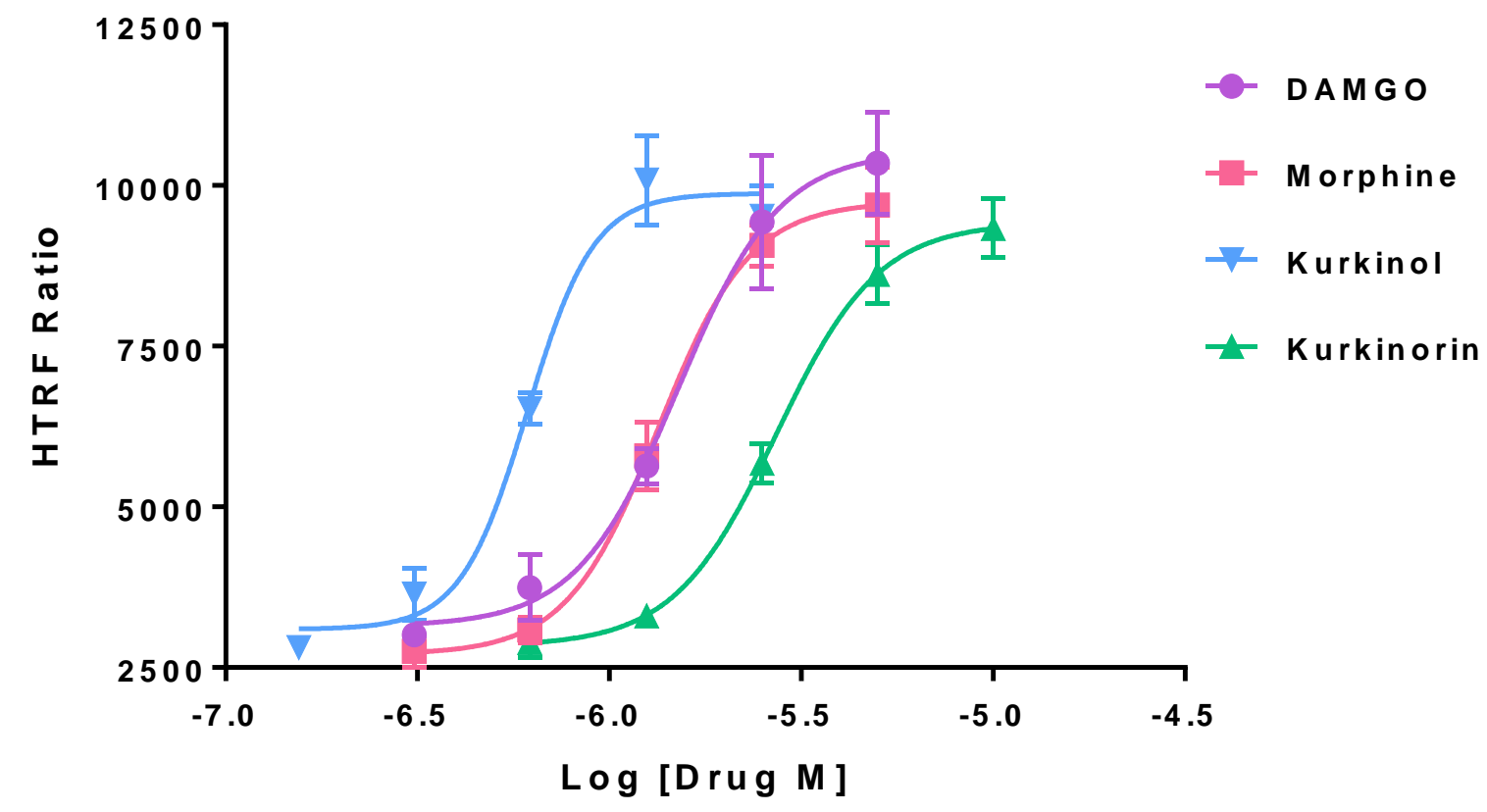

Figure 5.4 Kurkinol and Kurkinorin Significantly Differ in the Activation of pCREB

Kurkinol was found to be a potent activator of the $\beta$-arrestin2 dependent signalling molecule $P C R E B$ in comparison to morphine and DAMGO in $\mathrm{CHO}$ cells stably expressing the human $\mu$ receptor. A rightward shift in the dose response curve was identified with kurkinorin, indicating that it is a poor activator of CREB compared to DAMGO, morphine, and kurkinol. All data are presented as mean \pm SEM from a mean of 3 experimental replicates, each conducted in triplicate. 
Table 5.5 Potency and Efficacy of Kurkinol and Kurkinorin at Activating pCREB in CHO Cells Stably Expressing the Human Mu Opioid Receptor

\begin{tabular}{|c|c|c|c|c|c|c|c|c|c|c|c|}
\hline \multirow[b]{2}{*}{ DAMGO } & \multirow{2}{*}{$\begin{array}{c}\mathrm{EC}_{50} \pm \mathrm{SEM}(\mu \mathrm{M}) \\
1.51 \pm 0.0653\end{array}$} & \multicolumn{2}{|c|}{ P Value to DAMGO } & \multicolumn{2}{|c|}{ P Value to Morphine } & \multirow{2}{*}{ 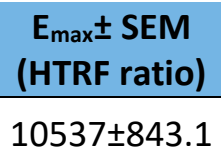 } & \multicolumn{2}{|c|}{ P Value to DAMGO } & \multicolumn{2}{|c|}{ P Value to Morphine } & \multirow{2}{*}{$\begin{array}{c}\mathbf{R}_{\mathbf{2}} \\
0.7213\end{array}$} \\
\hline & & - & - & n.s & n.s & & n.s & n.s & n.s & n.s & \\
\hline Morphine & $1.34 \pm 0.0353$ & n.s & n.s & - & - & $9747 \pm 456.2$ & n.s & n.s & n.s & n.s & 0.8649 \\
\hline Kurkinol & $0.61 \pm 0.0271$ & $<0.0001$ & $* * * *$ & $<0.0001$ & $* * * *$ & $9877 \pm 399.9$ & n.s & n.s & n.s & n.s & 0.8422 \\
\hline Kurkinorin & $2.72 \pm 0.03646$ & 0.0013 & $* *$ & 0.0001 & $* * *$ & $9424 \pm 420.3$ & n.s & n.s & n.s & n.s & 0.8799 \\
\hline
\end{tabular}

Nonlinear regression of the dose response effects on the activation of CREB was used to generate the $E C_{50}$ and $E_{\max }$ values for DAMGO, morphine, kurkinol, and kurkinorin in the human $\mu$ receptor $\mathrm{CHO}$ cell line. The $E C_{50}$ values were found to differ significantly between treatments with no effect on the $E_{\text {max. }}$ Oneway repeated measures ANOVA with Bonferroni multiple corrections test. All data are presented as mean \pm SEM from a mean of 3 experimental replicates, each conducted in triplicate. 


\subsubsection{Phosphorylation of ERK1/2 by Kurkinol and Kurkinorin}

To assess the timing of ERK $1 / 2$ phosphorylation $\mu$ receptor agonists were added to cells stably expressing the human $\mu$ receptor at 5, 10, 20, 30,60,120, and 180 min. Cells were lysed and protein samples run through electrophoresis gel before transfer to PVDF membranes and immunolabeling for pERK (Santa Cruz, sc-7383) and total ERK (Cell Signalling, Ab:9012). The western blots were analysed using ImageJ and the levels of pERK normalised total ERK before calculation of fold change from untreated cells. Full vehicle controls were run for both time course and antagonist experiments, with no significant change in pERK levels identified (Appendix 7.16).

Neither DAMGO nor morphine showed a significant change in pERK levels overtime (DAMGO: $F_{(8,45)}=1.615, p=0.1474$, morphine; $\left.F_{(8,45)}=1.073, p=0.3989\right)$. Both classical $\mu$ receptor agonists did show a trend towards biphasic ERK activation with two distinct activation peaks between 5-20 min and 150-180 $\mathrm{min}$ (Fig. $5.5 \mathrm{~A}, \mathrm{~B}$ ). Kurkinol induced potent ERK activation with significant differences between $5-20 \min \left(F_{(8,44)}=2.649, p=0.0183, p<0.05\right)$. From 30 min onward, pERK expression trends towards a decrease before the levels appear to rise again at $180 \mathrm{~min}$. Overall, the levels of pERK remain at a higher level over the 180 with kurkinol compared to DAMGO and morphine (Fig. 5.5 C). pERK immunolabeling was also significantly higher with kurkinorin treatment over the 180 min period and occurred in a biphasic manner, with early (10-30 $\mathrm{min}$ ) and late (120-150 $\mathrm{min}$ ) phases showing significant increases when compared to untreated cells $\left(\mathrm{F}_{(8,40)}=4.263, p=0.0009, p<0.05\right)$ (Fig. 5.4 D).

To investigate the role of $G$-protein and $\beta$-arrestin2 in the biphasic activation of pERK Hek293 cells stably expressing the human $\mu$ receptor were pre-treated with either $\operatorname{Px}\left(100 \mathrm{~nm} / \mathrm{ml}, 24 \mathrm{hrs}, \mathrm{G}_{\mathrm{i} / \mathrm{o}}\right.$ inhibitor) or dynosore (50 $\mu \mathrm{M}, 1 \mathrm{hr}, \beta$-arrestin2 inhibitor) before the treatment with vehicle or $\mu$ receptor agonists for 0,10 , or $180 \mathrm{~min}$ (Fig. 5.6). As with the full time course, the variation in the experiment masked any differences between treatments for DAMGO $\left(F_{(4,20)}=0.1329, p=0.9685\right)$ and morphine $\left(F_{(4,22)}=1.774, p=0.1700\right)$. While no obvious trend can be seen with DAMGO and antagonists (Fig. 5.6 A), morphine's induction of pERK at 10 min trends towards a decrease with Px, while dynosore appears to decrease activation at the $180 \mathrm{~min}$ time point (Fig. $5.6 \mathrm{~B}$ ). The effect of kurkinol on pERK levels was found to be reversed with dynosore at both 10 and $180 \mathrm{~min}$, while a large decrease was also seen with Px at $10 \mathrm{~min}\left(\mathrm{~F}_{(4,24)}=3.642, p=0.0190, p<0.05\right)$ (Fig. 5.6 C). No significant effect of pre-treatment with either antagonist was found for kurkinorin at 10 or $180 \mathrm{~min}$. A trend towards both pertussis and dynosore reducing the pERK levels at the 10 min time point, while only dynosore reduces activation at $180 \mathrm{~min}\left(\mathrm{~F}_{(4,22)}=1.654, p=0.1965\right)$ (Fig. $\left.5.6 \mathrm{D}\right)$. 

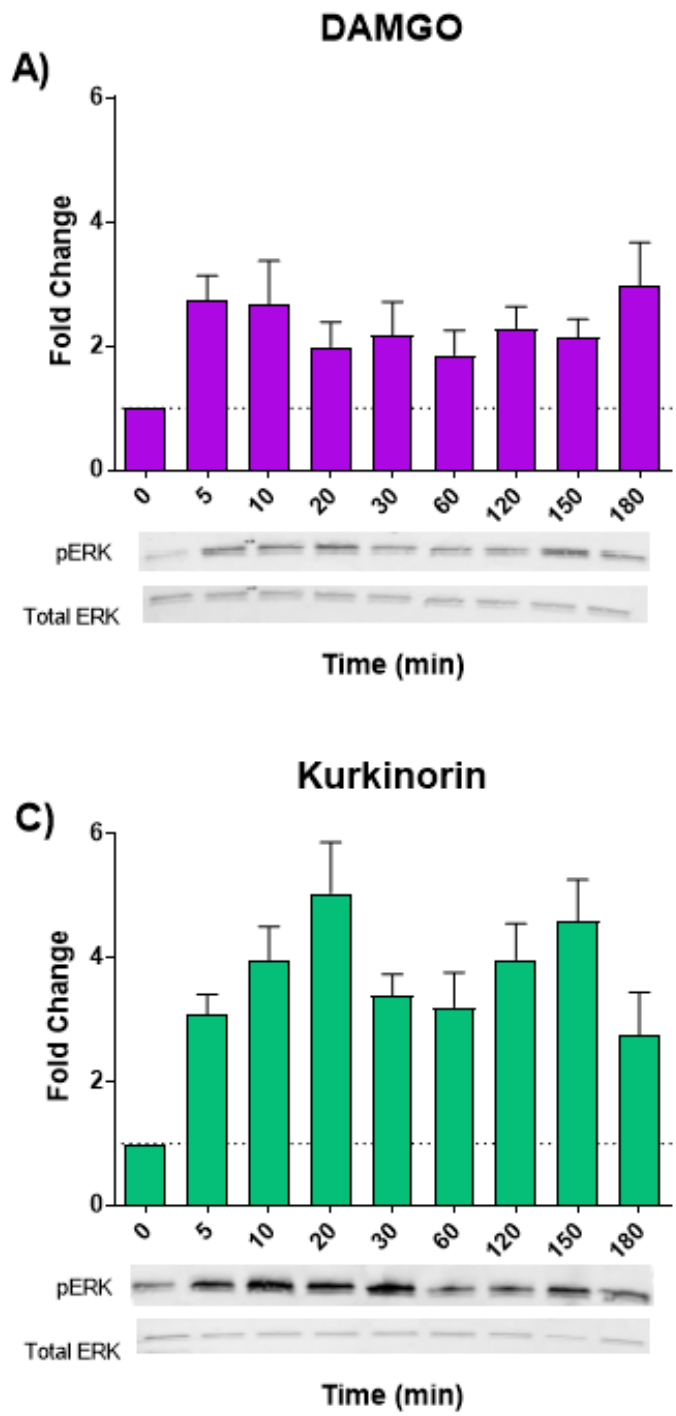

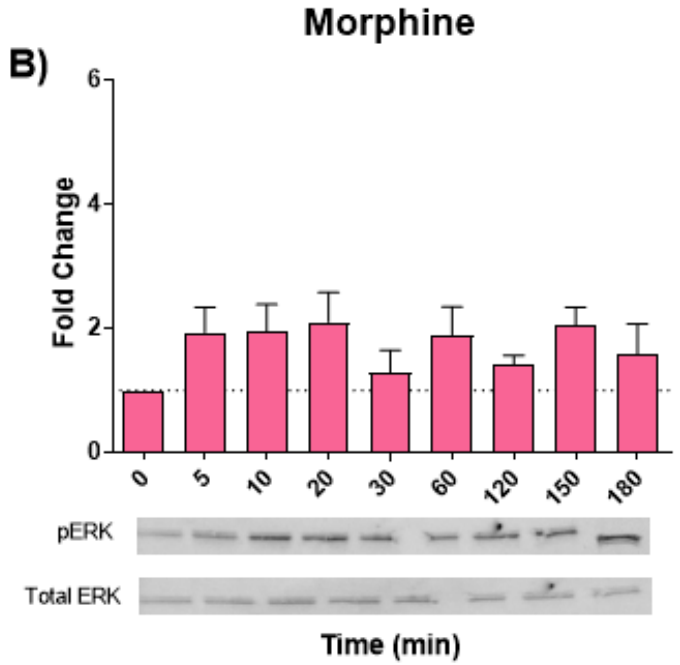

Kurkinol

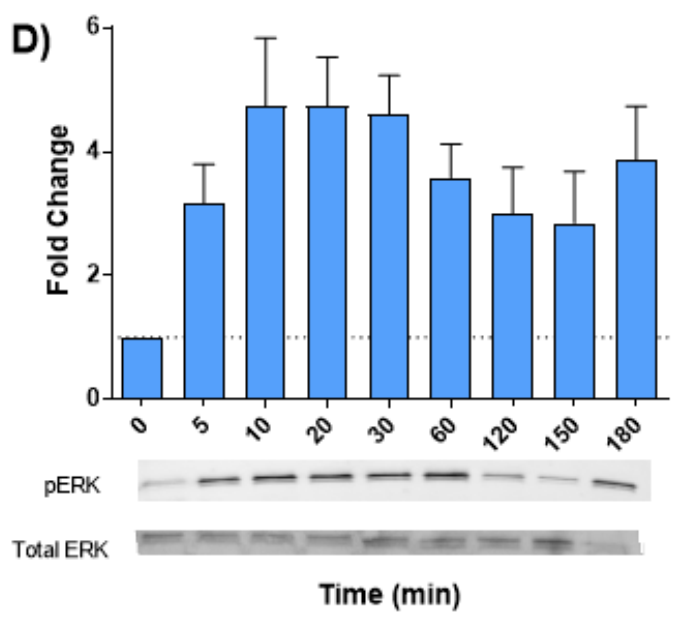

Figure 5.5 Kurkinol and Kurkinorin are Both Potent Bi-phasic Activators of pERK

Western blotting was used to assess the timing of ERK activation was assessed in Hek293 cells stably expressing the human $\mu$ receptor. (A) DAMGO $(1 \mu \mathrm{M})$ increases ERK phosphorylation over a $180 \mathrm{~min}$ time course. (B) Morphine $(10 \mu \mathrm{M})$ time course of ERK activation. (C) Kurkinol (10 $\mu \mathrm{M})$ significantly increases ERK1/2 activation from 5-20 min and appear to have a second phase starting at $180 \mathrm{~min}$. (D) Biphasic ERK phosphorylation can also be seen between 10-30 min and 120-150 min with kurkinorin $(10 \mu M)$. One-way mixed ANOVA with Bonferroni multiple corrections test. ${ }^{*} P<0.05, P<0.01, P<$ 0.001. All data is a mean of 4 experimental replicates. Data presented as mean \pm SEM. 


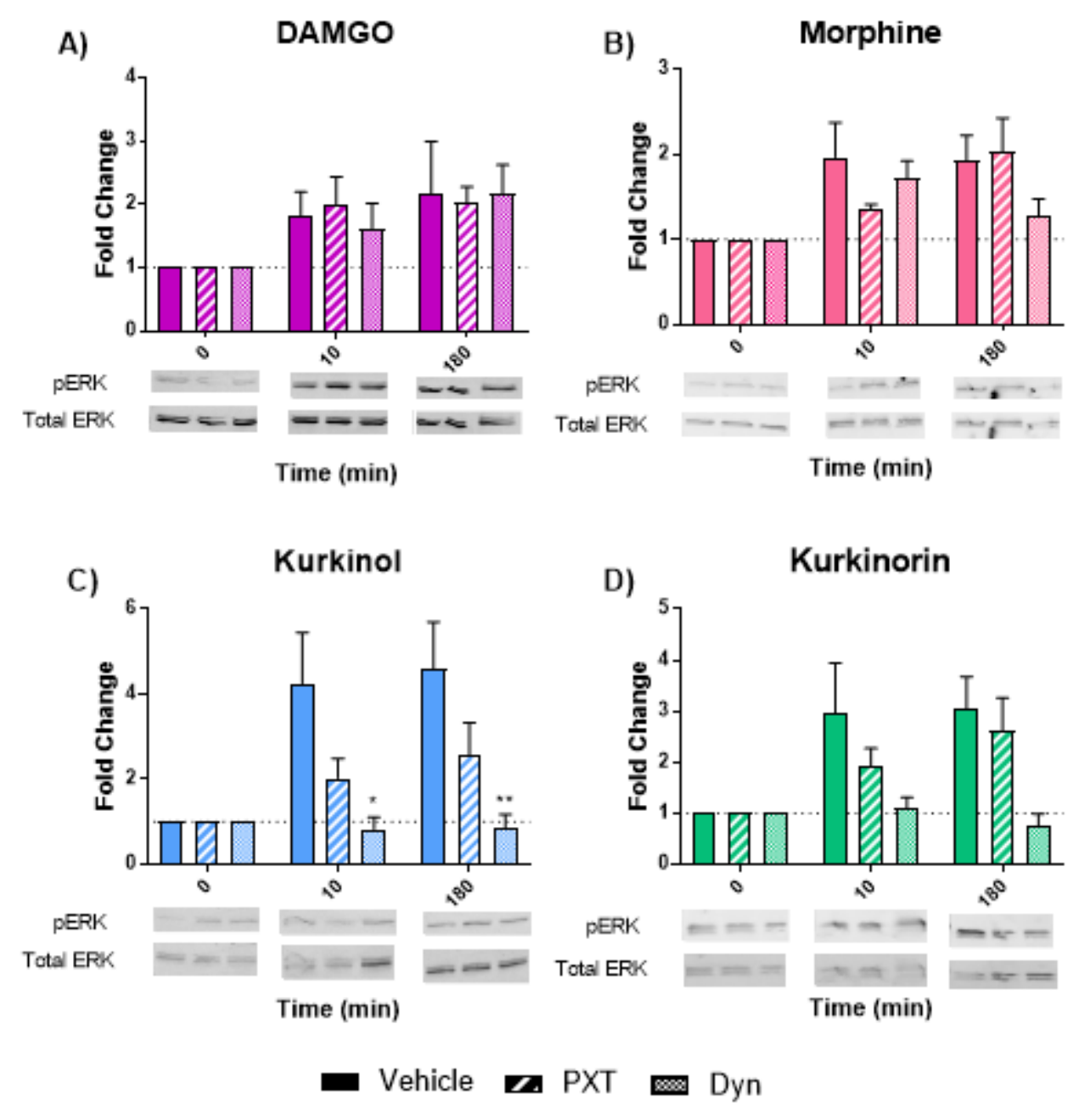

Figure 5.6 Dynosore Reduced both Early and Late Phase Activation of pERK in Hek293 Cells Stably Expressing the Human Mu Opioid Receptor

(A) The phosphorylation of ERK by DAMGO $(1 \mu \mathrm{M})$ is not significantly reduced by either pertussis toxin $(100 \mathrm{ng} / \mathrm{ml})$ or dynosore $(50 \mu \mathrm{M})$ at 10 or $180 \mathrm{~min}$. (B) pERK is also not significantly reduced when pertussis toxin or dynosore are applied alongside morphine $(10 \mu \mathrm{M})$. However, a trend towards decreased pERK with pertussis at $10 \mathrm{~min}$ and decreases with dynosore at $180 \mathrm{~min}$ can be seen. (C) Dynosore significantly reduces pERK levels at both 10 and $180 \mathrm{~min}$. (D) Both pertussis and dynosore appear to reduce ERK phosphorylation at $10 \mathrm{~min}$, while only dynosore reduced levels at $180 \mathrm{~min}$ of treatment. Two-way mixed ANOVA with Bonferroni multiple corrections test. ${ }^{*} P<0.05, P<0.001$. All data is a mean of 4 experimental replicates. Data presented as mean \pm SEM. 


\subsubsection{Generation of Bias Factors for Kurkinol and Kurkinorin}

When the selectivity of kurkinorin, kurkinol, DAMGO, and morphine were assessed for Gprotein and $\beta$-arrestin2 signalling pathway activation, kurkinol was shown to be a strong activator of the $\mathrm{G}$-protein dependent pathways with little to no activation of the $\beta$-arrestin2 pathways. Conversely, morphine appeared to show poor selectivity for all pathways measured. While kurkinorin appeared to be a more balanced agonist (Fig 5.7). This pathway selectivity data appears to indicate that kurkinol is an extremely G-protein biased agonist, while kurkinorin and morphine appear more balanced. To confirm the pattern of biased agonism for these compounds the dose response data were used to generate bias factors. To assess the effects of cell line and calculation type on bias factor generation we used both the simplified bias calculation (Crowley et al., 2020, Crowley et al., 2016) and the RA calculation (Gillis et al., 2020a, Rajagopal et al., 2010) (See Chapter 2, Section 2.4). To further add to the analysis we assessed the effect of using two different cell lines in the generation of bias factors and utilised non-amplifying G-protein dependent effectors signalling to remove some of the system bias.

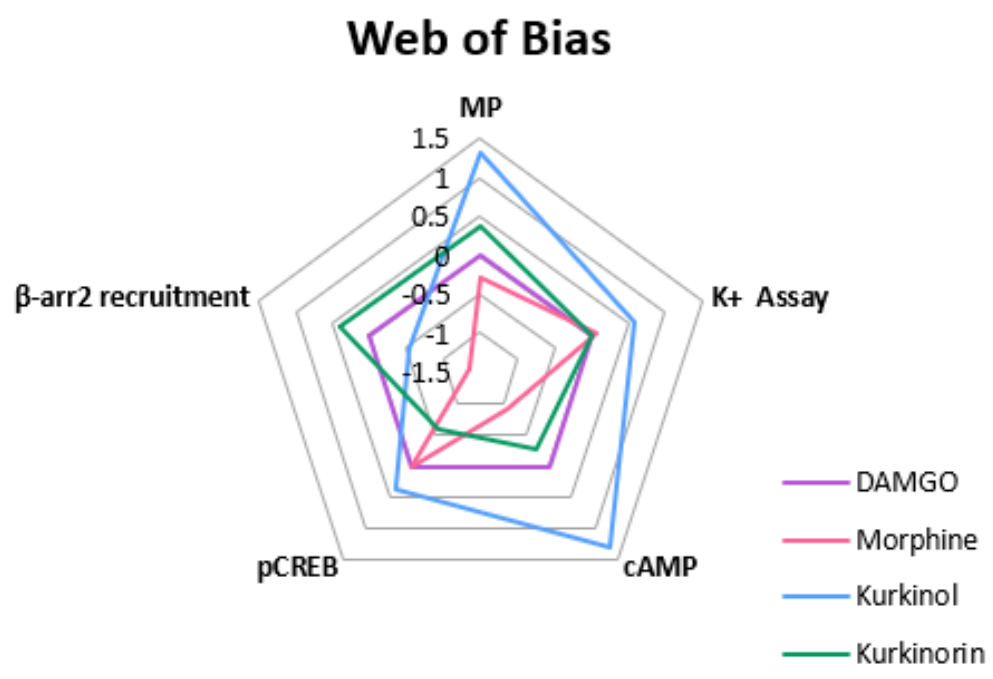

Figure 5.7 Kurkinol Shows Increased Selectivity for G-Protein Dependent Pathways

Radar plot of pathway selectivity, compared to DAMGO as a reference agonist, expressed as $\triangle \log \left(E_{\max } / E C_{50}\right)$ values for three G-protein dependent pathways and two $\beta$-arrestin2 dependent pathways in $\mathrm{CHO}$ cells stably expressing the human $\mu$ receptor.

\section{Abbreviations:}

$\beta$-arr2 $=\beta$-arrestin2

$c A M P=$ cyclic adenosine monophosphate $\mathrm{K}+=$ potassium
$M P=$ Membrane Potential

PCREB = phosphorylated $C A M P$ response element binding protein 
When the effect of the cell line was compared using the Membrane Potential assay in both Hek293 and $\mathrm{CHO}$ cells and bias calculated using the PCREB assay in $\mathrm{CHO}$ cells to evaluate the downstream $\beta$-arrestin2 signalling, large discrepancies were identified between the 2 cell lines. The simplified bias calculation identified all 3 of the test $\mu$ receptor agonists as $\beta$-arrestin2 biased, irrespective of the cell background (morphine; $\mathrm{CHO}=-0.30$, Hek293 $=-1.04$, kurkinol; $\mathrm{CHO}=0.88, \mathrm{Log}$ Hek293 $=-0.03$, kurkinorin $\mathrm{CHO}=0.68$, Hek293 = 0.36) (Table 5.6). When the $\mathrm{RA}_{\mathrm{i}}$ model was used to determine the pathway bias using the data from $\mathrm{CHO}$ cells a similar pattern of bias was observed as previously described, with morphine ( $\left.\log R A_{i}=0.78\right)$ showing the weakest G-protein bias, followed by kurkinorin ( $\log R A_{i}=1.77$ ) and then kurkinol having strong G-protein bias (Log $\left.R A_{i}=1.97\right)$ (Crowley et al., 2020, Crowley et al., 2016).

In contrast, when the Membrane Potential data from Hek293 cells was compared to the PCREB data from the CHO cells, all three compounds were strongly $\beta$-arrestin2 biased using the Log $R A_{i}$ (morphine Log $R A_{i}=-2.78$, kurkinol $\log R A_{i}=-1.78$, kurkinorin $\log R A_{i}=-1.37$ ) and simplified bias calculations (morphine $\beta=-1.04$, kurkinol $\beta=-0.03$, kurkinorin $\beta=0.36$ ). These results show significant signalling differences exist between cell lines, introducing inaccuracy that is not controlled by these equations (Table 5.6).

We further investigated the ability of bias calculations to account for differences in the subcellular pathways used to measure G-protein activation. With both calculations run using either the Membrane Potential or potassium assay in $\mathrm{CHO}$ cells vs. the phosphorylation of CREB in CHO cells stably expressing the human $\mu$ receptor (Table 5.6). The simplified bias calculation again identified morphine (GIRK; $\beta=0.03$ ), kurkinol (GIRK; $\beta=0.20$ ), and kurkinorin (GIRK; $\beta=0.31$ ) as having varying levels of $\beta$-arrestin2 bias, independent of the measure of $G$-protein activation (Table 5.6). When the $\mathrm{RA}_{\mathrm{i}}$ model was applied to the potassium assay data the degree of bias no longer followed the same pattern of bias. Instead, morphine ( $\left.\log R A_{i}=-0.28\right)$ and kurkinol $\left(\log R A_{i}=-0.10\right)$ were identified as moderately $\beta$-arrestin2 biased, while kurkinorin was very weakly G-protein biased ( $\log R A_{i}=0.0007$ ) (Table 5.6).

We hypothesised that by combining multiple subcellular signalling pathways associated with G-protein and $\beta$-arrestin2 recruitment we would be able to generate a bias factor that more accurately represented the signalling in vitro, providing an improved correlation to the behavioural effects in vivo. Therefore, the two measures of $\mathrm{G}$-protein activation in human $\mu$ receptor $\mathrm{CHO}$ cells were combined to give a single $\mathrm{G}$-protein $\mathrm{EC}_{50}$ and $\mathrm{E}_{\max }$ per compound. this was then compared to the level of CREB phosphorylation. When the simplified bias calculation was used all 3 test compounds were identified as $\beta$-arrestin2 biased (morphine; $\beta=-0.53$, kurkinol; $\beta=-0.21$, kurkinorin; $\beta=-0.10$ ). Whereas the $R A_{i}$ model identified all 3 test compounds as $\mathrm{G}$-protein biased with morphine showing the lowest level of $\beta$-arrestin2 bias ( $\left.\log R A_{i}=0.55\right)$, followed by kurkinol $\left(\log R A_{i}=0.87\right)$ and kurkinorin 
(Log $\left.R A_{i}=0.98\right)$ (Table. 5.6). Again, indicating that the $R A_{i}$ model provides a more robust assessment of signalling bias. 
Table 5.6 Comparison of the Bias Factors Generated from Two Different Cell Backgrounds

\begin{tabular}{|c|c|c|c|c|c|c|c|c|}
\hline & \multicolumn{3}{|c|}{ G-Protein Pathway } & \multicolumn{3}{|c|}{$\beta$-arrestin2 pathway } & \multirow{2}{*}{$\begin{array}{c}\text { Simplified } \\
\text { Bias } \\
\text { Factor }\end{array}$} & \multirow{2}{*}{$\begin{array}{c}\log R A_{i} \\
\text { Bias } \\
\text { Factor }\end{array}$} \\
\hline & Cell Line and Assay & $\mathrm{EC}_{50}(\mu \mathrm{M})$ & $E_{\max }(\%$ DAMGO) & Cell type & $\mathrm{EC}_{50}(\mu \mathrm{M})$ & $E_{\max }(\%$ DAMGO) & & \\
\hline DAMGO & $\begin{array}{c}\text { CHO Membrane Potential } \\
\text { Hek293 Membrane Potential } \\
\text { CHO Potassium } \\
\text { CHO combined }\end{array}$ & $\begin{array}{c}0.43 \pm 0.13 \\
11.24 \pm 8.41 \\
2.17 \pm 1.29 \\
1.303 \pm 0.13 \\
\end{array}$ & $\begin{array}{c}100 \pm 6 \\
100 \pm 8 \\
100 \pm 14 \\
100 \pm 10 \\
\end{array}$ & $\mathrm{CHO}$ & $1.51 \pm 0.0653$ & $100 \pm 8$ & $\begin{array}{l}1 \\
1 \\
1 \\
1\end{array}$ & $\begin{array}{l}0 \\
0 \\
0 \\
0\end{array}$ \\
\hline Morphine & $\begin{array}{c}\text { CHO Membrane Potential } \\
\text { Hek293 Membrane Potential } \\
\text { CHO Potassium } \\
\text { CHO combined }\end{array}$ & $\begin{array}{l}1.07 \pm 0.12 \\
81.03 \pm 6.17 \\
2.76 \pm 0.66 \\
1.91 \pm 0.12\end{array}$ & $\begin{array}{c}129 \pm 6 \\
68 \pm 6 \\
144 \pm 30 \\
136.5 \pm 18\end{array}$ & $\mathrm{CHO}$ & $1.34 \pm 0.0353$ & $93 \pm 4$ & $\begin{array}{l}-0.30 \\
-1.04 \\
0.03 \\
-0.53\end{array}$ & $\begin{array}{l}0.78 \\
-2.78 \\
-0.28 \\
0.55\end{array}$ \\
\hline Kurkinol & $\begin{array}{c}\text { CHO Membrane Potential } \\
\text { Hek293 Membrane Potential } \\
\text { CHO Potassium } \\
\text { CHO combined }\end{array}$ & $\begin{array}{c}0.028 \pm 0.017 \\
5.65 \pm 0.73 \\
0.68 \pm 0.25 \\
0.35 \pm 0.017\end{array}$ & $\begin{array}{c}115 \pm 8 \\
107 \pm 4 \\
118 \pm 17 \\
116.5 \pm 12.5\end{array}$ & $\mathrm{CHO}$ & $0.61 \pm 0.0271$ & $94 \pm 4$ & $\begin{array}{l}0.88 \\
-0.03 \\
0.20 \\
-0.21\end{array}$ & $\begin{array}{l}1.97 \\
-1.78 \\
-0.10 \\
0.87\end{array}$ \\
\hline Kurkinorin & $\begin{array}{c}\text { CHO Membrane Potential } \\
\text { Hek293 Membrane Potential } \\
\text { CHO Potassium } \\
\text { CHO combined }\end{array}$ & $\begin{array}{c}0.198 \pm 0.072 \\
9.04 \pm 0.89 \\
2.22 \pm 1.62 \\
1.21 \pm 0.072\end{array}$ & $\begin{array}{c}111 \pm 6 \\
92 \pm 5 \\
105 \pm 14 \\
108 \pm 10\end{array}$ & $\mathrm{CHO}$ & $2.72 \pm 0.03646$ & $89 \pm 4$ & $\begin{array}{l}0.68 \\
0.36 \\
0.31 \\
-0.10\end{array}$ & $\begin{array}{c}1.77 \\
-1.37 \\
0.0007 \\
0.98\end{array}$ \\
\hline
\end{tabular}

Simplified bias calculation; 1 is a balanced agonist, less than 1 is $\beta$-arrestin2 biased, greater than 1 is G-protein biased $\log R A_{i} ; O$ is balanced, less than $O$ is $\beta$-arrestin2 biased, greater than $O$ is $G$-protein biased 


\subsection{5. pCREB Efficacy Correlates to Therapeutic Window}

A simple linear regression analysis of the relationship between efficacy for the phosphorylation of CREB and the therapeutic window (tail-flick or PTX mechanical Vs charcoal meal) revealed a high correlation coefficient (Tail-flick $R^{2}=0.7071$, PTX mechanical $R^{2}=0.8407$ ) (Fig. $5.8 \mathrm{~A}$ B). This data suggests that CREB phosphorylation may be a good in vitro marker to predict the level of constipation $\mu$ receptor agonists may induce. A correlation was also identified between the different bias factors calculated with the RA $A_{i}$ model when PCREB was used to assess $\beta$-arrestin 2 signalling. This was found irrespective of the measure of G-protein signalling used when all in vitro studies were performed in $\mathrm{CHO}$ cells stably expressing the human $\mu$ receptor (Fig. 5.9 A-D). With $\mathrm{R}^{2}$ values ranging from 0.4341 to 0.6821 . Interestingly, a moderate positive correlation was also found between the therapeutic window and $\mathrm{RA}_{i}$ signalling bias when the data from Membrane Potential assay performed in Hek293 cells were used. Despite all $\mu$ receptor agonists being identified as $\beta$-arrestin2 biased, the closer towards balanced the agonist was the larger the therapeutic window. With $R^{2}$ values of 0.7168 and 0.5627 for the therapeutic windows generated with the tail-flick and mechanical PTX data respectively (Fig. 5.9 E, F). When the relationship between the therapeutic window and signalling bias was assessed using bias factors generated from the simplified method of bias a moderate to low correlation was identified, with $R^{2}$ values ranging from 0.0809 to 0.6821 (Appendix Fig. 7.17). This indicates that the simplified bias calculation does not produce bias factors that allow for the prediction of the behavioural effects of $\mu$ receptor agonists.

A)

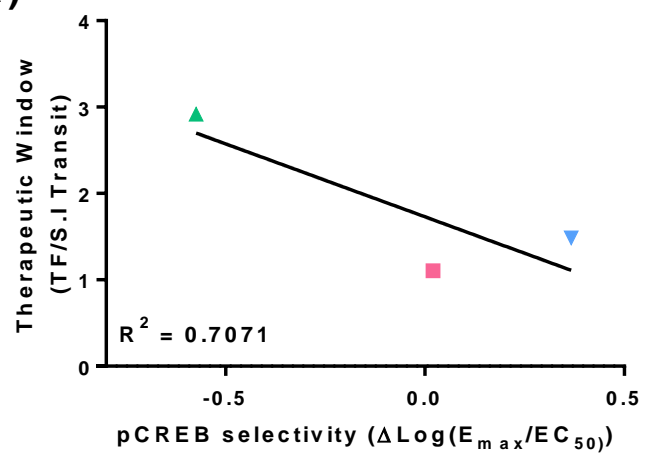

B )

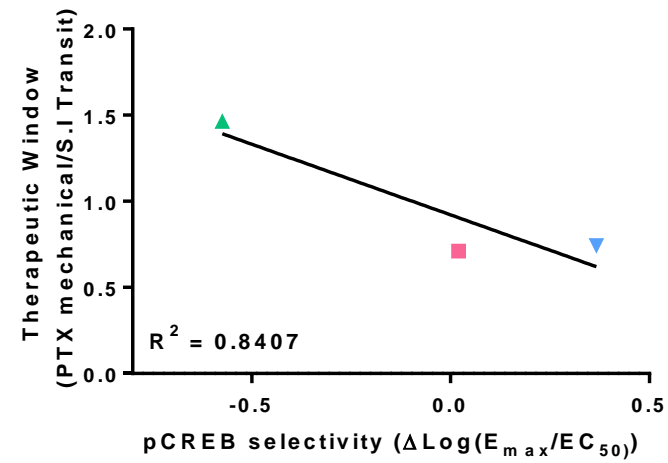

- Morphine $\nabla$ Kurkinol $\Delta$ Kurkinorin

Figure 5.8 Decreased CREB phosphorylation Selectivity Correlates to Improved Therapeutic Windows

$(A, B)$ The selectivity for $p C R E B$ activation $\left(\triangle \log \left(E_{\max } / E C_{50}\right)\right)$ was plotted against the therapeutic window generated using either the tail-flick or PTX induced mechanical allodynia as the measure of anti-nociception in comparison to the charcoal meal assay. Simple linear regression analysis showed that decreased CREB activation was strongly correlated to increased therapeutic windows generated using either (A) tail-flick and (B) paclitaxel mechanical dose response data. 
A)

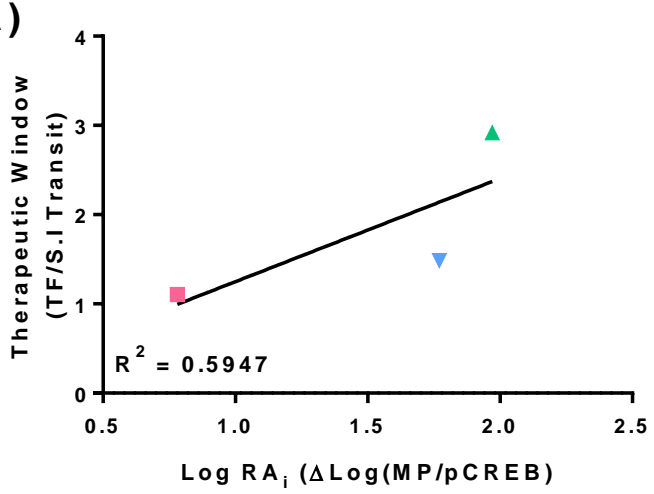

C)

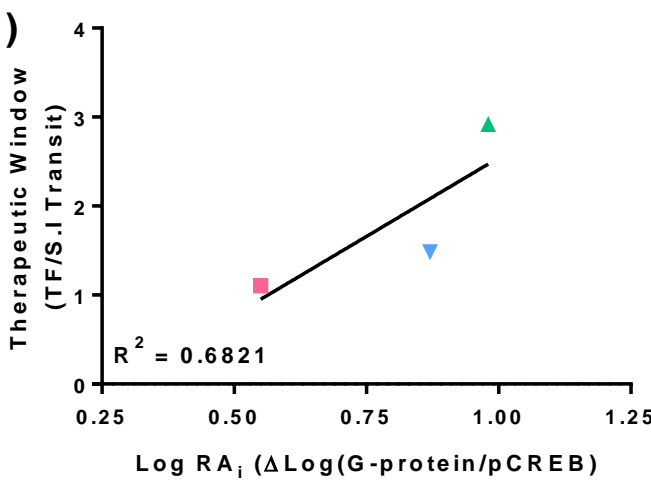

E)

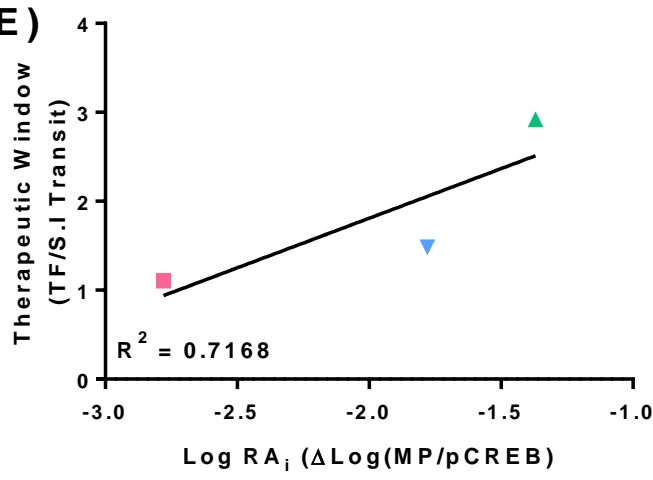

B)

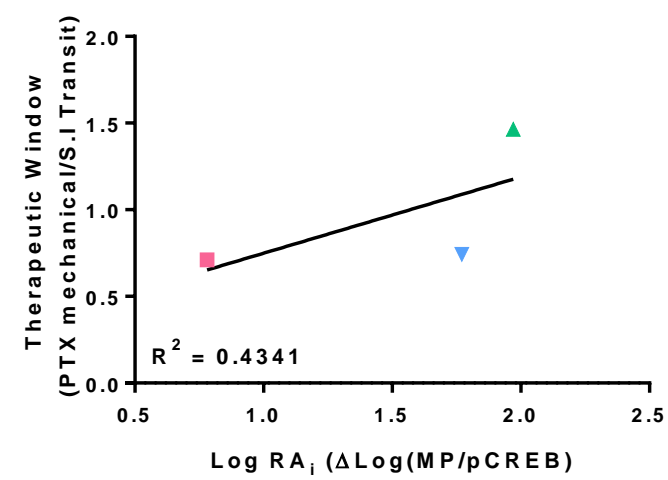

D)

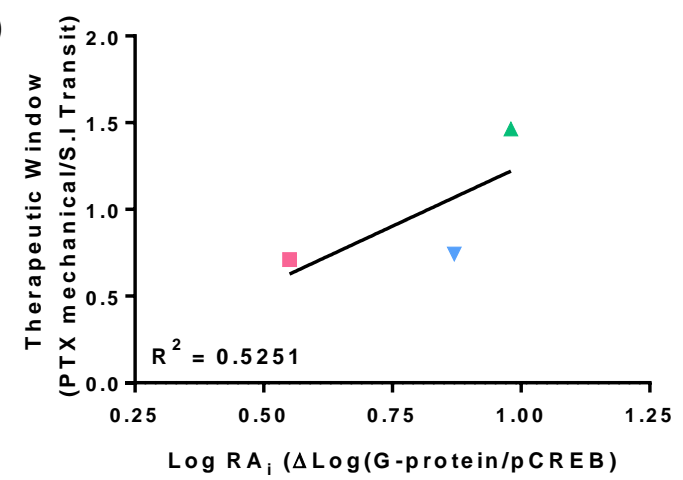

F)

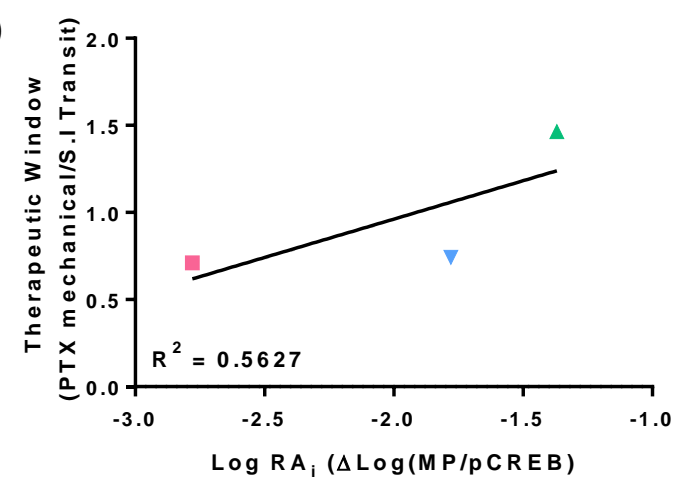

- Morphine $\checkmark$ Kurkinol $\Delta$ Kurkinorin

Figure 5.9 Increased Therapeutic Windows Correlate to Increased G-Protein Bias

Increased G-protein biased signalling as determined by the $\Delta \log R A_{i}$ equation using data from the (A, B) Membrane Potential assay, (C, D) combined G-protein (Membrane Potential and potassium assay) in $\mathrm{CHO}$ cells stably expressing the human $\mu$ receptor, and the (E, F) Membrane Potential assay in Hek293 cells vs the PCREB assay in CHO cells all showed moderately positive correlations to larger therapeutic windows. 


\subsection{Discussion}

The clinical utility that $\mu$ receptor G-protein biased agonist may have in the treatment of strong acute and chronic pain is not clear, this is in large part due to the bias factors calculated from in vitro data not translating to pre-clinical or clinical in vivo benefits. Many underlying factors have been identified as influencing the generation of bias factors, potentially identifying high rates of false positive G-protein biased agonists, explaining the disparity between bias factors and the behavioural profiles of $\mu$ receptor biased agonists. In this study, we aimed to assess the effects of some of these factors, including the role of cell background, non-amplifying measures of $\beta$-arretin2 signalling, and alternate subcellular signalling pathways have on the generation of bias. To achieve this, we conducted two non-amplifying assays of G-protein activation, the Molecular Devices Membrane Potential and Potassium Assay in addition to quantifying the $\beta$-arrestin2 dependent activation of CREB. These are in addition to the HitHunter cAMP and PathHunter $\beta$-arrestin2 recruitment assays previously used by our research team to assess signalling bias at the $\mu$ receptor (Crowley et al., 2020, Crowley et al., 2016). This enables signalling bias to be evaluated in greater depth. To further investigate how the bias calculation is affected by cell type the Membrane Potential assay was also conducted in $\mathrm{CHO}$ and Hek293 cells expressing the human $\mu$ receptor. This data was then run through 2 different calculations to determine signalling bias and to assess the reproducibility of bias factors calculated.

\subsubsection{G-Protein Dependent Pathway Activation Matches Pre- Determined Bias}

\subsubsection{Membrane Potential Assay of Adenylyl Cyclase Inhibition}

The Membrane Potential assay is the most common non-amplifying measure of cAMP inhibition used for the assessment of biased signalling at the $\mu$ receptor (Gillis et al., 2020a, Knapman et al., 2014a, Knapman et al., 2014b, Knapman et al., 2012). Upon the application of the AC activator, forskolin, the membrane hyperpolarises to induce efflux of the fluorescent dye. When forskolin is added in the presence of a $\mu$ receptor agonist, dose dependent inhibition of AC occurs. This is hypothesised to occur via the activation of PKA and $\mathrm{K}^{+}$channel inhibition resulting in the efflux of the fluorescent dye in both $\mathrm{CHO}$ and AT-1 cells, however, the exact mechanism is not known (Knapman et al., 2012, Knapman et al., 2014a, Knapman et al., 2014b).

Using this assay, we were able to show that the novel $\mu$ receptor agonists kurkinol and kurkinorin were full agonists at this pathway with $20 \%$ and $38 \%$ increases in potency in Hek293 cells stably expressing the human $\mu$ receptor respectively. We further investigated the effect of cell type on the inhibition of forskolin-induced membrane depolarisation by performing this assay in $\mathrm{CHO}$ cells stably expressing the human $\mu$ receptor. The same potency trends were identified in this cell line, with kurkinol $($ morphine $=85 \%$, DAMGO $=93 \%)$ and kurkinorin $($ morphine $=82 \%$, DAMGO $=93 \%)$ more 
potent than morphine and DAMGO (Fig 5.10). These effects on membrane potential were determined to be $\mu$ receptor dependent as pre-treatment with the $\mu$ receptor antagonist $\beta$-FNA reduced the response.

Despite the identification of the same pattern of potency, significant differences were identified between the $\mathrm{EC}_{50}$ values. With both $\mathrm{DAMGO}$ and morphine found to be more potent in $\mathrm{CHO}$ cells compared to the Hek293 cell line. This has large implications for the output of the bias calculations as a shift in the reference compound (DAMGO) will change what is defined as 'balanced' within the calculations. The shift in DAMGOs (reference) ability to activate subcellular signalling molecules in the $\mathrm{CHO}$ and Hek293 cells may explain the disparity between the bias factors for morphine (Hek293 = -2.78, CHO = 0.78), kurkinol (Hek293 = -1.78, CHO = 1.97), and kurkinorin (Hek293 $=-1.37, \mathrm{CHO}=1.77$ ), when the cell type is the only variable changed (Table 5.6). However, this analysis is complicated by the extremely high doses required to elicit a dose response in the Hek293 cell line. Not only are the $\mathrm{EC}_{50}$ values for morphine and DAMGO significantly higher than what has been published previously, but the highest doses would have also saturated the culture (Knapman et al., 2012, Knapman et al., 2014a, Knapman et al., 2014b). Resulting in the compounds acting directly on ion channels and influencing the $\mathrm{EC}_{50}$ values. Based on this, the data from this cell line cannot be taken as a direct measure of $\mu$ receptor signalling and the validity of any further analysis of this data is called into question.

\subsubsection{Kurkinol and Kurkinorin May Act A Partial Agonist at GIRK Channels}

To further explore the effects of the $\mu$ receptor agonism on Membrane Potential we quantified the ability of kurkinol and kurkinorin to activate the GIRK ( $\left.\mathrm{K}_{\mathrm{ir} 3}\right)$, a G-protein dependent signalling pathway, using the Molecular Devices Potassium Assay. As with the Membrane Potential assay, efflux of a $\mathrm{K}^{+}$channel sensitive dye allows the measurement of G-protein activation without signal amplification (Knapman et al., 2015).

Unlike previous measures of G-protein activation, no difference was identified between the potency or maximal effects induced by DAMGO, morphine, kurkinol, or kurkinorin. Upon further investigation we found that the $\mathrm{CHO}$ cell line contains low levels of endogenous potassium currents (Yu and Kerchner, 1998, Gamper, Stockand, and Shapiro., 2005). Therefore, in order for the sensitivity of this assay to be improved, and true effects of our novel compounds on potassium channels assessed, this experiment needs to be repeated with the co-transfection of $K_{\mathrm{ir} 3} \mathrm{GIRK}$ channels. Due to this error in experimental protocol the data from this experiment and any further analysis does not represent the true signalling and cannot be taken as accurate.

When performed following the correct experimental protocol Gillis et al. (2020a) showed the "G-protein" biased agonists TRV130, PZM21, SR-17018, and methadone are partial agonists for GIRK activation in the Potassium assay. Furthermore, herkinorin, the parent compound of kurkinol and 
kurkinorin, TRV130 and PZM21 show reduced GIRK2 activation in Hek293 cells. This led Yudin et al. (2019) to conclude that either $\beta$-arrestin2 is required for GIRK activation, or these drugs are partial agonists for this pathway. Despite this, no definitive answer can be given on the role partial agonism at GIRK channels plays in the behavioural profiles of kurkinol and kurkinorin. With partial agonism of the TRPM3 channels, VG Ca ${ }^{2+}{ }_{2.2}$ channels, and $\mathrm{G} \alpha$, recruitment upon $\mu$ receptor activation by TRV130 and PZM21. This inclusive signalling profile indicates that partial agonism of multiple G-protein dependent pathways, not just at GIRK channels, may be required for widening the therapeutic window (Gillis et al., 2020a, Yudin et al., 2019).

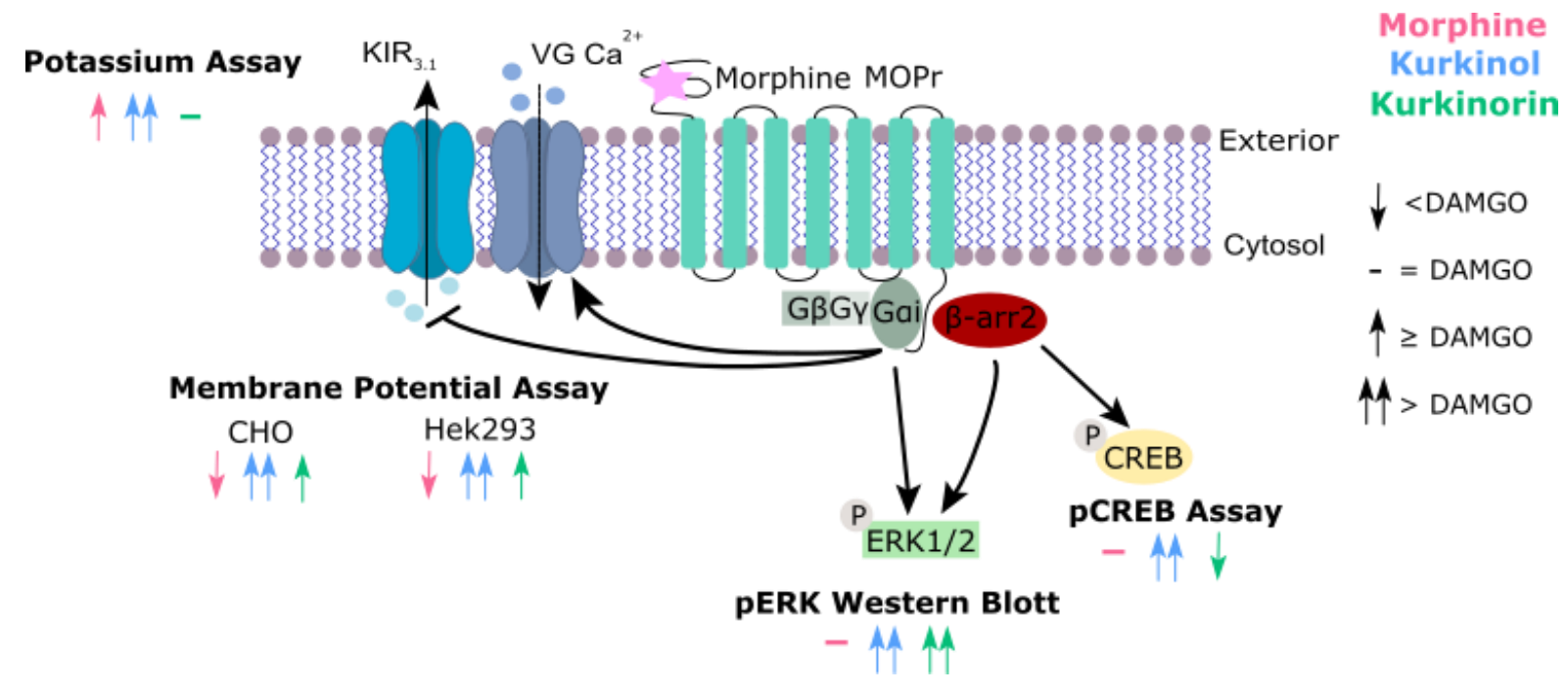

Figure 5.10 Summary of Pathway Selectivity at the Mu Opioid Receptor for Morphine, Kurkinol, and Kurkinorin

The selectivity in the Potassium assay, Membrane Potential assay, and pCREB assay were determined using the $\triangle \log \left(E_{\max }{ }^{\prime} E C_{50}\right)$ and the level of ERK phosphorylation by the fold change of $p E R K$ compared to vehicle. The level of selectivity for each pathway is presented as a slight increase ( $\uparrow$ ), large increase $(\uparrow \uparrow)$, decrease $(\downarrow)$, or no change (-) in comparison in the reference ligand DAMGO. 


\subsubsection{Kurkinorin is a Poor Activator of Mu Opioid Receptor-Induced CREB}

Phosphorylation of the transcription factor, CREB, is thought to play a major role in the rewarding properties of many drugs of abuse, including the $\mu$ receptor agonists morphine (Narita et al., 2010), Tramadol (Sadat-Shirazi et al., 2019), and the fentanyl analogue, ohmefentanyl (Gao et al., 2003). Further evidence supports the role of $\mu$ receptor-induced CREB activation modulating reward, with CREB mutant mice (reduced activity) showing no conditioned place preference to morphine (Walters et al., 2001). CREB phosphorylation has been shown to be dependent on $\beta$-arrestin signalling at the $\mathrm{k}$ receptor (Bruchas and Chavkin, 2010, Kang et al., 2005) while at the muscarinic receptor the induction of pCREB occurs in a $\beta$-arrestin-ERK dependent manner (Sabbir and Fernyhough, 2018). Furthermore, G-protein pathways, such as CAMP, correlate to decreased pCREB immunoreactivity within the catecholaminergic cell line of neuronal origin (Widnell et al., 1994). Based on this research on other GPCRs and the in vivo role of CREB on $\mu$ receptor-induced reward we chose to investigate the induction of CREB by DAMGO, morphine, kurkinol, and kurkinorin as a downstream measure of $\beta$ arrestin2 signalling.

We showed that although all the $\mu$ receptor agonists tested had the same efficacy in this pathway, there were significant differences in potency with kurkinol the most potent, followed by morphine, DAMGO, and finally kurkinorin (Fig. 5.10). Interestingly, kurkinorin (the second most potent and efficacious in the G-protein assays) was found to be the least potent activator of CREB. Furthermore, the level of PCREB showed a strong correlation to higher therapeutic windows generated from the tail-flick or CINP mechanical allodynia dose responses and inhibition of the small intestine gut motility. This correlation may be expandable to other $\mu$ receptor induced side effects as kurkinorin also induces less respiratory depression and motor coordination impairment compared to kurkinol and morphine. The correlation between the bias factors generated using the level of CREB phosphorylation further indicates that it provides a more accurate indication of the behavioural profile of novel $\mu$ receptor agonists in vivo. Although requiring further validation, this pathway holds promise for the development of better in vitro screens with improved translation to in vivo $\mu$ receptor dependent behaviours.

\subsubsection{Kurkinol and Kurkinorin are Potent Activators of ERK}

GPCR activation of ERK has been shown to occur in a biphasic manner, with early phase phosphorylation ( $<20 \mathrm{~min}$ ) associated with G-protein activation while later phases of phosphorylation (> $60 \mathrm{~min}$ ) are typically associated with $\beta$-arrestin2 signalling (Belcheva et al., 2005, Shenoy et al., 2006). Our data has shown that bi-phasic ERK phosphorylation occurs at the $\mu$ receptor, however, only kurkinol and kurkinorin showed significant increases in pERK from baseline. With kurkinol inducing increases between $5-30$ min while kurkinorin induced increases at both $10-30 \mathrm{~min}$ and $120-150$ 
$\min$ (Fig. 5.10). To further investigate the role of G-protein vs. $\beta$-arrestin2 dependent signalling pathways, ERK activation was assessed in the presence of $\mathrm{G}_{\mathrm{i} / \mathrm{o}}$ inhibitor or $\beta$-arrestin2 inhibitor at the selected time points of $10 \mathrm{~min}$ and $180 \mathrm{~min}$. Px is a well-established inhibitor of $\mathrm{G}_{\mathrm{i} / \mathrm{0}}$ protein dependent signalling, however, at the time this experiment was performed there were no readily available $\beta$ arrestin2 inhibitors. Therefore, in order to block $\beta$-arrestin2 dependent ERK activation we used a dynamin inhibitor, Dynosore, as $\beta$-arrestin has been shown to drive MAPK signalling in clathirin coated structures once internalised (Eichel et al., 2016, Garcia et al., 2009). Therefore, inhibiting these areas, with dynosore and similar compounds, has been proposed to inhibit arrestin dependent MAPK signalling. Once again, no significant difference for DAMGO or morphine was found when the inhibitors of G-protein or $\beta$-arrestin2 were present. Interestingly, kurkinol and kurkinorin showed a full reversal in the presence of both inhibitors. This indicates a flaw in the experimental procedure and provides no further indications on the role of $\mathrm{G}$-protein and $\beta$-arrestin2 dependent signalling in the biphasic nature of $\mathrm{pERK}$. This is most likely due to the low sensitivity of western blotting. When coupled with low reproducibility and high error (35-80\%), smaller changes in protein levels are often missed using this technique (Koller et al., 2005). To increase the sensitivity and speed of the measurement of ERK phosphorylation to identify its role in the behavioural profiles of $\mu$ receptor agonists the use of high throughput assays, such as the AlphaScreen ${ }^{\circledR}$ SureFire ${ }^{\circledR}$ pERK1/2 assay, would be required.

While research has shown the biphasic nature of pERK is due to G-protein vs $\beta$-arrestin2 signalling the link to the behavioural profiles is not clear. Interestingly, the current data indicates that high levels of ERK phosphorylation, irrespective of activation time, is associated with negative physiological outcomes. For example, high levels of pERK are typically seen within the shell of the nucleus accumbens (early and late phase) and the VTA (early phase) (mesolimbic system) following the administration of morphine and fentanyl and is associated with preference for drug paired chambers in mice and rats using the conditioned place preference assay (Lesscher et al., 2003, Rosas et al., 2016). While in the dorsal horn of the spinal cord, high levels of pERK are associated with antinociceptive tolerance and hyperalgesia (Bobeck et al., 2016, Deng et al., 2019). Furthermore, inhibition of MEK1/2 or ERK1/2 increases the anti-nociceptive properties of morphine in the $\mathrm{CCl}$ model of neuropathic pain (Popiolek-Barczyk et al., 2014), and alleviates hyperalgesia induced by inflammation (Ciruela et al., 2003, Ji et al., 2002, Ma et al., 2002, Obata et al., 2004a, Obata et al., 2004b, Sammons et al., 2000). The strong induction of pERK by the novel $\mu$ receptor agonists may throughout the $180 \mathrm{~min}$ time course, in part, explain the induction of hyperalgesia with kurkinol and tolerance in the CINP model with both novel compounds (Chapter 3, Section 3.3.4). However, this is not a perfect explanation as neither show tolerance to acute-thermal stimuli (Chapter 3, Section 3.3.1) nor do they have increased abuse liability in the CPP assay (Crowley et al., 2020), indicating other subcellular signalling pathways are involved in differentiating the $\mu$ receptor agonist properties of kurkinol and kurkinorin exerting these physiological and behavioural effects. 


\subsubsection{Experimental Bias Alters the Generation of Bias Factors}

Since the Black and Leff operational model of biased agonism (Black et al., 1983), many more operational models have been developed, such as the simplified operational model (Kenakin et al., 2012) and Slack and Hall operational model (Hall et al., 2018, Slack et al., 2012). The non-operational models that have been generated, allow the simplification of quantifying signalling bias. Such models include the simplified bias calculation (Crowley et al., 2020, Crowley et al., 2016, Paton et al., 2020b), the RA $A_{i}$ (Ehlert et al., 1999, Griffin et al., 2007) and the extended $R A_{i}$ model which calculates the bias factor from the Log $\mathrm{RA}_{\mathrm{i}}$ compared to a reference ligand in an 'equi-active comparison' (Rajagopal et al., 2010). Each model has been shown to have pros and cons concerning the other available models. Therefore, we chose to compare the bias factors generated by two different calculations, the simplified bias calculation and the $\mathrm{RA}_{\mathrm{i}}$ model. This not only allowed us to determine whether kurkinol and kurkinorin are biased agonists and if these values support the hypothesis that $\mathrm{G}$-protein biased $\mu$ receptor agonists display reduced side effects. But also allowed for the assessment of the reproducibility between equations. To ensure that this was performed in a robust fashion multiple cell signalling pathways, including the potential, GIRK, and PCREB assays in Hek293 and CHO cells stably expressing the human $\mu$ receptor cells to interrogate the role of cell background on bias factors.

The calculation of bias factors from dose response data has many caveats that must be accounted for to determine bias without the generation of false positives and negatives. We have accounted for system bias by selecting measures of both G-protein (Membrane Potential and potassium assays) and $\beta$-arrestin2 (pCREB assay) signalling that does not require amplification. However, a component of the potassium assay was missed, the concentrations required for the membrane potential assay in Hek293 cells were significantly higher than previously published concentrations, and further observational bias induced by the assay conditions were not fully controlled. During the generation of the human $\mu$ receptor stable cell lines, the expression levels were assessed and those determined to moderately express the receptor were selected to ensure the data was not impacted by the presence of large receptor reserves. Despite these efforts, all dose response experiments used for the generation of bias factors were performed without the presence of a $\mu$ receptor antagonist. Thus, receptor reserves were still present and may have masked the true cell signalling properties, incorrectly identifying bias resulting in inaccurate $\mathrm{EC}_{50}$ and $\mathrm{E}_{\max }$ values. Gillis et al. (2020a) were able to show this in the membrane potential assay, whereby PZM21, TRV130, and SR17018 were only partial agonists at this pathway in the presence of the $\mu$ receptor antagonist $\beta$ chlornaltrexamine, which also resulted in a rightward shift in their potencies. In terms of our data, in the presence of an antagonist, the potential partial agonism in the Potassium assay may be clearer and the potency differences teased apart more in all pathways assessed. Due to the recent publication of this work, time (COVID-19 delays), and reagent limitations, this was not performed and the possible implications of the inaccurate identification of signalling bias kept in mind when assessing these 
results. In future, it would be ideal to repeat these assays in the presence of the $\mu$ receptor antagonist $\beta$-FNA to remove all possibility of observational bias generating false results.

\subsubsection{Different Equations Give Different Bias Factors}

By comparing the two methods of generating bias factors we were able to show that bias factors generated by the same calculation are comparable, even when the measure of G-protein activation was changed. However, large variation was identified between the two equations when the same data was used. In general, the simplified bias equation identified all the $\mu$ receptor agonists as $\beta$-arrestin2 biased, while the $\mathrm{RA}_{i}$ model mainly identified G-protein bias. The lack of consistency between different calculations when using the same data is concerning and calls into question the reliability and accuracy of all bias calculations and the actual "signalling bias" of compounds. This is particularly concerning for the simplified bias equation which identified all agonists as $\beta$-arrestin 2 bias despite the selectivity analysis showing kurkinol and kurkinorin are highly selective for the G-protein pathways, with significantly lower levels of $\beta$-arrestin2 recruitment and pCREB activation (Fig. 5.7).

Furthermore, the original pattern of bias, generated using the simplified bias calculation and cAMP inhibition vs $\beta$-arrestin2 recruitment was not consistently replicated with this new data (Crowley et al., 2020, Crowley et al., 2016). The method of data generation originally used may be responsible for this inconsistency, as an amplifying G-protein (cAMP) assay vs non-amplifying $\beta$ arrestin2 (PathHunter $\beta$-arrestin2 recruitment) assay was used, immediately biasing the results towards the G-protein pathway. Additionally, the simplified bias calculation was utilised, which we have shown to correlate poorly to the behavioural data and therapeutic windows. Overall, this indicates that the bias factors generated from this method of data generation and analysis has a high chance of falsely identifying kurkinol and kurkinorin as G-protein biased and cannot be used as a reference for the bias factors generated in this thesis.

In terms of this study, it calls into question what bias factor accurately represents the signalling bias of these compounds. Based off previously discussed behavioural data (chapters 3,4 ) and the correlations to improved therapeutic windows, the $\mathrm{RA}_{\mathrm{i}}$ model and data from the Membrane Potential and pCREB assays conducted in $\mathrm{CHO}$ human $\mu$ receptor cells most accurately represents the behavioural profiles of kurkinol and kurkinorin. Whether this is to say this represents the best method of bias generation, or that signalling bias is correlated to the behavioural profiles, is not within the scope of this thesis. To answer this question a thorough investigation into the cellular mechanisms of behaviour and a more robust profile of the downstream signalling pathways may allow for the generation bias factors that accurately predict the behavioural profiles of $\mu$ receptor agonists. This can be further improved by utilising an operational model of determining signalling bias. As the kinetic measures of agonist receptor binding and receptor pathway activation remove more system bias from 
the analysis that may be altering the generation of bias factors and pushing the output towards a predefined answer.

\subsubsection{Different Cellular Backgrounds alter G-protein Bias}

One of the most common ways that bias is introduced into the calculation of signalling bias is assessing G-protein and $\beta$-arrestin2 dependent pathways in different cell lines. This commonly occurs due to the use of the DiscoverX assays that require the purchase of cell lines with the presence of the large subcellular reporters that are only available in $\mathrm{CHO}$ and Hek293 cell backgrounds. The lack of consistency between cell lines is not a surprising find due to the unusually high concentrations of all four compounds required to have an effect in the membrane potential assay when the Hek293 cell line was used. However, there have been numerous other studies that have shown that when the cell background is the only factor changed bias values vary greatly, showing cell-specific activation of subcellular signalling pathways, resulting in observational bias (Kenakin, 2019) (See table 5.1). Despite this, it is still common to see the use of different cell lines to generate bias factors, even from leaders in the field. With Bohn et al. (2017) determining signalling bias for the SR-compounds using CAMP in CHO cells or GTPYS in mouse membranes vs $\beta$-arrestin2 recruitment in U2OS cells (See table 5.1). While the bias of PZM21 has been determined using the cAMP inhibition in Hek293 cells Vs $\beta$-arrestin2 recruitment in $\mathrm{CHO}$ cells (Ehrlich, et al. 2019).

These cell-specific differences in the activation of subcellular signalling molecules is likely due to cell-specific expression of regulatory proteins, such as the RGS proteins. This is a family of GTPase accelerating proteins that increase the rate of GTP hydrolysis (100 fold) to negatively regulate the action of $\mu$ receptor activation and downstream signalling pathways (Tesmer et al., 1997). While there is some specificity for the expression of RGS proteins and their selectivity for receptors and G-proteins throughout the CNS, the regulatory role individual RGS proteins play in altering $\mu$ receptor signalling is not fully understood. For example, when RGS9 and the $\mu$ receptor were co-transfected into Hek293 cells the rates of receptor deactivation increased resulting in reduced cAMP inhibition by morphine (Masuho et al., 2013). While a global insensitivity of the $G \alpha_{0}$ G-protein subunit to RGS proteins in mice resulted in decreased coupling and activation of GIRK channels in response to DAMGO and fentanyl in the PAG rather than the expected increase (McPherson et al., 2018). These differential effects of RGS proteins is most likely due to their ability to form multiprotein signalling complexes, rather than act as GTPases. With different RGS proteins able to directly interact with GPCRs, AC, GIRK channels, and $\mathrm{Ca}^{2+}$ channels (Abramow-Newerly et al., 2006). Therefore, the differential expression of RGS proteins in $\mathrm{CHO}$ and Hek293 cells may play a role in the different potency in the Membrane Potential Assay, either via their GTPase activity or the formation of RGS signalling complexes differentially affecting the GIRK channels. Calling into question the validity and translatability to behavioural profiles in mice of the bias factors generated using this pathway as a measure of G-protein signalling. 
Overall, RGS proteins are important regulatory proteins that are poorly understood and often ignored when generating data for the determination of signalling bias, further simplifying a complex signalling system adding to the error, bias, and poor translation of $\mu$ receptor G-protein biased agonists.

\subsubsection{Limitations and Future Directions}

The largest limitations of this study lie in the lack of comparison between the cell lines in all assays. Ideally, the potassium assay (with GRIK channels transfected), pCREB, and pERK experiments would have all been performed in both cell lines. Unfortunately, due to the arrival of the different cell lines, time to perform the experiments due to the pandemic, and the inability to order in new reagents these experiments were only performed in one of the two cell lines. In future, the examination of both cell lines may be able to provide more robust evidence on how cell-specific signalling affects the generation of bias. Furthermore, the concentrations required to induce a response in the potassium and CREB assays may have been able to clarify if the high doses required in the membrane potential assay were due to low receptor expression or if other unknown factors were influencing that experiment.

A further limitation of this study is that the dose response effects on ERK phosphorylation at early and late time points were never assessed due to time restrictions. As previously discussed, the biphasic nature of ERK activation is thought to play a role in the different physiological effect of $\mu$ receptor agonists, with late-phase (or $\beta$-arrestin2 dependent) believed to be involved with abuse liability, tolerance, and hyperalgesia (Lesscher et al., 2003, Rosas et al., 2016). The dose response analysis of ERK phosphorylation at these time points would further add to the accuracy of the bias calculations and provide a better understanding of the role ERK in the behavioural effects of $\mu$ receptor agonists.

A major limitation in the field of biased agonism is the poor translation of bias factors generated in vitro, to pre-clinical models of anti-nociception and side effects, and then to human clinical trials. This is likely a major factor limiting the clinical translation of many G-protein biased $\mu$ receptor agonists. The use of immortalised cell lines from different species requiring the transfection of human $\mu$ receptor, mouse $\mu$ receptor, or rat $\mu$ receptor is thought to play a role in the poor translation. Especially with known differences in GPCR cell signalling in different species (Schattauer et al., 2017, Schattauer et al., 2012) and recruitment of non-cognate G-proteins (Chakrabarti et al., 2005, Seyedabadi et al., 2012, Wang et al., 2005b). A move towards a primary cell-based generation of cell signalling data and bias factors from multiple neuronal populations may help overcome these issues.

This has been conducted for the $\mathrm{k}$ receptor, with Schmid et al. (2013) were able to show selectivity for pERK over AKT for 6'-guanidinonaltrindole compared to U69,593 (reference). While Ho 
et al. (2018) observed that signalling bias for triazole 1.1 and 1.2 determined in CHO cells was maintained in primary striatal cultures. However, to quantify G-protein and $\beta$-arrestin2 dependent pathways in neurons, as Schmid et al. (2013) and Ho et al. (2016) have done, requires time consuming and low throughput experiments, such as confocal analysis of receptor internalisation and western blotting for ERK1/2 phosphorylation. Furthermore, these experiments have required the transfection of GFP-tagged receptors, introducing receptor reserves and system bias to their analysis. However, with the development of the live-cell CADDi CAMP reporter (Montana Molecular; Bozeman, USA) the level of cAMP inhibition in primary cultures can be assessed via plate reader in a high throughput manner. Recent work has also shown this method of determining real-time cAMP levels is compatible with primary neural stem cells, indicating it may be used successfully in primary striatal cultures (Yu et al., 2020). This experiment can be further strengthened by utilising the recently released $\beta$-arrestin2 CADDi (Montana Molecular). If co-transfected with the cAMP CADDi, the dose response effects of both signalling pathways could be assessed simultaneously in the same cell. Performing these experiments in primary cultures will allow for improved translation between in vitro screens and in vivo behavioural models conducted in rodents. While also reducing observational bias as $\mu$ receptor levels will intrinsically be at physiological levels. Unfortunately, there will still be a large system bias introduced by the need to constitutively activate $A C$ to allow $\mathrm{G}_{\mathrm{i} / 0}$ mediated decreases in cAMP to be detected.

To remove the system bias involved in the measurement of CAMP ex vivo, Muntean et al. (2018) developed a CAMP reporter mouse (CAMPER; CAMP encoded reporter) by knocking-in a fluorescent CAMP reporter. Allowing for the determination of increases and decreases in CAMP levels from baseline via a FRET signal given by the reporter. When used in primary cultures, the measurement of cAMP can be semi-high throughput by utilising high throughput confocal analysis. This still represents a decrease in data collection speed compared to the CADDis plate reader analysis. The CAMPER mouse does have one key advantage, the reporter is adeno-associated virus-Cre inducible allowing for the activation and determination of cAMP levels in a subset of neurons. Moreover, it is compatible with optogenetics allowing for the spatio-temporal dynamics of CAMP to be assessed over time in a specific subset of neurons. This can also be run in tandem with behavioural measures allowing direct correlations to be identified between in vivo cell signalling and behaviour.

With the ability of RGS proteins to regulate downstream signalling of $\mu$ receptor agonists in a cell dependent manner the use of primary cultures from different brain regions is also important. For example, RGS9 and RGS4 are localised within the PAG and dorsal horn of the spinal cord and have been implicated in regulating the anti-nociceptive effects of $\mu$ receptor agonists. While in the pons RGS4, 7, and 8 are localised and may play a role in regulating the induction of OIRD (Senese et al., 2020). Therefore, to fully investigate the downstream pathways activated by the $\mu$ receptor and their physiological and behavioural consequences the full regulatory mechanism must be in place. Thus, indicating that the bias of a compound must be determined in primary cultures from the areas 
associated with the behavioural effects. To translate the accurate generation of bias factors into a cell line-based system to decrease cost and time involved in primary cultures, more extensive research into the localisation and role of RGS and other regulatory proteins is required. Until we understand the cell-specific regulation of the $\mu$ receptor pathways the translation from in vitro screens through to clinical benefits will remain poor, and the success rate of clinical approval will remain low.

The role that heterodimers play in the differential signalling profiles of kurkinol and kurkinorin are unknown. the potential formation of $\mu$ receptor/k receptor heterodimers of most interest. Currently, few studies directly assessing the role $\mu$ receptor/K receptor heterodimers have been published. However, it has been shown that they occur in the spinal cord, producing robust antinociception in male mice and female mice dependent on the stage of their menstrual cycle (Chakrabarti et al., 2010). The mechanism of this sex difference was later identified as the differential regulation of oestrogen and progesterone receptors by the $\mu$ receptor/ $\mathrm{k}$ receptor heterodimer vs the individual opioid receptors (Liu et al., 2011). Indicating that the subcellular pathways are differentially regulated upon the formation of the heterodimer, possibly altering the signalling and bias of mixed agonists, including kurkinorin. Due to the lack of current research in the field and the potential benefit that mixed $\mu$ receptor/ $k$ receptor may provide clinically, this is an area of opioid signalling that warrants further investigation (Paton et al., 2020a). To speed up the investigation into the effects if $\mu$ receptor/ $\mathrm{k}$ receptor heterodimers have on cell signalling we can draw from the work into the effects if the $\delta$ receptor/ $\mu$ receptor heterodimer. This field is in a much more advanced state than any other GPCR heterodimer research. With changes in levels of ERK1/2 phosphorylation (Gomes et al., 2000), G-protein recruitment, the inhibition of cAMP (Gomes et al., 2004), and $\mathrm{Ca}^{2+}$ signalling (Charles et al., 2003) in the presence of the heterodimer vs either opioid receptor on their own. Due to the similarities between opioid receptors, these pose good first targets to assess the differential signalling of $\mu$ receptor/k receptor heterodimers may possess. 


\section{Chapter 6. General Discussion}

\subsection{Significance of the Study}

Chronic pain is a major problem worldwide, with 1 in 5 New Zealand adults affected. Current treatments are ineffective due to patient differences (gabapentin and anti-depressants) or the induction of tolerance and addiction ( $\mu$ receptor agonists such as morphine and fentanyl). Despite its prevalence, little progress has been made in the development of new analgesics. Particularly, in reducing the tolerance, addiction, and respiratory depression that occurs with $\mu$ receptor agonists. One avenue that has been explored is biased agonism. At the commencement of this thesis, it was believed that preferentially activating G-protein signalling over $\beta$-arrestin2 signalling would increase the therapeutic window of $\mu$ receptor agonists (Schmid et al., 2017). However, only one G-protein biased agonist, Olinvyk (TRV130), has gained FDA approval for the management of acute pain severe enough to require i.v. opioids when alternative treatments are inadequate (FDA, 2020). While preclinical analysis of other G-protein biased $\mu$ receptor agonists shows poor reproducibility and little overall improvement in side effect profiles compared to currently available options (Chapter 4, Table 4.3). Furthermore, recent studies have failed to replicate initial findings in $\beta$-arr ${ }^{-}-$mice, indicating biased agonism may not be responsible for the improved pre-clinical profile of "G-protein" biased agonists (Kliewer et al., 2020, Kliewer et al., 2019). This led to the identification of other potential cellular mechanisms for their behavioural profiles. With the main competing theory termed low intrinsic efficacy stating that partial agonism at G-protein and $\beta$-arrestin2 dependent pathways is responsible for the behavioural improvements of these compounds (Gillis et al., 2020a, Gillis et al., 2020b) (Fig $6.1 \mathrm{~A}$ ).

The rapid development of this field over the last three years has impacted the hypotheses of this thesis. We initially aimed to investigate the role of biased agonism in the anti-nociceptive and side effects using two novel $\mu$ receptor agonists with varying G-protein bias, kurkinol and kurkinorin. Our initial hypothesis was that the more G-protein biased kurkinol would show increased anti-nociception, decreased tolerance, and side effects compared to kurkinorin and morphine. We, therefore, assessed the potency, tolerance, and duration of action of these two novel compounds in acute thermal spinal and supraspinal pre-clinical models, and in the CINP model of chronic, neuropathic nociception in male and female C57BL/6J mice. While the induction of motor coordination impairment, constipation, urinary retention, hyperalgesia, and respiratory depression were used to assess their side effect profiles. With the change in the literature surrounding the role of $\mu$ receptor G-protein bias, we further investigated the role of $\beta$-arrestin2 in the induction of thermal nociceptive tolerance and respiratory depression in the $\beta$-arr2 ${ }^{-/}$mice and $\mathrm{Wt}$ controls. Furthermore, we investigated the cell signalling profile of kurkinol and kurkinorin using non-amplifying measures of G-protein (Membrane Potential assay and potassium assay) and $\beta$-arrestin2 signalling to probe the role of biased vs partial agonism at 
the $\mu$ receptor. This data was used to calculate bias factors utilising two different calculations to assess their reproducibility. The role different cell lines, and therefore regulatory proteins, on the translation issues of bias, were also probed utilising both $\mathrm{CHO}$ and Hek293 cells stably expressing the human $\mu$ receptor.

\subsection{Key Results}

\subsubsection{G-Protein Bias Correlates to the Anti-Nociceptive Profile but Not the Side Effect Profile}

In our hands, the degree of G-protein bias was found to correlate to the potency and tolerance in the tail-flick assay of acute thermal anti-nociception. Furthermore, we were able to repeat the initial work by Bohn et al. (1999) in $\beta$-arr $\%^{--}$mice, with the anti-nociceptive potency in the tail-flick higher than in the Wt controls while also displaying significantly reduced tolerance. The moderately biased kurkinorin also showed reduced tolerance in the $\beta$-arr2 ${ }^{-/}$mice indicating that $\beta$-arrestin2 is required for $\mu$ receptor desensitisation and tolerance. However, the correlation between G-protein bias and improved behavioural profiles does not translate to the other side effects assessed, with kurkinol inducing higher levels of motor incoordination, constipation, and respiratory depression than kurkinorin. Further, neither kurkinol nor kurkinorin showed any improvement in respiratory depression or small intestine transit in $\beta$-arr $2^{-/}$mice. In addition to this study, these key experiments in the field have also failed replication, with recent work by 3 groups. With (Kliewer et al., 2020) also finding the same level of respiratory depression and constipation in $\beta$-arr2 ${ }^{-/}$mice and their $\mathrm{Wt}$ littermate controls. This indicates an inverse correlation between signalling bias and the side effect profile. Furthermore, Kliewer et al. (2019) generated a series of $\mu$ receptor C-terminal mutations to dose dependently decrease the level of $\beta$-arrestin 2 recruitment in vivo. In doing so they observed a correlation between decreased $\beta$-arrestin2 and increases in anti-nociceptive potency and decreases in the level of tolerance. However, it was also correlated to increased levels of on-target side effects, including respiratory depression, constipation and opioid withdrawal. Together with previous literature, our results indicate that signalling bias does correlate to improved anti-nociceptive potency and tolerance, in acute models, as has been found with TRV130 and SR-17018 (Chapter 3, Table 3.6). The induction of side effects by G-protein biased agonists can also be seen with PZM21, TRV130, SR1150, -14969, -14968, and loperamide, with no 2 agonists showing the same side effect profile (Chapter 4, Table 4.3).

The correlation of improved anti-nociception with G-protein bias also does not translate to chronic models, as shown in the CINP model. This highlights the importance of determining the effect of biased agonism in the disease state it is developed to treat. Especially with the signalling changes that occur in chronic pain states, potentially altering the behavioural profiles of in acute vs chronic 
models of anti-nociception (Li et al., 2017, Samineni et al., 2017, Xiao et al., 2007, Xiao et al., 2011). Despite this, it is rarely examined during the pre-clinical development of G-protein biased $\mu$ receptor agonists (Chapter 3, Table 3.6).

The generation of Z-scores for the behavioural anti-nociceptive and side effects assessed showed that kurkinorin induced an equivalent anti-nociceptive profile and improved side effect profile compared to morphine, independent of signalling bias. Furthermore, kurkinol provided the largest therapeutic window between the potency in the tail-flick or potency in PTX-induced mechanical allodynia and the induction of constipation. While the therapeutic window could not be assessed for respiratory depression or motor coordination impairment due to a lack of doses for each drug, there does appear to be a correlation, with kurkinorin having a larger therapeutic window. Kurkinol on the other hand has a much smaller therapeutic window with the induction of multiple side effects, specifically respiratory depression. Previously these effects have been attributed to either $\beta$-arrestin2 recruitment or the efficacy for pathway activation. However, as mentioned above the level of $\beta$ arrestin2 recruitment does not correlate to the side effect profile, while neither compound showing partial agonism at the pathways assessed. Alternately, the background $\mathrm{k}$ receptor activation may be enough to mitigate the side effects induced by the $\mu$ receptor selectivity of kurkinorin. There is also the possibility that the issues with the measurement and calculation of bias may not correctly identify the signalling bias of these compounds.

\subsubsection{The Therapeutic Potential of Kurkinorin and Kurkinol}

The therapeutic potential of our novel $\mu$ receptor agonists do not match their signalling bias. As previously mentioned, kurkinol shows no improvement when compared to morphine in measures of motor incoordination, respiratory depression, or constipation. While it does not appear to have high abuse liability, the potent induction of these side effects would likely limit its clinical utility and likely. Due to the improvement in side effects following the administration of kurkinorin compared to morphine this, moderately G-protein biased agonist holds the most therapeutic potential. With improvements in tolerance to acute thermal noxious stimuli, reduced abuse liability, motor coordination impairment, constipation, and respiratory depression compared to morphine.

In comparison to other novel G-protein biased agonists, kurkinorin has a favorable behavioural profile. Compared to PZM21 kurkinorin is more potent in acute thermal nociception with improved levels of tolerance (Table 3.6). With both novel $\mu$ receptor agonists inducing similar levels of locomotor incoordination, G.I transit impairment, and respiratory depression (Hill et al., 2018, Kudla et al., 2019, Manglik. et al., 2016). However, the respiratory data for PZM21 is inconsistent between studies (Hill et al., 2018). While the SR-compounds are at an earlier stage of development, kurkinorin induces less respiratory depression compared to SR-11501, SR-14969, and SR-15968 (Schmid et al., 2017, Schwienteck et al., 2019) (Table 4.3). The strongest indicator of kurkinorins therapeutic potential is 
the similarity in behavioural profile to the FDA approved G-protein biased $\mu$ receptor agonist, Olynvik (TRV130). With both novel compounds showing improved therapeutic windows between acute thermal stimuli and measures of G.I transit (DeWire et al., 2013, Manglik. et al., 2016). Furthermore, in the initial studies TRV130 also showed reduced levels of respiratory depression, motor coordination, and abuse liability (DeWire et al., 2013, Manglik. et al., 2016). While, more recently TRV130 was shown to induce respiratory depression and have high abuse liability in pre-clinical rodent models (Altarifi et al., 2017, Zamarripa et al., 2018). This led to the failure of FDA approval with the first application, ultimately the re-evaluation in humans resulted in its approval for specific circumstances (FDA, 2020). Overall this highlights the importance of having the behavioural data of kurkinorin independently validated before any definite conclusion can be drawn on its therapeutic potential (Table $3.6 \&$ Table 4.3).

\subsubsection{The Role of Other Subcellular Pathways and Regulatory Proteins}

When assessing bias in vitro there has been a tendency to oversimplify the measurements of G-protein and $\beta$-arrestin2 signalling pathways. Common measures include $\beta$-arrestin2 recruitment, GTPYS binding, and inhibition of cAMP. However, bias has been identified between the different $\mathrm{Ga}_{\mathrm{i} / 0}$ subunits at the $\mu$ receptor in response to DAMGO, Met-enkephalin, and $\alpha$-neo in a cell dependent manner (Thompson et al., 2016). This indicates that activation of downstream G-protein dependent pathways may be differentially activated in both a cellular and ligand dependent manner. By only assessing the effects of upstream pathways, such as $\beta$-arrestin2 recruitment and GTPYS binding, the role of subcellular pathway molecules on bias factors and behaviour has been overlooked. While other signalling pathways are beginning to be analysed, such as the Membrane Potential assay that assesses the activation of ion channels. The role of other signalling molecules, such as activation of MAPK pathways, are often ignored. Previous investigation of the cellular pathways activated by 'biased' agonists may have been too narrow, possibly resulting in important correlations being missed. For instance, we have shown that the side effect profiles of kurkinol (potent activator) and kurkinorin (poor activator) may be correlated to the levels of PCREB, specifically the respiratory and motor coordination deficits. Further experimentation would be needed to confirm these results and prove a correlation between PCREB and the behavioural effects. The simplest way to assess this correlation would be to assess the level of PCREB in the brain regions associated with these effects with western blotting or IHC following administration of kurkinol and kurkinorin. If an increase is seen in these regions, further experiments may be carried out to assess the behavioural effects when PCREB is inhibited in these areas. Unfortunately, the correct Cre-Loc system does not exist to selectively remove CREB from $\mu$ receptor positive neurons. However, stereotaxic microinjection and siRNA knockdown may achieve the same results. Due to the location and role of the pre-Bötzinger in respiration, injection directly into this region would most likely not be possible. However, either i.c.v 
or injection into the $4^{\text {th }}$ ventricle may be optimized to result in knockdown in the pre-Bötzinger. Other downstream kinases of MAPK have also been associated with behavioural effects, such as pERK1/2 that is associated with the abuse liability (Lesscher et al., 2003, Rosas et al., 2016) and induction of hyperalgesia by $\mu$ receptor agonists (Bobeck et al., 2016, Deng et al., 2019). While the activation of p38 is linked to the induction of anti-nociceptive tolerance (Koch et al., 2004, Yang et al., 2010). As with PCREB, these molecules are often not measured or included in the analysis of signalling bias further limiting our ability to accurately predict bias and improve their translation.

Furthermore, regulatory proteins, such as RGS proteins and GRK proteins, can influence the regulation of the $\mu$ receptor and therefore the signalling bias that is observed. The phosphorylation of the $\mu$ receptor and subsequent increase in $\beta$-arrestin2 recruitment in response to GRK2 activation is well documented, with the overexpression of GRK2 in CHO, AtT20, and Hek293 cells known to result in increases in $\beta$-arrestin2 recruitment (Doll et al., 2012, Schulz et al., 2004, Thompson et al., 2016). While, RGS proteins are involved in the negative regulation of G-protein activity and the formation of signalling complexes that function to enhance the activation and deactivation of GIRK and VG $\mathrm{Ca}^{2+}$ channels (Senese et al., 2020) (Fig 6.1 B). This can have widespread, cell-specific impacts on signalling in response to the same agonist resulting in different behavioural profiles. This has been made clear by the generation of mutant mouse lines for multiple RGS proteins involved in the regulation of the $\mu$ receptor. This includes the constitutive RGS9-2 knockout mouse that shows increased anti-nociception and a decrease in tolerance to morphine in the hotplate assay. However, this also induced an increase in place preference and exacerbation of naloxone-precipitated withdrawal (Zachariou et al., 2003). In the inverse experiment the overexpression of RGD9-2 in the NAc, resulted in the opposite behavioural profile to morphine (Gaspari et al., 2014). Further, there appears to be ligand-specific regulation of the $\mu$ receptor by RGS9-2, as the administration of oxycodone, methadone, or fentanyl to the constitutive knockout mice resulted in decreased anti-nociception and increased tolerance in the hotplate assay (Gaspari et al., 2017, Psifogeorgou et al., 2011). Similar changes can be observed upon the genetic manipulation of RGS7, RGS4, and R7BP, while RGSz1 only negatively regulates the $\mu$ receptor (Table 6.1). To further complicate the role of regulatory proteins at the $\mu$ receptor, the constitutive knock in of RGS-insensitive $\mathrm{G} \alpha 0$ increased supraspinal anti-nociception measured in the hotplate assay but decreased spinal anti-nociception in the tail-flick assay. Despite the large behavioural effects, that regulatory proteins play in modulating the behavioural effects of $\mu$ receptor agonists, they are often ignored when signalling bias is assessed.

If the RGS and GRK2 expression does not match that of the in vivo target cells the translation of bias factors will remain poor. This further complicates the generation of bias factors for a receptor target such as the $\mu$ receptor that is widely expressed throughout the central and peripheral nervous system. Coupled with the poor understanding of the regulatory proteins signalling bias would need to be determined in every cell population where the binding and activation of $\mu$ receptor induces a 
behavioural response. If this is the case, then bias factors need to be determined for every cell type at a physiological level of the receptor in question with a range of different signalling molecules assessed using non-amplifying assays in primary cells ex vivo. This process would be time-consuming, expensive, and defeat the purpose of developing biased agonism to predict what novel compounds will show improved safety profiles in vivo. 
Table 6.1 Behavioural Effects of RGS Proteins that Regulate the Mu Opioid Receptor

\begin{tabular}{|c|c|c|c|c|c|c|}
\hline \multirow{2}{*}{ RGS Subtype } & \multirow{2}{*}{ Opioid } & \multirow{2}{*}{ Genetic Manipulation } & \multicolumn{4}{|c|}{ Behavioural Profile } \\
\hline & & & Anti-Nociception & Tolerance & Reward & Withdrawal \\
\hline RGS9-2 & $\begin{array}{c}\text { Morphine [1] } \\
\text { Morphine [2] } \\
\text { Methadone, Fentanyl [3] } \\
\text { Oxycodone [4] }\end{array}$ & $\begin{array}{c}\text { Constitutive } \mathrm{KO} \\
\text { AAV-RGS9-2 overexpression } \\
\text { Constitutive KO } \\
\text { Constitutive KO }\end{array}$ & $\begin{array}{l}\text { Increased } \\
\text { Decreased } \\
\text { Decreased } \\
\text { No change }\end{array}$ & $\begin{array}{c}\text { Delayed } \\
\text { Accelerated } \\
\text { n.d } \\
\text { Accelerated }\end{array}$ & $\begin{array}{l}\text { Increased } \\
\text { Decreased } \\
\text { n.d } \\
\text { Decreased }\end{array}$ & $\begin{array}{c}\text { Increased } \\
\text { Decreased } \\
\text { n.d } \\
\text { No change }\end{array}$ \\
\hline RGS-7 & Morphine [5] & Constitutive $\mathrm{KO}$ & Increased & Delayed & Increased & Increased \\
\hline RGS4 & $\begin{array}{c}\text { Morphine [6] } \\
\text { Fentanyl [6] } \\
\text { Morphine [7] } \\
\text { Methadone, Fentanyl [7] }\end{array}$ & $\begin{array}{c}\text { Antagonist CCG-63802 in vIPAG } \\
\text { Antagonist CCG-63802 in vIPAG } \\
\text { Constitutive KO } \\
\text { Constitutive KO }\end{array}$ & $\begin{array}{l}\text { Increased } \\
\text { No change } \\
\text { No change } \\
\text { Decreased }\end{array}$ & $\begin{array}{c}\text { n.d } \\
\text { n.d } \\
\text { No change } \\
\text { n.d }\end{array}$ & $\begin{array}{c}\text { n.d } \\
\text { n.d } \\
\text { Increased } \\
\text { n.d }\end{array}$ & $\begin{array}{c}\text { n.d } \\
\text { n.d } \\
\text { Increased } \\
\text { n.d }\end{array}$ \\
\hline RGSz1 & $\begin{array}{c}\text { Morphine [8] } \\
\text { Methadone, Fentanyl [8] } \\
\text { Morphine [8] }\end{array}$ & $\begin{array}{c}\text { Constitutive KO } \\
\text { Constitutive KO } \\
\text { Conditional vIPAG KD }\end{array}$ & $\begin{array}{l}\text { Increased } \\
\text { Increased } \\
\text { Increased }\end{array}$ & $\begin{array}{l}\text { Delayed } \\
\text { n.d } \\
\text { Delayed }\end{array}$ & $\begin{array}{c}\text { Increased } \\
\text { n.d } \\
\text { n.d }\end{array}$ & $\begin{array}{l}\text { n.d } \\
\text { n.d } \\
\text { n.d }\end{array}$ \\
\hline R7BP & Morphine [9] & Constitutive $\mathrm{KO}$ & Increased & Delayed & n.d & n.d \\
\hline RGS-insensitive Gao & $\begin{array}{l}\text { Morphine [10] } \\
\text { Morphine [10] }\end{array}$ & $\begin{array}{l}\text { Constitutive } \mathrm{KI} \\
\text { Constitutive } \mathrm{KI}\end{array}$ & $\begin{array}{c}\text { Increased (supraspinal) } \\
\text { Decreased (spinal) }\end{array}$ & $\begin{array}{l}\text { n.d } \\
\text { n.d }\end{array}$ & $\begin{array}{l}\text { n.d } \\
\text { n.d }\end{array}$ & $\begin{array}{l}\text { n.d } \\
\text { n.d }\end{array}$ \\
\hline
\end{tabular}

Data summarised from Senese et al. (2020)

\section{Abbreviations:}

$K O=$ knockout

$K D=$ knock down

$K I=$ knock in

\section{References:}

1. Zachariou, et al. 2003.

2. Gaspari, et al. 2014.

3. Psifogeorgou, et al. 2011.

4. Gaspari, et al. 2017.

5. Sutton, et al. 2016.
6. Morgan, et al. 2020

7. Han, et al. 2010.

8. Gaspari, et al. 2018.

9. Terzi, et al. 2012.

10. Lamberts, et al. 2013. 


\subsubsection{Issues with Bias Calculations}

Many confounding factors have been identified in the generation of bias factors over the last decade. Including system and observational bias that has been proposed as a partial explanation of the inconsistency between in vitro bias factors and in vivo behaviours. As well as the biases identified in data generation, the calculations have also shown inconsistencies between bias factors generated from the same data, with Rajagopal et al. (2011) finding that the $R A_{i}$ model did not always produce the same bias factors given by the Black and Leff operational model, particularly when variable cell signalling data resulted in poor curve fits. We have added to this previously identified issue with a lack of consistency between the simplified and Log $R A_{i}$ models of non-operational bias calculations. The inconsistency between operational and non-operational models of bias is the removal of kinetic parameters, such as the agonist-receptor dissociation constant, coupling of receptors to cellular pathways, and receptor population that are considered with operational models. Thus, making them more robust and a better representation of true signalling bias. We have also shown here that even within relative intrinsic efficacy-based models of generating bias factors there can be large discrepancies in the bias factors generated. Moving forward, the field of biased agonism needs to ensure that all biases are properly controlled for (system, operational, and cell type) and the effects on multiple signalling molecules measured before the use of the more robust operational models. Only when these factors are controlled for will we be able to delineate the true role of G-protein biased agonism at the $\mu$ receptor and the potential role of partial agonism. 
A)

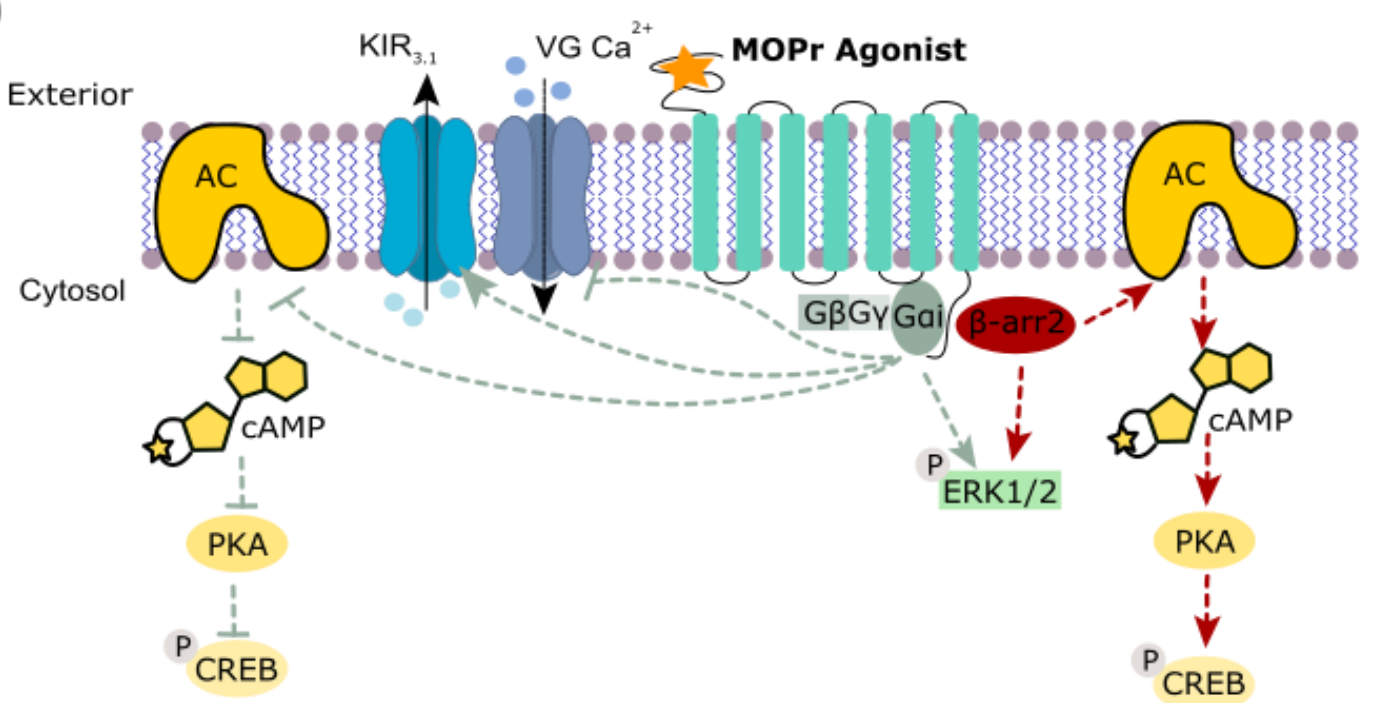

B)

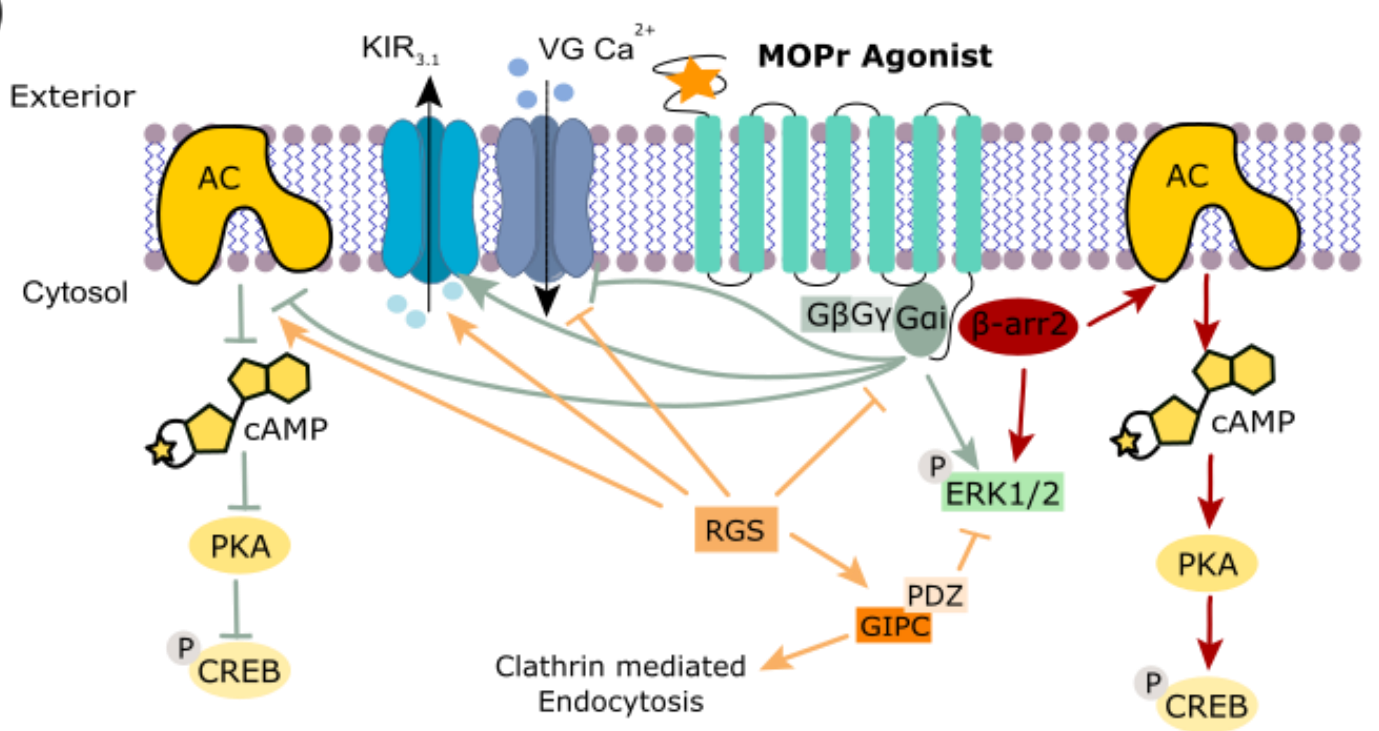

C)

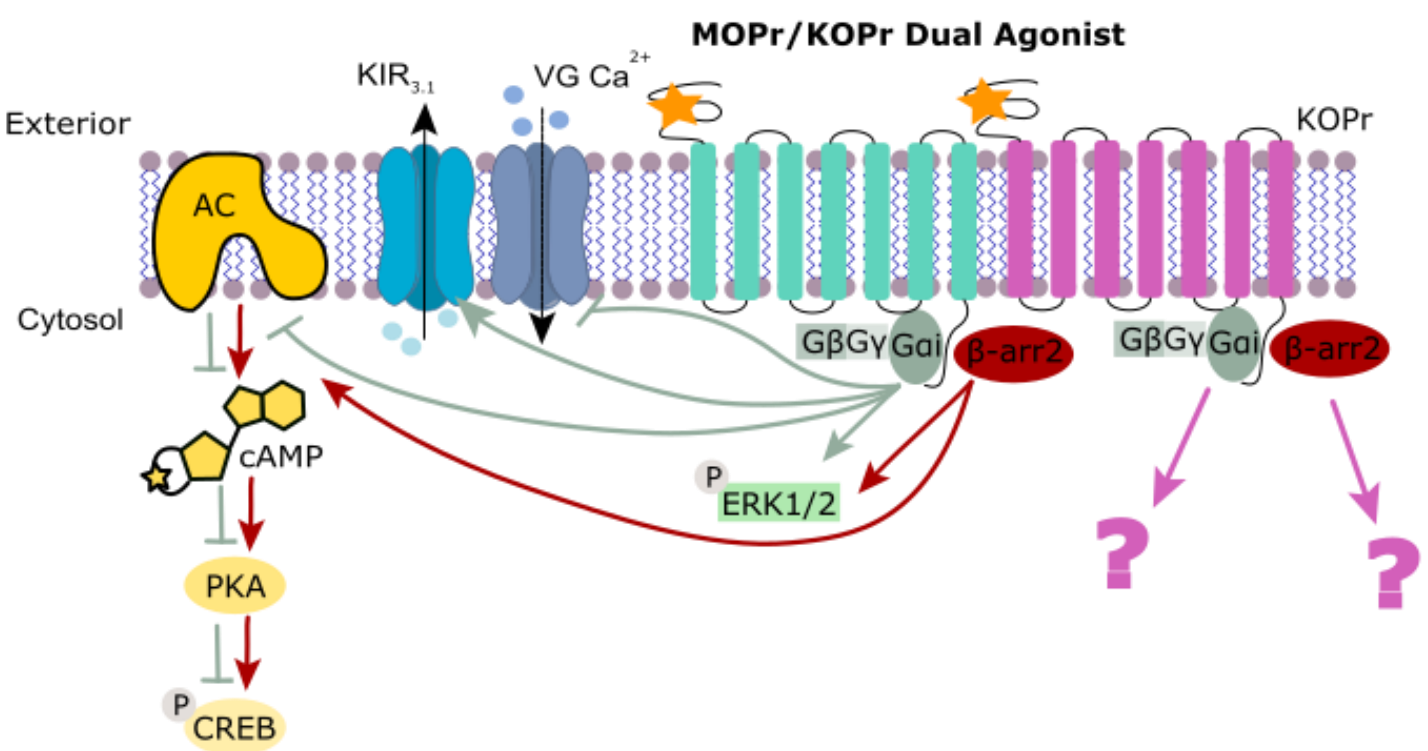


Figure 6.1 Alternate Theories to Explain the Behavioural Profiles of "G-Protein" Biased Mu Opioid Receptor Agonists

(A) Recent evidence has correlated partial agonism at both G-protein and $\beta$-arrestin2 dependent pathways to the increased therapeutic window of "G-protein biased" agonists. (B) The RGS family act as GAP proteins to inhibit the signalling G-protein signalling, they also inform multiprotein signalling complexes to modulate adenylyl cyclase, GIRK channels, voltage-gated calcium channels, pERK12, and endocytosis via clathrin-coated pits. Despite this, they are often ignored in the analysis of novel agonists for signalling bias. (C) The latent selectivity of kurkinorin for the $\mathrm{k}$ receptor may result in the formation and activation of $\mu$ receptor/k receptor heterodimers that may alter the downstream signalling molecules, therefore altering the behavioural profiles. 


\subsection{Alternative Paths to Non-Addictive Analgesics}

Due to the inconsistencies in the field of biased agonism and the work that needs to go into improving our ability to predict correlations between in vitro analyses and in vivo behaviour, alternate routes may provide a faster path to non-addictive analgesics. Other promising fields of research in this area include targeting regulators of $\mu$ receptor signalling, targeting the $\mathrm{k}$ receptor and $\delta$ receptor individually or as mixed agonists, and the targeting of voltage-gated $\mathrm{Na}^{+}$and $\mathrm{Ca}^{2+}$ channels.

Despite the lack of thought into the role of RGS proteins in the assessment of signalling bias, this protein family has become a target of interest for the treatment of chronic pain. With their activation and/or inhibition proposed as a mechanism to increase the therapeutic window of current $\mu$ receptor analgesics. The RGS4 and RGS9-2 are two RGS proteins that have been directly studied concerning anti-nociception, with their inhibition shown to increase thermal nociceptive thresholds after the administration of both endogenous and exogenous agonists (reviewed in Senese et al., 2020). This provides the 2 mechanisms through which RGS inhibitors have been proposed to treat chronic pain; as an independent treatment, leading to site-specific enhancement of anti-nociception by endogenous opioids, or as an adjunct to current $\mu$ receptor analgesics lowering the dose required to produce analgesia, thus increasing the therapeutic window. However, targeting these intracellular proteins has proven difficult due to their low receptor specificity. An example of this is the small molecule inhibitor, CCG-50014. This molecule has been shown to inhibit RGS1, 4, 5, 14, 17, and 19 (Blazer et al., 2011, Hayes et al., 2018). Despite this promiscuity it still enhances the anti-nociceptive effects of the $\mu$ receptor, indicating other RGS outside of RGS4 may be involved (Blazer et al., 2011, Yoon et al., 2015). This indicates that more basic research needs to be undertaken to understand the pathophysiological role of RGS protein before inhibitors can be developed for clinical use. But the promise of site-specific increases in $\mu$ receptor agonists, or even increasing the therapeutic window of current $\mu$ receptor agonists is worth the effort that is required.

The 2 other classical opioid receptors, $\mathrm{k}$ receptor and $\delta$ receptor, are also expressed in CNS regions associated with pain and have been observed to induce anti-nociception in pre-clinical rodent models (Gavériaux-Ruff et al., 2008, Nadal et al., 2006). While the traditional k receptor agonist $\mathrm{U} 50,488$ is heavily limited by the potent induction of side effects including sedation, aversion, and anxiety (Gallantine et al., 2008, Gillett et al., 2013, Land et al., 2009). Similarly, $\delta$ receptor agonists also induce side effects with seizures the most severe (Broom et al., 2002, Jutkiewicz et al., 2004). Due to the oppositional side effect profiles of these opioid systems the development of mixed opioid agonists has been proposed as a mechanism to reduce on-target $\mu$ receptor induced side effects. Specifically, the combination of the $\mathrm{k}$ receptor with either the $\mu$ receptor or $\delta$ receptor has been shown to induce favourable anti-nociceptive and side effect profiles, with a decrease in abuse liability a large attraction to this combination (Paton et al., 2020a). The 3 '-iodobenzoyl naltrexamine analogue, MP1104, is one such mixed opioid agonist (Váradi et al., 2015). In vitro analysis revealed MP1104 had 
3- and 13- fold higher selectivity for the $\mathrm{k}$ receptor than the $\mu$ receptor and $\delta$ receptor respectively, with full agonism at all three receptors (Váradi et al., 2015). While in vivo it has been shown to induce potent, long acting anti-nociception in the tail-flick assay in both rats and mice and attenuates cocaine self-administration in rats (Atigari et al., 2019, Váradi et al., 2015, Váradi et al., 2016). Furthermore, it does not induce anxiogenic or aversive effects as measured by elevated plus maze, forced swim test, and conditioned place aversion in rats (Atigari et al., 2019). Ultimately, these $\mu$ receptor/ $\mathrm{k}$ receptor mixed agonists appear to show the same pre-clinical behavioural profile as what biased agonism is trying to achieve (Paton et al., 2020a, Váradi et al., 2016). This can also be seen throughout this thesis with the significant improvements that kurkinorin has shown in terms of its side effect profile, which may be induced by latent selectivity for the $\mathrm{k}$ receptor. While we have yet to prove this, it is an intriguing path to follow in terms of kurkinorin mechanism of action and the cell signalling changes that occur in response to co-activation of the receptors. Current research has identified signalling changes in response to the co-activation of other $\mu$ receptor heterodimers, however, the $\mu$ receptor/ $\mathrm{K}$ receptor has been largely ignored in terms of its signalling profile. If kurkinorin does, in fact, coactivate $\mu$ receptor/ $\mathrm{k}$ receptor heterodimers it would be an interesting scaffold compound to probe these subcellular pathway changes and draw correlations back to the behavioural profile set out in this thesis. If the changes in cell signalling that occurs upon the activation of a $\mu$ receptor/ $\mathrm{k}$ receptor heterodimer does correlate to an improved therapeutic window the development of novel agonists with the same pharmacological profile it could pose a new avenue to generate non-addictive agonists for the treatment of chronic pain (Fig 6.1). Unfortunately, this is also not a straightforward method of generating improved analgesics as the issues of full, partial, or biased agonism and the discrepancies in bias calculations also exist at the $\mathrm{k}$ receptor (Mores et al., 2019, Schattauer et al., 2017, Schattauer et al., 2012). This means that large volumes of cell signalling research would be required for the action at the heterodimer to fully understand the correlation of heterodimer activation and behavioural profiles.

Another route for developing non-addictive analgesics for chronic pain that has renewed traction is the identification of ion channel blockers. This includes compounds such as gabapentin ( $\mathrm{Ca}^{2+}$ channel blocker), and Ziconotide ( $\mathrm{Ca}^{2+}$ channel blocker) and other novel compounds isolated from cone snails (conotoxins; $\chi-M r I A \&$ Contulakin-G), and spider venom (Ph $\alpha 1 \beta, \mathrm{Ca}^{2+}$ channel blocker) (Craig et al., 1999, Miljanich, 2004, Nielsen et al., 2005, Tonello et al., 2017, Vieira et al., 2005). In chronic pain states, they counteract the increase in neuronal excitability caused by the upregulation of the $\mathrm{Ca}^{2+}$ channels, either directly blocking their action, or targeting other channels involved in. As these compounds are fully independent of the opioid receptors, they induce none of the dose-limiting side effects. Unfortunately, they are also less effective at treating chronic pain compared to $\mu$ receptor analgesics. However, the co-administration of ion channel blockers with $\mu$ receptor agonists has been shown to reduce the dose of the $\mu$ receptor agonists required to induce the same level of anti- 
nociception in rodents (Bao et al., 2014, Papathanasiou et al., 2016). Therefore, increasing the therapeutic window and reducing the potential for accidental opioid overdose and abuse liability. 


\subsection{Final Conclusions}

There is a great need for the development of potent, non-addictive opioids for chronic pain highlighted by the unsatisfactory treatment in the eyes of patients and medical professionals due to tolerance, dose-limiting side effects, and addiction. This has contributed to a large portion of the opioid deaths in the United States, and the increased rates of opioid overdose seen around the world. The theory of biased agonism has been thoroughly investigated over the last 2 decades as a potential method of developing $\mu$ receptor agonists with improved anti-nociceptive and side effect profiles. Despite the initial promise this field showed, the lack of reproducibility and poor translation to improved clinical therapeutics has resulted in the development of alternate theories.

In this thesis, we investigated the behavioural and cell signalling effects of 2 novel $\mu$ receptor agonists, kurkinol and kurkinorin, with varying G-protein bias. Our results followed the trend seen in the more recent literature on biased agonism, whereby the anti-nociceptive and tolerance effects were correlated to G-protein bias in acute models of anti-nociception. We have also added to the literature by assessing the development of tolerance to chronic, neuropathic pain, an important step that is often missed. This allowed us to show that tolerance develops to G-protein biased agonists in disease states, where the pain pathways are damaged. Furthermore, the highly G-protein biased $\mu$ receptor agonist, kurkinol, induces more side effects than kurkinorin, adding to the literature evidence that correlates reduced $\beta$-arrestin2 recruitment to increased side effects.

The results of the in vitro work performed in this thesis reiterates the issues induced by observational and system bias on the generation of bias factors. As well as highlighting the issues that the assessment of a few select signalling molecules, rather than multiple subcellular pathways, may have led to correlations between signalling molecules and behaviour being missed. As well as the importance of considering the regulatory proteins and other as they are likely to change the direction and amplitude of signalling at both pathways, adding to the translatability issues of in vitro bias factors. Furthermore, the method of calculating bias needs to be optimized and standardized throughout the field as we and others have shown significant differences between the calculations. This includes accounting for the potency and efficacy of the compounds at the measured pathways and how the signalling may be altered overtime (kinetic bias). The theory of biased agonism still holds promise as a method to quickly identify compounds with improved safety profiles once the tools used to generate the bias factors have been further developed and the regulation of $G$ protein and $\beta$-arrestin pathways are fully understood. 


\section{Chapter 7. Appendix}

\subsection{Reagent recipes}

\subsubsection{CHO and Hek293 Growth Media}

$\begin{array}{ll}\text { DMEM/F12-K } & 35.6 \mathrm{ml} \\ \text { FCS } & 4 \mathrm{ml} \\ \text { PenStrep } & 400 \mu \mathrm{l}\end{array}$

\subsubsection{PBS - $1 \mathrm{~L}$}

$137 \mathrm{mM} \mathrm{NaCl}$

$8 \mathrm{~g}$

$2.7 \mathrm{mM} \mathrm{KCl}$

$0.2 \mathrm{~g}$

$4.3 \mathrm{mM} \mathrm{Na}_{2} \mathrm{HPO}_{4}$

$0.610 \mathrm{~g}$

$1.47 \mathrm{mM} \mathrm{KH}_{2} \mathrm{PO}_{4}$

$0.2 \mathrm{~g}$

\subsubsection{SOC media - $1 \mathrm{~L}$}

$\begin{array}{ll}0.5 \% \text { Yeast extract } & 5 \mathrm{~g} \\ 2 \% \text { Tryptone } & 20 \mathrm{~g} \\ 10 \mathrm{mM} \mathrm{NaCl} & 0.58 \mathrm{~g} \\ 2.5 \mathrm{mM} \mathrm{KCl} & 0.18 \mathrm{~g} \\ 10 \mathrm{mM} \mathrm{MgCl}_{2} & 2.03 \mathrm{~g} \\ 10 \mathrm{mM} \mathrm{MgSO}_{4} & 2.46 \mathrm{~g} \\ 20 \mathrm{mM} \mathrm{glucose}^{2.03 \mathrm{~g}}\end{array}$

\subsubsection{LB broth and LB agar - 1 L}

Bacto-tryptone

Yeast extract

$\mathrm{NaCl}$

Ampicillin

Agar
$10 \mathrm{~g}$

$5 \mathrm{~g}$

$10 \mathrm{~g}$

$100 \mu \mathrm{g} / \mathrm{ml}$

$10 \mathrm{~g}$ - added as required for the generation of LB agar

\subsubsection{TE Buffer- $50 \mathrm{ml}$}

$\begin{array}{ll}\text { EDTA }(0.5 \mathrm{M}) & 100 \mu \mathrm{l} \\ \text { Tris }(1 \mathrm{M}) & 500 \mu \mathrm{l} \\ \mathrm{dH}_{2} \mathrm{O} & 49.4 \mathrm{ml}\end{array}$




\subsection{Gel Electrophoresis and Western Blotting Solutions}

\subsubsection{RIPA Buffer (pH 7.5) - 1 L}

$\begin{array}{ll}\text { Tris } \mathrm{HCl} & 1.2 \mathrm{~g} \\ \mathrm{NaCl} & 8.766 \mathrm{~g} \\ \text { EDTA } & 0.37 \mathrm{~g} \\ \text { Triton-X-100 } & 10 \mathrm{ml} \\ \text { SDS } & 1 \mathrm{~g} \\ \text { Sodium deoxycholate } & 10 \mathrm{~g}\end{array}$

\subsubsection{5x Reducing Buffer $-1 \mathrm{~L}$}

$\begin{array}{ll}\text { Tris } \mathrm{HCl}(\mathrm{pH} \mathrm{6.8}) & 62.5 \mathrm{mM} \\ \mathrm{SDS} & 2 \%\end{array}$

Glycerol 20\%

Bromophenol Blue $\quad 1 \%$

\subsubsection{Gels (2x)}

\subsubsection{1. $10 \%$ Separating Gel}

$\begin{array}{ll}\mathrm{dH}_{2} \mathrm{O} & 8 \mathrm{ml} \\ 1.5 \mathrm{M} \text { Tris }(\mathrm{pH} \mathrm{8.8)} & 5 \mathrm{ml} \\ 10 \% \text { SDS } & 200 \mu \mathrm{l} \\ \text { Acrylamide } & 6.66 \mathrm{ml} \\ 10 \% \text { APS } & 100 \mu \mathrm{l} \\ \text { TEMED } & 10 \mu \mathrm{L}\end{array}$

Layered with $100 \%$ isopropanol while setting.

\subsubsection{4\% Stacking Gel}

$\begin{array}{ll}\mathrm{dH}_{2} \mathrm{O} & 6.1 \mathrm{ml} \\ 0.5 \mathrm{M} \text { Tris }(\mathrm{pH} \mathrm{6.8)} & 2.5 \mathrm{ml} \\ 10 \% \text { SDS } & 100 \mu \mathrm{l} \\ \text { Acrylamide } & 1.33 \mathrm{ml} \\ 10 \% \text { APS } & 50 \mu \mathrm{l} \\ \text { TEMED } & 10 \mu \mathrm{L}\end{array}$


7.2.4. 10x Running Buffer $-1 \mathrm{~L}$

SDS

$10 \mathrm{~g}$

Tris $\mathrm{HCl}$

$30.3 \mathrm{~g}$

Glycine

$144.1 \mathrm{~g}$

7.2.5. Western Transfer Buffer - $1 \mathrm{~L}$

Glycine $14.4 \mathrm{~g}$

Tris $\mathrm{HCl}$

$3.03 \mathrm{~g}$

Methanol

$200 \mathrm{ml}$

7.2.6. 10x TBS (pH 7.5) - 1 L

Tris $\mathrm{HCl}$

$60.5 \mathrm{~g}$

$\mathrm{NaCl}$

$87.6 \mathrm{~g}$

\subsubsection{T-TBS}

1x TBS with $0.1 \%$ Tween-20

\subsubsection{Stripping Buffer $-1 \mathrm{~L}$}

SDS

$20 \mathrm{~g}$

$0.05 \mathrm{M}$ Tris $\mathrm{HCl}, \mathrm{pH} 6.8$

$992.5 \mathrm{ml}$

$\beta$-mercaptoethanol

$7.5 \mathrm{~mL}$ 
7.3. Statistical Analysis

\begin{tabular}{|c|c|c|c|c|c|c|c|c|c|}
\hline Section & Experiment & Figure & Transformation & Normality & Homogeneity & Variance $<3$ & Sphericity & Test of Variance & $\begin{array}{c}\text { Three-Way } \\
\text { ANOVA }\end{array}$ \\
\hline 3.3.4 & $\begin{array}{l}\text { Hotplate: } \\
\text { Morphine }\end{array}$ & 1.5 & Log10 & Yes & No & $\begin{array}{c}>3 \text { at } 30 \text { and } \\
45 \mathrm{~min}\end{array}$ & yes & $\begin{array}{l}\text { No difference between } \\
\text { analysis with/out } 30 \text { and } \\
45 \mathrm{~min}\end{array}$ & yes \\
\hline 3.3.4 & Hotplate: Kurkinol & 1.6 & Square root & Yes & Yes & n.a & Yes & n.a & Yes \\
\hline 3.3.4 & $\begin{array}{l}\text { Hotplate: } \\
\text { Kurkinorin }\end{array}$ & 1.7 & Square root & Yes & No & $<3$ & Yes & n.a & Yes \\
\hline 3.3.7 & $\begin{array}{c}\text { PTX: mechanical } \\
\text { induction }\end{array}$ & $1.9 \mathrm{~A}$ & Square root & Yes & Yes & n.a & No & n.a & Yes \\
\hline 3.3.7 & $\begin{array}{l}\text { PTX: cold } \\
\text { induction }\end{array}$ & $1.9 \mathrm{~B}$ & Log10 & Yes & No & $<3$ & No & n.a & Yes \\
\hline 3.3.9 & $\begin{array}{c}\text { PTX mechanical: } \\
\text { Tolerance }\end{array}$ & 1.13 & Square root & Yes & No & $<3$ & No & n.a & Yes \\
\hline 3.3.9 & $\begin{array}{l}\text { PTX: cold } \\
\text { Tolerance }\end{array}$ & 1.14 & Square root & Yes & No & $<3$ & No & n.a & Yes \\
\hline 3.3.9 & $\begin{array}{c}\text { PTX: mechanical } \\
\text { Hyperalgesia }\end{array}$ & Appendix & Square root & Yes & No & $<3$ & No & n.a & Yes \\
\hline 31.3 .9 & $\begin{array}{c}\text { PTX: cold } \\
\text { Hyperalgesia }\end{array}$ & Appendix & Log10 & Yes & No & $<3$ & Yes & n.a & Yes \\
\hline
\end{tabular}

Table 7.1 Chapter 3 Statistical Test Assumption Information

Where sphericity was not meet the Greenhouse-Geisser correction was applied 


\begin{tabular}{|c|c|c|c|c|c|c|c|c|c|}
\hline Section & Experiment & Figure & Transformation & Normality & Homogeneity & $\begin{array}{c}\text { Variance } \\
\quad<3\end{array}$ & Sphericity & $\begin{array}{c}\text { Test of } \\
\text { Variance }\end{array}$ & $\begin{array}{c}\text { Three-Way } \\
\text { ANOVA }\end{array}$ \\
\hline 4.3 .2 & Rotarod: morphine & 4.3 & Square root & Yes & No & $<3$ & no & n.a & Yes \\
\hline 4.3.2 & Rotarod: morphine & 4.4 & Square root & Yes & No & $<3$ & no & n.a & Yes \\
\hline 4.1.1.5 & $\begin{array}{c}\text { Faecal Accumulation: } \\
\text { Morphine }\end{array}$ & $\begin{array}{c}4.6 \mathrm{~A}, \mathrm{~B}, \mathrm{G}, \mathrm{H} \\
\text { 4.7A }\end{array}$ & Square root & Yes & Yes & n.a & Yes & n.a & Yes \\
\hline 4.1.1.5 & $\begin{array}{l}\text { Faecal Accumulation: } \\
\text { Kurkinol }\end{array}$ & $\begin{array}{c}4.6 \mathrm{C}, \mathrm{D}, \mathrm{G}, \mathrm{H} \\
4.7 \mathrm{~B}\end{array}$ & Log10 & Yes & Yes & n.a & No & n.a & Yes \\
\hline 4.1.1.5 & $\begin{array}{c}\text { Faecal Accumulation: } \\
\text { Kurkinorin }\end{array}$ & $\begin{array}{c}4.6 \mathrm{E}, \mathrm{F}, \mathrm{G}, \mathrm{H} \\
4.7 \mathrm{C}\end{array}$ & Square root & Yes & Yes & n.a & Yes & n.a & Yes \\
\hline 4.1.1.7 & $\begin{array}{c}\text { Urinary Retention: } \\
\text { Morphine }\end{array}$ & $\begin{array}{c}4.10 \mathrm{~A}, \mathrm{~B}, \mathrm{G}, \mathrm{H} \\
4.11 \mathrm{~A}\end{array}$ & Square root & Yes & No & $<3$ & No & n.a & Yes \\
\hline 4.1.1.7 & Urinary Retention: Kurkinol & $\begin{array}{c}4.10 \mathrm{C}, \mathrm{D}, \mathrm{G}, \mathrm{H} \\
4.11 \mathrm{~B}\end{array}$ & Square root & Yes & No & $<3$ & No & n.a & Yes \\
\hline 4.1.1.7 & $\begin{array}{c}\text { Urinary Retention: } \\
\text { Kurkinorin }\end{array}$ & $\begin{array}{c}4.10 \mathrm{E}, \mathrm{F}, \mathrm{G}, \mathrm{H} \\
4.11 \mathrm{C}\end{array}$ & Reflect \& Log10 & Yes & No & $<3$ & No & n.a & Yes \\
\hline 4.1.1.8 & $\begin{array}{c}\text { Plethysmography: Kurkinol } \\
\text { Freq }\end{array}$ & $4.12 \mathrm{~A}, \mathrm{~B}, \mathrm{C}$ & Log10 & Yes & No & $<3$ & Yes & n.a & Yes \\
\hline 4.1.1.8 & $\begin{array}{c}\text { Plethysmography: Kurkinol } \\
T_{V}\end{array}$ & 4.12 D, E, F & Log10 & Yes & No & $<3$ & No & n.a & Yes \\
\hline 4.1.1.8 & $\begin{array}{l}\text { Plethysmography: Kurkinol } \\
\qquad V_{\min }\end{array}$ & $4.12 \mathrm{G}, \mathrm{H}, \mathrm{I}$ & Log10 & Yes & No & $<3$ & No & n.a & Yes \\
\hline 4.1.1.8 & $\begin{array}{l}\text { Plethysmography: } \\
\text { Kurkinorin Freq }\end{array}$ & $4.13 \mathrm{~A}, \mathrm{~B}, \mathrm{C}$ & Log10 & Yes & Yes & $<3$ & Yes & n.a & Yes \\
\hline 4.1.1.8 & $\begin{array}{c}\text { Plethysmography: } \\
\text { Kurkinorin } T_{V}\end{array}$ & $4.13 \mathrm{D}, \mathrm{E}, \mathrm{F}$ & Log10 & Yes & No & $<3$ & No & n.a & Yes \\
\hline 4.1.1.8 & $\begin{array}{l}\text { Plethysmography: } \\
\text { Kurkinorin } \mathrm{V}_{\min }\end{array}$ & $4.13 \mathrm{G}, \mathrm{H}, \mathrm{I}$ & Log10 & Yes & No & $<3$ & No & n.a & Yes \\
\hline
\end{tabular}

Table 7.2 Chapter 4 Statistical Test Assumption Information

Where sphericity was not meet the Greenhouse-Geisser correction was applied 


\subsection{Chemotherapy-Induced Neuropathic Pain Supplementary Data}

\subsubsection{Paclitaxel Induced Comparable Disease Between Treatment Groups}

A)

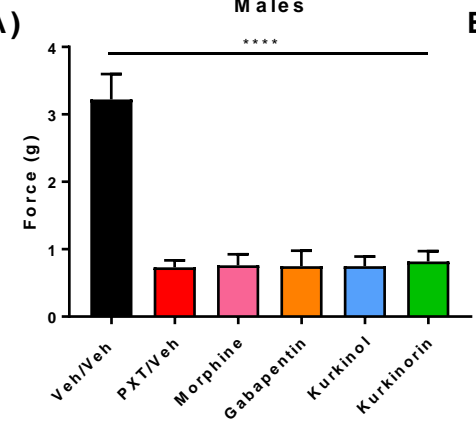

D)

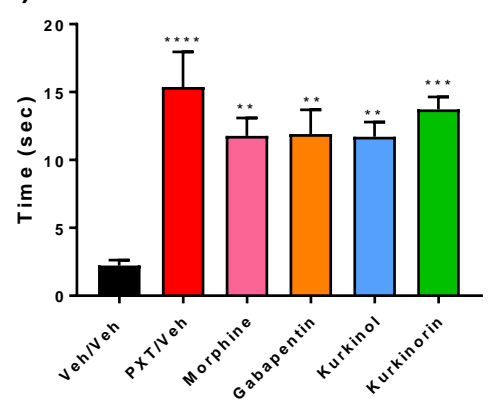

B)

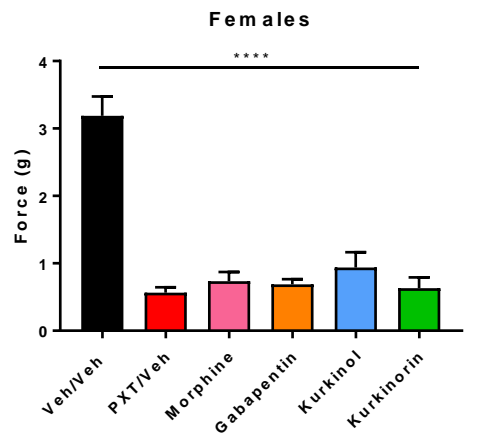

E)

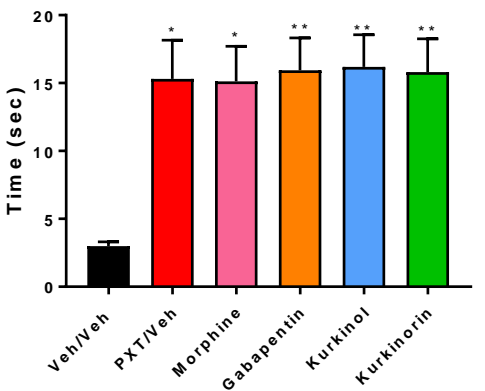

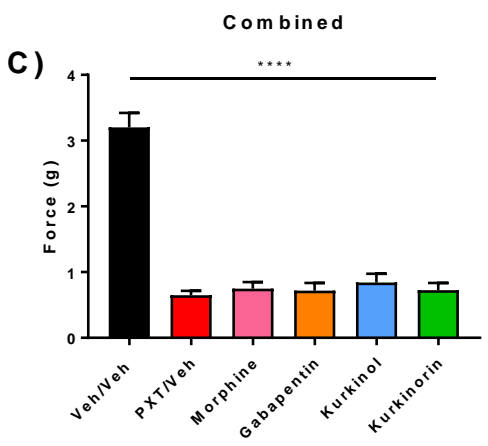

F)

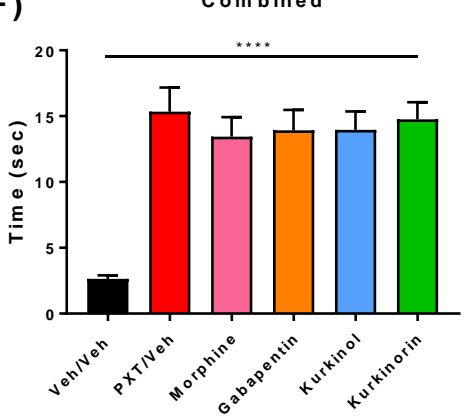

Figure 7.1 Treatment Groups Did Not Significantly Differ in the Induction of Neuropathic Pain on Day 15

Mice were placed into treatment groups on day 15 based on the induction of cold and mechanical allodynia.

(A) Male and (B) female mechanical allodynia scores on day 15 showed decreases to vehicle/vehicle-treated animals with no differences between the groups. (C) There were also no differences between treatment groups when the genders were combined. (D) Male and (E) female cold allodynia scores on day 15 showed an increase in time responding to cold stimuli compared to vehicle/vehicle with no differences between groups. (F) When the genders were combined there was no difference in cold allodynia baselines on day 15. One-Way ANOVA with Bonferroni Multiple corrections test. ${ }^{*} p<0.05,{ }^{* *} p<0.01,{ }^{* * *} p<0.001,{ }^{* * * *} p<0.0001$. Data presented as mean \pm SEM. $N=6$ females and 5/6 males per group, combined data $N=11-12$. 


\subsubsection{Induction of Hyperalgeisa by Kurkinol and Kurkinorin}

A)

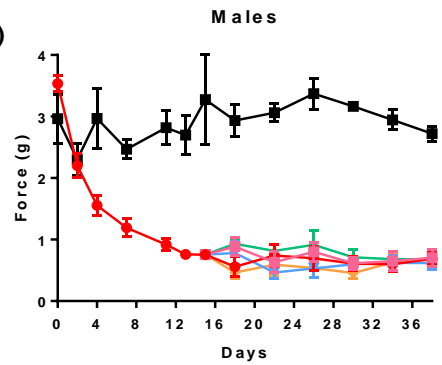

D)

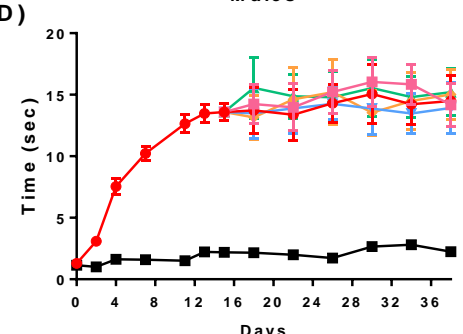

B )

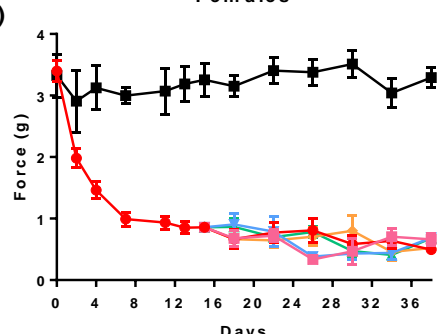

E)

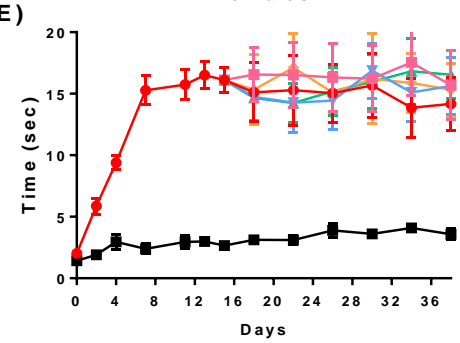

C

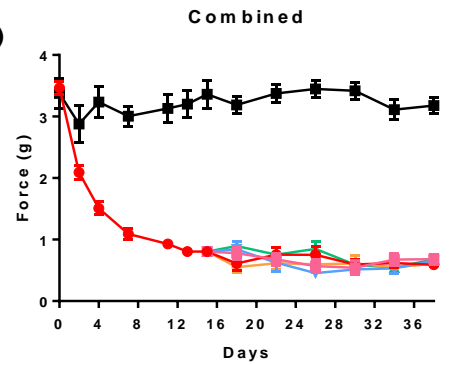

F)

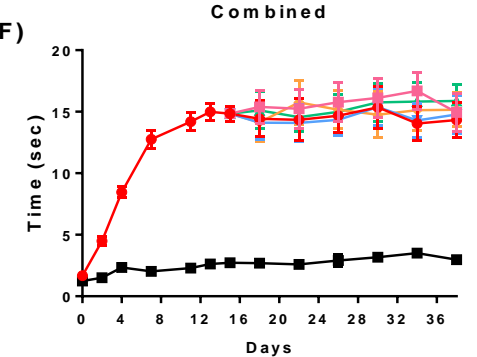

$\rightarrow$ Veh/Veh $\rightarrow$ PXT/Veh $\rightarrow$ Morphine $\neg$ Gabapentin $\rightarrow$ Kurkinol $\neg$ Kurkinorin

Figure 7.2 Chronic Treatment with Kurkinol or Kurkinorin did not Reverse Disease Progression or Induce Hyperalgesia to Mechanical or Cold Allodynia

On day 17 of chronic pain daily administration of vehicle, morphine, gabapentin, kurkinol, or kurkinorin was initiated with the effect on disease progression and/or hyperalgesia tracked every 4 days from day 18 . There was no reduction or increase in mechanical allodynia in (A) male, (B) female, or (C) overall when genders were combined. No change in cold allodynia was seen with chronic administration in (D) male, (E) female, or (F) overall when genders were combined. Three-way mixed ANOVA. Data presented as an average of measurements taken in quadruplicate. Data are shown as mean \pm SEM. ( $N=5-6$ males \& 6 females per group). Doses in brackets in $\mathrm{mg} / \mathrm{kg} / \mathrm{i} . \mathrm{p}$. 


\subsection{The Effect of Kurkinol and Kurkinorin on Core Body Temperature}

$\mu$ receptor agonists have the potential to negatively affect core body temperature. To assess the impact of kurkinol and kurkinorin on core temperature the rectal temperature of male mice was taken for 60 min post-administration. The core temperature following morphine administration was found to significantly decrease over time $\left(F_{(18,102)}=3.36, p<0.0001, p<0.05\right)$. Neither kurkinol nor kurkinorin showed significant decreases in rectal temperature, with both showing improvements from 45-60 min compared to morphine $(p<0.05)$. These temperatures were used during the calculation of respiratory measures from the wholebody plethysmography assay.

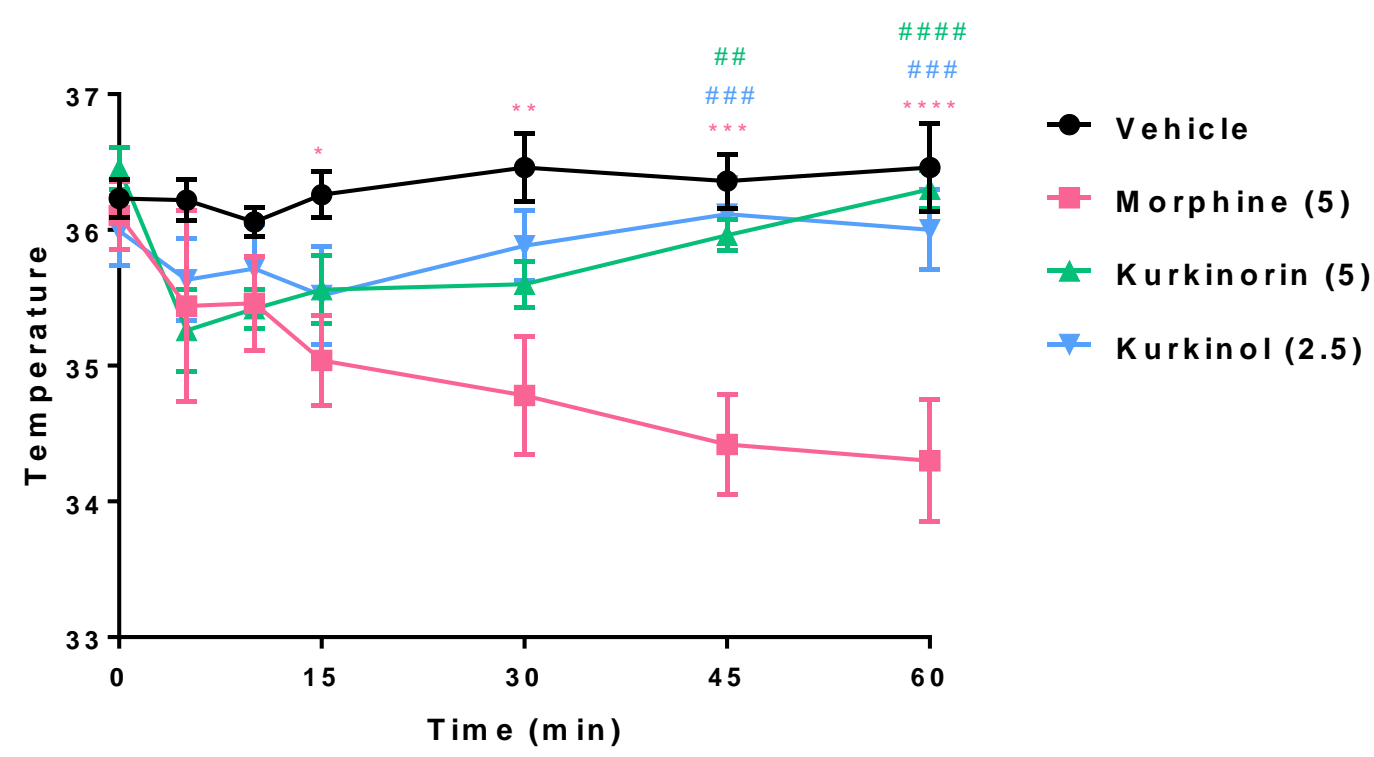

Figure 7.3 Hypothermic Effects of Kurkinorin \& Kurkinol

The change in rectal temperature in response to morphine (5 mg/kg/i.p.), kurkinol (2.5 mg/kg/i.p.), and kurkinorin $(5 \mathrm{mg} / \mathrm{kg})$ was monitored over a 60 min period. Morphine-induced a significant hypothermic reaction from 15 - 60 min. Neither kurkinorin nor kurkinol induced a change in rectal temperature, with both showing significant improvements to morphine from 45-60 min. Two-way repeated measures ANOVA with Bonferroni multiple corrections test, ${ }^{*} p<0.05,{ }^{* *} p<0.01,{ }^{* * *} p<0.001,{ }^{* * * *} p<0.0001$ compared to Vehicle, $\# \# p<0.01, \# \# \# p<0.001, \# \# \#$ $<0.0001$ compared to Morphine. Data are shown as mean \pm SEM $(N=5$ per group). Doses in brackets in $\mathrm{mg} / \mathrm{kg} / \mathrm{i} . \mathrm{p}$. 


\subsection{Whole-Body Plethysmography in Unrestrained Mice}

\subsubsection{Kurkinol Altered all Measures of Respiration}
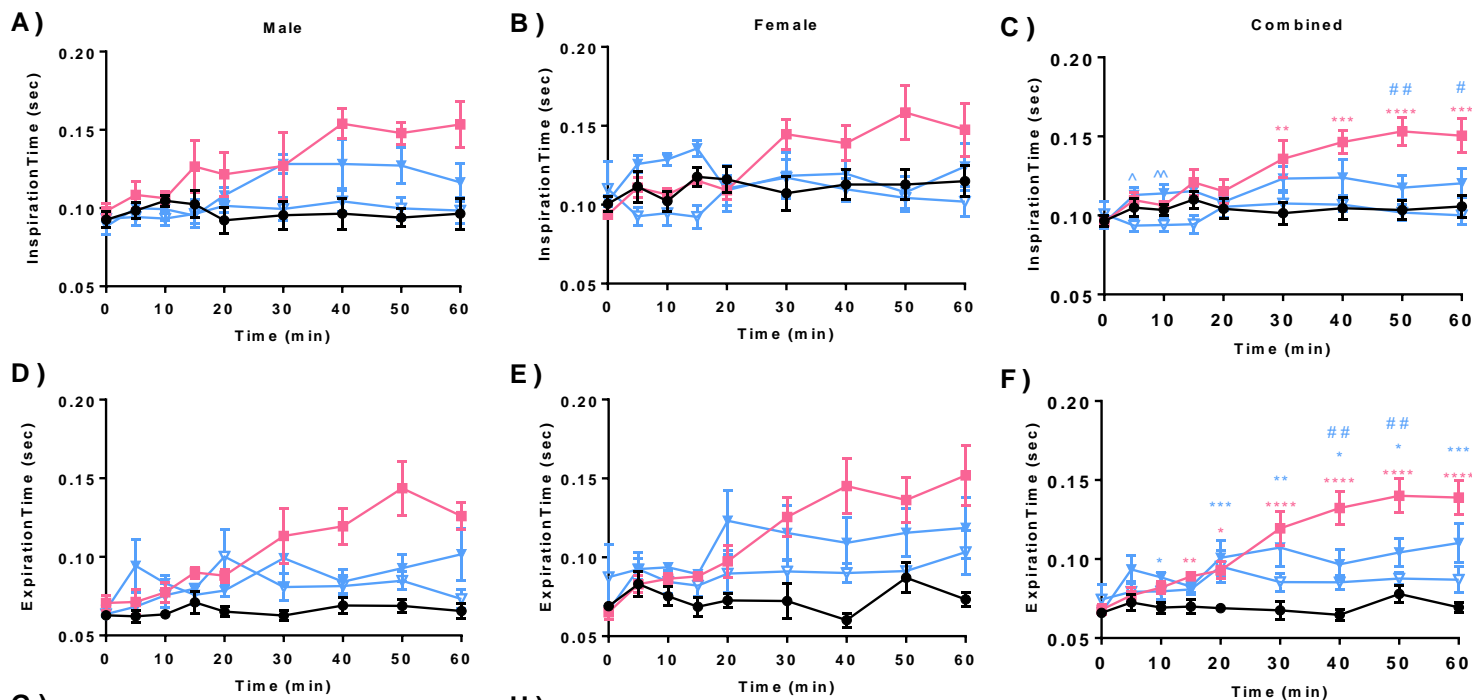

G )

E)

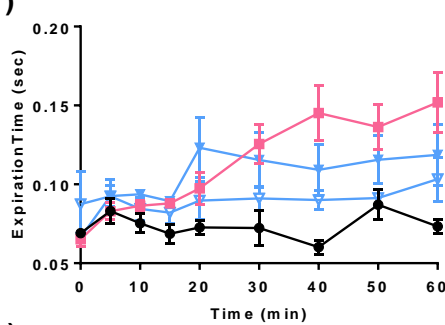

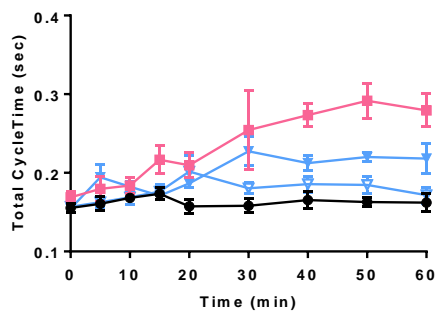

H)

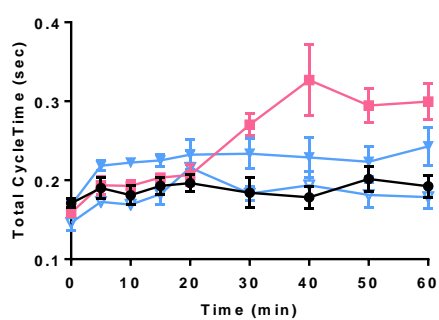

K)

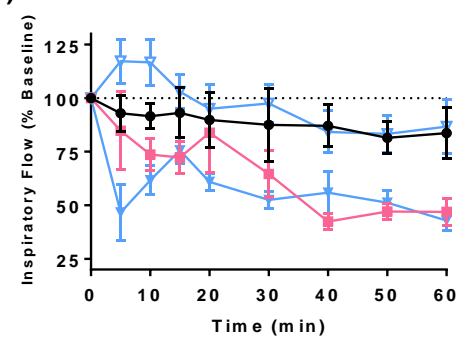

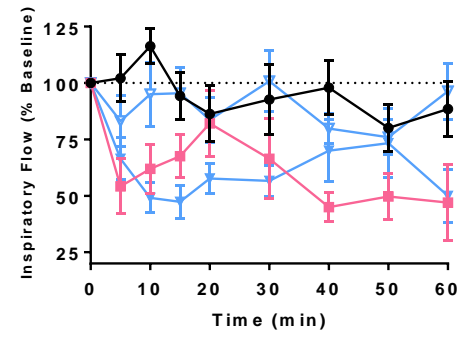

F)
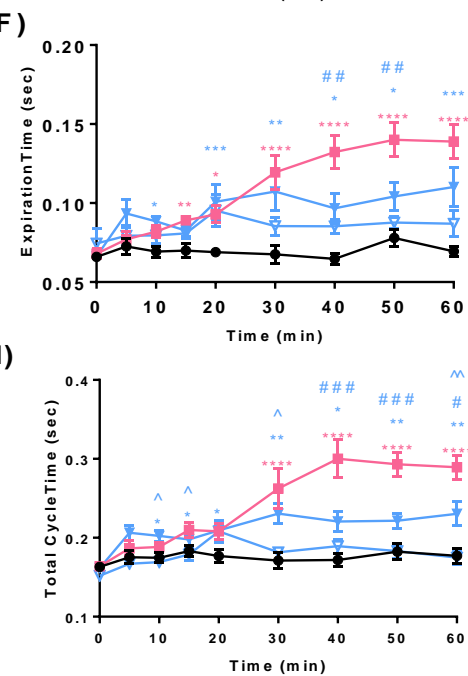

L)

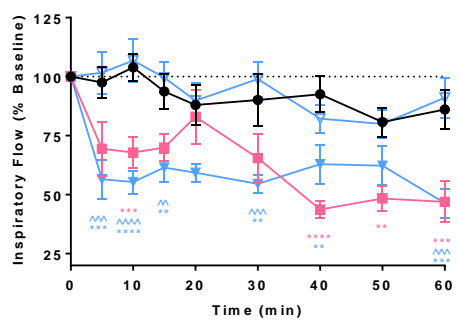

Vehicle $\rightarrow$ Morphine (5) $\neg$ Kurkinol (2.5)

Kurkinol (2.5) + PNA

Figure 7.4 Kurkinol Negatively Impacts Multiple Measures of Respiration in C57 BI/6 mice

(A) Effect on inspiration time in males and (B) females. (C) Kurkinol induced no increase in inspiration time. (D) Effect on expiration time in males and (E) females. (F) kurkinol increased expiration time compared to the vehicle with improvements to morphine between 40-50 min. (G) Effect on total cycle time in males and (H) females. (I) The total cycle time was increased in kurkinol treated mice, with improvements to morphine from 40-60 min. (J) Effect on inspiratory flow in males and (K) females. (L) Kurkinol had a faster onset of 5 min with a significant decrease between $5-60 \mathrm{~min}$. $\beta$-FNA pre-treatment reduced all effects. Three-way mixed ANOVA. Data presented as an average of measurements taken in quadruplicate. Data are shown as mean \pm SEM. ${ }^{*} p<0.05,{ }^{* *} p<0.01,{ }^{* * *} p<0.001,{ }^{* * * *} p<0.0001$ drug to vehicle, $\# p<0.05, \# \# p<0.01, \# \# \# p<0.001$, $\# \# \# \#<0.0001$ drug to morphine, $\wedge^{\wedge} p<0.05, \wedge^{\wedge} p<0.01, \wedge^{\wedge \wedge} p<0.001$, drug to $\beta$-FNA pre-treatment. $(N=7-8$ males \& 6-7 females per group). Doses in brackets in $\mathrm{mg} / \mathrm{kg} / \mathrm{i} . \mathrm{p}$. 


\subsubsection{Effects of Kurkinorin on Further Respiratory Measures}

A)

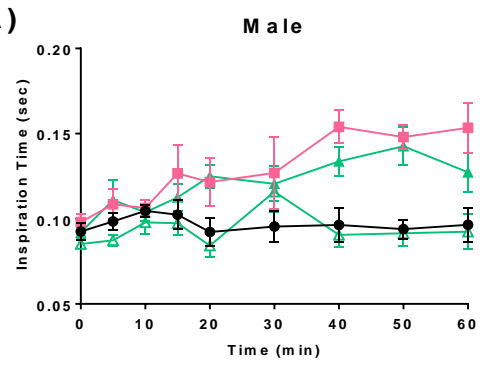

D)

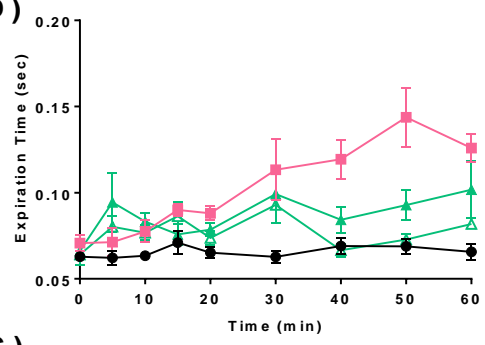

G)

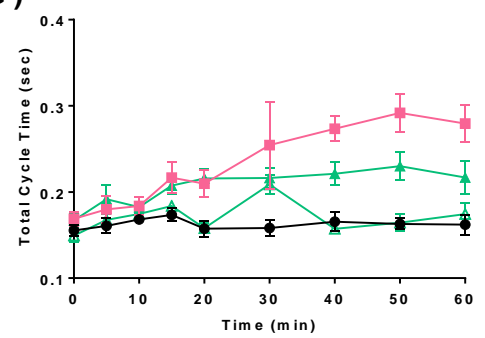

J)

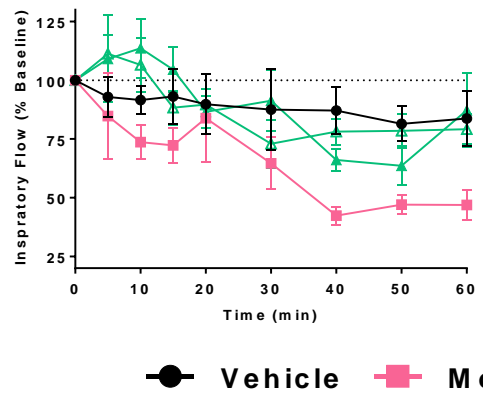

B)

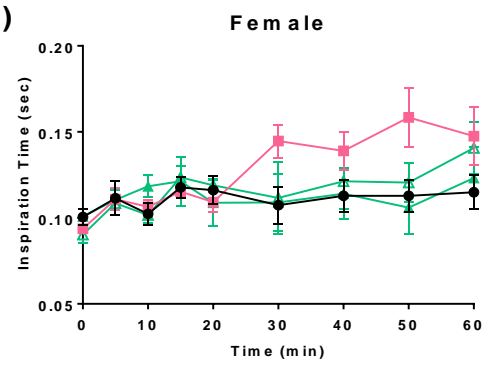

E)

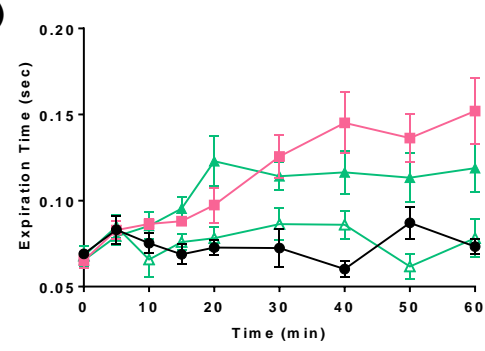

H)

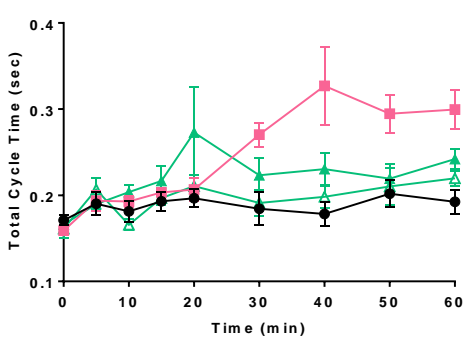

K)

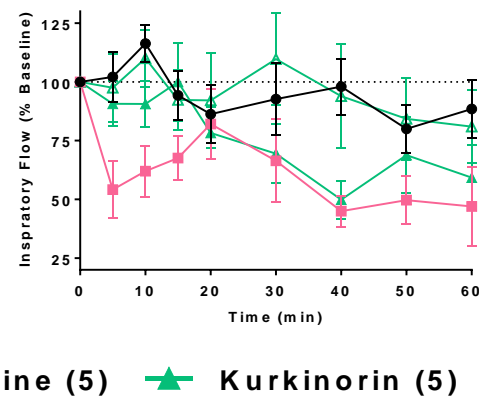

C)

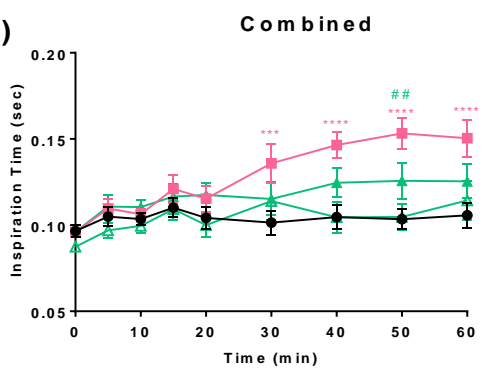

F)

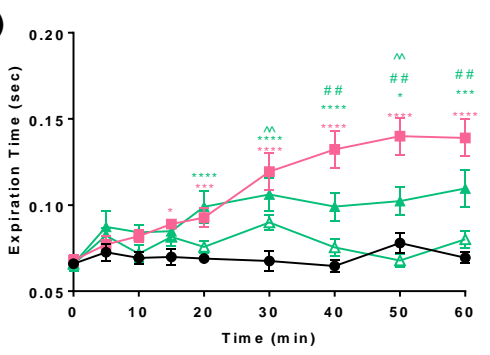

I)

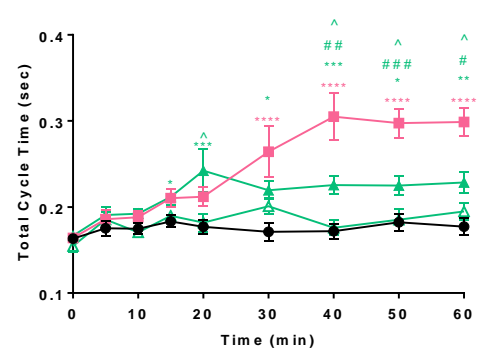

L)

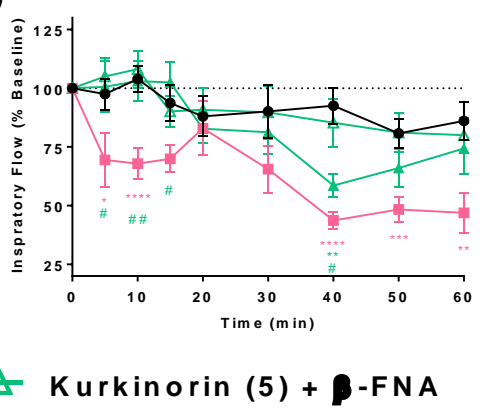

Figure 7.5 Kurkinorin has a Reduced Effect on Respiratory Measures in C57 BI/6 mice

(A) Effect on inspiration time in males and (B) females. (C) Kurkinorin did not affect inspiratory time when sexes were combined. (D) Kurkinorin reduced expiration time in males at 5, 15, 20, and 40 min, but not in (E) female. (F) kurkinorin showed improvements in expiration time to morphine from 40-60 min. (G) Effect on total cycle time in males and (H) females. (I) Total cycle time was increased in kurkinorin treated mice. (J) Effect on inspiratory flow in males and (K) females. (L) Kurkinorin decreased inspiratory flow at $40 \mathrm{~min}$. Threeway mixed ANOVA. Data presented as an average of measurements taken in quadruplicate. Data are shown as mean \pm SEM. ${ }^{*} p<0.05,{ }^{* *} p<0.01,{ }^{* * *} p<0.001, * * * * p<0.0001$ drug to vehicle, $\# p<0.05, \# \# p<0.01, \# \# \# p<$ $0.001, \# \# \#$, 0.0001 drug to morphine, $\wedge p<0.05, \wedge \wedge p<0.01, \wedge \wedge \wedge p<0.001, \wedge \wedge \wedge \wedge p<0.0001$ drug to $\beta$-FNA pre-treatment. ( $N=7-8$ males \& 6-7 females per group). Doses in brackets in $\mathrm{mg} / \mathrm{kg} / \mathrm{i}$. . 


\subsection{3. $\beta$-arrestin2 Knockout Vehicle Controls from Whole-body Plethysmography in Unrestrained Mice}

A)

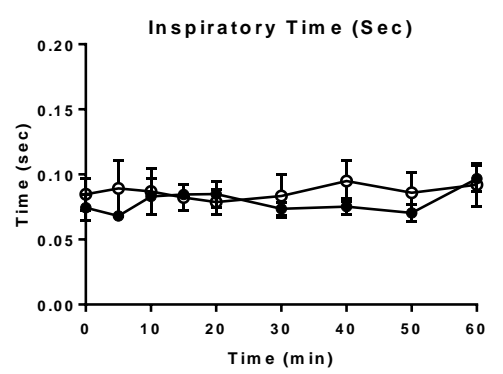

D )

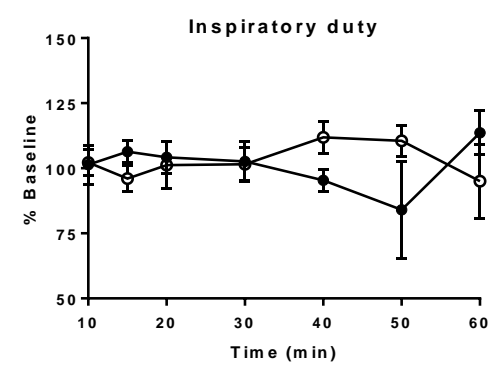

G)

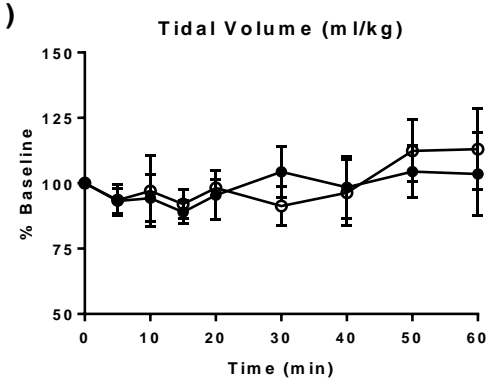

B )

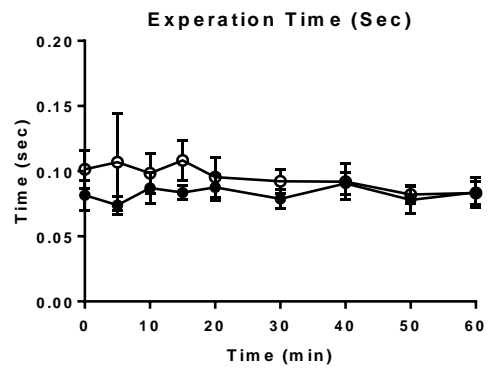

E)

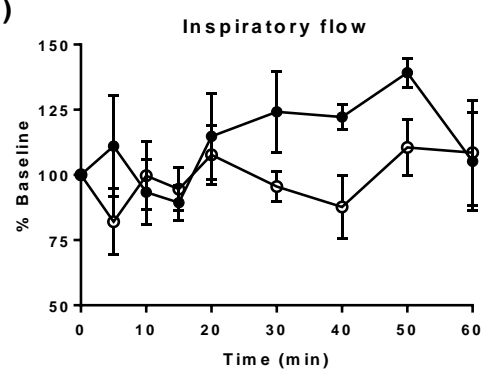

H)

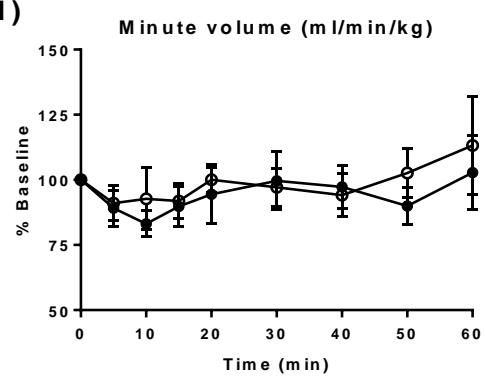

C)

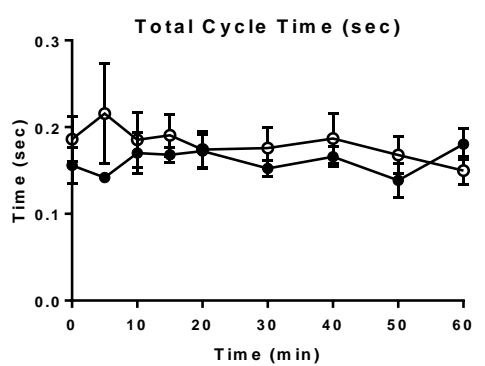

F)

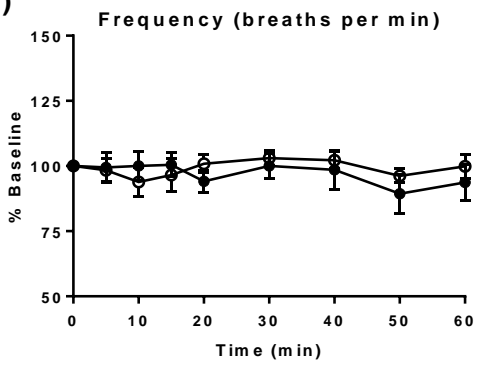

Figure 7.6 Respiratory Measures are not Affected by the Removal of $\beta$-arrestin2

The deletion of $\beta$-arr $2^{-/}$did not affect $(\boldsymbol{A})$ inspiratory time, (B) expiratory time, (C) total cycle time, (D) inspiratory duty, (E) inspiratory flow, (F) frequency, (G) tidal volume, and (H) minute volume. The vehicletreated animals were combined irrespective of genotype for furthers analyse to reduced error and simplify the interpretation. Two-way repeated measures ANOVA with Bonferroni multiple corrections test, Data are shown as mean \pm SEM ( $N=5-6$ per group). 


\subsubsection{Further Respiratory Effects of Morphine, Kurkinol, and Kurkinorin in $\beta$-arrestin2 Knock Out Mice}

A)

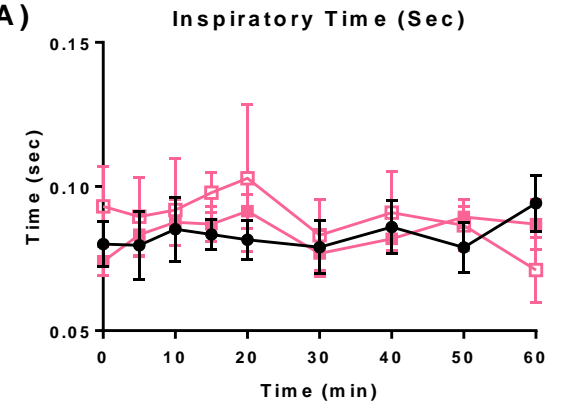

$\underset{\infty}{N}$
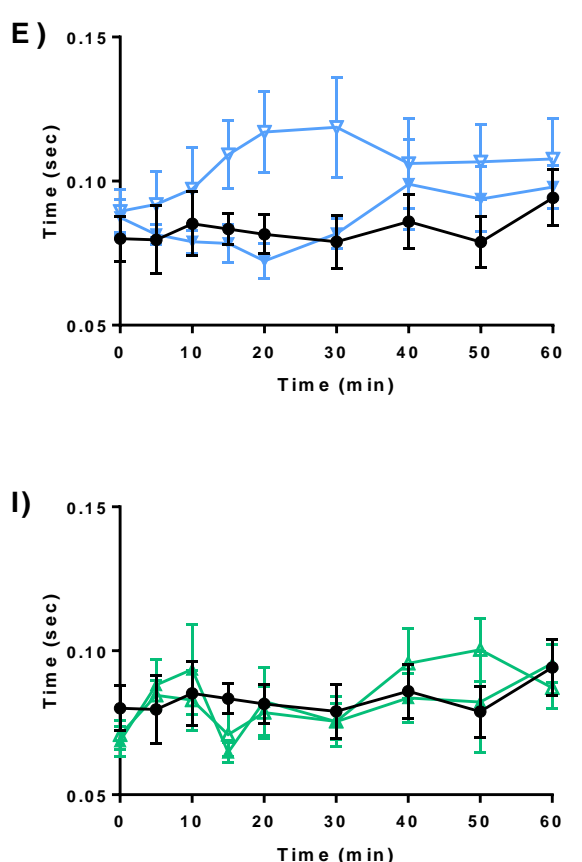

B )
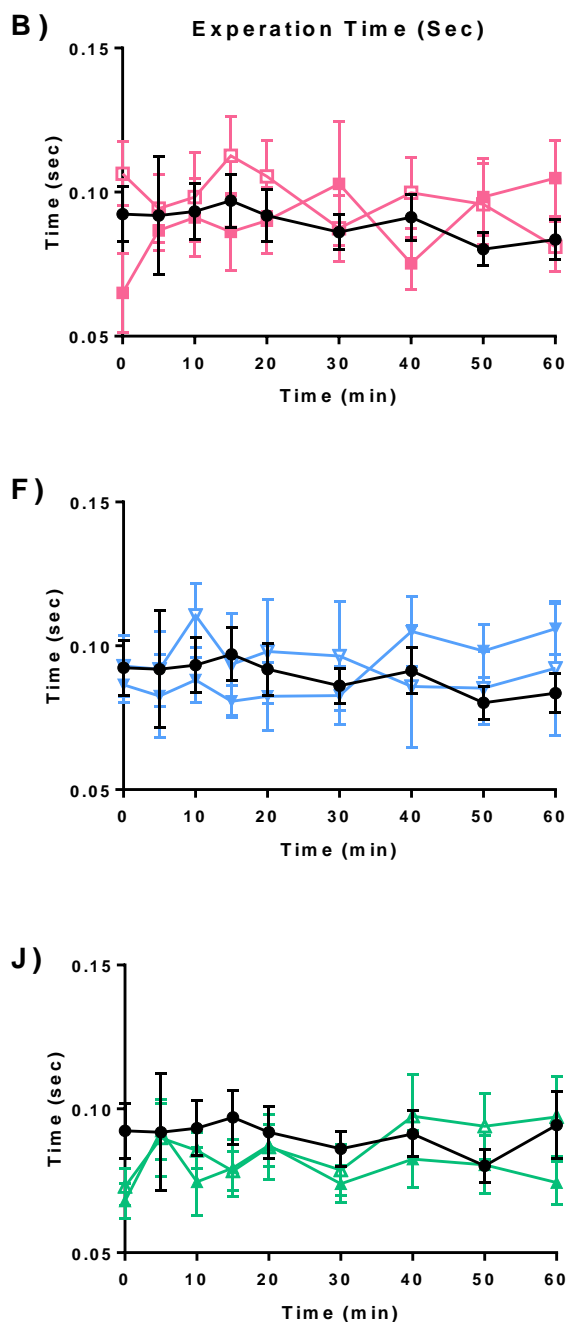

Vehicle

$=-$ Morphine (5)
C)
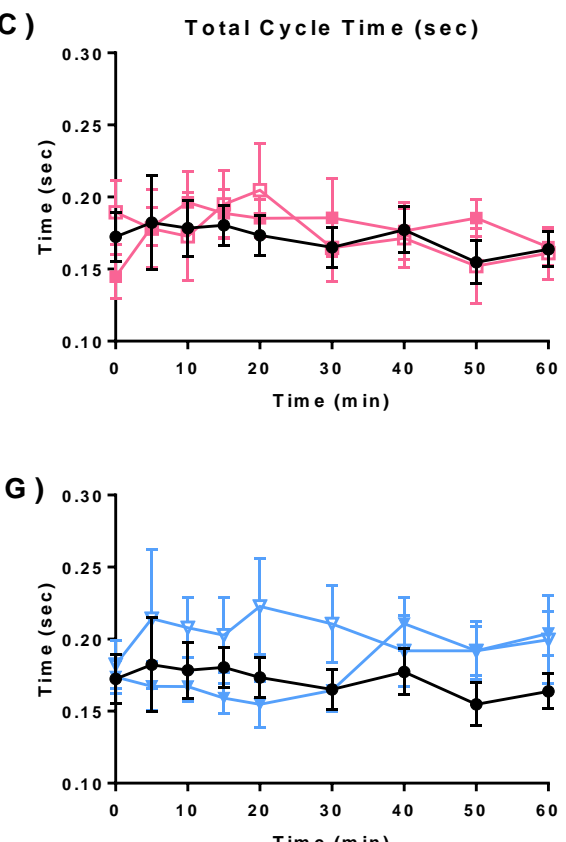

K) 0.

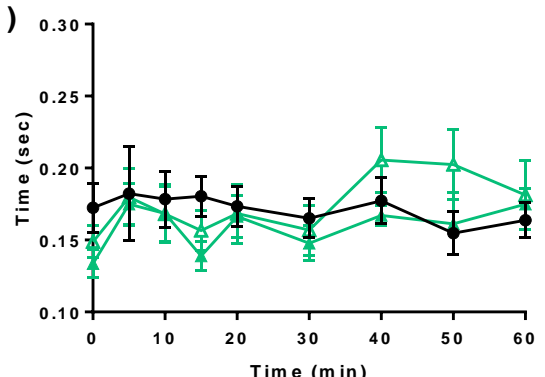

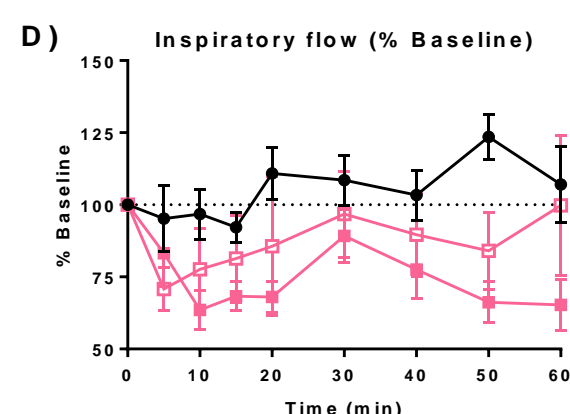

H)

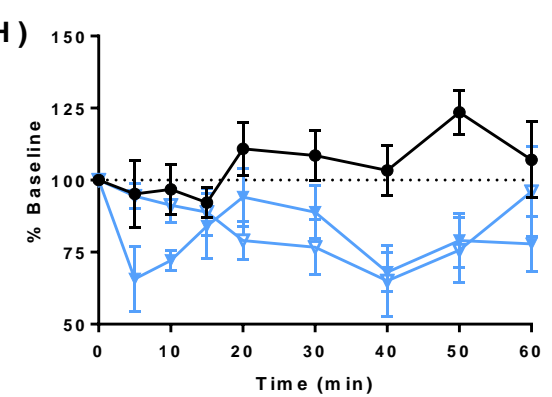

L)

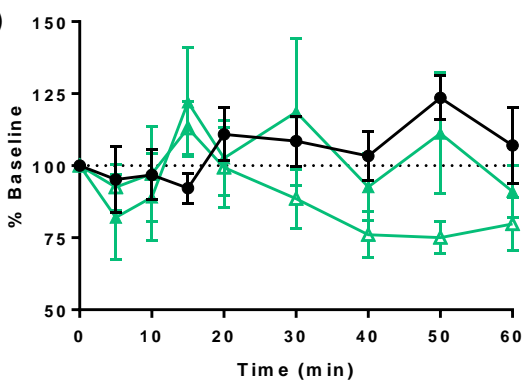

$\rightarrow$ Kurkinol (2.5)

$\rightarrow$ Kurkinorin (5)

$\square$ Morphine (5) (K/O)

$\nabla$ Kurkinol (2.5) (K/0) $\triangle$ Kurkinorin (5) $(K / 0)$ 
Figure 7.7 The Respiratory Effects of Morphine are not Affected by Deletion of $\beta$-arrestin2

The (A) inspiration time, (B) expiration time, (C) total cycle time and (D) inspiratory flow did not differ between Wt and $\beta$-arr ${ }^{-1}$ mice after morphine administration (5 mg/kg/i.p.). (E) When kurkinol (2.5 mg/kg/i.p.) was administered to $\beta$-arr $\%$ mice a trend towards increased inspiration and (F) total cycle time, with no change in (G) expiration time. (H) Kurkinol resulted in a significantly decreased inspiratory flow, irrespective of the genotype. (I) Inspiration time, (J) expiration time, (K) total cycle time and (L) inspiratory flow did not differ between Wt and $\beta$-arr ${ }^{-/}$mice after kurkinorin $(5 \mathrm{mg} / \mathrm{kg} / \mathrm{i} . \mathrm{p}$.) administration. Two-way repeated measures ANOVA with Bonferroni multiple corrections test. Data are shown as mean \pm SEM ( $N=5$ - 13 per group). 


\subsection{Plasmid Vector}

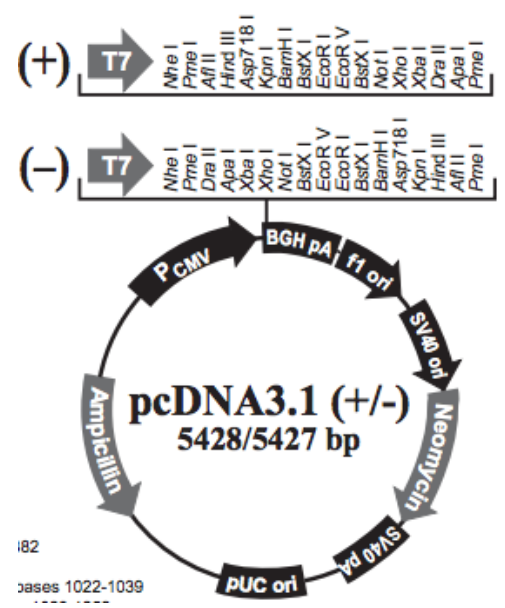

Figure 7.8 Human Mu Opioid Receptor Plasmid Vector Map

The human $\mu$ receptor plasmid was purchased from the cDNA resource centre (Bloomsburg, $P A$, United States) in a pcDNA3.1 (+/-) plasmid.

\subsection{Hek293 \&and CHO cells Stably Expressing the Human Mu Opioid Receptor}

\subsubsection{Hek293 Cells}

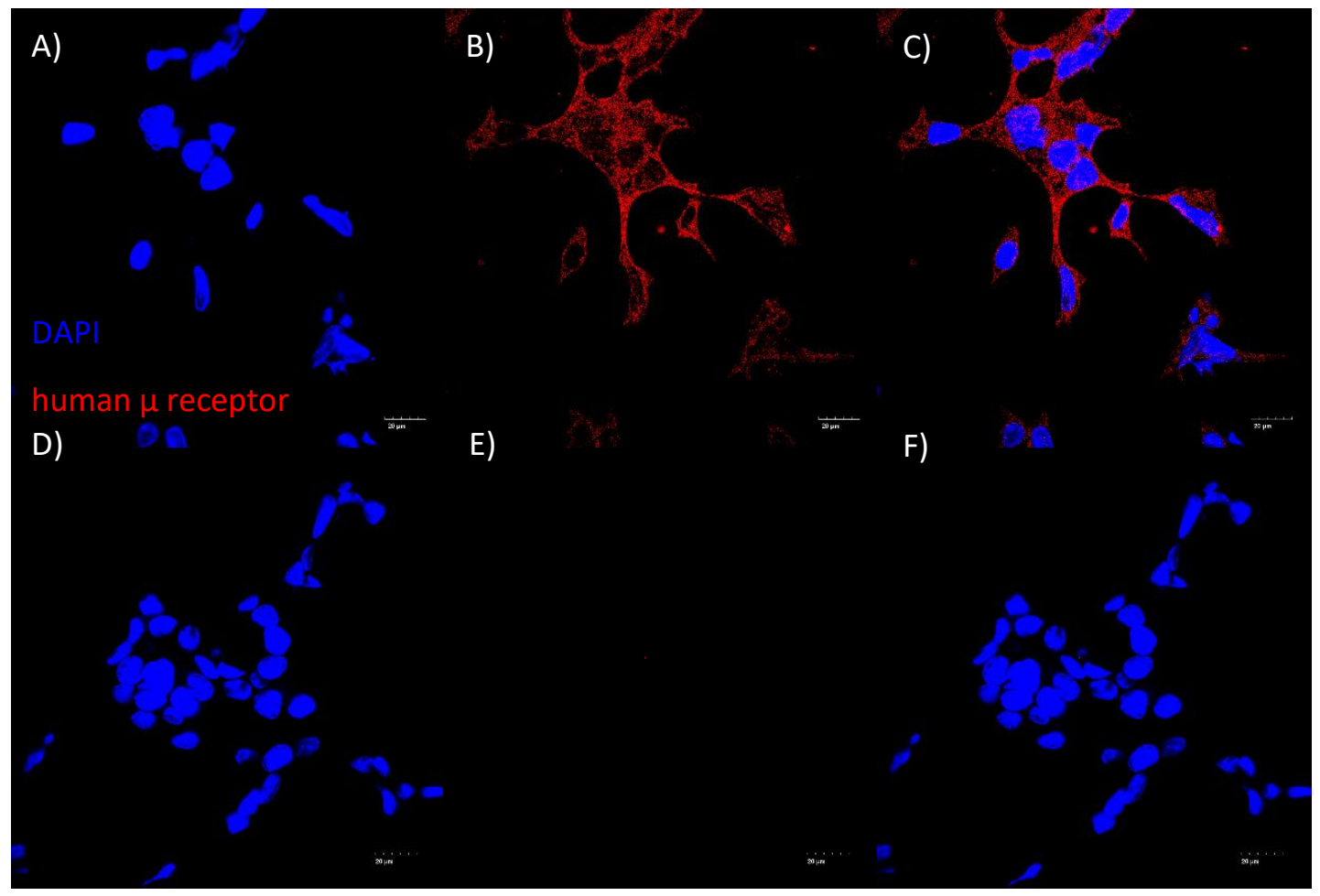

Figure 7.9 Hek293 Cells Stably Expressing the Human Mu Opioid Receptor

Confocal microscopy images of Hek293 cells stably expressing the human $\mu$ receptor. (A) DAPI stained nucleus, (B) Cy5 labelled human $\mu$ receptor, and (C) merged image. (D-E) Negative control images showing no background Cy5 fluorescence. 


\subsubsection{CHO Cells}

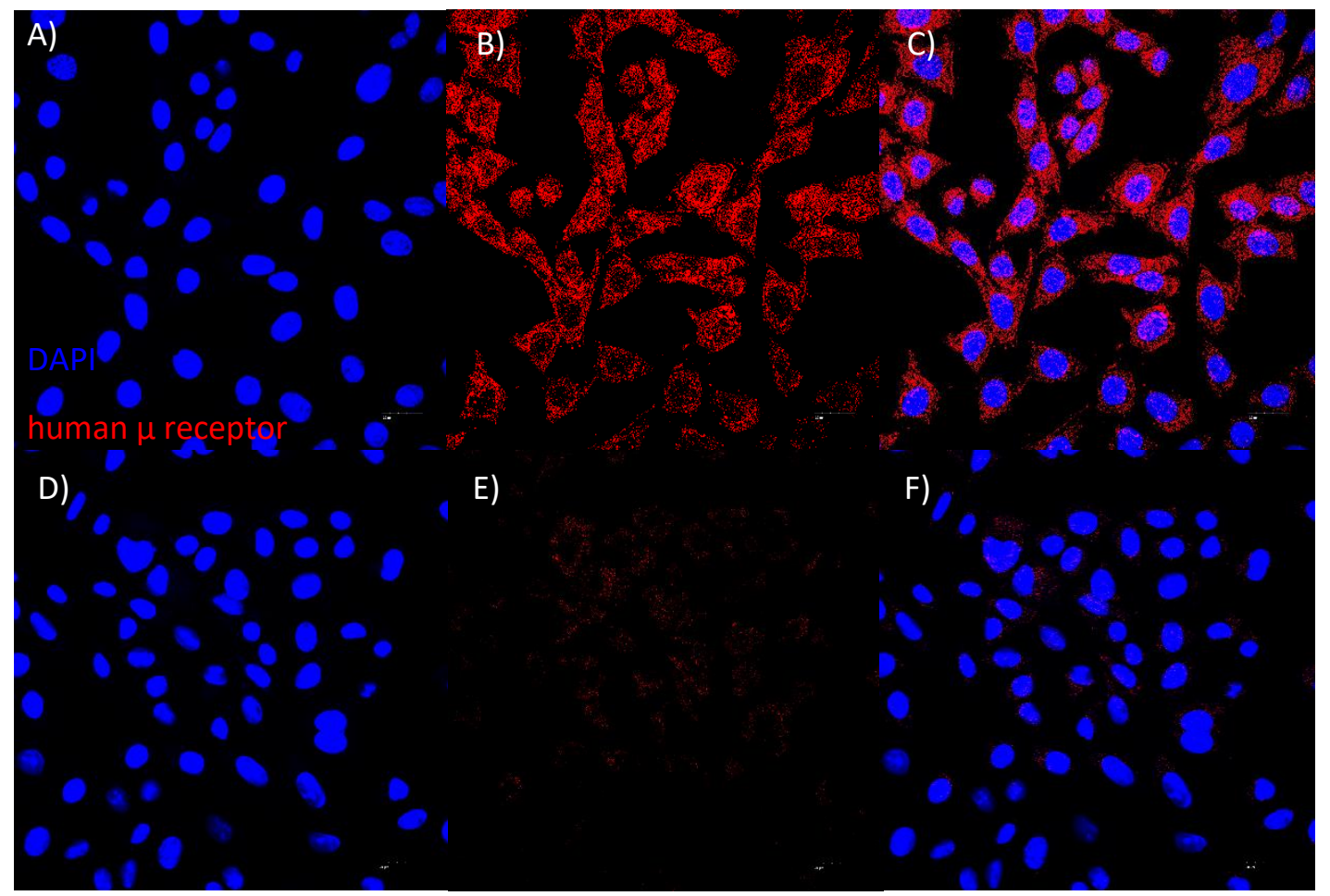

Figure 7.10 CHO Cells Stably Expressing the Human Mu Opioid Receptor

Confocal microscopy images of $\mathrm{CHO}$ cells stably expressing the human $\mu$ receptor. (A) DAPI stained nucleus, (B) Cy5 labelled human $\mu$ receptor, and (C) merged image. (D-E) Negative control images showing limited background Cy5 fluorescence.

\subsection{Membrane Potential Assay Optimisation}

\subsubsection{Optimisation of Forskolin}

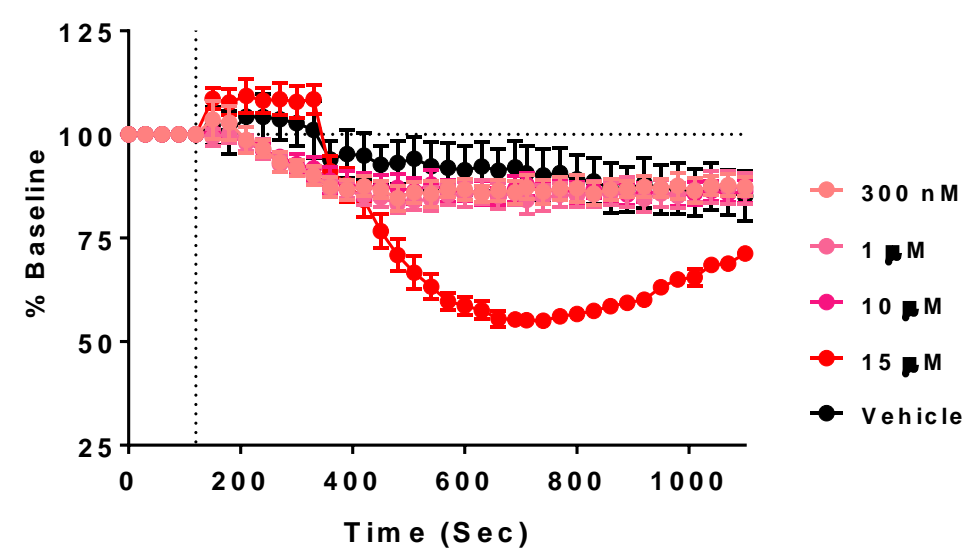

Figure 7.11 Dose Response effects of Forskolin on Membrane Potential

The dose of FSK was optimised to induce the largest change in the fluorescent. Baseline measurements

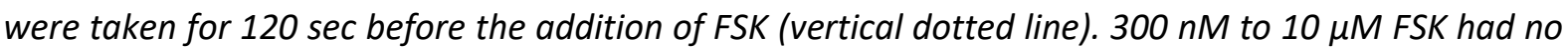
significant effect on the fluorescent signal. 15 MM FSK induced a strong efflux of the fluorescent dye via the activation of ion channels. This dose was used in future for all Molecular Devices FLIPR assays. 


\subsubsection{Optimisation of $\beta-F N A$}

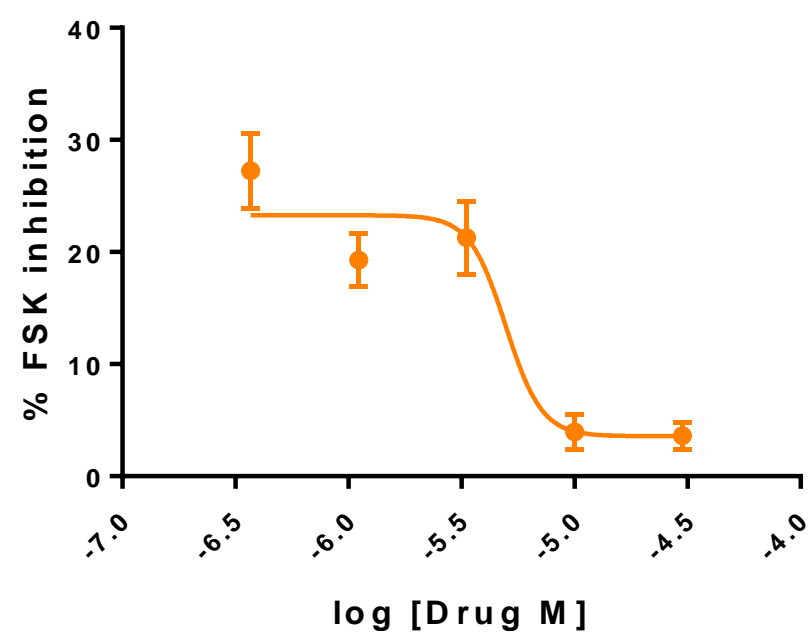

Figure 7.12 B-FNA Inhibition Curve

To ensure that the dose of $\beta$-FNA was enough to reduce the effects of $\mu$ receptor agonists on ion channels a dose response of the antagonist was applied to cells in tandem with the $50 \mu M$ DAMGO. The $I C_{80}$ of $30 \mu M$ was used to antagonise the $\mu$ receptor in future experiments. Data presented as mean \pm SEM. Each dose represents 2 replicates conducted in triplicate.

\subsection{Potassium Assay Optimisation}

\subsubsection{Optimisation of Thallium}

A)

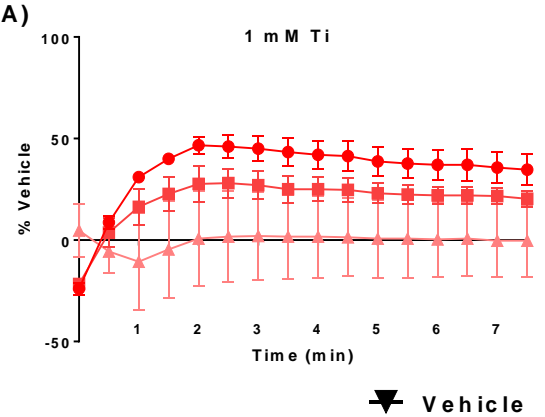

B)

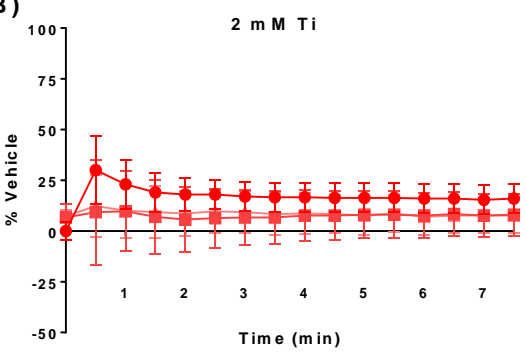

C)

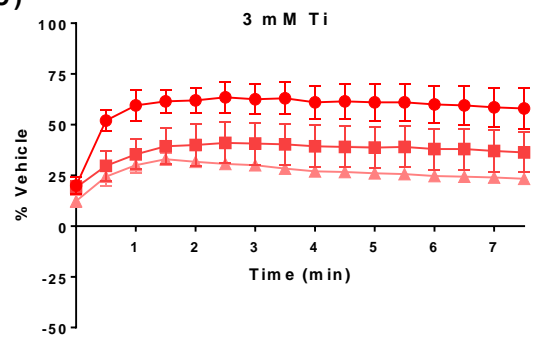

DAMGO 20 uM

Figure 7.13 Optimisation of Thallium for the Potassium Assay in Human Mu Opioid Receptor CHO Stable Cells

Due to the presence of organic anion transporters in $\mathrm{CHO}$ cells the addition of thallium is necessary to ensure the activation of the KIR3.1 channel can be detected. Three concentrations (1, 2, and $3 \mathrm{mM})$ suggested by the supplier (Molecular Devices) were trialled in duplicate over 2 experiments in response to 10, 20, and $30 \mu M D A M G O$. The best separation was seen with the $3 \mathrm{mM}$ concentration of Thallium, which was then used for all subsequent experiments. Data presented as mean \pm SEM. Each dose represents 2 replicates conducted in duplicate. Data presented as a percent of vehicle controls. 


\subsection{Western Blot Controls}

\subsubsection{Secondary Only Control}

$\mathrm{Mw}$

marker

A)

Protein lysate

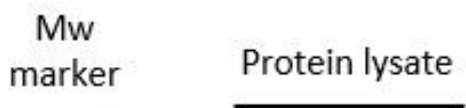

B)

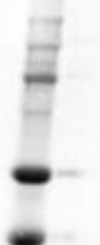

Figure 7.14. Secondary Only Control for Anti-mouse Cy5 and Anti-rabbit Cy5

Secondary only controls revealed no significant background staining for both (A) anti-mouse and (B) anti-rabbit Cy5.

Abbreviations: $M w=$ molecular weight

\subsubsection{Protein Loading Control}

Western blot protein loading control

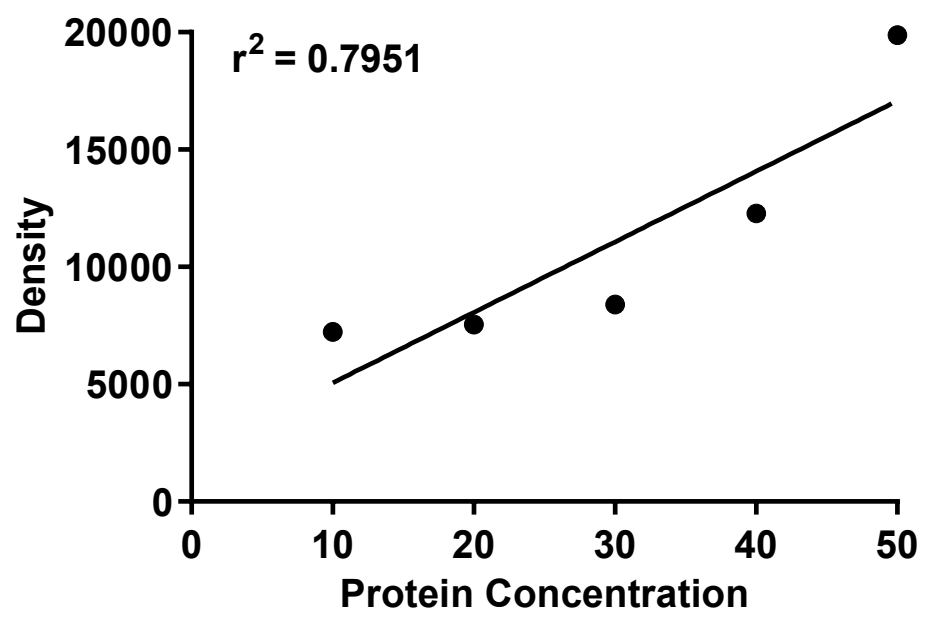

Figure 7.15 Protein Loading Analysis Shows a Strong Correlation between Density and Protein Concentration

When different amounts of protein were added to the wells a positive correlation $\left(R^{2}=0.7951\right)$ was identified between protein loading and band density. This indicates that protein levels utilised in this study were within the linear range of western blot experiments. 


\subsubsection{Quantification of the Molecular Weight of ERK}

A) Band Quantification

B)

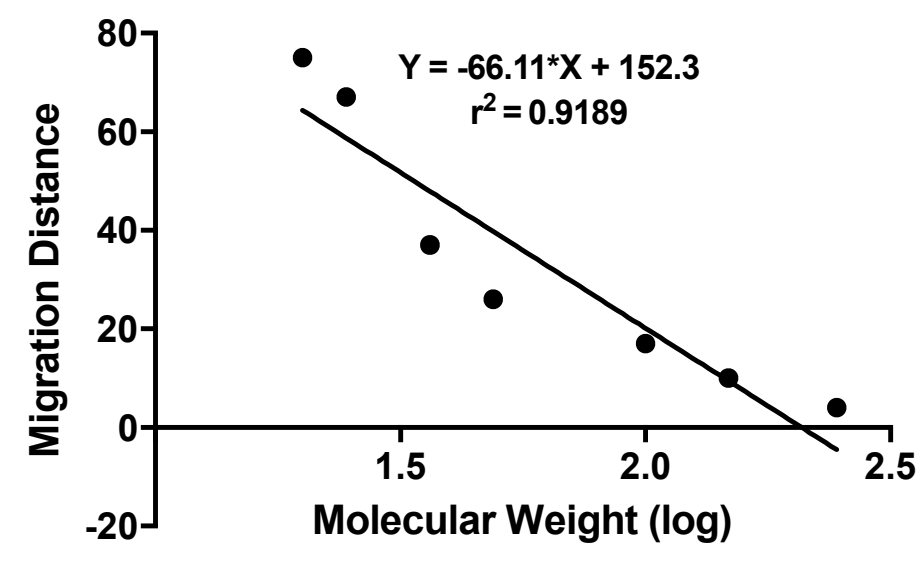

\begin{tabular}{c|c|c|c} 
Protein & $\begin{array}{c}\text { Distance } \\
(\mathbf{m m})\end{array}$ & $\begin{array}{c}\text { Calculated Molecular } \\
\text { Weight (kDa) }\end{array}$ & $\begin{array}{c}\text { Molecular Weight } \\
\text { (kDa) }\end{array}$ \\
\hline ERK1 & 44 & 43.5 & 42 \\
\hline ERK2 & 43 & 45 & 44
\end{tabular}

Figure 7.16 Quantification of the Molecular Weight the Protein Analysed

(A) To identify the size of the protein visualised the migration distance $(\mathrm{mm})$ of the molecular weight markers (Precision Plus Protein ${ }^{T M}$ Dual Colour Standards, \#1610374, Bio-Rad) were measured and plotted as a linear regression against the log of their molecular weights. (B) The distance ERK1 and ERK2 migrated was then used to estimate their molecular weight by substituting the values into the equation from the linear regression. A reverse log was then taken to determine the band size. Molecular weights (kDa) obtained. 


\subsubsection{Vehicle Controls}

A)

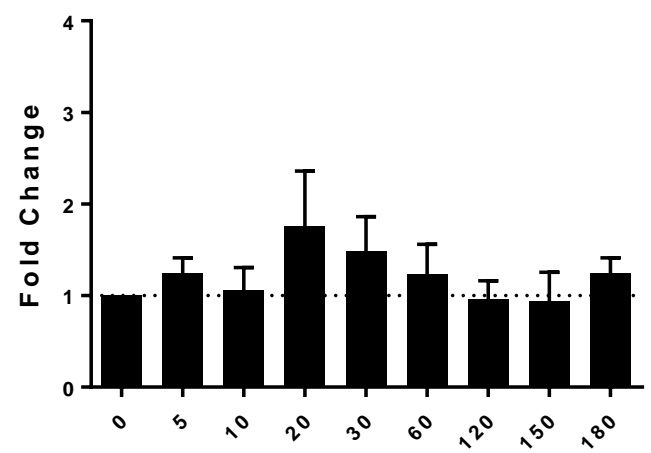

Tim e ( $m$ in $)$
B) Vehicle

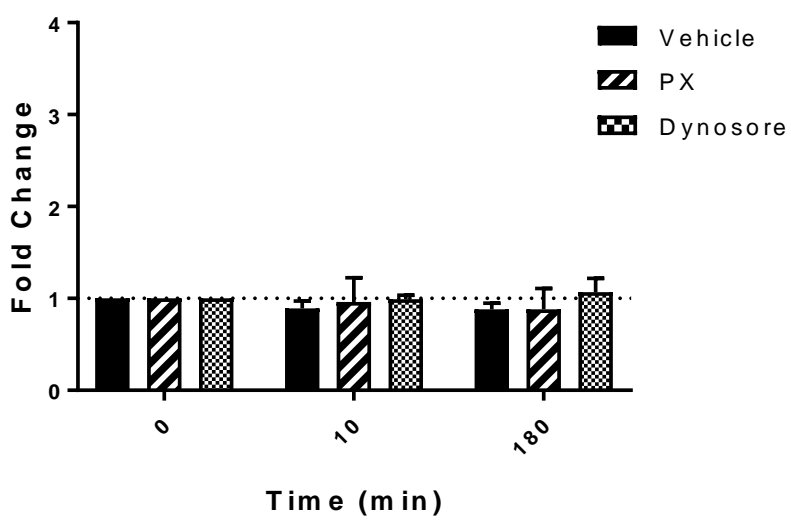

Figure 7.17 Vehicle Treated Cells Show No Significant Change in Baseline pERK levels

(A) Regular full ERK activation vehicle treatment. (B) vehicle from PX-vehicle and Dynosore vehicletreated cells. None influence activation over time. 


\subsubsection{Total and phosphorylate ERK Western Blot Scans}

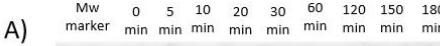

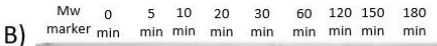
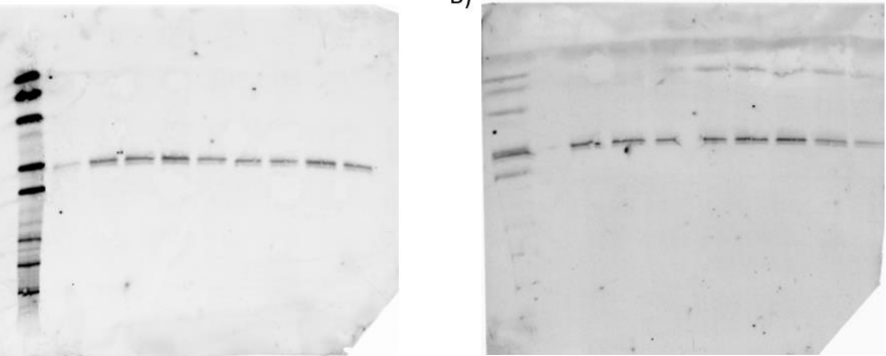

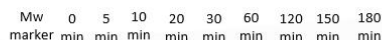

C)

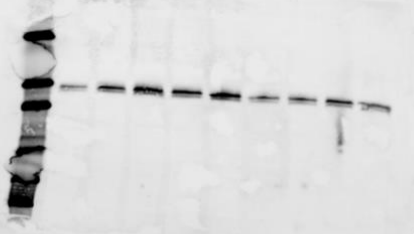

D)

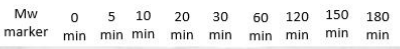

D)

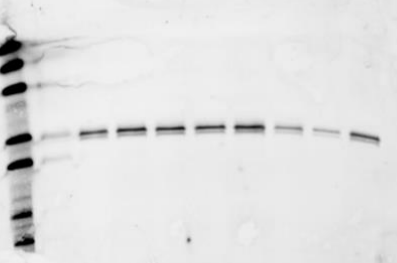

Figure 7.18 Time Course Experiment; Full Scans of pERK

$A=D A M G O, B=$ Morphine,$C=$ Kurkinol, $D=$ kurkinorin

Mw marker $=$ molecular weight marker

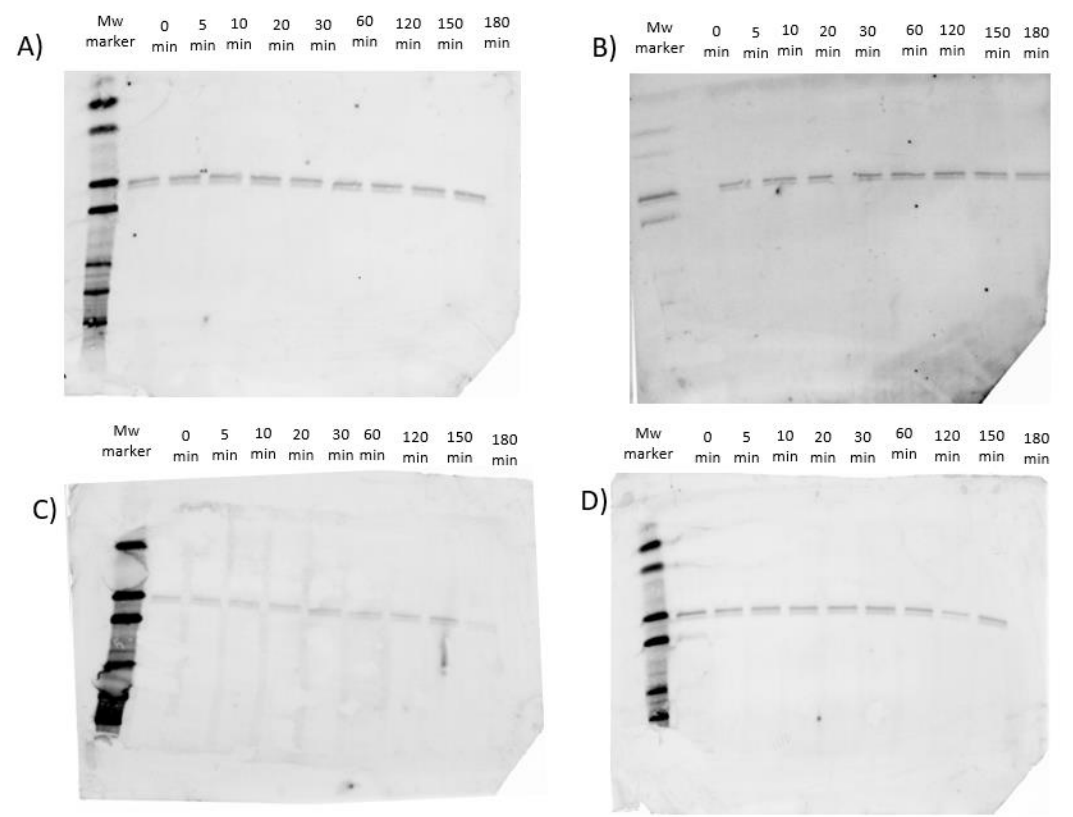

Figure 7.19 Time Course Experiment; Full Scans of Total ERK

$A=D A M G O, B=$ Morphine,$C=$ Kurkinol,$D=$ kurkinorin

Mw marker $=$ molecular weight marker 

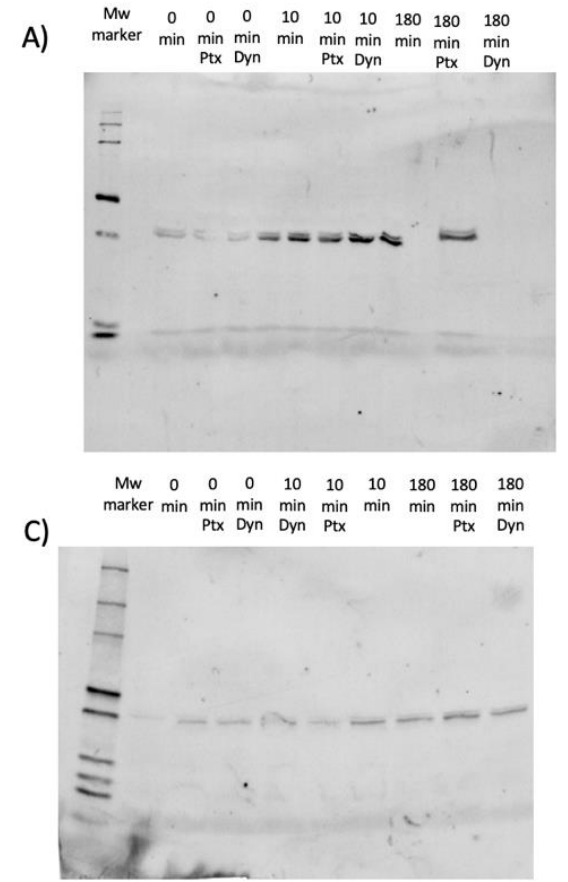

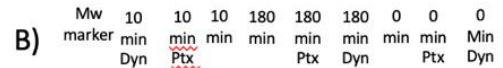

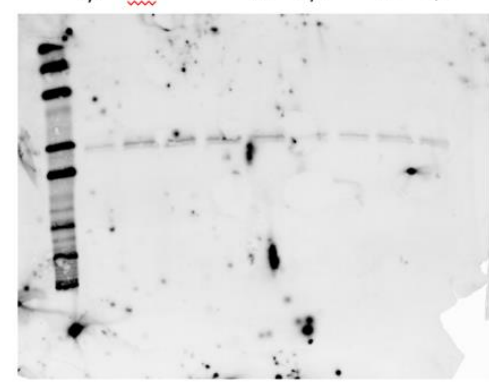

D)
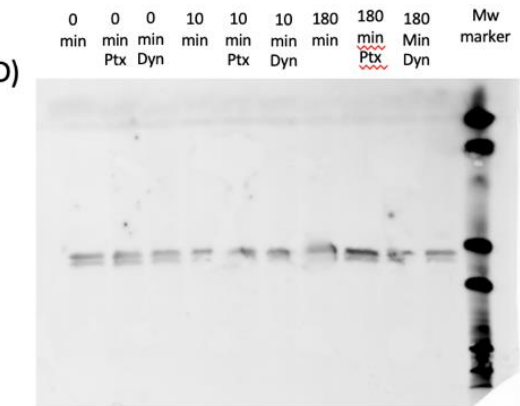

Figure 7.20 Antagonist Experiment; Full Scans of pERK

$A=D A M G O, B=$ Morphine,$C=$ Kurkinol, $D=$ kurkinorin

Mw marker $=$ molecular weight marker
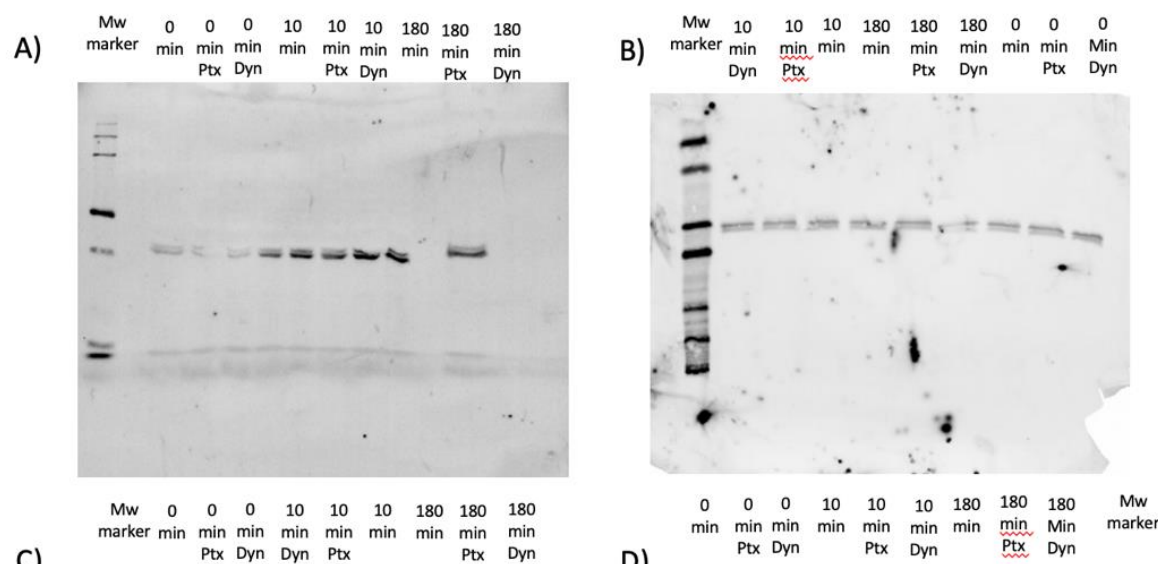

C)

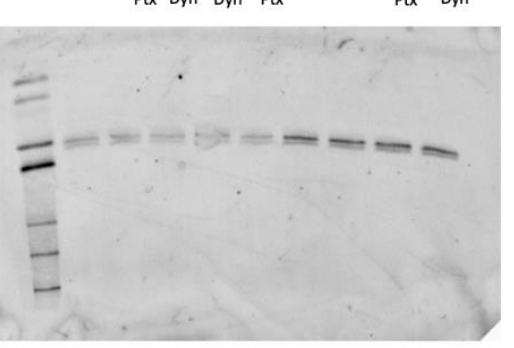

D)

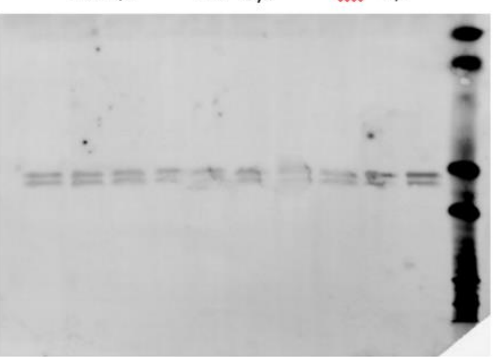

Figure 7.21 Antagonist Experiment; Full Scans of Total ERK

$A=D A M G O, B=$ Morphine,$C=$ Kurkinol, $D=$ kurkinorin

Mw marker $=$ molecular weight marker 


\subsection{Bias Factor Correlation}

A)

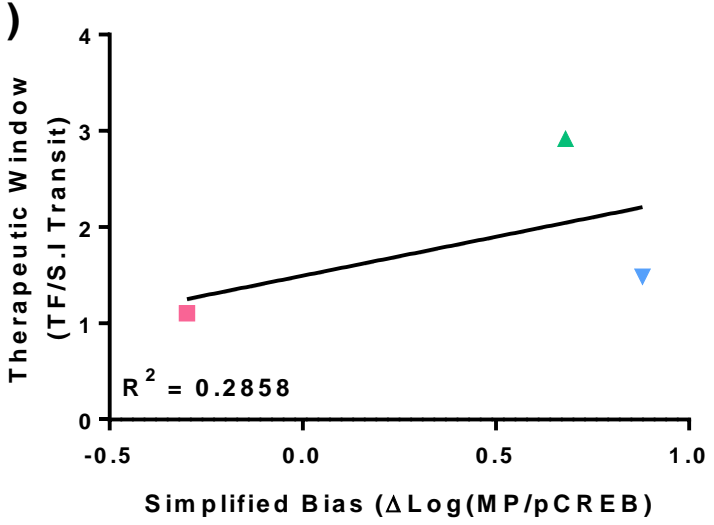

C)

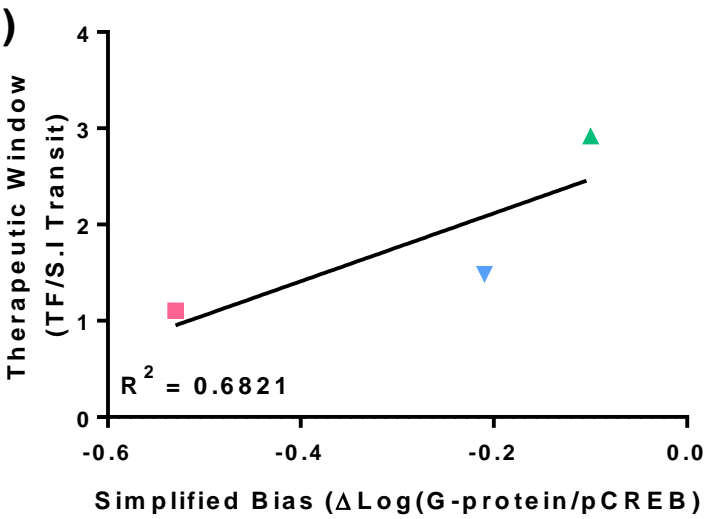

E)

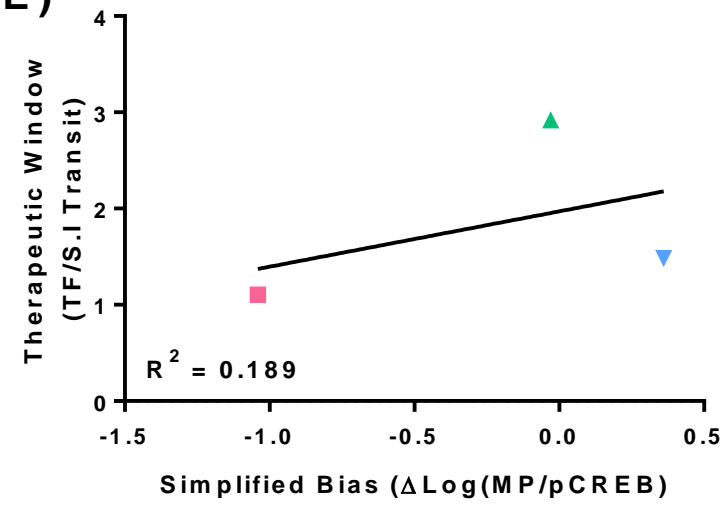

B )

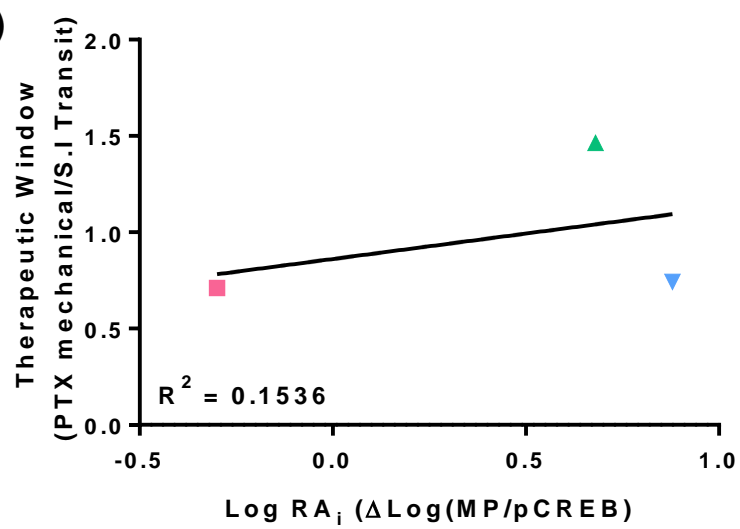

D)

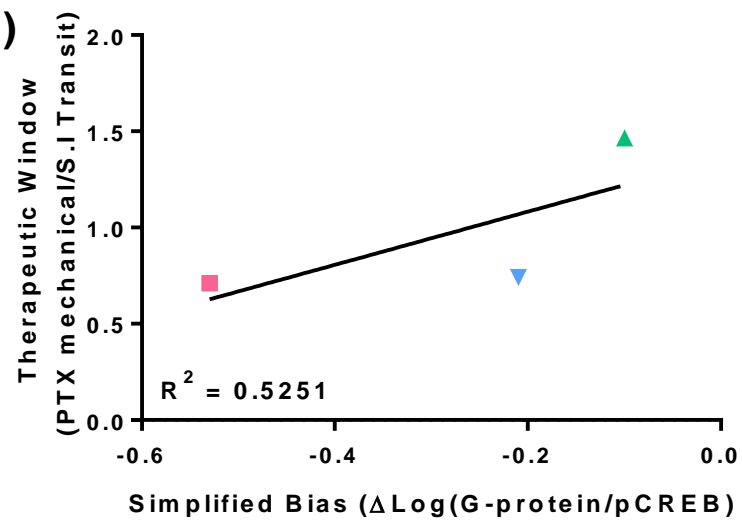

F )

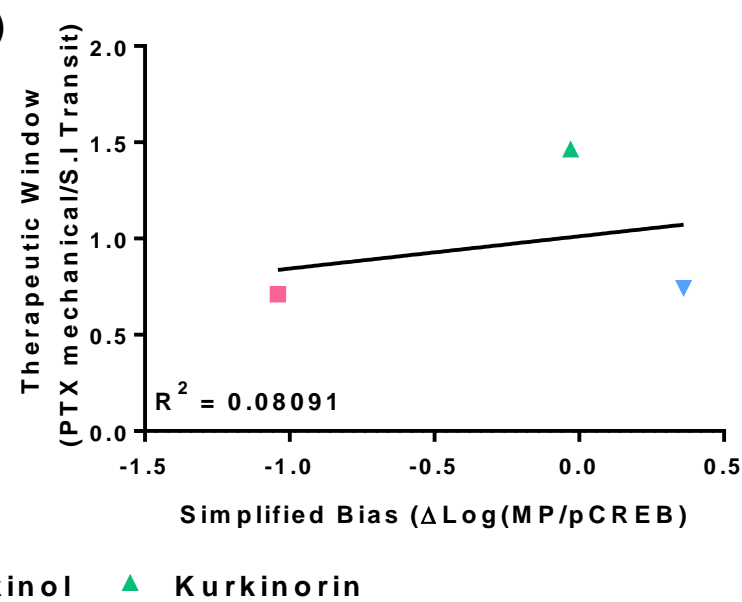

Figure 7.22 There is Poor Correlation Between Therapeutic Window and the Bias Factors Generated Using the Simplified Bias Equation

Increased G-protein biased signalling as determined by the simplified bias equation using data from the $(\boldsymbol{A}, \boldsymbol{B})$ membrane potential assay, $(\boldsymbol{C}, \boldsymbol{D})$ combined G-protein (membrane potential and potassium assay) in $\mathrm{CHO}$ cells stably expressing the human $\mu$ receptor, and the (E, F) membrane potential assay in Hek293 cells vs the PCREB assay in CHO cells all showed a weak positive correlation to the therapeutic window. 


\section{References}

Abdelhamid, E.E., Sultana, M., Portoghese, P.S. \& Takemori, A.E. 1991. Selective blockage of delta opioid receptors prevents the development of morphine tolerance and dependence in mice. J Pharmacol Exp Ther, 258, 299-303.

Abraham, A.D., Schattauer, S.S., Reichard, K.L., Cohen, J.H., Fontaine, H.M., Song, A.J., . . Chavkin, C. 2018. Estrogen Regulation of GRK2 Inactivates Kappa Opioid Receptor Signaling Mediating Analgesia, But Not Aversion. The Journal of neuroscience : the official journal of the Society for Neuroscience, 38, 8031-8043.

Abramow-Newerly, M., Roy, A.A., Nunn, C. \& Chidiac, P. 2006. RGS proteins have a signalling complex: Interactions between RGS proteins and GPCRs, effectors, and auxiliary proteins. Cellular Signalling, 18, 579-591.

Adams, J. E. (1976). "Naloxone reversal of analgesia produced by brain stimulation in the human." Pain 2(2): 161-166.

Adams, D.J., Smith, A.B., Schroeder, C.I., Yasuda, T. \& Lewis, R.J. 2003. Omega-conotoxin CVID inhibits a pharmacologically distinct voltage-sensitive calcium channel associated with transmitter release from preganglionic nerve terminals. J Biol Chem, 278, 4057-62.

Adamson, S.J., Deering, D.E., Sellman, J.D., Sheridan, J., Henderson, C., Robertson, R., . . Frampton, C.M. 2012. An estimation of the prevalence of opioid dependence in New Zealand. Int J Drug Policy, 23, 87-9.

Akbarali, H.I., Inkisar, A. \& Dewey, W.L. 2014. Site and mechanism of morphine tolerance in the gastrointestinal tract. Neurogastroenterology and motility : the official journal of the European Gastrointestinal Motility Society, 26, 1361-1367.

Akil, H., Meng, F., Devine, D.P. \& Watson, S.J. 1997. Molecular and neuroanatomical properties of the endogenous opioid system: Implications for treatment of opiate addiction. Seminars in the Neurosciences, 9, 70--83.

Al-Hasani, R. \& Bruchas, M.R. 2013. Molecular Mechanisms of Opioid Receptor-Dependent Signaling and Behavior. 115, 1363--1381.

Allchorne, A.J., Broom, D.C. \& Woolf, C.J. 2005. Detection of cold pain, cold allodynia and cold hyperalgesia in freely behaving rats. Mol Pain, 1, 36.

Alles, S.R.A. \& Smith, P.A. 2018. Etiology and Pharmacology of Neuropathic Pain. Pharmacological reviews, 70, 315-347.

Allescher, H., Ahmad, S., Kostka, P., Kwan, C. \& Daniel, E. 1989. Distribution of opioid receptors in canine small intestine: implications for function. American Journal of PhysiologyGastrointestinal and Liver Physiology, 256, G966-G974.

Altarifi, A.A., David, B., Muchhala, K.H., Blough, B.E., Akbarali, H. \& Negus, S.S. 2017. Effects of acute and repeated treatment with the biased mu opioid receptor agonist TRV130 (oliceridine) on measures of antinociception, gastrointestinal function, and abuse liability in rodents. Journal of Psychopharmacology, 130, 026988111668925.

Alter, M.J., Kruszon-Moran, D., Nainan, O.V., Mcquillan, G.M., Gao, F., Moyer, L.A., . . Margolis, H.S. 1999. The prevalence of hepatitis C virus infection in the United States, 1988 through 1994. N Engl J Med, 341, 556-62.

Andrews, N., Legg, E., Lisak, D., Issop, Y., Richardson, D., Harper, S., . . Rice, A.S. 2012. Spontaneous burrowing behaviour in the rat is reduced by peripheral nerve injury or inflammation associated pain. Eur J Pain, 16, 485-95.

Atigari, D.V., Uprety, R., Pasternak, G.W., Majumdar, S. \& Kivell, B.M. 2019. MP1104, a mixed kappadelta opioid receptor agonist has anti-cocaine properties with reduced side-effects in rats. Neuropharmacology, 150, 217-228.

Attal, N., Cruccu, G., Baron, R.A., Haanpää, M., Hansson, P., Jensen, T.S. \& Nurmikko, T. 2010. EFNS guidelines on the pharmacological treatment of neuropathic pain: 2010 revision. European journal of neurology, 17, 1113-e88.

Atwal, N., Casey, S.L., Mitchell, V.A. \& Vaughan, C.W. 2019. THC and gabapentin interactions in a mouse neuropathic pain model. Neuropharmacology, 144, 115-121. 
Aubrun F, Hrazdilova O, Langeron O, Coriat P, Riou B. A high initial VAS score and sedation after iv morphine titration are associated with the need for rescue analgesia. Can J Anaesth. 2004 Dec;51(10):969-74.

Austin Zamarripa, C., Edwards, S.R., Qureshi, H.N., Yi, J.N., Blough, B.E. \& Freeman, K.B. 2018. The Gprotein biased mu-opioid agonist, TRV130, produces reinforcing and antinociceptive effects that are comparable to oxycodone in rats. Drug Alcohol Depend, 192, 158-162.

Ayad, S., Demitrack, M.A., Burt, D.A., Michalsky, C., Wase, L., Fossler, M.J. \& Khanna, A.K. 2020. Evaluating the Incidence of Opioid-Induced Respiratory Depression Associated with Oliceridine and Morphine as Measured by the Frequency and Average Cumulative Duration of Dosing Interruption in Patients Treated for Acute Postoperative Pain. Clinical drug investigation, 40, 755-764.

Bagnol, D., Mansour, A., Akil, H. \& Watson, S.J. 1997. Cellular localization and distribution of the cloned mu and kappa opioid receptors in rat gastrointestinal tract. Neuroscience, 81, 579-591.

Bagriantsev, S.N., Gracheva, E.O. \& Gallagher, P.G. 2014. Piezo proteins: regulators of mechanosensation and other cellular processes. The Journal of biological chemistry, 289, 31673-31681.

Bai, L., et al. (2015). "Genetic Identification of an Expansive Mechanoreceptor Sensitive to Skin Stroking." Cell 163(7): 1783-1795.

Bali, A., Randhawa, P.K. \& Jaggi, A.S. 2015. Stress and opioids: Role of opioids in modulating stressrelated behavior and effect of stress on morphine conditioned place preference. Neuroscience \& Biobehavioral Reviews, 51, 138-150.

Bals-Kubik, R., Ableitner, A., Herz, A. \& Shippenberg, T.S. 1993. Neuroanatomical sites mediating the motivational effects of opioids as mapped by the conditioned place preference paradigm in rats. Journal of Pharmacology and Experimental Therapeutics, 264, 489-495.

Banach, M., Juranek, J.K. \& Zygulska, A.L. 2016. Chemotherapy-induced neuropathies-a growing problem for patients and health care providers. Brain and behavior, 7, e00558-e00558.

Bao, Y.-H., Zhou, Q.-H., Chen, R., Xu, H., Zeng, L.-L., Zhang, X., . . Du, D.-P. 2014. Gabapentin enhances the morphine anti-nociceptive effect in neuropathic pain via the interleukin-10-heme oxygenase-1 signalling pathway in rats. Journal of molecular neuroscience : $M N, 54,137-146$.

Bare, L.A., Mansson, E. \& Yang, D. 1994. Expression of two variants of the human mu opioid receptor mRNA in SK-N-SH cells and human brain. FEBS Lett, 354, 213-6.

Barrett, A.C., Smith, E.S. \& Picker, M.J. 2002. Sex-related differences in mechanical nociception and antinociception produced by mu- and kappa-opioid receptor agonists in rats. Eur J Pharmacol, 452, 163-73.

Bartley, E.J. \& Fillingim, R.B. 2013. Sex differences in pain: a brief review of clinical and experimental findings. British journal of anaesthesia, 111, 52-58.

Basbaum, A.I., Bautista, D.M., Scherrer, G. \& Julius, D. 2009. Cellular and molecular mechanisms of pain. Cell, 139, 267-284.

Basbaum, A.I. \& Fields, H.L. 1978. Endogenous pain control mechanisms: review and hypothesis. Ann Neurol, 4, 451-62.

Basbaum, A.I. \& Fields, H.L. 1984. Endogenous pain control systems: brainstem spinal pathways and endorphin circuitry. Annu Rev Neurosci, 7, 309-38.

Bauer, A.J., Sarr, M.G. \& Szurszewski, J.H. 1991. Opioids inhibit neuromuscular transmission in circular muscle of human and baboon jejunum. Gastroenterology, 101, 970-6.

Bautista, D.M., Movahed, P., Hinman, A., Axelsson, H.E., Sterner, O., Högestätt, E.D., . . Zygmunt, P.M. 2005. Pungent products from garlic activate the sensory ion channel TRPA1. Proceedings of the National Academy of Sciences, 102, 12248-12252.

Beaulieu, J.-M., Sotnikova, T.D., Marion, S., Lefkowitz, R.J., Gainetdinov, R.R. \& Caron, M.G. 2005. An $A k t / \beta$-arrestin 2/PP2A signaling complex mediates dopaminergic neurotransmission and behavior. Cell, 122, 261-273.

Beguin, C., Richards, M.R., Li, J.G., Wang, Y., Xu, W., Liu-Chen, L.Y., . . Cohen, B.M. 2006. Synthesis and in vitro evaluation of salvinorin $A$ analogues: effect of configuration at $C(2)$ and substitution at C(18). Bioorg Med Chem Lett, 16, 4679-85. 
Belin, A., Ran, C. Edvinsson, L. (2020). "Calcitonin Gene-Related Peptide (CGRP) and Cluster Headache." Brain Sci 10(1).

Belcheva, M.M., Clark, A.L., Haas, P.D., Serna, J.S., Hahn, J.W., Kiss, A. \& Coscia, C.J. 2005. Mu and kappa opioid receptors activate ERK/MAPK via different protein kinase $\mathrm{C}$ isoforms and secondary messengers in astrocytes. The Journal of biological chemistry, 280, 27662-27669.

Belcheva, M.M., Vogel, Z., Ignatova, E., Avidor-Reiss, T., Zippel, R., Levy, R., .. Coscia, C.J. 1998. Opioid modulation of extracellular signal-regulated protein kinase activity is ras-dependent and involves Gbetagamma subunits. J Neurochem, 70, 635-45.

Belluzzi, J.D., Grant, N., Garsky, V., Sarantakis, D., Wise, C.D. \& Stein, L. 1976. Analgesia induced in vivo by central administration of enkephalin in rat. Nature, 260, 625-6.

Belluzzi, J.D. \& Stein, L. 1977. Enkephalin may mediate euphoria and drive-reduction reward. Nature, 266, 556-558.

Ben Hamida, S., Boulos, L.-J., Mcnicholas, M., Charbogne, P. \& Kieffer, B.L. 2019. Mu opioid receptors in GABAergic neurons of the forebrain promote alcohol reward and drinking. Addiction biology, 24, 28-39.

Benbouzid, M., Pallage, V., Rajalu, M., Waltisperger, E., Doridot, S., Poisbeau, P., . . Barrot, M. 2008. Sciatic nerve cuffing in mice: a model of sustained neuropathic pain. European journal of pain, 12, 591-599.

Bennett, G.J. \& Xie, Y.-K. 1988. A peripheral mononeuropathy in rat that produces disorders of pain sensation like those seen in man. Pain, 33, 87-107.

Berger, B., Rothmaier, A.K., Wedekind, F., Zentner, J., Feuerstein, T.J. \& Jackisch, R. 2006. Presynaptic opioid receptors on noradrenergic and serotonergic neurons in the human as compared to the rat neocortex. British journal of pharmacology, 148, 795-806.

Bessou, P. \& Perl, E.R. 1969. Response of cutaneous sensory units with unmyelinated fibers to noxious stimuli. Journal of neurophysiology, 32, 1025-1043.

Bhargava, H.N., Thomas, P.T., Thorat, S. \& House, R.V. 1994. Effects of morphine tolerance and abstinence on cellular immune function. Brain Research, 642, 1-10.

Bijur, P.E., Esses, D., Birnbaum, A., Chang, A.K., Schechter, C. \& Gallagher, E.J. 2008. Response to morphine in male and female patients: analgesia and adverse events. Clin J Pain, 24, 192-8.

Black, J.W. \& Leff, P. 1983. Operational models of pharmacological agonism. Proc R Soc Lond B Biol Sci, 220, 141-62.

Blazer, L.L., Zhang, H., Casey, E.M., Husbands, S.M. \& Neubig, R.R. 2011. A nanomolar-potency small molecule inhibitor of regulator of G-protein signaling proteins. Biochemistry, 50, 3181-92.

Bloom, F.E., Rossier, J., Battenberg, E.L., Bayon, A., French, E., Henriksen, S.J., . . . Guillemin, R. 1978. beta-endorphin: cellular localization, electrophysiological and behavioral effects. Adv Biochem Psychopharmacol, 18, 89-109.

Bobeck, E.N., Ingram, S.L., Hermes, S.M., Aicher, S.A. \& Morgan, M.M. 2016. Ligand-biased activation of extracellular signal-regulated kinase $1 / 2$ leads to differences in opioid induced antinociception and tolerance. Behavioural brain research, 298, 17-24.

Boehmerle, W., Huehnchen, P., Peruzzaro, S., Balkaya, M. \& Endres, M. 2014. Electrophysiological, behavioral and histological characterization of paclitaxel, cisplatin, vincristine and bortezomib-induced neuropathy in C57BI/6 mice. Scientific reports, 4, 6370-6370.

Boehmerle, W., Splittgerber, U., Lazarus, M.B., Mckenzie, K.M., Johnston, D.G., Austin, D.J. \& Ehrlich, B.E. 2006. Paclitaxel induces calcium oscillations via an inositol 1,4,5-trisphosphate receptor and neuronal calcium sensor 1-dependent mechanism. Proc Natl Acad Sci U S A, 103, 1835661.

Boerner, K.E., Chambers, C.T., Gahagan, J., Keogh, E., Fillingim, R.B. \& Mogil, J.S. 2018. Conceptual complexity of gender and its relevance to pain. Pain, 159, 2137-2141.

Bohn, L.M., Dykstra, L.A., Lefkowitz, R.J., Caron, M.G. \& Barak, L.S. 2004. Relative opioid efficacy is determined by the complements of the $G$ protein-coupled receptor desensitization machinery. Molecular pharmacology, 66, 106--112. 
Bohn, L.M., Gainetdinov, R.R., Lin, F.T., Lefkowitz, R.J. \& Caron, M.G. 2000. Mu-opioid receptor desensitization by beta-arrestin-2 determines morphine tolerance but not dependence. Nature, 408, 720--723.

Bohn, L.M., Gainetdinov, R.R., Sotnikova, T.D., Medvedev, I.O., Lefkowitz, R.J., Dykstra, L.A. \& Caron, M.G. 2003. Enhanced rewarding properties of morphine, but not cocaine, in beta(arrestin)-2 knock-out mice. The Journal of neuroscience : the official journal of the Society for Neuroscience, 23, 10265--73.

Bohn, L.M., Lefkowitz, R.J. \& Caron, M.G. 2002. Differential mechanisms of morphine antinociceptive tolerance revealed in (beta)arrestin-2 knock-out mice. J Neurosci, 22, 10494--10500.

Bohn, L.M., Lefkowitz, R.J., Gainetdinov, R.R., Peppel, K., Caron, M.G. \& Lin, F.T. 1999. Enhanced morphine analgesia in mice lacking beta-arrestin 2. Science, 286, 2495--2498.

Bohn, L.M. \& Raehal, K.M. 2006. Opioid receptor signaling: relevance for gastrointestinal therapy. Current Opinion in Pharmacology, 6, 559-563.

Bonin, R.P. \& Bories, C.A. 2014. A Simplified Up-Down Method (SUDO) for Measuring Mechanical Nociception in Rodents Using von Frey Filaments. Molecular Pain, 10, 1744--8069--10--26.

Bourinet, E., Soong, T.W., Stea, A. \& Snutch, T.P. 1996. Determinants of the G protein-dependent opioid modulation of neuronal calcium channels. Proceedings of the National Academy of Sciences of the United States of America, 93, 1486--1491.

Bourne, S., Machado, A.G. \& Nagel, S.J. 2014. Basic Anatomy and Physiology of Pain Pathways. Neurosurgery Clinics of North America, 25, 629-638.

Breivik, H., Collett, B., Ventafridda, V., Cohen, R. \& Gallacher, D. 2006. Survey of chronic pain in Europe: prevalence, impact on daily life, and treatment. European journal of pain (London, England), 10, 287-333.

Brice-Tutt, A.C., Wilson, L.L., Eans, S.O., Stacy, H.M., Simons, C.A., Simpson, G.G., . . Mclaughlin, J.P. 2020. Multifunctional opioid receptor agonism and antagonism by a novel macrocyclic tetrapeptide prevents reinstatement of morphine-seeking behaviour. Br J Pharmacol.

Brookes, S.J. 2001. Classes of enteric nerve cells in the guinea-pig small intestine. Anat Rec, 262, 5870.

Broom, D.C., Jutkiewicz, E.M., Rice, K.C., Traynor, J.R. \& Woods, J.H. 2002. Behavioral effects of dopioid receptor agonists: potential antidepressants? Japanese journal of pharmacology, 90, 1-6.

Bruchas, M. and C. Chavkin. 2010. Kinase cascades and ligand-directed signaling at the kappa opioid receptor. Psychopharmacology 210: 137-147.

Bu, H., Liu, X., Tian, X., Yang, H. \& Gao, F. 2015. Enhancement of morphine analgesia and prevention of morphine tolerance by downregulation of $\beta$-arrestin 2 with antigene RNAs in mice. International Journal of Neuroscience, 125, 56-65.

Burgess, P.R. \& Perl, E.R. 1967. Myelinated afferent fibres responding specifically to noxious stimulation of the skin. The Journal of physiology, 190, 541-562.

Burgueño, J., Pujol, M., Monroy, X., Roche, D., Varela, M.J., Merlos, M. \& Giraldo, J. 2017. A Complementary Scale of Biased Agonism for Agonists with Differing Maximal Responses. Scientific Reports, 7, 15389.

Büscher, H.H., Hill, R.C., Römer, D., Cardinaux, F., Closse, A., Hauser, D. \& Pless, J. 1976. Evidence for analgesic activity of enkephalin in the mouse. Nature, 261, 423-425.

Bushnell, M.C., Case, L.K., Ceko, M., Cotton, V.A., Gracely, J.L., Low, L.A., . . Villemure, C. 2015. Effect of environment on the long-term consequences of chronic pain. Pain, 156 Suppl 1, S42-S49.

Bushnell, M.C., Ceko, M. \& Low, L.A. 2013. Cognitive and emotional control of pain and its disruption in chronic pain. Nature reviews. Neuroscience, 14, 502-511.

Busse JW, Wang L, Kamaleldin M, Craigie S, Riva JJ, Montoya L, Mulla SM, Lopes LC, Vogel N, Chen E, Kirmayr K, De Oliveira K, Olivieri L, Kaushal A, Chaparro LE, Oyberman I, Agarwal A, Couban R, Tsoi L, Lam T, Vandvik PO, Hsu S, Bala MM, Schandelmaier S, Scheidecker A, Ebrahim S, Ashoorion V, Rehman Y, Hong PJ, Ross S, Johnston BC, Kunz R, Sun X, Buckley N, Sessler DI, Guyatt GH. Opioids for Chronic Noncancer Pain: A Systematic Review and Meta-analysis. JAMA. 2018 Dec 18;320(23):2448-2460. 
Butelman, E.R., Prisinzano, T.E., Deng, H., Rus, S. \& Kreek, M.J. 2009. Unconditioned behavioral effects of the powerful kappa-opioid hallucinogen salvinorin $A$ in nonhuman primates: fast onset and entry into cerebrospinal fluid. J Pharmacol Exp Ther, 328, 588-97.

Butler, R.K. \& Finn, D.P. 2009. Stress-induced analgesia. Progress in neurobiology, 88, 184-202.

Cabot, P.J., Carter, L., Gaiddon, C., Zhang, Q., Schafer, M., Loeffler, J.P. \& Stein, C. 1997. Immune cellderived beta-endorphin. Production, release, and control of inflammatory pain in rats. $J$ Clin Invest, 100, 142-8.

Cahill, C.M., Cook, C. \& Pickens, S. 2014. Migraine and Reward System-Or Is It Aversive? Current Pain and Headache Reports, 18, 410.

Callaghan, B.C., Cheng, H.T., Stables, C.L., Smith, A.L. \& Feldman, E.L. 2012. Diabetic neuropathy: clinical manifestations and current treatments. Lancet Neurol, 11, 521-34.

Carleton, R.N., Afifi, T.O., Taillieu, T., Turner, S., El-Gabalawy, R., Sareen, J. \& Asmundson, G.J.G. 2018. Anxiety-related psychopathology and chronic pain comorbidity among public safety personnel. Journal of anxiety disorders, 55, 48-55.

Carlezon, W.A., Jr., Beguin, C., Dinieri, J.A., Baumann, M.H., Richards, M.R., Todtenkopf, M.S., . . . Cohen, B.M. 2006. Depressive-like effects of the kappa-opioid receptor agonist salvinorin A on behavior and neurochemistry in rats. J Pharmacol Exp Ther, 316, 440-7.

Carlton, S.M., Lekan, H.A., Kim, S.H. \& Chung, J.M. 1994. Behavioral manifestations of an experimental model for peripheral neuropathy produced by spinal nerve ligation in the primate. Pain, 56, $155-66$.

Cartmell, S.M., Gelgor, L. \& Mitchell, D. 1991. A revised rotarod procedure for measuring the effect of antinociceptive drugs on motor function in the rat. J Pharmacol Methods, 26, 149-59.

Castillo, R., Kissin, I. \& Bradley, E.L. 1986. Selective kappa opioid agonist for spinal analgesia without the risk of respiratory depression. Anesthesia and analgesia, 65, 350-354.

Castro-Junior, C.J., Milano, J., Souza, A.H., Silva, J.F., Rigo, F.K., Dalmolin, G., . . Gomez, M.V. 2013. Pha1 $\beta$ toxin prevents capsaicin-induced nociceptive behavior and mechanical hypersensitivity without acting on TRPV1 channels. Neuropharmacology, 71, 237-246.

Caterina, M. J., Schumacher, M. A. Tominaga, M. Rosen, T. A. Levine, J. D. Julius, D. (1997). "The capsaicin receptor: a heat-activated ion channel in the pain pathway." Nature 389(6653): 816-824.

Celik, M.O., Labuz, D., Henning, K., Busch-Dienstfertig, M., Gaveriaux-Ruff, C., Kieffer, B.L., . . . Machelska, H. 2016. Leukocyte opioid receptors mediate analgesia via $\mathrm{Ca}(2+)$-regulated release of opioid peptides. Brain Behav Immun, 57, 227-242.

Cepeda, M.S. \& Carr, D.B. 2003. Women experience more pain and require more morphine than men to achieve a similar degree of analgesia. Anesth Analg, 97, 1464-8.

Cerritelli, S., Hirschberg, S., Hill, R., Balthasar, N. \& Pickering, A.E. 2016. Activation of brainstem proopiomelanocortin neurons produces opioidergic analgesia, bradycardia and bradypnoea. PLOS One, 11.

Chakrabarti, S., Liu, N.-J. \& Gintzler, A.R. 2010. Formation of $\mu$-/k-opioid receptor heterodimer is sexdependent and mediates female-specific opioid analgesia. Proceedings of the National Academy of Sciences, 107, 20115-20119.

Chakrabarti, S., Regec, A. \& Gintzler, A.R. 2005. Biochemical demonstration of mu-opioid receptor association with Gsalpha: enhancement following morphine exposure. Brain Res Mol Brain Res, 135, 217-24.

Chaplan, S.R., Bach, F.W., Pogrel, J.W., Chung, J.M. \& Yaksh, T.L. 1994. Quantitative assessment of tactile allodynia in the rat paw. J Neurosci Methods, 53, 55-63.

Charles, A.C., Mostovskaya, N., Asas, K., Evans, C.J., Dankovich, M.L. \& Hales, T.G. 2003. Coexpression of delta-opioid receptors with micro receptors in $\mathrm{GH} 3$ cells changes the functional response to micro agonists from inhibitory to excitatory. Mol Pharmacol, 63, 89-95.

Charlton, S.J. \& Vauquelin, G. 2010. Elusive equilibrium: the challenge of interpreting receptor pharmacology using calcium assays. Br J Pharmacol, 161, 1250-65.

Chen, C. England, S. Akopian, A. N. Wood, J. N. (1998). "A sensory neuron-specific, proton-gated ion channel." Proceedings of the National Academy of Sciences 95(17): 10240-10245. 
Chen, X.-T., Pitis, P., Liu, G., Yuan, C., Gotchev, D., Cowan, C.L., . . Yamashita, D.S. 2013. StructureActivity Relationships and Discovery of a $G$ Protein Biased $\mu$ Opioid Receptor Ligand, [(3Methoxythiophen-2-yl)methyl](\{2-[(9R)-9-(pyridin-2-yl)-6-oxaspiro-[4.5]decan-9yl]ethyl\})amine (TRV130), for the Treatment of Acute Severe Pain. Journal of Medicinal Chemistry, 56, 8019-8031.

Chen., Mestek, A., Liu, J., Hurley, J.A. \& Yu, L. 1993. Molecular cloning and functional expression of a mu-opioid receptor from rat brain. Mol Pharmacol, 44, 8-12.

Chincholkar, M. 2018. Analgesic mechanisms of gabapentinoids and effects in experimental pain models: a narrative review. British Journal of Anaesthesia, 120, 1315-1334.

Choi, S.-I., Lim, J.Y., Yoo, S., Kim, H. \& Hwang, S.W. 2016. Emerging role of spinal cord TRPV1 in pain exacerbation. Neural plasticity, 2016.

Choi, Y., Yoon, Y.W., Na, H.S., Kim, S.H. \& Chung, J.M. 1994. Behavioral signs of ongoing pain and cold allodynia in a rat model of neuropathic pain. Pain, 59, 369-76.

Christensen, B.N. \& Perl, E.R. 1970. Spinal neurons specifically excited by noxious or thermal stimuli: marginal zone of the dorsal horn. J Neurophysiol, 33, 293-307.

Chu, L.-C., Tsaur, M.-L., Lin, C.-S., Hung, Y.-C., Wang, T.-Y., Chen, C.-C. \& Cheng, J.-K. 2011. Chronic intrathecal infusion of gabapentin prevents nerve ligation-induced pain in rats. British journal of anaesthesia, 106, 699-705.

Chu, L.F., Clark, D.J. \& Angst, M.S. 2006. Opioid tolerance and hyperalgesia in chronic pain patients after one month of oral morphine therapy: a preliminary prospective study. J Pain, 7, 43-8.

Cicero, T.J., Nock, B. \& Meyer, E.R. 1997. Sex-related differences in morphine's antinociceptive activity: relationship to serum and brain morphine concentrations. J Pharmacol Exp Ther, 282, 939-44.

Cichewicz, D.L. 2004. Synergistic interactions between cannabinoid and opioid analgesics. Life Sci, 74, 1317-24.

Ciruela, A., Dixon, A.K., Bramwell, S., Gonzalez, M.I., Pinnock, R.D. \& Lee, K. 2003. Identification of MEK1 as a novel target for the treatment of neuropathic pain. Br J Pharmacol, 138, 751-6.

Clayton, J.A. \& Collins, F.S. 2014. Policy: NIH to balance sex in cell and animal studies. Nature, 509, 282-283.

Cohen, K., Weizman, A. \& Weinstein, A. 2019. Positive and Negative Effects of Cannabis and Cannabinoids on Health. Clinical pharmacology and therapeutics, 105, 1139-1147.

Cohen, S.P. \& Mao, J. 2014. Neuropathic pain: mechanisms and their clinical implications. Bmj, 348, f7656.

Colleoni, M. \& Sacerdote, P. 2010. Murine models of human neuropathic pain. Biochimica et Biophysica Acta (BBA) - Molecular Basis of Disease, 1802, 924-933.

Collins, D., Reed, B., Zhang, Y. \& Kreek, M.J. 2016. Sex differences in responsiveness to the prescription opioid oxycodone in mice. Pharmacol Biochem Behav, 148, 99-105.

Compton, W.M. \& Volkow, N.D. 2006. Major increases in opioid analgesic abuse in the United States: concerns and strategies. Drug Alcohol Depend, 81, 103-7.

Conner, D.A., Mathier, M.A., Mortensen, R.M., Christe, M., Vatner, S.F., Seidman, C.E. \& Seidman, J.G. 1997. beta-Arrestin1 knockout mice appear normal but demonstrate altered cardiac responses to beta-adrenergic stimulation. Circulation research, 81, 1021-1026.

Connor, M., Bagley, E. E., Chieng, B. C., Christie, M. J. (2015). $\beta$-Arrestin-2 knockout prevents development of cellular $\mu$-opioid receptor tolerance but does not affect opioid-withdrawalrelated adaptations in single PAG neurons. British Journal of Pharmacology 172(2): 492-500.

Costa, A.R., Carvalho, P., Flik, G., Wilson, S.P., Reguenga, C., Martins, I. \& Tavares, I. 2019. Neuropathic Pain Induced Alterations in the Opioidergic Modulation of a Descending Pain Facilitatory Area of the Brain. Front Cell Neurosci, 13, 287.

Cowan, A., Lewis, J.W. \& Macfarlane, I.R. 1977. Agonist and antagonist properties of buprenorphine, a new antinociceptive agent. British journal of pharmacology, 60, 537-545.

Craft, R.M. 2007. Modulation of pain by estrogens. Pain, 132 Suppl 1, S3-12.

Craig, A.G., Norberg, T., Griffin, D., Hoeger, C., Akhtar, M., Schmidt, K., . . . Olivera, B.M. 1999. Contulakin-G, an O-glycosylated invertebrate neurotensin. J Biol Chem, 274, 13752-9.

Craig, K.D. 2015. Social communication model of pain. Pain, 156, 1198-9. 
Crain, S.M. \& Shen, K.F. 2000. Antagonists of excitatory opioid receptor functions enhance morphine's analgesic potency and attenuate opioid tolerance/dependence liability. Pain, 84, 121-31.

Crawford, C.A., Mcdougall, S.A., Bolanos, C.A., Hall, S. \& Berger, S.P. 1995. The effects of the kappa agonist U-50,488 on cocaine-induced conditioned and unconditioned behaviors and Fos immunoreactivity. Psychopharmacology (Berl), 120, 392-9.

Crowley, R.S., Riley, A.P., Alder, A.F., Anderson, R.J., 3rd, Luo, D., Kaska, S., . . Prisinzano, T.E. 2020. Synthetic Studies of Neoclerodane Diterpenes from Salvia divinorum: Design, Synthesis, and Evaluation of Analogues with Improved Potency and G-protein Activation Bias at the $\mu$-Opioid Receptor. ACS Chem Neurosci, 11, 1781-1790.

Crowley, R.S., Riley, A.P., Sherwood, A.M., Groer, C.E., Shivaperumal, N., Biscaia, M., . . Prisinzano, T.E. 2016. Synthetic Studies of Neoclerodane Diterpenes from Salvia divinorum: Identification of a Potent and Centrally Acting $\mu$ Opioid Analgesic with Reduced Abuse Liability. Journal of medicinal chemistry, 59, 11027-11038.

Cunha, F.Q., Poole, S., Lorenzetti, B.B. \& Ferreira, S.H. 1992. The pivotal role of tumour necrosis factor alpha in the development of inflammatory hyperalgesia. Br J Pharmacol, 107, 660-4.

Cvejic, S. \& Devi, L.A. 1997. Dimerization of the delta opioid receptor: implication for a role in receptor internalization. J Biol Chem, 272, 26959-64.

D'amour, F.E. \& Smith, D.L. 1941. A method for determining loss of pain sensation. J Pharmacol Exp Ther, 72, 74-9.

Dahan, A. 2006. Opioid-induced respiratory effects: new data on buprenorphine. Palliative medicine, $20,3-8$.

Dahan, A., Van Dam, C.J., Niesters, M., Van Velzen, M., Fossler, M.J., Demitrack, M.A. \& Olofsen, E. 2020. Benefit and Risk Evaluation of Biased $\mu$-Receptor Agonist Oliceridine versus Morphine. Anesthesiology: The Journal of the American Society of Anesthesiologists, 133, 559-568.

Dang, V. \& Christie, M. Beta-arrestin2 independent regulation of mu opioid receptor in locus coeruleus neurons. Soc Neurosci Abstr, 2006.

Dang, V.C., Chieng, B., Azriel, Y. \& Christie, M.J. 2011. Cellular morphine tolerance produced by Barrestin-2-dependent impairment of $\mu$-opioid receptor resensitization. The Journal of neuroscience : the official journal of the Society for Neuroscience, 31, 7122-7130.

Dang, V.C., Napier, I.A. \& Christie, M.J. 2009. Two distinct mechanisms mediate acute mu-opioid receptor desensitization in native neurons. The Journal of neuroscience : the official journal of the Society for Neuroscience, 29, 3322-3327.

De Deurwaerdère, P., Navailles, S., Berg, K.A., Clarke, W.P. \& Spampinato, U. 2004. Constitutive activity of the serotonin $2 \mathrm{C}$ receptor inhibits in vivo dopamine release in the rat striatum and nucleus accumbens. Journal of Neuroscience, 24, 3235-3241.

Di Forti M, Quattrone D, Freeman TP, Tripoli G, Gayer-Anderson C, Quigley H, Rodriguez V, Jongsma HE, Ferraro L, La Cascia C, La Barbera D, Tarricone I, Berardi D, Szöke A, Arango C, Tortelli A, Velthorst E, Bernardo M, Del-Ben CM, Menezes PR, Selten JP, Jones PB, Kirkbride JB, Rutten BP, de Haan L, Sham PC, van Os J, Lewis CM, Lynskey M, Morgan C, Murray RM; EU-GEI WP2 Group. The contribution of cannabis use to variation in the incidence of psychotic disorder across Europe (EU-GEI): a multicentre case-control study. Lancet Psychiatry. 2019 May;6(5):427-436. (19)30048-3. Epub 2019 Mar 19.

De Souza, A.H., Castro, C.J., Rigo, F.K., De Oliveira, S.M., Gomez, R.S., Diniz, D.M., . . Gomez, M.V. 2013. An Evaluation of the Antinociceptive Effects of Pha1 $\beta$, a Neurotoxin from the Spider Phoneutria nigriventer, and $\omega$-Conotoxin MVIIA, a Cone Snail Conus magus Toxin, in Rat Model of Inflammatory and Neuropathic Pain. Cellular and Molecular Neurobiology, 33, 59-67.

Deacon, R.M. 2009. Burrowing: a sensitive behavioural assay, tested in five species of laboratory rodents. Behav Brain Res, 200, 128-33.

Delfs, J.M., Kong, H., Mestek, A., Chen, Y., Yu, L., Reisine, T. \& Chesselet, M.F. 1994. Expression of mu opioid receptor mRNA in rat brain: an in situ hybridization study at the single cell level. Journal of Comparative Neurology, 345, 46-68.

Deloitte 2019. The Cost of Pain in Australia. 
Deng, M., Chen, S.-R., Chen, H., Luo, Y., Dong, Y. \& Pan, H.-L. 2019. Mitogen-activated protein kinase signaling mediates opioid-induced presynaptic NMDA receptor activation and analgesic tolerance. Journal of neurochemistry, 148, 275-290.

Deuis, J.R., Dvorakova, L.S. \& Vetter, I. 2017. Methods Used to Evaluate Pain Behaviors in Rodents. Frontiers in molecular neuroscience, 10, 284-284.

Deuis, J.R., Lim, Y.L., Rodrigues De Sousa, S., Lewis, R.J., Alewood, P.F., Cabot, P.J. \& Vetter, I. 2014. Analgesic effects of clinically used compounds in novel mouse models of polyneuropathy induced by oxaliplatin and cisplatin. Neuro Oncol, 16, 1324-32.

Deuis, J.R., Whately, E., Brust, A., Inserra, M.C., Asvadi, N.H., Lewis, R.J., . . Vetter, I. 2015. Activation of kappa Opioid Receptors in Cutaneous Nerve Endings by Conorphin-1, a Novel SubtypeSelective Conopeptide, Does Not Mediate Peripheral Analgesia. ACS Chem Neurosci, 6, 17518.

Devor, M. 1999. Unexplained peculiarities of the dorsal root ganglion. Pain, 82, S27-S35.

Dewire, S.M., Yamashita, D.S., Rominger, D.H., Liu, G., Cowan, C.L., Graczyk, T.M., . . Violin, J.D. 2013. A G protein-biased ligand at the \$ \mu\$-opioid receptor is potently analgesic with reduced gastrointestinal and respiratory dysfunction compared with morphines. Journal of Pharmacology and Experimental Therapeutics, 344, 708--717.

Di Chiara, G. \& Imperato, A. 1988a. Drugs abused by humans preferentially increase synaptic dopamine concentrations in the mesolimbic system of freely moving rats. Proc Natl Acad Sci U S A, 85, 5274-8.

Di Chiara, G. \& Imperato, A. 1988b. Opposite effects of mu and kappa opiate agonists on dopamine release in the nucleus accumbens and in the dorsal caudate of freely moving rats. J Pharmacol Exp Ther, 244, 1067-80.

Doll, C., Konietzko, J., Poll, F., Koch, T., Hollt, V. \& Schulz, S. 2011. Agonist-selective patterns of microopioid receptor phosphorylation revealed by phosphosite-specific antibodies. Br J Pharmacol, 164, 298-307.

Doll, C., Pöll, F., Peuker, K., Loktev, A., Glück, L. \& Schulz, S. 2012. Deciphering $\mu$ - opioid receptor phosphorylation and dephosphorylation in HEK293 cells. British journal of pharmacology, 167, 1259-1270.

Donthamsetti, P., Gallo, E.F., Buck, D.C., Stahl, E.L., Zhu, Y., Lane, J.R., . . Javitch, J.A. 2020. Arrestin recruitment to dopamine D2 receptor mediates locomotion but not incentive motivation. Molecular psychiatry, 25, 2086-2100.

Dosaka-Akita, K., Tortella, F.C., Holaday, J.W. \& Long, J.B. 1993. The kappa opioid agonist U-50,488H antagonizes respiratory effects of mu opioid receptor agonists in conscious rats. $J$ Pharmacol Exp Ther, 264, 631-7.

Dray, A. \& Metsch, R. 1984. Spinal opioid receptors and inhibition of urinary bladder motility in vivo. Neuroscience Letters, 47, 81-84.

Duan, B., Cheng, L. \& Ma, Q. 2018. Spinal Circuits Transmitting Mechanical Pain and Itch. Neuroscience bulletin, 34, 186-193.

Dubin, A.E. \& Patapoutian, A. 2010. Nociceptors: the sensors of the pain pathway. The Journal of clinical investigation, 120, 3760-3772.

Dutschmann, M. \& Herbert, H. 2006. The Kolliker-Fuse nucleus gates the postinspiratory phase of the respiratory cycle to control inspiratory off-switch and upper airway resistance in rat. Eur J Neurosci, 24, 1071-84.

Dutschmann, M., Morschel, M., Kron, M. \& Herbert, H. 2004. Development of adaptive behaviour of the respiratory network: implications for the pontine Kolliker-Fuse nucleus. Respir Physiol Neurobiol, 143, 155-65.

Ehlert, F.J., Griffin, M.T., Sawyer, G.W. \& Bailon, R. 1999. A simple method for estimation of agonist activity at receptor subtypes: comparison of native and cloned M3 muscarinic receptors in guinea pig ileum and transfected cells. J Pharmacol Exp Ther, 289, 981-92.

Ehrlich, A.T., Semache, M., Gross, F., Da Fonte, D.F., Runtz, L., Colley, C., . . Kieffer, B.L. 2019. Biased Signaling of the Mu Opioid Receptor Revealed in Native Neurons. iScience, 14, 47-57. 
Eichel, K., Jullié, D., \& von Zastrow, M. 2016. $\beta$-Arrestin drives MAP kinase signalling from clathrincoated structures after GPCR dissociation. Nature cell biology, 18(3), 303-310.

El Kouhen, R., Burd, A.L., Erickson-Herbrandson, L.J., Chang, C.Y., Law, P.Y. \& Loh, H.H. 2001. Phosphorylation of Ser363, Thr370, and Ser375 residues within the carboxyl tail differentially regulates mu-opioid receptor internalization. J Biol Chem, 276, 12774-80.

El-Gabalawy, R., Mackenzie, C.S., Shooshtari, S. \& Sareen, J. 2011. Comorbid physical health conditions and anxiety disorders: a population-based exploration of prevalence and health outcomes among older adults. General hospital psychiatry, 33, 556-564.

Emerich, D.F., Snodgrass, P., Pink, M., Bloom, F. \& Bartus, R.T. 1998. Central analgesic actions of loperamide following transient permeation of the blood brain barrier with Cereport ${ }^{\mathrm{TM}}$ (RMP7). Brain research, 801, 259-266.

Enck, P., Merlin, V., Erckenbrecht, J.F. \& Wienbeck, M. 1989. Stress effects on gastrointestinal transit in the rat. Gut, 30, 455-459.

Enyedi, P. \& Czirják, G. 2010. Molecular background of leak K+ currents: two-pore domain potassium channels. Physiological reviews, 90, 559-605.

Espejo, E.F. \& Mir, D. 1993. Structure of the rat's behaviour in the hot plate test. Behav Brain Res, 56, 171-6.

Fda 2020. FDA Approves New Opioid for Intravenous Use in Hospitals, Other Controlled Clinical Settings. U.S Food \& Drug Administration.

Ferracane, M.J., Brice-Tutt, A.C., Coleman, J.S., Simpson, G.G., Wilson, L.L., Eans, S.O., . . Aldrich, J.V. 2020. Design, Synthesis, and Characterization of the Macrocyclic Tetrapeptide cyclo[Pro-SarPhe-d-Phe]: A Mixed Opioid Receptor Agonist-Antagonist Following Oral Administration. ACS Chem Neurosci, 11, 1324-1336.

Ferreira, S.H., Lorenzetti, B.B., Bristow, A.F. \& Poole, S. 1988. Interleukin-1 beta as a potent hyperalgesic agent antagonized by a tripeptide analogue. Nature, 334, 698-700.

Ferrini, F., Lorenzo, L.-E., Godin, A.G., Quang, M.L. \& De Koninck, Y. 2017. Enhancing KCC2 function counteracts morphine-induced hyperalgesia. Scientific reports, 7, 3870-3870.

Ferris, C.F., Nodine, S., Pottala, T., Cai, X., Knox, T.M., Fofana, F.H., ... Hohmann, A.G. 2019. Alterations in brain neurocircuitry following treatment with the chemotherapeutic agent paclitaxel in rats. Neurobiology of pain (Cambridge, Mass.), 6, 100034-100034.

Field, M., Oles, R., Lewis, A., Mccleary, S., Hughes, J. \& Singh, L. 1997. Gabapentin (neurontin) and S -

$(+)-3$ - isobutylgaba represent a novel class of selective antihyperalgesic agents. British journal of pharmacology, 121, 1513-1522.

Fields, H.L., Bry, J., Hentall, I. \& Zorman, G. 1983. The activity of neurons in the rostral medulla of the rat during withdrawal from noxious heat. J Neurosci, 3, 2545-52.

Fields, H.L., Emson, P.C. \& Leigh, B.K. 1980. Multiple opiate receptor sites on primary afferent fibres.

Fields, H.L., Malick, A. \& Burstein, R. 1995. Dorsal horn projection targets of ON and OFF cells in the rostral ventromedial medulla. J Neurophysiol, 74, 1742-59.

Fisher, E., Moore, R. A. Fogarty, A. E. Finn, D. P. Finnerup, N. B. Gilron, I. Haroutounian, S. Krane, E. Rice, A. S. C. Rowbotham, M. Wallace, M. Eccleston, C. (2020). "Cannabinoids, cannabis, and cannabis-based medicine for pain management: a systematic review of randomised controlled trials." Pain.

Fredriksson, R., Lagerstrom, M.C., Lundin, L.G. \& Schioth, H.B. 2003. The G-protein-coupled receptors in the human genome form five main families. Phylogenetic analysis, paralogon groups, and fingerprints. Mol Pharmacol, 63, 1256-72.

Freedman, N.J. \& Lefkowitz, R.J. 1996. Desensitization of G protein-coupled receptors. Recent Prog Horm Res, 51, 319-51; discussion 352-3.

Gaginella, T.S. \& Wu, Z.C. 1983. [D-Ala2,D-Met5NH2]-enkephalin inhibits acetylcholine release from the submucosal plexus of rat colon. J Pharm Pharmacol, 35, 823-5.

Gallantine, E.L. \& Meert, T.F. 2008. Antinociceptive and Adverse Effects of $\mu$ - and K - Opioid Receptor Agonists: A Comparison of Morphine and U50488 - H. Basic \& clinical pharmacology \& toxicology, 103, 419-427. 
Galligan, J.J. \& North, R.A. 1991. Opioid, 5-HT1A and alpha 2 receptors localized to subsets of guineapig myenteric neurons. J Auton Nerv Syst, 32, 1-11.

Gamper N, Stockand JD, Shapiro MS. The use of Chinese hamster ovary (CHO) cells in the study of ion channels. J Pharmacol Toxicol Methods. 2005 May-June;51(3):177-85.

Gao, C., Che, L.W., Chen, J., Xu, X.J. \& Chi, Z.Q. 2003. Ohmefentanyl stereoisomers induce changes of CREB phosphorylation in hippocampus of mice in conditioned place preference paradigm. Cell Res, 13, 29-34.

Gao, T., Shi, T., Wiesenfeld-Hallin, Z., Li, T., Jiang, J.-D. \& Xu, X.-J. 2019. Sinomenine facilitates the efficacy of gabapentin or ligustrazine hydrochloride in animal models of neuropathic pain. European Journal of Pharmacology, 854, 101-108.

García Lopez, M. A., et al. (2009). "Inhibition of dynamin prevents CCL2-mediated endocytosis of CCR2 and activation of ERK1/2." Cellular Signalling 21(12): 1748-1757.

Gaskin, D.J. \& Richard, P. 2012. The economic costs of pain in the United States. J Pain, 13, 715-24.

Gaspari, S., Cogliani, V., Manouras, L., Anderson, E.M., Mitsi, V., Avrampou, K., . . Zachariou, V. 2017. RGS9-2 Modulates Responses to Oxycodone in Pain-Free and Chronic Pain States. Neuropsychopharmacology: official publication of the American College of Neuropsychopharmacology, 42, 1548-1556.

Gaspari, S., Papachatzaki, M.M., Koo, J.W., Carr, F.B., Tsimpanouli, M.-E., Stergiou, E., . . Zachariou, V. 2014. Nucleus Accumbens-Specific Interventions in RGS9-2 Activity Modulate Responses to Morphine. Neuropsychopharmacology, 39, 1968-1977.

Gaspari, S., Purushothaman, I., Cogliani, V., Sakloth, F., Neve, R.L., Howland, D., . . Zachariou, V. 2018. Suppression of RGSz1 function optimizes the actions of opioid analgesics by mechanisms that involve the Wnt/ $\beta$-catenin pathway. Proc Natl Acad Sci U S A, 115, E2085-e2094.

Gasser, H.S. 1941. The classification of nerve fibers. The Ohio Journal of Science, 41, 145-159.

Gasser, H.S. \& Erlanger, J. 1927. THE RÔLE PLAYED BY THE SIZES OF THE CONSTITUENT FIBERS OF A NERVE TRUNK IN DETERMINING THE FORM OF ITS ACTION POTENTIAL WAVE. American Journal of Physiology-Legacy Content, 80, 522-547.

Gavériaux-Ruff, C., Karchewski, L.A., Hever, X., Matifas, A. \& Kieffer, B.L. 2008. Inflammatory pain is enhanced in delta opioid receptor-knockout mice. Eur J Neurosci, 27, 2558-67.

Gemes, G., Koopmeiners, A., Rigaud, M., Lirk, P., Sapunar, D., Bangaru, M.L., . . Hogan, Q.H. 2013. Failure of action potential propagation in sensory neurons: mechanisms and loss of afferent filtering in C-type units after painful nerve injury. The Journal of physiology, 591, 1111-1131.

Giglio, C.A., Defino, H.L., Da-Silva, C.A., De-Souza, A.S. \& Del Bel, E.A. 2006. Behavioral and physiological methods for early quantitative assessment of spinal cord injury and prognosis in rats. Braz J Med Biol Res, 39, 1613-23.

Gillett, K., Harshberger, E. \& Valdez, G.R. 2013. Protracted withdrawal from ethanol and enhanced responsiveness stress: regulation via the dynorphin/kappa opioid receptor system. Alcohol (Fayetteville, N.Y.), 47, 359-365.

Gillis, A., Gondin, A.B., Kliewer, A., Sanchez, J., Lim, H.D., Alamein, C., . . Canals, M. 2020a. Low intrinsic efficacy for $G$ protein activation can explain the improved side effect profiles of new opioid agonists. Sci Signal, 13.

Gillis, A., Sreenivasan, V. \& Christie, M.J. 2020b. Intrinsic efficacy of opioid ligands and its importance for apparent bias, operational analysis and therapeutic window. Mol Pharmacol.

Glick, S.D., Maisonneuve, I.M., Raucci, J. \& Archer, S. 1995. Kappa opioid inhibition of morphine and cocaine self-administration in rats. Brain Res, 681, 147-52.

Glück, L., Loktev, A., Moulédous, L., Mollereau, C., Law, P.-Y. \& Schulz, S. 2014. Loss of morphine reward and dependence in mice lacking $G$ protein-coupled receptor kinase 5. Biological psychiatry, 76, 767-774.

Goldstein, A. \& Schulz, R. 1973. Morphine-tolerant longitudinal muscle strip from guinea-pig ileum. $\mathrm{Br}$ J Pharmacol, 48, 655-66. 
Gomes, I., Gupta, A., Filipovska, J., Szeto, H.H., Pintar, J.E. \& Devi, L.A. 2004. A role for heterodimerization of $\mathrm{mu}$ and delta opiate receptors in enhancing morphine analgesia. Proceedings of the National Academy of Sciences of the United States of America, 101, 51355139.

Gomes, I., Jordan, B.A., Gupta, A., Trapaidze, N., Nagy, V. \& Devi, L.A. 2000. Heterodimerization of mu and delta opioid receptors: A role in opiate synergy. $J$ Neurosci, 20, Rc110.

Goodman, O.B., Krupnick, J.G., Santini, F., Gurevich, V.V., Penn, R.B., Gagnon, A.W., . . Benovic, J.L. 1996. $\beta$-Arrestin acts as a clathrin adaptor in endocytosis of the $\beta 2$-adrenergic receptor. Nature, 383, 447-450.

Gornstein, E.L. \& Schwarz, T.L. 2017. Neurotoxic mechanisms of paclitaxel are local to the distal axon and independent of transport defects. Exp Neurol, 288, 153-166.

Grau, L.E., Dasgupta, N., Harvey, A.P., Irwin, K., Givens, A., Kinzly, M.L. \& Heimer, R. 2007. Illicit use of opioids: is OxyContin a "gateway drug"? Am J Addict, 16, 166-73.

Gray, A.C., Coupar, I.M. \& White, P.J. 2006. Comparison of opioid receptor distributions in the rat ileum. Life Sci, 78, 1610-6.

Grecksch, G., Just, S., Pierstorff, C., Imhof, A.-K., Glück, L., Doll, C., . . Schulz, S. 2011. Analgesic tolerance to high-efficacy agonists but not to morphine is diminished in phosphorylationdeficient S375A $\mu$-opioid receptor knock-in mice. The Journal of neuroscience : the official journal of the Society for Neuroscience, 31, 13890-13896.

Greenspan, J. D., Craft, R. M. LeResche, L. Arendt-Nielsen, L. Berkley, K. J. Fillingim, R. B. Gold, M. S. Holdcroft, A. Lautenbacher, S. Mayer, E. A. Mogil, J. S. Murphy, A. Z. Traub, R. J. (2007). "Studying sex and gender differences in pain and analgesia: a consensus report." Pain 132 Suppl 1(Suppl 1): S26-s45.

Gregus, A.M., Doolen, S., Dumlao, D.S., Buczynski, M.W., Takasusuki, T., Fitzsimmons, B.L., . . Yaksh, T.L. 2012. Spinal 12-lipoxygenase-derived hepoxilin A3 contributes to inflammatory hyperalgesia via activation of TRPV1 and TRPA1 receptors. Proc Natl Acad Sci U S A, 109, 67216.

Griffin, J.W. \& Thompson, W.J. 2008. Biology and pathology of nonmyelinating Schwann cells. Glia, 56, 1518-1531.

Griffin, M.T., Figueroa, K.W., Liller, S. \& Ehlert, F.J. 2007. Estimation of agonist activity at G proteincoupled receptors: analysis of M2 muscarinic receptor signaling through Gi/o,Gs, and G15. J Pharmacol Exp Ther, 321, 1193-207.

Grim, T.W., Schmid, C.L., Stahl, E.L., Pantouli, F., Ho, J.H., Acevedo-Canabal, A., . . Bohn, L.M. 2020. A $G$ protein signaling-biased agonist at the $\mu$-opioid receptor reverses morphine tolerance while preventing morphine withdrawal. Neuropsychopharmacology, 45, 416-425.

Groer, C.E., Schmid, C.L., Jaeger, A.M. \& Bohn, L.M. 2011. Agonist-directed interactions with specific beta-arrestins determine mu-opioid receptor trafficking, ubiquitination, and dephosphorylation. J Biol Chem, 286, 31731-41.

Groer, C.E., Tidgewell, K., Moyer, R.A., Harding, W.W., Rothman, R.B., Prisinzano, T.E. \& Bohn, L.M. 2007. An opioid agonist that does not induce mu-opioid receptor--arrestin interactions or receptor internalization. Mol Pharmacol, 71, 549-57.

Guilloux, J.P., Seney, M., Edgar, N. \& Sibille, E. 2011. Integrated behavioral z-scoring increases the sensitivity and reliability of behavioral phenotyping in mice: relevance to emotionality and sex. J Neurosci Methods, 197, 21-31.

Gunn, J.M., Ayton, D.R., Densley, K., Pallant, J.F., Chondros, P., Herrman, H.E. \& Dowrick, C.F. 2012. The association between chronic illness, multimorbidity and depressive symptoms in an Australian primary care cohort. Social psychiatry and psychiatric epidemiology, 47, 175-184. 
Gupta, A., Mulder, J., Gomes, I., Rozenfeld, R., Bushlin, I., Ong, E., . . Devi, L.A. 2010. Increased abundance of opioid receptor heteromers after chronic morphine administration. Science signaling, 3, ra54-ra54.

Hackler L, Zadina JE, Ge LJ, Kastin AJ. Isolation of relatively large amounts of endomorphin-1 and endomorphin-2 from human brain cortex. Peptides. 1997;18(10):1635-9

Hadjistavropoulos, T., Craig, K.D., Duck, S., Cano, A., Goubert, L., Jackson, P.L., . . Fitzgerald, T.D. 2011. A biopsychosocial formulation of pain communication. Psychol Bull, 137, 910-939.

Hall, D.A. \& Giraldo, J. 2018. A method for the quantification of biased signalling at constitutively active receptors. Br J Pharmacol, 175, 2046-2062.

Han, J.S., Bird, G.C., Li, W., Jones, J. \& Neugebauer, V. 2005. Computerized analysis of audible and ultrasonic vocalizations of rats as a standardized measure of pain-related behavior. J Neurosci Methods, 141, 261-9.

Han, M.-H., Renthal, W., Ring, R.H., Rahman, Z., Psifogeorgou, K., Howland, D., . . Zachariou, V. 2010. Brain Region Specific Actions of Regulator of G Protein Signaling 4 Oppose Morphine Reward and Dependence but Promote Analgesia. Biological Psychiatry, 67, 761-769.

Hanani, M. 2005. Satellite glial cells in sensory ganglia: from form to function. Brain research. Brain research reviews, 48, 457-476.

Hanson, S.M., Cleghorn, W.M., Francis, D.J., Vishnivetskiy, S.A., Raman, D., Song, X., . . Gurevich, V.V. 2008. Arrestin mobilizes signaling proteins to the cytoskeleton and redirects their activity. Cellular Pharmacology, 368, 375--387.

Harding, W.W., Tidgewell, K., Byrd, N., Cobb, H., Dersch, C.M., Butelman, E.R., . . Prisinzano, T.E. 2005. Neoclerodane diterpenes as a novel scaffold for ?? opioid receptor ligands. Journal of Medicinal Chemistry, 48, 4765--4771.

Hayashida, K.-I. \& Obata, H. 2019. Strategies to Treat Chronic Pain and Strengthen Impaired Descending Noradrenergic Inhibitory System. International journal of molecular sciences, 20, 822.

Hayes, M.P., Bodle, C.R. \& Roman, D.L. 2018. Evaluation of the Selectivity and Cysteine Dependence of Inhibitors across the Regulator of G Protein-Signaling Family. Mol Pharmacol, 93, 25-35.

Hayes, P., Meadows, H.J., Gunthorpe, M.J., Harries, M.H., Duckworth, D.M., Cairns, W., . . Davis, J.B. 2000. Cloning and functional expression of a human orthologue of rat vanilloid receptor-1. Pain, 88, 205-15.

He, S.Q., Yang, F., Perez, F.M., Xu, Q., Shechter, R., Cheong, Y.K., .. . Guan, Y. 2013. Tolerance develops to the antiallodynic effects of the peripherally acting opioid loperamide hydrochloride in nerve-injured rats. Pain, 154, 2477-86.

Heinricher, M.M., Barbaro, N.M. \& Fields, H.L. 1989. Putative nociceptive modulating neurons in the rostral ventromedial medulla of the rat: firing of on- and off-cells is related to nociceptive responsiveness. Somatosens Mot Res, 6, 427-39.

Herenbrink, C., Sykes, D.A., Donthamsetti, P., Canals, M., Coudrat, T., Shonberg, J., . . L Lane, J.R. 2016. The role of kinetic context in apparent biased agonism at GPCRs. Nat Commun, 7, 10842.

Hill, R., Disney, A., Conibear, A., Sutcliffe, K., Dewey, W., Husbands, S., . . Henderson, G. 2018. The novel $\mu$-opioid receptor agonist PZM21 depresses respiration and induces tolerance to antinociception. British journal of pharmacology, 175, 2653-2661.

Hille, B. 2001. Ion channels of excitable membranes (Sinauer, Sunderland, MA).

Hinder, L.M., Vincent, A.M., Hayes, J.M., Mclean, L.L. \& Feldman, E.L. 2013. Apolipoprotein E knockout as the basis for mouse models of dyslipidemia-induced neuropathy. Experimental neurology, 239, 102-110.

Hirabayashi, M., \& Alam, M. R. (1981). Enhancing effect of methamphetamine on ambulatory activity produced by repeated administration in mice. Pharmacology Biochemistry and Behavior, 15(6), 925-932.

Hisa, Y., Lyon., M.J, Malmgren L.T. (1985). "Central projection of the sensory component of the rat recurrent laryngeal nerve." Neurosci Lett 55(2): 185-190. 
Hisa, Y., Okamura, H., Tadaki, N., Taguchi, J., Uno, T., Ibata, Y. (1994). "Neuropeptide participation in canine laryngeal sensory innervation: immunohistochemistry and retrograde labeling." Annals of Otology, Rhinology \& Laryngology 103(10): 767-770.

Ho, J.H., Stahl, E.L., Schmid, C.L., Scarry, S.M., Aubé, J. \& Bohn, L.M. 2018. G protein signaling-biased agonism at the K-opioid receptor is maintained in striatal neurons. Sci Signal, 11.

Hodge, C.J., Jr. \& Apkarian, A.V. 1990. The spinothalamic tract. Crit Rev Neurobiol, 5, 363-97.

Hoeijmakers, J.G., Faber, C.G., Merkies, I.S. \& Waxman, S.G. 2015. Painful peripheral neuropathy and sodium channel mutations. Neurosci Lett, 596, 51-9.

Hosobuchi, Y., Hosobuchi, Y. Adams, J. E. Linchitz, R. (1977). "Pain relief by electrical stimulation of the central gray matter in humans and its reversal by naloxone." Science 197(4299): 183-186.

Hojo, M., Sudo, Y., Ando, Y., Minami, K., Takada, M., Matsubara, T., . . Uezono, Y. 2008. mu-Opioid receptor forms a functional heterodimer with cannabinoid CB1 receptor: electrophysiological and FRET assay analysis. J Pharmacol Sci, 108, 308-19.

Hole, K. \& Tjølsen, A. 1993. The tail-flick and formalin tests in rodents: changes in skin temperature as a confounding factor. Pain, 53, 247-54.

Hsia, J.A., Moss, J., Hewlett, E.L. \& Vaughan, M. 1984. ADP-ribosylation of adenylyl cyclase by pertussis toxin. Effects on inhibitory agonist binding. J Biol Chem, 259, 1086-90.

Huang, C.L., Slesinger, P.A., Casey, P.J., Jan, Y.N. \& Jan, L.Y. 1995. Evidence that direct binding of G beta gamma to the GIRK1 G protein-gated inwardly rectifying $\mathrm{K}+$ channel is important for channel activation. Neuron, 15, 1133--1143.

Hughes, J., Smith, T.W., Kosterlitz, H.W., Fothergill, L., Morgan, B. \& Morris, H.R. 1975. Identification of two related pentapeptides from the brain with potent opiate agonist activity. Nature, 258 , 577--579.

Hutchinson, M.R., Coats, B.D., Lewis, S.S., Zhang, Y., Sprunger, D.B., Rezvani, N., . . Watkins, L.R. 2008. Proinflammatory cytokines oppose opioid-induced acute and chronic analgesia. Brain, behavior, and immunity, 22, 1178-1189.

lasp. 1994. Part III: Pain Terms, A Current List with Definitions and Notes on Usage. Classification of Chronic Pain.

Igawa, Y., Westerling, D., Mattiasson, A. \& Andersson, K.E. 1993. Effects of morphine metabolites on micturition in normal, unanaesthetized rats. British journal of pharmacology, 110, 257-262.

Irwin, S., Houde, R.W., Bennett, D.R., Hendershot, L.C. \& Seevers, M.H. 1951. The effects of morphine methadone and meperidine on some reflex responses of spinal animals to nociceptive stimulation. J Pharmacol Exp Ther, 101, 132-43.

Isom, G.E. \& Elshowihy, R.M. 1982. Interaction of acute and chronic stress with respiration: Modification by naloxone. Pharmacology Biochemistry and Behavior, 16, 599-603.

Iyer V, Slivicki RA, Thomaz AC, Crystal JD, Mackie K, Hohmann AG. The cannabinoid CB $_{2}$ receptor agonist LY2828360 synergizes with morphine to suppress neuropathic nociception and attenuates morphine reward and physical dependence. Eur J Pharmacol. 2020 Nov 5;886:173544.

M lizuka, M Hirabayashi., 1983 Enhancing effect of morphine on ambulatory activity produced by repeated administration in mice. Nippon Yakurigaku Zasshi, 82, pp. 293-301

Jakobsen, J. \& Lundbaek, K. 1976. Neuropathy in experimental diabetes: an animal model. British medical journal, 2, 278-279.

Janczewski, W.A. \& Feldman, J.L. 2006. Distinct rhythm generators for inspiration and expiration in the juvenile rat. J Physiol, 570, 407-20.

Jensen, T.S. \& Yaksh, T.L. 1986. Comparison of the antinociceptive action of mu and delta opioid receptor ligands in the periaqueductal gray matter, medial and paramedial ventral medulla in the rat as studied by the microinjection technique. Brain Res, 372, 301-12.

Ji, R., Zhang, Q., Law, P., Low, H., Elde, R. \& Hokfelt, T. 1995. Expression of mu-, delta-, and kappaopioid receptor-like immunoreactivities in rat dorsal root ganglia after carrageenan-induced inflammation. The Journal of Neuroscience, 15, 8156-8166. 
Ji, R.R., Befort, K., Brenner, G.J. \& Woolf, C.J. 2002. ERK MAP kinase activation in superficial spinal cord neurons induces prodynorphin and NK-1 upregulation and contributes to persistent inflammatory pain hypersensitivity. J Neurosci, 22, 478-85.

Ji, R.R., Kohno, T., Moore, K.A. \& Woolf, C.J. 2003. Central sensitization and LTP: do pain and memory share similar mechanisms? Trends Neurosci, 26, 696-705.

Ji, Y., Murphy, A.Z. \& Traub, R.J. 2006. Sex differences in morphine-induced analgesia of visceral pain are supraspinally and peripherally mediated. Am J Physiol Regul Integr Comp Physiol, 291, R307-14.

Jiang, B., Shi, Y., Li, H., Kang, L. \& Ma, L. 2006. Decreased morphine analgesia in rat overexpressing beta-arrestin 2 at periaqueductal gray. Neurosci Lett, 400, 150-3.

Jin, L.Q., Wang, H.Y. \& Friedman, E. 2001. Stimulated D(1) dopamine receptors couple to multiple Galpha proteins in different brain regions. Journal of neurochemistry, 78, 981--990.

John, T.F., French, L.G. \& Erlichman, J.S. 2006. The antinociceptive effect of salvinorin A in mice. Eur J Pharmacol, 545, 129-33.

Johnston, I.N., Milligan, E.D., Wieseler-Frank, J., Frank, M.G., Zapata, V., Campisi, J., . . . Watkins, L.R. 2004. A role for proinflammatory cytokines and fractalkine in analgesia, tolerance, and subsequent pain facilitation induced by chronic intrathecal morphine. J Neurosci, 24, 7353-65.

Jordan, B.A. \& Devi, L.A. 1999. G-protein-coupled receptor heterodimerization modulates receptor function. Nature, 399, 697-700.

Jordt, S.-E., Bautista, D.M., Chuang, H.-H., Mckemy, D.D., Zygmunt, P.M., Högestätt, E.D., . . Julius, D. 2004. Mustard oils and cannabinoids excite sensory nerve fibres through the TRP channel ANKTM1. Nature, 427, 260-265.

Juarez-Salinas, D.L., Braz, J.M., Hamel, K.A. \& Basbaum, A.I. 2018. Pain relief by supraspinal gabapentin requires descending noradrenergic inhibitory controls. Pain reports, 3, e659-e659.

Jurna, I. 2003. [Serturner and morphine--a historical vignette]. Schmerz, 17, 280-3.

Jutkiewicz, E.M., Eller, E.B., Folk, J.E., Rice, K.C., Traynor, J.R. \& Woods, J.H. 2004. 8-Opioid agonists: differential efficacy and potency of SNC80, its 3-OH (SNC86) and 3-desoxy (SNC162) derivatives in Sprague-Dawley rats. Journal of Pharmacology and Experimental Therapeutics, 309, 173-181.

Kahanovitch, U., Tsemakhovich, V., Berlin, S., Rubinstein, M., Styr, B., Castel, R., . . Dascal, N. 2014. Recruitment of Gbetagamma controls the basal activity of G-protein coupled inwardly rectifying potassium (GIRK) channels: crucial role of distal C terminus of GIRK1. J.Physiol, 592, 5373--5390.

Kalia, M. and M. M. Mesulam (1980). "Brain stem projections of sensory and motor components of the vagus complex in the cat: II. Laryngeal, tracheobronchial, pulmonary, cardiac, and gastrointestinal branches." The Journal of comparative neurology 193(2): 467-508.

Kalso, E. 2005. Improving opioid effectiveness: from ideas to evidence. Eur J Pain, 9, 131-5.

Kandel, E.R., Schwartz, J.H., Jessell, T.M., Siegelbaum, S.A. \& Hudspeth, A.J. 2000. Principles of neural science, McGraw-hill New York.

Kang J, Shi Y, Xiang B, Qu B, Su W, Zhu M, Zhang M, Bao G, Wang F, Zhang X, Yang R, Fan F, Chen X, Pei G, Ma L. 2005. A nuclear function of beta-arrestin1 in GPCR signaling: regulation of histone acetylation and gene transcription. Cell. Dec 2;123(5):833-47.

Kane, B.E., Nieto, M.J., Mccurdy, C.R. \& Ferguson, D.M. 2006. A unique binding epitope for salvinorin A, a non-nitrogenous kappa opioid receptor agonist. Febs j, 273, 1966-74.

Kang, M., Maguma, H.T., Smith, T.H., Ross, G.R., Dewey, W.L. \& Akbarali, H.I. 2012. The Role of $\beta$ Arrestin2 in the Mechanism of Morphine Tolerance in the Mouse and Guinea Pig Gastrointestinal Tract. Journal of Pharmacology and Experimental Therapeutics, 340, 567-576.

Kang, Y., Zhou, X.E., Gao, X., He, Y., Liu, W., Ishchenko, A., . . Xu, H.E. 2015. Crystal structure of rhodopsin bound to arrestin by femtosecond X-ray laser. Nature, 523, 561-7.

Kaufman, D.L., Keith, D.E., Anton, B., Tian, J., Magendzo, K., Newman, D., . . . Xia, Y.-R. 1995. Characterization of the murine $\mu$ opioid receptor gene. Journal of Biological Chemistry, 270, 15877-15883.

Kelly, E. 2013. Efficacy and ligand bias at the mu-opioid receptor. Br J Pharmacol, 169, 1430-46. 
Kemp, T., Spike, R.C., Watt, C. \& Todd, A.J. 1996. The mu-opioid receptor (MOR1) is mainly restricted to neurons that do not contain GABA or glycine in the superficial dorsal horn of the rat spinal cord. Neuroscience, 75, 1231-8.

Kenakin, T. 1995a. Agonist-receptor efficacy I: mechanisms of efficacy and receptor promiscuity. Trends in Pharmacological Sciences, 16, 188--192.

Kenakin, T. 1995b. Agonist-receptor efficacy. II. Agonist trafficking of receptor signals. Trends Pharmacol Sci, 16, 232--238.

Kenakin, T. 2001. Inverse, protean, and ligand-selective agonism: matters of receptor conformation. FASEB journal : official publication of the Federation of American Societies for Experimental Biology, 15, 598-611.

Kenakin, T. 2017. A scale of agonism and allosteric modulation for assessment of selectivity, bias, and receptor mutation. Molecular pharmacology, 92, 414-424.

Kenakin, T. 2019. Biased Receptor Signaling in Drug Discovery. Pharmacol Rev, 71, 267-315.

Kenakin, T. \& Christopoulos, A. 2013. Signalling bias in new drug discovery: detection, quantification and therapeutic impact. Nat Rev Drug Discov, 12, 205-16.

Kenakin, T., Watson, C., Muniz-Medina, V., Christopoulos, A. \& Novick, S. 2012. A simple method for quantifying functional selectivity and agonist bias. ACS Chem Neurosci, 3, 193-203.

Kepler, K.L., Kest, B., Kiefel, J.M., Cooper, M.L. \& Bodnar, R.J. 1989. Roles of gender, gonadectomy and estrous phase in the analgesic effects of intracerebroventricular morphine in rats. Pharmacol Biochem Behav, 34, 119-27.

Kest, B., Wilson, S.G. \& Mogil, J.S. 1999. Sex differences in supraspinal morphine analgesia are dependent on genotype. J Pharmacol Exp Ther, 289, 1370-5.

Kidd, J.F., Pilkington, M.F., Schell, M.J., Fogarty, K.E., Skepper, J.N., Taylor, C.W. \& Thorn, P. 2002. Paclitaxel affects cytosolic calcium signals by opening the mitochondrial permeability transition pore. J Biol Chem, 277, 6504-10.

Kim, I.M., Tilley, D.G., Chen, J., Salazar, N.C., Whalen, E.J., Violin, J.D. \& Rockman, H.A. 2008. Betablockers alprenolol and carvedilol stimulate beta-arrestin-mediated EGFR transactivation. Proc Natl Acad Sci U S A, 105, 14555-60.

Kim. \& Moalem-Taylor, G. 2011. Detailed characterization of neuro-immune responses following neuropathic injury in mice. Brain Res, 1405, 95-108.

King, G.F. \& Hardy, M.C. 2013. Spider-Venom Peptides: Structure, Pharmacology, and Potential for Control of Insect Pests. Annual Review of Entomology, 58, 475-496.

Kivell, B.M., Ewald, A.W.M. \& Prisinzano, T.E. 2014. Salvinorin A analogs and other k-opioid receptor compounds as treatments for cocaine abuse. Advances in pharmacology (San Diego, Calif.), 69, 481-511.

Kliewer, A., Gillis, A., Hill, R., Schmidel, F., Bailey, C., Kelly, E., . . Schulz, S. 2020. Morphine-induced respiratory depression is independent of $\beta$-arrestin2 signalling. Br J Pharmacol.

Kliewer, A., Schmiedel, F., Sianati, S., Bailey, A., Bateman, J.T., Levitt, E.S., . . . Schulz, S. 2019. Phosphorylation-deficient G-protein-biased mu-opioid receptors improve analgesia and diminish tolerance but worsen opioid side effects. Nat Commun, 10, 367.

Knapman, A., Abogadie, F., Mcintrye, P. \& Connor, M. 2014a. A Real-Time, Fluorescence-Based Assay for Measuring $\mu$-Opioid Receptor Modulation of Adenylyl Cyclase Activity in Chinese Hamster Ovary Cells.

Knapman, A. \& Connor, M. 2015. Fluorescence-based, high-throughput assays for $\mu$-opioid receptor activation using a membrane potential-sensitive dye. Methods Mol Biol, 1230, 177-85.

Knapman, A., Santiago, M. \& Connor, M. 2014b. Buprenorphine signalling is compromised the N40D polymorphism of the human $\mu$ opioid receptor in vitro. Br J Pharmacol, 171, 4273-88.

Knapman, A., Santiago, M., Du, Y.P., Bennallack, P.R., Christie, M.J. \& Connor, M. 2012. A Continuous , Fluorescence-based Assay of $\mu$-Opioid Receptor Activation in AtT -20 Cells. 1--8.

Koblish, M., Carr, R., Siuda, E.R., Rominger, D.H., Gowen-Macdonald, W., Cowan, C.L., . . Lark, M.W. 2017. TRV0109101, a $\mathrm{G}$ protein-biased agonist of the $\mu$-opioid receptor, does not promote opioid-induced mechanical allodynia following chronic administration. Journal of Pharmacology and Experimental Therapeutics, 362, 254-262. 
Koch, T., Brandenburg, L.O., Liang, Y., Schulz, S., Beyer, A., Schröder, H. \& Höllt, V. 2004. Phospholipase D2 modulates agonist-induced mu-opioid receptor desensitization and resensitization. J Neurochem, 88, 680-8.

Koch, T. \& Hollt, V. 2008. Role of receptor internalization in opioid tolerance and dependence. Pharmacol Ther, 117, 199-206.

Koller, A. \& Wätzig, H. 2005. Precision and variance components in quantitative gel electrophoresis. Electrophoresis, 26, 2470-5.

König, M., Zimmer, A.M., Steiner, H., Holmes, P.V., Crawley, J.N., Brownstein, M.J. \& Zimmer, A. 1996. Pain responses, anxiety and aggression in mice deficient in pre-proenkephalin. Nature, 383, 535-538.

Kontani, H. \& Kawabata, Y. 1988. A study of morphine-induced urinary retention in anesthetized rats capable of micturition. Jpn J Pharmacol, 48, 31-6.

Kraus, M., Lintzeris, N., Maier, C. \& Savage, S. 2016. Recommendations for the Prevention, Detection, Treatment and Management of Prescription Opioid Analgesic Dependence: Outcomes From the Opioid Analgesic Dependence Education Nexus (OPEN) Meeting. Int J Ment Health Addict, 14, 313-321.

Krebs, E. E., Gravely, A., Nugent, S., Jensen, A. C., DeRonne, B., Goldsmith, E. S., Kroenke, K., Bair, M. J., \& Noorbaloochi, S. (2018). Effect of Opioid vs Nonopioid Medications on Pain-Related Function in Patients With Chronic Back Pain or Hip or Knee Osteoarthritis Pain: The SPACE Randomized Clinical Trial. JAMA, 319(9), 872-882.

Kremer, M., Salvat, E., Muller, A., Yalcin, I. \& Barrot, M. 2016. Antidepressants and gabapentinoids in neuropathic pain: Mechanistic insights. Neuroscience, 338, 183-206.

Kruger, L., Perl, E.R. \& Sedivec, M.J. 1981. Fine structure of myelinated mechanical nociceptor endings in cat hairy skin. The Journal of comparative neurology, 198, 137-154.

Kruse, A.C., Hu, J., Pan, A.C., Arlow, D.H., Rosenbaum, D.M., Rosemond, E., . . Kobilka, B.K. 2012. Structure and dynamics of the M3 muscarinic acetylcholine receptor. Nature, 482, 552-6.

Kruse, A.C., Ring, A.M., Manglik, A., Hu, J., Hu, K., Eitel, K., . . Kobilka, B.K. 2013. Activation and allosteric modulation of a muscarinic acetylcholine receptor. Nature, 504, 101-6.

Kudla, L., Bugno, R., Skupio, U., Wiktorowska, L., Solecki, W., Wojtas, A., . . . Przewlocki, R. 2019. Functional characterization of a novel opioid, PZM21, and its effects on the behavioural responses to morphine. British journal of pharmacology, 176, 4434-4445.

Kuhn, H. 1978. Light-regulated binding of rhodopsin kinase and other proteins to cattle photoreceptor membranes. Biochemistry, 17, 4389-95.

Kühn, H., Hall, S. \& Wilden, U. 1984. Light - induced binding of 48 - kDa protein to photoreceptor membranes is highly enhanced by phosphorylation of rhodopsin. FEBS letters, 176, 473-478.

Kuo, A., Wyse, B.D., Meutermans, W. \& Smith, M.T. 2015. In vivo profiling of seven common opioids for antinociception, constipation and respiratory depression: No two opioids have the same profile. British Journal of Pharmacology, 172, 532--548.

Kutrzeba, L.M., Karamyan, V.T., Speth, R.C., Williamson, J.S. \& Zjawiony, J.K. 2009. In vitro studies on metabolism of salvinorin A. Pharmaceutical Biology, 47, 1078-1084.

La Regina, A., Petrillo, P., Sbacchi, M. \& Tavani, A. 1988. Interaction of U-69,593 with mu-, alpha- and kappa-opioid binding sites and its analgesic and intestinal effects in rats. Life Sci, 42, 293-301.

Labots, M., Zheng, X., Moattari, G., Lozeman-Van't Klooster, J.G., Baars, J.M., Hesseling, P., . . Van Lith, H.A. 2016. Substrain and light regime effects on integrated anxiety-related behavioral zscores in male C57BL/6 mice-Hypomagnesaemia has only a small effect on avoidance behavior. Behavioural brain research, 306, 71-83.

Lalley, P.M. 2003. Mu-opioid receptor agonist effects on medullary respiratory neurons in the cat: evidence for involvement in certain types of ventilatory disturbances. Am J Physiol Regul Integr Comp Physiol, 285, R1287-304.

Lamb, K., Tidgewell, K., Simpson, D.S., Bohn, L.M. \& Prisinzano, T.E. 2012. Antinociceptive Effects of Herkinorin, a MOP Receptor Agonist Derived from Salvinorin A in the Formalin Test in Rats: New Concepts in Mu Opioid Receptor Pharmacology(). Drug and Alcohol Dependence, 121, 181-188. 
Lamont, L.A., Tranquilli, W.J. \& Grimm, K.A. 2000. Physiology of pain. Vet Clin North Am Small Anim Pract, 30, 703-28, v.

Lamotte, C. 1977. Distribution of the tract of Lissauer and the dorsal root fibres in the primate spinal cord. . J. comp. Neurol., 172, 59-562.

Land, B.B., Bruchas, M.R., Schattauer, S., Giardino, W.J., Aita, M., Messinger, D., . . Chavkin, C. 2009. Activation of the kappa opioid receptor in the dorsal raphe nucleus mediates the aversive effects of stress and reinstates drug seeking. Proc Natl Acad Sci U S A, 106, 19168-73.

Lane, J.R., May, L.T., Parton, R.G., Sexton, P.M. \& Christopoulos, A. 2017. A kinetic view of GPCR allostery and biased agonism. Nat Chem Biol, 13, 929-937.

Langford, D.J., Bailey, A.L., Chanda, M.L., Clarke, S.E., Drummond, T.E., Echols, S., . . Mogil, J.S. 2010. Coding of facial expressions of pain in the laboratory mouse. Nat Methods, 7, 447-9.

Laporte, S.A., Oakley, R.H., Holt, J.A., Barak, L.S. \& Caron, M.G. 2000. The interaction of beta-arrestin with the AP-2 adaptor is required for the clustering of beta2-adrenergic receptor into clathrincoated pits. Journal of Biological Chemistry, 275, 23120--23126.

Laporte, S.A., Oakley, R.H., Zhang, J., Holt, J.A., Ferguson, S.S., Caron, M.G. \& Barak, L.S. 1999. The beta2-adrenergic receptor/betaarrestin complex recruits the clathrin adaptor AP-2 during endocytosis. Proceedings of the National Academy of Sciences of the United States of America, 96, 3712--3717.

Latremoliere, A. \& Woolf, C.J. 2009. Central sensitization: a generator of pain hypersensitivity by central neural plasticity. The journal of pain : official journal of the American Pain Society, 10, 895-926.

Laugwitz, K.L., Allgeier, A., Offermanns, S. \& Spicher, K.A. 1996. The human thyrotropin receptor: a heptahelical receptor capable of stimulating members of all four $G$ protein families. Proceedings of the National Academy of Sciences of the United States of America, 93, 116-120.

Lay, J., Carbone, S.E., Dicello, J.J., Bunnett, N.W., Canals, M. \& Poole, D.P. 2016. Distribution and trafficking of the $\mu$-opioid receptor in enteric neurons of the guinea pig. Am J Physiol Gastrointest Liver Physiol, 311, G252-66.

Leach, K., Conigrave, A.D., Sexton, P.M. \& Christopoulos, A. 2015. Towards tissue-specific pharmacology: insights from the calcium-sensing receptor as a paradigm for GPCR (patho)physiological bias. Trends Pharmacol Sci, 36, 215-25.

Lefkowitz, R.J. 1998. G Protein-Coupled Receptors III. New roles for receptor kinases and betaarrestins in receptor signaling and desensitization. The Journal of biological chemistry, 273, 18677--18680.

Lesscher, H.M., Burbach, J.P., Van Ree, J.M. \& Gerrits, M.A. 2003. ERK1/2 activation in rat ventral tegmental area by the mu-opioid agonist fentanyl: an in vitro study. Neuroscience, 116, 13944.

Lever, I.J., Bradbury, E.J., Cunningham, J.R., Adelson, D.W., Jones, M.G., Mcmahon, S.B., ... Malcangio, M. 2001. Brain-derived neurotrophic factor is released in the dorsal horn by distinctive patterns of afferent fiber stimulation. Journal of Neuroscience, 21, 4469-4477.

Levitt, E.S., Abdala, A.P., Paton, J.F., Bissonnette, J.M. \& Williams, J.T. 2015. $\mu$ opioid receptor activation hyperpolarizes respiratory - controlling Kölliker-Fuse neurons and suppresses post - inspiratory drive. The Journal of physiology, 593, 4453-4469.

Lewanowitsch, T., Miller, J.H. \& Irvine, R.J. 2006. Reversal of morphine, methadone and heroin induced effects in mice by naloxone methiodide. Life Sciences, 78, 682--688.

Lewin, G.R., Rueff, A. \& Mendell, L.M. 1994. Peripheral and central mechanisms of NGF - induced hyperalgesia. European Journal of Neuroscience, 6, 1903-1912.

Lewis, V.A. \& Gebhart, G.F. 1977. Evaluation of the periaqueductal central gray (PAG) as a morphinespecific locus of action and examination of morphine-induced and stimulation-produced analgesia at coincident PAG loci. Brain Res, 124, 283-303. 
Li, A.-L., Lin, X., Dhopeshwarkar, A.S., Thomaz, A.C., Carey, L.M., Liu, Y., . . Hohmann, A.G. 2019. Cannabinoid CB2 Agonist AM1710 Differentially Suppresses Distinct Pathological Pain States and Attenuates Morphine Tolerance and Withdrawal. Molecular pharmacology, 95, 155-168.

Li, C., Sugam, J.A., Lowery-Gionta, E.G., Mcelligott, Z.A., Mccall, N.M., Lopez, A.J., . . Kash, T.L. 2016. Mu Opioid Receptor Modulation of Dopamine Neurons in the Periaqueductal Gray/Dorsal Raphe: A Role in Regulation of Pain. Neuropsychopharmacology, 41, 2122-2132.

Li, L.Y. \& Chang, K.J. 1996. The stimulatory effect of opioids on mitogen-activated protein kinase in Chinese hamster ovary cells transfected to express mu-opioid receptors. Mol Pharmacol, 50, 599-602.

Li, X.-Y. \& Toyoda, H. 2015. Role of leak potassium channels in pain signaling. Brain Research Bulletin, $119,73-79$.

Li, Y., Liu, X., Liu, C., Kang, J., Yang, J., Pei, G. \& Wu, C. 2009. Improvement of morphine-mediated analgesia by inhibition of $\beta$-arrestin2 expression in mice periaqueductal gray matter. International journal of molecular sciences, 10, 954-963.

Li, Y., Tatsui, C.E., Rhines, L.D., North, R.Y., Harrison, D.S., Cassidy, R.M., . . Dougherty, P.M. 2017. Dorsal root ganglion neurons become hyperexcitable and increase expression of voltagegated T-type calcium channels (Cav3.2) in paclitaxel-induced peripheral neuropathy. Pain, $158,417-429$.

Lieberman, A.R. 1976. Sensory ganglia. In: The peripheral nerve Chapman and Hall, 188-278.

Liles, J.H. \& Flecknell, P.A. 1992. The effects of buprenorphine, nalbuphine and butorphanol alone or following halothane anaesthesia on food and water consumption and locomotor movement in rats. Lab Anim, 26, 180-9.

Lim, R., Zavou, M.J., Milton, P.-L., Chan, S.T., Tan, J.L., Dickinson, H., ... Wallace, E.M. 2014. Measuring Respiratory Function in Mice Using Unrestrained Whole-body Plethysmography. Journal of Visualized Experiments, 1--11.

Lin, X., Dhopeshwarkar, A. S., Huibregtse, M., Mackie, K., \& Hohmann, A. G. (2018). Slowly Signaling G Protein-Biased $\mathrm{CB}_{2}$ Cannabinoid Receptor Agonist LY2828360 Suppresses Neuropathic Pain with Sustained Efficacy and Attenuates Morphine Tolerance and Dependence. Molecular pharmacology, 93(2), 49-62.

Lissaure, H. 1885. Beitrag zur pathologischen Anatomie der Tabes dorsalis und zum Faserverlauf im menschlichen Ruckenmark. Neurol. Zentbl., 4, 245-246.

Listos, J., Łupina, M., Talarek, S., Mazur, A., Orzelska-Górka, J. \& Kotlińska, J. 2019. The Mechanisms Involved in Morphine Addiction: An Overview. International journal of molecular sciences, 20, 4302.

Liu, B., Fan, L., Balakrishna, S., Sui, A., Morris, J.B. \& Jordt, S.E. 2013. TRPM8 is the principal mediator of menthol-induced analgesia of acute and inflammatory pain. Pain, 154, 2169-77.

Liu, N.J., Chakrabarti, S., Schnell, S., Wessendorf, M. \& Gintzler, A.R. 2011. Spinal synthesis of estrogen and concomitant signaling by membrane estrogen receptors regulate spinal $\kappa$ - and $\mu$-opioid receptor heterodimerization and female-specific spinal morphine antinociception. J Neurosci, 31, 11836-45.

Loeser, J.D. \& Melzack, R. 1999. Pain: an overview. The Lancet, 353, 1607-1609.

Loh, H.H., Tseng, L.F., Wei, E. \& Li, C.H. 1976. beta-endorphin is a potent analgesic agent. Proceedings of the National Academy of Sciences of the United States of America, 73, 2895-2898.

Lohse, M.J., Benovic, J.L., Codina, J., Caron, M.G. \& Lefkowitz, R.J. 1990. beta-Arrestin: a protein that regulates beta-adrenergic receptor function. Science, 248, 1547.

Lowinson, J.H. 2005. Substance abuse: A comprehensive textbook, Lippincott Williams \& Wilkins.

Loyd, D.R., Wang, X. \& Murphy, A.Z. 2008. Sex differences in micro-opioid receptor expression in the rat midbrain periaqueductal gray are essential for eliciting sex differences in morphine analgesia. J Neurosci, 28, 14007-17.

Lu, R. \& Schmidtko, A. 2013. Direct intrathecal drug delivery in mice for detecting in vivo effects of cGMP on pain processing. Methods Mol Biol, 1020, 215-21.

Lumsden, T. 1923. Observations on the respiratory centres in the cat. The Journal of Physiology, 57, 153-160. 
Lundberg, J.M., Hokfelt, T., Fahrenkrug, J., Nilsson, G. \& Terenius, L. 1979a. Peptides in the cat carotid body (glomus caroticum): VIP - , enkephalin - , and substance P - like immunoreactivity. Acta Physiologica Scandinavica, 107, 279-281.

Lundberg, J.M., Hökfelt, T., Kewenter, J., Pettersson, G., Ahlman, H., Edin, R., . . Uvnäs-Wallensten, K. 1979b. Substance P-, VIP-, and enkephalin-like immunoreactivity in the human vagus nerve. Gastroenterology, 77, 468-471.

Lundberg, J. M., et al. (1985). "Co-existence of substance P and calcitonin gene-related peptide-like immunoreactivities in sensory nerves in relation to cardiovascular and bronchoconstrictor effects of capsaicin." Eur J Pharmacol 108(3): 315-319.

Lupp, A., Richter, N., Doll, C., Nagel, F. \& Schulz, S. 2011. UMB-3, a novel rabbit monoclonal antibody, for assessing $\mu$-opioid receptor expression in mouse, rat and human formalin-fixed and paraffin-embedded tissues. Regulatory Peptides, 167, 9-13.

Luttrell, A.L.M., Ferguson, S.S.G., Daaka, Y., Miller, W.E., Maudsley, S., Della, G.J., . . L Lefkowitz, R.J. 1999. \$ \beta\$-Arrestin-Dependent Formation of \$ $\backslash$ beta\$2-Adrenergic Receptor-Src Protein Kinase Complexes. American Association for the Advancement of Science Stable, 283, 655-661.

Luttrell, L.M., Roudabush, F.L., Choy, E.W., Miller, W.E., Field, M.E., Pierce, K.L. \& Lefkowitz, R.J. 2001. Activation and targeting of extracellular signal-regulated kinases by $\beta$-arrestin scaffolds. Proceedings of the National Academy of Sciences, 98, 2449-2454.

Ma, M., Li, X., Tong, K., Cheng, J., Yu, Z., Ren, F., . . Shi, W. 2020. Discovery of Biased Mu-Opioid Receptor Agonists for the Treatment of Pain. ChemMedChem, 15, 155-161.

Ma, M., Sun, J., Li, M., Yu, Z., Cheng, J., Zhong, B. \& Shi, W. 2019. Synthesis and Evaluation of Novel Biased $\mu$-Opioid-Receptor ( $\mu \mathrm{OR}$ ) Agonists. Molecules (Basel, Switzerland), 24, 259.

Ma, W. \& Quirion, R. 2002. Partial sciatic nerve ligation induces increase in the phosphorylation of extracellular signal-regulated kinase (ERK) and c-Jun N-terminal kinase (JNK) in astrocytes in the lumbar spinal dorsal horn and the gracile nucleus. Pain, 99, 175-84.

Machelska, H. \& Celik, M.Ö. 2020. Opioid Receptors in Immune and Glial Cells-Implications for Pain Control. Frontiers in immunology, 11, 300-300.

Mackie, K. 2005. Distribution of cannabinoid receptors in the central and peripheral nervous system. Cannabinoids. Springer.

Maekawa, K., Minami, M., Yabuuchi, K., Toya, T., Katao, Y., Hosoi, Y., . . Satoh, M. 1994. In situ hybridization study of $\mu$-and $\mathrm{k}$-opioid receptor mRNAs in the rat spinal cord and dorsal root ganglia. Neuroscience letters, 168, 97-100.

Maguma, H.T., Dewey, W.L. \& Akbarali, H.I. 2012. Differences in the characteristics of tolerance to $\mu-$ opioid receptor agonists in the colon from wild type and $\beta$-arrestin2 knockout mice. European journal of pharmacology, 685, 133-140.

Maier, C., Baron, R., Tolle, T.R., Binder, A., Birbaumer, N., Birklein, F., . . . Treede, R.D. 2010 Quantitative sensory testing in the German Research Network on Neuropathic Pain (DFNS): somatosensory abnormalities in 1236 patients with different neuropathic pain syndromes. Pain, 150, 439-50.

Mangaiarkkarasi, A., Rameshkannan, S. \& Ali, R.M. 2015. Effect of Gabapentin and Pregabalin in Rat Model of Taxol Induced Neuropathic Pain. Journal of clinical and diagnostic research : JCDR, 9, FF11-FF14.

Manglik, A., Kruse, A.C., Kobilka, T.S., Thian, F.S., Mathiesen, J.M., Sunahara, R.K., . . . Granier, S. 2012. Crystal structure of the micro-opioid receptor bound to a morphinan antagonist. Nature, 485 , 321-6.

Manglik., A., Lin, H., Aryal, D.K., Mccorvy, J.D., Dengler, D., Corder, G., . . Shoichet, B.K. 2016. Structure-based discovery of opioid analgesics with reduced side effects. Nature, 537, 185190.

Manning, D.C., Raja, S.N., Meyer, R.A. \& Campbell, J.N. 1991. Pain and hyperalgesia after intradermal injection of bradykinin in humans. Clin Pharmacol Ther, 50, 721-9. 
Mansour, A., Khachaturian, H., Lewis, M.E., Akil, H. \& Watson, S.J. 1987. Autoradiographic differentiation of $\mathrm{mu}$, delta, and kappa opioid receptors in the rat forebrain and midbrain. The Journal of neuroscience : the official journal of the Society for Neuroscience, 7, 2445--64.

Manson, M. E., Corey, D. A., Rymut, S. M., \& Kelley, T. J. 2011. $\beta$-arrestin-2 regulation of the cAMP response element binding protein. Biochemistry, 50(27), 6022-6029.

Margolis, E.B., Hjelmstad, G.O., Fujita, W. \& Fields, H.L. 2014. Direct bidirectional $\mu$-opioid control of midbrain dopamine neurons. The Journal of neuroscience : the official journal of the Society for Neuroscience, 34, 14707-14716.

Marotta, P.L. \& Mccullagh, C.A. 2018. A cross-national analysis of the association between years of implementation of opioid substitution treatments and drug-related deaths in Europe from 1995 to 2013. European journal of epidemiology, 33, 679-688.

Marques, S.A., Garcez, V.F., Del Bel, E.A. \& Martinez, A.M.B. 2009. A simple, inexpensive and easily reproducible model of spinal cord injury in mice: morphological and functional assessment. Journal of neuroscience methods, 177, 183-193.

Martin, M., Matifas, A., Maldonado, R. \& Kieffer, B.L. 2003. Acute antinociceptive responses in single and combinatorial opioid receptor knockout mice: distinct $\mathrm{mu}$, delta and kappa tones. Eur J Neurosci, 17, 701-8.

Martin, W.R., Eades, J.A., Thompson, R.E., Huppler, R.E. \& Gilbert, P.E. 1976. The Effects of Morphine and Nalorphine-like drugs in the Nondependent and Morphine-dependent Chronic Spinal Dog. The Journal of pharmacology and experimental therapeutics, 197, 517--532.

Martins, S.S., Sampson, L., Cerdá, M. \& Galea, S. 2015. Worldwide Prevalence and Trends in Unintentional Drug Overdose: A Systematic Review of the Literature. American journal of public health, 105, e29-e49.

Masuho, I., Xie, K. \& Martemyanov, K.A. 2013. Macromolecular composition dictates receptor and G protein selectivity of regulator of G protein signaling (RGS) 7 and 9-2 protein complexes in living cells. The Journal of biological chemistry, 288, 25129-25142.

Matsumoto, S., Levendusky, M.C., Longhurst, P.A., Levin, R.M. \& Millington, W.R. 2004. Activation of $\mathrm{mu}$ opioid receptors in the ventrolateral periaqueductal gray inhibits reflex micturition in anesthetized rats. Neuroscience Letters, 363, 116-119.

Matthes, H.W., Maldonado, R., Simonin, F., Valverde, O., Slowe, S., Kitchen, I., . . Kieffer, B.L. 1996. Loss of morphine-induced analgesia, reward effect and withdrawal symptoms in mice lacking the mu-opioid-receptor gene. Nature, 383, 819-23.

Mayer, D.J. \& Liebeskind, J.C. 1974. Pain reduction by focal electrical stimulation of the brain: an anatomical and behavioral analysis. Brain Res, 68, 73-93.

Mayer, D.J., Wolfle, T.L., Akil, H., Carder, B. \& Liebeskind, J.C. 1971. Analgesia from electrical stimulation in the brainstem of the rat. Science, 174, 1351-4.

Mccracken, L.M. \& Iverson, G.L. 2002. Disrupted sleep patterns and daily functioning in patients with chronic pain. Pain research \& management, 7, 75-79.

Mccurdy, C.R., Sufka, K.J., Smith, G.H., Warnick, J.E. \& Nieto, M.J. 2006. Antinociceptive profile of salvinorin A, a structurally unique kappa opioid receptor agonist. Pharmacol Biochem Behav, 83, 109-13.

Mcdonald, P.H., Chow, C.-W., Miller, W.E., Laporte, S.A., Field, M.E., Lin, F.-T., . . Lefkowitz, R.J. 2000. $\beta$-Arrestin 2: a receptor-regulated MAPK scaffold for the activation of JNK3. Science, 290, 1574-1577.

Mcgregor, I.S., Lee, A.M. \& Westbrook, R.F. 1994. Stress-induced changes in respiratory quotient, energy expenditure and locomotor activity in rats: effects of midazolam. Psychopharmacology, 116, 475-482.

Mcpherson, K.B., Leff, E.R., Li, M.-H., Meurice, C., Tai, S., Traynor, J.R. \& Ingram, S.L. 2018. Regulators of G-Protein Signaling (RGS) Proteins Promote Receptor Coupling to G-Protein-Coupled Inwardly Rectifying Potassium (GIRK) Channels. The Journal of Neuroscience, 38, 8737.

Mellen, N.M., Janczewski, W.A., Bocchiaro, C.M. \& Feldman, J.L. 2003. Opioid-Induced Quantal Slowing Reveals Dual Networks for Respiratory Rhythm Generation. Neuron, 37, 821-826. 
Mello, N.K. \& Negus, S.S. 1998. Effects of kappa opioid agonists on cocaine- and food-maintained responding by rhesus monkeys. J Pharmacol Exp Ther, 286, 812-24.

Mendell, L.M. 2014. Constructing and deconstructing the gate theory of pain. Pain, 155, 210-216.

Mense, S. 1990. Structure-function relationships in identified afferent neurones. Anat Embryol, 181, 1-17.

Messlinger, K. and A. F. Russo (2019). "Current understanding of trigeminal ganglion structure and function in headache." Cephalalgia 39(13): 1661-1674.

Mestek, A., Hurley, J.H., Bye, L.S., Campbell, A.D., Chen, Y., Tian, M., . . Yu, L. 1995. The human mu opioid receptor: modulation of functional desensitization by calcium/calmodulin-dependent protein kinase and protein kinase C. J Neurosci, 15, 2396-406.

Miaskowski, C., Penko, J.M., Guzman, D., Mattson, J.E., Bangsberg, D.R. \& Kushel, M.B. 2011. Occurrence and characteristics of chronic pain in a community-based cohort of indigent adults living with HIV infection. J Pain, 12, 1004-16.

Michel, M.C. \& Charlton, S.J. 2018. Biased Agonism in Drug Discovery-Is It Too Soon to Choose a Path? Mol Pharmacol, 93, 259-265.

Miljanich, G.P. 2004. Ziconotide: neuronal calcium channel blocker for treating severe chronic pain. Curr Med Chem, 11, 3029-40.

Milligan, G. \& Kostenis, E. 2006. Heterotrimeric G-proteins: a short history. British journal of pharmacology, 147 Suppl 1, S46-S55.

Min, B.H., Augustin, L.B., Felsheim, R.F., Fuchs, J.A. \& Loh, H.H. 1994. Genomic structure analysis of promoter sequence of a mouse mu opioid receptor gene. Proceedings of the National Academy of Sciences, 91, 9081-9085.

Minneman, K.P. \& Iversen, I.L. 1976. Enkephalin and opiate narcotics increase cyclic GMP accumulation in slices of rat neostriatum. Nature, 262, 313-4.

Missale, C., Nash, S.R., Robinson, S.W., Jaber, M. \& Caron, M.G. 1998. Dopamine receptors: from structure to function. Physiological reviews, 78, 189-225.

Mohammed, W., Alhaddad, H., Marie, N., Tardy, F., Lamballais, F., Risde, P., . . Mgarbane, B. 2013. Comparison of tolerance to morphine-induced respiratory and analgesic effects in mice. Toxicology Letters, 217, 251--259.

Moore, D. \& Davies, P. 2018. The Problem of Chronic Pain and Scope for Improvements in Patient Outcomes. Report to the Faculty of Pain Medicine.

Morani, A.S., Ewald, A., Prevatt-Smith, K.M., Prisinzano, T.E. \& Kivell, B.M. 2013. The 2-methoxy methyl analogue of salvinorin $A$ attenuates cocaine-induced drug seeking and sucrose reinforcements in rats. Eur J Pharmacol, 720, 69-76.

Morani, A.S., Kivell, B., Prisinzano, T.E. \& Schenk, S. 2009. Effect of kappa-opioid receptor agonists U69593, U50488H, spiradoline and salvinorin A on cocaine-induced drug-seeking in rats. Pharmacol Biochem Behav, 94, 244-9.

Morani, A.S., Schenk, S., Prisinzano, T.E. \& Kivell, B.M. 2012. A single injection of a novel kappa opioid receptor agonist salvinorin $A$ attenuates the expression of cocaine-induced behavioral sensitization in rats. Behav Pharmacol, 23, 162-70.

Mores, K.L., Cummins, B.R., Cassell, R.J. \& Van Rijn, R.M. 2019. A Review of the Therapeutic Potential of Recently Developed G Protein-Biased Kappa Agonists. Frontiers in pharmacology, 10, 407407.

Morgan, M.M., Tran, A., Wescom, R.L. \& Bobeck, E.N. 2020. Differences in antinociceptive signalling mechanisms following morphine and fentanyl microinjections into the rat periaqueductal gray. Eur J Pain, 24, 617-624.

Mori, T., Nomura, M., Nagase, H., Narita, M. \& Suzuki, T. 2002. Effects of a newly synthesized kappaopioid receptor agonist, TRK-820, on the discriminative stimulus and rewarding effects of cocaine in rats. Psychopharmacology (Berl), 161, 17-22.

Morita, K. \& North, R.A. 1982. Opiate activation of potassium conductance in myenteric neurons: inhibition by calcium ion. Brain Res, 242, 145-50. 
Mousa, S.A., Shaqura, M., Khalefa, B.I., Zllner, C., Schaad, L., Schneider, J., . . Schfer, M. 2013. Rab7 silencing prevents \$mu\$-opioid receptor lysosomal targeting and rescues opioid responsiveness to strengthen diabetic neuropathic pain therapy. Diabetes, 62, 1308--1319.

Mulier, M., Vriens, J. \& Voets, T. 2017. TRP channel pores and local calcium signals. Cell Calcium, 66, 19-24.

Muntean, B.S., Zucca, S., Macmullen, C.M., Dao, M.T., Johnston, C., Iwamoto, H., . . Martemyanov, K.A. 2018. Interrogating the Spatiotemporal Landscape of Neuromodulatory GPCR Signaling by Real-Time Imaging of cAMP in Intact Neurons and Circuits. Cell reports, 22, 255-268.

Muraki, T. \& Kato, R. 1987. Genetic analysis of hypothermia induced by morphine in two strains of inbred mice. Pharmacol Biochem Behav, 27, 87-91.

Nadal, X., Baños, J.E., Kieffer, B.L. \& Maldonado, R. 2006. Neuropathic pain is enhanced in delta-opioid receptor knockout mice. Eur J Neurosci, 23, 830-4.

Nair, A.S., Poornachand, A. \& Kodisharapu, P.K. 2018. Ziconotide: Indications, Adverse Effects, and Limitations in Managing Refractory Chronic Pain. Indian journal of palliative care, 24, 118-119.

Naji-Esfahani, H., Vaseghi, G., Safaeian, L., Pilehvarian, A.A., Abed, A. \& Rafieian-Kopaei, M. 2016. Gender differences in a mouse model of chemotherapy-induced neuropathic pain. Lab Anim, 50, 15-20.

Narita, M., Matsushima, Y., Niikura, K., Narita, M., Takagi, S., Nakahara, K., . . Suzuki, T. 2010. Implication of dopaminergic projection from the ventral tegmental area to the anterior cingulate cortex in $\mu$-opioid-induced place preference. Addict Biol, 15, 434-47.

Neelakantan, H., Ward, S.J. \& Walker, E.A. 2015. Discriminative stimulus effects of morphine and oxycodone in the absence and presence of acetic acid in male and female $\mathrm{C} 57 \mathrm{BI} / 6$ mice. Exp Clin Psychopharmacol, 23, 217-27.

Nielsen, C.K., Lewis, R.J., Alewood, D., Drinkwater, R., Palant, E., Patterson, M., . . Smith, M.T. 2005. Anti-allodynic efficacy of the chi-conopeptide, Xen2174, in rats with neuropathic pain. Pain, $118,112-24$.

Niesters, M., Dahan, A., Kest, B., Zacny, J., Stijnen, T., Aarts, L. \& Sarton, E. 2010. Do sex differences exist in opioid analgesia? A systematic review and meta-analysis of human experimental and clinical studies. PAIN, 151, 61-68.

Nikolarakis, K.E., Almeida, O.F.X. \& Herz, A. 1986. Stimulation of hypothalamic $\beta$-endorphin and dynorphin release by corticotropin-releasing factor (in vitro). Brain Research, 399, 152-155.

Noda, M., Teranishi, Y., Takahashi, H., Toyosato, M., Notake, M., Nakanishi, S. \& Numa, S. 1982. Isolation and structural organization of the human preproenkephalin gene. Nature, 297, 4314.

Norn, S., Kruse, P.R. \& Kruse, E. 2005. [History of opium poppy and morphine]. Dan Medicinhist Arbog, $33,171-84$.

North, R.A. \& Williams, J.T. 1977. Extracellular recording from the guinea-pig myenteric plexus and the action of morphine. Eur J Pharmacol, 45, 23-33.

O'connor, A.B., Schwid, S.R., Herrmann, D.N., Markman, J.D. \& Dworkin, R.H. 2008. Pain associated with multiple sclerosis: systematic review and proposed classification. $P A I N^{\circledR}, 137,96-111$.

Obata, K., Yamanaka, H., Dai, Y., Mizushima, T., Fukuoka, T., Tokunaga, A. \& Noguchi, K. 2004a. Differential activation of MAPK in injured and uninjured DRG neurons following chronic constriction injury of the sciatic nerve in rats. Eur J Neurosci, 20, 2881-95.

Obata, K., Yamanaka, H., Kobayashi, K., Dai, Y., Mizushima, T., Katsura, H., ... Noguchi, K. 2004b. Role of mitogen-activated protein kinase activation in injured and intact primary afferent neurons for mechanical and heat hypersensitivity after spinal nerve ligation. J Neurosci, 24, 10211-22.

Ochiai, W., Kaneta, M., Nagae, M., Yuzuhara, A., Li, X., Suzuki, H., . . Sugiyama, K. 2016. Mice with neuropathic pain exhibit morphine tolerance due to a decrease in the morphine concentration in the brain. European Journal of Pharmaceutical Sciences, 92, 298-304.

Ogren, S.O. \& Berge, O.G. 1984. Test-dependent variations in the antinociceptive effect of pchloroamphetamine-induced release of 5-hydroxytryptamine. Neuropharmacology, 23, 91524. 
Okude, J., Ueda, T., Kofuku, Y., Sato, M., Nobuyama, N., Kondo, K., ... Shimada, I. 2015. Identification of a Conformational Equilibrium That Determines the Efficacy and Functional Selectivity of the $\mu$-Opioid Receptor. Angewandte Chemie (International ed. in English), 54, 15771-15776.

Okun, A., Defelice, M., Eyde, N., Ren, J., Mercado, R., King, T. \& Porreca, F. 2011. Transient inflammation-induced ongoing pain is driven by TRPV1 sensitive afferents. Mol Pain, 7, 4.

Onaran, H.O., Ambrosio, C., Uğur, Ö., Koncz, E.M., Grò, M.C., Vezzi, V., . . Costa, T. 2017. Systematic errors in detecting biased agonism: analysis of current methods and development of a new model-free approach. Scientific reports, 7, 1-17.

Onimaru, H. \& Homma, I. 2003. A novel functional neuron group for respiratory rhythm generation in the ventral medulla. J Neurosci, 23, 1478-86.

Ono, H., Nakamura, A., Matsumoto, K., Horie, S., Sakaguchi, G. \& Kanemasa, T. 2014. Circular muscle contraction in the mice rectum plays a key role in morphine-induced constipation. Neurogastroenterology \& Motility, 26, 1396-1407.

Ortega, A., Blount, J.F. \& Manchand, P.S. 1982. Salvinorin, a New trans-Neoclerodane Diterpene from Salvia divinorurn (Labiatae). Journal of the Chemical Society, Perkin Transactions I: Organic and Bio-Organic Chemistry, 2505--2508.

Ortiz-Miranda, S., Dayanithi, G., Custer, E., Treistman, S.N. \& Lemos, J.R. 2005. \$mu\$-opioid receptor preferentially inhibits oxytocin release from neurohypophysial terminals by blocking R-type Ca2+ channels. Journal of Neuroendocrinology, 17, 583--590.

Pacifici, R., Di Carlo, S., Bacosi, A., Pichini, S. \& Zuccaro, P. 2000. Pharmacokinetics and cytokine production in heroin and morphine-treated mice. Int J Immunopharmacol, 22, 603-14.

Pan, Y.X., Bolan, E. \& Pasternak, G.W. 2002. Dimerization of morphine and orphanin FQ/nociceptin receptors: generation of a novel opioid receptor subtype. Biochem Biophys Res Commun, 297, 659-63.

Papathanasiou, T., Juul, R.V., Heegaard, A.-M., Kreilgaard, M. \& Lund, T.M. 2016. Co-administration of morphine and gabapentin leads to dose dependent synergistic effects in a rat model of postoperative pain. European Journal of Pharmaceutical Sciences, 82, 97-105.

Pappagallo, M. 2001. Incidence, prevalence, and management of opioid bowel dysfunction. Am J Surg, $182,11 s-18 s$.

Pascual, D., Sánchez-Robles, E.M., García, M.M. \& Goicoechea, C. 2018. Chronic pain and cannabinoids. Great expectations or a christmas carol. Biochemical Pharmacology, 157, 3342.

Paton, K., Atigari, D., Kaska, S., Prisinzano, T.E. \& Kivell, B.M. 2020a. Strategies for developing kappa opioid receptor agonists for the treatment of pain with fewer side-effects. Journal of Pharmacology and Experimental Therapeutics, JPET-MR-2020-000134.

Paton, K.F., Biggerstaff, A., Kaska, S., Crowley, R.S., La Flamme, A.C., Prisinzano, T.E. \& Kivell, B.M. 2020b. Evaluation of Biased and Balanced Salvinorin A Analogs in Preclinical Models of Pain. Frontiers in neuroscience, 14, 765-765.

Paton, K.F., Kumar, N., Crowley, R.S., L.J, H., Prisinzano, T.E. \& B.M., K. 2017. The analgesic and antiinflammatory effects of Salvinorin A analogue b-tetrahydropyran Salvinorin B in mice. Eur $J$ Pain, 21, 1039--1050.

Paton, W.D.M. 1957. The action of morphine and related substances on contraction and on acetylcholine output of coaxially stimulated guinea-pig ileum. British Journal of Pharmacology and Chemotherapy, 12, 119-127.

Paqueron X, Lumbroso A, Mergoni P, Aubrun F, Langeron O, Coriat P, Riou B. Is morphine-induced sedation synonymous with analgesia during intravenous morphine titration? $\mathrm{Br} J$ Anaesth. $2002 \mathrm{Nov} ; 89(5): 697-701$

Peavy, K.M., Banta-Green, C.J., Kingston, S., Hanrahan, M., Merrill, J.O. \& Coffin, P.O. 2012. "Hooked on" prescription-type opiates prior to using heroin: results from a survey of syringe exchange clients. J Psychoactive Drugs, 44, 259-65.

Peckys, D. \& Landwehrmeyer, G.B. 1999. Expression of mu, kappa, and delta opioid receptor messenger RNA in the human CNS: a 33P in situ hybridization study. Neuroscience, 88, 10931135. 
Pedersen, S.F., Owsianik, G. \& Nilius, B. 2005. TRP channels: An overview. Cell Calcium, 38, 233-252.

Peirs, C. \& Seal, R.P. 2016. Neural circuits for pain: Recent advances and current views. Science, 354, 578-584.

Pellis, N.R., Harper, C. \& Dafny, N. 1986. Suppression of the induction of delayed hypersensitivity in rats by repetitive morphine treatments. Exp Neurol, 93, 92-7.

Peng, J., Sarkar, S. \& Chang, S.L. 2012. Opioid receptor expression in human brain and peripheral tissues using absolute quantitative real-time RT-PCR. Drug and alcohol dependence, 124, 223228.

Perl, E. R. (1968). "Myelinated afferent fibres innervating the primate skin and their response to noxious stimuli." J Physiol 197(3): 593-615.

Perry, S.J., Baillie, G.S., Kohout, T.A., Mcphee, I., Magiera, M.M., Ang, K.L., . . Houslay, M.D. 2002. Targeting of cyclic AMP degradation to $\beta 2$-adrenergic receptors by $\beta$-arrestins. Science, 298 , 834-836.

Pert, A. \& Yaksh, T. 1974. Sites of morphine induced analgesia in the primate brain: relation to pain pathways. Brain Res, 80, 135-40.

Pfeiffer, M., Kirscht, S., Stumm, R., Koch, T., Wu, D., Laugsch, M., . . . Schulz, S. 2003. Heterodimerization of substance $P$ and mu-opioid receptors regulates receptor trafficking and resensitization. J Biol Chem, 278, 51630-7.

Pfeiffer, M., Koch, T., Schroder, H., Laugsch, M., Hollt, V. \& Schulz, S. 2002. Heterodimerization of somatostatin and opioid receptors cross-modulates phosphorylation, internalization, and desensitization. J Biol Chem, 277, 19762-72.

Phillips, T.J.C., Cherry, C.L., Cox, S., Marshall, S.J. \& Rice, A.S.C. 2010. Pharmacological treatment of painful HIV-associated sensory neuropathy: a systematic review and meta-analysis of randomised controlled trials. PloS one, 5, e14433-e14433.

Pike, A., Hearn, L. \& Williams, A.C.D.C. 2016. Effectiveness of psychological interventions for chronic pain on health care use and work absence: systematic review and meta-analysis. Pain, 157, 777-785.

Pleuvry, B.J. \& Maddison, S.E. 1980. A sex difference in the effects of oral codeine and promethazine on the ventilatory response to carbon dioxide in human volunteers. $\mathrm{Br} J$ Clin Pharmacol, 9, 159-64.

Popiolek-Barczyk, K., Makuch, W., Rojewska, E., Pilat, D. \& Mika, J. 2014. Inhibition of intracellular signaling pathways NF-KB and MEK1/2 attenuates neuropathic pain development and enhances morphine analgesia. Pharmacol Rep, 66, 845-51.

Porreca, F., Heyman, J.S., Mosberg, H.I., Omnaas, J.R. \& Vaught, J.L. 1987. Role of mu and delta receptors in the supraspinal and spinal analgesic effects of [D-Pen2, D-Pen5]enkephalin in the mouse. J Pharmacol Exp Ther, 241, 393-400.

Porreca, F., Mosberg, H.I., Hurst, R., Hruby, V.J. \& Burks, T.F. 1984. Roles of mu, delta and kappa opioid receptors in spinal and supraspinal mediation of gastrointestinal transit effects and hot-plate analgesia in the mouse. J Pharmacol Exp Ther, 230, 341-8.

Posillico, C.K., Terasaki, L.S., Bilbo, S.D. \& Schwarz, J.M. 2015. Examination of sex and minocycline treatment on acute morphine-induced analgesia and inflammatory gene expression along the pain pathway in Sprague-Dawley rats. Biology of sex differences, 6, 33-33.

Powell, K.J., Quirion, R. \& Jhamandas, K. 2003. Inhibition of neurokinin-1-substance P receptor and prostanoid activity prevents and reverses the development of morphine tolerance in vivo and the morphine-induced increase in CGRP expression in cultured dorsal root ganglion neurons. Eur J Neurosci, 18, 1572-83.

Prkic, I., Mustapic, S., Radocaj, T., Stucke, A.G., Stuth, E.a.E., Hopp, F.A., . . Zuperku, E.J. 2012. Pontine $\mu$-opioid receptors mediate bradypnea caused by intravenous remifentanil infusions at clinically relevant concentrations in dogs. Journal of Neurophysiology, 108, 2430-2441.

Przewlocka, B., Sieja, A., Starowicz, K., Maj, M., Bilecki, W. \& Przewlocki, R. 2002. Knockdown of spinal opioid receptors by antisense targeting beta-arrestin reduces morphine tolerance and allodynia in rat. Neurosci Lett, 325, 107-10. 
Psifogeorgou, K., Terzi, D., Papachatzaki, M.M., Varidaki, A., Ferguson, D., Gold, S.J. \& Zachariou, V. 2011. A unique role of RGS9-2 in the striatum as a positive or negative regulator of opiate analgesia. The Journal of neuroscience : the official journal of the Society for Neuroscience, 31, 5617-5624.

Puck, T.T., Cieciura, S.J. \& Robinson, A. 1958. Genetics of somatic mammalian cells. III. Long-term cultivation of euploid cells from human and animal subjects. J Exp Med, 108, 945-56.

Putzke, J.D., Richards, J.S., Kezar, L., Hicken, B.L. \& Ness, T.J. 2002. Long-term use of gabapentin for treatment of pain after traumatic spinal cord injury. Clin J Pain, 18, 116-21.

Raehal, K.M. \& Bohn, L.M. 2011. The role of beta-arrestin2 in the severity of antinociceptive tolerance and physical dependence induced by different opioid pain therapeutics. Neuropharmacology, 60, 58-65.

Raehal, K.M., Walker, J.K.L. \& Bohn, L.M. 2005. Morphine side effects in beta-arrestin-2 knockout mice. The Journal of Pharmacology and Experimental Therapeutics, 314, 1195--1201.

Rajagopal, S., Ahn, S., Rominger, D.H., Gowen-Macdonald, W., Lam, C.M., Dewire, S.M., . . Lefkowitz, R.J. 2011. Quantifying ligand bias at seven-transmembrane receptors. Molecular pharmacology, 80, 367-377.

Rajagopal, S., Rajagopal, K. \& Lefkowitz, R.J. 2010. Teaching old receptors new tricks: biasing seventransmembrane receptors. Nature reviews. Drug discovery, 9, 373--86.

Ramer, M.S., Murphy, P.G., Richardson, P.M. \& Bisby, M.A. 1998. Spinal nerve lesion-induced mechanoallodynia and adrenergic sprouting in sensory ganglia are attenuated in interleukin6 knockout mice. Pain, 78, 115-121.

Ramon Y Cajal, S. 1909. Histologie du Systgme nerveux de l'Homme et des VertibrEs,. Paris: Maloine., 1.

Ramsey, I.S., Delling, M. \& Clapham, D.E. 2006. An introduction to TRP channels. Annu. Rev. Physiol., 68, 619-647.

Randall, L.O. \& Selitto, J.J. 1957. A method for measurement of analgesic activity on inflamed tissue. Arch Int Pharmacodyn Ther, 111, 409-19.

Ranson, S.W. 1913. The course within the spinal cord of the non-medullated fibres of the dorsal roots: A study of Lissauer's tract in the cat. J. comp. Neurol. , 23, 259/281.

Ranson, S.W. 1914. The tract of Lissauer and the substantia gelatinosa Rolandi. Am. J. Anat., 16, 97126.

Ray, S.B., Verma, D. \& Wadhwa, S. 2005. Acute analgesic effect of loperamide as compared to morphine after intrathecal administration in rat.

Ray, S.B. \& Yaksh, T.L. 2008. Spinal antinociceptive action of loperamide is mediated by opioid receptors in the formalin test in rats. Neurosci Lett, 448, 260-2.

Ren, X.-R., Reiter, E., Ahn, S., Kim, J., Chen, W. \& Lefkowitz, R.J. 2005. Different G protein-coupled receptor kinases govern $\mathrm{G}$ protein and beta-arrestin-mediated signaling of $\mathrm{V} 2$ vasopressin receptor. Proceedings of the National Academy of Sciences of the United States of America, $102,1448--1453$.

Rexed, B. 1952. The cytoarchitectonic organization of the spinal cord in the cat. J Comp Neurol, 96, 414-95.

Rezvani, A., Huidobro-Toro, J.P., Hu, J. \& Way, E.L. 1983. A rapid and simple method for the quantitative determination of tolerance development to opiates in the guinea-pig ileum in vitro. J Pharmacol Exp Ther, 225, 251-5.

Rigo, F.K., Trevisan, G., Rosa, F., Dalmolin, G.D., Otuki, M.F., Cueto, A.P., . . Richardson, M. 2013. Spider peptide Pha1 $\beta$ induces analgesic effect in a model of cancer pain. Cancer science, 104, 1226-1230.

Riley, A.P., Groer, C.E., Young, D., Ewald, A.W., Kivell, B.M. \& Prisinzano, T.E. 2014. Synthesis and kappa - Opioid Receptor Activity of Furan-Substituted Salvinorin A Analogues.

Rios, C., Gomes, I. \& Devi, L.A. 2006. mu opioid and CB1 cannabinoid receptor interactions: reciprocal inhibition of receptor signaling and neuritogenesis. Br J Pharmacol, 148, 387-95. 
Rojavin, M., Szabo, I., Bussiere, J.L., Rogers, T.J., Adler, M.W. \& Eisenstein, T.K. 1993. Morphine treatment in vitro or in vivo decreases phagocytic functions of murine macrophages. Life Sci, 53, 997-1006.

Romero, A., Gonzalez-Cuello, A., Laorden, M.L., Campillo, A., Vasconcelos, N., Romero-Alejo, E. \& Puig, M.M. 2012. Effects of surgery and/or remifentanil administration on the expression of pERK1/2, c-Fos and dynorphin in the dorsal root ganglia in mice. Naunyn Schmiedebergs Arch Pharmacol, 385, 397-409.

Roose, S.P. 2000. Considerations for the use of antidepressants in patients with cardiovascular disease. American Heart Journal, 140, S84-S88.

Rosas, M., Porru, S., Fenu, S., Ruiu, S., Peana, A.T., Papale, A., . . Acquas, E. 2016. Role of nucleus accumbens $\mu$ opioid receptors in the effects of morphine on ERK1/2 phosphorylation. Psychopharmacology (Berl), 233, 2943-54.

Rosen, S., Ham, B. Mogil, J. S. (2017). "Sex differences in neuroimmunity and pain." J Neurosci Res 95(1-2): 500-508.

Roskoski, R. 2012. ERK1/2 MAP kinases: Structure, function, and regulation. Pharmacological Research, 66, 105-143.

Roth, B.L., Lopez, E., Beischel, S., Westkaemper, R.B. \& Evans, J.M. 2004. Screening the receptorome to discover the molecular targets for plant-derived psychoactive compounds: a novel approach for CNS drug discovery. Pharmacology \& therapeutics, 102, 99-110.

Rowbotham MC, Wallace M. Evolution of Analgesic Tolerance and Opioid-Induced Hyperalgesia Over 6 Months: Double-Blind Randomized Trial Incorporating Experimental Pain Models. J Pain. 2020 Sep-Oct;21(9-10):1031-1046.

Roxburgh, A., Hall, W.D., Dobbins, T., Gisev, N., Burns, L., Pearson, S. \& Degenhardt, L. 2017. Trends in heroin and pharmaceutical opioid overdose deaths in Australia. Drug Alcohol Depend, 179, 291-298.

Roy, S., Liu, H.C. \& Loh, H.H. 1998. mu-Opioid receptor-knockout mice: the role of mu-opioid receptor in gastrointestinal transit. Brain Res Mol Brain Res, 56, 281-3.

Ruan, X. 2007. Drug-related side effects of long-term intrathecal morphine therapy. Pain Physician, $10,357$.

Rubinstein, M., Mogil, J.S., Japón, M., Chan, E.C., Allen, R.G. \& Low, M.J. 1996. Absence of opioid stressinduced analgesia in mice lacking beta-endorphin by site-directed mutagenesis. Proceedings of the National Academy of Sciences, 93, 3995-4000.

Russell, W.M.S. \& Burch, R.L. 1959. The principles of humane experimental technique, Methuen.

Rybak, I.A., Shevtsova, N.A., Paton, J.F., Dick, T.E., St-John, W.M., Morschel, M. \& Dutschmann, M. 2004. Modeling the ponto-medullary respiratory network. Respir Physiol Neurobiol, 143, 30719.

Saarto, T. \& Wiffen, P.J. 2007. Antidepressants for neuropathic pain. Cochrane Database of Systematic Reviews.

Sabbir, M. G. and P. Fernyhough. 2018. "Muscarinic receptor antagonists activate ERK-CREB signalling to augment neurite outgrowth of adult sensory neurons." Neuropharmacology 143: 268-281.

Sacerdote, P., Manfredi, B., Mantegazza, P. \& Panerai, A.E. 1997. Antinociceptive and immunosuppressive effects of opiate drugs: a structure-related activity study. British journal of pharmacology, 121, 834-840.

Sadat-Shirazi, M.S., Babhadi-Ashar, N., Khalifeh, S., Mahboubi, S., Ahmadian-Moghaddam, H. \& Zarrindast, M.R. 2019. Tramadol induces changes in $\Delta$-FosB, $\mu$-opioid receptor, and $p$-CREB level in the nucleus accumbens and prefrontal cortex of male Wistar rat. Am J Drug Alcohol Abuse, 45, 84-89.

Sadeque, A.J.M., Wandel, C., He, H., Shah, S. \& Wood, A.J.J. 2000. Increased drug delivery to the brain by P-glycoprotein inhibition. Clinical Pharmacology \& Therapeutics, 68, 231-237.

Sadja, R., Alagem, N. \& Reuveny, E. 2003. Gating of GIRK channels: Details of an intricate, membranedelimited signaling complex. Neuron, 39, 9--12.

Salzer, J.L. 2015. Schwann cell myelination. Cold Spring Harbor perspectives in biology, 7, a020529a020529. 
Samineni, V.K., Premkumar, L.S. \& Faingold, C.L. 2017. Neuropathic pain-induced enhancement of spontaneous and pain-evoked neuronal activity in the periaqueductal gray that is attenuated by gabapentin. Pain, 158, 1241-1253.

Sammons, M.J., Raval, P., Davey, P.T., Rogers, D., Parsons, A.A. \& Bingham, S. 2000. Carrageenaninduced thermal hyperalgesia in the mouse: role of nerve growth factor and the mitogenactivated protein kinase pathway. Brain Res, 876, 48-54.

Samur, D.N., Arslan, R., Aydın, S. \& Bektas, N. 2018. Valnoctamide: The effect on relieving of neuropathic pain and possible mechanisms. Eur J Pharmacol, 827, 208-214.

Sarton, E., Olofsen, E., Romberg, R., Den Hartigh, J., Kest, B., Nieuwenhuijs, D., . . Dahan, A. 2000. Sex differences in morphine analgesia: an experimental study in healthy volunteers. Anesthesiology, 93, 1245-54; discussion 6A.

Schafer, M., Mousa, S.A., Zhang, Q., Carter, L. \& Stein, C. 1996. Expression of corticotropin-releasing factor in inflamed tissue is required for intrinsic peripheral opioid analgesia. Proceedings of the National Academy of Sciences of the United States of America, 93, 6096-6100.

Schaible, H.-G., et al. (2011). "Update on peripheral mechanisms of pain: beyond prostaglandins and cytokines." Arthritis research \& therapy 13(2): 210-210.

Schattauer, S.S., Kuhar, J.R., Song, A. \& Chavkin, C. 2017. Nalfurafine is a G-protein biased agonist having significantly greater bias at the human than rodent form of the kappa opioid receptor. Cell Signal, 32, 59-65.

Schattauer, S.S., Miyatake, M., Shankar, H., Zietz, C., Levin, J.R., Liu-Chen, L.-Y., . . Chavkin, C. 2012. Ligand directed signaling differences between rodent and human k-opioid receptors. The Journal of biological chemistry, 287, 41595-41607.

Schenk, S., Partridge, B. \& Shippenberg, T.S. 1999. U69593, a kappa-opioid agonist, decreases cocaine self-administration and decreases cocaine-produced drug-seeking. Psychopharmacology (Berl), 144, 339-46.

Schmid, C.L., Kennedy, N.M., Ross, N.C., Lovell, K.M., Yue, Z., Morgenweck, J., . . Bohn, L.M. 2017. Bias Factor and Therapeutic Window Correlate to Predict Safer Opioid Analgesics. Cell, 171, 1165--1175.e13.

Schmid, C.L., Streicher, J.M., Groer, C.E., Munro, T.A., Zhou, L. \& Bohn, L.M. 2013. Functional selectivity of 6'-guanidinonaltrindole (6'-GNTI) at k-opioid receptors in striatal neurons. J Biol Chem, 288, 22387-98.

Schmidt, M.S., Prisinzano, T.E., Tidgewell, K., Harding, W., Butelman, E.R., Kreek, M.J. \& Murry, D.J. 2005. Determination of Salvinorin $A$ in body fluids by high performance liquid chromatography-atmospheric pressure chemical ionization. J Chromatogr B Analyt Technol Biomed Life Sci, 818, 221-5.

Schmidt, R.E., Dorsey, D.A., Beaudet, L.N., Frederick, K.E., Parvin, C.A., Plurad, S.B. \& Levisetti, M.G. 2003. Non-obese diabetic mice rapidly develop dramatic sympathetic neuritic dystrophy: a new experimental model of diabetic autonomic neuropathy. Am J Pathol, 163, 2077-91.

Schulz, S., Mayer, D., Pfeiffer, M., Stumm, R., Koch, T. \& Höllt, V. 2004. Morphine induces terminal $\mu$ - opioid receptor desensitization by sustained phosphorylation of serine - 375. The EMBO journal, 23, 3282-3289.

Schwienteck, K.L., Faunce, K.E., Rice, K.C., Obeng, S., Zhang, Y., Blough, B.E., . . Banks, M.L. 2019. Effectiveness comparisons of $\mathrm{G}$-protein biased and unbiased mu opioid receptor ligands in warm water tail-withdrawal and drug discrimination in male and female rats. Neuropharmacology, 150, 200-209.

Scott, A., Khan, K., Cook, J. \& Duronio, V. 2004. What is "inflammation"? Are we ready to move beyond Celsus? British journal of sports medicine, 38, 248-249.

Seltzer, Z.E., Dubner, R. \& Shir, Y. 1990. A novel behavioral model of neuropathic pain disorders produced in rats by partial sciatic nerve injury. Pain, 43, 205-218.

Senese, N.B., Kandasamy, R., Kochan, K.E. \& Traynor, J.R. 2020. Regulator of G-Protein Signaling (RGS) Protein Modulation of Opioid Receptor Signaling as a Potential Target for Pain Management. Front Mol Neurosci, 13, 5. 
Seo, J., Tsakem, E.L., Breitman, M. \& Gurevich, V.V. 2011. Identification of arrestin-3-specific residues necessary for JNK3 kinase activation. Journal of Biological Chemistry, 286, 27894--27901.

Seyedabadi, M., Ostad, S.N., Albert, P.R., Dehpour, A.R., Rahimian, R., Ghazi-Khansari, M. \& Ghahremani, M.H. 2012. Ser/Thr residues at $\alpha 3 / \beta 5$ loop of Gas are important in morphineinduced adenylyl cyclase sensitization but not mitogen-activated protein kinase phosphorylation. The FEBS Journal, 279, 650-660.

Shahid, M., Subhan, F., Ahmad, N., Ali, G., Akbar, S., Fawad, K. \& Sewell, R.D. 2017. Topical gabapentin gel alleviates allodynia and hyperalgesia in the chronic sciatic nerve constriction injury neuropathic pain model. Eur J Pain, 21, 668-680.

Shemesh, O.A. \& Spira, M.E. 2010. Paclitaxel induces axonal microtubules polar reconfiguration and impaired organelle transport: implications for the pathogenesis of paclitaxel-induced polyneuropathy. Acta Neuropathol, 119, 235-48.

Shenoy, S.K., Drake, M.T., Nelson, C.D., Houtz, D.A., Xiao, K., Madabushi, S., . . Lefkowitz, R.J. 2006. beta-arrestin-dependent, $\mathrm{G}$ protein-independent ERK1/2 activation by the beta2 adrenergic receptor. J Biol Chem, 281, 1261-73.

Shenoy, S.K., Mcdonald, P.H., Kohout, T.A. \& Lefkowitz, R.J. 2001. Regulation of receptor fate by ubiquitination of activated $\beta 2$-adrenergic receptor and $\beta$-arrestin. Science, 294, 1307-1313.

Sherrington, C. 1952. The integrative action of the nervous system, CUP Archive.

Sherrington, C.S. 1903. Qualitative difference of spinal reflex corresponding with qualitative difference of cutaneous stimulus. The Journal of physiology, 30, 39-46.

Sherwood, A.M., Crowley, R.S., Paton, K.F., Biggerstaff, A., Neuenswander, B., Day, V.W., . . . Prisinzano, T.E. 2017. Addressing Structural Flexibility at the A-Ring on Salvinorin A: Discovery of a Potent Kappa-Opioid Agonist with Enhanced Metabolic Stability. Journal of Medicinal Chemistry, acs.jmedchem.7b00148.

Shipton, E.E., Shipton, A.J., Williman, J.A. \& Shipton, E.A. 2017. Deaths from Opioid Overdosing: Implications of Coroners' Inquest Reports 2008-2012 and Annual Rise in Opioid Prescription Rates: A Population-Based Cohort Study. Pain and Therapy, 6, 203-215.

Shuey, D.L., Woodland, C., Tremblay, C., Gregson, R. \& Gerson, R.J. 2007. Oxymorphone hydrochloride, a potent opioid analgesic, is not carcinogenic in rats or mice. Toxicol Sci, 96, 162-73.

Shukla, A.K., Xiao, K. \& Lefkowitz, R.J. 2011. Emerging paradigms of \$ \beta\$-arrestin-dependent seven transmembrane receptor signaling. Trends Biochem Sci, 36, 457--469.

Siau, C., Xiao, W. \& Bennett, G.J. 2006. Paclitaxel- and vincristine-evoked painful peripheral neuropathies: loss of epidermal innervation and activation of Langerhans cells. Exp Neurol, 201, 507-14.

Simonson, B., Morani, A.S., Ewald, A.W., Walker, L., Kumar, N., Simpson, D., . . Kivell, B.M. 2015. Pharmacology and anti-addiction effects of the novel kappa opioid receptor agonist Mesyl Sal B, a potent and long-acting analogue of salvinorin A. Br J Pharmacol, 172, 515-31.

Simpkins, C.O., Dickey, C.A. \& Fink, M.P. 1984. Human neutrophil migration is enhanced by betaendorphin. Life Sci, 34, 2251-5.

Sindrup, S. H. Otto, M. Finnerup, N. B. Jensen, T. S. (2005). "Antidepressants in the treatment of neuropathic pain." Basic Clin Pharmacol Toxicol 96(6): 399-409.

Singhal, P.C., Sharma, P., Kapasi, A.A., Reddy, K., Franki, N. \& Gibbons, N. 1998. Morphine enhances macrophage apoptosis. J Immunol, 160, 1886-93.

Singla, N., Minkowitz, H.S., Soergel, D.G., Burt, D.A., Subach, R.A., Salamea, M.Y., . . S Skobieranda, F. 2017. A randomized, Phase llb study investigating oliceridine (TRV130), a novel micro-receptor G-protein pathway selective (mu-GPS) modulator, for the management of moderate to severe acute pain following abdominoplasty. J Pain Res, 10, 2413-2424.

Singla, N.K., Skobieranda, F., Soergel, D.G., Salamea, M., Burt, D.A., Demitrack, M.A. \& Viscusi, E.R. 2019. APOLLO-2: A Randomized, Placebo and Active-Controlled Phase III Study Investigating Oliceridine (TRV130), a G Protein-Biased Ligand at the mu-Opioid Receptor, for Management of Moderate to Severe Acute Pain Following Abdominoplasty. Pain Pract, 19, 715-731. 
Sisignano, M., Park, C.K., Angioni, C., Zhang, D.D., Von Hehn, C., Cobos, E.J., . . Brenneis, C. 2012. 5,6EET is released upon neuronal activity and induces mechanical pain hypersensitivity via TRPA1 on central afferent terminals. J Neurosci, 32, 6364-72.

Slack, R.J. \& Hall, D.A. 2012. Development of operational models of receptor activation including constitutive receptor activity and their use to determine the efficacy of the chemokine CCL17 at the CC chemokine receptor CCR4. Br J Pharmacol, 166, 1774-92.

Smith, B.H., Elliott, A.M., Chambers, W.A., Smith, W.C., Hannaford, P.C. \& Penny, K. 2001. The impact of chronic pain in the community. Family practice, 18, 292-299.

Smith, J.C., Ellenberger, H.H., Ballanyi, K., Richter, D.W. \& Feldman, J.L. 1991. Pre-Botzinger complex: a brainstem region that may generate respiratory rhythm in mammals. Science, 254, 726-9.

Smith, J.S., Nicholson, L.T., Suwanpradid, J., Glenn, R.A., Knape, N.M., Alagesan, P., . . Rajagopal, S. 2018. Biased agonists of the chemokine receptor CXCR3 differentially control chemotaxis and inflammation. Science signaling, 11, eaaq1075.

Smith, T.H., Grider, J.R., Dewey, W.L. \& Akbarali, H.I. 2012. Morphine Decreases Enteric Neuron Excitability via Inhibition of Sodium Channels. PLOS ONE, 7, e45251.

Soergel, D.G., Subach, R.A., Burnham, N., Lark, M.W., James, I.E., Sadler, B.M., . . Webster, L.R. 2014. Biased agonism of the mu-opioid receptor by TRV130 increases analgesia and reduces ontarget adverse effects versus morphine: A randomized, double-blind, placebo-controlled, crossover study in healthy volunteers. Pain, 155, 1829-35.

Sonnichsen, B.A. 2000. Distinct membrane domains on endosomes in the recycling pathway visualized by multicolor imaging of Rab4, Rab5, and Rab11. Journal of Cell Biology, 149, 901--913.

Sorge, R.E., Martin, L.J., Isbester, K.A., Sotocinal, S.G., Rosen, S., Tuttle, A.H., . . Kadoura, B. 2014. Olfactory exposure to males, including men, causes stress and related analgesia in rodents. Nature methods, 11, 629-632.

Souza, A.H., Ferreira, J., Cordeiro, M.D.N., Vieira, L.B., De Castro, C.J., Trevisan, G., . . . Gomez, M.V. 2008. Analgesic effect in rodents of native and recombinant Ph $\alpha 1 \beta$ toxin, a high-voltageactivated calcium channel blocker isolated from armed spider venom. PAIN, 140, 115-126.

Spanagel, R., Herz, A. \& Shippenberg, T.S. 1992. Opposing tonically active endogenous opioid systems modulate the mesolimbic dopaminergic pathway. Proceedings of the National Academy of Sciences, 89, 2046-2050.

Staff, N.P., Grisold, A., Grisold, W. \& Windebank, A.J. 2017. Chemotherapy-induced peripheral neuropathy: A current review. Annals of neurology, 81, 772-781.

Stahl, E.L., Ehlert, F.J. \& Bohn, L.M. 2019. Quantitating Ligand Bias Using the Competitive Model of Ligand Activity. Methods Mol Biol, 1957, 235-247.

Stahl, E.L., Zhou, L., Ehlert, F.J. \& Bohn, L.M. 2015. A novel method for analyzing extremely biased agonism at $\mathrm{G}$ protein-coupled receptors. Mol Pharmacol, 87, 866-77.

Starobova, H. \& Vetter, I. 2017. Pathophysiology of Chemotherapy-Induced Peripheral Neuropathy. Front Mol Neurosci, 10, 174.

Stein, C., Millan, M.J., Shippenberg, T.S., Peter, K. \& Herz, A. 1989. Peripheral opioid receptors mediating antinociception in inflammation. Evidence for involvement of mu, delta and kappa receptors. The Journal of Pharmacology and Experimental Therapeutics, 248, 1269--1275.

Stein, M.B., Cox, B.J., Afifi, T.O., Belik, S.-L. \& Sareen, J. 2006. Does co-morbid depressive illness magnify the impact of chronic physical illness? A population-based perspective. Psychological medicine, 36, 587-596.

Sternini, C., Patierno, S., Selmer, I.S. \& Kirchgessner, A. 2004. The opioid system in the gastrointestinal tract. Neurogastroenterol Motil, 16 Suppl 2, 3-16.

Stevenson, G.W., Wentland, M.P., Bidlack, J.M., Mello, N.K. \& Negus, S.S. 2004. Effects of the mixedaction kappa/mu opioid agonist 8-carboxamidocyclazocine on cocaine- and food-maintained responding in rhesus monkeys. Eur J Pharmacol, 506, 133-41.

Stone, L.S., German, J.P., Kitto, K.F., Fairbanks, C.A. \& Wilcox, G.L. 2014. Morphine and clonidine combination therapy improves therapeutic window in mice: synergy in antinociceptive but not in sedative or cardiovascular effects. PloS one, 9, e109903-e109903. 
Strathdee, S.A., Hallett, T.B., Bobrova, N., Rhodes, T., Booth, R., Abdool, R. \& Hankins, C.A. 2010. HIV and risk environment for injecting drug users: the past, present, and future. Lancet (London, England), 376, 268-284.

Suess, W.M., Alexander, A.B., Smith, D.D., Sweeney, H.W. \& Marion, R.J. 1980. The effects of psychological stress on respiration: a preliminary study of anxiety and hyperventilation. Psychophysiology, 17, 535-540.

Sullivan, L.C., Chavera, T.S., Jamshidi, R.J., Berg, K.A. \& Clarke, W.P. 2016. Constitutive Desensitization of Opioid Receptors in Peripheral Sensory Neurons. The Journal of pharmacology and experimental therapeutics, 359, 411-419.

Sullivan, M.J., Thorn, B., Haythornthwaite, J.A., Keefe, F., Martin, M., Bradley, L.A. \& Lefebvre, J.C. 2001. Theoretical perspectives on the relation between catastrophizing and pain. Clin J Pain, $17,52-64$.

Surprenant, A., Shen, K.Z., North, R.A. \& Tatsumi, H. 1990. Inhibition of calcium currents by noradrenaline, somatostatin and opioids in guinea-pig submucosal neurones. The Journal of physiology, 431, 585-608.

Sutton, L.P., Ostrovskaya, O., Dao, M., Xie, K., Orlandi, C., Smith, R., . . Martemyanov, K.A. 2016. Regulator of G-Protein Signaling 7 Regulates Reward Behavior by Controlling Opioid Signaling in the Striatum. Biol Psychiatry, 80, 235-45.

Szczepek, M., Beyrière, F., Hofmann, K.P., Elgeti, M., Kazmin, R., Rose, A., . . Scheerer, P. 2014. Crystal structure of a common GPCR-binding interface for $G$ protein and arrestin. Nature communications, 5, 4801-4801.

Tan-No, K., Niijima, F., Nakagawasai, O., Sato, T., Satoh, S. \& Tadano, T. 2003. Development of tolerance to the inhibitory effect of loperamide on gastrointestinal transit in mice. Eur J Pharm Sci, 20, 357-63.

Tanabe, M., Ono, K., Honda, M. \& Ono, H. 2009. Gabapentin and pregabalin ameliorate mechanical hypersensitivity after spinal cord injury in mice. European journal of pharmacology, 609, 6568.

Tao, R. \& Auerbach, S.B. 1994. Increased extracellular serotonin in rat brain after systemic or intraraphe administration of morphine. J Neurochem, 63, 517-24.

Taub, D.D., Eisenstein, T.K., Geller, E.B., Adler, M.W. \& Rogers, T.J. 1991. Immunomodulatory activity of mu- and kappa-selective opioid agonists. Proceedings of the National Academy of Sciences of the United States of America, 88, 360--4.

Taussig, R., Iniguez-Lluhi, J.A. \& Gilman, A.G. 1993. Inhibition of adenylyl cyclase by Gi alpha. Science, 261, 218-21.

Tayrouz, Y., Ganssmann, B., Ding, R., Klingmann, A., Aderjan, R., Burhenne, J., . . . Mikus, G. 2001. Ritonavir increases loperamide plasma concentrations without evidence for P-glycoprotein involvement. Clin Pharmacol Ther, 70, 405-14.

Terman, G.W., Jin, W., Cheong, Y.P., Lowe, J., Caron, M.G., Lefkowitz, R.J. \& Chavkin, C. 2004. G-protein receptor kinase 3 (GRK3) influences opioid analgesic tolerance but not opioid withdrawal. $\mathrm{Br}$ J Pharmacol, 141, 55-64.

Terskiy, A., Wannemacher, K M. Yadav, P. N. Tsai, M. Tian, B. Howells, R. D. (2007). "Search of the human proteome for endomorphin-1 and endomorphin-2 precursor proteins." Life Sciences 81(23): 1593-1601.

Tesmer, J.J., Berman, D.M., Gilman, A.G. \& Sprang, S.R. 1997. Structure of RGS4 bound to AlF4-activated G(i alpha1): stabilization of the transition state for GTP hydrolysis. Cell, 89, 251-61.

Thibault, K., Lin, W.K., Rancillac, A., Fan, M., Snollaerts, T., Sordoillet, V., . . . Pezet, S. 2014. BDNFdependent plasticity induced by peripheral inflammation in the primary sensory and the cingulate cortex triggers cold allodynia and reveals a major role for endogenous BDNF as a tuner of the affective aspect of pain. Journal of Neuroscience, 34, 14739-14751.

Thompson, G.L., Canals, M. \& Poole, D.P. 2014. Biological redundancy of endogenous GPCR ligands in the gut and the potential for endogenous functional selectivity. Front Pharmacol, 5, 262. 
Thompson, G.L., Lane, J.R., Coudrat, T., Sexton, P.M., Christopoulos, A. \& Canals, M. 2015. Biased Agonism of Endogenous Opioid Peptides at the mu-Opioid Receptor. Mol Pharmacol, 88, 335 $-346$.

Thompson, G.L., Lane, J.R., Coudrat, T., Sexton, P.M., Christopoulos, A. \& Canals, M. 2016. Systematic analysis of factors influencing observations of biased agonism at the mu-opioid receptor. Biochemical Pharmacology, 113, 70-87.

Tjølsen, A., Lund, A., Berge, O.G. \& Hole, K. 1989. An improved method for tail-flick testing with adjustment for tail-skin temperature. J Neurosci Methods, 26, 259-65.

Tjolsen, A., Rosland, J.H., Berge, O.G. \& Hole, K. 1991. The increasing-temperature hot-plate test: an improved test of nociception in mice and rats. J Pharmacol Methods, 25, 241-50.

Toblin, R.L., Mack, K.A., Perveen, G. \& Paulozzi, L.J. 2011. A population-based survey of chronic pain and its treatment with prescription drugs. Pain, 152, 1249-1255.

Tomaszewski, D., Bałkota, M., Truszczyński, A. \& Machowicz, A. 2014. Intrathecal morphine increases the incidence of urinary retention in orthopaedic patients under spinal anaesthesia. Anaesthesiol Intensive Ther, 46, 29-33.

Tompkins, D.A., Smith, M.T., Mintzer, M.Z., Campbell, C.M. \& Strain, E.C. 2014. A double blind, within subject comparison of spontaneous opioid withdrawal from buprenorphine versus morphine. Journal of Pharmacology and Experimental Therapeutics, 348, 217-226.

Tonello, R., Fusi, C., Materazzi, S., Marone, I.M., De Logu, F., Benemei, S., . . Nassini, R. 2017. The peptide Ph $\alpha 1 \beta$, from spider venom, acts as a TRPA1 channel antagonist with antinociceptive effects in mice. British journal of pharmacology, 174, 57-69.

Torrecilla, M., Marker, C.L., Cintora, S.C., Stoffel, M., Williams, J.T. \& Wickman, K. 2002. G-proteingated potassium channels containing Kir3.2 and Kir3.3 subunits mediate the acute inhibitory effects of opioids on locus ceruleus neurons. The Journal of neuroscience : the official journal of the Society for Neuroscience, 22, 4328--4334.

Treede, R.-D., Jensen, T.S., Campbell, J.N., Cruccu, G., Dostrovsky, J., Griffin, J., . . . Serra, J. 2008. Neuropathic pain: redefinition and a grading system for clinical and research purposes. Neurology, 70, 1630-1635.

Treede, R. D., Meyer, R. A., Campbell, J. N. (1998). "Myelinated mechanically insensitive afferents from monkey hairy skin: heat-response properties." Journal of neurophysiology 80(3): 10821093.

Treede, R.-D., Rief, W., Barke, A., Aziz, Q., Bennett, M.I., Benoliel, R., . . . Wang, S.-J. 2015. A classification of chronic pain for ICD-11. Pain, 156, 1003-1007.

Tsantoulas, C. 2015. Emerging potassium channel targets for the treatment of pain. Current opinion in supportive and palliative care, 9, 147-154.

Tsantoulas, C. \& Mcmahon, S.B. 2014. Opening paths to novel analgesics: the role of potassium channels in chronic pain. Trends in neurosciences, 37, 146-158.

Tseng, L.-F., Ostwald, T.J., Loh, H.H. \& Li, C.H. 1979. Behavioral activities of opioid peptides and morphine sulfate in golden hamsters and rats. Psychopharmacology, 64, 215-218.

Tseng, L.F. 2002. The antinociceptive properties of endomorphin-1 and endomorphin-2 in the mouse. Japanese journal of pharmacology, 89, 216-220.

Tubaro, E., Avico, U., Santiangeli, C., Zuccaro, P., Cavallo, G., Pacifici, R., . . Borelli, G. 1985. Morphine and methadone impact on human phagocytic physiology. Int J Immunopharmacol, 7, 865-74.

Tveita T, Thoner J, Klepstad P, Dale O, Jystad A, Borchgrevink PC. A controlled comparison between single doses of intravenous and intramuscular morphine with respect to analgesic effects and patient safety. Acta Anaesthesiol Scand. 2008 Aug;52(7):920-5.

Unett, D.J., Gatlin, J., Anthony, T.L., Buzard, D.J., Chang, S., Chen, C., . . Gaidarov, I. 2013. Kinetics of 5-HT2B receptor signaling: profound agonist-dependent effects on signaling onset and duration. J Pharmacol Exp Ther, 347, 645-59.

Urs, N.M., Daigle, T.L. \& Caron, M.G. 2011. A Dopamine D1 Receptor-Dependent $\beta$-Arrestin Signaling Complex Potentially Regulates Morphine-Induced Psychomotor Activation but not Reward in Mice. Neuropsychopharmacology, 36, 551-558. 
Urs, N.M., Gee, S.M., Pack, T.F., Mccorvy, J.D., Evron, T., Snyder, J.C., . . Caron, M.G. 2016. Distinct cortical and striatal actions of a $\beta$-arrestin-biased dopamine D2 receptor ligand reveal unique antipsychotic-like properties. Proceedings of the National Academy of Sciences of the United States of America, 113, E8178-E8186.

Valdes, L.J., Diaz, J.L. \& Paul, A.G. 1983. Ethnopharmacology of ska Maria Pastora (Salvia divinorum, Epling AND Jativa-M.). Journal of Ethnopharmacology, 7, 287--312.

Van Der Sluijs, P., Hull, M., Webster, P., Mle, P., Goud, B. \& Mellman, I. 1992. The small GTP-binding protein rab4 controls an early sorting event on the endocytic pathway. Cell, 70, 729--740.

Vankova, M.E., Weinger, M.B., Chen, D.-Y., Bronson, J.B., Motis, V. \& Koob, G.F. 1996. Role central mu, delta-1, and kappa-1 opioid receptors in opioid-induced muscle rigidity in the rat. Anesthesiology: The Journal of the American Society of Anesthesiologists, 85, 574-583.

Váradi, A., Marrone, G.F., Eans, S.O., Ganno, M.L., Subrath, J.J., Le Rouzic, V., . . Majumdar, S. 2015. Synthesis and characterization of a dual kappa-delta opioid receptor agonist analgesic blocking cocaine reward behavior. ACS Chem Neurosci, 6, 1813-24.

Váradi, A., Marrone, G.F., Palmer, T.C., Narayan, A., Szabó, M.R., Le Rouzic, V., . . Majumdar, S. 2016. Mitragynine/Corynantheidine Pseudoindoxyls As Opioid Analgesics with Mu Agonism and Delta Antagonism, Which Do Not Recruit $\beta$-Arrestin-2. Journal of medicinal chemistry, 59, 8381-8397.

Vaughan, C.W., Ingram, S.L., Connor, M.A. \& Christie, M.J. 1997. How opioids inhibit GABA-mediated neurotransmission. Nature, 390, 611-4.

Vieira, L.B., Kushmerick, C., Hildebrand, M.E., Garcia, E., Stea, A., Cordeiro, M.N., . . Snutch, T.P. 2005. Inhibition of high voltage-activated calcium channels by spider toxin PnTx3-6. Journal of Pharmacology and Experimental Therapeutics, 314, 1370-1377.

Viscusi, E.R., Webster, L., Kuss, M., Daniels, S., Bolognese, J.A., Zuckerman, S., . . Skobieranda, F. 2016. A randomized, phase 2 study investigating TRV130, a biased ligand of the mu-opioid receptor, for the intravenous treatment of acute pain. Pain, 157, 264-72.

Von Frey, M. 1896. Untersuchungen uber die Sinnesfunktionen der menschlichen Haut. Abh Sachs Ges (Akad) Wiss, 40, 175-266.

Vulchanova, L., et al. (1997). "Immunohistochemical study of the P2X2 and P2X3 receptor subunits in rat and monkey sensory neurons and their central terminals." Neuropharmacology 36(9): 1229-1242.

Vowles, K.E., Mcentee, M.L., Julnes, P.S., Frohe, T., Ney, J.P. \& Van Der Goes, D.N. 2015. Rates of opioid misuse, abuse, and addiction in chronic pain: a systematic review and data synthesis. Pain, 156, 569-76.

Wallace, V.C., Blackbeard, J., Pheby, T., Segerdahl, A.R., Davies, M., Hasnie, F., . . Rice, A.S. 2007. Pharmacological, behavioural and mechanistic analysis of HIV-1 gp120 induced painful neuropathy. Pain ${ }^{\circledR}, 133,47-63$.

Wallace, V.C., Norbury, T.A. \& Rice, A.S. 2005. Ultrasound vocalisation by rodents does not correlate with behavioural measures of persistent pain. Eur J Pain, 9, 445-52.

Walters, C.L. \& Blendy, J.A. 2001. Different requirements for CAMP response element binding protein in positive and negative reinforcing properties of drugs of abuse. The Journal of neuroscience : the official journal of the Society for Neuroscience, 21, 9438-9444.

Walwyn, W., Evans, C.J. \& Hales, T.G. 2007. Beta-arrestin2 and c-Src regulate the constitutive activity and recycling of mu opioid receptors in dorsal root ganglion neurons. The Journal of neuroscience : the official journal of the Society for Neuroscience, 27, 5092-5104.

Wang, D., Sun, X., Bohn, L.M. \& Sadee, W. 2005a. Opioid receptor homo- and heterodimerization in living cells by quantitative bioluminescence resonance energy transfer. Mol Pharmacol, 67, 2173-84.

Wang, H.L. 2000. A cluster of Ser/Thr residues at the C-terminus of mu-opioid receptor is required for G protein-coupled receptor kinase 2-mediated desensitization. Neuropharmacology, 39, 35363. 
Wang, H.Y., Friedman, E., Olmstead, M.C. \& Burns, L.H. 2005b. Ultra-low-dose naloxone suppresses opioid tolerance, dependence and associated changes in mu opioid receptor-G protein coupling and Gbetagamma signaling. Neuroscience, 135, 247-61.

Wang, J.B., Johnson, P.S., Persico, A.M., Hawkins, A.L., Griffin, C.A. \& Uhl, G.R. 1994. Human mu opiate receptor. cDNA and genomic clones, pharmacologic characterization and chromosomal assignment. FEBS Lett, 338, 217-22.

Wang, X., Traub, R.J. \& Murphy, A.Z. 2006. Persistent pain model reveals sex difference in morphine potency. Am J Physiol Regul Integr Comp Physiol, 291, R300-6.

Wang, Y., Chen, Y., Xu, W., Lee, D.Y.W., Ma, Z., Rawls, S.M., . . Liu-Chen, L.-Y. 2008. 2-Methoxymethylsalvinorin $B$ is a potent kappa opioid receptor agonist with longer lasting action in vivo than salvinorin A. The Journal of pharmacology and experimental therapeutics, 324, 1073-1083.

Wang, Y., Li, N., Zhao, Y. \& Zhang, L. 2018. Effects of female sex hormones on chemotherapeutic paclitaxel-induced neuropathic pain and involvement of inflammatory signal. Journal of biological regulators and homeostatic agents, 32, 1157-1163.

Wang, Y.J., Tao, Y.M., Li, F.Y., Wang, Y.H., Xu, X.J., Chen, J., . . . Liu, J.G. 2009. Pharmacological characterization of ATPM [(-)-3-aminothiazolo[5,4-b]-N-cyclopropylmethylmorphinan hydrochloride], a novel mixed kappa-agonist and mu-agonist/-antagonist that attenuates morphine antinociceptive tolerance and heroin self-administration behavior. J Pharmacol Exp Ther, 329, 306-13.

Wasserman, D.I., Wang, H.G., Rashid, A.J., Josselyn, S.A. \& Yeomans, J.S. 2013. Cholinergic control of morphine-induced locomotion in rostromedial tegmental nucleus versus ventral tegmental area sites. Eur J Neurosci, 38, 2774-85.

Wasson, R.G. 1962. A New Mexican Psychotropic Drug From the Mint Family. Botanical Museum Leaflets, Harvard University, 20, 77--84.

Webster, L.R. 2015. Opioid-Induced Constipation. Pain Med, 16 Suppl 1, S16-21.

Wemmie, J.A., Taugher, R.J. \& Kreple, C.J. 2013. Acid-sensing ion channels in pain and disease. Nature reviews. Neuroscience, 14, 461-471.

Wharton, J., Polak, J., Pearse, A., Mcgregor, G., Bryant, M.G., Bloom, S., . . Will, J. 1980. Enkephalin-, VIP-and substance P-like immunoreactivity in the carotid body. Nature, 284, 269-271.

Widnell, K.L., Russell, D.S. \& Nestler, E.J. 1994. Regulation of expression of cAMP response elementbinding protein in the locus coeruleus in vivo and in a locus coeruleus-like cell line in vitro. Proc Natl Acad Sci U S A, 91, 10947-51.

Wiese, A.D., Griffin, M.R., Schaffner, W., Stein, C.M., Greevy, R.A., Mitchel, E.F. \& Grijalva, C.G. 2019. Long-acting Opioid Use and the Risk of Serious Infections: A Retrospective Cohort Study. Clinical infectious diseases : an official publication of the Infectious Diseases Society of America, 68, 1862-1869.

Wiese, A.D., Griffin, M.R., Schaffner, W., Stein, C.M., Greevy, R.A., Mitchel, E.F., Jr. \& Grijalva, C.G. 2018. Opioid Analgesic Use and Risk for Invasive Pneumococcal Diseases: A Nested CaseControl Study. Annals of internal medicine, 168, 396-404.

Wilden, U., Hall, S. \& Kühn, H. 1986. Phosphodiesterase activation by photoexcited rhodopsin is quenched when rhodopsin is phosphorylated and binds the intrinsic 48-kDa protein of rod outer segments. Proceedings of the National Academy of Sciences, 83, 1174-1178.

Willer, J. C. and D. Albe-Fessard (1983). "Further studies on the role of afferent input from relatively large diameter fibers in transmission of nociceptive messages in humans. "Brain Res 278(12): 318-321.

Willer, J. C., Boureau, F. Albe-Fessard, D. (1978). "Role of large diameter cutaneous afferents in transmission of nociceptive messages: electrophysiological study in man." Brain Res 152(2): 358-364.

Williams, A. \& Craig, K.D. 2016. Updating the definition of pain. Pain, 157, 2420-2423.

Williams, C.L., Villar, R.G., Peterson, J.M. \& Burks, T.F. 1988. Stress-induced changes in intestinal transit in the rat: A model for irritable bowel syndrome. Gastroenterology, 94, 611-621. 
Williams, J.T., Ingram, S.L., Henderson, G., Chavkin, C., Von Zastrow, M., Schulz, S., . . Christie, M.J. 2013. Regulation of $\mathrm{Mu}$ Opioid Receptors;-Opioid Receptors: Desensitization, Phosphorylation, Internalization, and Tolerance. Pharmacological Reviews, 65, 223.

Willis, W.D. 1985. Nociceptive Pathways: Anatomy and Physiology of Nociceptive Ascending Pathways. Philosophical Transactions of the Royal Society of London. Series B, Biological Sciences, 308, 253-268.

Wilson, N., Kariisa, M., Seth, P., Smith, H.I. \& NI., D. 2020. Drug and Opioid-Involved Overdose Deaths - United States, 2017-2018. Morb Mortal Wkly Rep 69, 290-297.

Wisler, J.W., Dewire, S.M., Whalen, E.J., Violin, J.D., Drake, M.T., Ahn, S., . . Lefkowitz, R.J. 2007. A unique mechanism of beta-blocker action: carvedilol stimulates beta-arrestin signaling. Proc Natl Acad Sci U S A, 104, 16657-62.

Woolf, C.J. \& Ma, Q. 2007. Nociceptors-Noxious Stimulus Detectors. Neuron, 55, 353--364.

Woolf., Allchorne, A., Safieh-Garabedian, B. \& Poole, S. 1997. Cytokines, nerve growth factor and inflammatory hyperalgesia: the contribution of tumour necrosis factor alpha. British journal of pharmacology, 121, 417-424.

Woolfe, G. \& Macdonald, A. 1944. The evaluation of the analgesic action of pethidine hydrochloride (Demerol). Journal of Pharmacology and Experimental Therapeutics, 80, 300-307.

Wroblewski, B.M. \& Del Sel, H.J. 1980. Urethral instrumentation and deep sepsis in total hip replacement. Clin Orthop Relat Res, 209-12.

Xiao, W., Boroujerdi, A., Bennett, G.J. \& Luo, Z.D. 2007. Chemotherapy-evoked painful peripheral neuropathy: Analgesic effects of gabapentin and effects on expression of the alpha-2-delta type-1 calcium channel subunit. Neuroscience, 144, 714-720.

Xiao, W.H., Zheng, H., Zheng, F.Y., Nuydens, R., Meert, T.F. \& Bennett, G.J. 2011. Mitochondrial abnormality in sensory, but not motor, axons in paclitaxel-evoked painful peripheral neuropathy in the rat. Neuroscience, 199, 461-9.

Yaksh, T.L., Woller, S.A., Ramachandran, R. \& Sorkin, L.S. 2015. The search for novel analgesics: targets and mechanisms. F1000prime reports, 7, 56-56.

Yalcin, I., Charlet, A., Freund-Mercier, M.J., Barrot, M. \& Poisbeau, P. 2009. Differentiating thermal allodynia and hyperalgesia using dynamic hot and cold plate in rodents. $J$ Pain, 10, 767-73.

Yamdeu, R.S., Shaqura, M., Mousa, S.A., Schafer, M. \& Droese, J. 2011. p38 Mitogen-activated protein kinase activation by nerve growth factor in primary sensory neurons upregulates mu-opioid receptors to enhance opioid responsiveness toward better pain control. Anesthesiology, 114, 150--161.

Yang, C.H., Huang, H.W., Chen, K.H., Chen, Y.S., Sheen-Chen, S.M. \& Lin, C.R. 2011. Antinociceptive potentiation and attenuation of tolerance by intrathecal beta-arrestin 2 small interfering RNA in rats. Br J Anaesth, 107, 774-81.

Yang, L., Seifert, A., Wu, D., Wang, X., Rankovic, V., Schröder, H., . . . Koch, T. 2010. Role of phospholipase D2/phosphatidic acid signal transduction in micro- and delta-opioid receptor endocytosis. Mol Pharmacol, 78, 105-13.

Ye, L., Van Eps, N., Zimmer, M., Ernst, O.P. \& Prosser, R.S. 2016. Activation of the A2A adenosine Gprotein-coupled receptor by conformational selection. Nature, 533, 265-268.

Yeager, M.P., Colacchio, T.A., Yu, C.T., Hildebrandt, L., Howell, A.L., Weiss, J. \& Guyre, P.M. 1995. Morphine inhibits spontaneous and cytokine-enhanced natural killer cell cytotoxicity in volunteers. Anesthesiology, 83, 500-8.

Yin, D., Mufson, R.A., Wang, R. \& Shi, Y. 1999. Fas-mediated cell death promoted by opioids. Nature, $397,218$.

Yoon, S.Y., Woo, J., Park, J.O., Choi, E.J., Shin, H.S., Roh, D.H. \& Kim, K.S. 2015. Intrathecal RGS4 inhibitor, CCG50014, reduces nociceptive responses and enhances opioid-mediated analgesic effects in the mouse formalin test. Anesth Analg, 120, 671-7.

Yousry, I., et al. (2005). "Trigeminal ganglion and its divisions: detailed anatomic MR imaging with contrast-enhanced 3D constructive interference in the steady state sequences." AJNR Am J Neuroradiol 26(5): 1128-1135. 
Yu SP, Kerchner GA. Endogenous voltage-gated potassium channels in human embryonic kidney (HEK293) cells. J Neurosci Res. 1998 Jun 1;52(5):612-7.

Yu, J.Z., Wang, J., Sheridan, S.D., Perlis, R.H. \& Rasenick, M.M. 2020. N-3 polyunsaturated fatty acids promote astrocyte differentiation and neurotrophin production independent of CAMP in patient-derived neural stem cells. Mol Psychiatry.

Yudin, Y. \& Rohacs, T. 2019. The G-protein-biased agents PZM21 and TRV130 are partial agonists of mu-opioid receptor-mediated signalling to ion channels. Br J Pharmacol, 176, 3110-3125.

Zachariou, V., Georgescu, D., Sanchez, N., Rahman, Z., Dileone, R., Berton, O., . . . Nestler, E.J. 2003. Essential role for RGS9 in opiate action. Proceedings of the National Academy of Sciences of the United States of America, 100, 13656-13661.

Zadina JE, Hackler L, Ge LJ, Kastin AJ. A potent and selective endogenous agonist for the mu-opiate receptor. Nature. 1997 Apr 3;386(6624):499-502.

Zagon, I. S., McLaughlin PJ, Takemori AE, Portoghese PS (1985). " $\beta$-funaltrexamine ( $\beta$-FNA) and neural tumor response in mice." European Journal of Pharmacology 116(1): 165-169.

Zagon, I. S. and P. J. McLaughlin (1986). " $\beta$-Funaltrexamine ( $\beta$-FNA) and the regulation of body and brain development in rats." Brain Research Bulletin 17(1): 5-9.

Zajączkowska, R., Kocot-Kępska, M., Leppert, W., Wrzosek, A., Mika, J. \& Wordliczek, J. 2019. Mechanisms of Chemotherapy-Induced Peripheral Neuropathy. International journal of molecular sciences, 20, 1451.

Zaks-Zilberman, M., Zaks, T.Z. \& Vogel, S.N. 2001. Induction of proinflammatory and chemokine genes by lipopolysaccharide and paclitaxel (Taxol) in murine and human breast cancer cell lines. Cytokine, 15, 156-65.

Zamarripa, C., Edwards, S.R., Qureshi, H.N., Yi, J.N., Blough, B.E. \& Freeman, K.B. 2018. The G-protein biased mu-opioid agonist, TRV130, produces reinforcing and antinociceptive effects that are comparable to oxycodone in rats. Drug Alcohol Depend, 192, 158-162.

Zamponi, G.W. \& Snutch, T.P. 1998. Modulation of voltage-dependent calcium channels by G proteins. Current opinion in neurobiology, 8, 351--356.

Zerial, M. \& Mcbride, H. 2001. Rab proteins as membrane organizers. Nature reviews. Molecular cell biology, 2, 107--117.

Zhang, H., Li, Y., De Carvalho-Barbosa, M., Kavelaars, A., Heijnen, C.J., Albrecht, P.J. \& Dougherty, P.M. 2016. Dorsal Root Ganglion Infiltration by Macrophages Contributes to Paclitaxel Chemotherapy-Induced Peripheral Neuropathy. J Pain, 17, 775-86.

Zhang, J., Ferguson, S.S., Barak, L.S., Mnard, L. \& Caron, M.G. 1996. Dynamin and beta-arrestin reveal distinct mechanisms for $\mathrm{G}$ protein-coupled receptor internalization. The Journal of biological chemistry, 271, 18302--18305.

Zhang, Y., Butelman, E.R., Schlussman, S.D., Ho, A. \& Kreek, M.J. 2005. Effects of the plant-derived hallucinogen salvinorin $A$ on basal dopamine levels in the caudate putamen and in a conditioned place aversion assay in mice: agonist actions at kappa opioid receptors. Psychopharmacology (Berl), 179, 551-8.

Zhang, Z., Zhuang, J., Zhang, C. \& Xu, F. 2011. Activation of opioid 3-receptors in the commissural subdivision of the nucleus tractus solitarius abolishes the ventilatory response to hypoxia in anesthetized rats. Anesthesiology, 115, 353.

Zheng, H., Xiao, W.H. \& Bennett, G.J. 2011. Functional deficits in peripheral nerve mitochondria in rats with paclitaxel- and oxaliplatin-evoked painful peripheral neuropathy. Experimental neurology, 232, 154-161.

Zhu, L., Almaça, J., Dadi, P.K., Hong, H., Sakamoto, W., Rossi, M., ... Wess, J. 2017. $\beta$-arrestin-2 is an essential regulator of pancreatic $\beta$-cell function under physiological and pathophysiological conditions. Nature communications, 8, 14295-14295.

Zulazmi, N.A., Gopalsamy, B., Omar Farouk, A.A., Sulaiman, M.R., Bharatham, B.H. \& Perimal, E.K. 2015. Antiallodynic and antihyperalgesic effects of zerumbone on a mouse model of chronic constriction injury-induced neuropathic pain. Fitoterapia, 105, 215-221.

Zysman-Colman, Z. \& Lands, L.C. 2016. Whole Body Plethysmography: Practical Considerations. Paediatric Respiratory Reviews, 19, 39--41. 
